\title{
Gravitational Radiation from Post-Newtonian Sources and Inspiralling Compact Binaries
}

\author{
Luc Blanchet \\ Institut d'Astrophysique de Paris \\ 98 ${ }^{\text {bis }}$ Boulevard Arago \\ 75014 Paris, France \\ email: blanchet@iap.fr \\ http://www2.iap.fr/users/blanchet
}

Accepted: 27 January 2014

Published: 13 February 2014

Revised: 16 February 2016

(Update of lrr-2006-4)

\begin{abstract}
To be observed and analyzed by the network of gravitational wave detectors on ground (LIGO, VIRGO, etc.) and by the future detectors in space (eLISA, etc.), inspiralling compact binaries - binary star systems composed of neutron stars and/or black holes in their late stage of evolution - require high-accuracy templates predicted by general relativity theory. The gravitational waves emitted by these very relativistic systems can be accurately modelled using a high-order post-Newtonian gravitational wave generation formalism. In this article, we present the current state of the art on post-Newtonian methods as applied to the dynamics and gravitational radiation of general matter sources (including the radiation reaction back onto the source) and inspiralling compact binaries. We describe the post-Newtonian equations of motion of compact binaries and the associated Lagrangian and Hamiltonian formalisms, paying attention to the self-field regularizations at work in the calculations. Several notions of innermost circular orbits are discussed. We estimate the accuracy of the post-Newtonian approximation and make a comparison with numerical computations of the gravitational selfforce for compact binaries in the small mass ratio limit. The gravitational waveform and energy flux are obtained to high post-Newtonian order and the binary's orbital phase evolution is deduced from an energy balance argument. Some landmark results are given in the case of eccentric compact binaries - moving on quasi-elliptical orbits with non-negligible eccentricity. The spins of the two black holes play an important role in the definition of the gravitational wave templates. We investigate their imprint on the equations of motion and gravitational wave phasing up to high post-Newtonian order (restricting to spin-orbit effects which are linear in spins), and analyze the post-Newtonian spin precession equations as well as the induced precession of the orbital plane.
\end{abstract}

Keywords: Gravitational radiation, Post-Newtonian approximations, Multipolar expansion, Inspiralling compact binary

(c) The Author(s). This article is distributed under a Creative Commons Attribution 4.0 International License. http://creativecommons.org/licenses/by/4.0/ 


\section{Imprint / Terms of Use}

Living Reviews in Relativity is a peer-reviewed open access journal published by the Springer International Publishing AG, Gewerbestrasse 11, 6330 Cham, Switzerland. ISSN 1433-8351.

This article is distributed under the terms of the Creative Commons Attribution 4.0 International License (http://creativecommons.org/licenses/by/4.0/), which permits unrestricted use, distribution, and reproduction in any medium, provided you give appropriate credit to the original author(s) and the source, provide a link to the Creative Commons license, and indicate if changes were made. Figures that have been previously published elsewhere may not be reproduced without consent of the original copyright holders.

Luc Blanchet,

"Gravitational Radiation from Post-Newtonian Sources and Inspiralling Compact Binaries",

Living Rev. Relativity, 17, (2014), 2.

DOI 10.12942/lrr-2014-2. 


\section{Article Revisions}

Living Reviews supports two ways of keeping its articles up-to-date:

Fast-track revision. A fast-track revision provides the author with the opportunity to add short notices of current research results, trends and developments, or important publications to the article. A fast-track revision is refereed by the responsible subject editor. If an article has undergone a fast-track revision, a summary of changes will be listed here.

Major update. A major update will include substantial changes and additions and is subject to full external refereeing. It is published with a new publication number.

For detailed documentation of an article's evolution, please refer to the history document of the article's online version at http://dx.doi.org/10.12942/lrr-2014-2.

13 February 2014: Major revision, updated and expanded. About 180 new references. Improved introduction with added Section 1.2 on quadrupole formalism; More extensive description of asymptotic waveform and relation between radiative and source moments (Section 3.3); More complete discussion on radiation reaction effects (5.2) and explicit radiation reaction potentials (5.4); New Section 7.5 on the PN metric in the near zone; New sections on conservative dynamics: stability of orbits (8.2), first law of binary dynamics (8.3), comparison with self-force computations (8.4); New Section 9.5 on spherical-harmonic modes of gravitational waves; New sections on eccentric binaries: structure of the motion (10.1), quasi-Keplerian representation (10.2), averaged fluxes (10.3); New sections on spin effects: Lagrangian formalism (11.1), equations of motion (11.2), spin-orbit terms in the phase (11.3).

15 January 2016: Minor update. Added 13 new references. More accurate quotations on spins. Minor improvements in wording. Added new PN subdominant gravitational-wave modes and SF comparisons.

16 February 2016: Minor update. Added 12 new references. Added a discussion on $4 \mathrm{PN}$ equations of motion. 


\section{Contents}

1 Introduction $\quad 7$

1.1 Analytic approximations and wave generation formalism . . . . . . . . . . . . 8

1.2 The quadrupole moment formalism . . . . . . . . . . . . . . . . . . . . . 12

1.3 Problem posed by compact binary systems . . . . . . . . . . . . . . . . . . 14

1.4 Post-Newtonian equations of motion . . . . . . . . . . . . . . . . . . . . . . . . . . . . . . . . . . . .

1.5 Post-Newtonian gravitational radiation . . . . . . . . . . . . . . . . . . 20

$\begin{array}{ll}\text { Part A: Post-Newtonian Sources } & 23\end{array}$

2 Non-linear Iteration of the Vacuum Field Equations 23

2.1 Einstein's field equations . . . . . . . . . . . . . . . . . . . . . 23

2.2 Linearized vacuum equations . . . . . . . . . . . . . . . . . . . . . . . . . . . . . . . . . . . . .

2.3 The multipolar post-Minkowskian solution . . . . . . . . . . . . . . . . . . . . . . . 28

2.4 Generality of the MPM solution . . . . . . . . . . . . . . . . . . . . . . . . . . . . . . . . . . . . . . .

2.5 Near-zone and far-zone structures . . . . . . . . . . . . . . . . . . . . . 33

3 Asymptotic Gravitational Waveform $\quad 35$

3.1 The radiative multipole moments . . . . . . . . . . . . . . . . . . . . 35

3.2 Gravitational-wave tails and tails-of-tails . . . . . . . . . . . . . . . . . . . . . . . . . . . . . . . . . .

3.3 Radiative versus source moments . . . . . . . . . . . . . . . . . . . . 42

4 Matching to a Post-Newtonian Source $\quad 46$

4.1 The matching equation . . . . . . . . . . . . . . . . . . . . 46

4.2 General expression of the multipole expansion . . . . . . . . . . . . . . . . . . . . . . . . . . . . . . . . . .

4.3 Equivalence with the Will-Wiseman formalism . . . . . . . . . . . . . . . . 50

4.4 The source multipole moments . . . . . . . . . . . . . . . . . . 51

5 Interior Field of a Post-Newtonian Source $\quad \mathbf{5 3}$

5.1 Post-Newtonian iteration in the near zone . . . . . . . . . . . . . . . . 55

5.2 Post-Newtonian metric and radiation reaction effects . . . . . . . . . . . . . . . 57

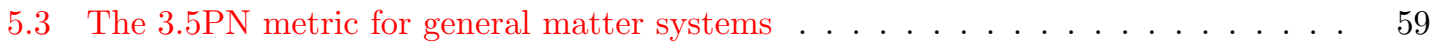

5.4 Radiation reaction potentials to $4 \mathrm{PN}$ order . . . . . . . . . . . . . 62

Part B: Compact Binary Systems $\quad 65$

6 Regularization of the Field of Point Particles $\quad 65$

6.1 Hadamard self-field regularization . . . . . . . . . . . . . . . . . . 66

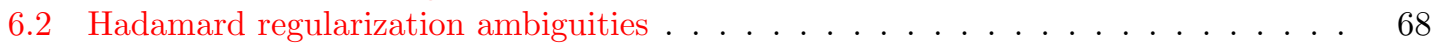

6.3 Dimensional regularization of the equations of motion . . . . . . . . . . . . . . . 72

6.4 Dimensional regularization of the radiation field . . . . . . . . . . . . . . 76

7 Newtonian-like Equations of Motion $\quad \mathbf{7 8}$

7.1 The 3PN acceleration and energy for particles . . . . . . . . . . . . . . . . . 78

7.2 Lagrangian and Hamiltonian formulations . . . . . . . . . . . . . . . . . . . 84

7.3 Equations of motion in the center-of-mass frame . . . . . . . . . . . . . . . . 90

7.4 Equations of motion and energy for quasi-circular orbits . . . . . . . . . . . . . . . 94

7.5 The $2.5 \mathrm{PN}$ metric in the near zone . . . . . . . . . . . . . . . . . . . 97 
8 Conservative Dynamics of Compact Binaries $\quad 101$

8.1 Concept of innermost circular orbit . . . . . . . . . . . . . . . . . . . . 101

8.2 Dynamical stability of circular orbits . . . . . . . . . . . . . . . . . . . 103

8.3 The first law of binary point-particle mechanics . . . . . . . . . . . . . . 108

8.4 Post-Newtonian approximation versus gravitational self-force . . . . . . . . . . . 112

9 Gravitational Waves from Compact Binaries 116

9.1 The binary's multipole moments . . . . . . . . . . . . . . . . . . . . . 117

9.2 Gravitational wave energy flux . . . . . . . . . . . . . . . . . . . . 120

9.3 Orbital phase evolution . . . . . . . . . . . . . . . . . . . . . . 123

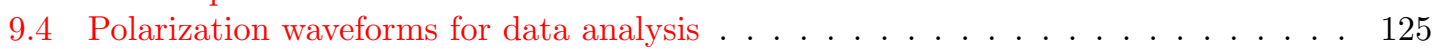

9.5 Spherical harmonic modes for numerical relativity . . . . . . . . . . . . . . 131

10 Eccentric Compact Binaries $\quad 135$

10.1 Doubly periodic structure of the motion of eccentric binaries . . . . . . . . . 135

10.2 Quasi-Keplerian representation of the motion . . . . . . . . . . . . . . . . . . 137

10.3 Averaged energy and angular momentum fluxes . . . . . . . . . . . . . . . . . . 140

11 Spinning Compact Binaries $\quad 146$

11.1 Lagrangian formalism for spinning point particles . . . . . . . . . . . . . . . . . . 148

11.2 Equations of motion and precession for spin-orbit effects . . . . . . . . . . . . . . 152

11.3 Spin-orbit effects in the gravitational wave flux and orbital phase . . . . . . . . . 157

$\begin{array}{lr}\text { References } & 161\end{array}$

\section{List of Tables}

1 Numerically determined value of the 3PN coefficient for the SF part of the redshift observable defined by Eq. (292) . . . . . . . . . . . . . . . . . . . . .

2 Numerically determined values of higher-order PN coefficients for the SF part of the redshift observable defined by Eq. (292) . . . . . . . . . . . . . . . . . .

3 Post-Newtonian contributions to the accumulated number of gravitational-wave cycles $\mathcal{N}_{\text {cycle }}$ for compact binaries detectable in the bandwidth of LIGO-VIRGO de-

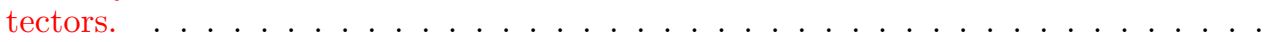

4 Spin-orbit contributions to the number of gravitational-wave cycles $\mathcal{N}_{\text {cycle }}$ for binaries detectable by ground-based detectors LIGO-VIRGO . . . . . . . . . . . . . . . 159 



\section{Introduction}

The theory of gravitational radiation from isolated sources, in the context of general relativity, is a fascinating science that can be explored by means of what was referred to in the XVIIIth century France as l'analyse sublime: The analytical (i.e., mathematical) method, and more specifically the resolution of partial differential equations. Indeed, the field equations of general relativity, when use is made of the harmonic-coordinate conditions, take the form of a quasi-linear hyperbolic differential system of equations, involving the famous wave operator or d'Alembertian [140]. The resolution of that system of equations constitutes a problème bien posé in the sense of Hadamard [236, 104], and which is amenable to an analytic solution using approximation methods.

Nowadays, the importance of the field lies in the exciting comparison of the theory with contemporary astrophysical observations, of binary pulsars like the historical Hulse-Taylor pulsar PSR 1913+16 [250], and, in a forthcoming future, of gravitational waves produced by massive and rapidly evolving systems such as inspiralling compact binaries. These should be routinely detected on Earth by the network of large-scale laser interferometers, including the advanced versions of the ground-based interferometers LIGO and VIRGO, with also GEO and the future cryogenic detector KAGRA. The first direct detection of a coalescence of two black holes has been achieved on September 14, 2015 by the advanced LIGO detector [1]. Further ahead, the space-based laser interferometer LISA (actually, the evolved version $e$ LISA) should be able to detect supermassive black-hole binaries at cosmological distances.

To prepare these experiments, the required theoretical work consists of carrying out a sufficiently general solution of the Einstein field equations, valid for a large class of matter systems, and describing the physical processes of the emission and propagation of the gravitational waves from the source to the distant detector, as well as their back-reaction onto the source. The solution should then be applied to specific sources like inspiralling compact binaries.

For general sources it is hopeless to solve the problem via a rigorous deduction within the exact theory of general relativity, and we have to resort to approximation methods. Of course the ultimate aim of approximation methods is to extract from the theory some firm predictions to be compared with the outcome of experiments. However, we have to keep in mind that such methods are often missing a clear theoretical framework and are sometimes not related in a very precise mathematical way to the first principles of the theory.

The flagship of approximation methods is the post-Newtonian approximation, which has been developed from the early days of general relativity [303]. This approximation is at the origin of many of the great successes of general relativity, and it gives wonderful answers to the problems of motion and gravitational radiation of systems of compact objects. Three crucial applications are:

1. The motion of $N$ point-like objects at the first post-Newtonian approximation level [184], is taken into account to describe the solar system dynamics (motion of the centers of mass of planets);

2. The gravitational radiation-reaction force, which appears in the equations of motion at the second-and-a-half post-Newtonian (2.5PN) order [148, 147, 143, 142], has been experimentally verified by the observation of the secular acceleration of the orbital motion of the Hulse-Taylor binary pulsar PSR 1913+16 [399, 400, 398];

3. The analysis of gravitational waves emitted by inspiralling compact binaries - two neutron stars or black holes driven into coalescence by emission of gravitational radiation - necessitates the prior knowledge of the equations of motion and radiation field up to very high post-Newtonian order.

This article reviews the current status of the post-Newtonian approach to the problems of the motion of inspiralling compact binaries and their emission of gravitational waves. When the 
two compact objects approach each other toward merger, the post-Newtonian expansion will lose accuracy and should be taken over by numerical-relativity computations [359, 116, 21]. We shall refer to other review articles like Refs. [233, 187] for discussions of numerical-relativity methods. Despite very intensive developments of numerical relativity, the post-Newtonian approximation remains indispensable for describing the inspiral phase of compact binaries to high accuracy, and for providing a powerful benchmark against which the numerical computations are tested.

Part A of the article deals with general post-Newtonian matter sources. The exterior field of the source is investigated by means of a combination of analytic post-Minkowskian and multipolar approximations. The physical observables in the far-zone of the source are described by a specific set of radiative multipole moments. By matching the exterior solution to the metric of the postNewtonian source in the near-zone the explicit expressions of the source multipole moments are obtained. The relationships between the radiative and source moments involve many non-linear multipole interactions, among them those associated with the tails (and tails-of-tails, etc.) of gravitational waves.

Part B is devoted to the application to compact binary systems, with particular emphasis on black hole binaries with spins. We present the equations of binary motion, and the associated Lagrangian and Hamiltonian, at the third post-Newtonian (3PN) order beyond the Newtonian acceleration. The gravitational-wave energy flux, taking consistently into account the relativistic corrections in the binary's moments as well as the various tail effects, is derived through 3.5PN order with respect to the quadrupole formalism. The binary's orbital phase, whose prior knowledge is crucial for searching and analyzing the signals from inspiralling compact binaries, is deduced from an energy balance argument (in the simple case of circular orbits).

All over the article we try to state the main results in a form that is simple enough to be understood without the full details; however, we also outline some of the proofs when they present some interest on their own. To emphasize the importance of some key results, we present them in the form of mathematical theorems. In applications we generally show the most up-to-date results up to the highest known post-Newtonian order. ${ }^{1}$

\subsection{Analytic approximations and wave generation formalism}

The basic problem we face is to relate the asymptotic gravitational-wave form $h_{i j}$ generated by some isolated source, at the location of a detector in the wave zone of the source, to the material content of the source, i.e., its stress-energy tensor $T^{\alpha \beta}$, using approximation methods in general relativity. $^{2}$ Therefore, a general wave-generation formalism must solve the field equations, and the non-linearity therein, by imposing some suitable approximation series in one or several small physical parameters. Some important approximations that we shall use in this article are the postNewtonian method (or non-linear 1/c-expansion), the post-Minkowskian method or non-linear iteration ( $G$-expansion), the multipole decomposition in irreducible representations of the rotation group (or equivalently $a$-expansion in the source radius), the far-zone expansion $(1 / R$-expansion in the distance to the source), and the perturbation in the small mass limit ( $\nu$-expansion in the mass ratio of a binary system). In particular, the post-Newtonian expansion has provided us in the past with our best insights into the problems of motion and radiation. The most successful wave-generation formalisms make a gourmet cocktail of these approximation methods. For reviews on analytic approximations and applications to the motion and the gravitational wave-generation

\footnotetext{
1 A few errata have been published in this intricate field; all formulas take into account the latest changes.

2 In this article Greek indices $\alpha \beta \ldots \mu \nu \ldots$ take space-time values $0,1,2,3$ and Latin indices $a b \ldots i j \ldots$ spatial values 1, 2, 3. Cartesian coordinates are assumed throughout and boldface notation is often used for ordinary Euclidean vectors. In Section 11 upper Latin letters $A B \ldots$ will refer to tetrad indices 0 , 1, 2, 3 with $a b \ldots$ the corresponding spatial values $1,2,3$. Our signature is +2 ; hence the Minkowski metric reads $\eta_{\alpha \beta}=$ $\operatorname{diag}(-1,+1,+1,+1)=\eta_{A B}$. As usual $G$ and $c$ are Newton's constant and the speed of light.
} 
see Refs. [404, 142, 144, 145, 405, 421, 46, 52, 378]. For reviews on black-hole pertubations and the self-force approach see Refs. [348, 373, 177, 23].

The post-Newtonian approximation is valid under the assumptions of a weak gravitational field inside the source (we shall see later how to model neutron stars and black holes), and of slow internal motions. ${ }^{3}$ The main problem with this approximation, is its domain of validity, which is limited to the near zone of the source - the region surrounding the source that is of small extent with respect to the wavelength of the gravitational waves. A serious consequence is the a priori inability of the post-Newtonian expansion to incorporate the boundary conditions at infinity, which determine the radiation reaction force in the source's local equations of motion.

The post-Minkowskian expansion, by contrast, is uniformly valid, as soon as the source is weakly self-gravitating, over all space-time. In a sense, the post-Minkowskian method is more fundamental than the post-Newtonian one; it can be regarded as an "upstream" approximation with respect to the post-Newtonian expansion, because each coefficient of the post-Minkowskian series can in turn be re-expanded in a post-Newtonian fashion. Therefore, a way to take into account the boundary conditions at infinity in the post-Newtonian series is to control first the post-Minkowskian expansion. Notice that the post-Minkowskian method is also upstream (in the previous sense) with respect to the multipole expansion, when considered outside the source, and with respect to the far-zone expansion, when considered far from the source.

The most "downstream" approximation that we shall use in this article is the post-Newtonian one; therefore this is the approximation that dictates the allowed physical properties of our matter source. We assume mainly that the source is at once slowly moving and weakly stressed, and we abbreviate this by saying that the source is post-Newtonian. For post-Newtonian sources, the parameter defined from the components of the matter stress-energy tensor $T^{\alpha \beta}$ and the source's Newtonian potential $U$ by

$$
\epsilon \equiv \max \left\{\left|\frac{T^{0 i}}{T^{00}}\right|,\left|\frac{T^{i j}}{T^{00}}\right|^{1 / 2},\left|\frac{U}{c^{2}}\right|^{1 / 2}\right\}
$$

is much less than one. This parameter represents essentially a slow motion estimate $\epsilon \sim v / c$, where $v$ denotes a typical internal velocity. By a slight abuse of notation, following Chandrasekhar et al. [122, 124, 123], we shall henceforth write formally $\epsilon \equiv 1 / c$, even though $\epsilon$ is dimensionless whereas $c$ has the dimension of a velocity. Thus, $1 / c \ll 1$ in the case of post-Newtonian sources. The small post-Newtonian remainders will be denoted $\mathcal{O}\left(1 / c^{n}\right)$. Furthermore, still following Refs. $[122,124,123]$, we shall refer to a small post-Newtonian term with formal order $\mathcal{O}\left(1 / c^{n}\right)$ relative to the Newtonian acceleration in the equations of motion, as $\frac{n}{2} \mathrm{PN}$.

We have $\left|U / c^{2}\right|^{1 / 2} \ll 1 / c$ for sources with negligible self-gravity, and whose dynamics are therefore driven by non-gravitational forces. However, we shall generally assume that the source is self-gravitating; in that case we see that it is necessarily weakly (but not negligibly) self-gravitating, i.e., $\left|U / c^{2}\right|^{1 / 2}=\mathcal{O}(1 / c) .{ }^{4}$ Note that the adjective "slow-motion" is a bit clumsy because we shall in fact consider very relativistic sources such as inspiralling compact binaries, for which $v / c$ can be as large as $50 \%$ in the last rotations, and whose description necessitates the control of high post-Newtonian approximations.

At the lowest-order in the Newtonian limit $1 / c \rightarrow 0$, the gravitational waveform of a postNewtonian matter source is generated by the time variations of the quadrupole moment of the source. We shall review in Section 1.2 the utterly important "Newtonian" quadrupole moment formalism [183, 285]. Taking into account higher post-Newtonian corrections in a wave generation

\footnotetext{
3 Establishing the post-Newtonian expansion rigorously has been the subject of numerous mathematically oriented works, see e.g., [361, 362, 363].

4 Note that for very eccentric binaries (with say $e \rightarrow 1^{-}$) the Newtonian potential $U$ can be numerically much larger than the estimate $\mathcal{O}\left(1 / c^{2}\right) \sim v^{2} / c^{2}$ at the apastron of the orbit.
} 
formalism will mean including into the waveform the contributions of higher multipole moments, beyond the quadrupole. Post-Newtonian corrections of order $\mathcal{O}\left(1 / c^{n}\right)$ beyond the quadrupole formalism will still be denoted as $\frac{n}{2} \mathrm{PN}$. Building a post-Newtonian wave generation formalism must be concomitant to understanding the multipole expansion in general relativity.

The multipole expansion is one of the most useful tools of physics, but its use in general relativity is difficult because of the non-linearity of the theory and the tensorial character of the gravitational interaction. In the stationary case, the multipole moments are determined by the expansion of the metric at spatial infinity [219, 238, 384], while, in the case of non-stationary fields, the moments, starting with the quadrupole, are defined at future null infinity. The multipole moments have been extensively studied in the linearized theory, which ignores the gravitational forces inside the source. Early studies have extended the Einstein quadrupole formula [given by Eq. (4) below] to include the current-quadrupole and mass-octupole moments [332, 333], and obtained the corresponding formulas for linear momentum [332, 333, 30, 358] and angular momentum [339, 134]. The general structure of the infinite multipole series in the linearized theory was investigated by several works [369, 367, 343, 403], from which it emerged that the expansion is characterized by two and only two sets of moments: Mass-type and current-type moments. Below we shall use a particular multipole decomposition of the linearized (vacuum) metric, parametrized by symmetric and trace-free (STF) mass and current moments, as given by Thorne [403]. The expressions of the multipole moments as integrals over the source, valid in the linearized theory but irrespective of a slow motion hypothesis, have been worked out in [309, 119, 118, 154]. In particular, Damour \& Iyer [154] obtained the complete closed-form expressions for the time-dependent mass and spin multipole moments (in STF guise) of linearized gravity.

In the full non-linear theory, the (radiative) multipole moments can be read off the coefficient of $1 / R$ in the expansion of the metric when $R \rightarrow+\infty$, with a null coordinate $T-R / c=$ const. The solutions of the field equations in the form of a far-field expansion (power series in $1 / R$ ) have been constructed, and their properties elucidated, by Bondi et al. [93] and Sachs [368]. The precise way under which such radiative space-times fall off asymptotically has been formulated geometrically by Penrose [337, 338] in the concept of an asymptotically simple space-time (see also Ref. [220]). The resulting Bondi-Sachs-Penrose approach is very powerful, but it can answer a priori only a part of the problem, because it gives information on the field only in the limit where $R \rightarrow+\infty$, which cannot be connected in a direct way to the actual matter content and dynamics of the source. In particular the multipole moments that one considers in this approach are those measured at infinity - we call them the radiative multipole moments. These moments are distinct, because of non-linearities, from some more natural source multipole moments, which are defined operationally by means of explicit integrals extending over the matter and gravitational fields.

An alternative way of defining the multipole expansion within the complete non-linear theory is that of Blanchet \& Damour [57, 41], following pioneering works by Bonnor and collaborators [94, 95, 96, 251] and Thorne [403]. In this approach the basic multipole moments are the source moments, rather than the radiative ones. In a first stage, the moments are left unspecified, as being some arbitrary functions of time, supposed to describe an actual physical source. They are iterated by means of a post-Minkowskian expansion of the vacuum field equations (valid in the source's exterior). Technically, the post-Minkowskian approximation scheme is greatly simplified by the assumption of a multipolar expansion, as one can consider separately the iteration of the different multipole pieces composing the exterior field. 5 In this "multipolar-post-Minkowskian" (MPM) formalism, which is physically valid over the entire weak-field region outside the source, and in particular in the wave zone (up to future null infinity), the radiative multipole moments are obtained in the form of some non-linear functionals of the more basic source moments. A priori, the method is not limited to post-Newtonian sources; however, we shall see that, in the current

\footnotetext{
5 Whereas, the direct attack of the post-Minkowskian expansion, valid at once inside and outside the source, faces some calculational difficulties [408, 136].
} 
situation, the closed-form expressions of the source multipole moments can be established only in the case where the source is post-Newtonian [44, 49]. The reason is that in this case the domain of validity of the post-Newtonian iteration (viz. the near zone) overlaps the exterior weak-field region, so that there exists an intermediate zone in which the post-Newtonian and multipolar expansions can be matched together. This is a standard application of the method of matched asymptotic expansions in general relativity [114, 113, 7, 357].

To be more precise, we shall show how a systematic multipolar and post-Minkowskian iteration scheme for the vacuum Einstein field equations yields the most general physically admissible solution of these equations [57]. The solution is specified once we give two and only two sets of time-varying (source) multipole moments. Some general theorems about the near-zone and farzone expansions of that general solution will be stated. Notably, we show [41] that the asymptotic behaviour of the solution at future null infinity is in agreement with the findings of the BondiSachs-Penrose [93, 368, 337, 338, 220] approach to gravitational radiation. However, checking that the asymptotic structure of the radiative field is correct is not sufficient by itself, because the ultimate aim, as we said, is to relate the far field to the properties of the source, and we are now obliged to ask: What are the multipole moments corresponding to a given stress-energy tensor $T^{\alpha \beta}$ describing the source? The general expression of the moments was obtained at the level of the second post-Newtonian (2PN) order in Ref. [44], and was subsequently proved to be in fact valid up to any post-Newtonian order in Ref. [49]. The source moments are given by some integrals extending over the post-Newtonian expansion of the total (pseudo) stress-energy tensor $\tau^{\alpha \beta}$, which is made of a matter part described by $T^{\alpha \beta}$ and a crucial non-linear gravitational source term $\Lambda^{\alpha \beta}$. These moments carry in front a particular operation of taking the finite part $(\mathcal{F} \mathcal{P}$ as we call it below), which makes them mathematically well-defined despite the fact that the gravitational part $\Lambda^{\alpha \beta}$ has a spatially infinite support, which would have made the bound of the integral at spatial infinity singular (of course the finite part is not added a posteriori to restore the well-definiteness of the integral, but is proved to be actually present in this formalism). The expressions of the moments had been obtained earlier at the 1PN level, albeit in different forms, in Ref. [59] for the mass-type moments [see Eq. (157a) below], and in Ref. [155] for the current-type ones.

The wave-generation formalism resulting from matching the exterior multipolar and postMinkowskian field [57, 41] to the post-Newtonian source [44, 49] is able to take into account, in principle, any post-Newtonian correction to both the source and radiative multipole moments (for any multipolarity of the moments). The relationships between the radiative and source moments include many non-linear multipole interactions, because the source moments mix with each other as they "propagate" from the source to the detector. Such multipole interactions include the famous effects of wave tails, corresponding to the coupling between the non-static moments with the total mass $\mathrm{M}$ of the source. The non-linear multipole interactions have been computed within the present wave-generation formalism up to the 3.5PN order in Refs. [60, 50, 48, 74, 197]. Furthermore, the back-reaction of the gravitational-wave emission onto the source, up to the $1.5 \mathrm{PN}$ order relative to the leading order of radiation reaction, has also been studied within this formalism $[58,43,47]$. Now, recall that the leading radiation reaction force, which is quadrupolar, occurs already at the $2.5 \mathrm{PN}$ order in the source's equations of motion. Therefore the $1.5 \mathrm{PN}$ "relative" order in the radiation reaction corresponds in fact to the $4 \mathrm{PN}$ order in the equations of motion, beyond the Newtonian acceleration. It has been shown that the gravitational-wave tails enter the radiation reaction at precisely the $1.5 \mathrm{PN}$ relative order, i.e., 4PN absolute order [58]. A systematic post-Newtonian iteration scheme for the near-zone field, formally taking into account all radiation reaction effects, has been obtained, fully consistent with the present formalism [357, 75].

A different wave-generation formalism has been devised by Will \& Wiseman [424] (see also Refs. [422, 335, 336]), after earlier attempts by Epstein \& Wagoner [185] and Thorne [403]. This formalism has exactly the same scope as the one of Refs. [57, 41, 44, 49], i.e., it applies to any isolated post-Newtonian sources, but it differs in the definition of the source multipole moments 
and in many technical details when properly implemented [424]. In both formalisms, the moments are generated by the post-Newtonian expansion of the pseudo-tensor $\tau^{\alpha \beta}$, but in the Will-Wiseman formalism they are defined by some compact-support integrals terminating at some finite radius enclosing the source, e.g., the radius $\mathcal{R}$ of the near zone. By contrast, in Refs. [44, 49], the moments are given by some integrals covering the whole space $\left(\mathbb{R}^{3}\right)$ and regularized by means of the finite part $\mathcal{F P}$. Nevertheless, in both formalisms the source multipole moments, which involve a whole series of relativistic corrections, must be coupled together in a complicated way in the true nonlinear solution; such non-linear couplings form an integral part of the radiative moments at infinity and thereby of the observed signal. We shall prove in Section 4.3 the complete equivalence, at the most general level, between the two formalisms.

\subsection{The quadrupole moment formalism}

The lowest-order wave generation formalism is the famous quadrupole formalism of Einstein [183] and Landau \& Lifshitz [285]. This formalism applies to a general isolated matter source which is post-Newtonian in the sense of existence of the small post-Newtonian parameter $\epsilon$ defined by Eq. (1). However, the quadrupole formalism is valid in the Newtonian limit $\epsilon \rightarrow 0$; it can rightly be qualified as "Newtonian" because the quadrupole moment of the matter source is Newtonian and its evolution obeys Newton's laws of gravity. In this formalism the gravitational field $h_{i j}^{\mathrm{TT}}$ is expressed in a transverse and traceless (TT) coordinate system covering the far zone of the source at retarded times, ${ }^{6}$ as

$$
h_{i j}^{\mathrm{TT}}=\frac{2 G}{c^{4} R} \mathcal{P}_{i j a b}(\boldsymbol{N})\left\{\frac{\mathrm{d}^{2} \mathrm{Q}_{a b}}{\mathrm{~d} T^{2}}(T-R / c)+\mathcal{O}\left(\frac{1}{c}\right)\right\}+\mathcal{O}\left(\frac{1}{R^{2}}\right),
$$

where $R=|\boldsymbol{X}|$ is the distance to the source, $T-R / c$ is the retarded time, $\boldsymbol{N}=\boldsymbol{X} / R$ is the unit direction from the source to the far away observer, and $\mathcal{P}_{i j a b}=\mathcal{P}_{i a} \mathcal{P}_{j b}-\frac{1}{2} \mathcal{P}_{i j} \mathcal{P}_{a b}$ is the TT projection operator, with $\mathcal{P}_{i j}=\delta_{i j}-N_{i} N_{j}$ being the projector onto the plane orthogonal to $\boldsymbol{N}$. The source's quadrupole moment takes the familiar Newtonian form

$$
\mathrm{Q}_{i j}(t)=\int_{\text {source }} \mathrm{d}^{3} \mathbf{x} \rho(\mathbf{x}, t)\left(x_{i} x_{j}-\frac{1}{3} \delta_{i j} \mathbf{x}^{2}\right),
$$

where $\rho$ is the Newtonian mass density. The total gravitational power emitted by the source in all directions around the source is given by the Einstein quadrupole formula

$$
\mathcal{F} \equiv\left(\frac{\mathrm{d} E}{\mathrm{~d} T}\right)^{\mathrm{GW}}=\frac{G}{c^{5}}\left\{\frac{1}{5} \frac{\mathrm{d}^{3} \mathrm{Q}_{a b}}{\mathrm{~d} T^{3}} \frac{\mathrm{d}^{3} \mathrm{Q}_{a b}}{\mathrm{~d} T^{3}}+\mathcal{O}\left(\frac{1}{c^{2}}\right)\right\} .
$$

Our notation $\mathcal{F}$ stands for the total gravitational energy flux or gravitational "luminosity" of the source. Similarly, the total angular momentum flux is given by

$$
\mathcal{G}_{i} \equiv\left(\frac{\mathrm{dJ}_{i}}{\mathrm{~d} T}\right)^{\mathrm{GW}}=\frac{G}{c^{5}}\left\{\frac{2}{5} \epsilon_{i a b} \frac{\mathrm{d}^{2} \mathrm{Q}_{a c}}{\mathrm{~d} T^{2}} \frac{\mathrm{d}^{3} \mathrm{Q}_{b c}}{\mathrm{~d} T^{3}}+\mathcal{O}\left(\frac{1}{c^{2}}\right)\right\},
$$

where $\epsilon_{a b c}$ denotes the standard Levi-Civita symbol with $\epsilon_{123}=1$.

Associated with the latter energy and angular momentum fluxes, there is also a quadrupole formula for the radiation reaction force, which reacts on the source's dynamics in consequence of the emission of waves. This force will inflect the time evolution of the orbital phase of the binary

6 The TT coordinate system can be extended to the near zone of the source as well; see for instance Ref. [282]. 
pulsar and inspiralling compact binaries. At the position $(\mathbf{x}, t)$ in a particular coordinate system covering the source, the reaction force density can be written as [114, 113, 319]

$$
F_{i}^{\text {reac }}=\frac{G}{c^{5}} \rho\left\{-\frac{2}{5} x^{a} \frac{\mathrm{d}^{5} \mathrm{Q}_{i a}}{\mathrm{~d} t^{5}}+\mathcal{O}\left(\frac{1}{c^{2}}\right)\right\} .
$$

This is the gravitational analogue of the damping force of electromagnetism. However, notice that gravitational radiation reaction is inherently gauge-dependent, so the expression of the force depends on the coordinate system which is used. Consider now the energy and angular momentum of a matter system made of some perfect fluid, say

$$
\begin{aligned}
E & =\int \mathrm{d}^{3} \mathbf{x} \rho\left[\frac{\mathbf{v}^{2}}{2}+\Pi-\frac{U}{2}\right]+\mathcal{O}\left(\frac{1}{c^{2}}\right), \\
\mathrm{J}_{i} & =\int \mathrm{d}^{3} \mathbf{x} \rho \epsilon_{i a b} x_{a} v_{b}+\mathcal{O}\left(\frac{1}{c^{2}}\right) .
\end{aligned}
$$

The specific internal energy of the fluid is denoted $\Pi$, and obeys the usual thermodynamic relation $\mathrm{d} \Pi=-P \mathrm{~d}(1 / \rho)$ where $P$ is the pressure; the gravitational potential obeys the Poisson equation $\Delta U=-4 \pi G \rho$. We compute the mechanical losses of energy and angular momentum from the time derivatives of $E$ and $\mathrm{J}_{i}$. We employ the usual Eulerian equation of motion $\rho \mathrm{d} v^{i} / \mathrm{d} t=-\partial_{i} P+$ $\rho \partial_{i} U+F_{i}^{\text {reac }}$ and continuity equation $\partial_{t} \rho+\partial_{i}\left(\rho v^{i}\right)=0$. Note that we add the small dissipative radiation-reaction contribution $F_{i}^{\text {reac }}$ in the equation of motion but neglect all conservative postNewtonian corrections. The result is

$$
\begin{aligned}
\frac{\mathrm{d} E}{\mathrm{~d} t} & =\int \mathrm{d}^{3} \mathbf{x} v^{i} F_{i}^{\mathrm{reac}}=-\mathcal{F}+\frac{\mathrm{d} f}{\mathrm{~d} t}, \\
\frac{\mathrm{d} \mathrm{J}_{i}}{\mathrm{~d} t} & =\int \mathrm{d}^{3} \mathbf{x} \epsilon_{i a b} x_{a} F_{b}^{\mathrm{reac}}=-\mathcal{G}_{i}+\frac{\mathrm{d} g_{i}}{\mathrm{~d} t},
\end{aligned}
$$

where one recognizes the fluxes at infinity given by Eqs. (4) and (5), and where the second terms denote some total time derivatives made of quadratic products of derivatives of the quadrupole moment. Looking only for secular effects, we apply an average over time on a typical period of variation of the system; the latter time derivatives will be in average numerically small in the case of quasi-periodic motion (see e.g., [103] for a discussion). Hence we obtain

$$
\begin{aligned}
& \left\langle\frac{\mathrm{d} E}{\mathrm{~d} t}\right\rangle=-\langle\mathcal{F}\rangle, \\
& \left\langle\frac{\mathrm{dJ}_{i}}{\mathrm{~d} t}\right\rangle=-\left\langle\mathcal{G}_{i}\right\rangle,
\end{aligned}
$$

where the brackets denote the time averaging over an orbit. These balance equations encode the secular decreases of energy and angular momentum by gravitational radiation emission.

The cardinal virtues of the Einstein-Landau-Lifshitz quadrupole formalism are: Its generality the only restrictions are that the source be Newtonian and bounded; its simplicity, as it necessitates only the computation of the time derivatives of the Newtonian quadrupole moment (using the Newtonian laws of motion); and, most importantly, its agreement with the observation of the dynamics of the binary pulsar PSR 1913+16 [399, 400, 398]. Indeed let us apply the balance equations (9) to a system of two point masses moving on an eccentric orbit modelling the binary pulsar PSR $1913+16$ - the classic references are [340, 339]; see also [186, 415]. We use the binary's Newtonian energy and angular momentum,

$$
E=-\frac{G m_{1} m_{2}}{2 a}
$$




$$
\mathrm{J}=m_{1} m_{2} \sqrt{\frac{G a\left(1-e^{2}\right)}{m_{1}+m_{2}}}
$$

where $a$ and $e$ are the semi-major axis and eccentricity of the orbit and $m_{1}$ and $m_{2}$ are the two masses. From the energy balance equation (9a) we obtain first the secular evolution of $a$; next changing from $a$ to the orbital period $P$ using Kepler's third law ${ }^{7}$ we get the secular evolution of the orbital period $P$ as

$$
\left\langle\frac{\mathrm{d} P}{\mathrm{~d} t}\right\rangle=-\frac{192 \pi}{5 c^{5}}\left(\frac{2 \pi G}{P}\right)^{5 / 3} \frac{m_{1} m_{2}}{\left(m_{1}+m_{2}\right)^{1 / 3}} \frac{1+\frac{73}{24} e^{2}+\frac{37}{96} e^{4}}{\left(1-e^{2}\right)^{7 / 2}} .
$$

The last factor, depending on the eccentricity, comes out from the orbital average and is known as the Peters \& Mathews [340] "enhancement" factor, so designated because in the case of the binary pulsar, which has a rather large eccentricity $e \simeq 0.617$, it enhances the effect by a factor $\sim 12$. Numerically, one finds $\langle\mathrm{d} P / \mathrm{d} t\rangle=-2.4 \times 10^{-12}$, a dimensionless number in excellent agreement with the observations of the binary pulsar [399, 400, 398]. On the other hand the secular evolution of the eccentricity $e$ is deduced from the angular momentum balance equation (9b) [together with the previous result (11)], as

$$
\left\langle\frac{\mathrm{d} e}{\mathrm{~d} t}\right\rangle=-\frac{608 \pi}{15 c^{5}} \frac{e}{P}\left(\frac{2 \pi G}{P}\right)^{5 / 3} \frac{m_{1} m_{2}}{\left(m_{1}+m_{2}\right)^{1 / 3}} \frac{1+\frac{121}{304} e^{2}}{\left(1-e^{2}\right)^{5 / 2}} .
$$

Interestingly, the system of equations (11) -(12) can be thoroughly integrated in closed analytic form. This yields the evolution of the eccentricity [339]:

$$
\frac{e^{2}}{\left(1-e^{2}\right)^{19 / 6}}\left(1+\frac{121}{304} e^{2}\right)^{145 / 121}=c_{0} P^{19 / 9}
$$

where $c_{0}$ denotes an integration constant to be determined by the initial conditions at the start of the binary evolution. When $e \ll 1$ the latter relation gives approximately $e^{2} \simeq c_{0} P^{19 / 9}$.

For a long while, it was thought that the various quadrupole formulas would be sufficient for sources of gravitational radiation to be observed directly on Earth - as they had proved to be amply sufficient in the case of the binary pulsar. However, further works [139] ${ }^{8}$ and $[87,138]$ showed that this is not true, as one has to include post-Newtonian corrections to the quadrupole formalism in order to prepare for the data analysis of future detectors, in the case of inspiralling compact binaries. From the beautiful test of the orbital decay (11) of the binary pulsar, we can say that the post-Newtonian corrections to the "Newtonian" quadrupole formalism - which we shall compute in this article - have already received a strong observational support.

\subsection{Problem posed by compact binary systems}

Inspiralling compact binaries, containing neutron stars and/or black holes, are likely to become the bread-and-butter sources of gravitational waves for the detectors LIGO, VIRGO, GEO and KAGRA on ground, and also eLISA in space. The two compact objects steadily lose their orbital binding energy by emission of gravitational radiation; as a result, the orbital separation between them decreases, and the orbital frequency increases. Thus, the frequency of the gravitational-wave

7 Namely $(G m)^{1}=\Omega^{2} a^{3}$, where $m=m_{1}+m_{2}$ is the total mass and $\Omega=2 \pi / P$ is the orbital frequency. This law is also appropriately called the 1-2-3 law [319].

8 This work entitled: "The last three minutes: Issues in gravitational-wave measurements of coalescing compact binaries" is sometimes coined the "3mn Caltech paper". 
signal, which equals twice the orbital frequency for the dominant harmonics, "chirps" in time (i.e., the signal becomes higher and higher pitched) until the two objects collide and merge.

The orbit of most inspiralling compact binaries can be considered to be circular, apart from the gradual inspiral, because the gravitational radiation reaction forces tend to circularize the motion rapidly. This effect is due to the emission of angular momentum by gravitational waves, resulting in a secular decrease of the eccentricity of the orbit, which has been computed within the quadrupole formalism in Eq. (12). For instance, suppose that the inspiralling compact binary was long ago (a few hundred million years ago) a system similar to the binary pulsar system, with an orbital frequency $\Omega_{0} \equiv 2 \pi / P_{0} \sim 10^{-4} \mathrm{rad} / \mathrm{s}$ and a rather large orbital eccentricity $e_{0} \sim 0.6$. When it becomes visible by the detectors on ground, i.e., when the gravitational wave signal frequency reaches about $f \equiv \Omega / \pi \sim 10 \mathrm{~Hz}$, the eccentricity of the orbit should be $e \sim 10^{-6}$ according to the formula (13). This is a very small eccentricity, even when compared to high-order relativistic corrections. Only non-isolated binary systems could have a non negligible eccentricity. For instance, the Kozai mechanism [283, 300] is one important scenario that produces eccentric binaries and involves the interaction between a pair of binaries in the dense cores of globular clusters [315]. If the mutual inclination angle of the inner binary is strongly tilted with respect to the outer compact star, then a resonance occurs and can increase the eccentricity of the inner binary to large values. This is one motivation for looking at the waves emitted by inspiralling binaries in non-circular, quasi-elliptical orbits (see Section 10).

The main point about modelling the inspiralling compact binary is that a model made of two structureless point particles, characterized solely by two mass parameters $m_{\mathrm{a}}$ and possibly two spins $S_{\mathrm{a}}$ (with a $=1,2$ labelling the particles), is sufficient in first approximation. Indeed, most of the non-gravitational effects usually plaguing the dynamics of binary star systems, such as the effects of a magnetic field, of an interstellar medium, of the internal structure of extended bodies, are dominated by gravitational effects. The main justification for a model of point particles is that the effects due to the finite size of the compact bodies are small. Consider for instance the influence of the Newtonian quadrupole moments $\mathrm{Q}_{\mathrm{a}}$ induced by tidal interaction between two neutron stars. Let $a_{\mathrm{a}}$ be the radius of the stars, and $r_{12}$ be the distance between the two centers of mass. We have, for tidal moments,

$$
\mathrm{Q}_{1}=k_{1} \frac{m_{2} a_{1}^{5}}{r_{12}^{3}} \text { and } \mathrm{Q}_{2}=k_{2} \frac{m_{1} a_{2}^{5}}{r_{12}^{3}},
$$

where $k_{\mathrm{a}}$ are the star's dimensionless (second) Love numbers [321], which depend on their internal structure, and are, typically, of the order unity. On the other hand, for compact objects, we can introduce their "compactness" parameters, defined by the dimensionless ratios

$$
K_{\mathrm{a}} \equiv \frac{G m_{\mathrm{a}}}{a_{\mathrm{a}} c^{2}}
$$

and equal $\sim 0.2$ for neutron stars (depending on their equation of state). The quadrupoles $\mathrm{Q}_{\mathrm{a}}$ will affect the Newtonian binding energy $E$ of the two bodies, and also the emitted total gravitational flux $\mathcal{F}$ as computed using the Newtonian quadrupole formula (4). It is known that for inspiralling compact binaries the neutron stars are not co-rotating because the tidal synchronization time is much larger than the time left till the coalescence. As shown by Kochanek [276] the best models for the fluid motion inside the two neutron stars are the so-called Roche-Riemann ellipsoids, which have tidally locked figures (the quadrupole moments face each other at any instant during the inspiral), but for which the fluid motion has zero circulation in the inertial frame. In the Newtonian approximation, using the energy balance equation (9a), we find that within such a model (in the case of two identical neutron stars with same mass $m$, compactness $K$ and Love number $k$ ), the orbital phase reads

$$
\phi^{\text {finite size }}-\phi_{0}=-\frac{1}{8 x^{5 / 2}}\left\{1+\operatorname{const} k\left(\frac{x}{K}\right)^{5}\right\},
$$


where "const" denotes a numerical coefficient of order one, $\phi_{0}$ is some initial phase, and $x \equiv$ $\left(G m \Omega / c^{3}\right)^{2 / 3}$ is a standard dimensionless post-Newtonian parameter of the order of $\sim 1 / c^{2}$ (with $\Omega=2 \pi / P$ the orbital frequency). The first term in the right-hand side of Eq. (16) corresponds to the gravitational-wave damping of two point masses without internal structure; the second term is the finite-size effect, which appears as a relative correction, proportional to $(x / K)^{5}$, to the latter radiation damping effect. Because the finite-size effect is purely Newtonian, its relative correction $\sim(x / K)^{5}$ should not depend on the speed of light $c$; and indeed the factors $1 / c^{2}$ cancel out in the ratio $x / K$. However, the compactness $K$ of neutron stars is of the order of 0.2 say, and by definition of compact objects we can consider that $K$ is formally of the order of unity or one half; therefore the factor $1 / c^{2}$ it contains in (15) should not be taken into account when estimating numerically the effect. So the real order of magnitude of the relative contribution of the finite-size effect in Eq. (16) is given by the factor $x^{5}$ alone. This means that for compact objects the finite-size effect should roughly be comparable, numerically, to a post-Newtonian correction of magnitude $x^{5} \sim 1 / c^{10}$ namely 5PN order. This is a much higher post-Newtonian order than the one at which we shall investigate the gravitational effects on the phasing formula. Using $k \sim 1, K \sim 0.2$ and the bandwidth of detectors between $10 \mathrm{~Hz}$ and $1000 \mathrm{~Hz}$, we find that the cumulative phase error due to the finite-size effect amounts to less that one orbital rotation over a total of $\sim 16000$ produced by the gravitational-wave damping of two neutron stars. The conclusion is that the finite-size effects can in general be neglected in comparison with purely gravitational-wave damping effects. The internal structure of the two compact bodies is "effaced" and their dynamics and radiation depend only, in first approximation, on the masses (and possibly spins); hence this property has been called the "effacement" principle of general relativity [142]. But note that for non-compact or moderately compact objects (such as white dwarfs for instance) the Newtonian tidal interaction dominates over the radiation damping. The constraints on the nuclear equation of state and the tidal deformability of neutron stars which can be inferred from gravitational wave observations of neutron star binary inspirals have been investigated in Refs. [320, 200, 414]. For numerical computations of the merger of two neutron stars see Refs. [187, 249].

Inspiralling compact binaries are ideally suited for application of a high-order post-Newtonian wave generation formalism. These systems are very relativistic, with orbital velocities as high as $0.5 \mathrm{c}$ in the last rotations (as compared to $\sim 10^{-3} \mathrm{c}$ for the binary pulsar), so it is not surprising that the quadrupole-moment formalism (2)-(6) constitutes a poor description of the emitted gravitational waves, since many post-Newtonian corrections are expected to play a substantial role. This expectation has been confirmed by measurement-analyses [139, 137, 198, 138, 393, 346, $350,284,157]$, which have demonstrated that the post-Newtonian precision needed to implement successfully the optimal filtering technique for the LIGO/VIRGO detectors corresponds grossly, in the case of neutron-star binaries, to the $3 \mathrm{PN}$ approximation, or $1 / c^{6}$ beyond the quadrupole moment approximation. Such a high precision is necessary because of the large number of orbital rotations that will be monitored in the detector's frequency bandwidth, giving the possibility of measuring very accurately the orbital phase of the binary. Thus, the $3 \mathrm{PN}$ order is required mostly to compute the time evolution of the orbital phase, which depends, via Eq. (9a), on the center-ofmass binding energy $E$ and the total gravitational-wave energy flux $\mathcal{F}$.

In summary, the theoretical problem is two-fold: On the one hand $E$, and on the other hand $\mathcal{F}$, are to be computed with $3 \mathrm{PN}$ precision or better. To obtain $E$ we must control the $3 \mathrm{PN}$ equations of motion of the binary in the case of general, not necessarily circular, orbits; as for $\mathcal{F}$ it necessitates the application of a $3 \mathrm{PN}$ wave generation formalism. It is remarkable that such high PN approximation is needed in preparation for the LIGO and VIRGO data analyses. As we shall see, the signal from compact binaries contains the signature of several non-linear effects which are specific to general relativity. We thus have the possibility of probing, experimentally, some aspects of the non-linear structure of Einstein's theory [84, 85, 15, 14]. 


\subsection{Post-Newtonian equations of motion}

By equations of motion we mean the explicit expression of the accelerations of the bodies in terms of the positions and velocities. In Newtonian gravity, writing the equations of motion for a system of $N$ particles is trivial; in general relativity, even writing the equations in the case $N=2$ is difficult. The first relativistic terms, at the 1PN order, were derived by Lorentz \& Droste [303]. Subsequently, Einstein, Infeld \& Hoffmann [184] obtained the 1PN corrections for $N$ particles by means of their famous "surface-integral" method, in which the equations of motion are deduced from the vacuum field equations, and are therefore applicable to any compact objects (be they neutron stars, black holes, or, perhaps, naked singularities). The $1 \mathrm{PN}$-accurate equations were also obtained, for the motion of the centers of mass of compact bodies, by Fock [201] (see also Refs. [341, 330]).

The 2PN approximation was tackled by Ohta et al. [324, 327, 326, 325], who considered the postNewtonian iteration of the Hamiltonian of $N$ point-particles. We refer here to the Hamiltonian as a "Fokker-type" Hamiltonian, which is obtained from the matter-plus-field Arnowitt-Deser-Misner (ADM) Hamiltonian by eliminating the field degrees of freedom. The 2.5PN equations of motion were obtained in harmonic coordinates by Damour \& Deruelle [148, 147, 175, 141, 142], building on a non-linear (post-Minkowskian) iteration of the metric of two particles initiated in Ref. [31]. The corresponding result for the ADM-Hamiltonian of two particles at the $2 \mathrm{PN}$ order was given in Ref. [169] (see also Refs. [375, 376]). The 2.5PN equations of motion have also been derived in the case of two extended compact objects [280, 234]. The 2.5PN equations of two point masses as well as the near zone gravitational field in harmonic-coordinate were computed in Ref. [76]. ${ }^{9}$

$\mathrm{Up}$ to the $2 \mathrm{PN}$ level the equations of motion are conservative. Only at the $2.5 \mathrm{PN}$ order does the first non-conservative effect appear, associated with the gravitational radiation emission. The equations of motion up to that level [148, 147, 175, 141, 142], have been used for the study of the radiation damping of the binary pulsar - its orbital $\dot{P}[142,143,173]$. The result was in agreement with the prediction of the quadrupole formalism given by (11). An important point is that the 2.5PN equations of motion have been proved to hold in the case of binary systems of strongly selfgravitating bodies [142]. This is via the effacing principle for the internal structure of the compact bodies. As a result, the equations depend only on the "Schwarzschild" masses, $m_{1}$ and $m_{2}$, of the neutron stars. Notably their compactness parameters $K_{1}$ and $K_{2}$, defined by Eq. (15), do not enter the equations of motion. This has also been explicitly verified up to the 2.5PN order by Kopeikin et al. [280, 234], who made a physical computation $\grave{a}$ la Fock, taking into account the internal structure of two self-gravitating extended compact bodies. The 2.5PN equations of motion have also been obtained by Itoh, Futamase \& Asada [256, 257] in harmonic coordinates, using a variant (but, much simpler and more developed) of the surface-integral approach of Einstein et al. [184], that is valid for compact bodies, independently of the strength of the internal gravity.

At the $3 \mathrm{PN}$ order the equations of motion have been worked out independently by several groups, by means of different methods, and with equivalent results:

1. Jaranowski \& Schäfer [261, 262, 263], and then with Damour [162, 164], employ the ADMHamiltonian canonical formalism of general relativity, following the line of research initiated in Refs. [324, 327, 326, 325, 169];

2. Blanchet \& Faye [69, 71, 70, 72], and with de Andrade [174] and Iyer [79], founding their approach on the post-Newtonian iteration initiated in Ref. [76], compute directly the equations of motion (instead of a Hamiltonian) in harmonic coordinates;

3. Itoh \& Futamase [255, 253] (see [213] for a review), continuing the surface-integral method

\footnotetext{
${ }^{9}$ All the works reviewed in this section concern general relativity. However, let us mention here that the equations of motion of compact binaries in scalar-tensor theories are known up to 2.5PN order [318].
} 
of Refs. [256, 257], obtain the complete 3PN equations of motion in harmonic coordinates, without need of a self-field regularization;

4. Foffa \& Sturani [203] derive the 3PN Lagrangian in harmonic coordinates within the effective field theory approach pioneered by Goldberger \& Rothstein [223].

It has been shown $[164,174]$ that there exists a unique "contact" transformation of the dynamical variables that changes the harmonic-coordinates Lagrangian of Ref. [174] (identical to the ones issued from Refs. [255, 253] and [203]) into a new Lagrangian, whose Legendre transform coincides with the ADM-Hamiltonian of Ref. [162]. The equations of motion are therefore physically equivalent. For a while, however, they depended on one unspecified numerical coefficient, which is due to some incompleteness of the Hadamard self-field regularization method. This coefficient has been fixed by means of a better regularization, dimensional regularization, both within the ADMHamiltonian formalism [163], and the harmonic-coordinates equations of motion [61]. These works have demonstrated the power of dimensional regularization and its adequateness to the classical problem of interacting point masses in general relativity. By contrast, notice that, interestingly, the surface-integral method [256, 257, 255, 253] by-passes the need of a regularization. We devote Section 6 to questions related to the use of self-field regularizations.

The effective field theory (EFT) approach to the problems of motion and radiation of compact binaries, has been extensively developed since the initial proposal [223] (see [206] for a review). It borrows techniques from quantum field theory and consists of treating the gravitational interaction between point particles as a classical limit of a quantum field theory, i.e., in the "tree level" approximation. The theory is based on the effective action, defined from a Feynman path integral that integrates over the degrees of freedom that mediate the gravitational interaction. ${ }^{10}$ The phase factor in the path integral is built from the standard Einstein-Hilbert action for gravity, augmented by a harmonic gauge fixing term and by the action of particles. The Feynman diagrams naturally show up as a perturbative technique for solving iteratively the Green's functions. Like traditional approaches [163, 61] the EFT uses the dimensional regularization.

Computing the equations of motion and radiation field using Feynman diagrams in classical general relativity is not a new idea by itself: Bertotti \& Plebanski [35] defined the diagrammatic tree-level perturbative expansion of the Green's functions in classical general relativity; Hari Dass \& Soni $[240]^{11}$ showed how to derive the classical energy-loss formula at Newtonian approximation using tree-level propagating gravitons; Feynman diagrams have been used for the equations of motion up to 2PN order in general relativity [324, 327, 326, 325] and in scalar-tensor theories [151]. Nevertheless, the systematic EFT treatment has proved to be powerful and innovative for the field, e.g., with the introduction of a decomposition of the metric into "Kaluza-Klein type" potentials [277], the interesting link with the renormalization group equation [222], and the systematization of the computation of diagrams [203].

The 3.5PN terms in the equations of motion correspond to the $1 \mathrm{PN}$ relative corrections in the radiation reaction force. They were derived by Iyer \& Will [258, 259] for point-particle binaries in a general gauge, relying on energy and angular momentum balance equations and the known expressions of the $1 \mathrm{PN}$ fluxes. The latter works have been extended to $2 \mathrm{PN}$ order [226] and to include the leading spin-orbit effects [428]. The result has been then established from first principles (i.e., not relying on balance equations) in various works at $1 \mathrm{PN}$ order [260, 336, 278, 322, 254]. The $1 \mathrm{PN}$ radiation reaction force has also been obtained for general extended fluid systems in a particular gauge [43, 47]. Known also is the contribution of gravitational-wave tails in the

\footnotetext{
10 The effective action should be equivalent, in the tree-level approximation, to the Fokker action [207], for which the field degrees of freedom (i.e., the metric), that are solutions of the field equations derived from the original matter + field action with gauge-fixing term, have been inserted back into the action, thus defining the Fokker action for the sole matter fields.

11 This reference has an eloquent title: "Feynman graph derivation of the Einstein quadrupole formula".
} 
equations of motion, which arises at the $4 \mathrm{PN}$ order and represents a $1.5 \mathrm{PN}$ modification of the radiation damping force [58]. This $1.5 \mathrm{PN}$ tail-induced correction to the radiation reaction force was also derived within the EFT approach [205, 215].

The state of the art on equations of motion is the $4 \mathrm{PN}$ approximation. Partial results on the equations of motion at the $4 \mathrm{PN}$ order have been obtained in [264, 265, 266] using the ADM Hamiltonian formalism, and in [204] using the EFT. The first derivation of the complete 4PN dynamics was accomplished in [166] by combining the local contributions [264, 265, 266] with a non-local contribution related to gravitational wave tails $[58,43]$, with the help of the result of an auxiliary analytical self-force calculation [36]. The non-local dynamics of [166] has been transformed in Ref. [167] into a local Hamiltonian containing an infinite series of even powers of the radial momentum. A second computation of the complete $4 \mathrm{PN}$ dynamics (including the same non-local interaction as in [166], but disagreeing on the local interaction) was accomplished in [33] using a Fokker Lagrangian in harmonic coordinates. Further works [39, 248] have given independent confirmations of the results of Refs. [166, 167]. More work is needed to understand the difference between the results of [166] and [33].

An important body of works concerns the effects of spins on the equations of motion of compact binaries. In this case we have in mind black holes rather than neutron stars, since astrophysical stellar-size black holes as well as super-massive galactic black holes have spins which can be close to maximal. The dominant effects are the spin-orbit (SO) coupling which is linear in spin, and the spin-spin (SS) coupling which is quadratic. For maximally spinning objects, and adopting a particular convention in which the spin is regarded as a $0.5 \mathrm{PN}$ quantity (see Section 11), the leading SO effect arises at the $1.5 \mathrm{PN}$ order while the leading SS effect appears at $2 \mathrm{PN}$ order. The leading SO and SS effects in the equations of motion have been determined by Barker \& O'Connell [27, 28] and Kidder, Will \& Wiseman [275, 271]. The next-to-leading SO effect, i.e., 1PN relative order corresponding to 2.5PN order, was obtained by Tagoshi, Ohashi \& Owen [394], then confirmed and completed by Faye, Blanchet \& Buonanno [194]. The results were also retrieved by two subsequent calculations, using the ADM Hamiltonian [165] and using EFT methods [292, 352]. The ADM calculation was later generalized to the $N$-body problem [241] and extended to the next-to-leading spin-spin effects (including both the coupling between different spins and spin square terms) in Refs. [387, 389, 388, 247, 243], and the next-to-next-to-leading SS interactions between different spins at the $4 \mathrm{PN}$ order [243]. In the meantime EFT methods progressed concurrently by computing the next-to-leading 3PN SS and spin-squared contributions [354, 356, 355, 293, 299], and the nextto-next-to-leading 4PN SS interactions for different spins [294] and for spin-squared [298]. Finally, the next-to-next-to-leading order $\mathrm{SO}$ effects, corresponding to $3.5 \mathrm{PN}$ order equivalent to $2 \mathrm{PN}$ relative order, were obtained in the ADM-coordinates Hamiltonian [242, 244] and in the harmoniccoordinates equations of motion [307, 90], with complete equivalence between the two approaches. Comparisons between the EFT and ADM Hamiltonian schemes for high-order SO and SS couplings can be found in Refs. [295, 299, 297]. We shall devote Section 11 to spin effects (focusing mainly on spin-orbit effects) in black hole binaries.

So far the status of post-Newtonian equations of motion is very satisfying. There is mutual agreement between all the results obtained by means of many different approaches and techniques, whenever they can be compared: point particles described by Dirac delta-functions or extended post-Newtonian fluids; surface-integrals methods; mixed post-Minkowskian and post-Newtonian expansions; direct post-Newtonian iteration and matching; EFT techniques versus traditional expansions; harmonic coordinates versus ADM-type coordinates; different processes or variants of the self-field regularization for point particles; different ways to including spins within the postNewtonian approximation. In Part B of this article, we present complete results for the 3.5PN equations of motion (including the $1 \mathrm{PN}$ radiation reaction), and discuss the conservative part of the equations in the case of quasi-circular orbits. Notably, the conservative part of the dynamics is compared with numerical results for the gravitational self-force in Section 8.4. 


\subsection{Post-Newtonian gravitational radiation}

The second problem, that of the computation of the gravitational waveform and the energy flux $\mathcal{F}$, has to be solved by application of a wave generation formalism (see Section 1.1). The earliest computations at the $1 \mathrm{PN}$ level beyond the quadrupole moment formalism were done by Wagoner \& Will [416], but based on some ill-defined expressions of the multipole moments [185, 403]. The computations were redone and confirmed by Blanchet \& Schäfer [86] applying the rigorous wave generation formalism of Refs. [57, 60]. Remember that at that time the post-Newtonian corrections to the emission of gravitational waves had only a purely academic interest.

The energy flux of inspiralling compact binaries was then completed to the $2 \mathrm{PN}$ order by Blanchet, Damour \& Iyer [64, 224], and, independently, by Will \& Wiseman [424, 422], using their own formalism; see Refs. [66, 82] for joint reports of these calculations. The energy flux has been computed using the EFT approach in Ref. [221] with results agreeing with traditional methods.

At the 1.5PN order in the radiation field, appears the first contribution of "hereditary" terms, which are a priori sensitive to the entire past history of the source, i.e., which depend on all previous times up to $t \rightarrow-\infty$ in the past [60]. This $1.5 \mathrm{PN}$ hereditary term represents the dominant contribution of tails in the wave zone. It has been evaluated for compact binaries in Refs. [426, 87] by application of the formula for tail integrals given in Ref. [60]. Higher-order tail effects at the 2.5PN and 3.5PN orders, as well as a crucial contribution of tails generated by the tails themselves (the so-called "tails of tails") at the $3 \mathrm{PN}$ order, were obtained in Refs [45, 48].

The 3PN approximation also involves, besides the tails of tails, many non-tail contributions coming from the relativistic corrections in the (source) multipole moments of the compact binary. Those have been almost completed in Refs. [81, 73, 80], in the sense that the result still involved one unknown numerical coefficient, due to the use of the Hadamard regularization. We shall review in Section 6 the computation of this parameter by means of dimensional regularization [62, 63], and shall present in Section 9 the most up-to-date results for the 3.5PN energy flux and orbital phase, deduced from the energy balance equation. In recent years all the results have been generalized to non-circular orbits, including both the fluxes of energy and angular momentum, and the associated balance equations $[10,9,12]$. The problem of eccentric orbits will be the subject of Section 10 .

Besides the problem of the energy flux there is the problem of the gravitational waveform itself, which includes higher-order amplitude corrections and correlatively higher-order harmonics of the orbital frequency, consistent with the post-Newtonian order. Such full post-Newtonian waveform is to be contrasted with the so-called "restricted" post-Newtonian waveform which retains only the leading-order harmonic of the signal at twice the orbital frequency, and is often used in practical data analysis when searching the signal. However, for parameter estimation the full waveform is to be taken into account. For instance it has been shown that using the full waveform in the data analysis of future space-based detectors like $e$ LISA will yield substantial improvements (with respect to the restricted waveform) of the angular resolution and the estimation of the luminosity distance of super-massive black hole binaries [16, 17, 410].

The full waveform has been obtained up to $2 \mathrm{PN}$ order in Ref. [82] by means of two independent wave generations (respectively those of Refs. [57, 44] and [424]), and it was subsequently extended up to the 3PN order in Refs. [11, 273, 272, 74]. At that order the signal contains the contributions of harmonics of the orbital frequency up to the eighth mode. The motivation is not only to build accurate templates for the data analysis of gravitational wave detectors, but also to facilitate the comparison and match of the high post-Newtonian prediction for the inspiral waveform with the numerically-generated waveforms for the merger and ringdown. For the latter application it is important to provide the post-Newtonian results in terms of a spin-weighted spherical harmonic decomposition suitable for a direct comparison with the results of numerical relativity. Recently the dominant quadrupole mode $(\ell, m)=(2,2)$ in the spin-weighted spherical harmonic decomposition has been obtained at the 3.5PN order [197]. Available results will be provided in Sections 9.4 
and 9.5 .

At the 2.5PN order in the waveform appears the dominant contribution of another hereditary effect called the "non-linear memory" effect (or sometimes Christodoulou effect) [128, 427, 406, 60, 50]. This effect was actually discovered using approximation methods in Ref. [42] (see [60] for a discussion). It implies a permanent change in the wave amplitude from before to after a burst of gravitational waves, which can be interpreted as the contribution of gravitons in the known formulas for the linear memory for massless particles [99]. Note that the non-linear memory takes the form of a simple anti-derivative of an "instantaneous" term, and therefore becomes instantaneous (i.e., non-hereditary) in the energy flux which is composed of the time-derivative of the waveform. In principle the memory contribution must be computed using some model for the evolution of the binary system in the past. Because of the cumulative effect of integration over the whole past, the memory term, though originating from $2.5 \mathrm{PN}$ order, finally contributes in the waveform at the Newtonian level $[427,11]$. It represents a part of the waveform whose amplitude steadily grows with time, but which is nearly constant over one orbital period. It is therefore essentially a zerofrequency effect (or DC effect), which has rather poor observational consequences in the case of the LIGO-VIRGO detectors, whose frequency bandwidth is always limited from below by some cut-off frequency $f_{\text {seismic }}>0$. Non-linear memory contributions in the waveform of inspiralling compact binaries have been thoroughly computed by Favata [189, 192].

The post-Newtonian results for the waveform and energy flux are in complete agreement (up to the 3.5PN order) with the results given by the very different technique of linear black-hole perturbations, valid when the mass of one of the bodies is small compared to the other. This is the test-mass limit $\nu \rightarrow 0$, in which we define the symmetric mass ratio to be the reduced mass divided by the total mass, $\nu \equiv \mu / m$ such that $\nu=1 / 4$ for equal masses. Linear black-hole perturbations, triggered by the geodesic motion of a small particle around the black hole, have been applied to this problem by Poisson [345] at the 1.5PN order (following the pioneering work [216]), by Tagoshi \& Nakamura [393], using a numerical code up to the 4PN order, and by Sasaki, Tagoshi \& Tanaka [372, 395, 397] (see also Ref. [316]), analytically up to the 5.5PN order. More recently the method has been improved and extended up to extremely high post-Newtonian orders: 14PN [209] and even 22PN [210] orders - but still for linear black-hole perturbations.

To successfully detect the gravitational waves emitted by spinning black hole binaries and to estimate the binary parameters, it is crucial to include spins effects in the templates, most importantly the spin-orbit effect which is linear in spins. The spins will affect the gravitational waves through a modulation of their amplitude, phase and frequency. Notably the orbital plane will precess in the case where the spins are not aligned or anti-aligned with the orbital angular momentum, see e.g., Ref. [8]. The leading SO and SS contributions in the waveform and flux of compact binaries are known from Refs. [275, 271, 314]; the next-to-leading SO terms at order 2.5PN were obtained in Ref. [53] after a previous attempt in [328]; the 3PN SO contribution is due to tails and was computed in Ref. [54], after intermediate results at the same order (but including SS terms) given in [353]. Finally, the next-to-next-to-leading SO contributions in the multipole moments and the energy flux, corresponding to 3.5PN order, and the next-to-leading SO tail corresponding to 4PN order, have been obtained in Refs. [89, 306]. The next-to-leading 3PN SS and spin-squared contributions in the radiation field were derived in Ref. [88]. In Section 11 we shall give full results for the contributions of spins (at SO linear level) in the energy flux and phase evolution up to $4 \mathrm{PN}$ order.

A related topic is the loss of linear momentum by gravitational radiation and the resulting gravitational recoil (or "kick") of black-hole binary systems. This phenomenon has potentially important astrophysical consequences [313]. In models of formation of massive black holes involving successive mergers of smaller "seed" black holes, a recoil with sufficient velocity could eject the system from the host galaxy and effectively terminate the process. Recoils could eject coalescing black holes from dwarf galaxies or globular clusters. Even in galaxies whose potential wells are 
deep enough to confine the recoiling system, displacement of the system from the center could have important dynamical consequences for the galactic core.

Post-Newtonian methods are not ideally suited to compute the recoil of binary black holes because most of the recoil is generated in the strong field regime close to the coalescence [199]. Nevertheless, after earlier computations of the dominant Newtonian effect [30, 199] $]^{12}$ and the 1 PN relative corrections [425], the recoil velocity has been obtained up to $2 \mathrm{PN}$ order for point particle binaries without spin [83], and is also known for the dominant spin effects [271]. Various estimations of the magnitude of the kick include a PN calculation for the inspiraling phase together with a treatment of the plunge phase [83], an application of the effective-one-body formalism [152], a close-limit calculation with Bowen-York type initial conditions [385], and a close-limit calculation with initial PN conditions for the ringdown phase [288, 290].

In parallel the problem of gravitational recoil of coalescing binaries has attracted considerable attention from the numerical relativity community. These computations led to increasingly accurate estimates of the kick velocity from the merger along quasicircular orbits of binary black holes without spins [115, 20] and with spins [117]. In particular these numerical simulations revealed the interesting result that very large kick velocities can be obtained in the case of spinning black holes for particular spin configurations.

\footnotetext{
12 In absence of a better terminology, we refer to the leading-order contribution to the recoil as "Newtonian", although it really corresponds to a 3.5PN subdominant radiation-reaction effect in the binary's equations of motion.
} 


\section{Part A: Post-Newtonian Sources}

\section{Non-linear Iteration of the Vacuum Field Equations}

\subsection{Einstein's field equations}

The field equations of general relativity are obtained by varying the space-time metric $g_{\alpha \beta}$ in the famous Einstein-Hilbert action,

$$
I_{\mathrm{EH}}=\frac{c^{3}}{16 \pi G} \int \mathrm{d}^{4} x \sqrt{-g} R+I_{\mathrm{mat}}\left[\Psi, g_{\alpha \beta}\right]
$$

They form a system of ten second-order partial differential equations obeyed by the metric,

$$
E^{\alpha \beta}\left[g, \partial g, \partial^{2} g\right]=\frac{8 \pi G}{c^{4}} T^{\alpha \beta}[\Psi, g]
$$

where the Einstein curvature tensor $E^{\alpha \beta} \equiv R^{\alpha \beta}-\frac{1}{2} R g^{\alpha \beta}$ is generated, through the gravitational coupling constant $\kappa=8 \pi G / c^{4}$, by the stress-energy tensor $T^{\alpha \beta} \equiv \frac{2}{\sqrt{-g}} \delta I_{\text {mat }} / \delta g_{\alpha \beta}$ of the matter fields $\Psi$. Among these ten equations, four govern, via the contracted Bianchi identity, the evolution of the matter system,

$$
\nabla_{\mu} E^{\alpha \mu}=0 \quad \Longrightarrow \quad \nabla_{\mu} T^{\alpha \mu}=0 .
$$

The matter equations can also be obtained by varying the matter action in (17) with respect to the matter fields $\Psi$. The space-time geometry is constrained by the six remaining equations, which place six independent constraints on the ten components of the metric $g_{\alpha \beta}$, leaving four of them to be fixed by a choice of the coordinate system.

In most of this paper we adopt the conditions of harmonic coordinates, sometimes also called de Donder coordinates. We define, as a basic variable, the gravitational-field amplitude

$$
h^{\alpha \beta} \equiv \sqrt{-g} g^{\alpha \beta}-\eta^{\alpha \beta},
$$

where $g^{\alpha \beta}$ denotes the contravariant metric (satisfying $g^{\alpha \mu} g_{\mu \beta}=\delta_{\beta}^{\alpha}$ ), where $g$ is the determinant of the covariant metric, $g \equiv \operatorname{det}\left(g_{\alpha \beta}\right)$, and where $\eta^{\alpha \beta}$ represents an auxiliary Minkowskian metric $\eta^{\alpha \beta} \equiv \operatorname{diag}(-1,1,1,1)$. The harmonic-coordinate condition, which accounts exactly for the four equations (19) corresponding to the conservation of the matter tensor, $\operatorname{reads}^{13}$

$$
\partial_{\mu} h^{\alpha \mu}=0
$$

Equation (21) introduces into the definition of our coordinate system a preferred Minkowskian structure, with Minkowski metric $\eta_{\alpha \beta}$. Of course, this is not contrary to the spirit of general relativity, where there is only one physical metric $g_{\alpha \beta}$ without any flat prior geometry, because the coordinates are not governed by geometry (so to speak), but rather can be chosen at convenience, depending on physical phenomena under study. The coordinate condition (21) is especially useful when studying gravitational waves as perturbations of space-time propagating on the fixed background metric $\eta_{\alpha \beta}$. This view is perfectly legitimate and represents a fruitful and rigorous way

\footnotetext{
13 Considering the coordinates $x^{\alpha}$ as a set of four scalars, a simple calculation shows that
}

$$
\partial_{\mu} h^{\alpha \mu}=\sqrt{-g} \square_{g} x^{\alpha},
$$

where $\square_{g} \equiv g^{\mu \nu} \nabla_{\mu} \nabla_{\nu}$ denotes the curved d'Alembertian operator. Hence the harmonic-coordinate condition tells that the coordinates $x^{\alpha}$ themselves, considered as scalars, are harmonic, i.e., obey the vacuum (curved) d'Alembertian equation. 
to think of the problem using approximation methods. Indeed, the metric $\eta_{\alpha \beta}$, originally introduced in the coordinate condition (21), does exist at any finite order of approximation (neglecting higher-order terms), and plays the role of some physical "prior" flat geometry at any order of approximation.

The Einstein field equations in harmonic coordinates can be written in the form of inhomogeneous flat d'Alembertian equations,

$$
\square h^{\alpha \beta}=\frac{16 \pi G}{c^{4}} \tau^{\alpha \beta},
$$

where $\square \equiv \square_{\eta}=\eta^{\mu \nu} \partial_{\mu} \partial_{\nu}$. The source term $\tau^{\alpha \beta}$ can rightly be interpreted as the stress-energy pseudo-tensor (actually, $\tau^{\alpha \beta}$ is a Lorentz-covariant tensor) of the matter fields, described by $T^{\alpha \beta}$, and the gravitational field, given by the gravitational source term $\Lambda^{\alpha \beta}$, i.e.,

$$
\tau^{\alpha \beta}=|g| T^{\alpha \beta}+\frac{c^{4}}{16 \pi G} \Lambda^{\alpha \beta} .
$$

The exact expression of $\Lambda^{\alpha \beta}$ in harmonic coordinates, including all non-linearities, reads ${ }^{14}$

$$
\begin{aligned}
\Lambda^{\alpha \beta} & =-h^{\mu \nu} \partial_{\mu \nu}^{2} h^{\alpha \beta}+\partial_{\mu} h^{\alpha \nu} \partial_{\nu} h^{\beta \mu}+\frac{1}{2} g^{\alpha \beta} g_{\mu \nu} \partial_{\lambda} h^{\mu \tau} \partial_{\tau} h^{\nu \lambda} \\
& -g^{\alpha \mu} g_{\nu \tau} \partial_{\lambda} h^{\beta \tau} \partial_{\mu} h^{\nu \lambda}-g^{\beta \mu} g_{\nu \tau} \partial_{\lambda} h^{\alpha \tau} \partial_{\mu} h^{\nu \lambda}+g_{\mu \nu} g^{\lambda \tau} \partial_{\lambda} h^{\alpha \mu} \partial_{\tau} h^{\beta \nu} \\
& +\frac{1}{8}\left(2 g^{\alpha \mu} g^{\beta \nu}-g^{\alpha \beta} g^{\mu \nu}\right)\left(2 g_{\lambda \tau} g_{\epsilon \pi}-g_{\tau \epsilon} g_{\lambda \pi}\right) \partial_{\mu} h^{\lambda \pi} \partial_{\nu} h^{\tau \epsilon} .
\end{aligned}
$$

As is clear from this expression, $\Lambda^{\alpha \beta}$ is made of terms at least quadratic in the gravitational-field strength $h$ and its first and second space-time derivatives. In the following, for the highest postNewtonian order that we shall consider, we will need the quadratic, cubic and quartic pieces of $\Lambda^{\alpha \beta}$; with obvious notation, we can write them as

$$
\Lambda^{\alpha \beta}=N^{\alpha \beta}[h, h]+M^{\alpha \beta}[h, h, h]+L^{\alpha \beta}[h, h, h, h]+\mathcal{O}\left(h^{5}\right) .
$$

These various terms can be straightforwardly computed from expanding Eq. (24); for instance the leading quadratic piece is explicitly given by ${ }^{15}$

$$
\begin{aligned}
N^{\alpha \beta}= & -h^{\mu \nu} \partial_{\mu \nu}^{2} h^{\alpha \beta}+\frac{1}{2} \partial^{\alpha} h_{\mu \nu} \partial^{\beta} h^{\mu \nu}-\frac{1}{4} \partial^{\alpha} h \partial^{\beta} h+\partial_{\nu} h^{\alpha \mu}\left(\partial^{\nu} h_{\mu}^{\beta}+\partial_{\mu} h^{\beta \nu}\right) \\
& -2 \partial^{(\alpha} h_{\mu \nu} \partial^{\mu} h^{\beta) \nu}+\eta^{\alpha \beta}\left[-\frac{1}{4} \partial_{\tau} h_{\mu \nu} \partial^{\tau} h^{\mu \nu}+\frac{1}{8} \partial_{\mu} h \partial^{\mu} h+\frac{1}{2} \partial_{\mu} h_{\nu \tau} \partial^{\nu} h^{\mu \tau}\right] .
\end{aligned}
$$

As we said, the condition (21) is equivalent to the matter equations of motion, in the sense of the conservation of the total pseudo-tensor $\tau^{\alpha \beta}$,

$$
\partial_{\mu} \tau^{\alpha \mu}=0 \Longleftrightarrow \nabla_{\mu} T^{\alpha \mu}=0 .
$$

In this article, we shall look for approximate solutions of the field equations (21) - (22) under the following four hypotheses:

1. The matter stress-energy tensor $T^{\alpha \beta}$ is of spatially compact support, i.e., can be enclosed into some time-like world tube, say $r \leqslant a$, where $r=|\mathbf{x}|$ is the harmonic-coordinate radial distance. Outside the domain of the source, when $r>a$, the gravitational source term, according to Eq. (27), is divergence-free,

$$
\partial_{\mu} \Lambda^{\alpha \mu}=0 \quad(\text { when } r>a) ;
$$

\footnotetext{
${ }^{14}$ In $d+1$ space-time dimensions, only one coefficient in this expression is modified; see Eq. (175) below.

15 See Eqs. (3.8) in Ref. [71] for the cubic and quartic terms. We denote e.g., $h_{\mu}^{\alpha}=\eta_{\mu \nu} h^{\alpha \nu}, h=\eta_{\mu \nu} h^{\mu \nu}$, and $\partial^{\alpha}=\eta^{\alpha \mu} \partial_{\mu}$. A parenthesis around a pair of indices denotes the usual symmetrization: $T^{(\alpha \beta)}=\frac{1}{2}\left(T^{\alpha \beta}+T^{\beta \alpha}\right)$.
} 
2. The matter distribution inside the source is smooth: $T^{\alpha \beta} \in C^{\infty}\left(\mathbb{R}^{3}\right) .{ }^{16}$ We have in mind a smooth hydrodynamical fluid system, without any singularities nor shocks (a priori), that is described by some Euler-type equations including high relativistic corrections. In particular, we exclude from the start the presence of any black holes; however, we shall return to this question in Part B when we look for a model describing compact objects;

3. The source is post-Newtonian in the sense of the existence of the small parameter defined by Eq. (1). For such a source we assume the legitimacy of the method of matched asymptotic expansions for identifying the inner post-Newtonian field and the outer multipolar decomposition in the source's exterior near zone;

4. The gravitational field has been independent of time (stationary) in some remote past, i.e., before some finite instant $-\mathcal{T}$ in the past, namely

$$
\frac{\partial}{\partial t}\left[h^{\alpha \beta}(\mathbf{x}, t)\right]=0 \quad \text { when } t \leqslant-\mathcal{T} .
$$

The latter condition is a means to impose, by brute force, the famous no-incoming radiation condition, ensuring that the matter source is isolated from the rest of the Universe and does not receive any radiation from infinity. Ideally, the no-incoming radiation condition should be imposed at past null infinity. As we shall see, this condition entirely fixes the radiation reaction forces inside the isolated source. We shall later argue (see Section 3.2) that our condition of stationarity in the past (29), although weaker than the ideal no-incoming radiation condition, does not entail any physical restriction on the general validity of the formulas we derive. Even more, the condition (29) is actually better suited in the case of real astrophysical sources like inspiralling compact binaries, for which we do not know the details of the initial formation and remote past evolution. In practice the initial instant $-\mathcal{T}$ can be set right after the explosions of the two supernovæ yielding the formation of the compact binary system.

Subject to the past-stationarity condition (29), the differential equations (22) can be written equivalently into the form of the integro-differential equations

$$
h^{\alpha \beta}=\frac{16 \pi G}{c^{4}} \square_{\mathrm{ret}}^{-1} \tau^{\alpha \beta}
$$

containing the usual retarded inverse d'Alembertian integral operator, given by

$$
\left(\square_{\mathrm{ret}}^{-1} \tau\right)(\mathbf{x}, t) \equiv-\frac{1}{4 \pi} \iiint \frac{\mathrm{d}^{3} \mathbf{x}^{\prime}}{\left|\mathbf{x}-\mathbf{x}^{\prime}\right|} \tau\left(\mathbf{x}^{\prime}, t-\left|\mathbf{x}-\mathbf{x}^{\prime}\right| / c\right),
$$

extending over the whole three-dimensional space $\mathbb{R}^{3}$.

\section{$2.2 \quad$ Linearized vacuum equations}

In what follows we solve the field equations (21) - (22), in the vacuum region outside the compactsupport source, in the form of a formal non-linearity or post-Minkowskian expansion, considering the field variable $h^{\alpha \beta}$ as a non-linear metric perturbation of Minkowski space-time. At the linearized level (or first-post-Minkowskian approximation), we write:

$$
h_{\mathrm{ext}}^{\alpha \beta}=G h_{(1)}^{\alpha \beta}+\mathcal{O}\left(G^{2}\right),
$$

$16 \mathbb{N}, \mathbb{Z}, \mathbb{R}$, and $\mathbb{C}$ are the usual sets of non-negative integers, integers, real numbers, and complex numbers; $C^{p}(\Omega)$ is the set of $p$-times continuously differentiable functions on the open domain $\Omega(p \leqslant+\infty)$. 
where the subscript "ext" reminds us that the solution is valid only in the exterior of the source, and where we have introduced Newton's constant $G$ as a book-keeping parameter, enabling one to label conveniently the successive post-Minkowskian approximations. Since $h^{\alpha \beta}$ is a dimensionless variable, with our convention the linear coefficient $h_{(1)}^{\alpha \beta}$ in Eq. (32) has the dimension of the inverse of $G$ (which should be a mass squared in a system of units where $\hbar=c=1$ ). In vacuum, the harmonic-coordinate metric coefficient $h_{(1)}^{\alpha \beta}$ satisfies

$$
\begin{aligned}
\square h_{(1)}^{\alpha \beta} & =0, \\
\partial_{\mu} h_{(1)}^{\alpha \mu} & =0 .
\end{aligned}
$$

We want to solve those equations by means of an infinite multipolar series valid outside a timelike world tube containing the source. Indeed the multipole expansion is the appropriate method for describing the physics of the source as seen from its exterior $(r>a)$. On the other hand, the post-Minkowskian series is physically valid in the weak-field region, which surely includes the exterior of any source, starting at a sufficiently large distance. For post-Newtonian sources the exterior weak-field region, where both multipole and post-Minkowskian expansions are valid, simply coincides with the exterior region $r>a$. It is therefore quite natural, and even, one would say inescapable when considering general sources, to combine the post-Minkowskian approximation with the multipole decomposition. This is the original idea of the "double-expansion" series of Bonnor and collaborators [94, 95, 96, 251], which combines the $G$-expansion (or $m$-expansion in their notation) with the $a$-expansion (equivalent to the multipole expansion, since the $\ell$-th order multipole moment scales with the source radius like $\left.a^{\ell}\right)$.

The multipolar-post-Minkowskian (MPM) method will be implemented systematically, using symmetric-trace-free (STF) harmonics to describe the multipole expansion [403], and looking for a definite algorithm for the approximation scheme [57]. The solution of the system of equations (33) takes the form of a series of retarded multipolar waves ${ }^{17}$

$$
h_{(1)}^{\alpha \beta}=\sum_{\ell=0}^{+\infty} \partial_{L}\left(\frac{K_{L}^{\alpha \beta}(t-r / c)}{r}\right),
$$

where $r=|\mathbf{x}|$, and where the functions $\mathrm{K}_{L}^{\alpha \beta} \equiv \mathrm{K}_{i_{1} \ldots i_{\ell}}^{\alpha \beta}$ are smooth functions of the retarded time $u \equiv t-r / c\left[\right.$ i.e., $\left.\mathrm{K}_{L}(u) \in C^{\infty}(\mathbb{R})\right]$, which become constant in the past, when $t \leqslant-\mathcal{T}$, see Eq. (29). Since a monopolar wave satisfies $\square\left(\mathrm{K}_{L}(u) / r\right)=0$ and the d'Alembertian commutes with the multi-derivative $\partial_{L}$, it is evident that Eq. (34) represents the most general solution of the wave equation (33a); but see Section 2 in Ref. [57] for a rigorous proof based on the Euler-PoissonDarboux equation. The gauge condition (33b), however, is not fulfilled in general, and to satisfy it we must algebraically decompose the set of functions $\mathrm{K}_{L}^{00}, \mathrm{~K}_{L}^{0 i}, \mathrm{~K}_{L}^{i j}$ into ten tensors which are STF with respect to all their indices, including the spatial indices $i, i j$. Imposing the condition (33b)

\footnotetext{
17 Our notation is the following: $L=i_{1} i_{2} \ldots i_{\ell}$ denotes a multi-index, made of $\ell$ (spatial) indices. Similarly, we write for instance $K=j_{1} \ldots j_{k}$ (in practice, we generally do not need to write explicitly the "carrier" letter $i$ or $j$ ), or $a L-1=a i_{1} \ldots i_{\ell-1}$. Always understood in expressions such as Eq. (34) are $\ell$ summations over the indices $i_{1}, \ldots, i_{\ell}$ ranging from 1 to 3 . The derivative operator $\partial_{L}$ is a short-hand for $\partial_{i_{1}} \ldots \partial_{i_{\ell}}$. The function $K_{L}$ (for any space-time indices $\alpha \beta$ ) is symmetric and trace-free (STF) with respect to the $\ell$ indices composing $L$. This means that for any pair of indices $i_{p}, i_{q} \in L$, we have $\mathrm{K} \ldots i_{p} \ldots i_{q} \ldots=\mathrm{K} \ldots i_{q} \ldots i_{p} \ldots$ and that $\delta_{i_{p} i_{q}} \mathrm{~K} \ldots i_{p} \ldots i_{q} \ldots=0$ (see Ref. [403] and Appendices A and B in Ref. [57] for reviews about the STF formalism). The STF projection is denoted with a hat, so $\mathrm{K}_{L} \equiv \hat{\mathrm{K}}_{L}$, or sometimes with carets around the indices, $\mathrm{K}_{L} \equiv \mathrm{K}_{\langle L\rangle}$. In particular, $\hat{n}_{L}=n_{\langle L\rangle}$ is the STF projection of the product of unit vectors $n_{L}=n_{i_{1}} \ldots n_{i_{\ell}}$, for instance $\hat{n}_{i j}=n_{\langle i j\rangle}=n_{i j}-\frac{1}{3} \delta_{i j}$ and $\hat{n}_{i j k}=n_{\langle i j k\rangle}=$ $n_{i j k}-\frac{1}{5}\left(\delta_{i j} n_{k}+\delta_{i k} n_{j}+\delta_{j k} n_{i}\right)$; an expansion into STF tensors $\hat{n}_{L}=\hat{n}_{L}(\theta, \phi)$ is equivalent to the usual expansion in spherical harmonics $\mathrm{Y}_{l m}=\mathrm{Y}_{l m}(\theta, \phi)$, see Eqs. (75) below. Similarly, we denote $x_{L}=x_{i_{1}} \ldots x_{i_{\ell}}=r^{l} n_{L}$ where $r=|\mathbf{x}|$, and $\hat{x}_{L}=x_{\langle L\rangle}=\operatorname{STF}\left[x_{L}\right]$. The Levi-Civita antisymmetric symbol is denoted $\epsilon_{i j k}\left(\right.$ with $\left.\epsilon_{123}=1\right)$. Parenthesis refer to symmetrization, $T_{(i j)}=\frac{1}{2}\left(T_{i j}+T_{j i}\right)$. Superscripts $(q)$ indicate $q$ successive time derivations.
} 
reduces the number of independent tensors to six, and we find that the solution takes an especially simple "canonical" form, parametrized by only two moments, plus some arbitrary linearized gauge transformation [403, 57].

Theorem 1. The most general solution of the linearized field equations (33) outside some time-like world tube enclosing the source $(r>a)$, and stationary in the past [see Eq. (29)], reads

$$
h_{(1)}^{\alpha \beta}=k_{(1)}^{\alpha \beta}+\partial^{\alpha} \varphi_{(1)}^{\beta}+\partial^{\beta} \varphi_{(1)}^{\alpha}-\eta^{\alpha \beta} \partial_{\mu} \varphi_{(1)}^{\mu} .
$$

The first term depends on two STF-tensorial multipole moments, $\mathrm{I}_{L}(u)$ and $\mathrm{J}_{L}(u)$, which are arbitrary functions of time except for the laws of conservation of the monopole: $\mathrm{I}=$ const, and dipoles: $\mathrm{I}_{i}=$ const, $\mathrm{J}_{i}=$ const. It is given by

$$
\begin{aligned}
& k_{(1)}^{00}=-\frac{4}{c^{2}} \sum_{\ell \geqslant 0} \frac{(-)^{\ell}}{\ell !} \partial_{L}\left(\frac{1}{r} \mathrm{I}_{L}(u)\right), \\
& k_{(1)}^{0 i}=\frac{4}{c^{3}} \sum_{\ell \geqslant 1} \frac{(-)^{\ell}}{\ell !}\left\{\partial_{L-1}\left(\frac{1}{r} \mathrm{I}_{i L-1}^{(1)}(u)\right)+\frac{\ell}{\ell+1} \epsilon_{i a b} \partial_{a L-1}\left(\frac{1}{r} \mathrm{~J}_{b L-1}(u)\right)\right\}, \\
& k_{(1)}^{i j}=-\frac{4}{c^{4}} \sum_{\ell \geqslant 2} \frac{(-)^{\ell}}{\ell !}\left\{\partial_{L-2}\left(\frac{1}{r} \mathrm{I}_{i j L-2}^{(2)}(u)\right)+\frac{2 \ell}{\ell+1} \partial_{a L-2}\left(\frac{1}{r} \epsilon_{a b(i} \mathrm{J}_{j) b L-2}^{(1)}(u)\right)\right\} .
\end{aligned}
$$

The other terms represent a linearized gauge transformation, with gauge vector $\varphi_{(1)}^{\alpha}$ parametrized by four other multipole moments, say $\mathrm{W}_{L}(u), \mathrm{X}_{L}(u), \mathrm{Y}_{L}(u)$ and $\mathrm{Z}_{L}(u)$ [see Eqs. (37)].

The conservation of the lowest-order moments gives the constancy of the total mass of the source, $\mathrm{M} \equiv \mathrm{I}=$ const, center-of-mass position, $\mathrm{X}_{i} \equiv \mathrm{I}_{i} / \mathrm{I}=$ const, total linear momentum $\mathrm{P}_{i} \equiv \mathrm{I}_{i}^{(1)}=0,{ }^{18}$ and total angular momentum, $\mathrm{J}_{i}=$ const. It is always possible to achieve $\mathrm{X}_{i}=0$ by translating the origin of our coordinates to the center of mass. The total mass M is the ADM mass of the Hamiltonian formulation of general relativity. Note that the quantities $\mathrm{M}, \mathrm{X}_{i}, \mathrm{P}_{i}$ and $\mathrm{J}_{i}$ include the contributions due to the waves emitted by the source. They describe the initial state of the source, before the emission of gravitational radiation.

The multipole functions $\mathrm{I}_{L}(u)$ and $\mathrm{J}_{L}(u)$, which thoroughly encode the physical properties of the source at the linearized level (because the other moments $W_{L}, \ldots, Z_{L}$ parametrize a gauge transformation), will be referred to as the mass-type and current-type source multipole moments. Beware, however, that at this stage the moments are not specified in terms of the stress-energy tensor $T^{\alpha \beta}$ of the source: Theorem 1 follows merely from the algebraic and differential properties of the vacuum field equations outside the source.

For completeness, we give the components of the gauge-vector $\varphi_{(1)}^{\alpha}$ entering Eq. (35):

$$
\begin{aligned}
\varphi_{(1)}^{0} & =\frac{4}{c^{3}} \sum_{\ell \geqslant 0} \frac{(-)^{\ell}}{\ell !} \partial_{L}\left(\frac{1}{r} \mathrm{~W}_{L}(u)\right), \\
\varphi_{(1)}^{i} & =-\frac{4}{c^{4}} \sum_{\ell \geqslant 0} \frac{(-)^{\ell}}{\ell !} \partial_{i L}\left(\frac{1}{r} \mathrm{X}_{L}(u)\right) \\
& -\frac{4}{c^{4}} \sum_{\ell \geqslant 1} \frac{(-)^{\ell}}{\ell !}\left\{\partial_{L-1}\left(\frac{1}{r} \mathrm{Y}_{i L-1}(u)\right)+\frac{\ell}{\ell+1} \epsilon_{i a b} \partial_{a L-1}\left(\frac{1}{r} \mathrm{Z}_{b L-1}(u)\right)\right\} .
\end{aligned}
$$

18 The constancy of the center of mass $\mathrm{X}_{i}$ - rather than a linear variation with time - results from our assumption of stationarity before the date $-\mathcal{T}$, see Eq. (29). Hence, $\mathrm{P}_{i}=0$. 
Because the theory is covariant with respect to non-linear diffeomorphisms and not merely with respect to linear gauge transformations, the moments $\mathrm{W}_{L}, \ldots, \mathrm{Z}_{L}$ do play a physical role starting at the non-linear level, in the following sense. If one takes these moments equal to zero and continues the post-Minkowskian iteration [see Section 2.3] one ends up with a metric depending on $\mathrm{I}_{L}$ and $\mathrm{J}_{L}$ only, but that metric will not describe the same physical source as the one which would have been constructed starting from the six moments $\mathrm{I}_{L}, \mathrm{~J}_{L}, \ldots, \mathrm{Z}_{L}$ altogether. In other words, the two non-linear metrics associated with the sets of multipole moments $\left\{\mathrm{I}_{L}, \mathrm{~J}_{L}, 0, \ldots, 0\right\}$ and $\left\{\mathrm{I}_{L}, \mathrm{~J}_{L}, \mathrm{~W}_{L}, \ldots, \mathrm{Z}_{L}\right\}$ are not physically equivalent - they are not isometric. We shall point out in Section 2.4 below that the full set of moments $\left\{\mathrm{I}_{L}, \mathrm{~J}_{L}, \mathrm{~W}_{L}, \ldots, \mathrm{Z}_{L}\right\}$ is in fact physically equivalent to some other reduced set of moments $\left\{\mathrm{M}_{L}, \mathrm{~S}_{L}, 0, \ldots, 0\right\}$, but with some moments $\mathrm{M}_{L}$, $\mathrm{S}_{L}$ that differ from $\mathrm{I}_{L}, \mathrm{~J}_{L}$ by non-linear corrections [see Eqs. (97)-(98)]. The moments $\mathrm{M}_{L}, \mathrm{~S}_{L}$ are called "canonical" moments; they play a useful role in intermediate calculations. All the multipole moments $\mathrm{I}_{L}, \mathrm{~J}_{L}, \mathrm{~W}_{L}, \mathrm{X}_{L}, \mathrm{Y}_{L}, \mathrm{Z}_{L}$ will be computed in Section 4.4.

\subsection{The multipolar post-Minkowskian solution}

By Theorem 1 we know the most general solution of the linearized equations in the exterior of the source. We then tackle the problem of the post-Minkowskian iteration of that solution. We consider the full post-Minkowskian series

$$
h_{\mathrm{ext}}^{\alpha \beta}=\sum_{n=1}^{+\infty} G^{n} h_{(n)}^{\alpha \beta},
$$

where the first term is composed of the result given by Eqs. (35)-(37). In this article, we shall always understand the infinite sums such as the one in Eq. (38) in the sense of formal power series, i.e., as an ordered collection of coefficients, $\left(h_{(n)}^{\alpha \beta}\right)_{n \in \mathbb{N}}$. We do not attempt to control the mathematical nature of the series and refer to the mathematical-physics literature for discussion of that point (see, in the present context, Refs. [130, 171, 361, 362, 363]).

We substitute the post-Minkowski ansatz (38) into the vacuum Einstein field equations (21) (22), i.e., with $\tau^{\alpha \beta}$ simply given by the gravitational source term $\Lambda^{\alpha \beta}$, and we equate term by term the factors of the successive powers of our book-keeping parameter $G$. We get an infinite set of equations for each of the $h_{(n)}^{\alpha \beta}$ 's: namely, $\forall n \geqslant 2$,

$$
\begin{aligned}
\square h_{(n)}^{\alpha \beta} & =\Lambda_{(n)}^{\alpha \beta}\left[h_{(1)}, h_{(2)}, \ldots, h_{(n-1)}\right], \\
\partial_{\mu} h_{(n)}^{\alpha \mu} & =0 .
\end{aligned}
$$

The right-hand side of the wave equation (39a) is obtained from inserting the previous iterations, known up to the order $n-1$, into the gravitational source term. In more details, the series of equations (39a) reads

$$
\begin{aligned}
\square h_{(2)}^{\alpha \beta} & =N^{\alpha \beta}\left[h_{(1)}, h_{(1)}\right] \\
\square h_{(3)}^{\alpha \beta} & =M^{\alpha \beta}\left[h_{(1)}, h_{(1)}, h_{(1)}\right]+N^{\alpha \beta}\left[h_{(1)}, h_{(2)}\right]+N^{\alpha \beta}\left[h_{(2)}, h_{(1)}\right], \\
\square h_{(4)}^{\alpha \beta} & =L^{\alpha \beta}\left[h_{(1)}, h_{(1)}, h_{(1)}, h_{(1)}\right] \\
& +M^{\alpha \beta}\left[h_{(1)}, h_{(1)}, h_{(2)}\right]+M^{\alpha \beta}\left[h_{(1)}, h_{(2)}, h_{(1)}\right]+M^{\alpha \beta}\left[h_{(2)}, h_{(1)}, h_{(1)}\right] \\
& +N^{\alpha \beta}\left[h_{(2)}, h_{(2)}\right]+N^{\alpha \beta}\left[h_{(1)}, h_{(3)}\right]+N^{\alpha \beta}\left[h_{(3)}, h_{(1)}\right]
\end{aligned}
$$


The quadratic, cubic and quartic pieces of $\Lambda^{\alpha \beta}$ are defined by Eq. (25)-(26).

Let us now proceed by induction. Some $n \in \mathbb{N}$ being given, we assume that we succeeded in constructing, starting from the linearized solution $h_{(1)}$, the sequence of post-Minkowskian solutions $h_{(2)}, h_{(3)}, \ldots, h_{(n-1)}$, and from this we want to infer the next solution $h_{(n)}$. The right-hand side of Eq. (39a), $\Lambda_{(n)}^{\alpha \beta}$, is known by induction hypothesis. Thus the problem is that of solving a flat wave equation whose source is given. The point is that this wave equation, instead of being valid everywhere in $\mathbb{R}^{3}$, is physically correct only outside the matter source $(r>a)$, and it makes no sense to solve it by means of the usual retarded integral. Technically speaking, the right-hand side of Eq. (39a) is composed of the product of many multipole expansions, which are singular at the origin of the spatial coordinates $r=0$, and which make the retarded integral divergent at that point. This does not mean that there are no solutions to the wave equation, but simply that the retarded integral does not constitute the appropriate solution in that context.

What we need is a solution which takes the same structure as the source term $\Lambda_{(n)}^{\alpha \beta}$, i.e., is expanded into multipole contributions, with a singularity at $r=0$, and satisfies the d'Alembertian equation as soon as $r>0$. Such a particular solution can be obtained, following the method of Ref. [57], by means of a mathematical trick, in which one first "regularizes" the source term $\Lambda_{(n)}^{\alpha \beta}$ by multiplying it by the factor $r^{B}$, where $r=|\mathbf{x}|$ is the spatial radial distance and $B$ is a complex number, $B \in \mathbb{C}$. Let us assume, for definiteness, that $\Lambda_{(n)}^{\alpha \beta}$ is composed of multipolar pieces with maximal multipolarity $\ell_{\max }$. This means that we start the iteration from the linearized metric (35) - (37) in which the multipolar sums are actually finite. ${ }^{19}$ The divergences when $r \rightarrow 0$ of the source term are typically power-like, say $1 / r^{k}$ (there are also powers of the logarithm of $r)$, and with the previous assumption there will exist a maximal order of divergency, say $k_{\max }$. Thus, when the real part of $B$ is large enough, i.e., $\Re(B)>k_{\max }-3$, the "regularized" source term $r^{B} \Lambda_{(n)}^{\alpha \beta}$ is regular enough when $r \rightarrow 0$ so that one can perfectly apply the retarded integral operator. This defines the $B$-dependent retarded integral, when $\Re(B)$ is large enough,

$$
I^{\alpha \beta}(B) \equiv \square_{\text {ret }}^{-1}\left[\widetilde{r}^{B} \Lambda_{(n)}^{\alpha \beta}\right]
$$

where the symbol $\square_{\text {ret }}^{-1}$ stands for the retarded integral defined by Eq. (31). It is convenient to introduce inside the regularizing factor some arbitrary constant length scale $r_{0}$ in order to make it dimensionless. Everywhere in this article we pose

$$
\widetilde{r} \equiv \frac{r}{r_{0}} .
$$

The fate of the constant $r_{0}$ in a detailed calculation will be interesting to follow, as we shall see. Now the point for our purpose is that the function $I^{\alpha \beta}(B)$ on the complex plane, which was originally defined only when $\Re(B)>k_{\max }-3$, admits a unique analytic continuation to all values of $B \in \mathbb{C}$ except at some integer values. Furthermore, the analytic continuation of $I^{\alpha \beta}(B)$ can be expanded, when $B \rightarrow 0$ (namely the limit of interest to us) into a Laurent expansion involving in general some multiple poles. The key idea, as we shall prove, is that the finite part, or the coefficient of the zeroth power of $B$ in that expansion, represents the particular solution we are looking for. We write the Laurent expansion of $I^{\alpha \beta}(B)$, when $B \rightarrow 0$, in the form

$$
I^{\alpha \beta}(B)=\sum_{p=p_{0}}^{+\infty} \iota_{p}^{\alpha \beta} B^{p},
$$

19 This assumption is justified because we are ultimately interested in the radiation field at some given finite post-Newtonian precision like $3 \mathrm{PN}$, and because only a finite number of multipole moments can contribute at any finite order of approximation. With a finite number of multipoles in the linearized metric (35)-(37), there is a maximal multipolarity $\ell_{\max }(n)$ at any post-Minkowskian order $n$, which grows linearly with $n$. 
where $p \in \mathbb{Z}$, and the various coefficients $\iota_{p}^{\alpha \beta}$ are functions of the field point $(\mathbf{x}, t)$. When $p_{0} \leqslant-1$ there are poles; and $-p_{0}$, which depends on $n$, refers to the maximal order of these poles. By applying the d'Alembertian operator onto both sides of Eq. (43), and equating the different powers of $B$, we arrive at

$$
\begin{aligned}
& p_{0} \leqslant p \leqslant-1 \Longrightarrow \square \iota_{p}^{\alpha \beta}=0, \\
& p \geqslant 0 \quad \Longrightarrow \quad \square \iota_{p}^{\alpha \beta}=\frac{(\ln r)^{p}}{p !} \Lambda_{(n)}^{\alpha \beta} .
\end{aligned}
$$

As we see, the case $p=0$ shows that the finite-part coefficient in Eq. (43), namely $\iota_{0}^{\alpha \beta}$, is a particular solution of the requested equation: $\square \iota_{0}^{\alpha \beta}=\Lambda_{(n)}^{\alpha \beta}$. Furthermore, we can prove that this solution, by its very construction, owns the same structure made of a multipolar expansion singular at $r=0$ as the corresponding source.

Let us forget about the intermediate name $\iota_{0}^{\alpha \beta}$, and denote, from now on, the latter solution by $u_{(n)}^{\alpha \beta} \equiv \iota_{0}^{\alpha \beta}$, or, in more explicit terms,

$$
u_{(n)}^{\alpha \beta}=\mathcal{F P}_{B=0} \square_{\text {ret }}^{-1}\left[\widetilde{r}^{B} \Lambda_{(n)}^{\alpha \beta}\right],
$$

where the finite-part symbol $\mathcal{F} \mathcal{P}_{B=0}$ means the previously detailed operations of considering the analytic continuation, taking the Laurent expansion, and picking up the finite-part coefficient when $B \rightarrow 0$. The story is not complete, however, because $u_{(n)}^{\alpha \beta}$ does not fulfill the constraint of harmonic coordinates (39b); its divergence, say $w_{(n)}^{\alpha}=\partial_{\mu} u_{(n)}^{\alpha \mu}$, is different from zero in general. From the fact that the source term is divergence-free in vacuum, $\partial_{\mu} \Lambda_{(n)}^{\alpha \mu}=0$ [see Eq. (28)], we find instead

$$
w_{(n)}^{\alpha}=\mathcal{F P}_{B=0} \square_{\text {ret }}^{-1}\left[B \widetilde{r}^{B} \frac{n_{i}}{r} \Lambda_{(n)}^{\alpha i}\right]
$$

The factor $B$ comes from the differentiation of the regularization factor $\widetilde{r}^{B}$. So, $w_{(n)}^{\alpha}$ is zero only in the special case where the Laurent expansion of the retarded integral in Eq. (46) does not develop any simple pole when $B \rightarrow 0$. Fortunately, when it does, the structure of the pole is quite easy to control. We find that it necessarily consists of an homogeneous solution of the source-free d'Alembertian equation, and, what is more (from its stationarity in the past), that solution is a retarded one. Hence, taking into account the index structure of $w_{(n)}^{\alpha}$, there must exist four STF-tensorial functions of $u=t-r / c$, say $N_{L}(u), P_{L}(u), Q_{L}(u)$ and $R_{L}(u)$, such that

$$
\begin{aligned}
w_{(n)}^{0} & =\sum_{l=0}^{+\infty} \partial_{L}\left[r^{-1} N_{L}(u)\right] \\
w_{(n)}^{i} & =\sum_{l=0}^{+\infty} \partial_{i L}\left[r^{-1} P_{L}(u)\right]+\sum_{l=1}^{+\infty}\left\{\partial_{L-1}\left[r^{-1} Q_{i L-1}(u)\right]+\epsilon_{i a b} \partial_{a L-1}\left[r^{-1} R_{b L-1}(u)\right]\right\} .
\end{aligned}
$$

From that expression we are able to find a new object, say $v_{(n)}^{\alpha \beta}$, which takes the same structure as $w_{(n)}^{\alpha}$ (a retarded solution of the source-free wave equation) and, furthermore, whose divergence is exactly the opposite of the divergence of $u_{(n)}^{\alpha \beta}$, i.e. $\partial_{\mu} v_{(n)}^{\alpha \mu}=-w_{(n)}^{\alpha}$. Such a $v_{(n)}^{\alpha \beta}$ is not unique, but we shall see that it is simply necessary to make a choice for $v_{(n)}^{\alpha \beta}$ (the simplest one) in order to obtain the general solution. The formulas that we adopt are

$$
v_{(n)}^{00}=-r^{-1} N^{(-1)}+\partial_{a}\left[r^{-1}\left(-N_{a}^{(-1)}+C_{a}^{(-2)}-3 P_{a}\right)\right],
$$




$$
\begin{aligned}
v_{(n)}^{0 i}=r^{-1}\left(-Q_{i}^{(-1)}\right. & \left.+3 P_{i}^{(1)}\right)-\epsilon_{i a b} \partial_{a}\left[r^{-1} R_{b}^{(-1)}\right]-\sum_{l=2}^{+\infty} \partial_{L-1}\left[r^{-1} N_{i L-1}\right] \\
v_{(n)}^{i j}=-\delta_{i j} r^{-1} P+ & \sum_{l=2}^{+\infty}\left\{2 \delta_{i j} \partial_{L-1}\left[r^{-1} P_{L-1}\right]-6 \partial_{L-2(i}\left[r^{-1} P_{j) L-2}\right]\right. \\
& \left.+\partial_{L-2}\left[r^{-1}\left(N_{i j L-2}^{(1)}+3 P_{i j L-2}^{(2)}-Q_{i j L-2}\right)\right]-2 \partial_{a L-2}\left[r^{-1} \epsilon_{a b(i} R_{j) b L-2}\right]\right\} .
\end{aligned}
$$

Notice the presence of anti-derivatives, denoted e.g., by $N^{(-1)}(u)=\int_{-\infty}^{u} \mathrm{~d} v N(v)$; there is no problem with the limit $v \rightarrow-\infty$ since all the corresponding functions are zero when $t \leqslant-\mathcal{T}$. The choice made in Eqs. (48) is dictated by the fact that the 00 component involves only some monopolar and dipolar terms, and that the spatial trace $i i$ is monopolar: $v_{(n)}^{i i}=-3 r^{-1} P$. Finally, if we pose

$$
h_{(n)}^{\alpha \beta}=u_{(n)}^{\alpha \beta}+v_{(n)}^{\alpha \beta},
$$

we see that we solve at once the d'Alembertian equation (39a) and the coordinate condition (39b). That is, we have succeeded in finding a solution of the field equations at the $n$-th post-Minkowskian order. By induction the same method applies to any order $n$, and, therefore, we have constructed a complete post-Minkowskian series (38) based on the linearized approximation $h_{(1)}^{\alpha \beta}$ given by Eqs. (35) - (37). The previous procedure constitutes an algorithm, which can be (and has recently been $[74,197]$ ) implemented by an algebraic computer programme. Again, note that this algorithm permits solving the full Einstein field equations together with the gauge condition (i.e., not only the relaxed field equations).

\subsection{Generality of the MPM solution}

We have a solution, but is that a general solution? The answer, "yes", is provided by the following result [57].

Theorem 2. The most general solution of the harmonic-coordinates Einstein field equations in the vacuum region outside an isolated source, admitting some post-Minkowskian and multipolar expansions, is given by the previous construction as

$$
h_{e x t}^{\alpha \beta}=\sum_{n=1}^{+\infty} G^{n} h_{(n)}^{\alpha \beta}\left[\mathrm{I}_{L}, \mathrm{~J}_{L}, \ldots, \mathrm{Z}_{L}\right] .
$$

It depends on two sets of arbitrary STF-tensorial functions of time $\mathrm{I}_{L}(u)$ and $\mathrm{J}_{L}(u)$ (satisfying the conservation laws) defined by Eqs. (36), and on four supplementary functions $\mathrm{W}_{L}(u), \ldots, \mathrm{Z}_{L}(u)$ parametrizing the gauge vector (37).

The proof is quite easy. With Eq. (49) we obtained a particular solution of the system of equations (39). To it we should add the most general solution of the corresponding homogeneous system of equations, which is obtained by setting $\Lambda_{(n)}^{\alpha \beta}=0$ into Eqs. (39). But this homogeneous system of equations is nothing but the linearized vacuum field equations (33), to which we know the most general solution $h_{(1)}^{\alpha \beta}$ given by Eqs. (35)-(37). Thus, we must add to our particular solution $h_{(n)}^{\alpha \beta}$ a general homogeneous solution that is necessarily of the type $h_{(1)}^{\alpha \beta}\left[\delta \mathrm{I}_{L}, \ldots, \delta \mathrm{Z}_{L}\right]$, where $\delta \mathrm{I}_{L}, \ldots, \delta \mathrm{Z}_{L}$ denote some corrections to the multipole moments at the $n$-th post-Minkowskian order (with the monopole $\delta \mathrm{I}$ and dipoles $\delta \mathrm{I}_{i}, \delta \mathrm{J}_{i}$ being constant). It is then clear, since precisely the linearized metric is a linear functional of all these moments, that the previous corrections to the moments can be absorbed into a re-definition of the original ones $\mathrm{I}_{L}, \ldots, \mathrm{Z}_{L}$ by posing

$$
\mathrm{I}_{L}^{\text {new }}=\mathrm{I}_{L}+G^{n-1} \delta \mathrm{I}_{L}
$$




$$
\mathrm{Z}_{L}^{\text {new }}=\mathrm{Z}_{L}+G^{n-1} \delta \mathrm{Z}_{L}
$$

After re-arranging the metric in terms of these new moments, taking into account the fact that the precision of the metric is limited to the $n$-th post-Minkowskian order, and dropping the superscript "new", we find exactly the same solution as the one we had before (indeed, the moments are arbitrary functions of time) - hence the proof.

The six sets of multipole moments $\mathrm{I}_{L}(u), \ldots, \mathrm{Z}_{L}(u)$ contain the physical information about any isolated source as seen in its exterior. However, as we now discuss, it is always possible to find two, and only two, sets of multipole moments, $\mathrm{M}_{L}(u)$ and $\mathrm{S}_{L}(u)$, for parametrizing the most general isolated source as well. The route for constructing such a general solution is to get rid of the moments $\mathrm{W}_{L}, \mathrm{X}_{L}, \mathrm{Y}_{L}, \mathrm{Z}_{L}$ at the linearized level by performing the linearized gauge transformation $\delta x^{\alpha}=\varphi_{(1)}^{\alpha}$, where $\varphi_{(1)}^{\alpha}$ is the gauge vector given by Eqs. (37). So, at the linearized level, we have only the two types of moments $\mathrm{M}_{L}$ and $\mathrm{S}_{L}$, parametrizing $k_{(1)}^{\alpha \beta}$ by the same formulas as in Eqs. (36). We must be careful to denote these moments with names different from $\mathrm{I}_{L}$ and $\mathrm{J}_{L}$ because they will ultimately correspond to a different physical source. Then we apply exactly the same postMinkowskian algorithm, following the formulas (45)-(49) as we did above, but starting from the gauge-transformed linear metric $k_{(1)}^{\alpha \beta}$ instead of $h_{(1)}^{\alpha \beta}$. The result of the iteration is therefore some

$$
k_{\mathrm{ext}}^{\alpha \beta}=\sum_{n=1}^{+\infty} G^{n} k_{(n)}^{\alpha \beta}\left[\mathrm{M}_{L}, \mathrm{~S}_{L}\right] .
$$

Obviously this post-Minkowskian algorithm yields some simpler calculations as we have only two multipole moments to iterate. The point is that one can show that the resulting metric (52) is isometric to the original one (50) if and only if the so-called canonical moments $\mathrm{M}_{L}$ and $\mathrm{S}_{L}$ are related to the source moments $\mathrm{I}_{L}, \mathrm{~J}_{L}, \ldots, \mathrm{Z}_{L}$ by some (quite involved) non-linear equations. We shall give in Eqs. (97) - (98) the most up to date relations we have between these moments. Therefore, the most general solution of the field equations, modulo a coordinate transformation, can be obtained by starting from the linearized metric $k_{(1)}^{\alpha \beta}\left[\mathrm{M}_{L}, \mathrm{~S}_{L}\right]$ instead of the more complicated $k_{(1)}^{\alpha \beta}\left[\mathrm{I}_{L}, \mathrm{~J}_{L}\right]+\partial^{\alpha} \varphi_{(1)}^{\beta}+\partial^{\beta} \varphi_{(1)}^{\alpha}-\eta^{\alpha \beta} \partial_{\mu} \varphi_{(1)}^{\mu}$, and continuing the post-Minkowskian calculation.

So why not consider from the start that the best description of the isolated source is provided by only the two types of multipole moments, $\mathrm{M}_{L}$ and $\mathrm{S}_{L}$, instead of the six types, $\mathrm{I}_{L}, \mathrm{~J}_{L}, \ldots, \mathrm{Z}_{L}$ ? The reason is that we shall determine in Theorem 6 below the explicit closed-form expressions of the six source moments $\mathrm{I}_{L}, \mathrm{~J}_{L}, \ldots, \mathrm{Z}_{L}$, but that, by contrast, it seems to be impossible to obtain some similar closed-form expressions for the canonical moments $\mathrm{M}_{L}$ and $\mathrm{S}_{L}$. The only thing we can do is to write down the explicit non-linear algorithm that computes $\mathrm{M}_{L}, \mathrm{~S}_{L}$ starting from $\mathrm{I}_{L}, \mathrm{~J}_{L}, \ldots, \mathrm{Z}_{L}$. In consequence, it is better to view the moments $\mathrm{I}_{L}, \mathrm{~J}_{L}, \ldots, \mathrm{Z}_{L}$ as more "fundamental" than $\mathrm{M}_{L}$ and $\mathrm{S}_{L}$, in the sense that they appear to be more tightly related to the description of the source, since they admit closed-form expressions as some explicit integrals over the source. Hence, we choose to refer collectively to the six moments $\mathrm{I}_{L}, \mathrm{~J}_{L}, \ldots, \mathrm{Z}_{L}$ as the multipole moments of the source. This being said, the moments $\mathrm{M}_{L}$ and $\mathrm{S}_{L}$ are generally very useful in practical computations because they yield a simpler post-Minkowskian iteration. Then, one can generally come back to the more fundamental source-rooted moments by using the fact that $\mathrm{M}_{L}$ and $\mathrm{S}_{L}$ differ from the corresponding $\mathrm{I}_{L}$ and $\mathrm{J}_{L}$ only by high-order post-Newtonian terms like $2.5 \mathrm{PN}$; see Eqs. (97) - (98) below. Indeed, this is to be expected because the physical difference between both types of moments stems only from non-linearities. 


\subsection{Near-zone and far-zone structures}

In our presentation of the post-Minkowskian algorithm (45)-(49) we have for the moment omitted a crucial recursive hypothesis, which is required in order to prove that at each post-Minkowskian order $n$, the inverse d'Alembertian operator can be applied in the way we did - notably that the $B$-dependent retarded integral can be analytically continued down to a neighbourhood of $B=0$. This hypothesis is that the "near-zone" expansion, i.e., when $r \rightarrow 0$, of each one of the postMinkowskian coefficients $h_{(n)}$ has a certain structure (here we often omit the space-time indices $\alpha \beta)$; this hypothesis is established as a theorem once the mathematical induction succeeds.

Theorem 3. The general structure of the expansion of the post-Minkowskian exterior metric in the near-zone (when $r \rightarrow 0$ ) is of the type: $\forall N \in \mathbb{N},{ }^{20}$

$$
h_{(n)}(\mathbf{x}, t)=\sum \hat{n}_{L} r^{m}(\ln r)^{p} F_{L, m, p, n}(t)+o\left(r^{N}\right),
$$

where $m \in \mathbb{Z}$, with $m_{0} \leqslant m \leqslant N$ (and $m_{0}$ becoming more and more negative as $n$ grows), $p \in \mathbb{N}$ with $p \leqslant n-1$. The functions $F_{L, m, p, n}$ are multilinear functionals of the source multipole moments $\mathrm{I}_{L}, \ldots, \mathrm{Z}_{L}$.

For the proof see Ref. [57]. As we see, the near-zone expansion involves, besides the simple powers of $r$, some powers of the logarithm of $r$, with a maximal power of $n-1$. As a corollary of that theorem, we find, by restoring all the powers of $c$ in Eq. (53) and using the fact that each $r$ goes into the combination $r / c$, that the general structure of the post-Newtonian expansion $(c \rightarrow+\infty)$ is necessarily of the type

$$
h_{(n)}(c) \simeq \sum_{p, q \in \mathbb{N}} \frac{(\ln c)^{p}}{c^{q}}
$$

where $p \leqslant n-1$ (and $q \geqslant 2$ ). The post-Newtonian expansion proceeds not only with the normal powers of $1 / c$ but also with powers of the logarithm of $c$ [57]. It is remarkable that there is no more complicated structure like for instance $\ln (\ln c)$.

Paralleling the structure of the near-zone expansion, we have a similar result concerning the structure of the far-zone expansion at Minkowskian future null infinity, i.e., when $r \rightarrow+\infty$ with $u=t-r / c=$ const: $\forall N \in \mathbb{N}$,

$$
h_{(n)}(\mathbf{x}, t)=\sum \frac{\hat{n}_{L}(\ln r)^{p}}{r^{k}} G_{L, k, p, n}(u)+o\left(\frac{1}{r^{N}}\right),
$$

where $k, p \in \mathbb{N}$, with $1 \leqslant k \leqslant N$, and where, likewise in the near-zone expansion (53), some powers of logarithms, such that $p \leqslant n-1$, appear. The appearance of logarithms in the far-zone expansion of the harmonic-coordinates metric has been known since the work of Fock [202]. One knows also that this is a coordinate effect, because the study of the "asymptotic" structure of space-time at future null infinity by Bondi et al. [93], Sachs [368], and Penrose [337, 338], has revealed the existence of other coordinate systems that avoid the appearance of any logarithms: the so-called radiative coordinates, in which the far-zone expansion of the metric proceeds with simple powers of the inverse radial distance. Hence, the logarithms are simply an artifact of the use of harmonic coordinates [252, 304, 41]. The following theorem, proved in Ref. [41], shows that our general construction of the metric in the exterior of the source, when developed at future null infinity, is consistent with the Bondi-Sachs-Penrose [93, 368, 337, 338] approach to gravitational radiation.

20 We employ the Landau symbol $o$ for remainders with its standard meaning. Thus, $f(r)=o[g(r)]$ when $r \rightarrow 0$ means that $f(r) / g(r) \rightarrow 0$ when $r \rightarrow 0$. Furthermore, we generally assume some differentiability properties such as $\mathrm{d}^{n} f(r) / \mathrm{d} r^{n}=o\left[g(r) / r^{n}\right]$ 
Theorem 4. The most general multipolar-post-Minkowskian solution, stationary in the past [see Eq. (29)], admits some radiative coordinates $(T, \mathbf{X})$, for which the expansion at future null infinity, $R \rightarrow+\infty$ with $U \equiv T-R / c=$ const, takes the form

$$
H_{(n)}(\mathbf{X}, T)=\sum \frac{\hat{N}_{L}}{R^{k}} K_{L, k, n}(U)+\mathcal{O}\left(\frac{1}{R^{N}}\right) .
$$

The functions $K_{L, k, n}$ are computable functionals of the source multipole moments. In radiative coordinates the retarded time $U$ is a null coordinate in the asymptotic limit. The metric $H_{\mathrm{ext}}^{\alpha \beta}=$ $\sum_{n \geqslant 1} G^{n} H_{(n)}^{\alpha \beta}$ is asymptotically simple in the sense of Penrose [337, 338, 220], perturbatively to any post-Minkowskian order.

Proof. We introduce a linearized "radiative" metric by performing a gauge transformation of the harmonic-coordinates metric defined by Eqs. (35)-(37), namely

$$
H_{(1)}^{\alpha \beta}=h_{(1)}^{\alpha \beta}+\partial^{\alpha} \xi_{(1)}^{\beta}+\partial^{\beta} \xi_{(1)}^{\alpha}-\eta^{\alpha \beta} \partial_{\mu} \xi_{(1)}^{\mu},
$$

where the gauge vector $\xi_{(1)}^{\alpha}$ is

$$
\xi_{(1)}^{\alpha}=\frac{2 \mathrm{M}}{c^{2}} \eta^{0 \alpha} \ln \left(\frac{r}{r_{0}}\right) .
$$

This gauge transformation is non-harmonic:

$$
\partial_{\mu} H_{(1)}^{\alpha \mu}=\square \xi_{(1)}^{\alpha}=\frac{2 \mathrm{M}}{c^{2} r^{2}} \eta^{0 \alpha}
$$

Its effect is to correct for the well-known logarithmic deviation of the retarded time in harmonic coordinates, with respect to the true space-time characteristic or light cones. After the change of gauge, the coordinate $u=t-r / c$ coincides with a null coordinate at the linearized level. ${ }^{21}$ This is the requirement to be satisfied by a linearized metric so that it can constitute the linearized approximation to a full (post-Minkowskian) radiative field [304]. One can easily show that, at the dominant order when $r \rightarrow+\infty$,

$$
k_{\mu} k_{\nu} H_{(1)}^{\mu \nu}=\mathcal{O}\left(\frac{1}{r^{2}}\right)
$$

where $k^{\mu}=\eta^{\mu \nu} k_{\nu}=(1, \mathbf{n})$ is the outgoing Minkowskian null vector. Given any $n \geqslant 2$, let us recursively assume that we have obtained all the previous radiative post-Minkowskian coefficients $H_{(m)}^{\alpha \beta}$, i.e. $\forall m \leqslant n-1$, and that all of them satisfy

$$
k_{\mu} k_{\nu} H_{(m)}^{\mu \nu}=\mathcal{O}\left(\frac{1}{r^{2}}\right)
$$

From this induction hypothesis one can prove that the $n$-th post-Minkowskian source term $\Lambda_{(n)}^{\alpha \beta}=$ $\Lambda_{(n)}^{\alpha \beta}\left(H_{(1)}, \ldots, H_{(n-1)}\right)$ is such that

$$
\Lambda_{(n)}^{\alpha \beta}=\frac{k^{\alpha} k^{\beta}}{r^{2}} \sigma_{(n)}(u, \mathbf{n})+\mathcal{O}\left(\frac{1}{r^{3}}\right) .
$$

To the leading order this term takes the classic form of the stress-energy tensor of massless particles, with $\sigma_{(n)}$ being proportional to the power in the massless waves. One can show that all the problems

\footnotetext{
21 In this proof the coordinates are considered as dummy variables denoted $(t, r)$. At the end, when we obtain the radiative metric, we shall denote the associated radiative coordinates by $(T, R)$.
} 
with the appearance of logarithms come from the retarded integral of the terms in Eq. (62) that behave like $1 / r^{2}$ : See indeed the integration formula (83), which behaves like $\ln r / r$ at infinity. But now, thanks to the particular index structure of the term (62), we can correct for the effect by adjusting the gauge at the $n$-th post-Minkowskian order. We pose, as a gauge vector,

$$
\xi_{(n)}^{\alpha}=\mathcal{F} \mathcal{P} \square_{\text {ret }}^{-1}\left[\frac{k^{\alpha}}{2 r^{2}} \int_{-\infty}^{u} \mathrm{~d} v \sigma_{(n)}(v, \mathbf{n})\right],
$$

where $\mathcal{F P}$ refers to the same finite part operation as in Eq. (45). This vector is such that the logarithms that will appear in the corresponding gauge terms cancel out the logarithms coming from the retarded integral of the source term (62); see Ref. [41] for the details. Hence, to the $n$-th post-Minkowskian order, we define the radiative metric as

$$
H_{(n)}^{\alpha \beta}=U_{(n)}^{\alpha \beta}+V_{(n)}^{\alpha \beta}+\partial^{\alpha} \xi_{(n)}^{\beta}+\partial^{\beta} \xi_{(n)}^{\alpha}-\eta^{\alpha \beta} \partial_{\mu} \xi_{(n)}^{\mu} .
$$

Here $U_{(n)}^{\alpha \beta}$ and $V_{(n)}^{\alpha \beta}$ denote the quantities that are the analogues of $u_{(n)}^{\alpha \beta}$ and $v_{(n)}^{\alpha \beta}$, which were introduced into the harmonic-coordinates algorithm: See Eqs. (45)-(48). In particular, these quantities are constructed in such a way that the sum $U_{(n)}^{\alpha \beta}+V_{(n)}^{\alpha \beta}$ is divergence-free, so we see that the radiative metric does not obey the harmonic-gauge condition, but instead

$$
\partial_{\mu} H_{(n)}^{\alpha \mu}=\square \xi_{(n)}^{\alpha}=\frac{k^{\alpha}}{2 r^{2}} \int_{-\infty}^{u} \mathrm{~d} v \sigma_{(n)}(v, \mathbf{n}) .
$$

The far-zone expansion of the latter metric is of the type (56), i.e., is free of any logarithms, and the retarded time in these coordinates tends asymptotically toward a null coordinate at future null infinity. The property of asymptotic simplicity, in the form given by Geroch \& Horowitz [220], is proved by introducing the usual conformal factor $\Omega=1 / R$ in radiative coordinates [41]. Finally, it can be checked that the metric so constructed, which is a functional of the source multipole moments $\mathrm{I}_{L}, \ldots, \mathrm{Z}_{L}$ (from the definition of the algorithm), is as general as the general harmoniccoordinate metric of Theorem 2, since it merely differs from it by a coordinate transformation $(t, \mathbf{x}) \longrightarrow(T, \mathbf{X})$, where $(t, \mathbf{x})$ are the harmonic coordinates and $(T, \mathbf{X})$ the radiative ones, together with a re-definition of the multipole moments.

\section{Asymptotic Gravitational Waveform}

\subsection{The radiative multipole moments}

The leading-order term $1 / R$ of the metric in radiative coordinates $(T, \mathbf{X})$ as given in Theorem 4 , neglecting $\mathcal{O}\left(1 / R^{2}\right)$, yields the operational definition of two sets of STF radiative multipole moments, mass-type $\mathrm{U}_{L}(U)$ and current-type $\mathrm{V}_{L}(U)$. As we have seen, radiative coordinates are such that the retarded time $U \equiv T-R / c$ becomes asymptotically a null coordinate at future null infinity. The radiative moments are defined from the spatial components $i j$ of the metric in a transverse-traceless (TT) radiative coordinate system. By definition, we have [403]

$$
\begin{aligned}
H_{i j}^{\mathrm{TT}}(U, \mathbf{X}) & =\frac{4 G}{c^{2} R} \mathcal{P}_{i j a b}(N) \sum_{\ell=2}^{+\infty} \frac{1}{c^{\ell} \ell !}\left\{N_{L-2} \mathrm{U}_{a b L-2}(U)-\frac{2 \ell}{c(\ell+1)} N_{c L-2} \epsilon_{c d(a} \mathrm{V}_{b) d L-2}(U)\right\} \\
& +\mathcal{O}\left(\frac{1}{R^{2}}\right) .
\end{aligned}
$$

We have formally re-summed the whole post-Minkowskian series in Eq. (56) from $n=1$ up to $+\infty$. As before we denote for instance $N_{L-2}=N_{i_{1}} \cdots N_{i_{\ell-2}}$ and so on, where $N_{i}=(\boldsymbol{N})_{i}$ and 
$\boldsymbol{N}=\boldsymbol{X} / R$. The TT algebraic projection operator $\mathcal{P}_{i j a b}$ has already been defined at the occasion of the quadrupole-moment formalism in Eq. (2); and obviously the multipole decomposition (66) represents the generalization of the quadrupole formalism. Notice that the meaning of Eq. (66) is for the moment rather empty, because we do not yet know how to relate the radiative moments to the actual source parameters. Only at the Newtonian level do we know this relation, which is

$$
\mathrm{U}_{i j}(U)=\mathrm{Q}_{i j}^{(2)}(U)+\mathcal{O}\left(\frac{1}{c^{2}}\right)
$$

where $\mathrm{Q}_{i j}$ is the Newtonian quadrupole moment (3). Associated to the asymptotic waveform (66) we can compute by standard methods the total energy flux $\mathcal{F}=(\mathrm{d} E / \mathrm{d} U)^{\mathrm{GW}}$ and angular momentum flux $\mathcal{G}_{i}=\left(\mathrm{dJ}_{i} / \mathrm{d} U\right)^{\mathrm{GW}}$ in gravitational waves [403]:

$$
\begin{aligned}
\mathcal{F} & =\sum_{\ell=2}^{+\infty} \frac{G}{c^{2 \ell+1}}\left\{\frac{(\ell+1)(\ell+2)}{(\ell-1) \ell \ell !(2 \ell+1) ! !} \mathrm{U}_{L}^{(1)} \mathrm{U}_{L}^{(1)}+\frac{4 \ell(\ell+2)}{c^{2}(\ell-1)(\ell+1) !(2 \ell+1) ! !} \mathrm{V}_{L}^{(1)} \mathrm{V}_{L}^{(1)}\right\} . \\
\mathcal{G}_{i} & =\epsilon_{i a b} \sum_{\ell=2}^{+\infty} \frac{G}{c^{2 \ell+1}}\left\{\frac{(\ell+1)(\ell+2)}{(\ell-1) \ell !(2 \ell+1) ! !} \mathrm{U}_{a L-1} \mathrm{U}_{b L-1}^{(1)}+\frac{4 \ell^{2}(\ell+2)}{c^{2}(\ell-1)(\ell+1) !(2 \ell+1) ! !} \mathrm{V}_{a L-1} \mathrm{~V}_{b L-1}^{(1)}\right\} .
\end{aligned}
$$

Next we introduce two unit polarization vectors $\boldsymbol{P}$ and $\boldsymbol{Q}$, orthogonal and transverse to the direction of propagation $\boldsymbol{N}$ (hence $N_{i} N_{j}+P_{i} P_{j}+Q_{i} Q_{j}=\delta_{i j}$ ). Our convention for the choice of $\boldsymbol{P}$ and $\boldsymbol{Q}$ will be clarified in Section 9.4. Then the two "plus" and "cross" polarization states of the asymptotic waveform are defined by

$$
\begin{aligned}
& h_{+}=\frac{1}{2}\left(P_{i} P_{j}-Q_{i} Q_{j}\right) H_{i j}^{\mathrm{TT}}, \\
& h_{\times}=\frac{1}{2}\left(P_{i} Q_{j}+P_{j} Q_{i}\right) H_{i j}^{\mathrm{TT}} .
\end{aligned}
$$

Although the multipole decomposition (66) is completely general, it will also be important, having in view the comparison between the post-Newtonian and numerical results (see for instance Refs. [107, 34, 237, 97, 98]), to consider separately the various modes $(\ell, m)$ of the asymptotic waveform as defined with respect to a basis of spin-weighted spherical harmonics of weight -2 . Those harmonics are function of the spherical angles $(\theta, \phi)$ defining the direction of propagation $\boldsymbol{N}$, and given by

$$
\begin{aligned}
Y_{(-2)}^{\ell m} & =\sqrt{\frac{2 \ell+1}{4 \pi}} d^{\ell m}(\theta) e^{\mathrm{i} m \phi}, \\
d^{\ell m} & =\sum_{k=k_{1}}^{k_{2}} \frac{(-)^{k}}{k !} e_{k}^{\ell m}\left(\cos \frac{\theta}{2}\right)^{2 \ell+m-2 k-2}\left(\sin \frac{\theta}{2}\right)^{2 k-m+2}, \\
e_{k}^{\ell m} & =\frac{\sqrt{(\ell+m) !(\ell-m) !(\ell+2) !(\ell-2) !}}{(k-m+2) !(\ell+m-k) !(\ell-k-2) !},
\end{aligned}
$$

where $k_{1}=\max (0, m-2)$ and $k_{2}=\min (\ell+m, \ell-2)$. We thus decompose $h_{+}$and $h_{\times}$onto the basis of such spin-weighted spherical harmonics, which means (see e.g., [107, 272])

$$
h_{+}-\mathrm{i} h_{\times}=\sum_{\ell=2}^{+\infty} \sum_{m=-\ell}^{\ell} h^{\ell m} Y_{(-2)}^{\ell m}(\theta, \phi) .
$$


Using the orthonormality properties of these harmonics we can invert the latter decomposition and obtain the separate modes $h^{\ell m}$ from a surface integral,

$$
h^{\ell m}=\int \mathrm{d} \Omega\left[h_{+}-\mathrm{i} h_{\times}\right] \bar{Y}_{(-2)}^{\ell m}(\theta, \phi)
$$

where the overline refers to the complex conjugation. On the other hand, we can also relate $h^{\ell m}$ to the radiative multipole moments $\mathrm{U}_{L}$ and $\mathrm{V}_{L}$. The result is

$$
h^{\ell m}=-\frac{G}{\sqrt{2} R c^{\ell+2}}\left[\mathrm{U}^{\ell m}-\frac{\mathrm{i}}{c} \mathrm{~V}^{\ell m}\right] \text {, }
$$

where $\mathrm{U}^{\ell m}$ and $\mathrm{V}^{\ell m}$ denote the radiative mass and current moments in standard (non-STF) guise. These are related to the STF moments by

$$
\begin{aligned}
\mathrm{U}^{\ell m} & =\frac{4}{\ell !} \sqrt{\frac{(\ell+1)(\ell+2)}{2 \ell(\ell-1)}} \alpha_{L}^{\ell m} \mathrm{U}_{L}, \\
\mathrm{~V}^{\ell m} & =-\frac{8}{\ell !} \sqrt{\frac{\ell(\ell+2)}{2(\ell+1)(\ell-1)}} \alpha_{L}^{\ell m} \mathrm{~V}_{L} .
\end{aligned}
$$

Here $\alpha_{L}^{\ell m}$ denotes the STF tensor connecting together the usual basis of spherical harmonics $Y^{\ell m}$ to the set of STF tensors $\hat{N}_{L}=N_{\left\langle i_{1}\right.} \ldots N_{\left.i_{\ell}\right\rangle}$ (where the brackets indicate the STF projection). Indeed both $Y^{\ell m}$ and $\hat{N}_{L}$ are basis of an irreducible representation of weight $\ell$ of the rotation group; the two basis are related by ${ }^{22}$

$$
\begin{aligned}
\hat{N}_{L}(\theta, \phi) & =\sum_{m=-\ell}^{\ell} \alpha_{L}^{\ell m} Y^{\ell m}(\theta, \phi), \\
Y^{\ell m}(\theta, \phi) & =\frac{(2 \ell+1) ! !}{4 \pi l !} \bar{\alpha}_{L}^{\ell m} \hat{N}_{L}(\theta, \phi) .
\end{aligned}
$$

In Section 9.5 we shall present all the modes $(\ell, m)$ of gravitational waves from inspiralling compact binaries up to $3 \mathrm{PN}$ order, and even $3.5 \mathrm{PN}$ order for the dominant mode $(2,2)$.

\subsection{Gravitational-wave tails and tails-of-tails}

We learned from Theorem 4 the general method which permits the computation of the radiative multipole moments $\mathrm{U}_{L}, \mathrm{~V}_{L}$ in terms of the source moments $\mathrm{I}_{L}, \mathrm{~J}_{L}, \ldots, \mathrm{Z}_{L}$, or in terms of the intermediate canonical moments $\mathrm{M}_{L}, \mathrm{~S}_{L}$ discussed in Section 2.4. We shall now show that the relation between $\mathrm{U}_{L}, \mathrm{~V}_{L}$ and $\mathrm{M}_{L}, \mathrm{~S}_{L}$ (say) includes tail effects starting at the relative $1.5 \mathrm{PN}$ order.

Tails are due to the back-scattering of multipolar waves off the Schwarzschild curvature generated by the total mass monopole $\mathrm{M}$ of the source. They correspond to the non-linear interaction between $\mathrm{M}$ and the multipole moments $\mathrm{M}_{L}$ and $\mathrm{S}_{L}$, and are given by some non-local integrals, extending over the past history of the source. At the $1.5 \mathrm{PN}$ order we find $[59,44]$

$$
\mathrm{U}_{L}(U)=\mathrm{M}_{L}^{(\ell)}(U)+\frac{2 G \mathrm{M}}{c^{3}} \int_{0}^{+\infty} \mathrm{d} \tau \mathrm{M}_{L}^{(\ell+2)}(U-\tau)\left[\ln \left(\frac{c \tau}{2 r_{0}}\right)+\kappa_{\ell}\right]+\mathcal{O}\left(\frac{1}{c^{5}}\right)
$$

22 The STF tensorial coefficient $\alpha_{L}^{\ell m}$ can be computed as $\alpha_{L}^{\ell m}=\int \mathrm{d} \Omega \hat{N}_{L} \bar{Y}^{\ell m}$. Our notation is related to that used in Refs. [403, 272] by $\mathcal{Y}_{L}^{\ell m}=\frac{(2 \ell+1) ! !}{4 \pi l !} \bar{\alpha}_{L}^{\ell m}$. 


$$
\mathrm{V}_{L}(U)=\mathrm{S}_{L}^{(\ell)}(U)+\frac{2 G \mathrm{M}}{c^{3}} \int_{0}^{+\infty} \mathrm{d} \tau \mathrm{S}_{L}^{(\ell+2)}(U-\tau)\left[\ln \left(\frac{c \tau}{2 r_{0}}\right)+\pi_{\ell}\right]+\mathcal{O}\left(\frac{1}{c^{5}}\right)
$$

where $r_{0}$ is the length scale introduced in Eq. (42), and the constants $\kappa_{\ell}$ and $\pi_{\ell}$ are given by

$$
\begin{aligned}
& \kappa_{\ell}=\frac{2 \ell^{2}+5 \ell+4}{\ell(\ell+1)(\ell+2)}+\sum_{k=1}^{\ell-2} \frac{1}{k}, \\
& \pi_{\ell}=\frac{\ell-1}{\ell(\ell+1)}+\sum_{k=1}^{\ell-1} \frac{1}{k} .
\end{aligned}
$$

Recall from the gauge vector $\xi_{(1)}^{\alpha}$ found in Eq. (58) that the retarded time $U=T-R / c$ in radiative coordinates is related to the retarded time $u=t-r / c$ in harmonic coordinates by

$$
U=u-\frac{2 G \mathrm{M}}{c^{3}} \ln \left(\frac{r}{r_{0}}\right)+\mathcal{O}\left(G^{2}\right)
$$

Inserting $U$ as given by Eq. (78) into Eqs. (76) we obtain the radiative moments expressed in terms of "source-rooted" harmonic coordinates $(t, r)$, e.g.,

$$
\mathrm{U}_{L}(U)=\mathrm{M}_{L}^{(\ell)}(u)+\frac{2 G \mathrm{M}}{c^{3}} \int_{0}^{+\infty} \mathrm{d} \tau \mathrm{M}_{L}^{(\ell+2)}(u-\tau)\left[\ln \left(\frac{c \tau}{2 r}\right)+\kappa_{\ell}\right]+\mathcal{O}\left(\frac{1}{c^{5}}\right) .
$$

The remainder $\mathcal{O}\left(G^{2}\right)$ in Eq. (78) is negligible here. This expression no longer depends on the constant $r_{0}$, i.e., we find that $r_{0}$ gets replaced by $r$. If we now replace the harmonic coordinates $(t, r)$ to some new ones, such as, for instance, some "Schwarzschild-like" coordinates $\left(t^{\prime}, r^{\prime}\right)$ such that $t^{\prime}=t$ and $r^{\prime}=r+G \mathrm{M} / c^{2}$ (and $u^{\prime}=u-G \mathrm{M} / c^{3}$ ), we get

$$
\mathrm{U}_{L}(U)=\mathrm{M}_{L}^{(\ell)}\left(u^{\prime}\right)+\frac{2 G \mathrm{M}}{c^{3}} \int_{0}^{+\infty} \mathrm{d} \tau \mathrm{M}_{L}^{(l+2)}\left(u^{\prime}-\tau\right)\left[\ln \left(\frac{c \tau}{2 r^{\prime}}\right)+\kappa_{\ell}^{\prime}\right]+\mathcal{O}\left(\frac{1}{c^{5}}\right),
$$

where $\kappa_{\ell}^{\prime}=\kappa_{\ell}+1 / 2$. This shows that the constant $\kappa_{\ell}$ (and $\pi_{\ell}$ as well) depends on the choice of source-rooted coordinates $(t, r)$ : For instance, we have $\kappa_{2}=11 / 12$ in harmonic coordinates from Eq. (77a), but $\kappa_{2}^{\prime}=17 / 12$ in Schwarzschild coordinates [345].

The tail integrals in Eqs. (76) involve all the instants from $-\infty$ in the past up to the current retarded time $U$. However, strictly speaking, they do not extend up to infinite past, since we have assumed in Eq. (29) that the metric is stationary before the date $-\mathcal{T}$. The range of integration of the tails is therefore limited a priori to the time interval $[-\mathcal{T}, U]$. But now, once we have derived the tail integrals, thanks to the latter technical assumption of stationarity in the past, we can argue that the results are in fact valid in more general situations for which the field has never been stationary. We have in mind the case of two bodies moving initially on some unbound (hyperbolic-like) orbit, and which capture each other, because of the loss of energy by gravitational radiation, to form a gravitationally bound system around time $-\mathcal{T}$.

In this situation let us check, using a simple Newtonian model for the behaviour of the multipole moment $\mathrm{M}_{L}(U-\tau)$ when $\tau \rightarrow+\infty$, that the tail integrals, when assumed to extend over the whole time interval $[-\infty, U]$, remain perfectly well-defined (i.e., convergent) at the integration bound $\tau=+\infty$. Indeed it can be shown [180] that the motion of initially free particles interacting gravitationally is given by $x^{i}(U-\tau)=V^{i} \tau+W^{i} \ln \tau+X^{i}+o(1)$, where $V^{i}, W^{i}$ and $X^{i}$ denote constant vectors, and $o(1) \rightarrow 0$ when $\tau \rightarrow+\infty$. From that physical assumption we find that the multipole moments behave when $\tau \rightarrow+\infty$ like

$$
\mathrm{M}_{L}(U-\tau)=A_{L} \tau^{\ell}+B_{L} \tau^{\ell-1} \ln \tau+C_{L} \tau^{\ell-1}+o\left(\tau^{\ell-1}\right),
$$


where $A_{L}, B_{L}$ and $C_{L}$ are constant tensors. We used the fact that the moment $\mathrm{M}_{L}$ will agree at the Newtonian level with the standard expression for the $\ell$-th mass multipole moment $\mathrm{Q}_{L}$. The appropriate time derivatives of the moment appearing in Eq. (76a) are therefore dominantly like

$$
\mathrm{M}_{L}^{(\ell+2)}(U-\tau)=\frac{D_{L}}{\tau^{3}}+o\left(\tau^{-3}\right),
$$

which ensures that the tail integral is convergent. This fact can be regarded as an a posteriori justification of our a priori too restrictive assumption of stationarity in the past. Thus, this assumption does not seem to yield any physical restriction on the applicability of the final formulas. However, once again, we emphasize that the past-stationarity is appropriate for real astrophysical sources of gravitational waves which have been formed at a finite instant in the past.

To obtain the results (76), we must implement in details the post-Minkowskian algorithm presented in Section 2.3. Let us flash here some results obtained with such algorithm. Consider first the case of the interaction between the constant mass monopole moment M (or ADM mass) and the time-varying quadrupole moment $\mathrm{M}_{i j}$. This coupling will represent the dominant non-static multipole interaction in the waveform. For these moments we can write the linearized metric using Eq. (35) in which by definition of the "canonical" construction we insert the canonical moments $\mathrm{M}_{i j}$ in place of $\mathrm{I}_{i j}$ (notice that $\mathrm{M}=\mathrm{I}$ ). We must plug this linearized metric into the quadraticorder part $N^{\alpha \beta}(h, h)$ of the gravitational source term (24) - (25) and explicitly given by Eq. (26). This yields many terms; to integrate these following the algorithm [cf. Eq. (45)], we need some explicit formulas for the retarded integral of an extended (non-compact-support) source having some definite multipolarity $\ell$. A thorough account of the technical formulas necessary for handling the quadratic and cubic interactions is given in the Appendices of Refs. [50] and [48]. For the present computation the most crucial formula, needed to control the tails, corresponds to a source term behaving like $1 / r^{2}$ :

$$
\square_{\text {ret }}^{-1}\left[\frac{\hat{n}_{L}}{r^{2}} \mathrm{~F}(t-r)\right]=-\hat{n}_{L} \int_{1}^{+\infty} \mathrm{d} x Q_{\ell}(x) \mathrm{F}(t-r x),
$$

where $\mathrm{F}$ is any smooth function representing a time derivative of the quadrupole moment, and $Q_{\ell}$ denotes the Legendre function of the second kind. ${ }^{23}$ Note that there is no need to include a finite part operation $\mathcal{F P}$ in Eq. (83) as the integral is convergent. With the help of this and other formulas we obtain successively the objects defined in this algorithm by Eqs. (45) - (48) and finally obtain the quadratic metric (49) for that multipole interaction. The result is $[60]^{24}$

$$
\begin{aligned}
h_{(2)}^{00} & =\frac{\mathrm{M} n_{a b}}{r^{4}}\left[-21 \mathrm{M}_{a b}-21 r \mathrm{M}_{a b}^{(1)}+7 r^{2} \mathrm{M}_{a b}^{(2)}+10 r^{3} \mathrm{M}_{a b}^{(3)}\right] \\
& +8 \mathrm{M} n_{a b} \int_{1}^{+\infty} \mathrm{d} x Q_{2}(x) \mathrm{M}_{a b}^{(4)}(t-r x), \\
h_{(2)}^{0 i} & =\frac{\mathrm{M} n_{i a b}}{r^{3}}\left[-\mathrm{M}_{a b}^{(1)}-r \mathrm{M}_{a b}^{(2)}-\frac{1}{3} r^{2} \mathrm{M}_{a b}^{(3)}\right] \\
& +\frac{\mathrm{M} n_{a}}{r^{3}}\left[-5 \mathrm{M}_{a i}^{(1)}-5 r \mathrm{M}_{a i}^{(2)}+\frac{19}{3} r^{2} \mathrm{M}_{a i}^{(3)}\right]
\end{aligned}
$$

${ }^{23}$ The function $Q_{\ell}$ is given in terms of the Legendre polynomial $P_{\ell}$ by

$$
Q_{\ell}(x)=\frac{1}{2} \int_{-1}^{1} \frac{\mathrm{d} z P_{\ell}(z)}{x-z}=\frac{1}{2} P_{\ell}(x) \ln \left(\frac{x+1}{x-1}\right)-\sum_{j=1}^{\ell} \frac{1}{j} P_{\ell-j}(x) P_{j-1}(x) .
$$

In the complex plane there is a branch cut from $-\infty$ to 1 . The first equality is known as the Neumann formula for the Legendre function.

${ }^{24}$ We pose $c=1$ until the end of this section. 


$$
\begin{aligned}
+ & +\mathrm{M} n_{a} \int_{1}^{+\infty} \mathrm{d} x Q_{1}(x) \mathrm{M}_{a i}^{(4)}(t-r x), \\
h_{(2)}^{i j} & =\frac{\mathrm{M} n_{i j a b}}{r^{4}}\left[-\frac{15}{2} \mathrm{M}_{a b}-\frac{15}{2} r \mathrm{M}_{a b}^{(1)}-3 r^{2} \mathrm{M}_{a b}^{(2)}-\frac{1}{2} r^{3} \mathrm{M}_{a b}^{(3)}\right] \\
& +\frac{\mathrm{M} \delta_{i j} n_{a b}}{r^{4}}\left[-\frac{1}{2} \mathrm{M}_{a b}-\frac{1}{2} r \mathrm{M}_{a b}^{(1)}-2 r^{2} \mathrm{M}_{a b}^{(2)}-\frac{11}{6} r^{3} \mathrm{M}_{a b}^{(3)}\right] \\
& +\frac{\mathrm{M} n_{a(i}}{r^{4}}\left[6 \mathrm{M}_{j) a}+6 r \mathrm{M}_{j) a}^{(1)}+6 r^{2} \mathrm{M}_{j) a}^{(2)}+4 r^{3} \mathrm{M}_{j) a}^{(3)}\right] \\
& +\frac{\mathrm{M}}{r^{4}}\left[-\mathrm{M}_{i j}-r \mathrm{M}_{i j}^{(1)}-4 r^{2} \mathrm{M}_{i j}^{(2)}-\frac{11}{3} r^{3} \mathrm{M}_{i j}^{(3)}\right] \\
& +8 \mathrm{M} \int_{1}^{+\infty} \mathrm{d} x Q_{0}(x) \mathrm{M}_{i j}^{(4)}(t-r x) .
\end{aligned}
$$

The metric is composed of two types of terms: "instantaneous" ones depending on the values of the quadrupole moment at the retarded time $u=t-r$, and "hereditary" tail integrals, depending on all previous instants $t-r x<u$.

Let us investigate now the cubic interaction between two mass monopoles $\mathrm{M}$ with the mass quadrupole $\mathrm{M}_{i j}$. Obviously, the source term corresponding to this interaction will involve [see Eq. (40b)] cubic products of three linear metrics, say $h_{\mathrm{M}} \times h_{\mathrm{M}} \times h_{\mathrm{M}_{i j}}$, and quadratic products between one linear metric and one quadratic, say $h_{\mathrm{M}^{2}} \times h_{\mathrm{M}_{i j}}$ and $h_{\mathrm{M}} \times h_{\mathrm{MM}_{i j}}$. The latter case is the most tricky because the tails present in $h_{\mathrm{MM}_{i j}}$, which are given explicitly by Eqs. (84), will produce in turn some tails of tails in the cubic metric $h_{\mathrm{M}^{2} \mathrm{M}_{i j}}$. The computation is rather involved [48] but can now be performed by an algebraic computer programme [74, 197]. Let us just mention the most difficult of the needed integration formulas for this calculation: ${ }^{25}$

$$
\begin{aligned}
\mathcal{F} \mathcal{P} \square_{\text {ret }}^{-1} & {\left[\frac{\hat{n}_{L}}{r} \int_{1}^{+\infty} \mathrm{d} x Q_{m}(x) \mathrm{F}(t-r x)\right]=\hat{n}_{L} \int_{1}^{+\infty} \mathrm{d} y \mathrm{~F}^{(-1)}(t-r y) } \\
& \times\left\{Q_{\ell}(y) \int_{1}^{y} \mathrm{~d} x Q_{m}(x) \frac{\mathrm{d} P_{\ell}}{\mathrm{d} x}(x)+P_{\ell}(y) \int_{y}^{+\infty} \mathrm{d} x Q_{m}(x) \frac{\mathrm{d} Q_{\ell}}{\mathrm{d} x}(x)\right\},
\end{aligned}
$$

where $\mathrm{F}^{(-1)}$ is the time anti-derivative of $\mathrm{F}$. With this formula and others given in Ref. [48] we are able to obtain the closed algebraic form of the cubic metric for the multipole interaction $\mathrm{M} \times \mathrm{M} \times \mathrm{M}_{i j}$, at the leading order when the distance to the source $r \rightarrow \infty$ with $u=$ const. The result is ${ }^{26}$

$$
\begin{aligned}
h_{(3)}^{00} & =\frac{\mathrm{M}^{2} n_{a b}}{r} \int_{0}^{+\infty} \mathrm{d} \tau \mathrm{M}_{a b}^{(5)}\left[-4 \ln ^{2}\left(\frac{\tau}{2 r}\right)-4 \ln \left(\frac{\tau}{2 r}\right)+\frac{116}{21} \ln \left(\frac{\tau}{2 r_{0}}\right)-\frac{7136}{2205}\right] \\
& +o\left(\frac{1}{r}\right), \\
h_{(3)}^{0 i} & =\frac{\mathrm{M}^{2} \hat{n}_{i a b}}{r} \int_{0}^{+\infty} \mathrm{d} \tau \mathrm{M}_{a b}^{(5)}\left[-\frac{2}{3} \ln \left(\frac{\tau}{2 r}\right)-\frac{4}{105} \ln \left(\frac{\tau}{2 r_{0}}\right)-\frac{716}{1225}\right]
\end{aligned}
$$

25 The equation (85) has been obtained using a not so well known mathematical relation between the Legendre functions and polynomials:

$$
\frac{1}{2} \int_{-1}^{1} \frac{\mathrm{d} z P_{\ell}(z)}{\sqrt{(x y-z)^{2}-\left(x^{2}-1\right)\left(y^{2}-1\right)}}=Q_{\ell}(x) P_{\ell}(y)
$$

where $1 \leqslant y<x$ is assumed. See Appendix A in Ref. [48] for the proof. This relation constitutes a generalization of the Neumann formula (see the footnote 23).

26 The neglected remainders are indicated by $o(1 / r)$ rather than $\mathcal{O}\left(1 / r^{2}\right)$ because they contain powers of the logarithm of $r$; in fact they could be more accurately written as $o\left(r^{\epsilon-2}\right)$ for some $\epsilon \ll 1$. 


$$
\begin{aligned}
& +\frac{\mathrm{M}^{2} n_{a}}{r} \int_{0}^{+\infty} \mathrm{d} \tau \mathrm{M}_{a i}^{(5)}\left[-4 \ln ^{2}\left(\frac{\tau}{2 r}\right)-\frac{18}{5} \ln \left(\frac{\tau}{2 r}\right)+\frac{416}{75} \ln \left(\frac{\tau}{2 r_{0}}\right)-\frac{22724}{7875}\right] \\
& +o\left(\frac{1}{r}\right) \\
h_{(3)}^{i j} & =\frac{\mathrm{M}^{2} \hat{n}_{i j a b}}{r} \int_{0}^{+\infty} \mathrm{d} \tau \mathrm{M}_{a b}^{(5)}\left[-\ln \left(\frac{\tau}{2 r}\right)-\frac{191}{210}\right] \\
& +\frac{\mathrm{M}^{2} \delta_{i j} n_{a b}}{r} \int_{0}^{+\infty} \mathrm{d} \tau \mathrm{M}_{a b}^{(5)}\left[-\frac{80}{21} \ln \left(\frac{\tau}{2 r}\right)-\frac{32}{21} \ln \left(\frac{\tau}{2 r_{0}}\right)-\frac{296}{35}\right] \\
& +\frac{\mathrm{M}^{2} \hat{n}_{a(i}}{r} \int_{0}^{+\infty} \mathrm{d} \tau \mathrm{M}_{j) a}^{(5)}\left[\frac{52}{7} \ln \left(\frac{\tau}{2 r}\right)+\frac{104}{35} \ln \left(\frac{\tau}{2 r_{0}}\right)+\frac{8812}{525}\right] \\
& +\frac{\mathrm{M}^{2}}{r} \int_{0}^{+\infty} \mathrm{d} \tau \mathrm{M}_{i j}^{(5)}\left[-4 \ln { }^{2}\left(\frac{\tau}{2 r}\right)-\frac{24}{5} \ln \left(\frac{\tau}{2 r}\right)+\frac{76}{15} \ln \left(\frac{\tau}{2 r_{0}}\right)-\frac{198}{35}\right] \\
& +o\left(\frac{1}{r}\right),
\end{aligned}
$$

where all the moments $\mathrm{M}_{a b}$ are evaluated at the instant $u-\tau=t-r-\tau$. Notice that the logarithms in Eqs. (86) contain either the ratio $\tau / r$ or $\tau / r_{0}$. We shall discuss in Eqs. (93)-(94) below the interesting fate of the arbitrary constant $r_{0}$.

From Theorem 4, the presence of logarithms of $r$ in Eqs. (86) is an artifact of the harmonic coordinates $x^{\alpha}$, and it is convenient to gauge them away by introducing radiative coordinates $X^{\alpha}$ at future null infinity. For controling the leading $1 / R$ term at infinity, it is sufficient to take into account the linearized logarithmic deviation of the light cones in harmonic coordinates: $X^{\alpha}=x^{\alpha}+G \xi_{(1)}^{\alpha}+\mathcal{O}\left(G^{2}\right)$, where $\xi_{(1)}^{\alpha}$ is the gauge vector defined by Eq. (58) [see also Eq. (78)]. With this coordinate change one removes the logarithms of $r$ in Eqs. (86) and we obtain the radiative (or Bondi-type [93]) logarithmic-free expansion

$$
\begin{aligned}
H_{(3)}^{00} & =\frac{\mathrm{M}^{2} N_{a b}}{R} \int_{0}^{+\infty} \mathrm{d} \tau \mathrm{M}_{a b}^{(5)}\left[-4 \ln ^{2}\left(\frac{\tau}{2 r_{0}}\right)+3221 \ln \left(\frac{\tau}{2 r_{0}}\right)-\frac{7136}{2205}\right] \\
& +\mathcal{O}\left(\frac{1}{R^{2}}\right) \\
H_{(3)}^{0 i} & =\frac{\mathrm{M}^{2} \hat{N}_{i a b}}{R} \int_{0}^{+\infty} \mathrm{d} \tau \mathrm{M}_{a b}^{(5)}\left[-\frac{74}{105} \ln \left(\frac{\tau}{2 r_{0}}\right)-\frac{716}{1225}\right] \\
& +\frac{\mathrm{M}^{2} N_{a}}{R} \int_{0}^{+\infty} \mathrm{d} \tau \mathrm{M}_{a i}^{(5)}\left[-4 \ln ^{2}\left(\frac{\tau}{2 r_{0}}\right)+\frac{146}{75} \ln \left(\frac{\tau}{2 r_{0}}\right)-\frac{22724}{7875}\right] \\
& +\mathcal{O}\left(\frac{1}{R^{2}}\right), \\
H_{(3)}^{i j} & =\frac{\mathrm{M}^{2} \hat{N}_{i j a b}}{R} \int_{0}^{+\infty} \mathrm{d} \tau \mathrm{M}_{a b}^{(5)}\left[-\ln \left(\frac{\tau}{2 r_{0}}\right)-\frac{191}{210}\right] \\
& +\frac{\mathrm{M}^{2} \delta_{i j} N_{a b}}{R} \int_{0}^{+\infty} \mathrm{d} \tau \mathrm{M}_{a b}^{(5)}\left[-\frac{16}{3} \ln \left(\frac{\tau}{2 r_{0}}\right)-\frac{296}{35}\right] \\
& +\frac{\mathrm{M}^{2} \hat{N}_{a(i}}{R} \int_{0}^{+\infty} \mathrm{d} \tau \mathrm{M}_{j) a}^{(5)}\left[\frac{52}{5} \ln \left(\frac{\tau}{2 r_{0}}\right)+\frac{8812}{525}\right] \\
& +\frac{\mathrm{M}^{2}}{R} \int_{0}^{+\infty} \mathrm{d} \tau \mathrm{M}_{i j}^{(5)}\left[-4 \ln \left(\frac{\tau}{2 r_{0}}\right)+\frac{4}{15} \ln \left(\frac{\tau}{2 r_{0}}\right)-\frac{198}{35}\right]
\end{aligned}
$$




$$
+\mathcal{O}\left(\frac{1}{R^{2}}\right)
$$

where the moments are evaluated at time $U-\tau=T-R-\tau$. It is trivial to compute the contribution of the radiative moments corresponding to that metric. We find the "tail of tail" term which will be reported in Eq. (91) below.

\subsection{Radiative versus source moments}

We first give the result for the radiative quadrupole moment $\mathrm{U}_{i j}$ expressed as a functional of the intermediate canonical moments $\mathrm{M}_{L}, \mathrm{~S}_{L}$ up to $3.5 \mathrm{PN}$ order included. The long calculation follows from implementing the explicit MPM algorithm of Section 2.3 and yields various types of terms:

$$
\mathrm{U}_{i j}=\mathrm{U}_{i j}^{\mathrm{inst}}+\mathrm{U}_{i j}^{\mathrm{tail}}+\mathrm{U}_{i j}^{\text {tail-tail }}+\mathrm{U}_{i j}^{\mathrm{mem}}+\mathcal{O}\left(\frac{1}{c^{8}}\right) .
$$

1. The instantaneous (i.e., non-hereditary) piece $\mathrm{U}_{i j}^{\text {inst }}$ up to $3.5 \mathrm{PN}$ order reads

$$
\begin{aligned}
& \mathrm{U}_{i j}^{\mathrm{inst}}= \mathrm{M}_{i j}^{(2)} \\
&+\frac{G}{c^{5}}\left[\frac{1}{7} \mathrm{M}_{a\langle i}^{(5)} \mathrm{M}_{j\rangle a}-\frac{5}{7} \mathrm{M}_{a\langle i}^{(4)} \mathrm{M}_{j\rangle a}^{(1)}-\frac{2}{7} \mathrm{M}_{a\langle i}^{(3)} \mathrm{M}_{j\rangle a}^{(2)}+\frac{1}{3} \epsilon_{a b\langle i} \mathrm{M}_{j\rangle a}^{(4)} \mathrm{S}_{b}\right] \\
&+\frac{G}{c^{7}}\left[-\frac{64}{63} \mathrm{~S}_{a\langle i}^{(2)} \mathrm{S}_{j\rangle a}^{(3)}+\frac{1957}{3024} \mathrm{M}_{i j a b}^{(3)} \mathrm{M}_{a b}^{(4)}+\frac{5}{2268} \mathrm{M}_{a b\langle i}^{(3)} \mathrm{M}_{j\rangle a b}^{(4)}+\frac{19}{648} \mathrm{M}_{a b}^{(3)} \mathrm{M}_{i j a b}^{(4)}\right. \\
&+\frac{16}{63} \mathrm{~S}_{a\langle i}^{(1)} \mathrm{S}_{j\rangle a}^{(4)}+\frac{1685}{1008} \mathrm{M}_{i j a b}^{(2)} \mathrm{M}_{a b}^{(5)}+\frac{5}{126} \mathrm{M}_{a b\langle i}^{(2)} \mathrm{M}_{j\rangle a b}^{(5)}-\frac{5}{756} \mathrm{M}_{a b}^{(2)} \mathrm{M}_{i j a b}^{(5)} \\
&+\frac{80}{63} \mathrm{~S}_{a\langle i} \mathrm{S}_{j\rangle a}^{(5)}+\frac{5}{42} \mathrm{~S}_{a} \mathrm{~S}_{i j a}^{(5)}+\frac{41}{28} \mathrm{M}_{i j a b}^{(1)} \mathrm{M}_{a b}^{(6)}+\frac{5}{189} \mathrm{M}_{a b\langle i}^{(1)} \mathrm{M}_{j\rangle a b}^{(6)} \\
&+\frac{1}{432} \mathrm{M}_{a b}^{(1)} \mathrm{M}_{i j a b}^{(6)}+\frac{91}{216} \mathrm{M}_{i j a b} \mathrm{M}_{a b}^{(7)}-\frac{5}{252} \mathrm{M}_{a b\langle i} \mathrm{M}_{j\rangle a b}^{(7)}-\frac{1}{432} \mathrm{M}_{a b} \mathrm{M}_{i j a b}^{(7)} \\
&+\epsilon_{a c\langle i}\left(\frac{32}{189} \mathrm{M}_{j\rangle b c}^{(3)} \mathrm{S}_{a b}^{(3)}-\frac{1}{6} \mathrm{M}_{a b}^{(3)} \mathrm{S}_{j\rangle b c}^{(3)}+\frac{3}{56} \mathrm{~S}_{j\rangle b c}^{(2)} \mathrm{M}_{a b}^{(4)}+\frac{10}{189} \mathrm{~S}_{a b}^{(2)} \mathrm{M}_{j\rangle b c}^{(4)}\right. \\
&+\frac{65}{189} \mathrm{M}_{j\rangle b c}^{(2)} \mathrm{S}_{a b}^{(4)}+\frac{1}{28} \mathrm{M}_{a b}^{(2)} \mathrm{S}_{j\rangle b c}^{(4)}+\frac{187}{168} \mathrm{~S}_{j\rangle b c}^{(1)} \mathrm{M}_{a b}^{(5)}-\frac{1}{189} \mathrm{~S}_{a b}^{(1)} \mathrm{M}_{j\rangle b c}^{(5)} \\
&-\frac{5}{189} \mathrm{M}_{j\rangle b c}^{(1)} \mathrm{S}_{a b}^{(5)}+\frac{1}{24} \mathrm{M}_{a b}^{(1)} \mathrm{S}_{j\rangle b c}^{(5)}+\frac{65}{84} \mathrm{~S}_{j\rangle b c} \mathrm{M}_{a b}^{(6)}+\frac{1}{189} \mathrm{~S}_{a b} \mathrm{M}_{j\rangle b c}^{(6)} \\
&\left.\left.-\frac{10}{63} \mathrm{M}_{j\rangle b c} \mathrm{~S}_{a b}^{(6)}+\frac{1}{168} \mathrm{M}_{a b} \mathrm{~S}_{j\rangle b c}^{(6)}\right)\right] \cdot
\end{aligned}
$$

The Newtonian term in this expression contains the Newtonian quadrupole moment $\mathrm{Q}_{i j}$ and recovers the standard quadrupole formalism [see Eq. (67)];

2. The hereditary tail integral $\mathrm{U}_{i j}^{\text {tail }}$ is made of the dominant tail term at $1.5 \mathrm{PN}$ order in agreement with Eq. (76a) above:

$$
\mathrm{U}_{i j}^{\mathrm{tail}}=\frac{2 G \mathrm{M}}{c^{3}} \int_{0}^{+\infty} \mathrm{d} \tau\left[\ln \left(\frac{c \tau}{2 r_{0}}\right)+\frac{11}{12}\right] \mathrm{M}_{i j}^{(4)}(U-\tau) .
$$

The length scale $r_{0}$ is the one that enters our definition of the finite-part operation $\mathcal{F} \mathcal{P}$ [see Eq. (42)] and it enters also the relation between the radiative and harmonic retarded times given by Eq. (78); 
3. The hereditary tail-of-tail term appears dominantly at 3PN order [48] and is issued from the radiative metric computed in Eqs. (87):

$$
\mathrm{U}_{i j}^{\text {tail-tail }}=2\left(\frac{G \mathrm{M}}{c^{3}}\right)^{2} \int_{0}^{+\infty} \mathrm{d} \tau\left[\ln ^{2}\left(\frac{c \tau}{2 r_{0}}\right)+\frac{57}{70} \ln \left(\frac{c \tau}{2 r_{0}}\right)+\frac{124627}{44100}\right] \mathrm{M}_{i j}^{(5)}(U-\tau)
$$

4. Finally the memory-type hereditary piece $\mathrm{U}_{i j}^{m e m}$ contributes at orders $2.5 \mathrm{PN}$ and $3.5 \mathrm{PN}$ and is given by

$$
\begin{aligned}
\mathrm{U}_{i j}^{\text {mem }}=\frac{G}{c^{5}}[ & \left.-\frac{2}{7} \int_{0}^{+\infty} \mathrm{d} \tau \mathrm{M}_{a\langle i}^{(3)} \mathrm{M}_{j\rangle a}^{(3)}(U-\tau)\right] \\
+\frac{G}{c^{7}}[ & -\frac{32}{63} \int_{0}^{+\infty} \mathrm{d} \tau \mathrm{S}_{a\langle i}^{(3)} \mathrm{S}_{j\rangle a}^{(3)}(U-\tau)-\frac{5}{756} \int_{0}^{+\infty} \mathrm{d} \tau \mathrm{M}_{a b}^{(4)} \mathrm{M}_{i j a b}^{(4)}(U-\tau) \\
& \left.-\frac{20}{189} \epsilon_{a b\langle i} \int_{0}^{+\infty} \mathrm{d} \tau \mathrm{S}_{a c}^{(3)} \mathrm{M}_{j\rangle b c}^{(4)}(U-\tau)+\frac{5}{42} \epsilon_{a b\langle i} \int_{0}^{+\infty} \mathrm{d} \tau \mathrm{M}_{a c}^{(3)} \mathrm{S}_{j\rangle b c}^{(4)}(U-\tau)\right]
\end{aligned}
$$

The 2.5PN non-linear memory integral - the first term inside the coefficient of $G / c^{5}$ - has been obtained using both post-Newtonian methods $[42,427,406,60,50]$ and rigorous studies of the field at future null infinity [128]. The expression (92) is in agreement with the more recent computation of the non-linear memory up to any post-Newtonian order in Refs. [189, 192].

Be careful to note that the latter post-Newtonian orders correspond to "relative" orders when counted in the local radiation-reaction force, present in the equations of motion: For instance, the 1.5PN tail integral in Eq. (90) is due to a $4 \mathrm{PN}$ radiative effect in the equations of motion [58]; similarly, the 3PN tail-of-tail integral is expected to be associated with some radiation-reaction terms occurring at the $5.5 \mathrm{PN}$ order.

Note that $\mathrm{U}_{i j}$, when expressed in terms of the intermediate moments $\mathrm{M}_{L}$ and $\mathrm{S}_{L}$, shows a dependence on the (arbitrary) length scale $r_{0}$; cf. the tail and tail-of-tail contributions (90)-(91). Most of this dependence comes from our definition of a radiative coordinate system as given by (78). Exactly as we have done for the $1.5 \mathrm{PN}$ tail term in Eq. (79), we can remove most of the $r_{0}$ 's by inserting $U=u-\frac{2 G \mathrm{M}}{c^{3}} \ln \left(r / r_{0}\right)$ back into (89)-(92), and expanding the result when $c \rightarrow \infty$, keeping the necessary terms consistently. In doing so one finds that there remains a $r_{0}$-dependent term at the $3 \mathrm{PN}$ order, namely

$$
\mathrm{U}_{i j}=\mathrm{M}_{i j}^{(2)}(u)-\frac{214}{105} \ln \left(\frac{r}{r_{0}}\right)\left(\frac{G \mathrm{M}}{c^{3}}\right)^{2} \mathrm{M}_{i j}^{(4)}(u)+\text { terms independent of } r_{0} .
$$

However, the latter dependence on $r_{0}$ is fictitious and should in fine disappear. The reason is that when we compute explicitly the mass quadrupole moment $\mathrm{M}_{i j}$ for a given matter source, we will find an extra contribution depending on $r_{0}$ occurring at the $3 \mathrm{PN}$ order which will cancel out the one in Eq. (93). Indeed we shall compute the source quadrupole moment $\mathrm{I}_{i j}$ of compact binaries at the $3 \mathrm{PN}$ order, and we do observe on the result (300) - (301) below the requested terms depending on $r_{0}$, namely ${ }^{27}$

$$
\mathrm{M}_{i j}=\mathrm{Q}_{i j}+\frac{214}{105} \ln \left(\frac{r_{12}}{r_{0}}\right)\left(\frac{G m}{c^{3}}\right)^{2} \mathrm{Q}_{i j}^{(2)}+\text { terms independent of } r_{0} .
$$

where $\mathrm{Q}_{i j}=\mu \hat{x}_{i j}$ denotes the Newtonian quadrupole, $r_{12}$ is the separation between the particles, and $m$ is the total mass differing from the ADM mass $\mathrm{M}$ by small post-Newtonian corrections.

27 The canonical moment $\mathrm{M}_{i j}$ differs from the source moment $\mathrm{I}_{i j}$ by small $2.5 \mathrm{PN}$ and $3.5 \mathrm{PN}$ terms; see Eq. (97). 
Combining Eqs. (93) and (94) we see that the $r_{0}$-dependent terms cancel as expected. The appearance of a logarithm and its associated constant $r_{0}$ at the $3 \mathrm{PN}$ order was pointed out in Ref. [7]; it was rederived within the present formalism in Refs. [58, 48]. Recently a result equivalent to Eq. (93) was obtained by means of the EFT approach using considerations related to the renormalization group equation [222].

The previous formulas for the $3.5 \mathrm{PN}$ radiative quadrupole moment permit to compute the dominant mode $(2,2)$ of the waveform up to order 3.5PN [197]; however, to control the full waveform one has also to take into account the contributions of higher-order radiative moments. Here we list the most accurate results we have for all the moments that permit the derivation of the waveform up to order $3 \mathrm{PN}[74]:^{28}$

$$
\begin{aligned}
& \mathrm{U}_{i j k}(U)=\mathrm{M}_{i j k}^{(3)}(U)+\frac{2 G M}{c^{3}} \int_{0}^{+\infty} \mathrm{d} \tau\left[\ln \left(\frac{c \tau}{2 r_{0}}\right)+\frac{97}{60}\right] \mathrm{M}_{i j k}^{(5)}(U-\tau) \\
& +\frac{G}{c^{5}}\left\{\int_{0}^{+\infty} \mathrm{d} \tau\left[-\frac{1}{3} \mathrm{M}_{a\langle i}^{(3)} \mathrm{M}_{j k\rangle a}^{(4)}-\frac{4}{5} \epsilon_{a b\langle i} \mathrm{M}_{j a}^{(3)} \mathrm{S}_{k\rangle b}^{(3)}\right](U-\tau)\right. \\
& -\frac{4}{3} \mathrm{M}_{a\langle i}^{(3)} \mathrm{M}_{j k\rangle a}^{(3)}-\frac{9}{4} \mathrm{M}_{a\langle i}^{(4)} \mathrm{M}_{j k\rangle a}^{(2)}+\frac{1}{4} \mathrm{M}_{a\langle i}^{(2)} \mathrm{M}_{j k\rangle a}^{(4)}-\frac{3}{4} \mathrm{M}_{a\langle i}^{(5)} \mathrm{M}_{j k\rangle a}^{(1)}+\frac{1}{4} \mathrm{M}_{a\langle i}^{(1)} \mathrm{M}_{j k\rangle a}^{(5)} \\
& +\frac{1}{12} \mathrm{M}_{a\langle i}^{(6)} \mathrm{M}_{j k\rangle a}+\frac{1}{4} \mathrm{M}_{a\langle i} \mathrm{M}_{j k\rangle a}^{(6)}+\frac{1}{5} \epsilon_{a b\langle i}\left[-12 \mathrm{~S}_{j a}^{(2)} \mathrm{M}_{k>b}^{(3)}-8 \mathrm{M}_{j a}^{(2)} \mathrm{S}_{k>b}^{(3)}-3 \mathrm{~S}_{j a}^{(1)} \mathrm{M}_{k>b}^{(4)}\right. \\
& \left.\left.-27 \mathrm{M}_{j a}^{(1)} \mathrm{S}_{k\rangle b}^{(4)}-\mathrm{S}_{j a} \mathrm{M}_{k\rangle b}^{(5)}-9 \mathrm{M}_{j a} \mathrm{~S}_{k\rangle b}^{(5)}-\frac{9}{4} \mathrm{~S}_{a} \mathrm{M}_{j k\rangle b}^{(5)}\right]+\frac{12}{5} \mathrm{~S}_{\langle i} \mathrm{S}_{j k\rangle}^{(4)}\right\} \\
& +\mathcal{O}\left(\frac{1}{c^{6}}\right) \\
& \mathrm{V}_{i j}(U)=\mathrm{S}_{i j}^{(2)}(U)+\frac{2 G M}{c^{3}} \int_{0}^{+\infty} \mathrm{d} \tau\left[\ln \left(\frac{c \tau}{2 r_{0}}\right)+\frac{7}{6}\right] \mathrm{S}_{i j}^{(4)}(U-\tau) \\
& +\frac{G}{7 c^{5}}\left\{4 \mathrm{~S}_{a\langle i}^{(2)} \mathrm{M}_{j\rangle a}^{(3)}+8 \mathrm{M}_{a\langle i}^{(2)} \mathrm{S}_{j\rangle a}^{(3)}+17 \mathrm{~S}_{a\langle i}^{(1)} \mathrm{M}_{j\rangle a}^{(4)}-3 \mathrm{M}_{a\langle i}^{(1)} \mathrm{S}_{j\rangle a}^{(4)}+9 \mathrm{~S}_{a\langle i} \mathrm{M}_{j\rangle a}^{(5)}\right. \\
& -3 \mathrm{M}_{a\langle i} \mathrm{S}_{j\rangle a}^{(5)}-\frac{1}{4} \mathrm{~S}_{a} \mathrm{M}_{i j a}^{(5)}-7 \epsilon_{a b\langle i} \mathrm{S}_{a} \mathrm{~S}_{j\rangle b}^{(4)}+\frac{1}{2} \epsilon_{a c\langle i}\left[3 \mathrm{M}_{a b}^{(3)} \mathrm{M}_{j\rangle b c}^{(3)}+\frac{353}{24} \mathrm{M}_{j\rangle b c}^{(2)} \mathrm{M}_{a b}^{(4)}\right. \\
& \left.\left.-\frac{5}{12} \mathrm{M}_{a b}^{(2)} \mathrm{M}_{j\rangle b c}^{(4)}+\frac{113}{8} \mathrm{M}_{j\rangle b c}^{(1)} \mathrm{M}_{a b}^{(5)}-\frac{3}{8} \mathrm{M}_{a b}^{(1)} \mathrm{M}_{j\rangle b c}^{(5)}+\frac{15}{4} \mathrm{M}_{j\rangle b c} \mathrm{M}_{a b}^{(6)}+\frac{3}{8} \mathrm{M}_{a b} \mathrm{M}_{j\rangle b c}^{(6)}\right]\right\} \\
& +\mathcal{O}\left(\frac{1}{c^{6}}\right) \text {. } \\
& \mathrm{U}_{i j k l}(U)=\mathrm{M}_{i j k l}^{(4)}(U)+\frac{G}{c^{3}}\left\{2 M \int_{0}^{+\infty} \mathrm{d} \tau\left[\ln \left(\frac{c \tau}{2 r_{0}}\right)+\frac{59}{30}\right] \mathrm{M}_{i j k l}^{(6)}(U-\tau)\right. \\
& \left.+\frac{2}{5} \int_{0}^{+\infty} \mathrm{d} \tau \mathrm{M}_{\langle i j}^{(3)} \mathrm{M}_{k l\rangle}^{(3)}(U-\tau)-\frac{21}{5} \mathrm{M}_{\langle i j}^{(5)} \mathrm{M}_{k l\rangle}-\frac{63}{5} \mathrm{M}_{\langle i j}^{(4)} \mathrm{M}_{k l\rangle}^{(1)}-\frac{102}{5} \mathrm{M}_{\langle i j}^{(3)} \mathrm{M}_{k l\rangle}^{(2)}\right\} \\
& +\mathcal{O}\left(\frac{1}{c^{5}}\right) \\
& \mathrm{V}_{i j k}(U)=\mathrm{S}_{i j k}^{(3)}(U)+\frac{G}{c^{3}}\left\{2 M \int_{0}^{+\infty} \mathrm{d} \tau\left[\ln \left(\frac{c \tau}{2 r_{0}}\right)+\frac{5}{3}\right] \mathrm{S}_{i j k}^{(5)}(U-\tau)\right. \\
& \left.+\frac{1}{10} \epsilon_{a b\langle i} \mathrm{M}_{j a}^{(5)} \mathrm{M}_{k\rangle b}-\frac{1}{2} \epsilon_{a b\langle i} \mathrm{M}_{j a}^{(4)} \mathrm{M}_{k\rangle b}^{(1)}-2 \mathrm{~S}_{\langle i} \mathrm{M}_{j k\rangle}^{(4)}\right\}
\end{aligned}
$$

\footnotetext{
${ }^{28}$ In all formulas below the STF projection \langle\rangle applies only to the "free" indices denoted $i j k l \ldots$ carried by the moments themselves. Thus the dummy indices such as $a b c \ldots$ are excluded from the STF projection.
} 


$$
\begin{aligned}
& +\mathcal{O}\left(\frac{1}{c^{5}}\right) \\
\mathrm{U}_{i j k l m}(U) & =\mathrm{M}_{i j k l m}^{(5)}(U)+\frac{G}{c^{3}}\left\{2 M \int_{0}^{+\infty} \mathrm{d} \tau\left[\ln \left(\frac{c \tau}{2 r_{0}}\right)+\frac{232}{105}\right] \mathrm{M}_{i j k l m}^{(7)}(U-\tau)\right. \\
& +\frac{20}{21} \int_{0}^{+\infty} \mathrm{d} \tau \mathrm{M}_{\langle i j}^{(3)} \mathrm{M}_{k l m\rangle}^{(4)}(U-\tau)-\frac{710}{21} \mathrm{M}_{\langle i j}^{(3)} \mathrm{M}_{k l m\rangle}^{(3)}-\frac{265}{7} \mathrm{M}_{\langle i j k}^{(2)} \mathrm{M}_{l m\rangle}^{(4)}-\frac{120}{7} \mathrm{M}_{\langle i j}^{(2)} \mathrm{M}_{k l m\rangle}^{(4)} \\
& \left.-\frac{155}{7} \mathrm{M}_{\langle i j k}^{(1)} \mathrm{M}_{l m\rangle}^{(5)}-\frac{41}{7} \mathrm{M}_{\langle i j}^{(1)} \mathrm{M}_{k l m\rangle}^{(5)}-\frac{34}{7} \mathrm{M}_{\langle i j k} \mathrm{M}_{l m\rangle}^{(6)}-\frac{15}{7} \mathrm{M}_{\langle i j} \mathrm{M}_{k l m\rangle}^{(6)}\right\} \\
& +\mathcal{O}\left(\frac{1}{c^{4}}\right), \\
\mathrm{V}_{i j k l}(U) & =\mathrm{S}_{i j k l}^{(4)}(U)+\frac{G}{c^{3}}\left\{2 \int_{0}^{+\infty} \mathrm{d} \tau\left[\ln \left(\frac{c \tau}{2 r_{0}}\right)+\frac{119}{60}\right] \mathrm{S}_{i j k l}^{(6)}(U-\tau)\right. \\
& -\frac{35}{3} \mathrm{~S}_{\langle i j}^{(2)} \mathrm{M}_{k l\rangle}^{(3)}-\frac{25}{3} \mathrm{M}_{\langle i j}^{(2)} \mathrm{S}_{k l\rangle}^{(3)}-\frac{65}{6} \mathrm{~S}_{\langle i j}^{(1)} \mathrm{M}_{k l\rangle}^{(4)}-\frac{25}{6} \mathrm{M}_{\langle i j}^{(1)} \mathrm{S}_{k l\rangle}^{(4)}-\frac{19}{6} \mathrm{~S}_{\langle i j} \mathrm{M}_{k l\rangle}^{(5)} \\
& -\frac{11}{6} \mathrm{M}_{\langle i j} \mathrm{S}_{k l\rangle}^{(5)}-\frac{11}{12} \mathrm{~S}_{\langle i} \mathrm{M}_{j k l\rangle}^{(5)}+\frac{1}{6} \epsilon_{a b\langle i}\left[-5 \mathrm{M}_{j a}^{(3)} \mathrm{M}_{k l\rangle b}^{(3)}-\frac{11}{2} \mathrm{M}_{j a}^{(4)} \mathrm{M}_{k l\rangle b}^{(2)}-\frac{5}{2} \mathrm{M}_{j a}^{(2)} \mathrm{M}_{k l\rangle b}^{(4)}\right. \\
& \left.\left.-\frac{1}{2} \mathrm{M}_{j a}^{(5)} \mathrm{M}_{k l\rangle b}^{(1)}+\frac{37}{10} \mathrm{M}_{j a}^{(1)} \mathrm{M}_{k l\rangle b}^{(5)}+\frac{3}{10} \mathrm{M}_{j a}^{(6)} \mathrm{M}_{k l\rangle b}+\frac{1}{2} \mathrm{M}_{j a} \mathrm{M}_{k l\rangle b}^{(6)}\right]\right\} \\
& +\mathcal{O}\left(\frac{1}{c^{4}}\right) \cdot
\end{aligned}
$$

For all the other multipole moments in the $3 \mathrm{PN}$ waveform, it is sufficient to assume the agreement between the radiative and canonical moments, namely

$$
\begin{aligned}
& \mathrm{U}_{L}(U)=\mathrm{M}_{L}^{(\ell)}(U)+\mathcal{O}\left(\frac{1}{c^{3}}\right), \\
& \mathrm{V}_{L}(U)=\mathrm{S}_{L}^{(\ell)}(U)+\mathcal{O}\left(\frac{1}{c^{3}}\right) .
\end{aligned}
$$

In a second stage of the general formalism, we must express the canonical moments $\left\{\mathrm{M}_{L}, \mathrm{~S}_{L}\right\}$ in terms of the six types of source moments $\left\{\mathrm{I}_{L}, \mathrm{~J}_{L}, \mathrm{~W}_{L}, \mathrm{X}_{L}, \mathrm{Y}_{L}, \mathrm{Z}_{L}\right\}$. For the control of the $(2,2)$ mode in the waveform up to $3.5 \mathrm{PN}$ order, we need to relate the canonical quadrupole moment $\mathrm{M}_{i j}$ to the corresponding source quadrupole moment $\mathrm{I}_{i j}$ up to that accuracy. We obtain [197]

$$
\begin{aligned}
& \mathrm{M}_{i j}=\mathrm{I}_{i j}+ \frac{4 G}{c^{5}}\left[\mathrm{~W}^{(2)} \mathrm{I}_{i j}-\mathrm{W}^{(1)} \mathrm{I}_{i j}^{(1)}\right] \\
&+\frac{4 G}{c^{7}}\left[\frac{4}{7} \mathrm{~W}_{a\langle i}^{(1)} \mathrm{I}_{j\rangle a}^{(3)}+\frac{6}{7} \mathrm{~W}_{a\langle i} \mathrm{I}_{j\rangle a}^{(4)}-\frac{1}{7} \mathrm{I}_{a\langle i} \mathrm{Y}_{j\rangle a}^{(3)}-\mathrm{Y}_{a\langle i} \mathrm{I}_{j\rangle a}^{(3)}-2 \mathrm{XI}_{i j}^{(3)}-\frac{5}{21} \mathrm{I}_{i j a} \mathrm{~W}_{a}^{(4)}\right. \\
&+\frac{1}{63} \mathrm{I}_{i j a}^{(1)} \mathrm{W}_{a}^{(3)}-\frac{25}{21} \mathrm{I}_{i j a} \mathrm{Y}_{a}^{(3)}-\frac{22}{63} \mathrm{I}_{i j a}^{(1)} \mathrm{Y}_{a}^{(2)}+\frac{5}{63} \mathrm{Y}_{a}^{(1)} \mathrm{I}_{i j a}^{(2)}+2 \mathrm{~W}_{i j} \mathrm{~W}^{(3)} \\
&+2 \mathrm{~W}_{i j}^{(1)} \mathrm{W}^{(2)}-\frac{4}{3} \mathrm{~W}_{\langle i} \mathrm{W}_{j\rangle}^{(3)}+2 \mathrm{Y}_{i j} \mathrm{~W}^{(2)}-4 \mathrm{~W}_{\langle i} \mathrm{Y}_{j\rangle}^{(2)} \\
&\left.+\epsilon_{a b\langle i}\left(\frac{1}{3} \mathrm{I}_{j\rangle a} \mathrm{Z}_{b}^{(3)}+\frac{4}{9} \mathrm{~J}_{j\rangle a} \mathrm{~W}_{b}^{(3)}-\frac{4}{9} \mathrm{~J}_{j\rangle a} \mathrm{Y}_{b}^{(2)}+\frac{8}{9} \mathrm{~J}_{j\rangle a}^{(1)} \mathrm{Y}_{b}^{(1)}+\mathrm{Z}_{a} \mathrm{I}_{j\rangle b}^{(3)}\right)\right]+\mathcal{O}\left(\frac{1}{c^{8}}\right) .
\end{aligned}
$$

Here, for instance, $\mathrm{W}$ denotes the monopole moment associated with the moment $\mathrm{W}_{L}$, and $\mathrm{Y}_{i}$ is the dipole moment corresponding to $\mathrm{Y}_{L}$. Notice that the difference between the canonical and source moments starts at the relatively high 2.5PN order. For the control of the full waveform 
up to $3 \mathrm{PN}$ order we need also the moments $\mathrm{M}_{i j k}$ and $\mathrm{S}_{i j}$, which admit similarly some correction terms starting at the $2.5 \mathrm{PN}$ order:

$$
\begin{aligned}
\mathrm{M}_{i j k} & =\mathrm{I}_{i j k}+\frac{4 G}{c^{5}}\left[\mathrm{~W}^{(2)} \mathrm{I}_{i j k}-\mathrm{W}^{(1)} \mathrm{I}_{i j k}^{(1)}+3 \mathrm{I}_{\langle i j} \mathrm{Y}_{k\rangle}^{(1)}\right]+\mathcal{O}\left(\frac{1}{c^{6}}\right) \\
\mathrm{S}_{i j} & =\mathrm{J}_{i j}+\frac{2 G}{c^{5}}\left[\epsilon_{a b\langle i}\left(-\mathrm{I}_{j\rangle b}^{(3)} \mathrm{W}_{a}-2 \mathrm{I}_{j\rangle b} \mathrm{Y}_{a}^{(2)}+\mathrm{I}_{j\rangle b}^{(1)} \mathrm{Y}_{a}^{(1)}\right)+3 \mathrm{~J}_{\langle i} \mathrm{Y}_{j\rangle}^{(1)}-2 \mathrm{~J}_{i j}^{(1)} \mathrm{W}^{(1)}\right] \\
& +\mathcal{O}\left(\frac{1}{c^{6}}\right)
\end{aligned}
$$

The remainders in Eqs. (98) are consistent with the 3PN approximation for the full waveform. Besides the mass quadrupole moment (97), and mass octopole and current quadrupole moments (98), we can state that, with the required $3 \mathrm{PN}$ precision, all the other moments $\mathrm{M}_{L}, \mathrm{~S}_{L}$ agree with their source counterparts $\mathrm{I}_{L}, \mathrm{~J}_{L}$ :

$$
\begin{aligned}
\mathrm{M}_{L} & =\mathrm{I}_{L}+\mathcal{O}\left(\frac{1}{c^{5}}\right) \\
\mathrm{S}_{L} & =\mathrm{J}_{L}+\mathcal{O}\left(\frac{1}{c^{5}}\right)
\end{aligned}
$$

With those formulas we have related the radiative moments $\left\{\mathrm{U}_{L}, \mathrm{~V}_{L}\right\}$ parametrizing the asymptotic waveform (66) to the six types of source multipole moments $\left\{\mathrm{I}_{L}, \mathrm{~J}_{L}, \mathrm{~W}_{L}, \mathrm{X}_{L}, \mathrm{Y}_{L}, \mathrm{Z}_{L}\right\}$. What is missing is the explicit dependence of the source moments as functions of the actual parameters of some matter source. We come to grips with this important question in the next section.

\section{Matching to a Post-Newtonian Source}

By Theorem 2 we control the most general class of solutions of the vacuum equations outside the source, in the form of non-linear functionals of the source multipole moments. For instance, these solutions include the Schwarzschild and Kerr solutions for black holes, as well as all their perturbations. By Theorem 4 we learned how to construct the radiative moments at infinity, which constitute the observables of the radiation field at large distances from the source, and we obtained in Section 3.3 explicit relationships between radiative and source moments. We now want to understand how a specific choice of matter stress-energy tensor $T^{\alpha \beta}$, i.e., a specific choice of some physical model describing the material source, selects a particular physical exterior solution among our general class, and therefore a given set of multipole moments for the source.

\subsection{The matching equation}

We shall provide the answer to that problem in the case of a post-Newtonian source for which the post-Newtonian parameter $\epsilon \sim 1 / c$ defined by Eq. (1) is small. The fundamental fact that permits the connection of the exterior field to the inner field of the source is the existence of a "matching" region, in which both the multipole expansion and the post-Newtonian expansion are valid. This region is nothing but the exterior part of the near zone, such that $r>a$ (exterior) and $r \ll \lambda$ (near zone); it always exists around post-Newtonian sources whose radius is much less than the emitted wavelength, $\frac{a}{\lambda} \sim \epsilon \ll 1$. In our formalism the multipole expansion is defined by the multipolarpost-Minkowskian (MPM) solution; see Section 2. Matching together the post-Newtonian and MPM solutions in this overlapping region is an application of the method of matched asymptotic expansions, which has frequently been applied in the present context, both for radiation-reaction $[114,113,7,58,43]$ and wave-generation [59, 155, 44, 49] problems. 
Let us denote by $\mathcal{M}(h)$ the multipole expansion of $h$ (for simplicity, we suppress the spacetime indices). By $\mathcal{M}(h)$ we really mean the MPM exterior metric that we have constructed in Sections 2.2 and 2.3:

$$
\mathcal{M}(h) \equiv h_{\mathrm{ext}}=\sum_{n=1}^{+\infty} G^{n} h_{(n)}\left[\mathrm{I}_{L}, \ldots, \mathrm{Z}_{L}\right] .
$$

This solution is formally defined for any radius $r>0$. Of course, the true solution $h$ agrees with its own multipole expansion in the exterior of the source, i.e.

$$
r>a \Longrightarrow \mathcal{M}(h)=h .
$$

By contrast, inside the source, $h$ and $\mathcal{M}(h)$ disagree with each other because $h$ is a fully-fledged solution of the field equations within the matter source, while $\mathcal{M}(h)$ is a vacuum solution becoming singular at $r=0$. Now let us denote by $\bar{h}$ the post-Newtonian expansion of $h$. We have already anticipated the general structure of this expansion which is given in Eq. (54). In the matching region, where both the multipolar and post-Newtonian expansions are valid, we write the numerical equality

$$
a<r \ll \lambda \quad \Longrightarrow \quad \mathcal{M}(h)=\bar{h} .
$$

This "numerical" equality is viewed here in a sense of formal expansions, as we do not control the convergence of the series. In fact, we should be aware that such an equality, though quite natural and even physically obvious, is probably not really justified within the approximation scheme (mathematically speaking), and we simply take it here as part of our fundamental assumptions.

We now transform Eq. (102) into a matching equation, by replacing in the left-hand side $\mathcal{M}(h)$ by its near-zone re-expansion $\overline{\mathcal{M}(h)}$, and in the right-hand side $\bar{h}$ by its multipole expansion $\mathcal{M}(\bar{h})$. The structure of the near-zone expansion $(r \rightarrow 0)$ of the exterior multipolar field has been found in Theorem 3, see Eq. (53). We denote the corresponding infinite series $\overline{\mathcal{M}(h)}$ with the same overbar as for the post-Newtonian expansion because it is really an expansion when $r / c \rightarrow 0$, equivalent to an expansion when $c \rightarrow \infty$. Concerning the multipole expansion of the post-Newtonian metric, $\mathcal{M}(\bar{h})$, we simply postulate for the moment its existence, but we shall show later how to construct it explicitly. Therefore, the matching equation is the statement that

$$
\overline{\mathcal{M}(h)}=\mathcal{M}(\bar{h})
$$

by which we really mean an infinite set of functional identities, valid $\forall(\mathbf{x}, t) \in \mathbb{R}_{*}^{3} \times \mathbb{R}$, between the coefficients of the series in both sides of the equation. Note that such a meaning is somewhat different from that of a numerical equality like Eq. (102), which is valid only when $\mathbf{x}$ belongs to some limited spatial domain. The matching equation (103) tells us that the formal near-zone expansion of the multipole decomposition is identical, term by term, to the multipole expansion of the post-Newtonian solution. However, the former expansion is nothing but the formal farzone expansion, when $r \rightarrow \infty$, of each of the post-Newtonian coefficients. Most importantly, it is possible to write down, within the present formalism, the general structure of these identical expansions as a consequence of Eq. (53):

$$
\overline{\mathcal{M}(h)}=\sum \hat{n}_{L} r^{m}(\ln r)^{p} F_{L, m, p}(t)=\mathcal{M}(\bar{h}),
$$

where the functions $F_{L, m, p}=\sum_{n \geqslant 1} G^{n} F_{L, m, p, n}$. The latter expansion can be interpreted either as the singular re-expansion of the multipole decomposition when $r \rightarrow 0$-i.e., the first equality in Eq. (104) -, or the singular re-expansion of the post-Newtonian series when $r \rightarrow+\infty-$ the second equality.

We recognize the beauty of singular perturbation theory, where two asymptotic expansions, taken formally outside their respective domains of validity, are matched together. Of course, the 
method works because there exists, physically, an overlapping region in which the two approximation series are expected to be numerically close to the exact solution. As we shall detail in Sections 4.2 and 5.2, the matching equation (103), supplemented by the condition of no-incoming radiation [say in the form of Eq. (29)], permits determining all the unknowns of the problem: On the one hand, the external multipolar decomposition $\mathcal{M}(h)$, i.e., the explicit expressions of the multipole moments therein (see Sections 4.2 and 4.4); on the other hand, the terms in the inner post-Newtonian expansion $\bar{h}$ that are associated with radiation-reaction effects, i.e., those terms which depend on the boundary conditions of the radiative field at infinity, and which correspond in the present case to a post-Newtonian source which is isolated from other sources in the Universe; see Section 5.2.

\subsection{General expression of the multipole expansion}

Theorem 5. Under the hypothesis of matching, Eq. (103), the multipole expansion of the solution of the Einstein field equation outside a post-Newtonian source reads

$$
\mathcal{M}\left(h^{\alpha \beta}\right)=\mathcal{F} \mathcal{P}_{B=0} \square_{\text {ret }}^{-1}\left[\widetilde{r}^{B} \mathcal{M}\left(\Lambda^{\alpha \beta}\right)\right]-\frac{4 G}{c^{4}} \sum_{\ell=0}^{+\infty} \frac{(-)^{\ell}}{\ell !} \partial_{L}\left\{\frac{1}{r} \mathcal{H}_{L}^{\alpha \beta}(t-r / c)\right\},
$$

where the "multipole moments" are given by

$$
\mathcal{H}_{L}^{\alpha \beta}(u)=\mathcal{F} \mathcal{P}_{B=0} \int \mathrm{d}^{3} \mathbf{x} \widetilde{r}^{B} x_{L} \bar{\tau}^{\alpha \beta}(\mathbf{x}, u)
$$

Here, $\bar{\tau}^{\alpha \beta}$ denotes the post-Newtonian expansion of the stress-energy pseudo-tensor in harmonic coordinates as defined by Eq. (23).

Proof (see Refs. [44, 49]): First notice where the physical restriction of considering a postNewtonian source enters this theorem: The multipole moments (106) depend on the post-Newtonian expansion $\bar{\tau}^{\alpha \beta}$ of the pseudo-tensor, rather than on $\tau^{\alpha \beta}$ itself. Consider $\Delta^{\alpha \beta}$, namely the difference between $h^{\alpha \beta}$, which is a solution of the field equations everywhere inside and outside the source, and the first term in Eq. (105), namely the finite part of the retarded integral of the multipole expansion $\mathcal{M}\left(\Lambda^{\alpha \beta}\right)$ :

$$
\Delta^{\alpha \beta} \equiv h^{\alpha \beta}-\mathcal{F P} \square_{\text {ret }}^{-1}\left[\mathcal{M}\left(\Lambda^{\alpha \beta}\right)\right] .
$$

From now on we shall generally abbreviate the symbols concerning the finite-part operation at $B=0$ by a mere $\mathcal{F P}$. According to Eq. $(30), h^{\alpha \beta}$ is given by the retarded integral of the pseudotensor $\tau^{\alpha \beta}$. So,

$$
\Delta^{\alpha \beta}=\frac{16 \pi G}{c^{4}} \square_{\mathrm{ret}}^{-1} \tau^{\alpha \beta}-\mathcal{F} \mathcal{P} \square_{\mathrm{ret}}^{-1}\left[\mathcal{M}\left(\Lambda^{\alpha \beta}\right)\right]
$$

In the second term the finite part plays a crucial role because the multipole expansion $\mathcal{M}\left(\Lambda^{\alpha \beta}\right)$ is singular at $r=0$. By contrast, the first term in Eq. (108), as it stands, is well-defined because we are considering only some smooth field distributions: $\tau^{\alpha \beta} \in C^{\infty}\left(\mathbb{R}^{4}\right)$. There is no need to include a finite part $\mathcal{F P}$ in the first term, but a contrario there is no harm to add one in front of it, because for convergent integrals the finite part simply gives back the value of the integral. The advantage of adding artificially the $\mathcal{F P}$ in the first term is that we can re-write Eq. (108) into the more interesting form

$$
\Delta^{\alpha \beta}=\frac{16 \pi G}{c^{4}} \mathcal{F} \mathcal{P} \square_{\text {ret }}^{-1}\left[\tau^{\alpha \beta}-\mathcal{M}\left(\tau^{\alpha \beta}\right)\right]
$$

in which we have also used the fact that $\mathcal{M}\left(\Lambda^{\alpha \beta}\right)=\frac{16 \pi G}{c^{4}} \mathcal{M}\left(\tau^{\alpha \beta}\right)$ because $T^{\alpha \beta}$ has a compact support. The interesting point about Eq. (109) is that $\Delta^{\alpha \beta}$ appears now to be the (finite part 
of a) retarded integral of a source with spatially compact support. This follows from the fact that the pseudo-tensor agrees numerically with its own multipole expansion when $r>a$ [by the same equation as Eq. (102)]. Therefore, $\mathcal{M}\left(\Delta^{\alpha \beta}\right)$ can be obtained from the known formula for the multipole expansion of the retarded solution of a wave equation with compact-support source. This formula, given in Appendix B of Ref. [59], yields the second term in Eq. (105),

$$
\mathcal{M}\left(\Delta^{\alpha \beta}\right)=-\frac{4 G}{c^{4}} \sum_{\ell=0}^{+\infty} \frac{(-)^{\ell}}{\ell !} \partial_{L}\left\{\frac{1}{r} \mathcal{H}_{L}^{\alpha \beta}(u)\right\},
$$

but in which the moments do not yet match the result (106); instead, ${ }^{29}$

$$
\mathcal{H}_{L}^{\alpha \beta}=\mathcal{F} \mathcal{P} \int \mathrm{d}^{3} \mathbf{x} x_{L}\left[\tau^{\alpha \beta}-\mathcal{M}\left(\tau^{\alpha \beta}\right)\right] .
$$

The reason is that we have not yet applied the assumption of a post-Newtonian source. Such sources are entirely covered by their own near zone (i.e., $a \ll \lambda$ ), and, in addition, for them the integral (111) has a compact support limited to the domain of the source. In consequence, we can replace the integrand in Eq. (111) by its post-Newtonian expansion, valid over all the near zone:

$$
\mathcal{H}_{L}^{\alpha \beta}=\mathcal{F} \mathcal{P} \int \mathrm{d}^{3} \mathbf{x} x_{L}\left[\bar{\tau}^{\alpha \beta}-\overline{\mathcal{M}\left(\tau^{\alpha \beta}\right)}\right] .
$$

Strangely enough, we do not get the expected result because of the presence of the second term in Eq. (112). Actually, this term is a bit curious, because the object $\overline{\mathcal{M}\left(\tau^{\alpha \beta}\right)}$ it contains is only known in the form of the formal series whose structure is given by the first equality in Eq. (104) (indeed $\tau$ and $h$ have the same type of structure). Happily - because we would not know what to do with this term in applications - we are now going to prove that the second term in Eq. (112) is in fact identically zero. The proof is based on the properties of the analytic continuation as applied to the formal structure (104) of $\overline{\mathcal{M}\left(\tau^{\alpha \beta}\right)}$. Each term of this series yields a contribution to Eq. (112) that takes the form, after performing the angular integration, of the integral $\mathcal{F} \mathcal{P}_{B=0} \int_{0}^{+\infty} \mathrm{d} r r^{B+b}(\ln r)^{p}$, and multiplied by some function of time. We want to prove that the radial integral $\int_{0}^{+\infty} \mathrm{d} r r^{B+b}(\ln r)^{p}$ is zero by analytic continuation $(\forall B \in \mathbb{C})$. First we can get rid of the logarithms by considering some repeated differentiations with respect to $B$; thus we need only to consider the simpler integral $\int_{0}^{+\infty} \mathrm{d} r r^{B+b}$. We split the integral into a "near-zone" integral $\int_{0}^{\mathcal{R}} \mathrm{d} r r^{B+b}$ and a "far-zone" one $\int_{\mathcal{R}}^{+\infty} \mathrm{d} r r^{B+b}$, where $\mathcal{R}$ is some constant radius. When $\Re(B)$ is a large enough positive number, the value of the near-zone integral is $\mathcal{R}^{B+b+1} /(B+b+1)$, while when $\Re(B)$ is a large negative number, the far-zone integral reads the opposite, $-\mathcal{R}^{B+b+1} /(B+b+1)$. Both obtained values represent the unique analytic continuations of the near-zone and far-zone integrals for any $B \in \mathbb{C}$ except $-b-1$. The complete integral $\int_{0}^{+\infty} \mathrm{d} r r^{B+b}$ is equal to the sum of these analytic continuations, and is therefore identically zero $(\forall B \in \mathbb{C}$, including the value $-b-1)$. At last we have completed the proof of Theorem 5 :

$$
\mathcal{H}_{L}^{\alpha \beta}=\mathcal{F} \mathcal{P} \int \mathrm{d}^{3} \mathbf{x} x_{L} \bar{\tau}^{\alpha \beta} .
$$

The latter proof makes it clear how crucial the analytic-continuation finite part $\mathcal{F} \mathcal{P}$ is, which we recall is the same as in our iteration of the exterior post-Minkowskian field [see Eq. (45)]. Without a finite part, the multipole moment (113) would be strongly divergent, because the pseudo-tensor $\bar{\tau}^{\alpha \beta}$ has a non-compact support owing to the contribution of the gravitational field, and the multipolar

29 Recall that our abbreviated notation $\mathcal{F P}$ includes the crucial regularization factor $\widetilde{r}^{B}$. 
factor $x_{L}$ behaves like $r^{\ell}$ when $r \rightarrow+\infty$. The latter divergence has plagued the field of postNewtonian expansions of gravitational radiation for many years. In applications such as in Part B of this article, we must carefully follow the rules for handling the $\mathcal{F P}$ operator.

The two terms in the right-hand side of Eq. (105) depend separately on the length scale $r_{0}$ that we have introduced into the definition of the finite part, through the analytic-continuation factor $\widetilde{r}^{B}=\left(r / r_{0}\right)^{B}$ introduced in Eq. (42). However, the sum of these two terms, i.e., the exterior multipolar field $\mathcal{M}(h)$ itself, is independent of $r_{0}$. To see this, the simplest way is to differentiate formally $\mathcal{M}(h)$ with respect to $r_{0}$; the differentiations of the two terms of Eq. (105) cancel each other. The independence of the field upon $r_{0}$ is quite useful in applications, since in general many intermediate calculations do depend on $r_{0}$, and only in the final stage does the cancellation of the $r_{0}$ 's occur. For instance, we have already seen in Eqs. (93) - (94) that the source quadrupole moment $\mathrm{I}_{i j}$ depends on $r_{0}$ starting from the $3 \mathrm{PN}$ level, but that this $r_{0}$ is compensated by another $r_{0}$ coming from the non-linear "tails of tails" at the $3 \mathrm{PN}$ order.

\subsection{Equivalence with the Will-Wiseman formalism}

Will \& Wiseman [424] (see also Refs. [422, 335]), extending previous work of Epstein \& Wagoner [185] and Thorne [403], have obtained a different-looking multipole decomposition, with different definitions for the multipole moments of a post-Newtonian source. They find, instead of our multipole decomposition given by Eq. (105),

$$
\mathcal{M}\left(h^{\alpha \beta}\right)=\square_{\mathrm{ret}}^{-1}\left[\mathcal{M}\left(\Lambda^{\alpha \beta}\right)\right]_{\mathcal{R}}-\frac{4 G}{c^{4}} \sum_{\ell=0}^{+\infty} \frac{(-)^{\ell}}{\ell !} \partial_{L}\left\{\frac{1}{r} \mathcal{W}_{L}^{\alpha \beta}(t-r / c)\right\} .
$$

There is no $\mathcal{F P}$ operation in the first term, but instead the retarded integral is truncated, as indicated by the subscript $\mathcal{R}$, to extend only in the "far zone": i.e., $\left|\mathbf{x}^{\prime}\right|>\mathcal{R}$ in the notation of Eq. (31), where $\mathcal{R}$ is a constant radius enclosing the source $(\mathcal{R}>a)$. The near-zone part of the retarded integral is thereby removed, and there is no problem with the singularity of the multipole expansion $\mathcal{M}\left(\Lambda^{\alpha \beta}\right)$ at the origin. The multipole moments $\mathcal{W}_{L}$ are then given, in contrast with our result (106), by an integral extending over the "near zone" only:

$$
\mathcal{W}_{L}^{\alpha \beta}(u)=\int_{|\mathbf{x}|<\mathcal{R}} \mathrm{d}^{3} \mathbf{x} x_{L} \bar{\tau}^{\alpha \beta}(\mathbf{x}, u) .
$$

Since the integrand is compact-supported there is no problem with the bound at infinity and the integral is well-defined (no need of a $\mathcal{F P}$ ).

Let us show that the two different formalisms are equivalent. We compute the difference between our moment $\mathcal{H}_{L}$ defined by Eq. (106), and the moment $\mathcal{W}_{L}$ given by Eq. (115). For the comparison we split $\mathcal{H}_{L}$ into far-zone and near-zone pieces corresponding to the radius $\mathcal{R}$. Since the finite part $\mathcal{F P}$ present in $\mathcal{H}_{L}$ deals only with the bound at infinity, it can be removed from the near-zone piece, which is then seen to reproduce $\mathcal{W}_{L}$ exactly. So the difference between the two moments is simply given by the far-zone piece:

$$
\mathcal{H}_{L}^{\alpha \beta}(u)-\mathcal{W}_{L}^{\alpha \beta}(u)=\mathcal{F} \mathcal{P} \int_{|\mathbf{x}|>\mathcal{R}} \mathrm{d}^{3} \mathbf{x} x_{L} \bar{\tau}^{\alpha \beta}(\mathbf{x}, u)
$$

We transform next this expression. Successively we write $\bar{\tau}^{\alpha \beta}=\mathcal{M}\left(\bar{\tau}^{\alpha \beta}\right)$ because we are outside the source, and $\mathcal{M}\left(\bar{\tau}^{\alpha \beta}\right)=\overline{\mathcal{M}\left(\tau^{\alpha \beta}\right)}$ thanks to the matching equation (103). At this stage, we recall from our reasoning right after Eq. (112) that the finite part of an integral over the whole space $\mathbb{R}^{3}$ of a quantity having the same structure as $\overline{\mathcal{M}\left(\tau^{\alpha \beta}\right)}$ is identically zero by analytic continuation. The main ingredient of the present proof is made possible by this fact, as it allows us to transform 
the far-zone integration $|\mathbf{x}|>\mathcal{R}$ in Eq. (116) into a near-zone one $|\mathbf{x}|<\mathcal{R}$, at the price of changing the overall sign in front of the integral. So,

$$
\mathcal{H}_{L}^{\alpha \beta}(u)-\mathcal{W}_{L}^{\alpha \beta}(u)=-\mathcal{F} \mathcal{P} \int_{|\mathbf{x}|<\mathcal{R}} \mathrm{d}^{3} \mathbf{x} x_{L} \overline{\mathcal{M}\left(\tau^{\alpha \beta}\right)}(\mathbf{x}, u) .
$$

Finally, it is straightforward to check that the right-hand side of this equation, when summed up over all multipolarities $\ell$, accounts exactly for the near-zone part that was removed from the retarded integral of $\mathcal{M}\left(\Lambda^{\alpha \beta}\right)$ in the first term in Eq. (114), so that the "complete" retarded integral as given by the first term in our own definition (105) is exactly reconstituted. In conclusion, the formalism of Ref. [424] is equivalent to the one of Refs. [44, 49].

\subsection{The source multipole moments}

In principle, the bridge between the exterior gravitational field generated by the post-Newtonian source and its inner field is provided by Theorem 5 ; however, we still have to make the connection with the explicit construction of the general multipolar and post-Minkowskian metric in Section 2. Namely, we must find the expressions of the six STF source multipole moments $\mathrm{I}_{L}, \mathrm{~J}_{L}, \ldots, \mathrm{Z}_{L}$ parametrizing the linearized metric $(35)-(37)$ at the basis of that construction. ${ }^{30}$

To do this we first find the equivalent of the multipole expansion given in Theorem 5, which was parametrized by non-trace-free multipole functions $\mathcal{H}_{L}^{\alpha \beta}$, in terms of new multipole functions $\mathcal{F}_{L}^{\alpha \beta}$ that are STF in all their indices $L$. The result is

$$
\mathcal{M}\left(h^{\alpha \beta}\right)=\mathcal{F} \mathcal{P} \square_{\text {ret }}^{-1}\left[\mathcal{M}\left(\Lambda^{\alpha \beta}\right)\right]-\frac{4 G}{c^{4}} \sum_{\ell=0}^{+\infty} \frac{(-)^{\ell}}{\ell !} \partial_{L}\left\{\frac{1}{r} \mathcal{F}_{L}^{\alpha \beta}(t-r / c)\right\}
$$

where the STF multipole functions (witness the multipolar factor $\hat{x}_{L} \equiv \mathrm{STF}\left[x_{L}\right]$ ) read

$$
\mathcal{F}_{L}^{\alpha \beta}(u)=\mathcal{F} \mathcal{P} \int \mathrm{d}^{3} \mathbf{x} \hat{x}_{L} \int_{-1}^{1} \mathrm{~d} z \delta_{\ell}(z) \bar{\tau}^{\alpha \beta}(\mathbf{x}, u+z r / c) .
$$

Notice the presence of an extra integration variable $z$, ranging from -1 to 1 . The $z$-integration involves the weighting function

$$
\delta_{\ell}(z)=\frac{(2 \ell+1) ! !}{2^{\ell+1} \ell !}\left(1-z^{2}\right)^{\ell}
$$

which approaches the Dirac delta-function (hence its name) in the limit of large multipolarities, $\lim _{\ell \rightarrow+\infty} \delta_{\ell}(z)=\delta(z)$, and is normalized in such a way that

$$
\int_{-1}^{1} d z \delta_{\ell}(z)=1
$$

The next step is to impose the harmonic-gauge conditions (21) onto the multipole decomposition (118), and to decompose the multipole functions $\mathcal{F}_{L}^{\alpha \beta}(u)$ into STF irreducible pieces with respect to both $L$ and their spatial indices contained into $\alpha \beta=00,0 i, i j$. This technical part of the calculation is identical to the one of the STF irreducible multipole moments of linearized gravity [154]. The formulas needed in this decomposition read

$$
\mathcal{F}_{L}^{00}=R_{L}
$$

\footnotetext{
30 Recall that in actual applications we need mostly the mass-type moment $\mathrm{I}_{L}$ and current-type one $\mathrm{J}_{L}$, because the other moments simply parametrize a linearized gauge transformation.
} 


$$
\begin{aligned}
\mathcal{F}_{L}^{0 i} & ={ }^{(+)} T_{i L}+\epsilon_{a i<i_{\ell}}{ }^{(0)} T_{L-1>a}+\delta_{i<i_{\ell}}{ }^{(-)} T_{L-1>}, \\
\mathcal{F}_{L}^{i j} & ={ }^{(+2)} U_{i j L}+\underset{L}{S T} F \operatorname{STF}\left[\epsilon_{a i j}{ }^{(+1)} U_{a j L-1}+\delta_{i i_{\ell}}{ }^{(0)} U_{j L-1}\right. \\
& \left.+\delta_{i i_{\ell}} \epsilon_{a j i_{\ell-1}}{ }^{(-1)} U_{a L-2}+\delta_{i i_{\ell}} \delta_{j i_{\ell-1}}{ }^{(-2)} U_{L-2}\right]+\delta_{i j} V_{L},
\end{aligned}
$$

where the ten tensors $R_{L},{ }^{(+)} T_{L+1}, \ldots,{ }^{(-2)} U_{L-2}, V_{L}$ are STF, and are uniquely given in terms of the $\mathcal{F}_{L}^{\alpha \beta}$ 's by some inverse formulas. Finally, the latter decompositions yield the following.

Theorem 6. The STF multipole moments $\mathrm{I}_{L}$ and $\mathrm{J}_{L}$ of a post-Newtonian source are given, formally up to any post-Newtonian order, by $(\ell \geqslant 2)$

$$
\begin{aligned}
\mathrm{I}_{L}(u) & =\mathcal{F P} \int \mathrm{d}^{3} \mathbf{x} \int_{-1}^{1} \mathrm{~d} z\left\{\delta_{\ell} \hat{x}_{L} \Sigma-\frac{4(2 \ell+1)}{c^{2}(\ell+1)(2 \ell+3)} \delta_{\ell+1} \hat{x}_{i L} \Sigma_{i}^{(1)}\right. \\
& \left.+\frac{2(2 \ell+1)}{c^{4}(\ell+1)(\ell+2)(2 \ell+5)} \delta_{\ell+2} \hat{x}_{i j L} \Sigma_{i j}^{(2)}\right\}(\mathbf{x}, u+z r / c), \\
\mathrm{J}_{L}(u) & =\mathcal{F} \mathcal{P} \int \mathrm{d}^{3} \mathbf{x} \int_{-1}^{1} \mathrm{~d} z \epsilon_{a b\left\langle i_{\ell}\right.}\left\{\delta_{\ell} \hat{x}_{L-1\rangle a} \Sigma_{b}-\frac{2 \ell+1}{c^{2}(\ell+2)(2 \ell+3)} \delta_{\ell+1} \hat{x}_{L-1\rangle a c} \Sigma_{b c}^{(1)}\right\}(\mathbf{x}, u+z r / c) .
\end{aligned}
$$

These moments are the ones that are to be inserted into the linearized metric $h_{(1)}^{\alpha \beta}$ that represents the lowest approximation to the post-Minkowskian field $h_{\mathrm{ext}}^{\alpha \beta}=\sum_{n \geqslant 1} G^{n} h_{(n)}^{\alpha \beta}$ defined in Eq. (50).

In these formulas the notation is as follows: Some convenient source densities are defined from the post-Newtonian expansion (denoted by an overbar) of the pseudo-tensor $\tau^{\alpha \beta}$ by

$$
\begin{aligned}
\Sigma & =\frac{\bar{\tau}^{00}+\bar{\tau}^{i i}}{c^{2}}, \\
\Sigma_{i} & =\frac{\bar{\tau}^{0 i}}{c}, \\
\Sigma_{i j} & =\bar{\tau}^{i j},
\end{aligned}
$$

(where $\bar{\tau}^{i i} \equiv \delta_{i j} \bar{\tau}^{i j}$ ). As indicated in Eqs. (123) all these quantities are to be evaluated at the spatial point $\mathbf{x}$ and at time $u+z r / c$.

For completeness, we give also the formulas for the four auxiliary source moments $\mathrm{W}_{L}, \ldots, \mathrm{Z}_{L}$, which parametrize the gauge vector $\varphi_{1}^{\alpha}$ as defined in Eqs. (37):

$$
\begin{aligned}
\mathrm{W}_{L}(u) & =\mathcal{F} \mathcal{P} \int \mathrm{d}^{3} \mathbf{x} \int_{-1}^{1} \mathrm{~d} z\left\{\frac{2 \ell+1}{(\ell+1)(2 \ell+3)} \delta_{\ell+1} \hat{x}_{i L} \Sigma_{i}\right. \\
- & \left.\frac{2 \ell+1}{2 c^{2}(\ell+1)(\ell+2)(2 \ell+5)} \delta_{\ell+2} \hat{x}_{i j L} \Sigma_{i j}^{(1)}\right\}, \\
\mathrm{X}_{L}(u) & =\mathcal{F} \mathcal{P} \int \mathrm{d}^{3} \mathbf{x} \int_{-1}^{1} \mathrm{~d} z\left\{\frac{2 \ell+1}{2(\ell+1)(\ell+2)(2 \ell+5)} \delta_{\ell+2} \hat{x}_{i j L} \Sigma_{i j}\right\}, \\
\mathrm{Y}_{L}(u) & =\mathcal{F} \mathcal{P} \int \mathrm{d}^{3} \mathbf{x} \int_{-1}^{1} \mathrm{~d} z\left\{-\delta_{\ell} \hat{x}_{L} \Sigma_{i i}+\frac{3(2 \ell+1)}{(\ell+1)(2 \ell+3)} \delta_{\ell+1} \hat{x}_{i L} \Sigma_{i}^{(1)}\right. \\
\mathrm{Z}_{L}(u) & =\mathcal{F} \mathcal{P} \int \mathrm{d}^{3} \mathbf{x} \int_{-1}^{1} \mathrm{~d} z \epsilon_{a b\left\langle i_{\ell}\right.}\left\{-\frac{2(2 \ell+1)}{(\ell+2)(2 \ell+3)} \delta_{\ell+1} \hat{x}_{L-1\rangle b c} \Sigma_{a c}\right\} .
\end{aligned}
$$


As discussed in Section 2, one can always find two intermediate "packages" of multipole moments, namely the canonical moments $\mathrm{M}_{L}$ and $\mathrm{S}_{L}$, which are some non-linear functionals of the source moments (123) and (125), and such that the exterior field depends only on them, modulo a change of coordinates. However, the canonical moments $\mathrm{M}_{L}, \mathrm{~S}_{L}$ do not admit general closed-form expressions like $(123)-(125){ }^{31}$

These source moments are physically valid for post-Newtonian sources and make sense only in the form of a post-Newtonian expansion, so in practice we need to know how to expand the $z$-integrals as series when $c \rightarrow+\infty$. Here is the appropriate formula:

$$
\int_{-1}^{1} \mathrm{~d} z \delta_{\ell}(z) \Sigma(\mathbf{x}, u+z r / c)=\sum_{k=0}^{+\infty} \frac{(2 \ell+1) ! !}{2^{k} k !(2 \ell+2 k+1) ! !}\left(\frac{r}{c} \frac{\partial}{\partial u}\right)^{2 k} \Sigma(\mathbf{x}, u) .
$$

Since the right-hand side involves only even powers of $1 / c$, the same result holds equally well for the advanced variable $u+z r / c$ or the retarded one $u-z r / c$. Of course, in the Newtonian limit, the moments $\mathrm{I}_{L}$ and $\mathrm{J}_{L}$ (and also $\mathrm{M}_{L}$ and $\mathrm{S}_{L}$ ) reduce to the standard Newtonian expressions. For instance, $\mathrm{I}_{i j}(u)=\mathrm{Q}_{i j}(u)+\mathcal{O}\left(1 / c^{2}\right)$ recovers the Newtonian quadrupole moment $(3) .^{32}$

Needless to say, the formalism becomes prohibitively difficult to apply at very high postNewtonian approximations. Some post-Newtonian order being given, we must first compute the relevant relativistic corrections to the pseudo stress-energy-tensor $\bar{\tau}^{\alpha \beta}$; this necessitates solving the field equations inside the matter, which we shall investigate in the next Section 5 . Then $\bar{\tau}^{\alpha \beta}$ is to be inserted into the source moments (123) and (125), where the formula (126) permits expressing all the terms up to that post-Newtonian order by means of more tractable integrals extending over $\mathbb{R}^{3}$. Given a specific model for the matter source we then have to find a way to compute all these spatial integrals; this is done in Section 9.1 for the case of point-mass binaries. Next, we must substitute the source multipole moments into the linearized metric (35)-(37), and iterate them until all the necessary multipole interactions taking place in the radiative moments $\mathrm{U}_{L}$ and $\mathrm{V}_{L}$ are under control. In fact, we have already worked out these multipole interactions for general sources in Section 3.3 up to the 3PN order in the full waveform, and 3.5PN order for the dominant $(2,2)$ mode. Only at this point does one have the physical radiation field at infinity, from which we can build the templates for the detection and analysis of gravitational waves. We advocate here that the complexity of the formalism simply reflects the complexity of the Einstein field equations. It is probably impossible to devise a different formalism, valid for general sources devoid of symmetries, that would be substantially simpler.

\section{$5 \quad$ Interior Field of a Post-Newtonian Source}

Theorem 6 solves in principle the question of the generation of gravitational waves by extended post-Newtonian matter sources. However, notice that this result has still to be completed by the precise procedure, i.e., an explicit "algorithm", for the post-Newtonian iteration of the nearzone field, analogous to the multipolar-post-Minkowskian algorithm we defined in Section 2. Such procedure will permit the systematic computation of the source multipole moments, which contain the full post-Newtonian expansion of the pseudo-tensor $\bar{\tau}^{\alpha \beta}$, and of the radiation reaction effects occurring within the matter source.

Before proceeding, let us recall that the "standard" post-Newtonian approximation, as it was used until, say, the early 1980's (see for instance Refs. [6, 181, 269, 270, 334] and also the earlier works $[344,122,124,123])$, was plagued with some apparently inherent difficulties, which croped

31 The work [65] provided some alternative expressions for all the multipole moments (123)-(125), useful for some applications, in the form of surface integrals extending on the outer part of the source's near zone.

32 The moments $\mathrm{W}_{L}, \ldots, \mathrm{Z}_{L}$ have also a Newtonian limit, but which is not particularly illuminating. 
up at some high post-Newtonian order. Historically these difficulties, even appearing at higher approximations, have cast a doubt on the actual soundness, from a theoretical point of view, of the post-Newtonian expansion. Practically speaking, they posed the question of the reliability of the approximation, when comparing the theory's predictions with very precise experimental results. This was one of the main reason for the famous radiation-reaction controversy raging at the time of the binary pulsar data $[182,418]$. In this section we assess the nature of these difficulties - are they purely technical or linked with some fundamental drawback of the approximation scheme? and eventually resolve them.

1. The first problem we face is that in higher approximations some divergent Poisson-type integrals appear. Indeed the post-Newtonian expansion replaces the resolution of a hyperboliclike d'Alembertian equation by a perturbatively equivalent hierarchy of elliptic-like Poisson equations. Rapidly it is found during the post-Newtonian iteration that the right-hand side of the Poisson equations acquires a non-compact support (it is distributed all over space $\mathbb{R}^{3}$ ), and that as a result the standard Poisson integral diverges at the bound of the integral at spatial infinity, i.e., when $r \equiv|\mathbf{x}| \rightarrow+\infty$, with $t=$ const.

2. The second problem is related with the limitation of the post-Newtonian approximation to the near zone - the region surrounding the source of small extent with respect to the wavelength of the emitted radiation: $r \ll \lambda$. As we have seen, the post-Newtonian expansion assumes from the start that all retardations $r / c$ are small, so it can rightly be viewed as a formal near-zone expansion, when $r \rightarrow 0$. Note that the fact which makes the Poisson integrals to become typically divergent, namely that the coefficients of the post-Newtonian series blow up at spatial infinity, when $r \rightarrow+\infty$, has nothing to do with the actual behaviour of the field at infinity. However, the serious consequence is that it is a priori impossible to implement within the post-Newtonian scheme alone the physical information that the matter system is isolated from the rest of the Universe. Most importantly, the no-incoming radiation condition, imposed at past null infinity, cannot be taken directly into account, $a$ priori, into the post-Newtonian scheme. In this sense the post-Newtonian approximation is not "self-supporting", because it necessitates some information taken from outside its own domain of validity.

The divergencies are linked to the fact that the post-Newtonian expansion is actually a singular perturbation, in the sense that the coefficients of the successive powers of $1 / c$ are not uniformly valid in space, since they typically blow up at spatial infinity like some powers of $r$. We know for instance that the post-Newtonian expansion cannot be "asymptotically flat" starting at the $2 \mathrm{PN}$ or 3PN level, depending on the adopted coordinate system [362]. The result is that the standard Poisson integrals are in general badly-behaving at infinity. Trying to solve the post-Newtonian equations by means of the Poisson integral does not make sense. However, this does not mean that there are no solutions to the problem, but simply that the Poisson integral does not constitute the appropriate solution of the Poisson equation in the context of post-Newtonian expansions.

Here we present, following Refs. [357, 75], a solution of both problems, in the form of a general expression for the near-zone gravitational field, developed to any post-Newtonian order, which has been determined from implementing the matching equation (103). This solution is free of the divergences of Poisson-type integrals we mentioned above, and yields, in particular, some general expression, valid up to any order, of the terms associated with the gravitational radiation reaction force inside the post-Newtonian source.

Though we shall focus our attention on the particular approach advocated in $[357,75]$, there are other ways to resolve the problems of the post-Newtonian approximation. Notably, an alternative solution to the problem of divergencies, proposed in Refs. [214, 211], is based on an initial-value formulation. In this method the problem of the appearance of divergencies is avoided because 
of the finiteness of the causal region of integration, between the initial Cauchy hypersurface and the considered field point. On the other hand, a different approach to the problem of radiation reaction, which does not use a matching procedure, is to work only within a post-Minkowskian iteration scheme without expanding the retardations, see e.g., Ref. [126].

\subsection{Post-Newtonian iteration in the near zone}

We perform the post-Newtonian iteration of the field equations in harmonic coordinates in the near zone of an isolated matter distribution. We deal with a general hydrodynamical fluid, whose stressenergy tensor is smooth, i.e., $T^{\alpha \beta} \in C^{\infty}\left(\mathbb{R}^{4}\right)$. Thus the scheme a priori excludes the presence of singularities and black holes; these will be dealt with in Part B of this article.

We shall now prove [357] that the post-Newtonian expansion can be indefinitely iterated without divergences. Like in Eq. (106) we denote by means of an overline the formal (infinite) postNewtonian expansion of the field inside the source's near-zone. The general structure of the post-Newtonian expansion is denoted (skipping the space-time indices $\alpha \beta$ ) as

$$
\bar{h}(\mathbf{x}, t, c)=\sum_{m=2}^{+\infty} \frac{1}{c^{m}} \underset{m}{\bar{h}}(\mathbf{x}, t ; \ln c) .
$$

The $m$-th post-Newtonian coefficient is naturally the factor of the $m$-th power of $1 / c$. However, we know from restoring the factors c's in Theorem 3 [see Eq. (53)], that the post-Newtonian expansion also involves powers of the logarithm of $c$; these are included for convenience here into the definition of the coefficients $\bar{h}_{m} \cdot{ }^{33}$ For the stress-energy pseudo-tensor appearing in Eq. (106) we have the same type of expansion,

$$
\bar{\tau}(\mathbf{x}, t, c)=\sum_{m=-2}^{+\infty} \frac{1}{c^{m}} \underset{m}{\bar{\tau}}(\mathbf{x}, t ; \ln c) .
$$

Note that the expansion starts with a term of order $c^{2}$ corresponding to the rest mass-energy $(\bar{\tau}$ has the dimension of an energy density). As usual we shall understand the infinite sums such as (127)(128) in the sense of formal series, i.e., merely as an ordered collection of coefficients. Because of our consideration of regular extended matter distributions the post-Newtonian coefficients are smooth functions of space-time, i.e., $\bar{h}_{m}(\mathbf{x}, t) \in C^{\infty}\left(\mathbb{R}^{4}\right)$.

Inserting the post-Newtonian ansatz (127) into the harmonic-coordinates Einstein field equation $(21)-(22)$ and equating together the powers of $1 / c$, results is an infinite set of Poisson-type equations $(\forall m \geqslant 2)$,

$$
\Delta \underset{m}{\bar{h}}=16 \pi G_{m-4} \bar{\tau}+\partial_{t}^{2} \bar{h}_{m-2},
$$

where the second term comes from the split of the d'Alembertian operator into a Laplacian and a second time derivative: $\square=\Delta-\frac{1}{c^{2}} \partial_{t}^{2}$ (this term is zero when $m=2$ and 3 ). We proceed by induction, i.e., we work at some given but arbitrary post-Newtonian order $m$, assume that we succeeded in constructing the sequence of previous coefficients $\bar{h}_{p}(\forall p \leqslant m-1)$, and from that show how to infer the next-order coefficient $\bar{h}_{m}$.

To cure the problem of divergencies we introduce a generalized solution of the Poisson equation with non-compact support source, in the form of an appropriate finite part of the usual Poisson integral obtained by regularization of the bound at infinity by means of a specific process of analytic continuation. For any source term like $\bar{\tau}_{m}$, we multiply it by the regularization factor $\widetilde{r}^{B}$ already extensively used in the construction of the exterior field, thus $B \in \mathbb{C}$ and $\widetilde{r}=r / r_{0}$ is given by Eq. (42). Only then do we apply the usual Poisson integral, which therefore defines a

\footnotetext{
33 For this argument we assume the validity of the matching equation (103) and that the post-Minkowskian series over $n=1, \ldots, \infty$ in Eq. (53) has been formally summed up.
} 
certain function of $B$. The well-definedness of that integral heavily relies on the behaviour of the integrand at the bound at infinity. There is no problem with the vicinity of the origin inside the source because of the smoothness of the pseudo-tensor. Then one can prove [357] that the latter function of $B$ generates a (unique) analytic continuation down to a neighbourhood of the value of interest $B=0$, except at $B=0$ itself, at which value it admits a Laurent expansion with multiple poles up to some finite order (but growing with the post-Newtonian order $m$ ). Then, we consider the Laurent expansion of that function when $B \rightarrow 0$ and pick up the finite part, or coefficient of the zero-th power of $B$, of that expansion. This defines our generalized Poisson integral:

$$
\Delta^{-1}\left[\begin{array}{c}
\bar{\tau} \\
m
\end{array}\right](\mathbf{x}, t) \equiv-\frac{1}{4 \pi} \mathcal{F} \mathcal{P}_{B=0} \int \frac{\mathrm{d}^{3} \mathbf{x}^{\prime}}{\left|\mathbf{x}-\mathbf{x}^{\prime}\right|} \widetilde{r}^{B} \underset{m}{\bar{\tau}}\left(\mathbf{x}^{\prime}, t\right) .
$$

The integral extends over all three-dimensional space but with the latter finite-part regularization at infinity denoted $\mathcal{F} \mathcal{P}_{B=0}$ or $\mathcal{F P}$ for short. The main properties of this generalized Poisson operator is that it solves the Poisson equation,

$$
\Delta\left(\Delta^{-1}\left[\begin{array}{c}
\bar{\tau} \\
m
\end{array}\right]\right)=\underset{m}{\bar{\tau}}
$$

and that the solution $\Delta^{-1} \bar{\tau}_{m}$ owns the same properties as its source $\bar{\tau}_{m}$, i.e., the smoothness and the same type of behaviour at infinity, as given by Eq. (104). Similarly, we define the generalized iterated Poisson integral as

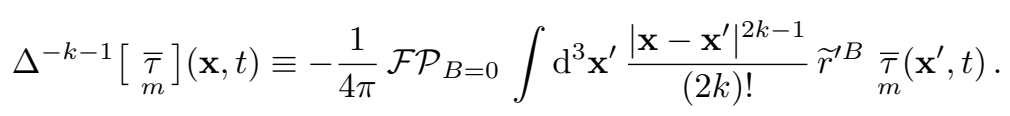

The most general solution of the Poisson equation will be obtained by application of the previous generalized Poisson operator to the right-hand side of Eq. (129), and augmented by the most general homogeneous solution of the Poisson equation. Thus, we can write

$$
\bar{h}=16 \pi G \Delta^{-1}\left[\begin{array}{c}
\bar{\tau} \\
m-4
\end{array}\right]+\partial_{t}^{2} \Delta^{-1}\left[\begin{array}{c}
\bar{h} \\
m-2
\end{array}\right]+\sum_{\ell=0}^{+\infty} \mathcal{B}_{m}(t) \hat{x}_{L} .
$$

The last term represents the most general solution of the Laplace equation that is regular at the origin $r=0$. It can be written in STF guise as a multipolar series of terms of the type $\hat{x}_{L}$, and multiplied by arbitrary STF-tensorial functions of time ${ }_{m} \mathcal{B}_{L}(t)$. These functions will be associated with the radiation reaction of the field onto the source; they will depend on which boundary conditions are to be imposed on the gravitational field at infinity from the source.

It is now trivial to iterate the process. We substitute for $\bar{h}_{m-2}$ in the right-hand side of Eq. (133) the same expression but with $m$ replaced by $m-2$, and similarly come down until we stop at either one of the coefficients $\bar{h}_{0}=0$ or $\bar{h}_{1}=0$. At this point $\bar{h}_{m}$ is expressed in terms of the previous $\bar{\tau}_{p}$ 's and ${ }_{p} \mathcal{B}_{L}$ 's with $p \leqslant m-2$. To finalize the process we introduce what we call the operator of the "instantaneous" potentials and denote $\square_{\text {inst }}^{-1}$. Our notation is chosen to contrast with the standard operator of the retarded potentials $\square_{\text {ret }}^{-1}$ defined by Eq. (31). However, beware of the fact that unlike $\square_{\text {ret }}^{-1}$ the operator $\square_{\text {inst }}^{-1}$ will be defined only when acting on a post-Newtonian series such as $\bar{\tau}$. Indeed, we pose

$$
\square_{\text {inst }}^{-1}[\bar{\tau}] \equiv \sum_{k=0}^{+\infty}\left(\frac{\partial}{c \partial t}\right)^{2 k} \Delta^{-k-1}[\bar{\tau}]
$$

where the $k$-th iteration of the generalized Poisson operator is defined by Eq. (132). This operator is instantaneous in the sense that it does not involve any integration over time. It is readily checked that in this way we have a solution of the source-free d'Alembertian equation,

$$
\square(\square \text { inst }[\bar{\tau}])=\bar{\tau} .
$$


On the other hand, the homogeneous solution in Eq. (133) will yield by iteration an homogeneous solution of the d'Alembertian equation that is necessarily regular at the origin. Hence it should be of the anti-symmetric type, i.e., be made of the difference between a retarded multipolar wave and the corresponding advanced wave. We shall therefore introduce a new definition for some STFtensorial functions $\mathcal{A}_{L}(t)$ parametrizing those advanced-minus-retarded free waves. It is very easy to relate if necessary the post-Newtonian expansion of $\mathcal{A}_{L}(t)$ to the functions ${ }_{m} \mathcal{B}_{L}(t)$ previously introduced in Eq. (133). Finally the most general post-Newtonian solution, iterated ad infinitum and without any divergences, is obtained into the form

$$
\bar{h}=\frac{16 \pi G}{c^{4}} \square_{\text {inst }}^{-1}[\bar{\tau}]-\frac{4 G}{c^{4}} \sum_{\ell=0}^{+\infty} \frac{(-)^{\ell}}{\ell !} \hat{\partial}_{L}\left\{\frac{\mathcal{A}_{L}(t-r / c)-\mathcal{A}_{L}(t+r / c)}{2 r}\right\} .
$$

We shall refer to the $\mathcal{A}_{L}(t)$ 's as the radiation-reaction functions. If we stay at the level of the post-Newtonian iteration which is confined into the near zone we cannot do more than Eq. (136): There is no means to compute the radiation-reaction functions $\mathcal{A}_{L}(t)$. We are here touching the second problem faced by the standard post-Newtonian approximation.

\subsection{Post-Newtonian metric and radiation reaction effects}

As we have understood this problem is that of the limitation to the near zone. Such limitation can be circumvented to the lowest post-Newtonian orders by considering retarded integrals that are formally expanded when $c \rightarrow+\infty$ as series of "instantaneous" Poisson-like integrals, see e.g., [6]. This procedure works well up to the $2.5 \mathrm{PN}$ level and has been shown to correctly fix the dominant radiation reaction term at the $2.5 \mathrm{PN}$ order [181, 269, 270, 334]. Unfortunately such a procedure assumes fundamentally that the gravitational field, after expansion of all retardations $r / c \rightarrow 0$, depends on the state of the source at a single time $t$, in keeping with the instantaneous character of the Newtonian interaction. However, we know that the post-Newtonian field (as well as the source's dynamics) will cease at some stage to be given by a functional of the source parameters at a single time, because of the imprint of gravitational-wave tails in the near zone field, in the form of the hereditary modification of the radiation reaction force at the $1.5 \mathrm{PN}$ relative order [58, 60,43$]$. Since the reaction force is itself of order $2.5 \mathrm{PN}$ this means that the formal post-Newtonian expansion of retarded Green functions is no longer valid starting at the $4 \mathrm{PN}$ order.

The solution of the problem resides in the matching of the near-zone field to the exterior field. We have already seen in Theorems 5 and 6 that the matching equation (103) yields the expression of the multipole expansion in the exterior domain. Now we prove that it also permits the full determinantion of the post-Newtonian metric in the near-zone, i.e., the radiation-reaction functions $\mathcal{A}_{L}$ which have been left unspecified in Eq. (136).

We find [357] that the radiation-reaction functions $\mathcal{A}_{L}$ are composed of the multipole moment functions $\mathcal{F}_{L}$ defined by Eq. (119), which will here characterize "linear-order" radiation reaction effects starting at $2.5 \mathrm{PN}$ order, and of an extra piece $\mathcal{R}_{L}$, which will be due to non-linear effets in the radiation reaction and turn out to arise at the $4 \mathrm{PN}$ order. Thus,

$$
\mathcal{A}_{L}(t)=\mathcal{F}_{L}(t)+\mathcal{R}_{L}(t) .
$$

The extra piece $\mathcal{R}_{L}$ is obtained from the multipole expansion of the pseudo-tensor $\mathcal{M}(\tau) .{ }^{34}$ Hence the radiation-reaction functions do depend on the behaviour of the field far away from the matter source (as physical intuition already told us). The explicit expression reads

$$
\mathcal{R}_{L}(t)=\mathcal{F P} \int \mathrm{d}^{3} \mathbf{x} \hat{x}_{L} \int_{1}^{+\infty} \mathrm{d} z \gamma_{\ell}(z) \mathcal{M}(\tau)(\mathbf{x}, t-z r / c) .
$$

\footnotetext{
${ }^{34}$ We mean the fully-fledge $\mathcal{M}(\tau)$; i.e., not the formal object $\mathcal{M}(\bar{\tau})$.
} 
The fact that the multipolar expansion $\mathcal{M}(\tau)$ is the source term for the function $\mathcal{R}_{L}$ is the consequence of the matching equation (103). The specific contributions due to $\mathcal{R}_{L}$ in the post-Newtonian metric (136) are associated with tails of waves [58, 43]. Notice that, remarkably, the $\mathcal{F} \mathcal{P}$ regularization deals with the bound of the integral at $r=0$, in contrast with Eq. (119) where it deals with the bound at $r=+\infty$. The weighting function $\gamma_{\ell}(z)$ therein, where $z$ extends up to infinity in contrast to the analogous function $\delta_{\ell}(z)$ in Eq. (119), is simply related to it by $\gamma_{\ell}(z) \equiv-2 \delta_{\ell}(z)$; such definition is motivated by the fact that the integral of that function is normalized to one: ${ }^{35}$

$$
\int_{1}^{+\infty} \mathrm{d} z \gamma_{\ell}(z)=1
$$

The post-Newtonian metric (136) is now fully determined. However, let us now prove a more interesting alternative formulation of it, derived in Ref. [75].

Theorem 7. The expression of the post-Newtonian field in the near zone of a post-Newtonian source, satisfying correct boundary conditions at infinity (no incoming radiation), reads

$$
\bar{h}^{\alpha \beta}=\frac{16 \pi G}{c^{4}} \square_{\mathrm{ret}}^{-1}\left[\bar{\tau}^{\alpha \beta}\right]-\frac{4 G}{c^{4}} \sum_{\ell=0}^{+\infty} \frac{(-)^{\ell}}{\ell !} \hat{\partial}_{L}\left\{\frac{\mathcal{R}_{L}^{\alpha \beta}(t-r / c)-\mathcal{R}_{L}^{\alpha \beta}(t+r / c)}{2 r}\right\}
$$

The first term represents a particular solution of the hierarchy of post-Newtonian equations, while the second one is a homogeneous multipolar solution of the wave equation, of the "anti-symmetric" type that is regular at the origin $r=0$ located inside the source, and parametrized by the multipolemoment functions (138).

Let us be more precise about the meaning of the first term in Eq. (140). Indeed such term is made of the formal expansion of the standard retarded integral (31) when $c \rightarrow \infty$, but acting on a post-Newtonian source term $\bar{\tau}$,

$$
\square_{\mathrm{ret}}^{-1}\left[\bar{\tau}^{\alpha \beta}\right](\mathbf{x}, t) \equiv-\frac{1}{4 \pi} \sum_{m=0}^{+\infty} \frac{(-)^{m}}{m !}\left(\frac{\partial}{c \partial t}\right)^{m} \mathcal{F} \mathcal{P} \int \mathrm{d}^{3} \mathbf{x}^{\prime}\left|\mathbf{x}-\mathbf{x}^{\prime}\right|^{m-1} \bar{\tau}^{\alpha \beta}\left(\mathbf{x}^{\prime}, t\right) .
$$

We emphasize that (141) constitutes the definition of a (formal) post-Newtonian expansion, each term of which being built from the post-Newtonian expansion of the pseudo-tensor. Crucial in the present formalism, is that each of the terms is regularized by means of the $\mathcal{F} \mathcal{P}$ operation in order to deal with the bound at infinity at which the post-Newtonian expansion is singular. Because of the presence of this regularization, the object (141) should carefully be distinguished from the "global" solution $\square_{\mathrm{ret}}^{-1}[\tau]$ defined by Eq. (31), with global non-expanded pseudo-tensor $\tau$.

The definition (141) is of interest because it corresponds to what one would intuitively think as the natural way of performing the post-Newtonian iteration, i.e., by formally Taylor expanding the retardations in Eq. (31), as was advocated by Anderson \& DeCanio [6]. Moreover, each of the terms of the series (141) is mathematically well-defined thanks to the finite part operation, and can therefore be implemented in practical computations. The point is that Eq. (141) solves the wave equation in a perturbative post-Newtonian sense,

$$
\square\left(\square_{\mathrm{ret}}^{-1}\left[\bar{\tau}^{\alpha \beta}\right]\right)=\bar{\tau}^{\alpha \beta}
$$

so constitutes a good prescription for a particular solution of the wave equation - as legitimate as the solution (134). Therefore the two solutions should differ by an homogeneous solution of the

\footnotetext{
35 Though the latter integral is a priori divergent, its value can be determined by invoking complex analytic continuation in $\ell \in \mathbb{C}$.
} 
wave equation which is necessarily of the anti-symmetric type (regular inside the source). Detailed investigations $[357,75]$ yield

$$
\square_{\mathrm{ret}}^{-1}\left[\bar{\tau}^{\alpha \beta}\right]=\square_{\mathrm{inst}}^{-1}\left[\bar{\tau}^{\alpha \beta}\right]-\frac{1}{4 \pi} \sum_{\ell=0}^{+\infty} \frac{(-)^{\ell}}{\ell !} \hat{\partial}_{L}\left\{\frac{\mathcal{F}_{L}^{\alpha \beta}(t-r / c)-\mathcal{F}_{L}^{\alpha \beta}(t+r / c)}{2 r}\right\},
$$

where the homogeneous solution is parametrized by the multipole-moments $\mathcal{F}_{L}(t)$. By combining Eqs. (140) and (143), we indeed become reconciled with the previous expression of the postNewtonian field found in Eq. (136).

For computations limited to the $3.5 \mathrm{PN}$ order (level of the $1 \mathrm{PN}$ correction to the radiation reaction force), the first term in Eq. (140) with the "intuitive" prescription (141) is sufficient. But because of the second term in (140) there is a fundamental breakdown of this scheme at the $4 \mathrm{PN}$ order where it becomes necessary to take into account non-linear radiation reaction effects associated with tails. The second term in (140) constitutes a generalization of the tail-transported radiation reaction arising at the $4 \mathrm{PN}$ order, i.e., $1.5 \mathrm{PN}$ order relative to the dominant radiation reaction order, as determined in Ref. [58]. The tail-transported radiation reaction is required by energy conservation and the presence of tails in the wave zone. The usual radiation reaction terms, up to $3.5 \mathrm{PN}$ order, are contained in the first term of Eq. (140), and are parametrized by the same multipole-moment functions $\mathcal{F}_{L}$ as the exterior multipolar field, as Eq. (143) explicitly shows. In Section 5.4 we shall give an explicit expression of the radiation reaction force showing the usual radiation reaction terms to $3.5 \mathrm{PN}$ order, issued from $\mathcal{F}_{L}$, and exhibiting the above tail-induced $4 \mathrm{PN}$ effect, issued from $\mathcal{R}_{L}$.

Finally note that the post-Newtonian solution, in either form (136) or (140), has been obtained without imposing the condition of harmonic coordinates (21) in an explicit way. We have simply matched together the post-Newtonian and multipolar expansions, satisfying the "relaxed" Einstein field equations (22) in their respective domains, and found that the matching determines uniquely the solution. An important check done in [357, 75], is therefore to verify that the harmonic coordinate condition (21) is indeed satisfied as a consequence of the conservation of the pseudotensor (27), so that we really grasp a solution of the full Einstein field equations.

\subsection{The 3.5PN metric for general matter systems}

The detailed calculations that are called for in applications necessitate having at one's disposal some explicit expressions of the metric coefficients $g_{\alpha \beta}$, in harmonic coordinates, at the highest possible post-Newtonian order. The 3.5PN metric that we present below can be viewed as an application of the formalism of the previous section. It is expressed by means of some particular retarded-type potentials, $V, V_{i}, \hat{W}_{i j}, \ldots$, whose main advantages are to somewhat minimize the number of terms, so that even at the $3.5 \mathrm{PN}$ order the metric is still tractable, and to delineate the different problems associated with the computation of different categories of terms. Of course, these potentials have no direct physical significance by themselves, but they offer a convenient parametrization of the $3.5 \mathrm{PN}$ metric.

The basic idea in our post-Newtonian iteration scheme is to use wherever possible a "direct" integration, with the help of some formulas like $\square_{\text {ret }}^{-1}\left(\partial_{\mu} V \partial^{\mu} V+V \square V\right)=V^{2} / 2$. The 3.5PN harmonic-coordinates metric reads [71]

$$
\begin{aligned}
g_{00} & =-1+\frac{2}{c^{2}} V-\frac{2}{c^{4}} V^{2}+\frac{8}{c^{6}}\left(\hat{X}+V_{i} V_{i}+\frac{V^{3}}{6}\right) \\
& +\frac{32}{c^{8}}\left(\hat{T}-\frac{1}{2} V \hat{X}+\hat{R}_{i} V_{i}-\frac{1}{2} V V_{i} V_{i}-\frac{1}{48} V^{4}\right)+\mathcal{O}\left(\frac{1}{c^{10}}\right), \\
g_{0 i} & =-\frac{4}{c^{3}} V_{i}-\frac{8}{c^{5}} \hat{R}_{i}-\frac{16}{c^{7}}\left(\hat{Y}_{i}+\frac{1}{2} \hat{W}_{i j} V_{j}+\frac{1}{2} V^{2} V_{i}\right)+\mathcal{O}\left(\frac{1}{c^{9}}\right),
\end{aligned}
$$




$$
\begin{aligned}
g_{i j} & =\delta_{i j}\left[1+\frac{2}{c^{2}} V+\frac{2}{c^{4}} V^{2}+\frac{8}{c^{6}}\left(\hat{X}+V_{k} V_{k}+\frac{V^{3}}{6}\right)\right]+\frac{4}{c^{4}} \hat{W}_{i j} \\
& +\frac{16}{c^{6}}\left(\hat{Z}_{i j}+\frac{1}{2} V \hat{W}_{i j}-V_{i} V_{j}\right)+\mathcal{O}\left(\frac{1}{c^{8}}\right) .
\end{aligned}
$$

All the potentials are generated by the matter stress-energy tensor $T^{\alpha \beta}$ through some convenient definitions recalling Eqs. (124),

$$
\begin{aligned}
\sigma & =\frac{T^{00}+T^{i i}}{c^{2}}, \\
\sigma_{i} & =\frac{T^{0 i}}{c}, \\
\sigma_{i j} & =T^{i j} .
\end{aligned}
$$

Starting at Newtonian and $1 \mathrm{PN}$ orders, $V$ and $V_{i}$ represent some retarded versions of the usual Newtonian and gravitomagnetic potentials,

$$
\begin{aligned}
V & =\square_{\text {ret }}^{-1}[-4 \pi G \sigma], \\
V_{i} & =\square_{\text {ret }}^{-1}\left[-4 \pi G \sigma_{i}\right] .
\end{aligned}
$$

From the $2 \mathrm{PN}$ order we have the potentials

$$
\begin{aligned}
\hat{X} & =\square_{\text {ret }}^{-1}\left[-4 \pi G V \sigma_{i i}+\hat{W}_{i j} \partial_{i j} V+2 V_{i} \partial_{t} \partial_{i} V+V \partial_{t}^{2} V+\frac{3}{2}\left(\partial_{t} V\right)^{2}-2 \partial_{i} V_{j} \partial_{j} V_{i}\right], \\
\hat{R}_{i} & =\square_{\text {ret }}^{-1}\left[-4 \pi G\left(V \sigma_{i}-V_{i} \sigma\right)-2 \partial_{k} V \partial_{i} V_{k}-\frac{3}{2} \partial_{t} V \partial_{i} V\right], \\
\hat{W}_{i j} & =\square_{\text {ret }}^{-1}\left[-4 \pi G\left(\sigma_{i j}-\delta_{i j} \sigma_{k k}\right)-\partial_{i} V \partial_{j} V\right] .
\end{aligned}
$$

Some parts of these potentials are directly generated by compact-support matter terms, while other parts are made of non-compact-support products of $V$-type potentials. There exists also an important cubically non-linear term generated by the coupling between $\hat{W}_{i j}$ and $V$, see the second term in the $\hat{X}$-potential. Note the important point that here and below the retarded integral operator $\square_{\text {ret }}^{-1}$ is really meant to be the one given by Eq. (141); thus it involves in principle the finite part regularization $\mathcal{F P}$ to deal with (IR-type) divergences occurring at high post-Newtonian orders for non-compact-support integrals. For instance, such finite part regularization is important to take into account in the computation of the near zone metric at the 3PN order [68].

At the next level, 3PN, we have even more complicated potentials, namely

$$
\begin{aligned}
& \hat{T}=\square_{\mathrm{ret}}^{-1} {\left[-4 \pi G\left(\frac{1}{4} \sigma_{i j} \hat{W}_{i j}+\frac{1}{2} V^{2} \sigma_{i i}+\sigma V_{i} V_{i}\right)+\hat{Z}_{i j} \partial_{i j} V+\hat{R}_{i} \partial_{t} \partial_{i} V-2 \partial_{i} V_{j} \partial_{j} \hat{R}_{i}-\partial_{i} V_{j} \partial_{t} \hat{W}_{i j}\right.} \\
&+\left.V V_{i} \partial_{t} \partial_{i} V+2 V_{i} \partial_{j} V_{i} \partial_{j} V+\frac{3}{2} V_{i} \partial_{t} V \partial_{i} V+\frac{1}{2} V^{2} \partial_{t}^{2} V+\frac{3}{2} V\left(\partial_{t} V\right)^{2}-\frac{1}{2}\left(\partial_{t} V_{i}\right)^{2}\right], \quad(148 \mathrm{a}) \\
& \hat{Y}_{i}=\square_{\mathrm{ret}}^{-1}\left[-4 \pi G\left(-\sigma \hat{R}_{i}-\sigma V V_{i}+\frac{1}{2} \sigma_{k} \hat{W}_{i k}+\frac{1}{2} \sigma_{i k} V_{k}+\frac{1}{2} \sigma_{k k} V_{i}\right)\right. \\
&+ \hat{W}_{k l} \partial_{k l} V_{i}-\partial_{t} \hat{W}_{i k} \partial_{k} V+\partial_{i} \hat{W}_{k l} \partial_{k} V_{l}-\partial_{k} \hat{W}_{i l} \partial_{l} V_{k}-2 \partial_{k} V \partial_{i} \hat{R}_{k}-\frac{3}{2} V_{k} \partial_{i} V \partial_{k} V \\
&\left.-\frac{3}{2} V \partial_{t} V \partial_{i} V-2 V \partial_{k} V \partial_{k} V_{i}+V \partial_{t}^{2} V_{i}+2 V_{k} \partial_{k} \partial_{t} V_{i}\right] \\
& \hat{Z}_{i j}=\square_{\mathrm{ret}}^{-1}\left[-4 \pi G V\left(\sigma_{i j}-\delta_{i j} \sigma_{k k}\right)-2 \partial_{(i} V \partial_{t} V_{j)}+\partial_{i} V_{k} \partial_{j} V_{k}+\partial_{k} V_{i} \partial_{k} V_{j}-2 \partial_{(i} V_{k} \partial_{k} V_{j)}\right.
\end{aligned}
$$




$$
\left.-\frac{3}{4} \delta_{i j}\left(\partial_{t} V\right)^{2}-\delta_{i j} \partial_{k} V_{m}\left(\partial_{k} V_{m}-\partial_{m} V_{k}\right)\right]
$$

These involve many types of compact-support contributions, as well as quadratic-order and cubicorder parts; but, surprisingly, there are no quartically non-linear terms. Indeed it has been possible to "integrate directly" all the quartic contributions in the 3PN metric; see the terms composed of $V^{4}$ and $V \hat{X}$ in the first of Eqs. (144).

Note that the 3PN metric (144) does represent the inner post-Newtonian field of an isolated system, because it contains, to this order, the correct radiation-reaction terms corresponding to outgoing radiation. These terms come from the expansions of the retardations in the retarded potentials (146)-(148); we elaborate more on radiation-reaction effects in the next Section 5.4.

The above potentials are not independent: They are linked together by some differential identities issued from the harmonic gauge conditions, which are equivalent, via the Bianchi identities, to the equations of motion of the matter fields; see Eq. (27). These identities read

$$
\begin{aligned}
& \partial_{t}\left\{V+\frac{1}{c^{2}}\left[\frac{1}{2} \hat{W}_{k k}+2 V^{2}\right]+\frac{4}{c^{4}}\left[\hat{X}+\frac{1}{2} \hat{Z}_{k k}+\frac{1}{2} V \hat{W}_{k k}+\frac{2}{3} V^{3}\right]\right\} \\
& +\partial_{i}\left\{V_{i}+\frac{2}{c^{2}}\left[\hat{R}_{i}+V V_{i}\right]+\frac{4}{c^{4}}\left[\hat{Y}_{i}-\frac{1}{2} \hat{W}_{i j} V_{j}+\frac{1}{2} \hat{W}_{k k} V_{i}+V \hat{R}_{i}+V^{2} V_{i}\right]\right\}=\mathcal{O}\left(\frac{1}{c^{6}}\right) \\
& \partial_{t}\left\{V_{i}+\frac{2}{c^{2}}\left[\hat{R}_{i}+V V_{i}\right]\right\}+\partial_{j}\left\{\hat{W}_{i j}-\frac{1}{2} \hat{W}_{k k} \delta_{i j}+\frac{4}{c^{2}}\left[\hat{Z}_{i j}-\frac{1}{2} \hat{Z}_{k k} \delta_{i j}\right]\right\}=\mathcal{O}\left(\frac{1}{c^{4}}\right)
\end{aligned}
$$

For latter applications to systems of compact objects, let us give the geodesic equations of a particle moving in the $3.5 \mathrm{PN}$ metric $(144) .{ }^{36}$ It is convenient to write these equations as

$$
\frac{\mathrm{d} P^{i}}{\mathrm{~d} t}=F^{i}
$$

where the "linear momentum density" $P^{i}$ and the "force density" $F^{i}$ of the particle are given by

$$
\begin{aligned}
P^{i} & =c \frac{g_{i \mu} v^{\mu}}{\sqrt{-g_{\rho \sigma} v^{\rho} v^{\sigma}}}, \\
F^{i} & =\frac{c}{2} \frac{\partial_{i} g_{\mu \nu} v^{\mu} v^{\nu}}{\sqrt{-g_{\rho \sigma} v^{\rho} v^{\sigma}}},
\end{aligned}
$$

where $v^{\mu}=\left(c, v^{i}\right)$ with $v^{i}=\mathrm{d} x^{i} / \mathrm{d} t$ being the particle's ordinary coordinate velocity, and where the metric components are taken at the location of the particle. Notice that we are here viewing the particle as moving in the fixed background metric (144). In Part B of this article, the metric will be generated by the system of particles itself, and we shall have to supplement the computation of the metric at the location of one of these particles by a suitable self-field regularization.

The expressions of both $P^{i}$ and $F^{i}$ in terms of the non-linear potentials follow from insertion of the 3.5PN metric coefficients (144). We obtain some complicated-looking (but useful in applications) sums of products of potentials given by

$$
\begin{aligned}
P^{i} & =v^{i}+\frac{1}{c^{2}}\left(\frac{1}{2} v^{2} v^{i}+3 V v^{i}-4 V_{i}\right) \\
& +\frac{1}{c^{4}}\left(\frac{3}{8} v^{4} v^{i}+\frac{7}{2} V v^{2} v^{i}-4 V_{j} v^{i} v^{j}-2 V_{i} v^{2}+\frac{9}{2} V^{2} v^{i}-4 V V_{i}+4 \hat{W}_{i j} v^{j}-8 \hat{R}_{i}\right)
\end{aligned}
$$

\footnotetext{
${ }^{36}$ Of course the geodesic equations are appropriate for the motion of particles without spins; for spinning particles
} one has also to take into account the coupling of the spin to the space-time curvature, see Eq. (377). 


$$
\begin{aligned}
& +\frac{1}{c^{6}}\left(\frac{5}{16} v^{6} v^{i}+\frac{33}{8} V v^{4} v^{i}-\frac{3}{2} V_{i} v^{4}-6 V_{j} v^{i} v^{j} v^{2}+\frac{49}{4} V^{2} v^{2} v^{i}+2 \hat{W}_{i j} v^{j} v^{2}\right. \\
& +2 \hat{W}_{j k} v^{i} v^{j} v^{k}-10 V V_{i} v^{2}-20 V V_{j} v^{i} v^{j}-4 \hat{R}_{i} v^{2}-8 \hat{R}_{j} v^{i} v^{j}+\frac{9}{2} V^{3} v^{i}+12 V_{j} V_{j} v^{i} \\
& \left.+12 \hat{W}_{i j} V v^{j}+12 \hat{X} v^{i}+16 \hat{Z}_{i j} v^{j}-10 V^{2} V_{i}-8 \hat{W}_{i j} V_{j}-8 V \hat{R}_{i}-16 \hat{Y}_{i}\right)+\mathcal{O}\left(\frac{1}{c^{8}}\right), \\
& F^{i}=\partial_{i} V+\frac{1}{c^{2}}\left(-V \partial_{i} V+\frac{3}{2} \partial_{i} V v^{2}-4 \partial_{i} V_{j} v^{j}\right) \\
& +\frac{1}{c^{4}}\left(\frac{7}{8} \partial_{i} V v^{4}-2 \partial_{i} V_{j} v^{j} v^{2}+\frac{9}{2} V \partial_{i} V v^{2}+2 \partial_{i} \hat{W}_{j k} v^{j} v^{k}\right. \\
& \left.-4 V_{j} \partial_{i} V v^{j}-4 V \partial V_{j} v^{j}-8 \partial_{i} \hat{R}_{j} v^{j}+\frac{1}{2} V^{2} \partial_{i} V+8 V_{j} \partial_{i} V_{j}+4 \partial_{i} \hat{X}\right) \\
& +\frac{1}{c^{6}}\left(\frac{11}{16} v^{6} \partial_{i} V-\frac{3}{2} \partial_{i} V_{j} v^{j} v^{4}+\frac{49}{8} V \partial_{i} V v^{4}+\partial_{i} \hat{W}_{j k} v^{2} v^{j} v^{k}-10 V_{j} \partial_{i} V v^{2} v^{j}-10 V \partial_{i} V_{j} v^{2} v^{j}\right. \\
& -4 \partial_{i} \hat{R}_{j} v^{2} v^{j}+\frac{27}{4} V^{2} \partial_{i} V v^{2}+12 V_{j} \partial_{i} V_{j} v^{2}+6 \hat{W}_{j k} \partial_{i} V v^{j} v^{k}+6 V \partial_{i} \hat{W}_{j k} v^{j} v^{k} \\
& +6 \partial_{i} \hat{X} v^{2}+8 \partial_{i} \hat{Z}_{j k} v^{j} v^{k}-20 V_{j} V \partial_{i} V v^{j}-10 V^{2} \partial_{i} V_{j} v^{j}-8 V_{k} \partial_{i} \hat{W}_{j k} v^{j}-8 \hat{W}_{j k} \partial_{i} V_{k} v^{j} \\
& -8 \hat{R}_{j} \partial_{i} V v^{j}-8 V \partial_{i} \hat{R}_{j} v^{j}-16 \partial_{i} \hat{Y}_{j} v^{j}-\frac{1}{6} V^{3} \partial_{i} V-4 V_{j} V_{j} \partial_{i} V+16 \hat{R}_{j} \partial_{i} V_{j}+16 V_{j} \partial_{i} \hat{R}_{j} \\
& \left.-8 V V_{j} \partial_{i} V_{j}-4 \hat{X} \partial_{i} V-4 V \partial_{i} \hat{X}+16 \partial_{i} \hat{T}\right)+\mathcal{O}\left(\frac{1}{c^{8}}\right) \text {. }
\end{aligned}
$$

Note that it will be supposed that all the accelerations appearing in the potentials and in the final expression of the equations of motion are order-reduced by means of the equations of motion themselves. For instance, we see from Eq. (152a) that when computing the time-derivative of $P_{i}$ we shall meet an acceleration at $1 \mathrm{PN}$ order which is therefore to be replaced by the explicit $2.5 \mathrm{PN}$ equations of motion. The order-reduction is a crucial aspect of the post-Newtonian method. It is justified by the fact that the matter equations of motion, say $\nabla_{\mu} T^{\alpha \mu}=0$, represent four out of the ten Einstein field equations, see Section 2.1 for discussion. In the harmonic-coordinate approach the equations of motion are equivalent to the harmonic gauge conditions $\partial_{\mu} h^{\alpha \mu}=0$. Thus, each time we get an acceleration in some PN expression (including the PN expression of the acceleration itself), we have also another equation (or the same equation) which tells that the acceleration is given by another PN expression. The post-Newtonian method assumes that it is legitimate to replace that acceleration and to re-expand consistently with the PN order. Post-Newtonian predictions based on such consistent PN order-reduction have been very successful. ${ }^{37}$

\subsection{Radiation reaction potentials to $4 \mathrm{PN}$ order}

We said that the metric (144) contains the correct radiation-reaction terms appropriate for an isolated system up to the $3.5 \mathrm{PN}$ level included. The metric can even be generalized to include the radiation-reaction terms up to $4 \mathrm{PN}$ order. To show this we shall use a particular non-harmonic coordinate system to describe the radiation reaction terms up to $4 \mathrm{PN}$ order, which constitutes a natural generalization of the Burke \& Thorne $[114,113]$ coordinate system at 2.5PN order. Recall that at the lowest $2.5 \mathrm{PN}$ order the radiation reaction force takes the simple form of Eq. (6), in

\footnotetext{
37 Note, however, that the operation of order-reduction is illicit at the level of the Lagrangian. In fact, it is known that the elimination of acceleration terms in a Lagrangian by substituting the equations of motion derived from that Lagrangian, results in a different Lagrangian whose equations of motion differ from those of the original Lagrangian by a gauge transformation [374].
} 
which the force $F_{i}^{\text {reac }}=\rho \partial_{i} V^{\text {reac }}$ involves only a scalar potential given by

$$
V^{\text {reac }}(\mathbf{x}, t)=-\frac{G}{5 c^{5}} x^{i} x^{j} \mathrm{Q}_{i j}^{(5)}(t)+\mathcal{O}\left(\frac{1}{c^{7}}\right)
$$

At such dominant 2.5PN level ("Newtonian" radiation reaction) the source quadrupole moment $\mathrm{Q}_{i j}$ is simply given by the usual Newtonian expression (3).

The novel feature when one extends the Newtonian radiation reaction to include the $1 \mathrm{PN}$ corrections is that the reaction force is no longer composed of a single scalar depending on the mass-type multipole moments, but involves also a vectorial component depending in particular on the current-type quadrupole moment. This was noticed in the physically restricted case where the dominant quadrupolar radiation from the source is suppressed [56]. The vectorial component of the reaction force could be important in some astrophysical situations like rotating neutron stars undergoing gravitational instabilities. Here we report the results of the extension to $1.5 \mathrm{PN}$ order of the lowest-order Burke \& Thorne scalar radiation reaction potential (153), in some appropriate coordinate system, following Refs. [43, 47].

At that level (corresponding to $4 \mathrm{PN}$ order in the metric), and in this particular coordinate system, it suffices to incorporate some radiation-reaction contributions into the scalar and vectorial potentials $V$ and $V_{i}$ which parametrize the metric in Eq. (144). We thus pose

$$
\begin{gathered}
\mathcal{V}=V^{\text {inst }}+V^{\text {reac }}, \\
\mathcal{V}_{i}=V_{i}^{\text {inst }}+V_{i}^{\text {reac }} .
\end{gathered}
$$

Then the metric, accurate to $4 \mathrm{PN}$ order regarding the radiation-reaction contributions - we indicate this by using the symbol $\mathcal{O}^{\text {reac }}$ for the remainders - reads

$$
\begin{aligned}
& g_{00}=-1+\frac{2}{c^{2}} \mathcal{V}-\frac{2}{c^{4}} \mathcal{V}^{2}+\mathcal{O}^{\text {reac }}\left(\frac{1}{c^{11}}\right), \\
& g_{0 i}=-\frac{4}{c^{3}} \mathcal{V}_{i}+\mathcal{O}^{\text {reac }}\left(\frac{1}{c^{10}}\right), \\
& g_{i j}=\delta_{i j}\left[1+\frac{2}{c^{2}} \mathcal{V}\right]+\mathcal{O}^{\text {reac }}\left(\frac{1}{c^{9}}\right) .
\end{aligned}
$$

The other contributions, which are conservative (i.e., non radiative), are given up to $3 \mathrm{PN}$ order by the metric (144) in which all the potentials take the same form as in Eqs. (146) - (148), but where one neglects all the retardations, which means that the retarded integral operator $\square_{\text {ret }}^{-1}$ should be replaced by the operator of the instantaneous potentials $\square_{\text {inst }}^{-1}$ defined by Eq. (134). This is for instance what we have indicated in Eqs. (154) by writing $V^{\text {inst }}$ and $V_{i}^{\text {inst }}$. Up to $3.5 \mathrm{PN}$ order, in this particular coordinate system, the effect of all these retardations gets replaced by the effect of the radiation-reaction potentials $V^{\text {reac }}$ and $V_{i}^{\text {reac }}$; furthermore, at the $4 \mathrm{PN}$ order there is a modification of the scalar radiation-reaction potential that is imposed by gravitational-wave tails propagating in the wave zone [58]. The explicit form of these potentials is $[43,47]^{38}$

$$
\begin{aligned}
V^{\text {reac }}(\mathbf{x}, t)= & -\frac{G}{5 c^{5}} x^{i j} \mathrm{I}_{i j}^{(5)}(t)+\frac{G}{c^{7}}\left[\frac{1}{189} x^{i j k} \mathrm{I}_{i j k}^{(7)}(t)-\frac{1}{70} \mathbf{x}^{2} x^{i j} \mathrm{I}_{i j}^{(7)}(t)\right] \\
& -\frac{4 G^{2} \mathrm{M}}{5 c^{8}} x^{i j} \int_{0}^{+\infty} \mathrm{d} \tau \mathrm{I}_{i j}^{(7)}(t-\tau)\left[\ln \left(\frac{c \tau}{2 r_{0}}\right)+\frac{11}{12}\right]+\mathcal{O}\left(\frac{1}{c^{9}}\right),
\end{aligned}
$$

\footnotetext{
38 Recall the footnote 17 for our notation. In particular $\hat{x}^{i j k}$ in the vector potential denotes the STF combination $\hat{x}^{i j k}=x^{i j k}-\frac{r^{2}}{5}\left(x^{i} \delta^{j k}+x^{j} \delta^{k i}+x^{k} \delta^{i j}\right)$ with $x^{i j k}=x^{i} x^{j} x^{k}$.
} 


$$
V_{i}^{\text {reac }}(\mathbf{x}, t)=\frac{G}{c^{5}}\left[\frac{1}{21} \hat{x}^{i j k} \mathrm{I}_{j k}^{(6)}(t)-\frac{4}{45} \epsilon_{i j k} x^{j l} \mathrm{~J}_{k l}^{(5)}(t)\right]+\mathcal{O}\left(\frac{1}{c^{7}}\right),
$$

where the multipole moments $\mathrm{I}_{L}$ and $\mathrm{J}_{L}$ denote the source multipole moments defined in Eqs. (123). Witness the tail integral at 4PN order characterized by a logarithmic kernel; see Section 3.2.

The scalar potential $V^{\text {reac }}$ will obviously reproduce Eq. (153) at the dominant order. However, note that it is crucial to include in Eq. (156a) the $1 \mathrm{PN}$ correction in the source quadrupole moment $\mathrm{I}_{i j}$. The mass-type moments $\mathrm{I}_{L}$ to $1 \mathrm{PN}$ order (and the current-type $\mathrm{J}_{L}$ to Newtonian order), read

$$
\begin{aligned}
\mathrm{I}_{L} & =\int \mathrm{d}^{3} \mathbf{x}\left\{\hat{x}_{L} \sigma+\frac{1}{2 c^{2}(2 \ell+3)} \mathbf{x}^{2} \hat{x}_{L} \partial_{t}^{2} \sigma-\frac{4(2 \ell+1)}{c^{2}(\ell+1)(2 \ell+3)} \hat{x}_{i L} \partial_{t} \sigma_{i}\right\}+\mathcal{O}\left(\frac{1}{c^{4}}\right), \\
\mathrm{J}_{L} & =\int \mathrm{d}^{3} \mathbf{x} \epsilon_{a b<i_{\ell}} \hat{x}_{L-1>a} \sigma_{b}+\mathcal{O}\left(\frac{1}{c^{2}}\right) .
\end{aligned}
$$

The matter source densities $\sigma$ and $\sigma_{i}$ are given in Eqs. (145). Note that the mass multipole moments $\mathrm{I}_{L}$ extend only over the compact support of the source even at the $1 \mathrm{PN}$ order. Only at the $2 \mathrm{PN}$ order will they involve some non-compact supported contributions - i.e., some integrals extending up to infinity [44].

The 3.5PN radiation reaction force in the equations of motion of compact binary systems has been derived by Iyer \& Will [258, 259] in an arbitrary gauge, based on the energy and angular momentum balance equations at the relative $1 \mathrm{PN}$ order. As demonstrated in Ref. [259] the expressions of the radiation scalar and vector radiation-reaction potentials (156), which are valid in a particular gauge but are here derived from first principles, are fully consistent with the works [258, 259].

With the radiation-reaction potentials (156) in hands, one can prove [47] the energy balance equation up to $1.5 \mathrm{PN}$ order, namely

$$
\begin{aligned}
\frac{\mathrm{d} E^{4 \mathrm{PN}}}{\mathrm{d} t}= & -\frac{G}{5 c^{5}}\left(\mathrm{I}_{i j}^{(3)}+\frac{2 G \mathrm{M}}{c^{3}} \int_{0}^{+\infty} \mathrm{d} \tau \mathrm{I}_{i j}^{(5)}(t-\tau)\left[\ln \left(\frac{c \tau}{2 r_{0}}\right)+\frac{11}{12}\right]\right)^{2} \\
& -\frac{G}{c^{7}}\left[\frac{1}{189} \mathrm{I}_{i j k}^{(4)} \mathrm{I}_{i j k}^{(4)}+\frac{16}{45} \mathrm{~J}_{i j}^{(3)} \mathrm{J}_{i j}^{(3)}\right]+\mathcal{O}\left(\frac{1}{c^{9}}\right) .
\end{aligned}
$$

One recognizes in the right-hand side the known positive-definite expression for the energy flux at $1.5 \mathrm{PN}$ order. Indeed the effective quadrupole moment which appears in the parenthesis of (158) agrees with the tail-modified radiative quadrupole moment $\mathrm{U}_{i j}$ parametrizing the field in the far zone; see Eq. (90) where we recall that $\mathrm{M}_{L}$ and $\mathrm{I}_{L}$ are identical up to $2.5 \mathrm{PN}$ order. 


\section{Part B: Compact Binary Systems}

The problem of the motion and gravitational radiation of compact objects in post-Newtonian approximations is of crucial importance, for at least three reasons listed in the Introduction of this article: Motion of $N$ planets in the solar system; gravitational radiation reaction force in binary pulsars; direct detection of gravitational waves from inspiralling compact binaries. As discussed in Section 1.3, the appropriate theoretical description of inspiralling compact binaries is by two structureless point-particles, characterized solely by their masses $m_{1}$ and $m_{2}$ (and possibly their spins), and moving on a quasi-circular orbit.

Strategies to detect and analyze the very weak signals from compact binary inspiral involve matched filtering of a set of accurate theoretical template waveforms against the output of the detectors. Many analyses [139, 137, 198, 138, 393, 346, 350, 284, 157, 158, 159, 156, 105, 106, 3, 18, 111] have shown that, in order to get sufficiently accurate theoretical templates, one must include postNewtonian effects up to the 3PN level or higher. Recall that in practice, the post-Newtonian templates for the inspiral phase have to be matched to numerical-relativity results for the subsequent merger and ringdown phases. The match proceeds essentially through two routes: Either the socalled Hybrid templates obtained by direct matching between the PN expanded waveform and the numerical computations [4, 371], or the Effective-One-Body (EOB) templates [108, 109, 161, 168] that build on post-Newtonian results and extend their realm of validity to facilitate the analytical comparison with numerical relativity $[112,329]$. Note also that various post-Newtonian resummation techniques, based on Padé approximants, have been proposed to improve the efficiency of PN templates [157, 158, 161].

\section{Regularization of the Field of Point Particles}

Our aim is to compute the metric (and its gradient needed in the equations of motion) at the $3 \mathrm{PN}$ order (say) for a system of two point-like particles. A priori one is not allowed to use directly some metric expressions like Eqs. (144) above, which have been derived under the assumption of a continuous (smooth) matter distribution. Applying them to a system of point particles, we find that most of the integrals become divergent at the location of the particles, i.e., when $\mathbf{x} \rightarrow \boldsymbol{y}_{1}(t)$ or $\boldsymbol{y}_{2}(t)$, where $\boldsymbol{y}_{1}(t)$ and $\boldsymbol{y}_{2}(t)$ denote the two trajectories. Consequently, we must supplement the calculation by a prescription for how to remove the infinite part of these integrals. At this stage different choices for a "self-field" regularization (which will take care of the infinite self-field of point particles) are possible. In this section we review the:

1. Hadamard self-field regularization, which has proved to be very convenient for doing practical computations (in particular, by computer), but suffers from the important drawback of yielding some ambiguity parameters, which cannot be determined within this regularization, starting essentially at the $3 \mathrm{PN}$ order;

2. Dimensional self-field regularization, an extremely powerful regularization which is free of any ambiguities (at least up to the $3 \mathrm{PN}$ level), and therefore permits to uniquely fix the values of the ambiguity parameters coming from Hadamard's regularization. However, dimensional regularization has not yet been implemented to the present problem in the general case (i.e., for an arbitrary space dimension $d \in \mathbb{C}$ ).

The why and how the final results are unique and independent of the employed self-field regularization (in agreement with the physical expectation) stems from the effacing principle of general relativity [142] - namely that the internal structure of the compact bodies makes a contribution only at the formal 5PN approximation. However, we shall review several alternative computations, independent of the self-field regularization, which confirm the end results. 


\subsection{Hadamard self-field regularization}

In most practical computations we employ the Hadamard regularization [236, 381] (see Ref. [382] for an entry to the mathematical literature). Let us present here an account of this regularization, as well as a theory of generalized functions (or pseudo-functions) associated with it, following the detailed investigations in Refs. [70, 72].

Consider the class $\mathcal{F}$ of functions $F(\mathbf{x})$ which are smooth $\left(C^{\infty}\right)$ on $\mathbb{R}^{3}$ except for the two points $\boldsymbol{y}_{1}$ and $\boldsymbol{y}_{2}$, around which they admit a power-like singular expansion of the type: ${ }^{39}$

$$
\forall \mathcal{N} \in \mathbb{N}, \quad F(\mathbf{x})=\sum_{a_{0} \leqslant a \leqslant \mathcal{N}} r_{1}^{a} f_{a}\left(\mathbf{n}_{1}\right)+o\left(r_{1}^{\mathcal{N}}\right)
$$

and similarly for the other point 2 . Here $r_{1}=\left|\mathbf{x}-\boldsymbol{y}_{1}\right| \rightarrow 0$, and the coefficients ${ }_{1} f_{a}$ of the various powers of $r_{1}$ depend on the unit direction $\mathbf{n}_{1}=\left(\mathbf{x}-\boldsymbol{y}_{1}\right) / r_{1}$ of approach to the singular point. The powers $a$ of $r_{1}$ are real, range in discrete steps [i.e., $a \in\left(a_{i}\right)_{i \in \mathbb{N}}$ ], and are bounded from below $\left(a_{0} \leqslant a\right)$. The coefficients ${ }_{1} f_{a}$ (and ${ }_{2} f_{a}$ ) for which $a<0$ can be referred to as the singular coefficients of $F$. If $F$ and $G$ belong to $\mathcal{F}$ so does the ordinary product $F G$, as well as the ordinary gradient $\partial_{i} F$. We define the Hadamard partie finie of $F$ at the location of the point 1 where it is singular as

$$
(F)_{1}=\int \frac{\mathrm{d} \Omega_{1}}{4 \pi} f_{0}\left(\mathbf{n}_{1}\right)
$$

where $\mathrm{d} \Omega_{1}=\mathrm{d} \Omega\left(\mathbf{n}_{1}\right)$ denotes the solid angle element centered on $\boldsymbol{y}_{1}$ and of direction $\mathbf{n}_{1}$. Notice that because of the angular integration in Eq. (160), the Hadamard partie finie is "non-distributive" in the sense that

$$
(F G)_{1} \neq(F)_{1}(G)_{1} \quad \text { in general }
$$

The non-distributivity of Hadamard's partie finie is the main source of the appearance of ambiguity parameters at the 3PN order, as discussed in Section 6.2.

The second notion of Hadamard partie finie (Pf) concerns that of the integral $\int \mathrm{d}^{3} \mathbf{x} F$, which is generically divergent at the location of the two singular points $\boldsymbol{y}_{1}$ and $\boldsymbol{y}_{2}$ (we assume that the integral converges at infinity). It is defined by

$$
\operatorname{Pf}_{s_{1} s_{2}} \int \mathrm{d}^{3} \mathbf{x} F=\lim _{s \rightarrow 0}\left\{\int_{\mathcal{S}(s)} \mathrm{d}^{3} \mathbf{x} F+4 \pi \sum_{a+3<0} \frac{s^{a+3}}{a+3}\left(\frac{F}{r_{1}^{a}}\right)_{1}+4 \pi \ln \left(\frac{s}{s_{1}}\right)\left(r_{1}^{3} F\right)_{1}+1 \leftrightarrow 2\right\} .
$$

The first term integrates over a domain $\mathcal{S}(s)$ defined as $\mathbb{R}^{3}$ from which the two spherical balls $r_{1} \leqslant s$ and $r_{2} \leqslant s$ of radius $s$ and centered on the two singularities, denoted $\mathcal{B}\left(\boldsymbol{y}_{1}, s\right)$ and $\mathcal{B}\left(\boldsymbol{y}_{2}, s\right)$, are excised: $\mathcal{S}(s) \equiv \mathbb{R}^{3} \backslash \mathcal{B}\left(\boldsymbol{y}_{1}, s\right) \cup \mathcal{B}\left(\boldsymbol{y}_{2}, s\right)$. The other terms, where the value of a function at point 1 takes the meaning (160), are precisely such that they cancel out the divergent part of the first term in the limit where $s \rightarrow 0$ (the symbol $1 \leftrightarrow 2$ means the same terms but corresponding to the other point 2). The Hadamard partie-finie integral depends on two strictly positive constants $s_{1}$ and $s_{2}$, associated with the logarithms present in Eq. (162). We shall look for the fate of these constants in the final equations of motion and radiation field. See Ref. [70] for alternative expressions of the partie-finie integral.

We now come to a specific variant of Hadamard's regularization called the extended Hadamard regularization (EHR) and defined in Refs. [70, 72]. The basic idea is to associate to any $F \in \mathcal{F}$

39 The function $F(\mathbf{x})$ depends also on (coordinate) time $t$, through for instance its dependence on the velocities $\boldsymbol{v}_{1}(t)$ and $\boldsymbol{v}_{2}(t)$, but the time $t$ is purely "spectator" in the regularization process, and thus will not be indicated. See the footnote 20 for the definition of the Landau symbol $o$ for remainders. 
a pseudo-function, called the partie finie pseudo-function $\operatorname{Pf} F$, namely a linear form acting on functions $G$ of $\mathcal{F}$, and which is defined by the duality bracket

$$
\forall G \in \mathcal{F}, \quad\langle\operatorname{Pf} F, G\rangle=\operatorname{Pf} \int \mathrm{d}^{3} \mathbf{x} F G .
$$

When restricted to the set $\mathcal{D}$ of smooth functions, i.e., $C^{\infty}\left(\mathbb{R}^{4}\right)$, with compact support (obviously we have $\mathcal{D} \subset \mathcal{F}$ ), the pseudo-function $\operatorname{Pf} F$ is a distribution in the sense of Schwartz [381]. The product of pseudo-functions coincides, by definition, with the ordinary point-wise product, namely $\operatorname{Pf} F \cdot \operatorname{Pf} G=\operatorname{Pf}(F G)$. In practical computations, we use an interesting pseudo-function, constructed on the basis of the Riesz delta function [365], which plays a role analogous to the Dirac measure in distribution theory, $\delta_{1}(\mathbf{x}) \equiv \delta\left(\mathbf{x}-\boldsymbol{y}_{1}\right)$. This is the delta-pseudo-function $\operatorname{Pf} \delta_{1}$ defined by

$$
\forall F \in \mathcal{F}, \quad\left\langle\operatorname{Pf} \delta_{1}, F\right\rangle=\operatorname{Pf} \int \mathrm{d}^{3} \mathbf{x} \delta_{1} F=(F)_{1},
$$

where $(F)_{1}$ is the partie finie of $F$ as given by Eq. (160). From the product of $\operatorname{Pf} \delta_{1}$ with any $\operatorname{Pf} F$ we obtain the new pseudo-function $\operatorname{Pf}\left(F \delta_{1}\right)$, that is such that

$$
\forall G \in \mathcal{F}, \quad\left\langle\operatorname{Pf}\left(F \delta_{1}\right), G\right\rangle=(F G)_{1}
$$

As a general rule, we are not allowed, in consequence of the "non-distributivity" of the Hadamard partie finie, Eq. (161), to replace $F$ within the pseudo-function $\operatorname{Pf}\left(F \delta_{1}\right)$ by its regularized value: $\operatorname{Pf}\left(F \delta_{1}\right) \neq(F)_{1} \operatorname{Pf} \delta_{1}$ in general. It should be noticed that the object $\operatorname{Pf}\left(F \delta_{1}\right)$ has no equivalent in distribution theory.

Next, we treat the spatial derivative of a pseudo-function of the type $\operatorname{Pf} F$, namely $\partial_{i}(\operatorname{Pf} F)$. Essentially, we require [70] that the rule of integration by parts holds. By this we mean that we are allowed to freely operate by parts any duality bracket, with the all-integrated ("surface") terms always being zero, as in the case of non-singular functions. This requirement is motivated by our will that a computation involving singular functions be as much as possible the same as if we were dealing with regular functions. Thus, by definition,

$$
\forall F, G \in \mathcal{F}, \quad\left\langle\partial_{i}(\operatorname{Pf} F), G\right\rangle=-\left\langle\partial_{i}(\operatorname{Pf} G), F\right\rangle .
$$

Furthermore, we assume that when all the singular coefficients of $F$ vanish, the derivative of $\operatorname{Pf} F$ reduces to the ordinary derivative, i.e., $\partial_{i}(\operatorname{Pf} F)=\operatorname{Pf}\left(\partial_{i} F\right)$. Then it is trivial to check that the rule (166) contains as a particular case the standard definition of the distributional derivative [381]. Notably, we see that the integral of a gradient is always zero: $\left\langle\partial_{i}(\operatorname{Pf} F), 1\right\rangle=0$. This should certainly be the case if we want to compute a quantity like a Hamiltonian density which is defined only modulo a total divergence. We pose

$$
\partial_{i}(\operatorname{Pf} F)=\operatorname{Pf}\left(\partial_{i} F\right)+\mathrm{D}_{i}[F]
$$

where $\operatorname{Pf}\left(\partial_{i} F\right)$ represents the "ordinary" derivative and $\mathrm{D}_{i}[F]$ is the distributional term. The following solution of the basic relation (166) was obtained in Ref. [70]:

$$
\mathrm{D}_{i}[F]=4 \pi \operatorname{Pf}\left(n_{1}^{i}\left[\frac{1}{2} r_{1} f_{-1}+\sum_{k \geqslant 0} \frac{1}{r_{1}^{k}} f_{-2-k}\right] \delta_{1}\right)+1 \leftrightarrow 2,
$$

where for simplicity we assume that the powers $a$ in the expansion (159) of $F$ are relative integers. The distributional term (168) is of the form $\operatorname{Pf}\left(G \delta_{1}\right)$ plus $1 \leftrightarrow 2$; it is generated solely by the 
singular coefficients of $F .^{40}$ The formula for the distributional term associated with the $\ell$-th distributional derivative, i.e. $\mathrm{D}_{L}[F]=\partial_{L} \operatorname{Pf} F-\operatorname{Pf} \partial_{L} F$, where $L=i_{1} i_{2} \cdots i_{\ell}$, reads

$$
\mathrm{D}_{L}[F]=\sum_{k=1}^{\ell} \partial_{i_{1} \ldots i_{k-1}} \mathrm{D}_{i_{k}}\left[\partial_{i_{k+1} \ldots i_{\ell}} F\right]
$$

We refer to Theorem 4 in Ref. [70] for the definition of another derivative operator, representing the most general derivative satisfying the same properties as the one defined by Eq. (168), and, in addition, the commutation of successive derivatives (or Schwarz lemma). ${ }^{41}$

The distributional derivative defined by (167) - (168) does not satisfy the Leibniz rule for the derivation of a product, in accordance with a general result of Schwartz [380]. Rather, the investigation of Ref. [70] suggests that, in order to construct a consistent theory (using the ordinary point-wise product for pseudo-functions), the Leibniz rule should be weakened, and replaced by the rule of integration by part, Eq. (166), which is in fact nothing but an integrated version of the Leibniz rule. However, the loss of the Leibniz rule stricto sensu constitutes one of the reasons for the appearance of the ambiguity parameters at 3PN order, see Section 6.2.

The Hadamard regularization $(F)_{1}$ is defined by Eq. (160) in a preferred spatial hypersurface $t=$ const of a coordinate system, and consequently is not a priori compatible with the Lorentz invariance. Thus we expect that the equations of motion in harmonic coordinates (which manifestly preserve the global Lorentz invariance) should exhibit at some stage a violation of the Lorentz invariance due to the latter regularization. In fact this occurs exactly at the $3 \mathrm{PN}$ order. Up to the 2.5PN level, the use of the regularization $(F)_{1}$ is sufficient to get some unambiguous equations of motion which are Lorentz invariant [76]. This problem can be dealt with within Hadamard's regularization, by introducing a Lorentz-invariant variant of this regularization, denoted $[F]_{1}$ in Ref. [72]. It consists of performing the Hadamard regularization within the spatial hypersurface that is geometrically orthogonal (in a Minkowskian sense) to the four-velocity of the particle. The regularization $[F]_{1}$ differs from the simpler regularization $(F)_{1}$ by relativistic corrections of order $1 / c^{2}$ at least. See [72] for the formulas defining this regularization in the form of some infinite power series in $1 / c^{2}$. The regularization $[F]_{1}$ plays a crucial role in obtaining the equations of motion at the $3 \mathrm{PN}$ order in Refs. $[69,71]$. In particular, the use of the Lorentz-invariant regularization $[F]_{1}$ permits to obtain the value of the ambiguity parameter $\omega_{\text {kinetic }}$ in Eq. (170a) below.

\subsection{Hadamard regularization ambiguities}

The standard Hadamard regularization yields some ambiguous results for the computation of certain integrals at the 3PN order, as noticed by Jaranowski \& Schäfer [261, 262, 263] in their computation of the equations of motion within the ADM-Hamiltonian formulation of general relativity. By standard Hadamard regularization we mean the regularization based solely on the definitions of the partie finie of a singular function, Eq. (160), and the partie finie of a divergent integral, Eq. (162), and without using a theory of pseudo-functions and generalized distributional derivatives as in Refs. [70, 72]. It was shown in Refs. [261, 262, 263] that there are two and only two types of ambiguous terms in the 3PN Hamiltonian, which were then parametrized by two unknown numerical coefficients called $\omega_{\text {static }}$ and $\omega_{\text {kinetic }}$.

Progressing concurrently, Blanchet \& Faye [70, 72] introduced the "extended" Hadamard regularization - the one we outlined in Section 6.1 - and obtained $[69,71]$ the 3PN equations of motion

\footnotetext{
40 The sum over $k$ in Eq. (168) is always finite since there is a maximal order $a_{0}$ of divergency in Eq. (159).

41 It was shown in Ref. [71] that using one or the other of these derivatives results in some equations of motion that differ by a coordinate transformation, and the redefinition of some ambiguity parameter. This indicates that the distributional derivatives introduced in Ref. [70] constitute some technical tools devoid of physical meaning besides precisely the appearance of Hadamard's ambiguity parameters.
} 
complete except for one and only one unknown numerical constant, called $\lambda$. The new extended Hadamard regularization is mathematically well-defined and yields unique results for the computation of any integral in the problem; however, it turned out to be in a sense "incomplete" as it could not determine the value of this constant. The comparison of the result with the work [261, 262], on the basis of the computation of the invariant energy of compact binaries moving on circular orbits, revealed [69] that

$$
\begin{aligned}
\omega_{\text {kinetic }} & =\frac{41}{24}, \\
\omega_{\text {static }} & =-\frac{11}{3} \lambda-\frac{1987}{840} .
\end{aligned}
$$

Therefore, the ambiguity $\omega_{\text {kinetic }}$ is fixed, while $\lambda$ is equivalent to the other ambiguity $\omega_{\text {static }}$. Notice that the value (170a) for the kinetic ambiguity parameter $\omega_{\text {kinetic }}$, which is in factor of some velocity dependent terms, is the only one for which the $3 \mathrm{PN}$ equations of motion are Lorentz invariant. Fixing up this value was possible because the extended Hadamard regularization [70, 72] was defined in such a way that it keeps the Lorentz invariance.

The value of $\omega_{\text {kinetic }}$ given by Eq. (170a) was recovered in Ref. [162] by directly proving that such value is the unique one for which the global Poincaré invariance of the ADM-Hamiltonian formalism is verified. Since the coordinate conditions associated with the ADM formalism do not manifestly respect the Poincaré symmetry, it was necessary to prove that the $3 \mathrm{PN}$ Hamiltonian is compatible with the existence of generators for the Poincaré algebra. By contrast, the harmoniccoordinate conditions preserve the Poincare invariance, and therefore the associated equations of motion at 3PN order are manifestly Lorentz-invariant, as was found to be the case in Refs. [69, 71].

The appearance of one and only one physical unknown coefficient $\lambda$ in the equations of motion constitutes a quite striking fact, that is related specifically with the use of a Hadamard-type regularization. ${ }^{42}$ Technically speaking, the presence of the ambiguity parameter $\lambda$ is associated with the non-distributivity of Hadamard's regularization, in the sense of Eq. (161). Mathematically speaking, $\lambda$ is probably related to the fact that it is impossible to construct a distributional derivative operator, such as Eqs. (167) - (168), satisfying the Leibniz rule for the derivation of the product [380]. The Einstein field equations can be written in many different forms, by shifting the derivatives and operating some terms by parts with the help of the Leibniz rule. All these forms are equivalent in the case of regular sources, but since the derivative operator (167) - (168) violates the Leibniz rule they become inequivalent for point particles.

Physically speaking, let us also argue that $\lambda$ has its root in the fact that in a complete computation of the equations of motion valid for two regular extended weakly self-gravitating bodies, many non-linear integrals, when taken individually, start depending, from the 3PN order, on the internal structure of the bodies, even in the "compact-body" limit where the radii tend to zero. However, when considering the full equations of motion, one expects that all the terms depending on the internal structure can be removed, in the compact-body limit, by a coordinate transformation (or by some appropriate shifts of the central world lines of the bodies), and that finally $\lambda$ is given by a pure number, for instance a rational fraction, independent of the details of the internal structure of the compact bodies. From this argument (which could be justified by the effacing principle in general relativity) the value of $\lambda$ is necessarily the one we compute below, Eq. (172), and will be valid for any compact objects, for instance black holes.

The ambiguity parameter $\omega_{\text {static }}$, which is in factor of some static, velocity-independent term, and hence cannot be derived by invoking Lorentz invariance, was computed by Damour, Jaranowski

\footnotetext{
42 Note also that the harmonic-coordinates $3 \mathrm{PN}$ equations of motion [69, 71] depend, in addition to $\lambda$, on two arbitrary constants $r_{1}^{\prime}$ and $r_{2}^{\prime}$ parametrizing some logarithmic terms. These constants are closely related to the constants $s_{1}$ and $s_{2}$ in the partie-finie integral (162); see Ref. [71] and Eq. (185) below for the precise definition. However, $r_{1}^{\prime}$ and $r_{2}^{\prime}$ are not "physical" in the sense that they can be removed by a coordinate transformation.
} 
\& Schäfer [163] by means of dimensional regularization, instead of some Hadamard-type one, within the ADM-Hamiltonian formalism. Their result is

$$
\omega_{\text {static }}=0 .
$$

As argued in [163], clearing up the static ambiguity is made possible by the fact that dimensional regularization, contrary to Hadamard's regularization, respects all the basic properties of the algebraic and differential calculus of ordinary functions: Associativity, commutativity and distributivity of point-wise addition and multiplication, Leibniz's rule, and the Schwarz lemma. In this respect, dimensional regularization is certainly superior to Hadamard's one, which does not respect the distributivity of the product [recall Eq. (161)] and unavoidably violates at some stage the Leibniz rule for the differentiation of a product.

The ambiguity parameter $\lambda$ is fixed from the result (171) and the necessary link (170b) provided by the equivalence between the harmonic-coordinates and ADM-Hamiltonian formalisms [69, 164]. However, $\lambda$ has also been computed directly by Blanchet, Damour \& Esposito-Farèse [61] applying dimensional regularization to the $3 \mathrm{PN}$ equations of motion in harmonic coordinates (in the line of Refs. [69, 71]). The end result,

$$
\lambda=-\frac{1987}{3080},
$$

is in full agreement with Eq. (171). ${ }^{43}$ Besides the independent confirmation of the value of $\omega_{\text {static }}$ or $\lambda$, the work [61] provides also a confirmation of the consistency of dimensional regularization, since the explicit calculations are entirely different from the ones of Ref. [163]: Harmonic coordinates instead of ADM-type ones, work at the level of the equations of motion instead of the Hamiltonian, a different form of Einstein's field equations which is solved by a different iteration scheme.

Let us comment that the use of a self-field regularization, be it dimensional or based on Hadamard's partie finie, signals a somewhat unsatisfactory situation on the physical point of view, because, ideally, we would like to perform a complete calculation valid for extended bodies, taking into account the details of the internal structure of the bodies (energy density, pressure, internal velocity field, etc.). By considering the limit where the radii of the objects tend to zero, one should recover the same result as obtained by means of the point-mass regularization. This would demonstrate the suitability of the regularization. This program was undertaken at the $2 \mathrm{PN}$ order in Refs. [280, 234] which derived the equations of motion of two extended fluid balls, and obtained equations of motion depending only on the two masses $m_{1}$ and $m_{2}$ of the compact bodies. ${ }^{44}$ At the $3 \mathrm{PN}$ order we expect that the extended-body program should give the value of the regularization parameter $\lambda$-probably after a coordinate transformation to remove the terms depending on the internal structure. Ideally, its value should also be confirmed by independent and more physical methods like those of Refs. [407, 281, 172].

An important work, in several aspects more physical than the formal use of regularizations, is the one of Itoh \& Futamase [255, 253, 254], following previous investigations in Refs. [256, 257]. These authors derived the 3PN equations of motion in harmonic coordinates by means of a particular variant of the famous "surface-integral" method à la Einstein, Infeld \& Hoffmann [184]. The aim is to describe extended relativistic compact binary systems in the so-called strong-field point particle limit which has been defined in Ref. [212]. This approach is interesting because it is based on the physical notion of extended compact bodies in general relativity, and is free of the problems of ambiguities. The end result of Refs. [255, 253] is in agreement with the 3PN harmonic

\footnotetext{
43 One may wonder why the value of $\lambda$ is a complicated rational fraction while $\omega_{\text {static }}$ is so simple. This is because $\omega_{\text {static }}$ was introduced precisely to measure the amount of ambiguities of certain integrals, while by contrast, $\lambda$ was introduced [see Eq. (185)] as an unknown constant entering the relation between the arbitrary scales $r_{1}^{\prime}, r_{2}^{\prime}$ on the one hand, and $s_{1}, s_{2}$ on the other hand, which has a priori nothing to do with the ambiguous part of integrals.

44 See however some comments on the latter work in Ref. [145], pp. 168-169.
} 
coordinates equations of motion $[69,71]$ and is unambiguous, as it does directly determine the ambiguity parameter $\lambda$ to exactly the value (172).

The 3PN equations of motion in harmonic coordinates or, more precisely, the associated 3PN Lagrangian, were also derived by Foffa \& Sturani [203] using another important approach, coined the effective field theory (EFT) [223]. Their result is fully compatible with the value (172) for the ambiguity parameter $\lambda$; however, in contrast with the surface-integral method of Refs. [255, 253], this does not check the method of regularization because the EFT approach is also based on dimensional self-field regularization.

We next consider the problem of the binary's radiation field, where the same phenomenon occurs, with the appearance of some Hadamard regularization ambiguity parameters at 3PN order. More precisely, Blanchet, Iyer \& Joguet [81], computing the 3PN compact binary's mass quadrupole moment $\mathrm{I}_{i j}$, found it necessary to introduce three Hadamard regularization constants $\xi, \kappa$, and $\zeta$, which are independent of the equation-of-motion related constant $\lambda$. The total gravitational-wave flux at $3 \mathrm{PN}$ order, in the case of circular orbits, was found to depend on a single combination of the latter constants, $\theta=\xi+2 \kappa+\zeta$, and the binary's orbital phase, for circular orbits, involved only the linear combination of $\theta$ and $\lambda$ given by $\hat{\theta}=\theta-\frac{7}{3} \lambda$, as shown in [73].

Dimensional regularization (instead of Hadamard's) has next been applied in Refs. [62, 63] to the computation of the $3 \mathrm{PN}$ radiation field of compact binaries, leading to the following unique determination of the ambiguity parameters: ${ }^{45}$

$$
\begin{aligned}
\xi & =-\frac{9871}{9240}, \\
\kappa & =0 \\
\zeta & =-\frac{7}{33} .
\end{aligned}
$$

These values represent the end result of dimensional regularization. However, several alternative calculations provide a check, independent of dimensional regularization, for all the parameters (173). One computes [80] the 3PN binary's mass dipole moment $\mathrm{I}_{i}$ using Hadamard's regularization, and identifies $\mathrm{I}_{i}$ with the $3 \mathrm{PN}$ center of mass vector position $\mathrm{G}_{i}$, already known as a conserved integral associated with the Poincaré invariance of the 3PN equations of motion in harmonic coordinates [174]. This yields $\xi+\kappa=-9871 / 9240$ in agreement with Eqs. (173). Next, one considers [65] the limiting physical situation where the mass of one of the particles is exactly zero (say, $m_{2}=0$ ), and the other particle moves with uniform velocity. Technically, the $3 \mathrm{PN}$ quadrupole moment of a boosted Schwarzschild black hole is computed and compared with the result for $\mathrm{I}_{i j}$ in the limit $m_{2}=0$. The result is $\zeta=-7 / 33$, and represents a direct verification of the global Poincaré invariance of the wave generation formalism (the parameter $\zeta$ representing the analogue for the radiation field of the parameter $\left.\omega_{\text {kinetic }}\right)$. Finally, one proves [63] that $\kappa=0$ by showing that there are no dangerously divergent diagrams corresponding to non-zero $\kappa$-values, where a diagram is meant here in the sense of Ref. [151].

The determination of the parameters (173) completes the problem of the general relativistic prediction for the templates of inspiralling compact binaries up to 3.5PN order. The numerical values of these parameters indicate, following measurement-accuracy analyses [105, 106, 159, 156], that the 3.5PN order should provide an excellent approximation for both the on-line search and the subsequent off-line analysis of gravitational wave signals from inspiralling compact binaries in the LIGO and VIRGO detectors.

45 The result for $\xi$ happens to be amazingly related to the one for $\lambda$ by a cyclic permutation of digits; compare $3 \xi=-9871 / 3080$ with $\lambda=-1987 / 3080$. 


\subsection{Dimensional regularization of the equations of motion}

As reviewed in Section 6.2, work at 3PN order using Hadamard's self-field regularization showed the appearance of ambiguity parameters, due to an incompleteness of the Hadamard regularization employed for curing the infinite self field of point particles. We give here more details on the determination using dimensional regularization of the ambiguity parameter $\lambda$ [or equivalently $\omega_{\text {static }}$, see Eq. (170b)] which appeared in the 3PN equations of motion.

Dimensional regularization was invented as a means to preserve the gauge symmetry of perturbative quantum field theories [391, 91, 100, 131]. Our basic problem here is to respect the gauge symmetry associated with the diffeomorphism invariance of the classical general relativistic description of interacting point masses. Hence, we use dimensional regularization not merely as a trick to compute some particular integrals which would otherwise be divergent, but as a powerful tool for solving in a consistent way the Einstein field equations with singular point-mass sources, while preserving its crucial symmetries. In particular, we shall prove that dimensional regularization determines the kinetic ambiguity parameter $\omega_{\text {kinetic }}$ (and its radiation-field analogue $\zeta$ ), and is therefore able to correctly keep track of the global Lorentz-Poincaré invariance of the gravitational field of isolated systems. The dimensional regularization is also an important ingredient of the EFT approach to equations of motion and gravitational radiation [223].

The Einstein field equations in $d+1$ space-time dimensions, relaxed by the condition of harmonic coordinates $\partial_{\mu} h^{\alpha \mu}=0$, take exactly the same form as given in Eqs. (18)-(23). In particular the box operator $\square$ now denotes the flat space-time d'Alembertian operator in $d+1$ dimensions with signature $(-1,1,1, \cdots)$. The gravitational constant $G$ is related to the usual three-dimensional Newton's constant $G_{\mathrm{N}}$ by

$$
G=G_{\mathrm{N}} \ell_{0}^{d-3}
$$

where $\ell_{0}$ denotes an arbitrary length scale. The explicit expression of the gravitational source term $\Lambda^{\alpha \beta}$ involves some $d$-dependent coefficients, and is given by

$$
\begin{aligned}
\Lambda^{\alpha \beta}= & -h^{\mu \nu} \partial_{\mu \nu}^{2} h^{\alpha \beta}+\partial_{\mu} h^{\alpha \nu} \partial_{\nu} h^{\beta \mu}+\frac{1}{2} g^{\alpha \beta} g_{\mu \nu} \partial_{\lambda} h^{\mu \tau} \partial_{\tau} h^{\nu \lambda} \\
& -g^{\alpha \mu} g_{\nu \tau} \partial_{\lambda} h^{\beta \tau} \partial_{\mu} h^{\nu \lambda}-g^{\beta \mu} g_{\nu \tau} \partial_{\lambda} h^{\alpha \tau} \partial_{\mu} h^{\nu \lambda}+g_{\mu \nu} g^{\lambda \tau} \partial_{\lambda} h^{\alpha \mu} \partial_{\tau} h^{\beta \nu} \\
& +\frac{1}{4}\left(2 g^{\alpha \mu} g^{\beta \nu}-g^{\alpha \beta} g^{\mu \nu}\right)\left(g_{\lambda \tau} g_{\epsilon \pi}-\frac{1}{d-1} g_{\tau \epsilon} g_{\lambda \pi}\right) \partial_{\mu} h^{\lambda \pi} \partial_{\nu} h^{\tau \epsilon} .
\end{aligned}
$$

When $d=3$ we recover Eq. (24). In the following we assume, as usual in dimensional regularization, that the dimension of space is a complex number, $d \in \mathbb{C}$, and prove many results by invoking complex analytic continuation in $d$. We shall often pose $\varepsilon \equiv d-3$.

We parametrize the $3 \mathrm{PN}$ metric in $d$ dimensions by means of some retarded potentials $V, V_{i}$, $\hat{W}_{i j}, \ldots$, which are straightforward $d$-dimensional generalizations of the potentials used in three dimensions and which were defined in Section 5.3. Those are obtained by post-Newtonian iteration of the $d$-dimensional field equations, starting from appropriate definitions of matter source densities generalizing Eqs. (145):

$$
\begin{aligned}
\sigma & =\frac{2}{d-1} \frac{(d-2) T^{00}+T^{i i}}{c^{2}}, \\
\sigma_{i} & =\frac{T^{0 i}}{c}, \\
\sigma_{i j} & =T^{i j} .
\end{aligned}
$$

As a result all the expressions of Section 5.3 acquire some explicit $d$-dependent coefficients. For instance we find [61]

$$
V=\square_{\text {ret }}^{-1}[-4 \pi G \sigma]
$$




$$
\hat{W}_{i j}=\square_{\mathrm{ret}}^{-1}\left[-4 \pi G\left(\sigma_{i j}-\delta_{i j} \frac{\sigma_{k k}}{d-2}\right)-\frac{d-1}{2(d-2)} \partial_{i} V \partial_{j} V\right]
$$

Here $\square_{\text {ret }}^{-1}$ means the retarded integral in $d+1$ space-time dimensions, which admits, though, no simple expression generalizing Eq. (31) in physical $(t, \mathbf{x})$ space. $^{46}$

As reviewed in Section 6.1, the generic functions $F(\mathbf{x})$ we have to deal with in 3 dimensions, are smooth on $\mathbb{R}^{3}$ except at $\boldsymbol{y}_{1}$ and $\boldsymbol{y}_{2}$, around which they admit singular Laurent-type expansions in powers and inverse powers of $r_{1} \equiv\left|\mathbf{x}-\boldsymbol{y}_{1}\right|$ and $r_{2} \equiv\left|\mathbf{x}-\boldsymbol{y}_{2}\right|$, given by Eq. (178). In $d$ spatial dimensions, there is an analogue of the function $F$, which results from the post-Newtonian iteration process performed in $d$ dimensions as we just outlined. Let us call this function $F^{(d)}(\mathbf{x})$, where $\mathbf{x} \in \mathbb{R}^{d}$. When $r_{1} \rightarrow 0$ the function $F^{(d)}$ admits a singular expansion which is more involved than in 3 dimensions, as it reads

$$
F^{(d)}(\mathbf{x})=\sum_{\substack{p_{0} \leqslant p \leqslant \mathcal{N} \\ q_{0} \leqslant q \leqslant q_{1}}} r_{1}^{p+q \varepsilon} f_{p, q}^{(\varepsilon)}\left(\mathbf{n}_{1}\right)+o\left(r_{1}^{\mathcal{N}}\right)
$$

The coefficients $f_{1}{ }_{p, q}^{(\varepsilon)}\left(\mathbf{n}_{1}\right)$ depend on $\varepsilon=d-3$, and the powers of $r_{1}$ involve the relative integers $p$ and $q$ whose values are limited by some $p_{0}, q_{0}$ and $q_{1}$ as indicated. Here we will be interested in functions $F^{(d)}(\mathbf{x})$ which have no poles as $\varepsilon \rightarrow 0$ (this will always be the case at $3 \mathrm{PN}$ order). Therefore, we can deduce from the fact that $F^{(d)}(\mathbf{x})$ is continuous at $d=3$ the constraint

$$
\sum_{q=q_{0}}^{q_{1}} f_{1}^{(\varepsilon=0)}\left(\mathbf{n}_{1}\right)=f_{1}\left(\mathbf{n}_{1}\right)
$$

For the problem at hand, we essentially have to deal with the regularization of Poisson integrals, or iterated Poisson integrals (and their gradients needed in the equations of motion), of the generic function $F^{(d)}$. The Poisson integral of $F^{(d)}$, in $d$ dimensions, is given by the Green's function for the Laplace operator,

$$
P^{(d)}\left(\mathbf{x}^{\prime}\right)=\Delta^{-1}\left[F^{(d)}(\mathbf{x})\right] \equiv-\frac{\tilde{k}}{4 \pi} \int \frac{\mathrm{d}^{d} \mathbf{x}}{\left|\mathbf{x}-\mathbf{x}^{\prime}\right|^{d-2}} F^{(d)}(\mathbf{x}),
$$

where $\tilde{k}$ is a constant related to the usual Eulerian $\Gamma$-function by ${ }^{47}$

$$
\tilde{k}=\frac{\Gamma\left(\frac{d-2}{2}\right)}{\pi^{\frac{d-2}{2}}} .
$$

We need to evaluate the Poisson integral at the point $\mathbf{x}^{\prime}=\boldsymbol{y}_{1}$ where it is singular; this is quite easy in dimensional regularization, because the nice properties of analytic continuation allow simply to get $\left[P^{(d)}\left(\mathbf{x}^{\prime}\right)\right]_{\mathbf{x}^{\prime}=\boldsymbol{y}_{1}}$ by replacing $\mathbf{x}^{\prime}$ by $\boldsymbol{y}_{1}$ inside the explicit integral (180). So we simply have

$$
P^{(d)}\left(\boldsymbol{y}_{1}\right)=-\frac{\tilde{k}}{4 \pi} \int \frac{\mathrm{d}^{d} \mathbf{x}}{r_{1}^{d-2}} F^{(d)}(\mathbf{x}) .
$$

\footnotetext{
${ }^{46}$ In higher approximations there will be also IR divergences and one should really employ the $d$-dimensional version of Eq. (141).

${ }^{47}$ We have $\lim _{d \rightarrow 3} \tilde{k}=1$. Notice that $\tilde{k}$ is closely linked to the volume $\Omega_{d-1}$ of the sphere with $d-1$ dimensions (i.e. embedded into Euclidean $d$-dimensional space):

$$
\tilde{k} \Omega_{d-1}=\frac{4 \pi}{d-2} .
$$
}


It is not possible at present to compute the equations of motion in the general $d$-dimensional case, but only in the limit where $\varepsilon \rightarrow 0[163,61]$. The main technical step of our strategy consists of computing, in the limit $\varepsilon \rightarrow 0$, the difference between the $d$-dimensional Poisson potential (182), and its Hadamard 3-dimensional counterpart given by $(P)_{1}$, where the Hadamard partie finie is defined by Eq. (160). But we must be precise when defining the Hadamard partie finie of a Poisson integral. Indeed, the definition (160) stricto sensu is applicable when the expansion of the function $F$, for $r_{1} \rightarrow 0$, does not involve logarithms of $r_{1}$; see Eq. (160). However, the Poisson integral $P\left(\mathbf{x}^{\prime}\right)$ of $F(\mathbf{x})$ will typically involve such logarithms at the $3 \mathrm{PN}$ order, namely some $\ln r_{1}^{\prime}$ where $r_{1}^{\prime} \equiv\left|\mathbf{x}^{\prime}-\boldsymbol{y}_{1}\right|$ formally tends to zero (hence $\ln r_{1}^{\prime}$ is formally infinite). The proper way to define the Hadamard partie finie in this case is to include the $\ln r_{1}^{\prime}$ into its definition; we arrive at [70]

$$
(P)_{1}=-\frac{1}{4 \pi} \mathrm{Pf}_{r_{1}^{\prime}, s_{2}} \int \frac{\mathrm{d}^{3} \mathbf{x}}{r_{1}} F(\mathbf{x})-\left(r_{1}^{2} F\right)_{1} .
$$

The first term follows from Hadamard's partie finie integral (162); the second one is given by Eq. (160). Notice that in this result the constant $s_{1}$ entering the partie finie integral (162) has been "replaced" by $r_{1}^{\prime}$, which plays the role of a new regularization constant (together with $r_{2}^{\prime}$ for the other particle), and which ultimately parametrizes the final Hadamard regularized 3PN equations of motion. It was shown that $r_{1}^{\prime}$ and $r_{2}^{\prime}$ are unphysical, in the sense that they can be removed by a coordinate transformation $[69,71]$. On the other hand, the constant $s_{2}$ remaining in the result (183) is the source for the appearance of the physical ambiguity parameter $\lambda$. Denoting the difference between the dimensional and Hadamard regularizations by means of the script letter $\mathcal{D}$, we pose (for what concerns the point 1)

$$
\mathcal{D} P_{1} \equiv P^{(d)}\left(\boldsymbol{y}_{1}\right)-(P)_{1} .
$$

That is, $\mathcal{D} P_{1}$ is what we shall have to add to the Hadamard-regularization result in order to get the $d$-dimensional result. However, we shall only compute the first two terms of the Laurent expansion of $\mathcal{D} P_{1}$ when $\varepsilon \rightarrow 0$, say $\mathcal{D} P_{1}=a_{-1} \varepsilon^{-1}+a_{0}+\mathcal{O}(\varepsilon)$. This is the information we need to clear up the ambiguity parameter. We insist that the difference $\mathcal{D} P_{1}$ comes exclusively from the contribution of terms developing some poles $\propto 1 / \varepsilon$ in the $d$-dimensional calculation.

Next we outline the way we obtain, starting from the computation of the "difference", the $3 \mathrm{PN}$ equations of motion in dimensional regularization, and show how the ambiguity parameter $\lambda$ is determined. By contrast to $r_{1}^{\prime}$ and $r_{2}^{\prime}$ which are pure gauge, $\lambda$ is a genuine physical ambiguity, introduced in Refs. [70, 71] as the single unknown numerical constant parametrizing the ratio between $s_{2}$ and $r_{2}^{\prime}$ [where $s_{2}$ is the constant left in Eq. (183)] as

$$
\ln \left(\frac{r_{2}^{\prime}}{s_{2}}\right)=\frac{159}{308}+\lambda \frac{m_{1}+m_{2}}{m_{2}} \quad(\operatorname{and} 1 \leftrightarrow 2),
$$

where $m_{1}$ and $m_{2}$ are the two masses. The terms corresponding to the $\lambda$-ambiguity in the acceleration $\boldsymbol{a}_{1}=d \boldsymbol{v}_{1} / d t$ of particle 1 read simply

$$
\Delta \boldsymbol{a}_{1}[\lambda]=-\frac{44 \lambda}{3} \frac{G_{\mathrm{N}}^{4} m_{1} m_{2}^{2}\left(m_{1}+m_{2}\right)}{r_{12}^{5} c^{6}} \boldsymbol{n}_{12},
$$

where the relative distance between particles is denoted $\boldsymbol{y}_{1}-\boldsymbol{y}_{2} \equiv r_{12} \boldsymbol{n}_{12}$ (with $\boldsymbol{n}_{12}$ being the unit vector pointing from particle 2 to particle 1 ). We start from the end result of Ref. [71] for the $3 \mathrm{PN}$ harmonic coordinates acceleration $\boldsymbol{a}_{1}$ in Hadamard's regularization, abbreviated as HR. Since the result was obtained by means of the specific extended variant of Hadamard's regularization (in short EHR, see Section 6.1) we write it as

$$
\boldsymbol{a}_{1}^{(\mathrm{HR})}=\boldsymbol{a}_{1}^{(\mathrm{EHR})}+\Delta \boldsymbol{a}_{1}[\lambda]
$$


where $\boldsymbol{a}_{1}^{(\mathrm{EHR})}$ is a fully determined functional of the masses $m_{1}$ and $m_{2}$, the relative distance $r_{12} \boldsymbol{n}_{12}$, the coordinate velocities $\boldsymbol{v}_{1}$ and $\boldsymbol{v}_{2}$, and also the gauge constants $r_{1}^{\prime}$ and $r_{2}^{\prime}$. The only ambiguous term is the second one and is given by Eq. (186).

Our strategy is to extract from both the dimensional and Hadamard regularizations their common core part, obtained by applying the so-called "pure-Hadamard-Schwartz" (pHS) regularization. Following the definition in Ref. [61], the pHS regularization is a specific, minimal Hadamard-type regularization of integrals, based on the partie finie integral (162), together with a minimal treatment of "contact" terms, in which the definition (162) is applied separately to each of the elementary potentials $V, V_{i}$, etc. (and gradients) that enter the post-Newtonian metric. Furthermore, the regularization of a product of these potentials is assumed to be distributive, i.e., $(F G)_{1}=(F)_{1}(G)_{1}$ in the case where $F$ and $G$ are given by such elementary potentials; this is thus in contrast with Eq. (161). The pHS regularization also assumes the use of standard Schwartz distributional derivatives [381]. The interest of the pHS regularization is that the dimensional regularization is equal to it plus the "difference"; see Eq. (190).

To obtain the pHS-regularized acceleration we need to substract from the EHR result a series of contributions, which are specific consequences of the use of EHR [70, 72]. For instance, one of these contributions corresponds to the fact that in the EHR the distributional derivative is given by Eqs. (167) - (168) which differs from the Schwartz distributional derivative in the pHS regularization. Hence we define

$$
\boldsymbol{a}_{1}^{(\mathrm{pHS})}=\boldsymbol{a}_{1}^{(\mathrm{EHR})}-\sum \delta \boldsymbol{a}_{1}
$$

where the $\delta \boldsymbol{a}_{1}$ 's denote the extra terms following from the EHR prescriptions. The pHS-regularized acceleration (188) constitutes essentially the result of the first stage of the calculation of $\boldsymbol{a}_{1}$, as reported in Ref. [193].

The next step consists of evaluating the Laurent expansion, in powers of $\varepsilon=d-3$, of the difference between the dimensional regularization and the pHS (3-dimensional) computation. As we reviewed above, this difference makes a contribution only when a term generates a pole $\sim 1 / \varepsilon$, in which case the dimensional regularization adds an extra contribution, made of the pole and the finite part associated with the pole [we consistently neglect all terms $\mathcal{O}(\varepsilon)$ ]. One must then be especially wary of combinations of terms whose pole parts finally cancel but whose dimensionally regularized finite parts generally do not, and must be evaluated with care. We denote the above defined difference by

$$
\mathcal{D} \boldsymbol{a}_{1}=\sum \mathcal{D} P_{1}
$$

It is made of the sum of all the individual differences of Poisson or Poisson-like integrals as computed in Eq. (184). The total difference (189) depends on the Hadamard regularization scales $r_{1}^{\prime}$ and $s_{2}$ (or equivalently on $\lambda$ and $r_{1}^{\prime}, r_{2}^{\prime}$ ), and on the parameters associated with dimensional regularization, namely $\varepsilon$ and the characteristic length scale $\ell_{0}$ introduced in Eq. (174). Finally, the result is the explicit computation of the $\varepsilon$-expansion of the dimensional regularization (DR) acceleration as

$$
\boldsymbol{a}_{1}^{(\mathrm{DR})}=\boldsymbol{a}_{1}^{(\mathrm{pHS})}+\mathcal{D} \boldsymbol{a}_{1}
$$

With this result we can prove two theorems [61].

Theorem 8. The pole part $\propto 1 / \varepsilon$ of the DR acceleration (190) can be re-absorbed (i.e. renormalized) into some shifts of the two "bare" world-lines: $\boldsymbol{y}_{1} \rightarrow \boldsymbol{y}_{1}+\boldsymbol{\xi}_{1}$ and $\boldsymbol{y}_{2} \rightarrow \boldsymbol{y}_{2}+\boldsymbol{\xi}_{2}$, with $\boldsymbol{\xi}_{1,2} \propto 1 / \varepsilon$ say, so that the result, expressed in terms of the "dressed" quantities, is finite when $\varepsilon \rightarrow 0$.

The situation in harmonic coordinates is to be contrasted with the calculation in ADM-type coordinates within the Hamiltonian formalism, where it was shown that all pole parts directly cancel 
out in the total 3PN Hamiltonian: No renormalization of the world-lines is needed [163]. The central result is then:

Theorem 9. The renormalized (finite) DR acceleration is physically equivalent to the Hadamardregularized (HR) acceleration (end result of Ref. [71]), in the sense that

$$
\boldsymbol{a}_{1}^{(\mathrm{HR})}=\lim _{\varepsilon \rightarrow 0}\left[\boldsymbol{a}_{1}^{(\mathrm{DR})}+\delta_{\boldsymbol{\xi}} \boldsymbol{a}_{1}\right]
$$

where $\delta_{\boldsymbol{\xi}} \boldsymbol{a}_{1}$ denotes the effect of the shifts on the acceleration, if and only if the HR ambiguity parameter $\lambda$ entering the harmonic-coordinates equations of motion takes the unique value (172).

The precise shifts $\boldsymbol{\xi}_{1}$ and $\boldsymbol{\xi}_{2}$ needed in Theorem 9 involve not only a pole contribution $\propto 1 / \varepsilon$, but also a finite contribution when $\varepsilon \rightarrow 0$. Their explicit expressions read: ${ }^{48}$

$$
\left.\boldsymbol{\xi}_{1}=\frac{11}{3} \frac{G_{\mathrm{N}}^{2} m_{1}^{2}}{c^{6}}\left[\frac{1}{\varepsilon}-2 \ln \left(\frac{r_{1}^{\prime} \bar{q}^{1 / 2}}{\ell_{0}}\right)-\frac{327}{1540}\right] \boldsymbol{a}_{1}^{\mathrm{N}} \quad \text { (together with } 1 \leftrightarrow 2\right),
$$

where $G_{\mathrm{N}}$ is Newton's constant, $\ell_{0}$ is the characteristic length scale of dimensional regularization, cf. Eq. (174), where $\boldsymbol{a}_{1}^{\mathrm{N}}$ is the Newtonian acceleration of the particle 1 in $d$ dimensions, and $\bar{q} \equiv 4 \pi e^{\gamma_{\mathrm{E}}}$ depends on Euler's constant $\gamma_{\mathrm{E}} \simeq 0.577$.

\subsection{Dimensional regularization of the radiation field}

We now address the similar problem concerning the binary's radiation field - to 3PN order beyond Einstein's quadrupole formalism (2) - (3). As reviewed in Section 6.2, three ambiguity parameters: $\xi, \kappa$ and $\zeta$, have been shown to appear in the 3PN expression of the quadrupole moment [81, 80].

To apply dimensional regularization, we must use as in Section 6.3 the $d$-dimensional postNewtonian iteration leading to potentials such as those in Eqs. (177); and we have to generalize to $d$ dimensions some key results of the wave generation formalism of Part A. Essentially, we need the $d$-dimensional analogues of the multipole moments of an isolated source $\mathrm{I}_{L}$ and $\mathrm{J}_{L}$ in Eqs. (123). Here we report the result we find in the case of the mass-type moment:

$$
\begin{aligned}
\mathrm{I}_{L}^{(d)}(t)=\frac{d-1}{2(d-2)} \mathcal{F} \mathcal{P} \int \mathrm{d}^{d} \mathbf{x} & \left\{\hat{x}_{L} \sum_{[\ell]}(\mathbf{x}, t)-\frac{4(d+2 \ell-2)}{c^{2}(d+\ell-2)(d+2 \ell)} \hat{x}_{a L} \sum_{[\ell+1]}^{\sum^{(1)}(\mathbf{x}, t)}\right. \\
+ & \frac{2(d+2 \ell-2)}{c^{4}(d+\ell-1)(d+\ell-2)(d+2 \ell+2)} \hat{x}_{a b L} \sum_{[\ell+2]}^{\left.\sum^{(2)}(\mathbf{x}, t)\right\},}
\end{aligned}
$$

in which we denote, generalizing Eqs. (124),

$$
\begin{aligned}
\Sigma & =\frac{2}{d-1} \frac{(d-2) \bar{\tau}^{00}+\bar{\tau}^{i i}}{c^{2}}, \\
\Sigma_{i} & =\frac{\bar{\tau}^{0 i}}{c}, \\
\Sigma_{i j} & =\bar{\tau}^{i j},
\end{aligned}
$$

and where for any source densities the underscript $[\ell]$ means the infinite series

$$
\sum_{[\ell]}(\mathbf{x}, t)=\sum_{k=0}^{+\infty} \frac{1}{2^{2 k} k !} \frac{\Gamma\left(\frac{d}{2}+\ell\right)}{\Gamma\left(\frac{d}{2}+\ell+k\right)}\left(\frac{r}{c} \frac{\partial}{\partial t}\right)^{2 k} \Sigma(\mathbf{x}, t) .
$$

48 When working at the level of the equations of motion (not considering the metric outside the world-lines), the effect of shifts can be seen as being induced by a coordinate transformation of the bulk metric as in Ref. [71]. 
The latter definition represents the $d$-dimensional version of the post-Newtonian expansion series (126). At Newtonian order, the expression (193) reduces to the standard result $\mathrm{I}_{L}^{(d)}=$ $\int \mathrm{d}^{d} \mathbf{x} \rho \hat{x}_{L}+\mathcal{O}\left(1 / c^{2}\right)$ with $\rho=T^{00} / c^{2}$ denoting the usual Newtonian density.

The ambiguity parameters $\xi, \kappa$ and $\zeta$ come from the Hadamard regularization of the mass quadrupole moment $\mathrm{I}_{i j}$ at the $3 \mathrm{PN}$ order. The terms corresponding to these ambiguities were found to be

$$
\Delta \mathrm{I}_{i j}[\xi, \kappa, \zeta]=\frac{44}{3} \frac{G_{\mathrm{N}}^{2} m_{1}^{3}}{c^{6}}\left[\left(\xi+\kappa \frac{m_{1}+m_{2}}{m_{1}}\right) y_{1}^{\langle i} a_{1}^{j\rangle}+\zeta v_{1}^{\langle i} v_{1}^{j\rangle}\right]+1 \leftrightarrow 2,
$$

where $\boldsymbol{y}_{1}, \boldsymbol{v}_{1}$ and $\boldsymbol{a}_{1}$ denote the first particle's position, velocity and acceleration (and the brackets \langle\rangle surrounding indices refer to the STF projection). Like in Section 6.3, we express both the Hadamard and dimensional results in terms of the more basic pHS regularization. The first step of the calculation [80] is therefore to relate the Hadamard-regularized quadrupole moment $\mathrm{I}_{i j}^{(\mathrm{HR})}$, for general orbits, to its pHS part:

$$
\mathrm{I}_{i j}^{(\mathrm{HR})}=\mathrm{I}_{i j}^{(\mathrm{pHS})}+\Delta \mathrm{I}_{i j}\left[\xi+\frac{1}{22}, \kappa, \zeta+\frac{9}{110}\right] .
$$

In the right-hand side we find both the pHS part, and the effect of adding the ambiguities, with some numerical shifts of the ambiguity parameters $(\xi \rightarrow \xi+1 / 22, \zeta \rightarrow \zeta+9 / 110)$ due to the difference between the specific Hadamard-type regularization scheme used in Ref. [81] and the pHS one. The pHS part is free of ambiguities but depends on the gauge constants $r_{1}^{\prime}$ and $r_{2}^{\prime}$ introduced in the harmonic-coordinates equations of motion [69, 71].

We next use the $d$-dimensional moment (193) to compute the difference between the dimensional regularization (DR) result and the pHS one $[62,63]$. As in the work on equations of motion, we find that the ambiguities arise solely from the terms in the integration regions near the particles, that give rise to poles $\propto 1 / \varepsilon$, corresponding to logarithmic ultra-violet (UV) divergences in 3 dimensions. The infra-red (IR) region at infinity, i.e., $|\mathrm{x}| \rightarrow+\infty$, does not contribute to the difference between DR and pHS. The compact-support terms in the integrand of Eq. (193), proportional to the matter source densities $\sigma, \sigma_{a}$, and $\sigma_{a b}$, are also found not to contribute to the difference. We are therefore left with evaluating the difference linked with the computation of the non-compact terms in the expansion of the integrand of (193) near the singularities that produce poles in $d$ dimensions.

Let $F^{(d)}(\mathbf{x})$ be the non-compact part of the integrand of the quadrupole moment (193) (with indices $L=i j$ ), where $F^{(d)}$ includes the appropriate multipolar factors such as $\hat{x}_{i j}$, so that

$$
\mathrm{I}_{i j}^{(d)}=\int \mathrm{d}^{d} \mathbf{x} F^{(d)}(\mathbf{x})
$$

We do not indicate that we are considering here only the non-compact part of the moments. Near the singularities the function $F^{(d)}(\mathbf{x})$ admits a singular expansion of the type (178). In practice, the various coefficients ${ }_{1} f_{p, q}^{(\varepsilon)}$ are computed by specializing the general expressions of the non-linear retarded potentials $V, V_{a}, \hat{W}_{a b}$, etc. (valid for general extended sources) to point particles in $d$ dimensions. On the other hand, the analogue of Eq. (198) in 3 dimensions is

$$
\mathrm{I}_{i j}=\operatorname{Pf} \int \mathrm{d}^{3} \mathbf{x} F(\mathbf{x})
$$

where Pf refers to the Hadamard partie finie defined in Eq. (162). The difference $\mathcal{D I}_{i j}$ between the DR evaluation of the $d$-dimensional integral (198) and its corresponding three-dimensional evaluation (199), reads then

$$
\mathcal{D} \mathrm{I}_{i j}=\mathrm{I}_{i j}^{(d)}-\mathrm{I}_{i j}
$$


Such difference depends only on the UV behaviour of the integrands, and can therefore be computed "locally", i.e., in the vicinity of the particles, when $r_{1} \rightarrow 0$ and $r_{2} \rightarrow 0$. We find that Eq. (200) depends on two constant scales $s_{1}$ and $s_{2}$ coming from Hadamard's partie finie (162), and on the constants belonging to dimensional regularization, i.e., $\varepsilon=d-3$ and $\ell_{0}$ defined by Eq. (174). The dimensional regularization of the $3 \mathrm{PN}$ quadrupole moment is then obtained as the sum of the pHS part, and of the difference computed according to Eq. (200), namely

$$
\mathrm{I}_{i j}^{(\mathrm{DR})}=\mathrm{I}_{i j}^{(\mathrm{pHS})}+\mathcal{D} \mathrm{I}_{i j}
$$

An important fact, hidden in our too-compact notation (201), is that the sum of the two terms in the right-hand side of Eq. (201) does not depend on the Hadamard regularization scales $s_{1}$ and $s_{2}$. Therefore it is possible without changing the sum to re-express these two terms (separately) by means of the constants $r_{1}^{\prime}$ and $r_{2}^{\prime}$ instead of $s_{1}$ and $s_{2}$, where $r_{1}^{\prime}, r_{2}^{\prime}$ are the two fiducial scales entering the Hadamard-regularization result (197). This replacement being made the pHS term in Eq. (201) is exactly the same as the one in Eq. (197). At this stage all elements are in place to prove the following theorem $[62,63]$.

Theorem 10. The DR quadrupole moment (201) is physically equivalent to the Hadamard-regularized one (end result of Refs. [81, 80]), in the sense that

$$
\mathrm{I}_{i j}^{(\mathrm{HR})}=\lim _{\varepsilon \rightarrow 0}\left[\mathrm{I}_{i j}^{(\mathrm{DR})}+\delta_{\xi} \mathrm{I}_{i j}\right],
$$

where $\delta_{\boldsymbol{\xi}} \mathrm{I}_{i j}$ denotes the effect of the same shifts as determined in Theorems 8 and 9, if and only if the HR ambiguity parameters $\xi, \kappa$ and $\zeta$ take the unique values reported in Eqs. (173). Moreover, the poles $1 / \varepsilon$ separately present in the two terms in the brackets of Eq. (202) cancel out, so that the physical ("dressed") DR quadrupole moment is finite and given by the limit when $\varepsilon \rightarrow 0$ as shown in Eq. (202).

This theorem finally provides an unambiguous determination of the $3 \mathrm{PN}$ radiation field by dimensional regularization. Furthermore, as reviewed in Section 6.2, several checks of this calculation could be done, which provide independent confirmations for the ambiguity parameters. Such checks also show the powerfulness of dimensional regularization and its validity for describing the classical general-relativistic dynamics of compact bodies.

\section{Newtonian-like Equations of Motion}

\subsection{The 3PN acceleration and energy for particles}

We present the acceleration of one of the particles, say the particle 1, at the 3PN order, as well as the $3 \mathrm{PN}$ energy of the binary, which is conserved in the absence of radiation reaction. To get this result we used essentially a "direct" post-Newtonian method (issued from Ref. [76]), which consists of reducing the 3PN metric of an extended regular source, worked out in Eqs. (144), to the case where the matter tensor is made of delta functions, and then curing the self-field divergences by means of the Hadamard regularization technique. The equations of motion are simply the 3PN geodesic equations explicitly provided in Eqs. (150)-(152); the metric therein is the regularized metric generated by the system of particles itself. Hadamard's regularization permits to compute all the terms but one, and the Hadamard ambiguity parameter $\lambda$ is obtained from dimensional regularization; see Section 6.3. We also add the 3.5PN terms in harmonic coordinates which are known from Refs. [258, 259, 260, 336, 278, 322, 254]. These correspond to radiation reaction effects at relative $1 \mathrm{PN}$ order (see Section 5.4 for discussion on radiation reaction up to $1.5 \mathrm{PN}$ order). 
Though the successive post-Newtonian approximations are really a consequence of general relativity, the final equations of motion must be interpreted in a Newtonian-like fashion. That is, once a convenient general-relativistic (Cartesian) coordinate system is chosen, we should express the results in terms of the coordinate positions, velocities, and accelerations of the bodies, and view the trajectories of the particles as taking place in the absolute Euclidean space of Newton. But because the equations of motion are actually relativistic, they must:

1. Stay manifestly invariant - at least in harmonic coordinates - when we perform a global post-Newtonian-expanded Lorentz transformation;

2. Possess the correct "perturbative" limit, given by the geodesics of the (post-Newtonianexpanded) Schwarzschild metric, when one of the masses tends to zero;

3. Be conservative, i.e., to admit a Lagrangian or Hamiltonian formulation, when the gravitational radiation reaction is turned off.

We denote by $r_{12}=\left|\boldsymbol{y}_{1}(t)-\boldsymbol{y}_{2}(t)\right|$ the harmonic-coordinate distance between the two particles, with $\boldsymbol{y}_{1}=\left(y_{1}^{i}\right)$ and $\boldsymbol{y}_{2}=\left(y_{2}^{i}\right)$, by $\boldsymbol{n}_{12}=\left(\boldsymbol{y}_{1}-\boldsymbol{y}_{2}\right) / r_{12}$ the corresponding unit direction, and by $\boldsymbol{v}_{1}=\mathrm{d} \boldsymbol{y}_{1} / \mathrm{d} t$ and $\boldsymbol{a}_{1}=\mathrm{d} \boldsymbol{v}_{1} / \mathrm{d} t$ the coordinate velocity and acceleration of the particle 1 (and idem for 2). Sometimes we pose $\boldsymbol{v}_{12}=\boldsymbol{v}_{1}-\boldsymbol{v}_{2}$ for the relative velocity. The usual Euclidean scalar product of vectors is denoted with parentheses, e.g., $\left(n_{12} v_{1}\right)=\boldsymbol{n}_{12} \cdot \boldsymbol{v}_{1}$ and $\left(v_{1} v_{2}\right)=\boldsymbol{v}_{1} \cdot \boldsymbol{v}_{2}$. The equations of the body 2 are obtained by exchanging all the particle labels $1 \leftrightarrow 2$ (remembering that $\boldsymbol{n}_{12}$ and $\boldsymbol{v}_{12}$ change sign in this operation):

$$
\begin{aligned}
\boldsymbol{a}_{1}=- & \frac{G m_{2}}{r_{12}^{2}} \boldsymbol{n}_{12} \\
+\frac{1}{c^{2}}\{ & {\left[\frac{5 G^{2} m_{1} m_{2}}{r_{12}^{3}}+\frac{4 G^{2} m_{2}^{2}}{r_{12}^{3}}+\frac{G m_{2}}{r_{12}^{2}}\left(\frac{3}{2}\left(n_{12} v_{2}\right)^{2}-v_{1}^{2}+4\left(v_{1} v_{2}\right)-2 v_{2}^{2}\right)\right] \boldsymbol{n}_{12} } \\
& \left.+\frac{G m_{2}}{r_{12}^{2}}\left(4\left(n_{12} v_{1}\right)-3\left(n_{12} v_{2}\right)\right) \boldsymbol{v}_{12}\right\} \\
+\frac{1}{c^{4}}\{ & {\left[-\frac{57 G^{3} m_{1}^{2} m_{2}}{4 r_{12}^{4}}-\frac{69 G^{3} m_{1} m_{2}^{2}}{2 r_{12}^{4}}-\frac{9 G^{3} m_{2}^{3}}{r_{12}^{4}}\right.} \\
& +\frac{G m_{2}}{r_{12}^{2}}\left(-\frac{15}{8}\left(n_{12} v_{2}\right)^{4}+\frac{3}{2}\left(n_{12} v_{2}\right)^{2} v_{1}^{2}-6\left(n_{12} v_{2}\right)^{2}\left(v_{1} v_{2}\right)-2\left(v_{1} v_{2}\right)^{2}+\frac{9}{2}\left(n_{12} v_{2}\right)^{2} v_{2}^{2}\right. \\
& +\frac{G^{2} m_{1} m_{2}}{r_{12}^{3}}\left(\frac{39}{2}\left(n_{12} v_{1}\right)^{2}-39\left(n_{12} v_{1}\right)\left(n_{12} v_{2}\right)+\frac{17}{2}\left(n_{12} v_{2}\right)^{2}-\frac{15}{4} v_{1}^{2}-\frac{5}{2}\left(v_{1} v_{2}\right)+\frac{5}{4} v_{2}^{2}\right) \\
& \left.+\frac{G^{2} m_{2}^{2}}{r_{12}^{3}}\left(2\left(n_{12} v_{1}\right)^{2}-4\left(n_{12} v_{1}\right)\left(n_{12} v_{2}\right)-6\left(n_{12} v_{2}\right)^{2}-8\left(v_{1} v_{2}\right)+4 v_{2}^{2}\right)\right] \boldsymbol{n}_{12} \\
+ & {\left[\frac{G^{2} m_{2}^{2}}{r_{12}^{3}}\left(-2\left(n_{12} v_{1}\right)-2\left(n_{12} v_{2}\right)\right)+\frac{G^{2} m_{1} m_{2}}{r_{12}^{3}}\left(-\frac{63}{4}\left(n_{12} v_{1}\right)+\frac{55}{4}\left(n_{12} v_{2}\right)\right)\right.} \\
& +\frac{G m_{2}}{r_{12}^{2}}\left(-6\left(n_{12} v_{1}\right)\left(n_{12} v_{2}\right)^{2}+\frac{9}{2}\left(n_{12} v_{2}\right)^{3}+\left(n_{12} v_{2}\right) v_{1}^{2}-4\left(n_{12} v_{1}\right)\left(v_{1} v_{2}\right)\right.
\end{aligned}
$$




$$
\begin{aligned}
& +\frac{1}{c^{5}}\left\{\left[\frac{208 G^{3} m_{1} m_{2}^{2}}{15 r_{12}^{4}}\left(n_{12} v_{12}\right)-\frac{24 G^{3} m_{1}^{2} m_{2}}{5 r_{12}^{4}}\left(n_{12} v_{12}\right)+\frac{12 G^{2} m_{1} m_{2}}{5 r_{12}^{3}}\left(n_{12} v_{12}\right) v_{12}^{2}\right] \boldsymbol{n}_{12}\right. \\
& \left.+\left[\frac{8 G^{3} m_{1}^{2} m_{2}}{5 r_{12}^{4}}-\frac{32 G^{3} m_{1} m_{2}^{2}}{5 r_{12}^{4}}-\frac{4 G^{2} m_{1} m_{2}}{5 r_{12}^{3}} v_{12}^{2}\right] \boldsymbol{v}_{12}\right\} \\
& +\frac{1}{c^{6}}\left\{\left[\frac { G m _ { 2 } } { r _ { 1 2 } ^ { 2 } } \left(\frac{35}{16}\left(n_{12} v_{2}\right)^{6}-\frac{15}{8}\left(n_{12} v_{2}\right)^{4} v_{1}^{2}+\frac{15}{2}\left(n_{12} v_{2}\right)^{4}\left(v_{1} v_{2}\right)+3\left(n_{12} v_{2}\right)^{2}\left(v_{1} v_{2}\right)^{2}\right.\right.\right. \\
& -\frac{15}{2}\left(n_{12} v_{2}\right)^{4} v_{2}^{2}+\frac{3}{2}\left(n_{12} v_{2}\right)^{2} v_{1}^{2} v_{2}^{2}-12\left(n_{12} v_{2}\right)^{2}\left(v_{1} v_{2}\right) v_{2}^{2}-2\left(v_{1} v_{2}\right)^{2} v_{2}^{2} \\
& \left.+\frac{15}{2}\left(n_{12} v_{2}\right)^{2} v_{2}^{4}+4\left(v_{1} v_{2}\right) v_{2}^{4}-2 v_{2}^{6}\right) \\
& +\frac{G^{2} m_{1} m_{2}}{r_{12}^{3}}\left(-\frac{171}{8}\left(n_{12} v_{1}\right)^{4}+\frac{171}{2}\left(n_{12} v_{1}\right)^{3}\left(n_{12} v_{2}\right)-\frac{723}{4}\left(n_{12} v_{1}\right)^{2}\left(n_{12} v_{2}\right)^{2}\right. \\
& +\frac{383}{2}\left(n_{12} v_{1}\right)\left(n_{12} v_{2}\right)^{3}-\frac{455}{8}\left(n_{12} v_{2}\right)^{4}+\frac{229}{4}\left(n_{12} v_{1}\right)^{2} v_{1}^{2} \\
& -\frac{205}{2}\left(n_{12} v_{1}\right)\left(n_{12} v_{2}\right) v_{1}^{2}+\frac{191}{4}\left(n_{12} v_{2}\right)^{2} v_{1}^{2}-\frac{91}{8} v_{1}^{4}-\frac{229}{2}\left(n_{12} v_{1}\right)^{2}\left(v_{1} v_{2}\right) \\
& +244\left(n_{12} v_{1}\right)\left(n_{12} v_{2}\right)\left(v_{1} v_{2}\right)-\frac{225}{2}\left(n_{12} v_{2}\right)^{2}\left(v_{1} v_{2}\right)+\frac{91}{2} v_{1}^{2}\left(v_{1} v_{2}\right) \\
& -\frac{177}{4}\left(v_{1} v_{2}\right)^{2}+\frac{229}{4}\left(n_{12} v_{1}\right)^{2} v_{2}^{2}-\frac{283}{2}\left(n_{12} v_{1}\right)\left(n_{12} v_{2}\right) v_{2}^{2} \\
& \left.+\frac{259}{4}\left(n_{12} v_{2}\right)^{2} v_{2}^{2}-\frac{91}{4} v_{1}^{2} v_{2}^{2}+43\left(v_{1} v_{2}\right) v_{2}^{2}-\frac{81}{8} v_{2}^{4}\right) \\
& +\frac{G^{2} m_{2}^{2}}{r_{12}^{3}}\left(-6\left(n_{12} v_{1}\right)^{2}\left(n_{12} v_{2}\right)^{2}+12\left(n_{12} v_{1}\right)\left(n_{12} v_{2}\right)^{3}+6\left(n_{12} v_{2}\right)^{4}\right. \\
& +4\left(n_{12} v_{1}\right)\left(n_{12} v_{2}\right)\left(v_{1} v_{2}\right)+12\left(n_{12} v_{2}\right)^{2}\left(v_{1} v_{2}\right)+4\left(v_{1} v_{2}\right)^{2} \\
& \left.-4\left(n_{12} v_{1}\right)\left(n_{12} v_{2}\right) v_{2}^{2}-12\left(n_{12} v_{2}\right)^{2} v_{2}^{2}-8\left(v_{1} v_{2}\right) v_{2}^{2}+4 v_{2}^{4}\right) \\
& +\frac{G^{3} m_{2}^{3}}{r_{12}^{4}}\left(-\left(n_{12} v_{1}\right)^{2}+2\left(n_{12} v_{1}\right)\left(n_{12} v_{2}\right)+\frac{43}{2}\left(n_{12} v_{2}\right)^{2}+18\left(v_{1} v_{2}\right)-9 v_{2}^{2}\right) \\
& +\frac{G^{3} m_{1} m_{2}^{2}}{r_{12}^{4}}\left(\frac{415}{8}\left(n_{12} v_{1}\right)^{2}-\frac{375}{4}\left(n_{12} v_{1}\right)\left(n_{12} v_{2}\right)+\frac{1113}{8}\left(n_{12} v_{2}\right)^{2}-\frac{615}{64}\left(n_{12} v_{12}\right)^{2} \pi^{2}\right. \\
& \left.+18 v_{1}^{2}+\frac{123}{64} \pi^{2} v_{12}^{2}+33\left(v_{1} v_{2}\right)-\frac{33}{2} v_{2}^{2}\right) \\
& +\frac{G^{3} m_{1}^{2} m_{2}}{r_{12}^{4}}\left(-\frac{45887}{168}\left(n_{12} v_{1}\right)^{2}+\frac{24025}{42}\left(n_{12} v_{1}\right)\left(n_{12} v_{2}\right)-\frac{10469}{42}\left(n_{12} v_{2}\right)^{2}+\frac{48197}{840} v_{1}^{2}\right. \\
& \left.-\frac{36227}{420}\left(v_{1} v_{2}\right)+\frac{36227}{840} v_{2}^{2}+110\left(n_{12} v_{12}\right)^{2} \ln \left(\frac{r_{12}}{r_{1}^{\prime}}\right)-22 v_{12}^{2} \ln \left(\frac{r_{12}}{r_{1}^{\prime}}\right)\right) \\
& +\frac{16 G^{4} m_{2}^{4}}{r_{12}^{5}}+\frac{G^{4} m_{1}^{2} m_{2}^{2}}{r_{12}^{5}}\left(175-\frac{41}{16} \pi^{2}\right)+\frac{G^{4} m_{1}^{3} m_{2}}{r_{12}^{5}}\left(-\frac{3187}{1260}+\frac{44}{3} \ln \left(\frac{r_{12}}{r_{1}^{\prime}}\right)\right) \\
& \left.+\frac{G^{4} m_{1} m_{2}^{3}}{r_{12}^{5}}\left(\frac{110741}{630}-\frac{41}{16} \pi^{2}-\frac{44}{3} \ln \left(\frac{r_{12}}{r_{2}^{\prime}}\right)\right)\right] \boldsymbol{n}_{12} \\
& +\left[\frac { G m _ { 2 } } { r _ { 1 2 } ^ { 2 } } \left(\frac{15}{2}\left(n_{12} v_{1}\right)\left(n_{12} v_{2}\right)^{4}-\frac{45}{8}\left(n_{12} v_{2}\right)^{5}-\frac{3}{2}\left(n_{12} v_{2}\right)^{3} v_{1}^{2}+6\left(n_{12} v_{1}\right)\left(n_{12} v_{2}\right)^{2}\left(v_{1} v_{2}\right)\right.\right.
\end{aligned}
$$




$$
\begin{aligned}
& -6\left(n_{12} v_{2}\right)^{3}\left(v_{1} v_{2}\right)-2\left(n_{12} v_{2}\right)\left(v_{1} v_{2}\right)^{2}-12\left(n_{12} v_{1}\right)\left(n_{12} v_{2}\right)^{2} v_{2}^{2}+12\left(n_{12} v_{2}\right)^{3} v_{2}^{2} \\
& +\left(n_{12} v_{2}\right) v_{1}^{2} v_{2}^{2}-4\left(n_{12} v_{1}\right)\left(v_{1} v_{2}\right) v_{2}^{2}+8\left(n_{12} v_{2}\right)\left(v_{1} v_{2}\right) v_{2}^{2}+4\left(n_{12} v_{1}\right) v_{2}^{4} \\
& \left.-7\left(n_{12} v_{2}\right) v_{2}^{4}\right) \\
& +\frac{G^{2} m_{2}^{2}}{r_{12}^{3}}\left(-2\left(n_{12} v_{1}\right)^{2}\left(n_{12} v_{2}\right)+8\left(n_{12} v_{1}\right)\left(n_{12} v_{2}\right)^{2}+2\left(n_{12} v_{2}\right)^{3}+2\left(n_{12} v_{1}\right)\left(v_{1} v_{2}\right)\right. \\
& \left.+4\left(n_{12} v_{2}\right)\left(v_{1} v_{2}\right)-2\left(n_{12} v_{1}\right) v_{2}^{2}-4\left(n_{12} v_{2}\right) v_{2}^{2}\right) \\
& +\frac{G^{2} m_{1} m_{2}}{r_{12}^{3}}\left(-\frac{243}{4}\left(n_{12} v_{1}\right)^{3}+\frac{565}{4}\left(n_{12} v_{1}\right)^{2}\left(n_{12} v_{2}\right)-\frac{269}{4}\left(n_{12} v_{1}\right)\left(n_{12} v_{2}\right)^{2}\right. \\
& -\frac{95}{12}\left(n_{12} v_{2}\right)^{3}+\frac{207}{8}\left(n_{12} v_{1}\right) v_{1}^{2}-\frac{137}{8}\left(n_{12} v_{2}\right) v_{1}^{2}-36\left(n_{12} v_{1}\right)\left(v_{1} v_{2}\right) \\
& \left.+\frac{27}{4}\left(n_{12} v_{2}\right)\left(v_{1} v_{2}\right)+\frac{81}{8}\left(n_{12} v_{1}\right) v_{2}^{2}+\frac{83}{8}\left(n_{12} v_{2}\right) v_{2}^{2}\right) \\
& +\frac{G^{3} m_{2}^{3}}{r_{12}^{4}}\left(4\left(n_{12} v_{1}\right)+5\left(n_{12} v_{2}\right)\right) \\
& +\frac{G^{3} m_{1} m_{2}^{2}}{r_{12}^{4}}\left(-\frac{307}{8}\left(n_{12} v_{1}\right)+\frac{479}{8}\left(n_{12} v_{2}\right)+\frac{123}{32}\left(n_{12} v_{12}\right) \pi^{2}\right) \\
& \left.\left.+\frac{G^{3} m_{1}^{2} m_{2}}{r_{12}^{4}}\left(\frac{31397}{420}\left(n_{12} v_{1}\right)-\frac{36227}{420}\left(n_{12} v_{2}\right)-44\left(n_{12} v_{12}\right) \ln \left(\frac{r_{12}}{r_{1}^{\prime}}\right)\right)\right] \boldsymbol{v}_{12}\right\} \\
& +\frac{1}{c^{7}}\left\{\left[\frac{G^{4} m_{1}^{3} m_{2}}{r_{12}^{5}}\left(\frac{3992}{105}\left(n_{12} v_{1}\right)-\frac{4328}{105}\left(n_{12} v_{2}\right)\right)\right.\right. \\
& +\frac{G^{4} m_{1}^{2} m_{2}^{2}}{r_{12}^{6}}\left(-\frac{13576}{105}\left(n_{12} v_{1}\right)+\frac{2872}{21}\left(n_{12} v_{2}\right)\right)-\frac{3172}{21} \frac{G^{4} m_{1} m_{2}^{3}}{r_{12}^{6}}\left(n_{12} v_{12}\right) \\
& +\frac{G^{3} m_{1}^{2} m_{2}}{r_{12}^{4}}\left(48\left(n_{12} v_{1}\right)^{3}-\frac{696}{5}\left(n_{12} v_{1}\right)^{2}\left(n_{12} v_{2}\right)+\frac{744}{5}\left(n_{12} v_{1}\right)\left(n_{12} v_{2}\right)^{2}-\frac{288}{5}\left(n_{12} v_{2}\right)^{3}\right. \\
& -\frac{4888}{105}\left(n_{12} v_{1}\right) v_{1}^{2}+\frac{5056}{105}\left(n_{12} v_{2}\right) v_{1}^{2}+\frac{2056}{21}\left(n_{12} v_{1}\right)\left(v_{1} v_{2}\right) \\
& \left.-\frac{2224}{21}\left(n_{12} v_{2}\right)\left(v_{1} v_{2}\right)-\frac{1028}{21}\left(n_{12} v_{1}\right) v_{2}^{2}+\frac{5812}{105}\left(n_{12} v_{2}\right) v_{2}^{2}\right) \\
& +\frac{G^{3} m_{1} m_{2}^{2}}{r_{12}^{4}}\left(-\frac{582}{5}\left(n_{12} v_{1}\right)^{3}+\frac{1746}{5}\left(n_{12} v_{1}\right)^{2}\left(n_{12} v_{2}\right)-\frac{1954}{5}\left(n_{12} v_{1}\right)\left(n_{12} v_{2}\right)^{2}\right. \\
& +158\left(n_{12} v_{2}\right)^{3}+\frac{3568}{105}\left(n_{12} v_{12}\right) v_{1}^{2}-\frac{2864}{35}\left(n_{12} v_{1}\right)\left(v_{1} v_{2}\right) \\
& \left.+\frac{10048}{105}\left(n_{12} v_{2}\right)\left(v_{1} v_{2}\right)+\frac{1432}{35}\left(n_{12} v_{1}\right) v_{2}^{2}-\frac{5752}{105}\left(n_{12} v_{2}\right) v_{2}^{2}\right) \\
& +\frac{G^{2} m_{1} m_{2}}{r_{12}^{3}}\left(-56\left(n_{12} v_{12}\right)^{5}+60\left(n_{12} v_{1}\right)^{3} v_{12}^{2}-180\left(n_{12} v_{1}\right)^{2}\left(n_{12} v_{2}\right) v_{12}^{2}\right. \\
& +174\left(n_{12} v_{1}\right)\left(n_{12} v_{2}\right)^{2} v_{12}^{2}-54\left(n_{12} v_{2}\right)^{3} v_{12}^{2}-\frac{246}{35}\left(n_{12} v_{12}\right) v_{1}^{4} \\
& +\frac{1068}{35}\left(n_{12} v_{1}\right) v_{1}^{2}\left(v_{1} v_{2}\right)-\frac{984}{35}\left(n_{12} v_{2}\right) v_{1}^{2}\left(v_{1} v_{2}\right)-\frac{1068}{35}\left(n_{12} v_{1}\right)\left(v_{1} v_{2}\right)^{2}
\end{aligned}
$$




$$
\begin{aligned}
&+ \frac{180}{7}\left(n_{12} v_{2}\right)\left(v_{1} v_{2}\right)^{2}-\frac{534}{35}\left(n_{12} v_{1}\right) v_{1}^{2} v_{2}^{2}+\frac{90}{7}\left(n_{12} v_{2}\right) v_{1}^{2} v_{2}^{2} \\
&+ \frac{984}{35}\left(n_{12} v_{1}\right)\left(v_{1} v_{2}\right) v_{2}^{2}-\frac{732}{35}\left(n_{12} v_{2}\right)\left(v_{1} v_{2}\right) v_{2}^{2}-\frac{204}{35}\left(n_{12} v_{1}\right) v_{2}^{4} \\
&+\left.\left.\frac{24}{7}\left(n_{12} v_{2}\right) v_{2}^{4}\right)\right] \boldsymbol{n}_{12} \\
&+\left[-\frac{184}{21} \frac{G^{4} m_{1}^{3} m_{2}}{r_{12}^{5}}+\frac{6224}{105} \frac{G^{4} m_{1}^{2} m_{2}^{2}}{r_{12}^{6}}+\frac{6388}{105} \frac{G^{4} m_{1} m_{2}^{3}}{r_{12}^{6}}\right. \\
&+\frac{G^{3} m_{1}^{2} m_{2}}{r_{12}^{4}}\left(\frac{52}{15}\left(n_{12} v_{1}\right)^{2}-\frac{56}{15}\left(n_{12} v_{1}\right)\left(n_{12} v_{2}\right)-\frac{44}{15}\left(n_{12} v_{2}\right)^{2}-\frac{132}{35} v_{1}^{2}+\frac{152}{35}\left(v_{1} v_{2}\right)\right. \\
&\left.\quad-\frac{48}{35} v_{2}^{2}\right) \\
&+\frac{G^{3} m_{1} m_{2}^{2}}{r_{12}^{4}}\left(\frac{454}{15}\left(n_{12} v_{1}\right)^{2}-\frac{372}{5}\left(n_{12} v_{1}\right)\left(n_{12} v_{2}\right)+\frac{854}{15}\left(n_{12} v_{2}\right)^{2}-\frac{152}{21} v_{1}^{2}\right. \\
&\left.+\frac{2864}{105}\left(v_{1} v_{2}\right)-\frac{1768}{105} v_{2}^{2}\right) \\
&+\frac{G^{2} m_{1} m_{2}}{r_{12}^{3}}\left(60\left(n_{12} v_{12}\right)^{4}-\frac{348}{5}\left(n_{12} v_{1}\right)^{2} v_{12}^{2}+\frac{684}{5}\left(n_{12} v_{1}\right)\left(n_{12} v_{2}\right) v_{12}^{2}\right. \\
&-66\left(n_{12} v_{2}\right)^{2} v_{12}^{2}+\frac{334}{35} v_{1}^{4}-\frac{1336}{35} v_{1}^{2}\left(v_{1} v_{2}\right)+\frac{1308}{35}\left(v_{1} v_{2}\right)^{2}+\frac{654}{35} v_{1}^{2} v_{2}^{2} \\
&\left.\left.\left.-\frac{1252}{35}\left(v_{1} v_{2}\right) v_{2}^{2}+\frac{292}{35} v_{2}^{4}\right)\right] \boldsymbol{v}_{12}\right\}+\mathcal{O}\left(\frac{1}{c^{8}}\right) \cdot
\end{aligned}
$$

The $2.5 \mathrm{PN}$ and $3.5 \mathrm{PN}$ terms are associated with gravitational radiation reaction. ${ }^{49}$ The $3 \mathrm{PN}$ harmonic-coordinates equations of motion depend on two arbitrary length scales $r_{1}^{\prime}$ and $r_{2}^{\prime}$ associated with the logarithms present at the 3PN order. It has been proved in Ref. [71] that $r_{1}^{\prime}$ and $r_{2}^{\prime}$ are merely linked with the choice of coordinates - we can refer to $r_{1}^{\prime}$ and $r_{2}^{\prime}$ as "gauge constants". In our approach $[69,71]$, the harmonic coordinate system is not uniquely fixed by the coordinate condition $\partial_{\mu} h^{\alpha \mu}=0$. In fact there are infinitely many "locally-defined" harmonic coordinate systems. For general smooth matter sources, as in the general formalism of Part A, we expect the existence and uniqueness of a global harmonic coordinate system. But here we have some pointparticles, with delta-function singularities, and in this case we do not have the notion of a global coordinate system. We can always change the harmonic coordinates by means of the gauge vector $\eta^{\alpha}=\delta x^{\alpha}$, satisfying $\Delta \eta^{\alpha}=0$ except at the location of the two particles (we assume that the transformation is at the $3 \mathrm{PN}$ level, so we can consider simply a flat-space Laplace equation). More precisely, we can show that the logarithms appearing in Eq. (203), together with the constants $r_{1}^{\prime}$ and $r_{2}^{\prime}$ therein, can be removed by the coordinate transformation associated with the 3PN gauge vector (with $r_{1}=\left|\mathbf{x}-\boldsymbol{y}_{1}(t)\right|$ and $r_{2}=\left|\mathbf{x}-\boldsymbol{y}_{2}(t)\right|$; and $\partial^{\alpha}=\eta^{\alpha \mu} \partial_{\mu}$ ):

$$
\eta^{\alpha}=-\frac{22}{3} \frac{G^{2} m_{1} m_{2}}{c^{6}} \partial^{\alpha}\left[\frac{G m_{1}}{r_{2}} \ln \left(\frac{r_{12}}{r_{1}^{\prime}}\right)+\frac{G m_{2}}{r_{1}} \ln \left(\frac{r_{12}}{r_{2}^{\prime}}\right)\right] .
$$

Therefore, the arbitrariness in the choice of the constants $r_{1}^{\prime}$ and $r_{2}^{\prime}$ is innocuous on the physical

49 Notice the dependence upon the irrational number $\pi^{2}$. Technically, the $\pi^{2}$ terms arise from non-linear interactions involving some integrals such as

$$
\frac{1}{\pi} \int \frac{\mathrm{d}^{3} \mathbf{x}}{r_{1}^{2} r_{2}^{2}}=\frac{\pi^{2}}{r_{12}} .
$$


point of view, because the physical results must be gauge invariant. Indeed we shall verify that $r_{1}^{\prime}$ and $r_{2}^{\prime}$ cancel out in our final results.

When retaining the "even" relativistic corrections at the $1 \mathrm{PN}, 2 \mathrm{PN}$ and $3 \mathrm{PN}$ orders, and neglecting the "odd" radiation reaction terms at the $2.5 \mathrm{PN}$ and $3.5 \mathrm{PN}$ orders, we find that the equations of motion admit a conserved energy (and a Lagrangian, as we shall see); that energy can be straightforwardly obtained by guess-work starting from Eq. (203), with the result

$$
\begin{aligned}
& E=\frac{m_{1} v_{1}^{2}}{2}-\frac{G m_{1} m_{2}}{2 r_{12}} \\
& +\frac{1}{c^{2}}\left\{\frac{G^{2} m_{1}^{2} m_{2}}{2 r_{12}^{2}}+\frac{3 m_{1} v_{1}^{4}}{8}+\frac{G m_{1} m_{2}}{r_{12}}\left(-\frac{1}{4}\left(n_{12} v_{1}\right)\left(n_{12} v_{2}\right)+\frac{3}{2} v_{1}^{2}-\frac{7}{4}\left(v_{1} v_{2}\right)\right)\right\} \\
& +\frac{1}{c^{4}}\left\{-\frac{G^{3} m_{1}^{3} m_{2}}{2 r_{12}^{3}}-\frac{19 G^{3} m_{1}^{2} m_{2}^{2}}{8 r_{12}^{3}}+\frac{5 m_{1} v_{1}^{6}}{16}\right. \\
& +\frac{G m_{1} m_{2}}{r_{12}}\left(\frac{3}{8}\left(n_{12} v_{1}\right)^{3}\left(n_{12} v_{2}\right)+\frac{3}{16}\left(n_{12} v_{1}\right)^{2}\left(n_{12} v_{2}\right)^{2}-\frac{9}{8}\left(n_{12} v_{1}\right)\left(n_{12} v_{2}\right) v_{1}^{2}\right. \\
& -\frac{13}{8}\left(n_{12} v_{2}\right)^{2} v_{1}^{2}+\frac{21}{8} v_{1}^{4}+\frac{13}{8}\left(n_{12} v_{1}\right)^{2}\left(v_{1} v_{2}\right)+\frac{3}{4}\left(n_{12} v_{1}\right)\left(n_{12} v_{2}\right)\left(v_{1} v_{2}\right) \\
& \left.-\frac{55}{8} v_{1}^{2}\left(v_{1} v_{2}\right)+\frac{17}{8}\left(v_{1} v_{2}\right)^{2}+\frac{31}{16} v_{1}^{2} v_{2}^{2}\right) \\
& \left.+\frac{G^{2} m_{1}^{2} m_{2}}{r_{12}^{2}}\left(\frac{29}{4}\left(n_{12} v_{1}\right)^{2}-\frac{13}{4}\left(n_{12} v_{1}\right)\left(n_{12} v_{2}\right)+\frac{1}{2}\left(n_{12} v_{2}\right)^{2}-\frac{3}{2} v_{1}^{2}+\frac{7}{4} v_{2}^{2}\right)\right\} \\
& +\frac{1}{c^{6}}\left\{\frac{35 m_{1} v_{1}^{8}}{128}\right. \\
& +\frac{G m_{1} m_{2}}{r_{12}}\left(-\frac{5}{16}\left(n_{12} v_{1}\right)^{5}\left(n_{12} v_{2}\right)-\frac{5}{16}\left(n_{12} v_{1}\right)^{4}\left(n_{12} v_{2}\right)^{2}-\frac{5}{32}\left(n_{12} v_{1}\right)^{3}\left(n_{12} v_{2}\right)^{3}\right. \\
& +\frac{19}{16}\left(n_{12} v_{1}\right)^{3}\left(n_{12} v_{2}\right) v_{1}^{2}+\frac{15}{16}\left(n_{12} v_{1}\right)^{2}\left(n_{12} v_{2}\right)^{2} v_{1}^{2}+\frac{3}{4}\left(n_{12} v_{1}\right)\left(n_{12} v_{2}\right)^{3} v_{1}^{2} \\
& +\frac{19}{16}\left(n_{12} v_{2}\right)^{4} v_{1}^{2}-\frac{21}{16}\left(n_{12} v_{1}\right)\left(n_{12} v_{2}\right) v_{1}^{4}-2\left(n_{12} v_{2}\right)^{2} v_{1}^{4} \\
& +\frac{55}{16} v_{1}^{6}-\frac{19}{16}\left(n_{12} v_{1}\right)^{4}\left(v_{1} v_{2}\right)-\left(n_{12} v_{1}\right)^{3}\left(n_{12} v_{2}\right)\left(v_{1} v_{2}\right) \\
& -\frac{15}{32}\left(n_{12} v_{1}\right)^{2}\left(n_{12} v_{2}\right)^{2}\left(v_{1} v_{2}\right)+\frac{45}{16}\left(n_{12} v_{1}\right)^{2} v_{1}^{2}\left(v_{1} v_{2}\right) \\
& +\frac{5}{4}\left(n_{12} v_{1}\right)\left(n_{12} v_{2}\right) v_{1}^{2}\left(v_{1} v_{2}\right)+\frac{11}{4}\left(n_{12} v_{2}\right)^{2} v_{1}^{2}\left(v_{1} v_{2}\right)-\frac{139}{16} v_{1}^{4}\left(v_{1} v_{2}\right) \\
& -\frac{3}{4}\left(n_{12} v_{1}\right)^{2}\left(v_{1} v_{2}\right)^{2}+\frac{5}{16}\left(n_{12} v_{1}\right)\left(n_{12} v_{2}\right)\left(v_{1} v_{2}\right)^{2}+\frac{41}{8} v_{1}^{2}\left(v_{1} v_{2}\right)^{2}+\frac{1}{16}\left(v_{1} v_{2}\right)^{3} \\
& \left.-\frac{45}{16}\left(n_{12} v_{1}\right)^{2} v_{1}^{2} v_{2}^{2}-\frac{23}{32}\left(n_{12} v_{1}\right)\left(n_{12} v_{2}\right) v_{1}^{2} v_{2}^{2}+\frac{79}{16} v_{1}^{4} v_{2}^{2}-\frac{161}{32} v_{1}^{2}\left(v_{1} v_{2}\right) v_{2}^{2}\right) \\
& +\frac{G^{2} m_{1}^{2} m_{2}}{r_{12}^{2}}\left(-\frac{49}{8}\left(n_{12} v_{1}\right)^{4}+\frac{75}{8}\left(n_{12} v_{1}\right)^{3}\left(n_{12} v_{2}\right)-\frac{187}{8}\left(n_{12} v_{1}\right)^{2}\left(n_{12} v_{2}\right)^{2}\right. \\
& +\frac{247}{24}\left(n_{12} v_{1}\right)\left(n_{12} v_{2}\right)^{3}+\frac{49}{8}\left(n_{12} v_{1}\right)^{2} v_{1}^{2}+\frac{81}{8}\left(n_{12} v_{1}\right)\left(n_{12} v_{2}\right) v_{1}^{2} \\
& -\frac{21}{4}\left(n_{12} v_{2}\right)^{2} v_{1}^{2}+\frac{11}{2} v_{1}^{4}-\frac{15}{2}\left(n_{12} v_{1}\right)^{2}\left(v_{1} v_{2}\right)-\frac{3}{2}\left(n_{12} v_{1}\right)\left(n_{12} v_{2}\right)\left(v_{1} v_{2}\right)
\end{aligned}
$$




$$
\left.\left.\begin{array}{rl} 
& +\frac{21}{4}\left(n_{12} v_{2}\right)^{2}\left(v_{1} v_{2}\right)-27 v_{1}^{2}\left(v_{1} v_{2}\right)+\frac{55}{2}\left(v_{1} v_{2}\right)^{2}+\frac{49}{4}\left(n_{12} v_{1}\right)^{2} v_{2}^{2} \\
& \left.-\frac{27}{2}\left(n_{12} v_{1}\right)\left(n_{12} v_{2}\right) v_{2}^{2}+\frac{3}{4}\left(n_{12} v_{2}\right)^{2} v_{2}^{2}+\frac{55}{4} v_{1}^{2} v_{2}^{2}-28\left(v_{1} v_{2}\right) v_{2}^{2}+\frac{135}{16} v_{2}^{4}\right) \\
+ & \frac{3 G^{4} m_{1}^{4} m_{2}}{8 r_{12}^{4}}+\frac{G^{4} m_{1}^{3} m_{2}^{2}}{r_{12}^{4}}\left(\frac{9707}{420}-\frac{22}{3} \ln \left(\frac{r_{12}}{r_{1}^{\prime}}\right)\right) \\
+ & \frac{G^{3} m_{1}^{2} m_{2}^{2}}{r_{12}^{3}}\left(\frac{547}{12}\left(n_{12} v_{1}\right)^{2}-\frac{3115}{48}\left(n_{12} v_{1}\right)\left(n_{12} v_{2}\right)-\frac{123}{64}\left(n_{12} v_{1}\right)\left(n_{12} v_{12}\right) \pi^{2}-\frac{575}{18} v_{1}^{2}\right. \\
& \left.+\frac{41}{64} \pi^{2}\left(v_{1} v_{12}\right)+\frac{4429}{144}\left(v_{1} v_{2}\right)\right) \\
+ & \frac{G^{3} m_{1}^{3} m_{2}}{r_{12}^{3}}\left(-\frac{44627}{840}\left(n_{12} v_{1}\right)^{2}+\frac{32027}{840}\left(n_{12} v_{1}\right)\left(n_{12} v_{2}\right)+\frac{3}{2}\left(n_{12} v_{2}\right)^{2}+\frac{24187}{2520} v_{1}^{2}\right. \\
& \left.-\frac{27967}{2520}\left(v_{1} v_{2}\right)+\frac{5}{4} v_{2}^{2}+22\left(n_{12} v_{1}\right)\left(n_{12} v_{12}\right) \ln \left(\frac{r_{12}}{r_{1}^{\prime}}\right)-\frac{22}{3}\left(v_{1} v_{12}\right) \ln \left(\frac{r_{12}}{r_{1}^{\prime}}\right)\right)
\end{array}\right\}\right)
$$

To the terms given above, we must add the same terms but corresponding to the relabelling $1 \leftrightarrow 2$. Actually, this energy is not conserved because of the radiation reaction. Thus its time derivative, as computed by means of the 3PN equations of motion themselves (i.e., by order-reducing all the accelerations), is purely equal to the $2.5 \mathrm{PN}$ effect,

$$
\begin{aligned}
\frac{\mathrm{d} E}{\mathrm{~d} t} & =\frac{4}{5} \frac{G^{2} m_{1}^{2} m_{2}}{c^{5} r_{12}^{3}}\left[\left(v_{1} v_{12}\right)\left(-v_{12}^{2}+2 \frac{G m_{1}}{r_{12}}-8 \frac{G m_{2}}{r_{12}}\right)+\left(n_{12} v_{1}\right)\left(n_{12} v_{12}\right)\left(3 v_{12}^{2}-6 \frac{G m_{1}}{r_{12}}+\frac{52}{3} \frac{G m_{2}}{r_{12}}\right)\right] \\
& +1 \leftrightarrow 2+\mathcal{O}\left(\frac{1}{c^{7}}\right) .
\end{aligned}
$$

The resulting energy balance equation can be better expressed by transfering to the left-hand side certain $2.5 \mathrm{PN}$ terms so that we recognize in the right-hand side the familiar form of a total energy flux. Posing

$$
E^{2.5 \mathrm{PN}}=E+\frac{4 G^{2} m_{1}^{2} m_{2}}{5 c^{5} r_{12}^{2}}\left(n_{12} v_{1}\right)\left[v_{12}^{2}-\frac{2 G\left(m_{1}-m_{2}\right)}{r_{12}}\right]+1 \leftrightarrow 2
$$

we find agreement with the standard Einstein quadrupole formula (4):

$$
\frac{\mathrm{d} E^{2.5 \mathrm{PN}}}{\mathrm{d} t}=-\frac{G}{5 c^{5}} \frac{\mathrm{d}^{3} \mathrm{Q}_{i j}}{\mathrm{~d} t^{3}} \frac{\mathrm{d}^{3} \mathrm{Q}_{i j}}{\mathrm{~d} t^{3}}+\mathcal{O}\left(\frac{1}{c^{7}}\right),
$$

where the Newtonian trace-free quadrupole moment reads $\mathrm{Q}_{i j}=m_{1}\left(y_{1}^{i} y_{1}^{j}-\frac{1}{3} \delta^{i j} \boldsymbol{y}_{1}^{2}\right)+1 \leftrightarrow 2$. We refer to $[258,259]$ for the discussion of the energy balance equation up to the next $3.5 \mathrm{PN}$ order. See also Eq. (158) for the energy balance equation at relative 1.5PN order for general fluid systems.

\subsection{Lagrangian and Hamiltonian formulations}

The conservative part of the equations of motion in harmonic coordinates (203) is derivable from a generalized Lagrangian, depending not only on the positions and velocities of the bodies, but also on their accelerations: $\boldsymbol{a}_{1}=\mathrm{d} \boldsymbol{v}_{1} / \mathrm{d} t$ and $\boldsymbol{a}_{2}=\mathrm{d} \boldsymbol{v}_{2} / \mathrm{d} t$. As shown in Ref. [147], the accelerations in the harmonic-coordinates Lagrangian occur already from the $2 \mathrm{PN}$ order. This fact is in 
accordance with a general result [308] that $N$-body equations of motion cannot be derived from an ordinary Lagrangian beyond the $1 \mathrm{PN}$ level, provided that the gauge conditions preserve the manifest Lorentz invariance. Note that we can always arrange for the dependence of the Lagrangian upon the accelerations to be linear, at the price of adding some so-called "multi-zero" terms to the Lagrangian, which do not modify the equations of motion (see, e.g., Ref. [169]). At the 3PN level, we find that the Lagrangian also depends on accelerations. It is notable that these accelerations are sufficient - there is no need to include derivatives of accelerations. Note also that the Lagrangian is not unique because we can always add to it a total time derivative $\mathrm{d} F / \mathrm{d} t$, where $F$ is any function depending on the positions and velocities, without changing the dynamics. We find [174]

$$
\begin{aligned}
& L^{\text {harm }}=\frac{G m_{1} m_{2}}{2 r_{12}}+\frac{m_{1} v_{1}^{2}}{2} \\
& +\frac{1}{c^{2}}\left\{-\frac{G^{2} m_{1}^{2} m_{2}}{2 r_{12}^{2}}+\frac{m_{1} v_{1}^{4}}{8}+\frac{G m_{1} m_{2}}{r_{12}}\left(-\frac{1}{4}\left(n_{12} v_{1}\right)\left(n_{12} v_{2}\right)+\frac{3}{2} v_{1}^{2}-\frac{7}{4}\left(v_{1} v_{2}\right)\right)\right\} \\
& +\frac{1}{c^{4}}\left\{\frac{G^{3} m_{1}^{3} m_{2}}{2 r_{12}^{3}}+\frac{19 G^{3} m_{1}^{2} m_{2}^{2}}{8 r_{12}^{3}}\right. \\
& +\frac{G^{2} m_{1}^{2} m_{2}}{r_{12}^{2}}\left(\frac{7}{2}\left(n_{12} v_{1}\right)^{2}-\frac{7}{2}\left(n_{12} v_{1}\right)\left(n_{12} v_{2}\right)+\frac{1}{2}\left(n_{12} v_{2}\right)^{2}+\frac{1}{4} v_{1}^{2}-\frac{7}{4}\left(v_{1} v_{2}\right)+\frac{7}{4} v_{2}^{2}\right) \\
& +\frac{G m_{1} m_{2}}{r_{12}}\left(\frac{3}{16}\left(n_{12} v_{1}\right)^{2}\left(n_{12} v_{2}\right)^{2}-\frac{7}{8}\left(n_{12} v_{2}\right)^{2} v_{1}^{2}+\frac{7}{8} v_{1}^{4}+\frac{3}{4}\left(n_{12} v_{1}\right)\left(n_{12} v_{2}\right)\left(v_{1} v_{2}\right)\right. \\
& \left.-2 v_{1}^{2}\left(v_{1} v_{2}\right)+\frac{1}{8}\left(v_{1} v_{2}\right)^{2}+\frac{15}{16} v_{1}^{2} v_{2}^{2}\right)+\frac{m_{1} v_{1}^{6}}{16} \\
& \left.+G m_{1} m_{2}\left(-\frac{7}{4}\left(a_{1} v_{2}\right)\left(n_{12} v_{2}\right)-\frac{1}{8}\left(n_{12} a_{1}\right)\left(n_{12} v_{2}\right)^{2}+\frac{7}{8}\left(n_{12} a_{1}\right) v_{2}^{2}\right)\right\} \\
& +\frac{1}{c^{6}}\left\{\frac { G ^ { 2 } m _ { 1 } ^ { 2 } m _ { 2 } } { r _ { 1 2 } ^ { 2 } } \left(\frac{13}{18}\left(n_{12} v_{1}\right)^{4}+\frac{83}{18}\left(n_{12} v_{1}\right)^{3}\left(n_{12} v_{2}\right)-\frac{35}{6}\left(n_{12} v_{1}\right)^{2}\left(n_{12} v_{2}\right)^{2}-\frac{245}{24}\left(n_{12} v_{1}\right)^{2} v_{1}^{2}\right.\right. \\
& +\frac{179}{12}\left(n_{12} v_{1}\right)\left(n_{12} v_{2}\right) v_{1}^{2}-\frac{235}{24}\left(n_{12} v_{2}\right)^{2} v_{1}^{2}+\frac{373}{48} v_{1}^{4}+\frac{529}{24}\left(n_{12} v_{1}\right)^{2}\left(v_{1} v_{2}\right) \\
& -\frac{97}{6}\left(n_{12} v_{1}\right)\left(n_{12} v_{2}\right)\left(v_{1} v_{2}\right)-\frac{719}{24} v_{1}^{2}\left(v_{1} v_{2}\right)+\frac{463}{24}\left(v_{1} v_{2}\right)^{2}-\frac{7}{24}\left(n_{12} v_{1}\right)^{2} v_{2}^{2} \\
& \left.-\frac{1}{2}\left(n_{12} v_{1}\right)\left(n_{12} v_{2}\right) v_{2}^{2}+\frac{1}{4}\left(n_{12} v_{2}\right)^{2} v_{2}^{2}+\frac{463}{48} v_{1}^{2} v_{2}^{2}-\frac{19}{2}\left(v_{1} v_{2}\right) v_{2}^{2}+\frac{45}{16} v_{2}^{4}\right) \\
& +\frac{5 m_{1} v_{1}^{8}}{128} \\
& +G m_{1} m_{2}\left(\frac{3}{8}\left(a_{1} v_{2}\right)\left(n_{12} v_{1}\right)\left(n_{12} v_{2}\right)^{2}+\frac{5}{12}\left(a_{1} v_{2}\right)\left(n_{12} v_{2}\right)^{3}+\frac{1}{8}\left(n_{12} a_{1}\right)\left(n_{12} v_{1}\right)\left(n_{12} v_{2}\right)^{3}\right. \\
& +\frac{1}{16}\left(n_{12} a_{1}\right)\left(n_{12} v_{2}\right)^{4}+\frac{11}{4}\left(a_{1} v_{1}\right)\left(n_{12} v_{2}\right) v_{1}^{2}-\left(a_{1} v_{2}\right)\left(n_{12} v_{2}\right) v_{1}^{2} \\
& -2\left(a_{1} v_{1}\right)\left(n_{12} v_{2}\right)\left(v_{1} v_{2}\right)+\frac{1}{4}\left(a_{1} v_{2}\right)\left(n_{12} v_{2}\right)\left(v_{1} v_{2}\right) \\
& +\frac{3}{8}\left(n_{12} a_{1}\right)\left(n_{12} v_{2}\right)^{2}\left(v_{1} v_{2}\right)-\frac{5}{8}\left(n_{12} a_{1}\right)\left(n_{12} v_{1}\right)^{2} v_{2}^{2}+\frac{15}{8}\left(a_{1} v_{1}\right)\left(n_{12} v_{2}\right) v_{2}^{2} \\
& -\frac{15}{8}\left(a_{1} v_{2}\right)\left(n_{12} v_{2}\right) v_{2}^{2}-\frac{1}{2}\left(n_{12} a_{1}\right)\left(n_{12} v_{1}\right)\left(n_{12} v_{2}\right) v_{2}^{2} \\
& \left.-\frac{5}{16}\left(n_{12} a_{1}\right)\left(n_{12} v_{2}\right)^{2} v_{2}^{2}\right)
\end{aligned}
$$




$$
\begin{aligned}
& +\frac{G^{2} m_{1}^{2} m_{2}}{r_{12}}\left(-\frac{235}{24}\left(a_{2} v_{1}\right)\left(n_{12} v_{1}\right)-\frac{29}{24}\left(n_{12} a_{2}\right)\left(n_{12} v_{1}\right)^{2}-\frac{235}{24}\left(a_{1} v_{2}\right)\left(n_{12} v_{2}\right)\right. \\
& -\frac{17}{6}\left(n_{12} a_{1}\right)\left(n_{12} v_{2}\right)^{2}+\frac{185}{16}\left(n_{12} a_{1}\right) v_{1}^{2}-\frac{235}{48}\left(n_{12} a_{2}\right) v_{1}^{2} \\
& \left.-\frac{185}{8}\left(n_{12} a_{1}\right)\left(v_{1} v_{2}\right)+\frac{20}{3}\left(n_{12} a_{1}\right) v_{2}^{2}\right) \\
& +\frac{G m_{1} m_{2}}{r_{12}}\left(-\frac{5}{32}\left(n_{12} v_{1}\right)^{3}\left(n_{12} v_{2}\right)^{3}+\frac{1}{8}\left(n_{12} v_{1}\right)\left(n_{12} v_{2}\right)^{3} v_{1}^{2}+\frac{5}{8}\left(n_{12} v_{2}\right)^{4} v_{1}^{2}\right. \\
& -\frac{11}{16}\left(n_{12} v_{1}\right)\left(n_{12} v_{2}\right) v_{1}^{4}+\frac{1}{4}\left(n_{12} v_{2}\right)^{2} v_{1}^{4}+\frac{11}{16} v_{1}^{6} \\
& -\frac{15}{32}\left(n_{12} v_{1}\right)^{2}\left(n_{12} v_{2}\right)^{2}\left(v_{1} v_{2}\right)+\left(n_{12} v_{1}\right)\left(n_{12} v_{2}\right) v_{1}^{2}\left(v_{1} v_{2}\right) \\
& +\frac{3}{8}\left(n_{12} v_{2}\right)^{2} v_{1}^{2}\left(v_{1} v_{2}\right)-\frac{13}{16} v_{1}^{4}\left(v_{1} v_{2}\right)+\frac{5}{16}\left(n_{12} v_{1}\right)\left(n_{12} v_{2}\right)\left(v_{1} v_{2}\right)^{2} \\
& +\frac{1}{16}\left(v_{1} v_{2}\right)^{3}-\frac{5}{8}\left(n_{12} v_{1}\right)^{2} v_{1}^{2} v_{2}^{2}-\frac{23}{32}\left(n_{12} v_{1}\right)\left(n_{12} v_{2}\right) v_{1}^{2} v_{2}^{2}+\frac{1}{16} v_{1}^{4} v_{2}^{2} \\
& \left.-\frac{1}{32} v_{1}^{2}\left(v_{1} v_{2}\right) v_{2}^{2}\right) \\
& -\frac{3 G^{4} m_{1}^{4} m_{2}}{8 r_{12}^{4}}+\frac{G^{4} m_{1}^{3} m_{2}^{2}}{r_{12}^{4}}\left(-\frac{9707}{420}+\frac{22}{3} \ln \left(\frac{r_{12}}{r_{1}^{\prime}}\right)\right) \\
& +\frac{G^{3} m_{1}^{2} m_{2}^{2}}{r_{12}^{3}}\left(\frac{383}{24}\left(n_{12} v_{1}\right)^{2}-\frac{889}{48}\left(n_{12} v_{1}\right)\left(n_{12} v_{2}\right)-\frac{123}{64}\left(n_{12} v_{1}\right)\left(n_{12} v_{12}\right) \pi^{2}-\frac{305}{72} v_{1}^{2}\right. \\
& \left.+\frac{41}{64} \pi^{2}\left(v_{1} v_{12}\right)+\frac{439}{144}\left(v_{1} v_{2}\right)\right) \\
& +\frac{G^{3} m_{1}^{3} m_{2}}{r_{12}^{3}}\left(-\frac{8243}{210}\left(n_{12} v_{1}\right)^{2}+\frac{15541}{420}\left(n_{12} v_{1}\right)\left(n_{12} v_{2}\right)+\frac{3}{2}\left(n_{12} v_{2}\right)^{2}+\frac{15611}{1260} v_{1}^{2}\right. \\
& -\frac{17501}{1260}\left(v_{1} v_{2}\right)+\frac{5}{4} v_{2}^{2}+22\left(n_{12} v_{1}\right)\left(n_{12} v_{12}\right) \ln \left(\frac{r_{12}}{r_{1}^{\prime}}\right) \\
& \left.\left.-\frac{22}{3}\left(v_{1} v_{12}\right) \ln \left(\frac{r_{12}}{r_{1}^{\prime}}\right)\right)\right\}+1 \leftrightarrow 2+\mathcal{O}\left(\frac{1}{c^{7}}\right)
\end{aligned}
$$

Witness the accelerations occurring at the 2PN and 3PN orders; see also the gauge-dependent logarithms of $r_{12} / r_{1}^{\prime}$ and $r_{12} / r_{2}^{\prime}$. We refer to [174] for the explicit expressions of the ten conserved quantities corresponding to the integrals of energy [also given in Eq. (205)], linear and angular momenta, and center-of-mass position. Notice that while it is strictly forbidden to replace the accelerations by the equations of motion in the Lagrangian, this can and should be done in the final expressions of the conserved integrals derived from that Lagrangian.

Now we want to exhibit a transformation of the particles' dynamical variables - or contact transformation, as it is called in the jargon - which transforms the 3PN harmonic-coordinates Lagrangian (209) into a new Lagrangian, valid in some ADM or ADM-like coordinate system, and such that the associated Hamiltonian coincides with the 3PN Hamiltonian that has been obtained by Jaranowski \& Schäfer [261, 262]. In ADM coordinates the Lagrangian will be ordinary, depending only on the positions and velocities of the bodies. Let this contact transformation be $\boldsymbol{Y}_{1}(t)=\boldsymbol{y}_{1}(t)+\delta \boldsymbol{y}_{1}(t)$ and $1 \leftrightarrow 2$, where $\boldsymbol{Y}_{1}$ and $\boldsymbol{y}_{1}$ denote the trajectories in ADM and harmonic coordinates, respectively. For this transformation to be able to remove all the accelerations in the 
initial Lagrangian $L^{\text {harm }}$ up to the $3 \mathrm{PN}$ order, we determine [174] it to be necessarily of the form

$$
\delta \boldsymbol{y}_{1}=\frac{1}{m_{1}}\left[\frac{\partial L^{\text {harm }}}{\partial \boldsymbol{a}_{1}}+\frac{\partial F}{\partial \boldsymbol{v}_{1}}+\frac{1}{c^{6}} \boldsymbol{X}_{1}\right]+\mathcal{O}\left(\frac{1}{c^{8}}\right) \quad(\text { and idem } 1 \leftrightarrow 2),
$$

where $F$ is a freely adjustable function of the positions and velocities, made of $2 \mathrm{PN}$ and $3 \mathrm{PN}$ terms, and where $\boldsymbol{X}_{1}$ represents a special correction term, that is purely of order $3 \mathrm{PN}$. The point is that once the function $F$ is specified there is a unique determination of the correction term $\boldsymbol{X}_{1}$ for the contact transformation to work (see Ref. [174] for the details). Thus, the freedom we have is entirely encoded into the function $F$, and the work then consists in showing that there exists a unique choice of $F$ for which our Lagrangian $L^{\text {harm }}$ is physically equivalent, via the contact transformation (210), to the ADM Hamiltonian of Refs. [261, 262]. An interesting point is that not only the transformation must remove all the accelerations in $L^{\text {harm }}$, but it should also cancel out all the logarithms $\ln \left(r_{12} / r_{1}^{\prime}\right)$ and $\ln \left(r_{12} / r_{2}^{\prime}\right)$, because there are no logarithms in ADM coordinates. The result we find, which can be checked to be in full agreement with the expression of the gauge vector in Eq. (204), is that $F$ involves the logarithmic terms

$$
F=\frac{22}{3} \frac{G^{3} m_{1} m_{2}}{c^{6} r_{12}^{2}}\left[m_{1}^{2}\left(n_{12} v_{1}\right) \ln \left(\frac{r_{12}}{r_{1}^{\prime}}\right)-m_{2}^{2}\left(n_{12} v_{2}\right) \ln \left(\frac{r_{12}}{r_{2}^{\prime}}\right)\right]+\cdots,
$$

together with many other non-logarithmic terms (indicated by dots) that are entirely specified by the isometry of the harmonic and ADM descriptions of the motion. For this particular choice of $F$ the ADM Lagrangian reads

$$
L^{\mathrm{ADM}}=L^{\mathrm{harm}}+\frac{\delta L^{\text {harm }}}{\delta y_{1}^{i}} \delta y_{1}^{i}+\frac{\delta L^{\text {harm }}}{\delta y_{2}^{i}} \delta y_{2}^{i}+\frac{\mathrm{d} F}{\mathrm{~d} t}+\mathcal{O}\left(\frac{1}{c^{8}}\right) .
$$

Inserting into this equation all our explicit expressions we find

$$
\begin{aligned}
& L^{\mathrm{ADM}}=\frac{G m_{1} m_{2}}{2 R_{12}}+\frac{1}{2} m_{1} V_{1}^{2} \\
& +\frac{1}{c^{2}}\left\{-\frac{G^{2} m_{1}^{2} m_{2}}{2 R_{12}^{2}}+\frac{1}{8} m_{1} V_{1}^{4}+\frac{G m_{1} m_{2}}{R_{12}}\left(-\frac{1}{4}\left(N_{12} V_{1}\right)\left(N_{12} V_{2}\right)+\frac{3}{2} V_{1}^{2}-\frac{7}{4}\left(V_{1} V_{2}\right)\right)\right\} \\
& +\frac{1}{c^{4}}\left\{\frac{G^{3} m_{1}^{3} m_{2}}{4 R_{12}^{3}}+\frac{5 G^{3} m_{1}^{2} m_{2}^{2}}{8 R_{12}^{3}}+\frac{m_{1} V_{1}^{6}}{16}\right. \\
& +\frac{G^{2} m_{1}^{2} m_{2}}{R_{12}^{2}}\left(\frac{15}{8}\left(N_{12} V_{1}\right)^{2}+\frac{11}{8} V_{1}^{2}-\frac{15}{4}\left(V_{1} V_{2}\right)+2 V_{2}^{2}\right) \\
& +\frac{G m_{1} m_{2}}{R_{12}}\left(\frac{3}{16}\left(N_{12} V_{1}\right)^{2}\left(N_{12} V_{2}\right)^{2}-\frac{1}{4}\left(N_{12} V_{1}\right)\left(N_{12} V_{2}\right) V_{1}^{2}-\frac{5}{8}\left(N_{12} V_{2}\right)^{2} V_{1}^{2}+\frac{7}{8} V_{1}^{4}\right. \\
& \left.\left.+\frac{3}{4}\left(N_{12} V_{1}\right)\left(N_{12} V_{2}\right)\left(V_{1} V_{2}\right)-\frac{7}{4} V_{1}^{2}\left(V_{1} V_{2}\right)+\frac{1}{8}\left(V_{1} V_{2}\right)^{2}+\frac{11}{16} V_{1}^{2} V_{2}^{2}\right)\right\} \\
& +\frac{1}{c^{6}}\left\{\frac{5 m_{1} V_{1}^{8}}{128}-\frac{G^{4} m_{1}^{4} m_{2}}{8 R_{12}^{4}}+\frac{G^{4} m_{1}^{3} m_{2}^{2}}{R_{12}^{4}}\left(-\frac{227}{24}+\frac{21}{32} \pi^{2}\right)\right. \\
& +\frac{G m_{1} m_{2}}{R_{12}}\left(-\frac{5}{32}\left(N_{12} V_{1}\right)^{3}\left(N_{12} V_{2}\right)^{3}+\frac{3}{16}\left(N_{12} V_{1}\right)^{2}\left(N_{12} V_{2}\right)^{2} V_{1}^{2}\right. \\
& +\frac{9}{16}\left(N_{12} V_{1}\right)\left(N_{12} V_{2}\right)^{3} V_{1}^{2}-\frac{3}{16}\left(N_{12} V_{1}\right)\left(N_{12} V_{2}\right) V_{1}^{4}-\frac{5}{16}\left(N_{12} V_{2}\right)^{2} V_{1}^{4} \\
& +\frac{11}{16} V_{1}^{6}-\frac{15}{32}\left(N_{12} V_{1}\right)^{2}\left(N_{12} V_{2}\right)^{2}\left(V_{1} V_{2}\right)+\frac{3}{4}\left(N_{12} V_{1}\right)\left(N_{12} V_{2}\right) V_{1}^{2}\left(V_{1} V_{2}\right)
\end{aligned}
$$




$$
\begin{aligned}
- & \frac{1}{16}\left(N_{12} V_{2}\right)^{2} V_{1}^{2}\left(V_{1} V_{2}\right)-\frac{21}{16} V_{1}^{4}\left(V_{1} V_{2}\right)+\frac{5}{16}\left(N_{12} V_{1}\right)\left(N_{12} V_{2}\right)\left(V_{1} V_{2}\right)^{2} \\
+ & \frac{1}{8} V_{1}^{2}\left(V_{1} V_{2}\right)^{2}+\frac{1}{16}\left(V_{1} V_{2}\right)^{3}-\frac{5}{16}\left(N_{12} V_{1}\right)^{2} V_{1}^{2} V_{2}^{2} \\
& \left.-\frac{9}{32}\left(N_{12} V_{1}\right)\left(N_{12} V_{2}\right) V_{1}^{2} V_{2}^{2}+\frac{7}{8} V_{1}^{4} V_{2}^{2}-\frac{15}{32} V_{1}^{2}\left(V_{1} V_{2}\right) V_{2}^{2}\right) \\
+\frac{G^{2} m_{1}^{2} m_{2}}{R_{12}^{2}}( & -\frac{5}{12}\left(N_{12} V_{1}\right)^{4}-\frac{13}{8}\left(N_{12} V_{1}\right)^{3}\left(N_{12} V_{2}\right)-\frac{23}{24}\left(N_{12} V_{1}\right)^{2}\left(N_{12} V_{2}\right)^{2} \\
& +\frac{13}{16}\left(N_{12} V_{1}\right)^{2} V_{1}^{2}+\frac{1}{4}\left(N_{12} V_{1}\right)\left(N_{12} V_{2}\right) V_{1}^{2}+\frac{5}{6}\left(N_{12} V_{2}\right)^{2} V_{1}^{2}+\frac{21}{16} V_{1}^{4} \\
& -\frac{1}{2}\left(N_{12} V_{1}\right)^{2}\left(V_{1} V_{2}\right)+\frac{1}{3}\left(N_{12} V_{1}\right)\left(N_{12} V_{2}\right)\left(V_{1} V_{2}\right)-\frac{97}{16} V_{1}^{2}\left(V_{1} V_{2}\right) \\
& +\frac{341}{48}\left(V_{1} V_{2}\right)^{2}+\frac{29}{24}\left(N_{12} V_{1}\right)^{2} V_{2}^{2}-\left(N_{12} V_{1}\right)\left(N_{12} V_{2}\right) V_{2}^{2}+\frac{43}{12} V_{1}^{2} V_{2}^{2} \\
& \left.-\frac{71}{8}\left(V_{1} V_{2}\right) V_{2}^{2}+\frac{47}{16} V_{2}^{4}\right) \\
+\frac{G^{3} m_{1}^{2} m_{2}^{2}}{R_{12}^{3}} & \frac{73}{16}\left(N_{12} V_{1}\right)^{2}-11\left(N_{12} V_{1}\right)\left(N_{12} V_{2}\right)+\frac{3}{64} \pi^{2}\left(N_{12} V_{1}\right)\left(N_{12} V_{12}\right) \\
& \left.-\frac{265}{48} V_{1}^{2}-\frac{1}{64} \pi^{2}\left(V_{1} V_{12}\right)+\frac{59}{8}\left(V_{1} V_{2}\right)\right) \\
+\frac{G^{3} m_{1}^{3} m_{2}}{R_{12}^{3}}( & \left.\left.-5\left(N_{12} V_{1}\right)^{2}-\frac{1}{8}\left(N_{12} V_{1}\right)\left(N_{12} V_{2}\right)+\frac{173}{48} V_{1}^{2}-\frac{27}{8}\left(V_{1} V_{2}\right)+\frac{13}{8} V_{2}^{2}\right)\right\} \\
+2+\mathcal{O}\left(\frac{1}{c^{7}}\right) & \cdot
\end{aligned}
$$

The notation is the same as in Eq. (209), except that we use upper-case letters to denote the ADMcoordinates positions and velocities; thus, for instance $\boldsymbol{N}_{12}=\left(\boldsymbol{Y}_{1}-\boldsymbol{Y}_{2}\right) / R_{12}$ and $\left(N_{12} V_{1}\right)=\boldsymbol{N}_{12} \cdot \boldsymbol{V}_{1}$. The Hamiltonian is simply deduced from the latter Lagrangian by applying the usual Legendre transformation. Posing $\boldsymbol{P}_{1}=\partial L^{\mathrm{ADM}} / \partial \boldsymbol{V}_{1}$ and $1 \leftrightarrow 2$, we get $[261,262,263,162,174]$

$$
\begin{aligned}
H^{\mathrm{ADM}}=- & \frac{G m_{1} m_{2}}{2 R_{12}}+\frac{P_{1}^{2}}{2 m_{1}} \\
+ & \frac{1}{c^{2}}\left\{-\frac{P_{1}^{4}}{8 m_{1}^{3}}+\frac{G^{2} m_{1}^{2} m_{2}}{2 R_{12}^{2}}+\frac{G m_{1} m_{2}}{R_{12}}\left(\frac{1}{4} \frac{\left(N_{12} P_{1}\right)\left(N_{12} P_{2}\right)}{m_{1} m_{2}}-\frac{3}{2} \frac{P_{1}^{2}}{m_{1}^{2}}+\frac{7}{4} \frac{\left(P_{1} P_{2}\right)}{m_{1} m_{2}}\right)\right\} \\
+ & \frac{1}{c^{4}}\left\{\frac{P_{1}^{6}}{16 m_{1}^{5}}-\frac{G^{3} m_{1}^{3} m_{2}}{4 R_{12}^{3}}-\frac{5 G^{3} m_{1}^{2} m_{2}^{2}}{8 R_{12}^{3}}\right. \\
& +\frac{G^{2} m_{1}^{2} m_{2}}{R_{12}^{2}}\left(-\frac{3}{2} \frac{\left(N_{12} P_{1}\right)\left(N_{12} P_{2}\right)}{m_{1} m_{2}}+\frac{19}{4} \frac{P_{1}^{2}}{m_{1}^{2}}-\frac{27}{4} \frac{\left(P_{1} P_{2}\right)}{m_{1} m_{2}}+\frac{5 P_{2}^{2}}{2 m_{2}^{2}}\right) \\
& +\frac{G m_{1} m_{2}}{R_{12}}\left(-\frac{3}{16} \frac{\left(N_{12} P_{1}\right)^{2}\left(N_{12} P_{2}\right)^{2}}{m_{1}^{2} m_{2}^{2}}+\frac{5}{8} \frac{\left(N_{12} P_{2}\right)^{2} P_{1}^{2}}{m_{1}^{2} m_{2}^{2}}\right. \\
& \left.\left.+\frac{5}{8} \frac{P_{1}^{4}}{m_{1}^{4}}-\frac{3}{4} \frac{\left(N_{12} P_{1}\right)\left(N_{12} P_{2}\right)\left(P_{1} P_{2}\right)}{m_{1}^{2} m_{2}^{2}}-\frac{1}{8} \frac{\left(P_{1} P_{2}\right)^{2}}{m_{1}^{2} m_{2}^{2}}-\frac{11}{16} \frac{P_{1}^{2} P_{2}^{2}}{m_{1}^{2} m_{2}^{2}}\right)\right\} \\
+ & \frac{1}{c^{6}}\left\{-\frac{5 P_{1}^{8}}{128 m_{1}^{7}}+\frac{G^{4} m_{1}^{4} m_{2}}{8 R_{12}^{4}}+\frac{G^{4} m_{1}^{3} m_{2}^{2}}{R_{12}^{4}}\left(\frac{227}{24}-\frac{21}{32} \pi^{2}\right)\right.
\end{aligned}
$$




$$
\begin{aligned}
& +\frac{G^{3} m_{1}^{2} m_{2}^{2}}{R_{12}^{3}}\left(-\frac{43}{16} \frac{\left(N_{12} P_{1}\right)^{2}}{m_{1}^{2}}+\frac{119}{16} \frac{\left(N_{12} P_{1}\right)\left(N_{12} P_{2}\right)}{m_{1} m_{2}}-\frac{3}{64} \pi^{2} \frac{\left(N_{12} P_{1}\right)^{2}}{m_{1}^{2}}\right. \\
& +\frac{3}{64} \pi^{2} \frac{\left(N_{12} P_{1}\right)\left(N_{12} P_{2}\right)}{m_{1} m_{2}}-\frac{473}{48} \frac{P_{1}^{2}}{m_{1}^{2}}+\frac{1}{64} \pi^{2} \frac{P_{1}^{2}}{m_{1}^{2}}+\frac{143}{16} \frac{\left(P_{1} P_{2}\right)}{m_{1} m_{2}} \\
& \left.-\frac{1}{64} \pi^{2} \frac{\left(P_{1} P_{2}\right)}{m_{1} m_{2}}\right) \\
& +\frac{G^{3} m_{1}^{3} m_{2}}{R_{12}^{3}}\left(\frac{5}{4} \frac{\left(N_{12} P_{1}\right)^{2}}{m_{1}^{2}}+\frac{21}{8} \frac{\left(N_{12} P_{1}\right)\left(N_{12} P_{2}\right)}{m_{1} m_{2}}-\frac{425}{48} \frac{P_{1}^{2}}{m_{1}^{2}}+\frac{77}{8} \frac{\left(P_{1} P_{2}\right)}{m_{1} m_{2}}-\frac{25 P_{2}^{2}}{8 m_{2}^{2}}\right) \\
& +\frac{G^{2} m_{1}^{2} m_{2}}{R_{12}^{2}}\left(\frac{5}{12} \frac{\left(N_{12} P_{1}\right)^{4}}{m_{1}^{4}}-\frac{3}{2} \frac{\left(N_{12} P_{1}\right)^{3}\left(N_{12} P_{2}\right)}{m_{1}^{3} m_{2}}+\frac{10}{3} \frac{\left(N_{12} P_{1}\right)^{2}\left(N_{12} P_{2}\right)^{2}}{m_{1}^{2} m_{2}^{2}}\right. \\
& +\frac{17}{16} \frac{\left(N_{12} P_{1}\right)^{2} P_{1}^{2}}{m_{1}^{4}}-\frac{15}{8} \frac{\left(N_{12} P_{1}\right)\left(N_{12} P_{2}\right) P_{1}^{2}}{m_{1}^{3} m_{2}}-\frac{55}{12} \frac{\left(N_{12} P_{2}\right)^{2} P_{1}^{2}}{m_{1}^{2} m_{2}^{2}} \\
& +\frac{P_{1}^{4}}{16 m_{1}^{4}}-\frac{11}{8} \frac{\left(N_{12} P_{1}\right)^{2}\left(P_{1} P_{2}\right)}{m_{1}^{3} m_{2}}+\frac{125}{12} \frac{\left(N_{12} P_{1}\right)\left(N_{12} P_{2}\right)\left(P_{1} P_{2}\right)}{m_{1}^{2} m_{2}^{2}} \\
& -\frac{115}{16} \frac{P_{1}^{2}\left(P_{1} P_{2}\right)}{m_{1}^{3} m_{2}}+\frac{25}{48} \frac{\left(P_{1} P_{2}\right)^{2}}{m_{1}^{2} m_{2}^{2}}-\frac{193}{48} \frac{\left(N_{12} P_{1}\right)^{2} P_{2}^{2}}{m_{1}^{2} m_{2}^{2}}+\frac{371}{48} \frac{P_{1}^{2} P_{2}^{2}}{m_{1}^{2} m_{2}^{2}} \\
& \left.-\frac{27}{16} \frac{P_{2}^{4}}{m_{2}^{4}}\right) \\
& +\frac{G m_{1} m_{2}}{R_{12}}\left(\frac{5}{32} \frac{\left(N_{12} P_{1}\right)^{3}\left(N_{12} P_{2}\right)^{3}}{m_{1}^{3} m_{2}^{3}}+\frac{3}{16} \frac{\left(N_{12} P_{1}\right)^{2}\left(N_{12} P_{2}\right)^{2} P_{1}^{2}}{m_{1}^{4} m_{2}^{2}}\right. \\
& -\frac{9}{16} \frac{\left(N_{12} P_{1}\right)\left(N_{12} P_{2}\right)^{3} P_{1}^{2}}{m_{1}^{3} m_{2}^{3}}-\frac{5}{16} \frac{\left(N_{12} P_{2}\right)^{2} P_{1}^{4}}{m_{1}^{4} m_{2}^{2}}-\frac{7}{16} \frac{P_{1}^{6}}{m_{1}^{6}} \\
& +\frac{15}{32} \frac{\left(N_{12} P_{1}\right)^{2}\left(N_{12} P_{2}\right)^{2}\left(P_{1} P_{2}\right)}{m_{1}^{3} m_{2}^{3}}+\frac{3}{4} \frac{\left(N_{12} P_{1}\right)\left(N_{12} P_{2}\right) P_{1}^{2}\left(P_{1} P_{2}\right)}{m_{1}^{4} m_{2}^{2}} \\
& +\frac{1}{16} \frac{\left(N_{12} P_{2}\right)^{2} P_{1}^{2}\left(P_{1} P_{2}\right)}{m_{1}^{3} m_{2}^{3}}-\frac{5}{16} \frac{\left(N_{12} P_{1}\right)\left(N_{12} P_{2}\right)\left(P_{1} P_{2}\right)^{2}}{m_{1}^{3} m_{2}^{3}} \\
& +\frac{1}{8} \frac{P_{1}^{2}\left(P_{1} P_{2}\right)^{2}}{m_{1}^{4} m_{2}^{2}}-\frac{1}{16} \frac{\left(P_{1} P_{2}\right)^{3}}{m_{1}^{3} m_{2}^{3}}-\frac{5}{16} \frac{\left(N_{12} P_{1}\right)^{2} P_{1}^{2} P_{2}^{2}}{m_{1}^{4} m_{2}^{2}} \\
& \left.\left.+\frac{7}{32} \frac{\left(N_{12} P_{1}\right)\left(N_{12} P_{2}\right) P_{1}^{2} P_{2}^{2}}{m_{1}^{3} m_{2}^{3}}+\frac{1}{2} \frac{P_{1}^{4} P_{2}^{2}}{m_{1}^{4} m_{2}^{2}}+\frac{1}{32} \frac{P_{1}^{2}\left(P_{1} P_{2}\right) P_{2}^{2}}{m_{1}^{3} m_{2}^{3}}\right)\right\} \\
& +1 \leftrightarrow 2+\mathcal{O}\left(\frac{1}{c^{7}}\right)
\end{aligned}
$$

Arguably, the results given by the ADM-Hamiltonian formalism (for the problem at hand) look simpler than their harmonic-coordinate counterparts. Indeed, the ADM Lagrangian is ordinary - no accelerations - and there are no logarithms nor associated gauge constants $r_{1}^{\prime}$ and $r_{2}^{\prime}$. ${ }^{50}$ Of course, one is free to describe the binary motion in whatever coordinates one likes, and the two formalisms, harmonic (209) and ADM (213)-(214), describe rigorously the same physics. On the other hand, the higher complexity of the harmonic-coordinates Lagrangian (209) enables one to perform more tests of the computations, notably by inquiring about the future of the constants $r_{1}^{\prime}$ and $r_{2}^{\prime}$, that we know must disappear from physical quantities such as the center-of-mass energy and the total gravitational-wave flux.

\footnotetext{
${ }^{50}$ On the other hand, the ADM-Hamiltonian formalism provides a limited description of the gravitational radiation field, compared to what will be done using harmonic coordinates in Section 9.
} 


\subsection{Equations of motion in the center-of-mass frame}

In this section we translate the origin of coordinates to the binary's center-of-mass by imposing the vanishing of the binary's mass dipole moment: $\mathrm{I}_{i}=0$ in the notation of Part A. Actually the dipole moment is computed as the center-of-mass conserved integral associated with the boost symmetry of the $3 \mathrm{PN}$ equations of motion $[174,79]$. This condition results in the $3 \mathrm{PN}$-accurate relationship between the individual positions in the center-of-mass frame $\boldsymbol{y}_{1}$ and $\boldsymbol{y}_{2}$, and the relative position $\boldsymbol{x} \equiv \boldsymbol{y}_{1}-\boldsymbol{y}_{2}$ and velocity $\boldsymbol{v} \equiv \boldsymbol{v}_{1}-\boldsymbol{v}_{2}=\mathrm{d} \boldsymbol{x} / \mathrm{d} t$ (formerly denoted $\boldsymbol{y}_{12}$ and $\boldsymbol{v}_{12}$ ). We shall also use the orbital separation $r \equiv|\boldsymbol{x}|$, together with $\boldsymbol{n}=\boldsymbol{x} / r$ and $\dot{\boldsymbol{r}} \equiv \boldsymbol{n} \cdot \boldsymbol{v}$. Mass parameters are: The total mass $m=m_{1}+m_{2}$ (to be distinguished from the ADM mass denoted by M in Part A); the relative mass difference $\Delta=\left(m_{1}-m_{2}\right) / m$; the reduced mass $\mu=m_{1} m_{2} / m$; and the very useful symmetric mass ratio

$$
\nu \equiv \frac{\mu}{m} \equiv \frac{m_{1} m_{2}}{\left(m_{1}+m_{2}\right)^{2}}
$$

The usefulness of this ratio lies in its interesting range of variation: $0<\nu \leqslant 1 / 4$, with $\nu=1 / 4$ in the case of equal masses, and $\nu \rightarrow 0$ in the test-mass limit for one of the bodies. Thus $\nu$ is numerically rather small and may be viewed as a small expansion parameter. We also pose $X_{1}=m_{1} / m$ and $X_{2}=m_{2} / m$ so that $\Delta=X_{1}-X_{2}$ and $\nu=X_{1} X_{2}$.

For reference we give the $3 \mathrm{PN}$-accurate expressions of the individual positions in the center-ofmass frame in terms of relative variables. They are in the form

$$
\begin{aligned}
& \boldsymbol{y}_{1}=\left[X_{2}+\nu \Delta \mathcal{P}\right] \boldsymbol{x}+\nu \Delta \mathcal{Q} \boldsymbol{v}+\mathcal{O}\left(\frac{1}{c^{7}}\right), \\
& \boldsymbol{y}_{2}=\left[-X_{1}+\nu \Delta \mathcal{P}\right] \boldsymbol{x}+\nu \Delta \mathcal{Q} \boldsymbol{v}+\mathcal{O}\left(\frac{1}{c^{7}}\right),
\end{aligned}
$$

where all post-Newtonian corrections, beyond Newtonian order, are proportional to the mass ratio $\nu$ and the mass difference $\Delta$. The two dimensionless coefficients $\mathcal{P}$ and $\mathcal{Q}$ read

$$
\begin{aligned}
\mathcal{P}= & \frac{1}{c^{2}}\left\{\frac{v^{2}}{2}-\frac{G m}{2 r}\right\} \\
+ & \frac{1}{c^{4}}\left\{\frac{3 v^{4}}{8}-\frac{3 \nu v^{4}}{2}+\frac{G m}{r}\left(-\frac{\dot{r}^{2}}{8}+\frac{3 \dot{r}^{2} \nu}{4}+\frac{19 v^{2}}{8}+\frac{3 \nu v^{2}}{2}\right)+\frac{G^{2} m^{2}}{r^{2}}\left(\frac{7}{4}-\frac{\nu}{2}\right)\right\} \\
+ & \frac{1}{c^{6}}\left\{\frac{5 v^{6}}{16}-\frac{11 \nu v^{6}}{4}+6 \nu^{2} v^{6}\right. \\
& +\frac{G m}{r}\left(\frac{\dot{r}^{4}}{16}-\frac{5 \dot{r}^{4} \nu}{8}+\frac{21 \dot{r}^{4} \nu^{2}}{16}-\frac{5 \dot{r}^{2} v^{2}}{16}+\frac{21 \dot{r}^{2} \nu v^{2}}{16}\right. \\
& +\frac{G^{2} m^{2}}{r^{2}}\left(-\frac{71 \dot{r}^{2} \nu^{2} v^{2}}{3}+\frac{53 v^{4}}{16}-7 \frac{7 \dot{r}^{2} \nu}{8}+\frac{15 \nu^{2} v^{4}}{2}\right) \\
& \left.+\frac{G^{3} m^{3}}{r^{3}}\left(-\frac{14351}{1260}+\frac{\nu}{8}-\frac{\nu^{2}}{2}+\frac{22}{3} \ln \left(\frac{r}{r_{0}^{\prime \prime}}\right)\right)\right\} \\
\mathcal{Q}= & \frac{1}{c^{4}}\left\{-\frac{7 G m \dot{r}}{4}\right\}+\frac{1}{c^{5}}\left\{\frac{4 G m v^{2}}{5}-\frac{8 G^{2} m^{2}}{5 r}\right\} \\
+ & \frac{1}{c^{6}}\left\{G m \dot{r}\left(\frac{5 \dot{r}^{2}}{12}-\frac{19 \dot{r}^{2} \nu}{24}-\frac{15 v^{2}}{8}+\frac{21 \nu v^{2}}{4}\right)+\frac{G^{2} m^{2} \dot{r}}{r}\left(-\frac{235}{24}-\frac{21 \nu}{4}\right)\right\}
\end{aligned}
$$


Up to 2.5PN order there is agreement with the circular-orbit limit of Eqs. (6.4) in Ref. [45]. Notice the $2.5 \mathrm{PN}$ radiation-reaction term entering the coefficient $\mathcal{Q}$; such $2.5 \mathrm{PN}$ term is explicitly displayed for circular orbits in Eqs. (224) below. In Eqs. (217) the logarithms at the 3PN order appear only in the coefficient $\mathcal{P}$. They contain a particular combination $r_{0}^{\prime \prime}$ of the two gauge-constants $r_{1}^{\prime}$ and $r_{2}^{\prime}$ defined by

$$
\Delta \ln r_{0}^{\prime \prime}=X_{1}^{2} \ln r_{1}^{\prime}-X_{2}^{2} \ln r_{2}^{\prime},
$$

and which happens to be different from a similar combination $r_{0}^{\prime}$ we shall find in the equations of relative motion, see Eq. (221).

The 3PN and even 3.5PN center-of-mass equations of motion are obtained by replacing in the $3.5 \mathrm{PN}$ equations of motion (203) in a general frame, the positions and velocities by their centerof-mass expressions (216)-(217), applying as usual the order-reduction of all accelerations where necessary. We write the relative acceleration in the center-of-mass frame in the form

$$
\frac{\mathrm{d} \boldsymbol{v}}{\mathrm{d} t}=-\frac{G m}{r^{2}}[(1+\mathcal{A}) \boldsymbol{n}+\mathcal{B} \boldsymbol{v}]+\mathcal{O}\left(\frac{1}{c^{8}}\right),
$$

and find that the coefficients $\mathcal{A}$ and $\mathcal{B}$ are [79]

$$
\begin{aligned}
& \mathcal{A}=\frac{1}{c^{2}}\left\{-\frac{3 \dot{r}^{2} \nu}{2}+v^{2}+3 \nu v^{2}-\frac{G m}{r}(4+2 \nu)\right\} \\
&+\frac{1}{c^{4}}\left\{\frac{15 \dot{r}^{4} \nu}{8}-\frac{45 \dot{r}^{4} \nu^{2}}{8}-\frac{9 \dot{r}^{2} \nu v^{2}}{2}+6 \dot{r}^{2} \nu^{2} v^{2}+3 \nu v^{4}-4 \nu^{2} v^{4}\right. \\
&+\left.\frac{G m}{r}\left(-2 \dot{r}^{2}-25 \dot{r}^{2} \nu-2 \dot{r}^{2} \nu^{2}-\frac{13 \nu v^{2}}{2}+2 \nu^{2} v^{2}\right)+\frac{G^{2} m^{2}}{r^{2}}\left(9+\frac{87 \nu}{4}\right)\right\} \\
&+\frac{1}{c^{5}}\left\{-\frac{24 \dot{r} \nu v^{2}}{5} \frac{G m}{r}-\frac{136 \dot{r} \nu}{15} \frac{G^{2} m^{2}}{r^{2}}\right\} \\
&+\frac{1}{c^{6}}\{-\frac{35 \dot{r}^{6} \nu}{16}+\frac{175 \dot{r}^{6} \nu^{2}}{16}-\frac{175 \dot{r}^{6} \nu^{3}}{16}+\frac{15 \dot{r}^{4} \nu v^{2}}{2}-\frac{135 \dot{r}^{4} \nu^{2} v^{2}}{4}+\frac{255 \dot{r}^{4} \nu^{3} v^{2}}{8} \\
&-\frac{15 \dot{r}^{2} \nu v^{4}}{2}+\frac{237 \dot{r}^{2} \nu^{2} v^{4}}{8}-\frac{45 \dot{r}^{2} \nu^{3} v^{4}}{2}+\frac{11 \nu v^{6}}{4}-\frac{49 \nu^{2} v^{6}}{4}+13 \nu^{3} v^{6} \\
&+\frac{G m}{r}\left(79 \dot{r}^{4} \nu-\frac{69 \dot{r}^{4} \nu^{2}}{2}-30 \dot{r}^{4} \nu^{3}-121 \dot{r}^{2} \nu v^{2}+16 \dot{r}^{2} \nu^{2} v^{2}+20 \dot{r}^{2} \nu^{3} v^{2}+\frac{75 \nu v^{4}}{4}\right. \\
&++\frac{G^{2} m^{2}}{r^{2}} \dot{r}\left(\frac{692}{35} \nu v^{2}-\frac{724}{15} v^{2} \nu^{2}+\frac{294}{5} \nu \dot{r}^{2}+\frac{376}{5} \nu^{2} \dot{r}^{2}\right) \\
&+\frac{1}{c^{7}}\{\left.\frac{G m}{r} \dot{r}\left(\frac{396}{35} \nu+\frac{184}{5} \nu^{2}\right)\right\}, \\
&+ \frac{G^{2} m^{2}}{r^{2}}\left(\dot{r}^{2}+\frac{32573 \dot{r}^{2} \nu}{168}+\frac{11 \dot{r}^{2} \nu^{2}}{8}-7 \dot{r}^{2} \nu^{3}+\frac{615 \dot{r}^{2} \nu \pi^{2}}{64}-\frac{26987 \nu v^{2}}{840}+\nu^{3} v^{2}\right. \\
&\left.-\frac{123 \nu \pi^{2} v^{2}}{64}-110 \dot{r}^{2} \nu \ln \left(\frac{r}{r_{0}^{\prime}}\right)+22 \nu v^{2} \ln \left(\frac{r}{r_{0}^{\prime}}\right)\right)
\end{aligned}
$$




$$
\begin{aligned}
\mathcal{B}= & \frac{1}{c^{2}}\{-4 \dot{r}+2 \dot{r} \nu\} \\
+ & \frac{1}{c^{4}}\left\{\frac{9 \dot{r}^{3} \nu}{2}+3 \dot{r}^{3} \nu^{2}-\frac{15 \dot{r} \nu v^{2}}{2}-2 \dot{r} \nu^{2} v^{2}+\frac{G m}{r}\left(2 \dot{r}+\frac{41 \dot{r} \nu}{2}+4 \dot{r} \nu^{2}\right)\right\} \\
+ & \frac{1}{c^{5}}\left\{\frac{8 \nu v^{2}}{5} \frac{G m}{r}+\frac{24 \nu}{5} \frac{G^{2} m^{2}}{r^{2}}\right\} \\
+\frac{1}{c^{6}} & \left\{-\frac{45 \dot{r}^{5} \nu}{8}+15 \dot{r}^{5} \nu^{2}+\frac{15 \dot{r}^{5} \nu^{3}}{4}+12 \dot{r}^{3} \nu v^{2}-\frac{111 \dot{r}^{3} \nu^{2} v^{2}}{4}-12 \dot{r}^{3} \nu^{3} v^{2}-\frac{65 \dot{r} \nu v^{4}}{8}\right. \\
& +19 \dot{r} \nu^{2} v^{4}+6 \dot{r} \nu^{3} v^{4} \\
& +\frac{G m}{r}\left(\frac{329 \dot{r}^{3} \nu}{6}+\frac{59 \dot{r}^{3} \nu^{2}}{2}+18 \dot{r}^{3} \nu^{3}-15 \dot{r} \nu v^{2}-27 \dot{r} \nu^{2} v^{2}-10 \dot{r} \nu^{3} v^{2}\right) \\
& \left.+\frac{G^{2} m^{2}}{r^{2}}\left(-4 \dot{r}-\frac{18169 \dot{r} \nu}{840}+25 \dot{r} \nu^{2}+8 \dot{r} \nu^{3}-\frac{123 \dot{r} \nu \pi^{2}}{32}+44 \dot{r} \nu \ln \left(\frac{r}{r_{0}^{\prime}}\right)\right)\right\} \\
+ & \frac{G m}{c^{7}}\left(-\frac{626}{35} \nu v^{4}-\frac{12}{5} \nu^{2} v^{4}+\frac{678}{5} \nu v^{2} \dot{r}^{2}+\frac{12}{5} \nu^{2} v^{2} \dot{r}^{2}-120 \nu \dot{r}^{4}\right) \\
& +\frac{G^{2} m^{2}}{r^{2}}\left(\frac{164}{21} \nu v^{2}+\frac{148}{5} \nu^{2} v^{2}-\frac{82}{3} \nu \dot{r}^{2}-\frac{848}{15} \nu^{2} \dot{r}^{2}\right) \\
& \left.+\frac{G^{3} m^{3}}{r^{3}}\left(-\frac{1060}{21} \nu-\frac{104}{5} \nu^{2}\right)\right\} \cdot
\end{aligned}
$$

Up to the 2.5PN order the result agrees with Ref. [302]. The 3.5PN term is issued from Refs. [258, $259,260,336,278,322,254]$. At the $3 \mathrm{PN}$ order we have some gauge-dependent logarithms containing a constant $r_{0}^{\prime}$ which is the "logarithmic barycenter" of the two constants $r_{1}^{\prime}$ and $r_{2}^{\prime}$ :

$$
\ln r_{0}^{\prime}=X_{1} \ln r_{1}^{\prime}+X_{2} \ln r_{2}^{\prime} .
$$

The logarithms in Eqs. (220), together with the constant $r_{0}^{\prime}$ therein, can be removed by applying the gauge transformation (204), while still staying within the class of harmonic coordinates. The resulting modification of the equations of motion will affect only the coefficients of the $3 \mathrm{PN}$ order in Eqs. (220); let us denote them by $\mathcal{A}_{3 \mathrm{PN}}$ and $\mathcal{B}_{3 \mathrm{PN}}$. The new values of these coefficients, obtained after removal of the logarithms by the latter harmonic gauge transformation, will be denoted $\mathcal{A}_{3 \mathrm{PN}}^{\mathrm{MH}}$ and $\mathcal{B}_{3 \mathrm{PN}}^{\mathrm{MH}}$. Here $\mathrm{MH}$ stands for the modified harmonic coordinate system, differing from the $\mathrm{SH}$ (standard harmonic) coordinate system containing logarithms at the $3 \mathrm{PN}$ order in the coefficients $\mathcal{A}_{3 \mathrm{PN}}$ and $\mathcal{B}_{3 \mathrm{PN}}$. See Ref. [9] for a full description of the coordinate transformation between $\mathrm{SH}$ and $\mathrm{MH}$ coordinates for various quantities. We have [320, 9]

$$
\begin{aligned}
\mathcal{A}_{3 \mathrm{PN}}^{\mathrm{MH}}= & \frac{1}{c^{6}}\left\{-\frac{35 \dot{r}^{6} \nu}{16}+\frac{175 \dot{r}^{6} \nu^{2}}{16}-\frac{175 \dot{r}^{6} \nu^{3}}{16}+\frac{15 \dot{r}^{4} \nu v^{2}}{2}-\frac{135 \dot{r}^{4} \nu^{2} v^{2}}{4}+\frac{255 \dot{r}^{4} \nu^{3} v^{2}}{8}-\frac{15 \dot{r}^{2} \nu v^{4}}{2}\right. \\
& +\frac{237 \dot{r}^{2} \nu^{2} v^{4}}{8}-\frac{45 \dot{r}^{2} \nu^{3} v^{4}}{2}+\frac{11 \nu v^{6}}{4}-\frac{49 \nu^{2} v^{6}}{4}+13 \nu^{3} v^{6} \\
& +\frac{G m}{r}\left(79 \dot{r}^{4} \nu-\frac{69 \dot{r}^{4} \nu^{2}}{2}-30 \dot{r}^{4} \nu^{3}-121 \dot{r}^{2} \nu v^{2}+16 \dot{r}^{2} \nu^{2} v^{2}+20 \dot{r}^{2} \nu^{3} v^{2}+\frac{75 \nu v^{4}}{4}\right. \\
& \left.+8 \nu^{2} v^{4}-10 \nu^{3} v^{4}\right) \\
& +\frac{G^{2} m^{2}}{r^{2}}\left(\dot{r}^{2}+\frac{22717 \dot{r}^{2} \nu}{168}+\frac{11 \dot{r}^{2} \nu^{2}}{8}-7 \dot{r}^{2} \nu^{3}+\frac{615 \dot{r}^{2} \nu \pi^{2}}{64}-\frac{20827 \nu v^{2}}{840}+\nu^{3} v^{2}\right. \\
& \left.-\frac{123 \nu \pi^{2} v^{2}}{64}\right)
\end{aligned}
$$




$$
\begin{aligned}
&\left.+\frac{G^{3} m^{3}}{r^{3}}\left(-16-\frac{1399 \nu}{12}-\frac{71 \nu^{2}}{2}+\frac{41 \nu \pi^{2}}{16}\right)\right\}, \\
& \mathcal{B}_{3 \mathrm{PN}}^{\mathrm{MH}}=\frac{1}{c^{6}}\left\{-\frac{45 \dot{r}^{5} \nu}{8}+15 \dot{r}^{5} \nu^{2}+\frac{15 \dot{r}^{5} \nu^{3}}{4}+12 \dot{r}^{3} \nu v^{2}-\frac{111 \dot{r}^{3} \nu^{2} v^{2}}{4}-12 \dot{r}^{3} \nu^{3} v^{2}-\frac{65 \dot{r} \nu v^{4}}{8}\right. \\
&+19 \dot{r} \nu^{2} v^{4}+6 \dot{r} \nu^{3} v^{4} \\
&+\frac{G m}{r}\left(\frac{329 \dot{r}^{3} \nu}{6}+\frac{59 \dot{r}^{3} \nu^{2}}{2}+18 \dot{r}^{3} \nu^{3}-15 \dot{r} \nu v^{2}-27 \dot{r} \nu^{2} v^{2}-10 \dot{r} \nu^{3} v^{2}\right) \\
&\left.+\frac{G^{2} m^{2}}{r^{2}}\left(-4 \dot{r}-\frac{5849 \dot{r} \nu}{840}+25 \dot{r} \nu^{2}+8 \dot{r} \nu^{3}-\frac{123 \dot{r} \nu \pi^{2}}{32}\right)\right\} .
\end{aligned}
$$

Again, the other terms in the equations of motion (219)-(220) are unchanged. These gaugetransformed coefficients in $\mathrm{MH}$ coordinates are useful because they do not yield the usual complications associated with logarithms. However, they must be handled with care in applications such as in Ref. [320], because one must ensure that all other quantities in the problem (energy, angular momentum, gravitational-wave fluxes, etc.) are defined in the same specific $\mathrm{MH}$ gauge avoiding logarithms. In the following we shall no longer use the $\mathrm{MH}$ coordinate system leading to Eqs. (222), except when constructing the generalized quasi-Keplerian representation of the 3PN motion in Section 10.2. Therefore all expressions we shall derive below, notably all those concerning the radiation field, are valid in the $\mathrm{SH}$ coordinate system in which the equations of motion are fully given by Eq. (203) or, in the center-of-mass frame, by Eqs. (219) - (220).

For future reference let also give the 3PN center-of-mass Hamiltonian in ADM coordinates derived in Refs. [261, 262, 162]. In the center-of-mass frame the conjugate variables are the relative separation $\boldsymbol{X}=\boldsymbol{Y}_{1}-\boldsymbol{Y}_{2}$ and the conjugate momentum (per unit reduced mass) $\boldsymbol{P}$ such that $\mu \boldsymbol{P}=\boldsymbol{P}_{1}=-\boldsymbol{P}_{2}$ where $\boldsymbol{P}_{1}$ and $\boldsymbol{P}_{2}$ are defined in Section 7.2). Posing $\boldsymbol{N} \equiv \boldsymbol{X} / R$ with $R \equiv|\boldsymbol{X}|$, together with $P^{2} \equiv \boldsymbol{P}^{2}$ and $P_{R} \equiv \boldsymbol{N} \cdot \boldsymbol{P}$, we have

$$
\begin{aligned}
& \frac{H^{\mathrm{ADM}}}{\mu}=\frac{P^{2}}{2}-\frac{G m}{R} \\
& +\frac{1}{c^{2}}\left\{-\frac{P^{4}}{8}+\frac{3 \nu P^{4}}{8}+\frac{G m}{R}\left(-\frac{P_{R}^{2} \nu}{2}-\frac{3 P^{2}}{2}-\frac{\nu P^{2}}{2}\right)+\frac{G^{2} m^{2}}{2 R^{2}}\right\} \\
& +\frac{1}{c^{4}}\left\{\frac{P^{6}}{16}-\frac{5 \nu P^{6}}{16}+\frac{5 \nu^{2} P^{6}}{16}\right. \\
& +\frac{G m}{R}\left(-\frac{3 P_{R}^{4} \nu^{2}}{8}-\frac{P_{R}^{2} P^{2} \nu^{2}}{4}+\frac{5 P^{4}}{8}-\frac{5 \nu P^{4}}{2}-\frac{3 \nu^{2} P^{4}}{8}\right) \\
& +\frac{G^{2} m^{2}}{R^{2}}\left(\frac{3 P_{R}^{2} \nu}{2}+\frac{5 P^{2}}{2}+4 \nu P^{2}\right) \\
& \left.+\frac{G^{3} m^{3}}{R^{3}}\left(-\frac{1}{4}-\frac{3 \nu}{4}\right)\right\} \\
& +\frac{1}{c^{6}}\left\{-\frac{5 P^{8}}{128}+\frac{35 \nu P^{8}}{128}-\frac{35 \nu^{2} P^{8}}{64}+\frac{35 \nu^{3} P^{8}}{128}\right. \\
& +\frac{G m}{R}\left(-\frac{5 P_{R}{ }^{6} \nu^{3}}{16}+\frac{3 P_{R}{ }^{4} P^{2} \nu^{2}}{16}-\frac{3 P_{R}{ }^{4} P^{2} \nu^{3}}{16}+\frac{P_{R}{ }^{2} P^{4} \nu^{2}}{8}\right. \\
& \left.-\frac{3 P_{R}^{2} P^{4} \nu^{3}}{16}-\frac{7 P^{6}}{16}+\frac{21 \nu P^{6}}{8}-\frac{53 \nu^{2} P^{6}}{16}-\frac{5 \nu^{3} P^{6}}{16}\right) \\
& +\frac{G^{2} m^{2}}{R^{2}}\left(\frac{5 P_{R}{ }^{4} \nu}{12}+\frac{43 P_{R}{ }^{4} \nu^{2}}{12}+\frac{17 P_{R}^{2} P^{2} \nu}{16}\right.
\end{aligned}
$$




$$
\begin{array}{r}
\left.+\frac{15 P_{R}^{2} P^{2} \nu^{2}}{8}-\frac{27 P^{4}}{16}+\frac{17 \nu P^{4}}{2}+\frac{109 \nu^{2} P^{4}}{16}\right) \\
+\frac{G^{3} m^{3}}{R^{3}}\left(-\frac{85 P_{R}^{2} \nu}{16}-\frac{7 P_{R}^{2} \nu^{2}}{4}-\frac{25 P^{2}}{8}-\frac{335 \nu P^{2}}{48}\right. \\
\left.-\frac{23 \nu^{2} P^{2}}{8}-\frac{3 P_{R}^{2} \nu \pi^{2}}{64}+\frac{\nu P^{2} \pi^{2}}{64}\right) \\
\left.+\frac{G^{4} m^{4}}{R^{4}}\left(\frac{1}{8}+\frac{109 \nu}{12}-\frac{21 \nu \pi^{2}}{32}\right)\right\}+\mathcal{O}\left(\frac{1}{c^{8}}\right) .
\end{array}
$$

\subsection{Equations of motion and energy for quasi-circular orbits}

Most inspiralling compact binaries will have been circularized by the time they become visible by the detectors LIGO and VIRGO; see Section 1.2. In the case of orbits that are circular - apart from the gradual radiation-reaction inspiral - the complicated equations of motion simplify drastically, since we have $\dot{r}=(n v)=\mathcal{O}\left(1 / c^{5}\right)$. For circular orbits, up to the $2.5 \mathrm{PN}$ order, the relation between center-of-mass variables and the relative ones reads

$$
\begin{aligned}
& \boldsymbol{y}_{1}=\boldsymbol{x}\left[X_{2}+3 \gamma^{2} \nu \Delta\right]-\frac{4}{5} \frac{G^{2} \nu m^{2} \Delta}{r c^{5}} \boldsymbol{v}+\mathcal{O}\left(\frac{1}{c^{6}}\right), \\
& \boldsymbol{y}_{2}=\boldsymbol{x}\left[-X_{1}+3 \gamma^{2} \nu \Delta\right]-\frac{4}{5} \frac{G^{2} \nu m^{2} \Delta}{r c^{5}} \boldsymbol{v}+\mathcal{O}\left(\frac{1}{c^{6}}\right),
\end{aligned}
$$

where we recall $X_{1}=m_{1} / m, X_{2}=m_{2} / m$ and $\Delta=X_{1}-X_{2}$. See Eqs. (216) $-(217)$ for more general formulas. To conveniently display the successive post-Newtonian corrections, we employ the post-Newtonian parameter

$$
\gamma \equiv \frac{G m}{r c^{2}}=\mathcal{O}\left(\frac{1}{c^{2}}\right) \text {. }
$$

Notice that there are no corrections of order 1PN in Eqs. (224) for circular orbits; the dominant term is of order $2 \mathrm{PN}$, i.e., is proportional to $\gamma^{2}=\mathcal{O}\left(1 / c^{4}\right)$. See Ref. [79] for a systematic calculation of Eqs. (224) to higher order.

The relative acceleration $\boldsymbol{a} \equiv \boldsymbol{a}_{1}-\boldsymbol{a}_{2}$ of two bodies moving on a circular orbit at the $3.5 \mathrm{PN}$ order is then given by

$$
\boldsymbol{a}=-\Omega^{2} \boldsymbol{x}-\frac{32}{5} \frac{G^{3} m^{3} \nu}{c^{5} r^{4}}\left[1+\gamma\left(-\frac{743}{336}-\frac{11}{4} \nu\right)\right] \boldsymbol{v}+\mathcal{O}\left(\frac{1}{c^{8}}\right),
$$

where $\boldsymbol{x} \equiv \boldsymbol{y}_{1}-\boldsymbol{y}_{2}$ is the relative separation (in harmonic coordinates) and $\Omega$ denotes the angular frequency of the circular motion. The second term in Eq. (226), opposite to the velocity $\boldsymbol{v} \equiv \boldsymbol{v}_{1}-\boldsymbol{v}_{2}$, represents the radiation reaction force up to $3.5 \mathrm{PN}$ order, which comes from the reduction of the coefficients of $1 / c^{5}$ and $1 / c^{7}$ in Eqs. (220). The radiation-reaction force is responsible for the secular decrease of the separation $r$ and increase of the orbital frequency $\Omega$ :

$$
\begin{aligned}
\dot{r} & =-\frac{64}{5} \frac{G^{3} m^{3} \nu}{r^{3} c^{5}}\left[1+\gamma\left(-\frac{1751}{336}-\frac{7}{4} \nu\right)\right], \\
\dot{\Omega} & =\frac{96}{5} \frac{G m \nu}{r^{3}} \gamma^{5 / 2}\left[1+\gamma\left(-\frac{2591}{336}-\frac{11}{12} \nu\right)\right] .
\end{aligned}
$$

Concerning conservative effects, the main content of the $3 \mathrm{PN}$ equations (226) is the relation between the frequency $\Omega$ and the orbital separation $r$, which is given by the following generalized 
version of Kepler's third law:

$$
\begin{aligned}
\Omega^{2}=\frac{G m}{r^{3}} & \left\{1+(-3+\nu) \gamma+\left(6+\frac{41}{4} \nu+\nu^{2}\right) \gamma^{2}\right. \\
+ & \left.\left(-10+\left[-\frac{75707}{840}+\frac{41}{64} \pi^{2}+22 \ln \left(\frac{r}{r_{0}^{\prime}}\right)\right] \nu+\frac{19}{2} \nu^{2}+\nu^{3}\right) \gamma^{3}\right\}+\mathcal{O}\left(\frac{1}{c^{8}}\right) .
\end{aligned}
$$

The length scale $r_{0}^{\prime}$ is given in terms of the two gauge-constants $r_{1}^{\prime}$ and $r_{2}^{\prime}$ by Eq. (221). As for the energy, it is inferred from the circular-orbit reduction of the general result (205). We have

$$
\begin{aligned}
E=-\frac{\mu c^{2} \gamma}{2} & \left\{1+\left(-\frac{7}{4}+\frac{1}{4} \nu\right) \gamma+\left(-\frac{7}{8}+\frac{49}{8} \nu+\frac{1}{8} \nu^{2}\right) \gamma^{2}\right. \\
+ & \left.\left(-\frac{235}{64}+\left[\frac{46031}{2240}-\frac{123}{64} \pi^{2}+\frac{22}{3} \ln \left(\frac{r}{r_{0}^{\prime}}\right)\right] \nu+\frac{27}{32} \nu^{2}+\frac{5}{64} \nu^{3}\right) \gamma^{3}\right\}+\mathcal{O}\left(\frac{1}{c^{8}}\right)
\end{aligned}
$$

This expression is that of a physical observable $E$; however, it depends on the choice of a coordinate system, as it involves the post-Newtonian parameter $\gamma$ defined from the harmonic-coordinate separation $r$. But the numerical value of $E$ should not depend on the choice of a coordinate system, so $E$ must admit a frame-invariant expression, the same in all coordinate systems. To find it we re-express $E$ with the help of the following frequency-related parameter $x$, instead of the post-Newtonian parameter $\gamma:{ }^{51}$

$$
x \equiv\left(\frac{G m \Omega}{c^{3}}\right)^{2 / 3}=\mathcal{O}\left(\frac{1}{c^{2}}\right) .
$$

We readily obtain from Eq. (228) the expression of $\gamma$ in terms of $x$ at 3PN order,

$$
\begin{aligned}
\gamma= & x\left\{1+\left(1-\frac{\nu}{3}\right) x+\left(1-\frac{65}{12} \nu\right) x^{2}\right. \\
& \left.+\left(1+\left[-\frac{2203}{2520}-\frac{41}{192} \pi^{2}-\frac{22}{3} \ln \left(\frac{r}{r^{\prime}{ }_{0}}\right)\right] \nu+\frac{229}{36} \nu^{2}+\frac{1}{81} \nu^{3}\right) x^{3}+\mathcal{O}\left(\frac{1}{c^{8}}\right)\right\},
\end{aligned}
$$

that we substitute back into Eq. (229), making all appropriate post-Newtonian re-expansions. As a result, we gladly discover that the logarithms together with their associated gauge constant $r_{0}^{\prime}$ have cancelled out. Therefore, our result is [160, 69]

$$
\begin{aligned}
E=-\frac{\mu c^{2} x}{2} & \left\{1+\left(-\frac{3}{4}-\frac{1}{12} \nu\right) x+\left(-\frac{27}{8}+\frac{19}{8} \nu-\frac{1}{24} \nu^{2}\right) x^{2}\right. \\
+ & \left.\left(-\frac{675}{64}+\left[\frac{34445}{576}-\frac{205}{96} \pi^{2}\right] \nu-\frac{155}{96} \nu^{2}-\frac{35}{5184} \nu^{3}\right) x^{3}\right\}+\mathcal{O}\left(\frac{1}{c^{8}}\right) .
\end{aligned}
$$

For circular orbits one can check that there are no terms of order $x^{7 / 2}$ in Eq. (232), so this result is actually valid up to the $3.5 \mathrm{PN}$ order. We shall discuss in Section 11 how the effects of the spins of the two black holes affect the latter formula.

The formula (232) has been extended to include the logarithmic terms $\propto \ln x$ at the $4 \mathrm{PN}$ and $5 \mathrm{PN}$ orders $[67,289]$, that are due to tail effects occurring in the near zone, see Sections 5.2 and

\footnotetext{
51 This parameter is an invariant in a large class of coordinate systems - those for which the metric becomes asymptotically Minkowskian far from the system: $g_{\alpha \beta} \rightarrow \operatorname{diag}(-1,1,1,1)$.
} 
5.4. Adding also the Schwarzschild test-mass limit ${ }^{52}$ up to $5 \mathrm{PN}$ order, we get:

$$
\begin{aligned}
E=-\frac{\mu c^{2} x}{2} & \left\{1+\left(-\frac{3}{4}-\frac{\nu}{12}\right) x+\left(-\frac{27}{8}+\frac{19}{8} \nu-\frac{\nu^{2}}{24}\right) x^{2}\right. \\
+ & \left(-\frac{675}{64}+\left[\frac{34445}{576}-\frac{205}{96} \pi^{2}\right] \nu-\frac{155}{96} \nu^{2}-\frac{35}{5184} \nu^{3}\right) x^{3} \\
+ & \left(-\frac{3969}{128}+\nu e_{4}(\nu)+\frac{448}{15} \nu \ln x\right) x^{4} \\
+ & \left.\left(-\frac{45927}{512}+\nu e_{5}(\nu)+\left[-\frac{4988}{35}-\frac{656}{5} \nu\right] \nu \ln x\right) x^{5}\right\}+\mathcal{O}\left(\frac{1}{c^{12}}\right) .
\end{aligned}
$$

We can write also a similar expression for the angular momentum,

$$
\begin{aligned}
\mathrm{J}=\frac{G \mu m}{c x^{1 / 2}} & \left\{1+\left(\frac{3}{2}+\frac{\nu}{6}\right) x+\left(\frac{27}{8}-\frac{19}{8} \nu+\frac{\nu^{2}}{24}\right) x^{2}\right. \\
+ & \left(\frac{135}{16}+\left[-\frac{6889}{144}+\frac{41}{24} \pi^{2}\right] \nu+\frac{31}{24} \nu^{2}+\frac{7}{1296} \nu^{3}\right) x^{3} \\
+ & \left(\frac{2835}{128}+\nu j_{4}(\nu)-\frac{64}{3} \nu \ln x\right) x^{4} \\
+ & \left.\left(\frac{15309}{256}+\nu j_{5}(\nu)+\left[\frac{9976}{105}+\frac{1312}{15} \nu\right] \nu \ln x\right) x^{5}\right\}+\mathcal{O}\left(\frac{1}{c^{12}}\right) .
\end{aligned}
$$

For circular orbits the energy $E$ and angular momentum $J$ are known to be linked together by the so-called "thermodynamic" relation

$$
\frac{\partial E}{\partial \Omega}=\Omega \frac{\partial \mathrm{J}}{\partial \Omega}
$$

which is actually just one aspect of the "first law of binary black hole mechanics" that we shall discuss in more details in Section 8.3.

We have introduced in Eqs. (233) - (234) some non-logarithmic 4PN and $5 \mathrm{PN}$ coefficients $e_{4}(\nu)$, $j_{4}(\nu)$ and $e_{5}(\nu), j_{5}(\nu)$, which can however be proved to be polynomials in the symmetric mass ratio $\nu .{ }^{53}$ Recent works on the $4 \mathrm{PN}$ approximation to the equations of motion by means of both EFT methods [204] and the traditional ADM-Hamiltonian approach [264, 265], and complemented by an analytic computation of the gravitational self-force in the small mass ratio $\nu$ limit [36], have yielded the next-order $4 \mathrm{PN}$ coefficient as ( $\gamma_{\mathrm{E}}$ being Euler's constant)

$$
\begin{aligned}
e_{4}(\nu) & =-\frac{123671}{5760}+\frac{9037}{1536} \pi^{2}+\frac{1792}{15} \ln 2+\frac{896}{15} \gamma_{\mathrm{E}} \\
& +\left[-\frac{498449}{3456}+\frac{3157}{576} \pi^{2}\right] \nu+\frac{301}{1728} \nu^{2}+\frac{77}{31104} \nu^{3} .
\end{aligned}
$$

The numerical value $e_{4}(0) \simeq 153.88$ was predicted before thanks to a comparison with numerical self-force calculations [289, 287].

52 Namely,

$$
E^{\mathrm{Schw}}=\mu c^{2}\left[\frac{1-2 x}{\sqrt{1-3 x}}-1\right]
$$

53 From the thermodynamic relation (235) we necessarily have the relations

$$
\begin{aligned}
& j_{4}(\nu)=-\frac{5}{7} e_{4}(\nu)+\frac{64}{35}, \\
& j_{5}(\nu)=-\frac{2}{3} e_{5}(\nu)-\frac{4988}{945}-\frac{656}{135} \nu .
\end{aligned}
$$




\subsection{The 2.5PN metric in the near zone}

The near-zone metric is given by Eqs. (144) for general post-Newtonian matter sources. For pointparticles binaries all the potentials $V, V_{i}, \cdots$ parametrizing the metric must be computed and iterated for delta-function sources. Up to the $2.5 \mathrm{PN}$ order it is sufficient to cure the divergences due to singular sources by means of the Hadamard self-field regularization. Let us point out that the computation is greatly helped - and indeed is made possible at all - by the existence of the following solution $g$ of the elementary Poisson equation

$$
\Delta g=\frac{1}{r_{1} r_{2}},
$$

which takes the very nice closed analytic form [202]

$$
\begin{aligned}
& g=\ln S, \\
& S \equiv r_{1}+r_{2}+r_{12},
\end{aligned}
$$

where $r_{\mathrm{a}}=\left|\boldsymbol{x}-\boldsymbol{y}_{\mathrm{a}}\right|$ and $r_{12}=\left|\boldsymbol{y}_{1}-\boldsymbol{y}_{2}\right|$. Furthermore, to obtain the metric at the 2.5PN order, the solutions of even more difficult elementary Poisson equations are required. Namely we meet

$$
\begin{aligned}
& \Delta K_{1}=2 \partial_{i} \partial_{j}\left(\frac{1}{r_{2}}\right) \partial_{i} \partial_{j} \ln r_{1}, \\
& \Delta H_{1}=2 \partial_{i} \partial_{j}\left(\frac{1}{r_{1}}\right) \underset{1}{\partial_{i} \partial_{j} g},
\end{aligned}
$$

with ${ }_{\mathrm{a}} \partial_{i}$ denoting the partial derivatives with respect to the source points $y_{\mathrm{a}}^{i}$ (and as usual $\partial_{i}$ being the partial derivative with respect to the field point $x^{i}$ ). It is quite remarkable that the solutions of the latter equations are known in closed analytic form. By combining several earlier results from Refs. [120, 324, 377], one can write these solutions into the form [64, 76]

$$
\begin{aligned}
K_{1} & =\left(\frac{1}{2} \Delta-\Delta_{1}\right)\left[\frac{\ln r_{1}}{r_{2}}\right]+\frac{1}{2} \Delta_{2}\left[\frac{\ln r_{12}}{r_{2}}\right]+\frac{r_{2}}{2 r_{12}^{2} r_{1}^{2}}+\frac{1}{r_{12}^{2} r_{2}}, \\
H_{1} & =\frac{1}{2} \Delta_{1}\left[\frac{g}{r_{1}}+\frac{\ln r_{1}}{r_{12}}-\Delta_{1}\left(\frac{r_{1}+r_{12}}{2} g\right)\right]-\frac{r_{2}}{2 r_{1}^{2} r_{12}^{2}} \\
& +\partial_{2} \partial_{i}\left[\frac{\ln r_{12}}{r_{1}}+\frac{\ln r_{1}}{2 r_{12}}\right]+\frac{1}{2} \Delta_{2}\left[\frac{\ln r_{12}}{r_{1}}\right],
\end{aligned}
$$

where $\Delta_{\mathrm{a}}$ are the Laplacians with respect to the two source points.

We report here the complete expression of the 2.5PN metric in harmonic coordinates valid at any field point in the near zone. Posing $g_{\alpha \beta}=\eta_{\alpha \beta}+k_{\alpha \beta}$ we have [76]

$$
\begin{aligned}
k_{00}=\frac{2 G m_{1}}{c^{2} r_{1}}+ & \frac{1}{c^{4}}\left[\frac{G m_{1}}{r_{1}}\left(-\left(n_{1} v_{1}\right)^{2}+4 v_{1}^{2}\right)-2 \frac{G^{2} m_{1}^{2}}{r_{1}^{2}}\right. \\
& \left.+G^{2} m_{1} m_{2}\left(-\frac{2}{r_{1} r_{2}}-\frac{r_{1}}{2 r_{12}^{3}}+\frac{r_{1}^{2}}{2 r_{2} r_{12}^{3}}-\frac{5}{2 r_{2} r_{12}}\right)\right]+\frac{4 G^{2} m_{1} m_{2}}{3 c^{5} r_{12}^{2}}\left(n_{12} v_{12}\right) \\
+ & \frac{1}{c^{6}}\left[\frac{G m_{1}}{r_{1}}\left(\frac{3}{4}\left(n_{1} v_{1}\right)^{4}-3\left(n_{1} v_{1}\right)^{2} v_{1}^{2}+4 v_{1}^{4}\right)+\frac{G^{2} m_{1}^{2}}{r_{1}^{2}}\left(3\left(n_{1} v_{1}\right)^{2}-v_{1}^{2}\right)+2 \frac{G^{3} m_{1}^{3}}{r_{1}^{3}}\right. \\
+ & G^{2} m_{1} m_{2}\left(v _ { 1 } ^ { 2 } \left(\frac{3 r_{1}^{3}}{8 r_{12}^{5}}-\frac{3 r_{1}^{2} r_{2}}{8 r_{12}^{5}}-\frac{3 r_{1} r_{2}^{2}}{8 r_{12}^{5}}+\frac{3 r_{2}^{3}}{8 r_{12}^{5}}-\frac{37 r_{1}}{8 r_{12}^{3}}+\frac{r_{1}^{2}}{r_{2} r_{12}^{3}}+\frac{3 r_{2}}{8 r_{12}^{3}}\right.\right. \\
& \left.+\frac{2 r_{2}^{2}}{r_{1} r_{12}^{3}}+\frac{6}{r_{1} r_{12}}-\frac{5}{r_{2} r_{12}}-\frac{8 r_{12}}{r_{1} r_{2} S}+\frac{16}{r_{12} S}\right)
\end{aligned}
$$




$$
\begin{aligned}
& +\left(v_{1} v_{2}\right)\left(\frac{8}{r_{1} r_{2}}-\frac{3 r_{1}^{3}}{4 r_{12}^{5}}+\frac{3 r_{1}^{2} r_{2}}{4 r_{12}^{5}}+\frac{13 r_{1}}{4 r_{12}^{3}}-\frac{2 r_{1}^{2}}{r_{2} r_{12}^{3}}-\frac{6}{r_{1} r_{12}}-\frac{16}{r_{1} S}-\frac{12}{r_{12} S}\right) \\
& +\left(n_{12} v_{1}\right)^{2}\left(-\frac{15 r_{1}^{3}}{8 r_{12}^{5}}+\frac{15 r_{1}^{2} r_{2}}{8 r_{12}^{5}}+\frac{15 r_{1} r_{2}^{2}}{8 r_{12}^{5}}-\frac{15 r_{2}^{3}}{8 r_{12}^{5}}+\frac{57 r_{1}}{8 r_{12}^{3}}-\frac{3 r_{1}^{2}}{4 r_{2} r_{12}^{3}}-\frac{33 r_{2}}{8 r_{12}^{3}}\right. \\
& \left.+\frac{7}{4 r_{2} r_{12}}-\frac{16}{S^{2}}-\frac{16}{r_{12} S}\right) \\
& +\left(n_{12} v_{1}\right)\left(n_{12} v_{2}\right)\left(\frac{15 r_{1}^{3}}{4 r_{12}^{5}}-\frac{15 r_{1}^{2} r_{2}}{4 r_{12}^{5}}-\frac{9 r_{1}}{4 r_{12}^{3}}+\frac{12}{S^{2}}+\frac{12}{r_{12} S}\right) \\
& +\left(n_{1} v_{1}\right)^{2}\left(\frac{2}{r_{1} r_{2}}-\frac{r_{1}}{4 r_{12}^{3}}-\frac{3 r_{2}^{2}}{4 r_{1} r_{12}^{3}}+\frac{7}{4 r_{1} r_{12}}-\frac{8}{S^{2}}-\frac{8}{r_{1} S}\right) \\
& +\left(n_{1} v_{1}\right)\left(n_{1} v_{2}\right)\left(\frac{r_{1}}{r_{12}^{3}}+\frac{16}{S^{2}}+\frac{16}{r_{1} S}\right)-\left(n_{1} v_{2}\right)^{2}\left(\frac{8}{S^{2}}+\frac{8}{r_{1} S}\right) \\
& +\left(n_{12} v_{1}\right)\left(n_{1} v_{1}\right)\left(-\frac{3 r_{1}^{2}}{r_{12}^{4}}+\frac{3 r_{2}^{2}}{2 r_{12}^{4}}+\frac{3}{2 r_{12}^{2}}+\frac{16}{S^{2}}\right)+\frac{16\left(n_{1} v_{2}\right)\left(n_{2} v_{1}\right)}{S^{2}} \\
& +\left(n_{12} v_{2}\right)\left(n_{1} v_{1}\right)\left(\frac{3 r_{1}^{2}}{r_{12}^{4}}-\frac{3 r_{2}^{2}}{2 r_{12}^{4}}+\frac{13}{2 r_{12}^{2}}-\frac{40}{S^{2}}\right)-\frac{12\left(n_{1} v_{1}\right)\left(n_{2} v_{2}\right)}{S^{2}} \\
& \left.+\left(n_{12} v_{1}\right)\left(n_{1} v_{2}\right)\left(\frac{3 r_{1}^{2}}{2 r_{12}^{4}}+\frac{4}{r_{12}^{2}}+\frac{16}{S^{2}}\right)+\left(n_{12} v_{2}\right)\left(n_{1} v_{2}\right)\left(\frac{-3 r_{1}^{2}}{2 r_{12}^{4}}-\frac{3}{r_{12}^{2}}+\frac{16}{S^{2}}\right)\right) \\
& +G^{3} m_{1}^{2} m_{2}\left(\frac{4}{r_{1}^{3}}+\frac{1}{2 r_{2}^{3}}+\frac{9}{2 r_{1}^{2} r_{2}}-\frac{r_{1}^{3}}{4 r_{12}^{6}}+\frac{3 r_{1}^{4}}{16 r_{2} r_{12}^{6}}-\frac{r_{1}^{2} r_{2}}{8 r_{12}^{6}}+\frac{r_{1} r_{2}^{2}}{4 r_{12}^{6}}-\frac{r_{2}^{3}}{16 r_{12}^{6}}+\frac{5 r_{1}}{4 r_{12}^{4}}\right. \\
& -\frac{23 r_{1}^{2}}{8 r_{2} r_{12}^{4}}+\frac{43 r_{2}}{8 r_{12}^{4}}-\frac{5 r_{2}^{2}}{2 r_{1} r_{12}^{4}}-\frac{3}{r_{12}^{3}}+\frac{3 r_{1}}{r_{2} r_{12}^{3}}+\frac{r_{2}}{r_{1} r_{12}^{3}}-\frac{5 r_{2}^{2}}{r_{1}^{2} r_{12}^{3}}+\frac{4 r_{2}^{3}}{r_{1}^{3} r_{12}^{3}}+\frac{3}{2 r_{1} r_{12}^{2}} \\
& \left.\left.-\frac{r_{1}^{2}}{4 r_{2}^{3} r_{12}^{2}}+\frac{3}{16 r_{2} r_{12}^{2}}+\frac{15 r_{2}}{4 r_{1}^{2} r_{12}^{2}}-\frac{4 r_{2}^{2}}{r_{1}^{3} r_{12}^{2}}+\frac{5}{r_{1}^{2} r_{12}}+\frac{5}{r_{1} r_{2} r_{12}}-\frac{4 r_{2}}{r_{1}^{3} r_{12}}-\frac{r_{12}^{2}}{4 r_{1}^{2} r_{2}^{3}}\right)\right] \\
& +\frac{1}{c^{7}}\left[G ^ { 2 } m _ { 1 } m _ { 2 } \left(\left(n_{12} v_{12}\right)^{2}\left(n_{1} v_{1}\right)\left(-\frac{8 r_{1}^{3}}{r_{12}^{5}}-\frac{16 r_{1}}{r_{12}^{3}}\right)+\left(n_{12} v_{12}\right)^{2}\left(n_{1} v_{2}\right)\left(\frac{8 r_{1}^{3}}{r_{12}^{5}}+\frac{5 r_{1}}{r_{12}^{3}}\right)\right.\right. \\
& +\left(n_{12} v_{12}\right)^{3}\left(-\frac{7 r_{1}^{4}}{2 r_{12}^{6}}+\frac{7 r_{1}^{2} r_{2}^{2}}{2 r_{12}^{6}}-\frac{11 r_{1}^{2}}{r_{12}^{4}}-\frac{37}{4 r_{12}^{2}}\right) \\
& +\left(n_{12} v_{1}\right)\left(n_{12} v_{12}\right)^{2}\left(\frac{20 r_{1}^{2}}{r_{12}^{4}}-\frac{11}{2 r_{12}^{2}}\right)-4\left(n_{12} v_{12}\right)\left(n_{1} v_{1}\right)^{2} \frac{r_{1}^{2}}{r_{12}^{4}} \\
& +4\left(n_{12} v_{12}\right)\left(n_{1} v_{1}\right)\left(n_{1} v_{2}\right) \frac{r_{1}^{2}}{r_{12}^{4}}+\left(n_{12} v_{1}\right)^{2}\left(n_{1} v_{1}\right) \frac{r_{1}}{r_{12}^{3}} \\
& +22\left(n_{12} v_{1}\right)\left(n_{12} v_{12}\right)\left(n_{1} v_{1}\right) \frac{r_{1}}{r_{12}^{3}}-\left(n_{12} v_{1}\right)^{2}\left(n_{1} v_{2}\right) \frac{r_{1}}{r_{12}^{3}} \\
& +4\left(n_{12} v_{1}\right)\left(n_{12} v_{12}\right)\left(n_{1} v_{2}\right) \frac{r_{1}}{r_{12}^{3}}+11\left(n_{12} v_{1}\right)^{2}\left(n_{12} v_{12}\right) \frac{1}{2 r_{12}^{2}} \\
& +\left(n_{1} v_{2}\right) v_{12}^{2}\left(-\frac{8 r_{1}^{3}}{5 r_{12}^{5}}-\frac{2 r_{1}}{3 r_{12}^{3}}\right)+\left(n_{1} v_{1}\right) v_{12}^{2}\left(\frac{8 r_{1}^{3}}{5 r_{12}^{5}}+\frac{11 r_{1}}{3 r_{12}^{3}}\right) \\
& -\left(n_{12} v_{1}\right) v_{12}^{2}\left(\frac{4 r_{1}^{2}}{r_{12}^{4}}+\frac{5}{2 r_{12}^{2}}\right)-\left(n_{12} v_{12}\right) v_{1}^{2}\left(\frac{12 r_{1}^{2}}{r_{12}^{4}}+\frac{5}{2 r_{12}^{2}}\right) \\
& +\left(n_{12} v_{12}\right) v_{12}^{2}\left(\frac{3 r_{1}^{4}}{2 r_{12}^{6}}-\frac{3 r_{1}^{2} r_{2}^{2}}{2 r_{12}^{6}}+\frac{7 r_{1}^{2}}{r_{12}^{4}}+\frac{27}{4 r_{12}^{2}}\right)
\end{aligned}
$$




$$
\begin{aligned}
& -29\left(n_{1} v_{1}\right) v_{1}^{2} \frac{r_{1}}{3 r_{12}^{3}}+\left(n_{1} v_{2}\right) v_{1}^{2} \frac{r_{1}}{r_{12}^{3}}+5\left(n_{12} v_{1}\right) v_{1}^{2} \frac{1}{r_{12}^{2}} \\
& +\left(n_{12} v_{12}\right)\left(v_{1} v_{2}\right)\left(\frac{12 r_{1}^{2}}{r_{12}^{4}}+\frac{3}{r_{12}^{2}}\right)+8\left(n_{1} v_{1}\right)\left(v_{1} v_{2}\right) \frac{r_{1}}{r_{12}^{3}} \\
& \left.+2\left(n_{1} v_{2}\right)\left(v_{1} v_{2}\right) \frac{r_{1}}{3 r_{12}^{3}}-5\left(n_{12} v_{1}\right)\left(v_{1} v_{2}\right) \frac{1}{r_{12}^{2}}\right) \\
& +G^{3} m_{1}^{2} m_{2}\left(\left(n_{1} v_{12}\right)\left(-\frac{8 r_{1}^{3}}{15 r_{12}^{6}}+\frac{8 r_{1} r_{2}^{2}}{15 r_{12}^{6}}-\frac{16 r_{1}}{3 r_{12}^{4}}+\frac{8}{r_{12}^{3}}-\frac{8 r_{2}^{2}}{r_{1}^{2} r_{12}^{3}}+\frac{8}{r_{1}^{2} r_{12}}\right)\right. \\
& +\left(n_{12} v_{1}\right)\left(-\frac{4 r_{1}^{2}}{3 r_{12}^{5}}+\frac{4 r_{2}^{2}}{3 r_{12}^{5}}+\frac{20}{3 r_{12}^{3}}\right)+8\left(n_{1} v_{1}\right) \frac{r_{1}}{3 r_{12}^{4}} \\
& +\left(n_{12} v_{12}\right)\left(\frac{3}{r_{1}^{3}}-\frac{4 r_{1}^{2}}{3 r_{12}^{5}}+\frac{68 r_{2}^{2}}{15 r_{12}^{5}}+\frac{3 r_{1}}{r_{12}^{4}}-\frac{6 r_{2}^{2}}{r_{1} r_{12}^{4}}+\frac{3 r_{2}^{4}}{r_{1}^{3} r_{12}^{4}}-\frac{76}{3 r_{12}^{3}}\right. \\
& \left.\left.\left.+\frac{2}{r_{1} r_{12}^{2}}-\frac{6 r_{2}^{2}}{r_{1}^{3} r_{12}^{2}}\right)\right)\right]+1 \leftrightarrow 2+\mathcal{O}\left(\frac{1}{c^{8}}\right) \\
& k_{0 i}=-\frac{4 G m_{1}}{c^{3} r_{1}} v_{1}^{i}+\frac{1}{c^{5}}\left[n _ { 1 } ^ { i } \left(-\frac{G^{2} m_{1}^{2}}{r_{1}^{2}}\left(n_{1} v_{1}\right)+\frac{G^{2} m_{1} m_{2}}{S^{2}}\left(-16\left(n_{12} v_{1}\right)+12\left(n_{12} v_{2}\right)\right.\right.\right. \\
& \left.\left.-16\left(n_{2} v_{1}\right)+12\left(n_{2} v_{2}\right)\right)\right) \\
& +n_{12}^{i} G^{2} m_{1} m_{2}\left(-6\left(n_{12} v_{12}\right) \frac{r_{1}}{r_{12}^{3}}-4\left(n_{1} v_{1}\right) \frac{1}{r_{12}^{2}}+12\left(n_{1} v_{1}\right) \frac{1}{S^{2}}\right. \\
& \left.-16\left(n_{1} v_{2}\right) \frac{1}{S^{2}}+4\left(n_{12} v_{1}\right) \frac{1}{S}\left(\frac{1}{S}+\frac{1}{r_{12}}\right)\right) \\
& +v_{1}^{i}\left(\frac{G m_{1}}{r_{1}}\left(2\left(n_{1} v_{1}\right)^{2}-4 v_{1}^{2}\right)+\frac{G^{2} m_{1}^{2}}{r_{1}^{2}}+G^{2} m_{1} m_{2}\left(\frac{3 r_{1}}{r_{12}^{3}}-\frac{2 r_{2}}{r_{12}^{3}}\right)\right. \\
& \left.\left.+G^{2} m_{1} m_{2}\left(-\frac{r_{2}^{2}}{r_{1} r_{12}^{3}}-\frac{3}{r_{1} r_{12}}+\frac{8}{r_{2} r_{12}}-\frac{4}{r_{12} S}\right)\right)\right] \\
& +\frac{1}{c^{6}}\left[n _ { 1 2 } ^ { i } \left(G^{2} m_{1} m_{2}\left(-10\left(n_{12} v_{12}\right)^{2} \frac{r_{1}^{2}}{r_{12}^{4}}-12\left(n_{12} v_{12}\right)\left(n_{1} v_{1}\right) \frac{r_{1}}{r_{12}^{3}}+2 v_{12}^{2} \frac{r_{1}^{2}}{r_{12}^{4}}-4 \frac{v_{1}^{2}}{r_{12}^{2}}\right)\right.\right. \\
& \left.+G^{3} m_{1}^{2} m_{2}\left(\frac{2 r_{1}^{2}}{3 r_{12}^{5}}-\frac{2 r_{2}^{2}}{3 r_{12}^{5}}-\frac{2}{r_{12}^{3}}\right)\right) \\
& +v_{1}^{i} \frac{G^{2} m_{1} m_{2}}{r_{12}^{3}}\left(\frac{16\left(n_{1} v_{12}\right) r_{1}}{3}-4\left(n_{12} v_{2}\right) r_{12}\right) \\
& \left.+v_{12}^{i} \frac{G^{2} m_{1} m_{2}}{r_{12}^{2}}\left(-2\left(n_{12} v_{1}\right)+6\left(n_{12} v_{12}\right) \frac{r_{1}^{2}}{r_{12}^{2}}\right)\right]+1 \leftrightarrow 2+\mathcal{O}\left(\frac{1}{c^{7}}\right), \\
& k_{i j}=\frac{2 G m_{1}}{c^{2} r_{1}} \delta^{i j}+\frac{1}{c^{4}}\left[\delta ^ { i j } \left(-\frac{G m_{1}}{r_{1}}\left(n_{1} v_{1}\right)^{2}+\frac{G^{2} m_{1}^{2}}{r_{1}^{2}}\right.\right. \\
& \left.+G^{2} m_{1} m_{2}\left(\frac{2}{r_{1} r_{2}}-\frac{r_{1}}{2 r_{12}^{3}}+\frac{r_{1}^{2}}{2 r_{2} r_{12}^{3}}-\frac{5}{2 r_{1} r_{12}}+\frac{4}{r_{12} S}\right)\right) \\
& +4 \frac{G m_{1}}{r_{1}} v_{1}^{i} v_{1}^{j}+\frac{G^{2} m_{1}^{2}}{r_{1}^{2}} n_{1}^{i} n_{1}^{j}-4 G^{2} m_{1} m_{2} n_{12}^{i} n_{12}^{j}\left(\frac{1}{S^{2}}+\frac{1}{r_{12} S}\right) \\
& \left.+\frac{4 G^{2} m_{1} m_{2}}{S^{2}}\left(n_{1}^{(i} n_{2}^{j)}+2 n_{1}^{(i} n_{12}^{j)}\right)\right]
\end{aligned}
$$




$$
+\frac{G^{2} m_{1} m_{2}}{c^{5} r_{12}^{2}}\left(-\frac{2}{3}\left(n_{12} v_{12}\right) \delta^{i j}-6\left(n_{12} v_{12}\right) n_{12}^{i} n_{12}^{j}+8 n_{12}^{(i} v_{12}^{j)}\right)+1 \leftrightarrow 2+\mathcal{O}\left(\frac{1}{c^{6}}\right)
$$

Here we pose $S=r_{1}+r_{2}+r_{12}$ and $1 \leftrightarrow 2$ refers to the same quantity but with all particle labels exchanged. To higher order one needs the solution of elementary equations still more intricate than (239) and the 3PN metric valid in closed form all over the near zone is not currently known.

Let us also display the latter $2.5 \mathrm{PN}$ metric computed at the location of the particle 1 for instance, thanks to the Hadamard self-field regularization, i.e., in the sense of the Hadamard partie finie defined by Eq. (160). We get

$$
\begin{aligned}
& \left(k_{00}\right)_{1}=\frac{2 G m_{2}}{c^{2} r_{12}}+\frac{G m_{2}}{c^{4} r_{12}}\left(4 v_{2}^{2}-\left(n_{12} v_{2}\right)^{2}-3 \frac{G m_{1}}{r_{12}}-2 \frac{G m_{2}}{r_{12}}\right) \\
& +\frac{8 G^{2} m_{1} m_{2}}{3 c^{5} r_{12}^{2}}\left(n_{12} v_{12}\right)+\frac{G m_{2}}{c^{6} r_{12}}\left(\frac{3}{4}\left(n_{12} v_{2}\right)^{4}-3\left(n_{12} v_{2}\right)^{2} v_{2}^{2}+4 v_{2}^{4}\right) \\
& +\frac{G^{2} m_{1} m_{2}}{c^{6} r_{12}^{2}}\left(-\frac{87}{4}\left(n_{12} v_{1}\right)^{2}+\frac{47}{2}\left(n_{12} v_{1}\right)\left(n_{12} v_{2}\right)-\frac{55}{4}\left(n_{12} v_{2}\right)^{2}+\frac{23}{4} v_{1}^{2}-\frac{39}{2}\left(v_{1} v_{2}\right)\right) \\
& +\frac{47}{4} \frac{G^{2} m_{1} m_{2}}{c^{6} r_{12}^{2}} v_{2}^{2}+\frac{G m_{2}}{c^{6} r_{12}}\left\{\frac{G m_{2}}{r_{12}}\left[3\left(n_{12} v_{2}\right)^{2}-v_{2}^{2}\right]-\frac{G^{2} m_{1}^{2}}{r_{12}^{2}}+\frac{17}{2} \frac{G^{2} m_{1} m_{2}}{r_{12}^{2}}+2 \frac{G^{2} m_{2}^{2}}{r_{12}^{2}}\right\} \\
& +\frac{G^{2} m_{1} m_{2}}{c^{7} r_{12}^{2}}\left\{-20\left(n_{12} v_{1}\right)^{3}+40\left(n_{12} v_{1}\right)^{2}\left(n_{12} v_{2}\right)-36\left(n_{12} v_{1}\right)\left(n_{12} v_{2}\right)^{2}+16\left(n_{12} v_{2}\right)^{3}\right. \\
& +\frac{296}{15}\left(n_{12} v_{1}\right) v_{1}^{2}-\frac{116}{15}\left(n_{12} v_{2}\right) v_{1}^{2}-\frac{104}{5}\left(n_{12} v_{1}\right)\left(v_{1} v_{2}\right)+\frac{232}{15}\left(n_{12} v_{2}\right)\left(v_{1} v_{2}\right) \\
& +\frac{56}{15}\left(n_{12} v_{1}\right) v_{2}^{2}-\frac{52}{5}\left(n_{12} v_{2}\right) v_{2}^{2}+\frac{G m_{1}}{r_{12}}\left(-\frac{64}{5}\left(n_{12} v_{1}\right)+\frac{104}{5}\left(n_{12} v_{2}\right)\right) \\
& \left.+\frac{G m_{2}}{r_{12}}\left(-\frac{144}{5}\left(n_{12} v_{1}\right)+\frac{392}{15}\left(n_{12} v_{2}\right)\right)\right\}+\mathcal{O}\left(\frac{1}{c^{8}}\right) \\
& \left(k_{0 i}\right)_{1}=-\frac{4 G m_{2}}{c^{3} r_{12}} v_{2}^{i}+\frac{G m_{2}}{c^{5} r_{12}}\left\{n_{12}^{i}\left[\frac{G m_{1}}{r_{12}}\left(10\left(n_{12} v_{1}\right)+2\left(n_{12} v_{2}\right)\right)-\frac{G m_{2}}{r_{12}}\left(n_{12} v_{2}\right)\right]\right\} \\
& +\frac{G m_{2}}{c^{5} r_{12}}\left\{4 \frac{G m_{1}}{r_{12}} v_{1}^{i}+v_{2}^{i}\left(2\left(n_{12} v_{2}\right)^{2}-4 v_{2}^{2}-2 \frac{G m_{1}}{r_{12}}+\frac{G m_{2}}{r_{12}}\right)\right\} \\
& +\frac{G^{2} m_{1} m_{2}}{c^{6} r_{12}^{2}}\left\{n _ { 1 2 } ^ { i } \left(10\left(n_{12} v_{1}\right)^{2}-8\left(n_{12} v_{1}\right)\left(n_{12} v_{2}\right)-2\left(n_{12} v_{2}\right)^{2}-6 v_{1}^{2}\right.\right. \\
& \left.+4\left(v_{1} v_{2}\right)+2 v_{2}^{2}-\frac{8}{3} \frac{G m_{1}}{r_{12}}+\frac{4}{3} \frac{G m_{2}}{r_{12}}\right) \\
& \left.-8\left(n_{12} v_{1}\right) v_{1}^{i}+v_{2}^{i}\left(\frac{20}{3}\left(n_{12} v_{1}\right)+\frac{4}{3}\left(n_{12} v_{2}\right)\right)\right\}+\mathcal{O}\left(\frac{1}{c^{7}}\right), \\
& \left(k_{i j}\right)_{1}=\frac{2 G m_{2}}{c^{2} r_{12}} \delta^{i j}+\frac{G m_{2}}{c^{4} r_{12}} \delta^{i j}\left(-\left(n_{12} v_{2}\right)^{2}+\frac{G m_{1}}{r_{12}}+\frac{G m_{2}}{r_{12}}\right) \\
& +\frac{G m_{2}}{c^{4} r_{12}}\left\{n_{12}^{i j}\left(-8 \frac{G m_{1}}{r_{12}}+\frac{G m_{2}}{r_{12}}\right)+4 v_{2}^{i j}\right\}-\frac{4 G^{2} m_{1} m_{2}}{3 c^{5} r_{12}^{2}} \delta^{i j}\left(n_{12} v_{12}\right) \\
& +\frac{G^{2} m_{1} m_{2}}{c^{5} r_{12}^{2}}\left\{-12 n_{12}^{i j}\left(n_{12} v_{12}\right)+16 n_{12}^{(i} v_{12}^{j)}\right\}+\mathcal{O}\left(\frac{1}{c^{6}}\right) \text {. }
\end{aligned}
$$

When regularized at the location of the particles, the metric can be computed to higher order, for instance 3PN. We shall need it when we compute the so-called redshift observable in Sections (8.3) and (8.4); indeed, see Eq. (276). 


\section{Conservative Dynamics of Compact Binaries}

\subsection{Concept of innermost circular orbit}

Having in hand the conserved energy $E(x)$ for circular orbits given by Eq. (232), or even more accurate by (233), we define the innermost circular orbit (ICO) as the minimum, when it exists, of the energy function $E(x)$ - see e.g., Ref. [51]. Notice that the ICO is not defined as a point of dynamical general-relativistic unstability. Hence, we prefer to call this point the ICO rather than, strictly speaking, an innermost stable circular orbit or ISCO. A study of the dynamical stability of circular binary orbits in the post-Newtonian approximation is reported in Section 8.2.

The previous definition of the ICO is motivated by the comparison with some results of numerical relativity. Indeed we shall confront the prediction of the standard (Taylor-based) post-Newtonian approximation with numerical computations of the energy of binary black holes under the assumptions of conformal flatness for the spatial metric and of exactly circular orbits $[228,232,133,121]$. The latter restriction is implemented by requiring the existence of an "helical" Killing vector (HKV), which is time-like inside the light cylinder associated with the circular motion, and space-like outside. The HKV will be defined in Eq. (273) below. In the numerical approaches of Refs. [228, 232, 133, 121] there are no gravitational waves, the field is periodic in time, and the gravitational potentials tend to zero at spatial infinity within a restricted model equivalent to solving five out of the ten Einstein field equations (the so-called Isenberg-WilsonMathews approximation; see Ref. [228] for a discussion). Considering an evolutionary sequence of equilibrium configurations the circular-orbit energy $E(\Omega)$ and the ICO of binary black holes are obtained numerically (see also Refs. [92, 229, 301] for related calculations of binary neutron stars and strange quark stars).

Since the numerical calculations [232, 133] have been performed in the case of two corotating black holes, which are spinning essentially with the orbital angular velocity, we must for the comparison include within our post-Newtonian formalism the effects of spins appropriate to two Kerr black holes rotating at the orbital rate. The total relativistic masses of the two Kerr black holes (with a $=1,2$ labelling the black holes) are given by ${ }^{54}$

$$
m_{\mathrm{a}}^{2}=\mu_{\mathrm{a}}^{2}+\frac{S_{\mathrm{a}}^{2}}{4 \mu_{\mathrm{a}}^{2}} .
$$

We assume the validity of the Christodoulou mass formula for Kerr black holes [127, 129]; i.e., we neglect the influence of the companion. Here $S_{\mathrm{a}}$ is the spin, related to the usual Kerr parameter by $S_{\mathrm{a}}=m_{\mathrm{a}} a_{\mathrm{a}}$, and $\mu_{\mathrm{a}} \equiv m_{\mathrm{a}}^{\mathrm{irr}}$ is the irreducible mass, not to be confused with the reduced mass of the binary system, and given by $4 \pi \mu_{\mathrm{a}}=\sqrt{A_{\mathrm{a}}}\left(A_{\mathrm{a}}\right.$ is the hole's surface area). The angular velocity of the black hole, defined by the angular velocity of the outgoing photons that remain for ever at the location of the light-like horizon, is

$$
\omega_{\mathrm{a}}=\left.\frac{\partial m_{\mathrm{a}}}{\partial S_{\mathrm{a}}}\right|_{\mu_{\mathrm{a}}}=\frac{S_{\mathrm{a}}}{4 m_{\mathrm{a}} \mu_{\mathrm{a}}^{2}} .
$$

We shall give in Eq. (284) below a more general formulation of the "internal structure" of the black holes. Combining Eqs. (243) - (244) we obtain $m_{\mathrm{a}}$ and $S_{\mathrm{a}}$ as functions of $\mu_{\mathrm{a}}$ and $\omega_{\mathrm{a}}$,

$$
\begin{aligned}
& m_{\mathrm{a}}=\frac{\mu_{\mathrm{a}}}{\sqrt{1-4 \mu_{\mathrm{a}}^{2} \omega_{\mathrm{a}}^{2}}}, \\
& S_{\mathrm{a}}=\frac{4 \mu_{\mathrm{a}}^{3} \omega_{\mathrm{a}}}{\sqrt{1-4 \mu_{\mathrm{a}}^{2} \omega_{\mathrm{a}}^{2}}} .
\end{aligned}
$$

\footnotetext{
${ }^{54}$ In all of Section 8 we pose $G=1=c$.
} 
In the limit of slow rotation we get

$$
\begin{aligned}
S_{\mathrm{a}} & =I_{\mathrm{a}} \omega_{\mathrm{a}}+\mathcal{O}\left(\omega_{\mathrm{a}}^{3}\right), \\
m_{\mathrm{a}} & =\mu_{\mathrm{a}}+\frac{1}{2} I_{\mathrm{a}} \omega_{\mathrm{a}}^{2}+\mathcal{O}\left(\omega_{\mathrm{a}}^{4}\right),
\end{aligned}
$$

where $I_{\mathrm{a}}=4 \mu_{\mathrm{a}}^{3}$ is the moment of inertia of the black hole. We see that the total mass-energy $m_{\mathrm{a}}$ involves the irreducible mass augmented by the usual kinetic energy of the spin.

We now need the relation between the rotation frequency $\omega_{\mathrm{a}}$ of each of the corotating black holes and the orbital frequency $\Omega$ of the binary system. Indeed $\Omega$ is the basic variable describing each equilibrium configuration calculated numerically in Refs. [232, 133], with the irreducible masses held constant along the numerical evolutionary sequences. Here we report the result of an investigation of the condition for corotation based on the first law of mechanics for spinning black holes [55], which concluded that the corotation condition at $2 \mathrm{PN}$ order reads

$$
\omega_{\mathrm{a}}=\Omega\left\{1-\nu x+\nu\left(-\frac{3}{2}+\frac{\nu}{3}\right) x^{2}+\mathcal{O}\left(x^{3}\right)\right\},
$$

where $x$ denotes the post-Newtonian parameter (230) and $\nu$ the symmetric mass ratio (215). The condition (247) is issued from the general relation which will be given in Eq. (285). Interestingly, notice that $\omega_{1}=\omega_{2}$ up to the rather high $2 \mathrm{PN}$ order. In the Newtonian limit $x \rightarrow 0$ or the test-particle limit $\nu \rightarrow 0$ we simply have $\omega_{\mathrm{a}}=\Omega$, in agreement with physical intuition.

To take into account the spin effects our first task is to replace all the masses entering the energy function (232) by their equivalent expressions in terms of $\omega_{\mathrm{a}}$ and the irreducible masses $\mu_{\mathrm{a}}$, and then to replace $\omega_{\mathrm{a}}$ in terms of $\Omega$ according to the corotation prescription (247). ${ }^{55}$ It is clear that the leading contribution is that of the spin kinetic energy given in Eq. (246b), and it comes from the replacement of the rest mass-energy $m=m_{1}+m_{2}$. From Eq. (246b) this effect is of order $\Omega^{2}$ in the case of corotating binaries, which means by comparison with Eq. (232) that it is equivalent to an "orbital" effect at the $2 \mathrm{PN}$ order (i.e., $\propto x^{2}$ ). Higher-order corrections in Eq. (246b), which behave at least like $\Omega^{4}$, will correspond to the orbital $5 \mathrm{PN}$ order at least and are negligible for the present purpose. In addition there will be a subdominant contribution, of the order of $\Omega^{8 / 3}$ equivalent to $3 \mathrm{PN}$ order, which comes from the replacement of the masses into the Newtonian part, proportional to $x \propto \Omega^{2 / 3}$, of the energy $E$; see Eq. (232). With the 3PN accuracy we do not need to replace the masses that enter into the post-Newtonian corrections in $E$, so in these terms the masses can be considered to be the irreducible ones.

Our second task is to include the specific relativistic effects due to the spins, namely the spinorbit (SO) interaction and the spin-spin (SS) one. In the case of spins $S_{1}$ and $S_{2}$ aligned parallel to the orbital angular momentum (and right-handed with respect to the sense of motion) the SO energy reads

$$
E_{\mathrm{SO}}=-m \nu(m \Omega)^{5 / 3}\left[\left(\frac{4}{3} \frac{m_{1}^{2}}{m^{2}}+\nu\right) \frac{S_{1}}{m_{1}^{2}}+\left(\frac{4}{3} \frac{m_{2}^{2}}{m^{2}}+\nu\right) \frac{S_{2}}{m_{2}^{2}}\right] .
$$

We shall review in Section 11 the most up-to-date results for the spin-orbit energy and related quantities; here we are simply employing the leading-order formula obtained in Refs. [27, 28, 275, 271] and given by the first term in Eq. (415). We immediately infer from this formula that in the case of corotating black holes the SO effect is equivalent to a $3 \mathrm{PN}$ orbital effect and thus must be retained with the present accuracy. With this approximation, the masses in Eq. (248) can be replaced by the irreducible ones. As for the SS interaction (still in the case of spins aligned with

\footnotetext{
55 Note that this is an iterative process because the masses in Eq. (247) are themselves to be replaced by the irreducible masses.
} 
the orbital angular momentum) it is given by

$$
E_{\mathrm{SS}}=\mu \nu(m \Omega)^{2} \frac{S_{1} S_{2}}{m_{1}^{2} m_{2}^{2}} .
$$

The SS effect can be neglected here because it is of order 5PN for corotating systems. Summing up all the spin contributions to $3 \mathrm{PN}$ order we find that the supplementary energy due to the corotating spins is $[51,55]^{56}$

$$
\Delta E^{\mathrm{corot}}=\mu x_{\mu}\left[(2-6 \eta) x_{\mu}^{2}+\eta(-10+25 \eta) x_{\mu}^{3}+\mathcal{O}\left(x_{\mu}^{4}\right)\right] .
$$

The total mass $\mu=\mu_{1}+\mu_{2}$, the symmetric mass ratio $\eta=\mu_{1} \mu_{2} / \mu^{2}$, and the dimensionless invariant post-Newtonian parameter $x_{\mu}=(\mu \Omega)^{2 / 3}$ are now expressed in terms of the irreducible masses $\mu_{\mathrm{a}}$, rather than the masses $m_{\mathrm{a}}$. The complete 3PN energy of the corotating binary is finally given by the sum of Eqs. (232) and (250), in which all the masses are now understood as being the irreducible ones, which must be assumed to stay constant when the binary evolves for the comparison with the numerical calculation.

The left panel of Figure 1 shows the results for $E_{\mathrm{ICO}}$ in the case of irrotational and corotational binaries. Since $\Delta E^{\text {corot }}$, given by Eq. (250), is at least of order $2 \mathrm{PN}$, the result for $1 \mathrm{PN}^{\text {corot }}$ is the same as for $1 \mathrm{PN}$ in the irrotational case; then, obviously, $2 \mathrm{PN}^{\text {corot }}$ takes into account only the leading $2 \mathrm{PN}$ corotation effect, i.e., the spin kinetic energy given by Eq. (246b), while $3 \mathrm{PN}^{\text {corot }}$ involves also, in particular, the corotational SO coupling at the $3 \mathrm{PN}$ order. In addition we present the numerical point obtained by numerical relativity under the assumptions of conformal flatness and of helical symmetry $[228,232]$. As we can see the $3 \mathrm{PN}$ points, and even the $2 \mathrm{PN}$ ones, are in good agreement with the numerical value. The fact that the $2 \mathrm{PN}$ and $3 \mathrm{PN}$ values are so close to each other is a good sign of the convergence of the expansion. In fact one might say that the role of the $3 \mathrm{PN}$ approximation is merely to "confirm" the value already given by the $2 \mathrm{PN}$ one (but of course, had we not computed the $3 \mathrm{PN}$ term, we would not be able to trust very much the $2 \mathrm{PN}$ value). As expected, the best agreement we obtain is for the $3 \mathrm{PN}$ approximation and in the case of corotation, i.e., the point $3 \mathrm{PN}^{\text {corot }}$. However, the $1 \mathrm{PN}$ approximation is clearly not precise enough, but this is not surprising in the highly relativistic regime of the ICO. The right panel of Figure 1 shows other very interesting comparisons with numerical relativity computations [133, 121], done not only for the case of corotational binaries but also in the irrotational (non-spinning) case. Witness in particular the almost perfect agreement between the standard 3PN point (PN standard, shown with a green triangle) and the numerical quasi-equilibrium point (QE, red triangle) in the case of irrotational non-spinning (NS) binaries.

However, we recall that the numerical works $[228,232,133,121]$ assume that the spatial metric is conformally flat, which is incompatible with the post-Newtonian approximation starting from the 2PN order (see [196] for a discussion). Nevertheless, the agreement found in Figure 1 constitutes an appreciable improvement of the previous situation, because the first estimations of the ICO in post-Newtonian theory [274] and numerical relativity [132, 342, 29] disagreed with each other, and do not match with the present $3 \mathrm{PN}$ results.

\subsection{Dynamical stability of circular orbits}

In this section, following Ref. [79], we shall investigate the problem of the stability, against dynamical perturbations, of circular orbits at the 3PN order. We propose to use two different methods, one based on a linear perturbation at the level of the center-of-mass equations of motion (219) (220) in (standard) harmonic coordinates, the other one consisting of perturbing the Hamiltonian

\footnotetext{
56 In Ref. [51] it was assumed that the corotation condition was given by the leading-order result $\omega_{\mathrm{a}}=\Omega$. The 1PN correction in Eq. (247) modifies the 3PN terms in Eq. (250) with respect to the result of Ref. [51].
} 

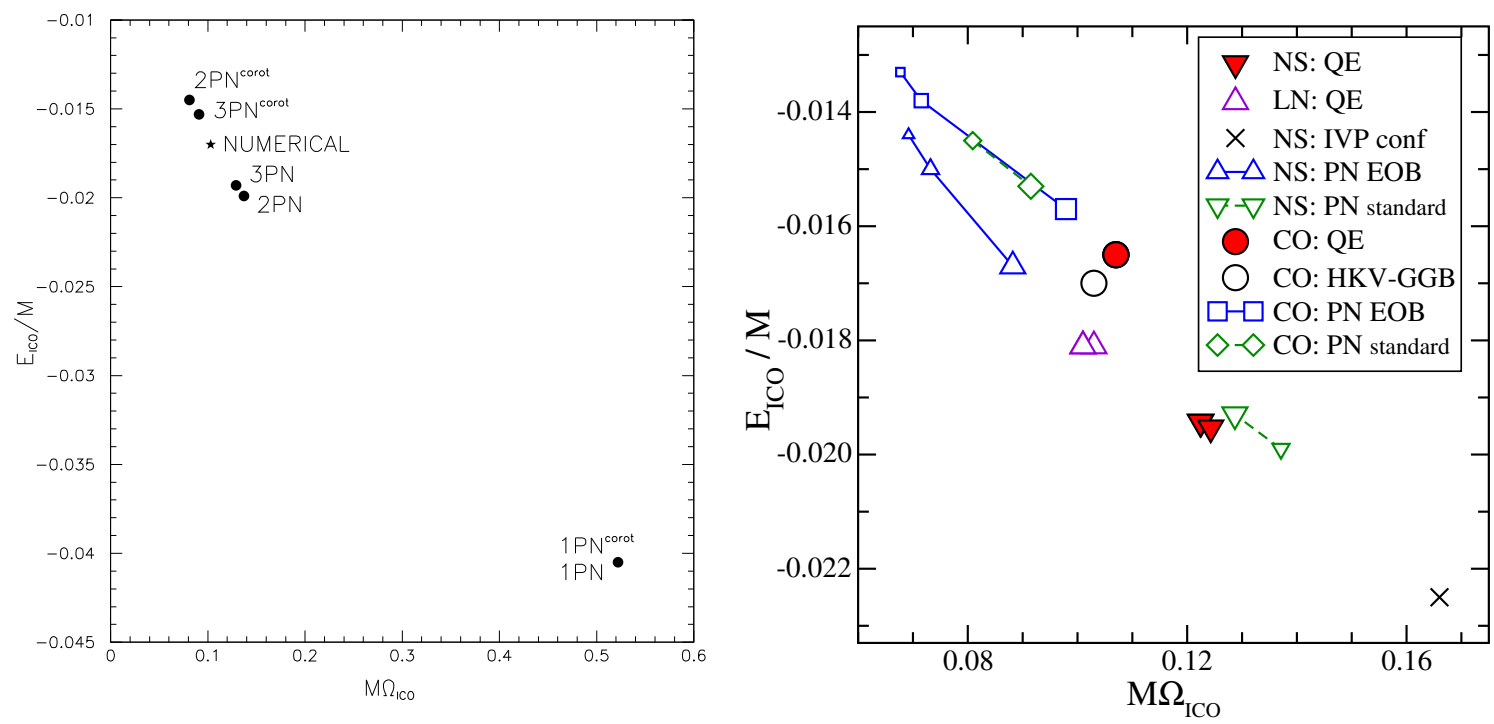

Figure 1: The binding energy $E_{\mathrm{ICO}}$ versus $\Omega_{\mathrm{ICO}}$ in the equal-mass case $(\nu=1 / 4)$. Left panel: Comparison with the numerical relativity result of Gourgoulhon, Grandclément et al. [228, 232] valid in the corotating case (marked by a star). Points indicated by $n \mathrm{PN}$ are computed from the minimum of Eq. (232), and correspond to irrotational binaries. Points denoted by $n \mathrm{PN}^{\text {corot }}$ come from the minimum of the sum of Eqs. (232) and (250), and describe corotational binaries. Note the very good convergence of the standard (Taylor-expanded) PN series. Right panel: Numerical relativity results of Cook, Pfeiffer et al. [133, 121] for quasi-equilibrium (QE) configurations and various boundary conditions for the lapse function, in the non-spinning (NS), leading-order non spinning (LN) and corotating (CO) cases. The point from [228, 232] (HKV-GGB) is also reported as in the left panel, together with IVP, the initial value approach with effective potential [132, 342], as well as standard PN predictions from the left panel and non-standard (EOB) ones. The agreement between the QE computation and the standard non-resummed 3PN point is excellent especially in the irrotational NS case.

equations in ADM coordinates for the center-of-mass Hamiltonian (223). We shall find a criterion for the stability of circular orbits and shall present it in an invariant way - the same in different coordinate systems. We shall check that our two methods agree on the result.

We deal first with the perturbation of the equations of motion, following Kidder, Will \& Wiseman [275] (see their Section III.A). We introduce polar coordinates $(r, \varphi)$ in the orbital plane and pose $u \equiv \dot{r}$ and $\Omega \equiv \dot{\varphi}$. Then Eq. (219) yields the system of equations

$$
\begin{aligned}
\dot{r} & =u, \\
\dot{u} & =-\frac{G m}{r^{2}}[1+\mathcal{A}+\mathcal{B} u]+r \Omega^{2}, \\
\dot{\Omega} & =-\Omega\left[\frac{G m}{r^{2}} \mathcal{B}+\frac{2 u}{r}\right],
\end{aligned}
$$

where $\mathcal{A}$ and $\mathcal{B}$ are given by Eqs. (220) as functions of $r, u$ and $\Omega$ (through $v^{2}=u^{2}+r^{2} \Omega^{2}$ ).

In the case of an orbit that is circular apart from the adiabatic inspiral at the $2.5 \mathrm{PN}$ order (we neglect the radiation-reaction damping effects), we have $\dot{r}_{0}=\dot{u}_{0}=\dot{\Omega}_{0}=0$ hence $u_{0}=0$. In this section we shall indicate quantities associated with the circular orbit, which constitutes the zero-th approximation in our perturbation scheme, using the subscript 0. Hence Eq. (251b) gives 
the angular velocity $\Omega_{0}$ of the circular orbit as

$$
\Omega_{0}^{2}=\frac{G m}{r_{0}^{3}}\left(1+\mathcal{A}_{0}\right) \text {. }
$$

Solving iteratively this relation at the $3 \mathrm{PN}$ order using the equations of motion (219) - (220), we obtain $\Omega_{0}$ as a function of the circular-orbit radius $r_{0}$ in standard harmonic coordinates; the result agrees with Eq. (228). ${ }^{57}$

We now investigate the linear perturbation around the circular orbit defined by the constants $r_{0}, u_{0}=0$ and $\Omega_{0}$. We pose

$$
\begin{aligned}
r & =r_{0}+\delta r, \\
u & =\delta u, \\
\Omega & =\Omega_{0}+\delta \Omega,
\end{aligned}
$$

where $\delta r, \delta u$ and $\delta \Omega$ denote the linear perturbations of the circular orbit. Then a system of linear equations readily follows:

$$
\begin{aligned}
\dot{\delta r} & =\delta u \\
\dot{\delta u} & =\alpha_{0} \delta r+\beta_{0} \delta \Omega, \\
\dot{\delta} \Omega & =\gamma_{0} \delta u
\end{aligned}
$$

where the coefficients, which solely depend on the unperturbed circular orbit (hence the added subscript 0), read as [275]

$$
\begin{aligned}
\alpha_{0} & =3 \Omega_{0}^{2}-\frac{G m}{r_{0}^{2}}\left(\frac{\partial \mathcal{A}}{\partial r}\right)_{0}, \\
\beta_{0} & =2 r_{0} \Omega_{0}-\frac{G m}{r_{0}^{2}}\left(\frac{\partial \mathcal{A}}{\partial \Omega}\right)_{0}, \\
\gamma_{0} & =-\Omega_{0}\left[\frac{2}{r_{0}}+\frac{G m}{r_{0}^{2}}\left(\frac{\partial \mathcal{B}}{\partial u}\right)_{0}\right] .
\end{aligned}
$$

In obtaining these equations we use the fact that $\mathcal{A}$ is a function of the square $u^{2}$ through $v^{2}=u^{2}+r^{2} \Omega^{2}$, so that $\partial \mathcal{A} / \partial u$ is proportional to $u$ and thus vanishes in the unperturbed configuration (because $u=\delta u$ ). On the other hand, since the radiation reaction is neglected, $\mathcal{B}$ is also proportional to $u$ [see Eq. (220b)], so only $\partial \mathcal{B} / \partial u$ can contribute at the zero-th perturbative order. Now by examining the fate of perturbations that are proportional to some $e^{i \sigma t}$, we arrive at the condition for the frequency $\sigma$ of the perturbation to be real, and hence for stable circular orbits to exist, as being [275]

$$
\hat{C}_{0} \equiv-\alpha_{0}-\beta_{0} \gamma_{0}>0 .
$$

Substituting into this $\mathcal{A}$ and $\mathcal{B}$ at the $3 \mathrm{PN}$ order we then arrive at the orbital-stability criterion

$$
\begin{aligned}
\hat{C}_{0}=\frac{G m}{r_{0}^{3}}\{1 & +\frac{G m}{r_{0} c^{2}}(-9+\nu)+\frac{G^{2} m^{2}}{r_{0}^{2} c^{4}}\left(30+\frac{65}{4} \nu+\nu^{2}\right) \\
& \left.+\frac{G^{3} m^{3}}{r_{0}^{3} c^{6}}\left(-70+\left[-\frac{29927}{840}-\frac{451}{64} \pi^{2}+22 \ln \left(\frac{r_{0}}{r_{0}^{\prime}}\right)\right] \nu+\frac{19}{2} \nu^{2}+\nu^{3}\right)+\mathcal{O}\left(\frac{1}{c^{8}}\right)\right\},
\end{aligned}
$$

57 One should not confuse the circular-orbit radius $r_{0}$ with the constant $r_{0}^{\prime}$ entering the logarithm at the 3PN order in Eq. (228) and which is defined by Eq. (221). 
where we recall that $r_{0}$ is the radius of the orbit in harmonic coordinates.

Our second method is to use the Hamiltonian equations associated with the 3PN center-of-mass Hamiltonian in ADM coordinates $H^{\mathrm{ADM}}$ given by Eq. (223). We introduce the polar coordinates $(R, \Psi)$ in the orbital plane - we assume that the orbital plane is equatorial, given by $\Theta=\frac{\pi}{2}$ in the spherical coordinate system $(R, \Theta, \Psi)$ - and make the substitution

$$
P^{2}=P_{R}^{2}+\frac{P_{\Psi}^{2}}{R^{2}}
$$

This yields a reduced Hamiltonian that is a function of $R, P_{R}$ and $P_{\Psi}$, and describes the motion in polar coordinates in the orbital plane; henceforth we denote it by $\mathcal{H}=\mathcal{H}\left[R, P_{R}, P_{\Psi}\right] \equiv H^{\mathrm{ADM}} / \mu$. The Hamiltonian equations then read

$$
\begin{aligned}
\frac{\mathrm{d} R}{\mathrm{~d} t} & =\frac{\partial \mathcal{H}}{\partial P_{R}}, \\
\frac{\mathrm{d} \Psi}{\mathrm{d} t} & =\frac{\partial \mathcal{H}}{\partial P_{\Psi}}, \\
\frac{\mathrm{d} P_{R}}{\mathrm{~d} t} & =-\frac{\partial \mathcal{H}}{\partial R}, \\
\frac{\mathrm{d} P_{\Psi}}{\mathrm{d} t} & =0 .
\end{aligned}
$$

Evidently the constant $P_{\Psi}$ is nothing but the conserved angular-momentum integral. For circular orbits we have $R=R_{0}$ (a constant) and $P_{R}=0$, so

$$
\frac{\partial \mathcal{H}}{\partial R}\left[R_{0}, 0, P_{\Psi}^{0}\right]=0
$$

which gives the angular momentum $P_{\Psi}^{0}$ of the circular orbit as a function of $R_{0}$, and

$$
\Omega_{0} \equiv\left(\frac{\mathrm{d} \Psi}{\mathrm{d} t}\right)_{0}=\frac{\partial \mathcal{H}}{\partial P_{\Psi}}\left[R_{0}, 0, P_{\Psi}^{0}\right],
$$

which yields the angular frequency of the circular orbit $\Omega_{0}$, which is evidently the same numerical quantity as in Eq. (252), but is here expressed in terms of the separation $R_{0}$ in ADM coordinates. The last equation, which is equivalent to $R=$ const $=R_{0}$, is

$$
\frac{\partial \mathcal{H}}{\partial P_{R}}\left[R_{0}, 0, P_{\Psi}^{0}\right]=0 \text {. }
$$

It is automatically verified because $\mathcal{H}$ is a quadratic function of $P_{R}$ and hence $\partial \mathcal{H} / \partial P_{R}$ is zero for circular orbits.

We consider now a perturbation of the circular orbit defined by

$$
\begin{aligned}
P_{R} & =\delta P_{R}, \\
P_{\Psi} & =P_{\Psi}^{0}+\delta P_{\Psi}, \\
R & =R_{0}+\delta R, \\
\Omega & =\Omega_{0}+\delta \Omega .
\end{aligned}
$$

The Hamiltonian equations (259), worked out at the linearized order, read as

$$
\delta \dot{P}_{R}=-\pi_{0} \delta R-\rho_{0} \delta P_{\Psi},
$$




$$
\begin{aligned}
\delta \dot{P}_{\Psi} & =0, \\
\dot{\delta R} & =\sigma_{0} \delta P_{R}, \\
\delta \Omega & =\rho_{0} \delta R+\tau_{0} \delta P_{\Psi},
\end{aligned}
$$

where the coefficients, which depend on the unperturbed orbit, are given by

$$
\begin{aligned}
\pi_{0} & =\frac{\partial^{2} \mathcal{H}}{\partial R^{2}}\left[R_{0}, 0, P_{\Psi}^{0}\right], \\
\rho_{0} & =\frac{\partial^{2} \mathcal{H}}{\partial R \partial P_{\Psi}}\left[R_{0}, 0, P_{\Psi}^{0}\right], \\
\sigma_{0} & =\frac{\partial^{2} \mathcal{H}}{\partial P_{R}^{2}}\left[R_{0}, 0, P_{\Psi}^{0}\right], \\
\tau_{0} & =\frac{\partial^{2} \mathcal{H}}{\partial P_{\Psi}^{2}}\left[R_{0}, 0, P_{\Psi}^{0}\right] .
\end{aligned}
$$

By looking to solutions proportional to some $e^{i \sigma t}$ one obtains some real frequencies, and therefore one finds stable circular orbits, if and only if

$$
\hat{C}_{0} \equiv \pi_{0} \sigma_{0}>0 \text {. }
$$

Using explicitly the Hamiltonian (223) we readily obtain

$$
\begin{aligned}
\hat{C}_{0}=\frac{G m}{R_{0}^{3}}\{1 & +\frac{G m}{R_{0} c^{2}}(-9+\nu)+\frac{G^{2} m^{2}}{R_{0}^{2} c^{4}}\left(\frac{117}{4}+\frac{43}{8} \nu+\nu^{2}\right) \\
& \left.+\frac{G^{3} m^{3}}{R_{0}^{3} c^{6}}\left(-61+\left[\frac{4777}{48}-\frac{325}{64} \pi^{2}\right] \nu-\frac{31}{8} \nu^{2}+\nu^{3}\right)+\mathcal{O}\left(\frac{1}{c^{8}}\right)\right\} .
\end{aligned}
$$

This result does not look the same as our previous result (257), but this is simply due to the fact that it depends on the ADM radial separation $R_{0}$ instead of the harmonic one $r_{0}$. Fortunately we have derived in Section 7.2 the material needed to connect $R_{0}$ to $r_{0}$ with the 3PN accuracy. Indeed, with Eqs. (210) we have the relation valid for general orbits in an arbitrary frame between the separation vectors in both coordinate systems. Specializing that relation to circular orbits we find

$R_{0}=r_{0}\left\{1+\frac{G^{2} m^{2}}{r_{0}^{2} c^{4}}\left(-\frac{1}{4}-\frac{29}{8} \nu\right)+\frac{G^{3} m^{3}}{r_{0}^{3} c^{6}}\left(\left[\frac{3163}{1680}+\frac{21}{32} \pi^{2}-\frac{22}{3} \ln \left(\frac{r_{0}}{r_{0}^{\prime}}\right)\right] \nu+\frac{3}{8} \nu^{2}\right)+\mathcal{O}\left(\frac{1}{c^{8}}\right)\right\}$.

Note that the difference between $R_{0}$ and $r_{0}$ starts only at $2 \mathrm{PN}$ order. That relation easily permits to perfectly reconcile both expressions (257) and (267).

Finally let us give to $\hat{C}_{0}$ an invariant meaning by expressing it with the help of the orbital frequency $\Omega_{0}$ of the circular orbit, or, more conveniently, of the frequency-related parameter $x_{0} \equiv$ $\left(G m \Omega_{0} / c^{3}\right)^{2 / 3}$ - cf. Eq. (230). This allows us to write the criterion for stability as $C_{0}>0$, where $C_{0}=\frac{G^{2} m^{2}}{x_{0}^{3}} \hat{C}_{0}$ admits the gauge-invariant form

$$
C_{0}=1-6 x_{0}+14 \nu x_{0}^{2}+\left(\left[\frac{397}{2}-\frac{123}{16} \pi^{2}\right] \nu-14 \nu^{2}\right) x_{0}^{3}+\mathcal{O}\left(x_{0}^{4}\right) .
$$

This form is more interesting than the coordinate-dependent expressions (257) or (267), not only because of its invariant form, but also because as we see the 1PN term yields exactly the Schwarzschild 
result that the innermost stable circular orbit or ISCO of a test particle (i.e., in the limit $\nu \rightarrow 0$ ) is located at $x_{\mathrm{ISCO}}=\frac{1}{6}$. Thus we find that, at the $1 \mathrm{PN}$ order, but for any mass ratio $\nu$,

$$
x_{\mathrm{ISCO}}^{\mathrm{PPN}}=\frac{1}{6}
$$

One could have expected that some deviations of the order of $\nu$ already occur at the $1 \mathrm{PN}$ order, but it turns out that only from the $2 \mathrm{PN}$ order does one find the occurrence of some nonSchwarzschildean corrections proportional to $\nu$. At the $2 \mathrm{PN}$ order we obtain

$$
x_{\mathrm{ISCO}}^{2 \mathrm{PN}}=\frac{3}{14 \nu}\left(1-\sqrt{1-\frac{14 \nu}{9}}\right) .
$$

For equal masses this gives $x_{\mathrm{ISCO}}^{2 \mathrm{PN}} \simeq 0.187$. Notice also that the effect of the finite mass corrections is to increase the frequency of the ISCO with respect to the Schwarzschild result, i.e., to make it more inward: 58

$$
x_{\mathrm{ISCO}}^{2 \mathrm{PN}}=\frac{1}{6}\left[1+\frac{7}{18} \nu+\mathcal{O}\left(\nu^{2}\right)\right]
$$

Finally, at the 3PN order, for equal masses $\nu=\frac{1}{4}$, we find that according to our criterion all the circular orbits are stable. More generally, we find that at the $3 \mathrm{PN}$ order all orbits are stable when the mass ratio $\nu$ is larger than some critical value $\nu_{c} \simeq 0.183$.

The stability criterion (269) has been compared in great details to various other stability criteria by Favata [191] and shown to perform very well, and has also been generalized to spinning black hole binaries in Ref. [190]. Note that this criterion is based on the physical requirement that a stable perturbation should have a real frequency. It gives an innermost stable circular orbit, when it exists, which differs from the innermost circular orbit or ICO defined in Section 8.1; see Ref. [378] for a discussion on the difference between an ISCO and the ICO in the PN context. Note also that the criterion (269) is based on systematic post-Newtonian expansions, without resorting for instance to Padé approximants. Nevertheless, it performs better than other criteria based on various resummation techniques, as discussed in Ref. [191].

\subsection{The first law of binary point-particle mechanics}

In this section we shall review a very interesting relation for binary systems of point particles modelling black hole binaries and moving on circular orbits, known as the "first law of pointparticle mechanics". This law was obtained using post-Newtonian methods in Ref. [289], but is actually a particular case of a more general law, valid for systems of black holes and extended fluid balls, derived by Friedman, Uryū \& Shibata [208].

Before tackling the problem it is necessary to make more precise the notion of circular orbits. These are obtained from the conservative part of the dynamics, neglecting the dissipative radiation-reaction force responsible for the gravitational-wave inspiral. In post-Newtonian theory this means neglecting the radiation-reaction force at $2.5 \mathrm{PN}$ and $3.5 \mathrm{PN}$ orders, i.e., considering only the conservative dynamics at the even-parity $1 \mathrm{PN}, 2 \mathrm{PN}$ and $3 \mathrm{PN}$ orders. We have seen in Sections 5.2 and 5.4 that this clean separation between conservative even-parity and dissipative odd-parity terms breaks at 4PN order, because of a contribution originating from gravitationalwave tails in the radiation-reaction force. We expect that at any higher order $4 \mathrm{PN}, 4.5 \mathrm{PN}, 5 \mathrm{PN}$, etc. there will be a mixture of conservative and dissipative effects; here we assume that at any higher order we can neglect the radiation-reaction dissipation effects.

58 This tendency is in agreement with numerical and analytical self-force calculations [24, 287]. 
Consider a system of two compact objects moving on circular orbits. We examine first the case of non-spinning objects. With exactly circular orbits the geometry admits a helical Killing vector (HKV) field $K^{\alpha}$, satisfying the Killing equation $\nabla^{\alpha} K^{\beta}+\nabla^{\beta} K^{\alpha}=0$. Imposing the existence of the HKV is the rigorous way to implement the notion of circular orbits. A Killing vector is only defined up to an overall constant factor. The helical Killing vector $K^{\alpha}$ extends out to a large distance where the geometry is essentially flat. There,

$$
K^{\alpha} \partial_{\alpha}=\partial_{t}+\Omega \partial_{\varphi}
$$

in any natural coordinate system which respects the helical symmetry [370]. We let this equality define the overall constant factor, thereby specifying the Killing vector field uniquely. In Eq. (273) $\Omega$ denotes the angular frequency of the binary's circular motion.

An observer moving with one of the particles (say the particle 1), while orbiting around the other particle, would detect no change in the local geometry. Thus the four-velocity $u_{1}^{\alpha}$ of that particle is tangent to the Killing vector $K^{\alpha}$ evaluated at the location of the particle, which we denote by $K_{1}^{\alpha}$. A physical quantity is then defined as the constant of proportionality $u_{1}^{T}$ between these two vectors, namely

$$
u_{1}^{\alpha}=u_{1}^{T} K_{1}^{\alpha} .
$$

The four-velocity of the particle is normalized by $\left(g_{\mu \nu}\right)_{1} u_{1}^{\mu} u_{1}^{\nu}=-1$, where $\left(g_{\mu \nu}\right)_{1}$ denotes the metric at the location of the particle. For a self-gravitating compact binary system, the metric at point 1 is generated by the two particles and has to be regularized according to one of the self-field regularizations discussed in Section 6. It will in fact be sometimes more convenient to work with the inverse of $u_{1}^{T}$, denoted $z_{1} \equiv 1 / u_{1}^{T}$. From Eq. (274) we get

$$
z_{1}=-\left(u_{1} K_{1}\right)
$$

where $\left(u_{1} K_{1}\right)=\left(g_{\mu \nu}\right)_{1} u_{1}^{\mu} K_{1}^{\nu}$ denotes the usual space-time dot product. Thus we can regard $z_{1}$ as the Killing energy of the particle that is associated with the HKV field $K^{\alpha}$. The quantity $z_{1}$ represents also the redshift of light rays emitted from the particle and received on the helical symmetry axis perpendicular to the orbital plane at large distances from it [176]. In the following we shall refer to $z_{1}$ as the redshift observable.

If we choose a coordinate system such that Eq. (273) is satisfied everywhere, then in particular $K_{1}^{t}=1$, thus $u_{1}^{T}$ simply agrees with $u_{1}^{t}$, the $t$-component of the four-velocity of the particle. The Killing vector on the particle is then $K_{1}^{\alpha}=u_{1}^{\alpha} / u_{1}^{t}$, and simply reduces to the particle's ordinary coordinate velocity: $K_{1}^{\alpha}=v_{1}^{\alpha} / c$ where $v_{1}^{\alpha}=\mathrm{d} y_{1}^{\alpha} / \mathrm{d} t$ and $y_{1}^{\alpha}(t)=\left[c t, \boldsymbol{y}_{1}(t)\right]$ denotes the particle's trajectory in that coordinate system. The redshift observable we are thus considering is

$$
z_{1}=\frac{1}{u_{1}^{T}}=\sqrt{-\left(g_{\mu \nu}\right)_{1} v_{1}^{\mu} v_{1}^{\nu} / c^{2}}
$$

It is important to note that for circular orbits this quantity does not depend upon the choice of coordinates; in a perturbative approach in which the perturbative parameter is the particles' mass ratio $\nu \ll 1$, it does not depend upon the choice of perturbative gauge with respect to the background metric. We shall be interested in the invariant scalar function $z_{1}(\Omega)$, where $\Omega$ is the angular frequency of the circular orbit introduced when imposing Eq. (273).

We have obtained in Section 7.4 the expressions of the post-Newtonian binding energy $E$ and angular momentum $\mathrm{J}$ for point-particle binaries on circular orbits. We shall now show that there are some differential and algebraic relations linking $E$ and $\mathrm{J}$ to the redshift observables $z_{1}$ and $z_{2}$ associated with the two individual particles. Here we prefer to introduce instead of $E$ the total relativistic $(\mathrm{ADM})$ mass of the binary system

$$
\mathrm{M}=m+\frac{E}{c^{2}},
$$


where $m$ is the sum of the two post-Newtonian individual masses $m_{1}$ and $m_{2}$ - those which have been used up to now, for instance in Eq. (203). Note that in the spinning case such post-Newtonian masses acquire some spin contributions given, e.g., by Eqs. (243) - (246).

For point particles without spins, the ADM mass M, angular momentum J, and redshifts $z_{\mathrm{a}}$, are functions of three independent variables, namely the orbital frequency $\Omega$ that is imposed by the existence of the HKV, and the individual masses $m_{\mathrm{a}}$. For spinning point particles, we have also the two spins $S_{\mathrm{a}}$ which are necessarily aligned with the orbital angular momentum. We first recover that the ADM quantities obey the "thermodynamical" relation already met in Eq. (235),

$$
\frac{\partial \mathrm{M}}{\partial \Omega}=\Omega \frac{\partial \mathrm{J}}{\partial \Omega}
$$

Such relation is commonly used in post-Newtonian theory (see e.g., [160, 51]). It states that the gravitational-wave energy and angular momentum fluxes are strictly proportional for circular orbits, with $\Omega$ being the coefficient of proportionality. This relation is used in computations of the binary evolution based on a sequence of quasi-equilibrium configurations [228, 232, 133, 121], as discussed in Section 8.1.

The first law will be a thermodynamical generalization of Eq. (278), describing the changes in the ADM quantities not only when the orbital frequency $\Omega$ varies with fixed masses, but also when the individual masses $m_{\mathrm{a}}$ of the particles vary with fixed orbital frequency. That is, one compares together different conservative dynamics with different masses but the same frequency. This situation is answered by the differential equations

$$
\frac{\partial \mathrm{M}}{\partial m_{\mathrm{a}}}-\Omega \frac{\partial \mathrm{J}}{\partial m_{\mathrm{a}}}=z_{\mathrm{a}} \quad(\mathrm{a}=1,2) .
$$

Finally the three relations $(278)-(279)$ can be summarized in the following result.

Theorem 11. The changes in the ADM mass and angular momentum of a binary system made of point particles in response to infinitesimal variations of the individual masses of the point particles, are related together by the first law of binary point-particle mechanics as [208, 289]

$$
\delta \mathrm{M}-\Omega \delta \mathrm{J}=\sum_{\mathrm{a}} z_{\mathrm{a}} \delta m_{\mathrm{a}} .
$$

This law was proved in a very general way in Ref. [208] for systems of black holes and extended bodies under some arbitrary Killing symmetry. The particular form given in Eq. (280) is a specialization to the case of point particle binaries with helical Killing symmetry. It has been proved directly in this form in Ref. [289] up to high post-Newtonian order, namely 3PN order plus the logarithmic contributions occurring at $4 \mathrm{PN}$ and $5 \mathrm{PN}$ orders.

The first law of binary point-particle mechanics (280) is of course reminiscent of the celebrated first law of black hole mechanics $\delta \mathrm{M}-\omega_{\mathrm{H}} \delta \mathrm{J}=\frac{\kappa}{8 \pi} \delta A$, which holds for any non-singular, asymptotically flat perturbation of a stationary and axisymmetric black hole of mass $\mathrm{M}$, intrinsic angular momentum (or spin) $\mathrm{J} \equiv \mathrm{M} a$, surface area $A$, uniform surface gravity $\kappa$, and angular frequency $\omega_{\mathrm{H}}$ on the horizon [26, 417]; see Ref. [289] for a discussion.

An interesting by-product of the first law (280) is the remarkably simple algebraic relation

$$
\mathrm{M}-2 \Omega \mathrm{J}=\sum_{\mathrm{a}} z_{\mathrm{a}} m_{\mathrm{a}}
$$

which can be seen as a first integral of the differential relation (280). Note that the existence of such a simple algebraic relation between the local quantities $z_{1}$ and $z_{2}$ on one hand, and the globally defined quantities $\mathrm{M}$ and $\mathrm{J}$ on the other hand, is not trivial. 
Next, we report the result of a generalization of the first law applicable to systems of point particles with spins (moving on circular orbits). ${ }^{59}$ This result is valid through linear order in the spin of each particle, but holds also for the quadratic coupling between different spins (interaction spin terms $S_{1} \times S_{2}$ in the language of Section 11). To be consistent with the HKV symmetry, we must assume that the two spins $S_{\mathrm{a}}$ are aligned or anti-aligned with the orbital angular momentum. We introduce the total (ADM-like) angular momentum J which is related to the orbital angular momentum $L$ by $\mathrm{J}=L+\sum_{\mathrm{a}} S_{\mathrm{a}}$ for aligned or anti-aligned spins. The first law now becomes [55]

$$
\delta \mathrm{M}-\Omega \delta \mathrm{J}=\sum_{\mathrm{a}}\left[z_{\mathrm{a}} \delta m_{\mathrm{a}}+\left(\Omega_{\mathrm{a}}-\Omega\right) \delta S_{\mathrm{a}}\right],
$$

where $\Omega_{\mathrm{a}}=\left|\boldsymbol{\Omega}_{\mathrm{a}}\right|$ denotes the precession frequency of the spins. This law has been derived in Ref. [55] from the canonical Hamiltonian formalism. The spin variables used here are the canonical spins $\boldsymbol{S}_{\mathrm{a}}$, that are easily seen to obey, from the algebra satisfied by the canonical variables, the usual Newtonian-looking precession equations $\mathrm{d} \boldsymbol{S}_{\mathrm{a}} / \mathrm{d} t=\boldsymbol{\Omega}_{\mathrm{a}} \times \boldsymbol{S}_{\mathrm{a}}$. These variables are identical to the "constant-in-magnitude" spins which will be defined and extensively used in Section 11. Similarly, to Eq. (281) we have also a first integral associated with the variational law (282):

$$
\mathrm{M}-2 \Omega \mathrm{J}=\sum_{\mathrm{a}}\left[z_{\mathrm{a}} m_{\mathrm{a}}-2\left(\Omega-\Omega_{\mathrm{a}}\right) S_{\mathrm{a}}\right] .
$$

Notice that the relation (282) has been derived for point particles and arbitrary aligned spins. We would like now to derive the analogous relation for binary black holes. The key difference is that black holes are extended finite-size objects while point particles have by definition no spatial extension. For point particle binaries the spins can have arbitrary magnitude and still be compatible with the HKV. In this case the law (282) would describe also (super-extremal) naked singularities. For black hole binaries the HKV constraints the rotational state of each black hole and the binary system must be corotating.

Let us derive, in a heuristic way, the analogue of the first law (282) for black holes by introducing some "constitutive relations" $m_{\mathrm{a}}\left(\mu_{\mathrm{a}}, S_{\mathrm{a}}, \cdots\right)$ specifying the energy content of the bodies, i.e., the relations linking their masses $m_{\mathrm{a}}$ to the spins $S_{\mathrm{a}}$ and to some irreducible masses $\mu_{\mathrm{a}}$. More precisely, we define for each spinning particle the analogue of an irreducible mass $\mu_{\mathrm{a}} \equiv m_{\mathrm{a}}^{\text {irr }}$ via the variational relation $\delta m_{\mathrm{a}}=c_{\mathrm{a}} \delta \mu_{\mathrm{a}}+\omega_{\mathrm{a}} \delta S_{\mathrm{a}},{ }^{60}$ in which the "response coefficient" $c_{\mathrm{a}}$ of the body and its proper rotation frequency $\omega_{\mathrm{a}}$ are associated with the internal structure:

$$
\begin{gathered}
\left.c_{\mathrm{a}} \equiv \frac{\partial m_{\mathrm{a}}}{\partial \mu_{\mathrm{a}}}\right|_{S_{\mathrm{a}}}, \\
\left.\omega_{\mathrm{a}} \equiv \frac{\partial m_{\mathrm{a}}}{\partial S_{\mathrm{a}}}\right|_{\mu_{\mathrm{a}}} .
\end{gathered}
$$

For instance, using the Christodoulou mass formula (243) for Kerr black holes, we obtain the rotation frequency $\omega_{\mathrm{a}}$ given by Eq. (244). On the other hand, the response coefficient $c_{\mathrm{a}}$ differs from 1 only because of spin effects, and we can check that $c_{\mathrm{a}}=1+\mathcal{O}\left(S_{\mathrm{a}}^{2}\right)$.

Within the latter heuristic model a condition for the corotation of black hole binaries has been proposed in Ref. [55] as

$$
z_{\mathrm{a}} \omega_{\mathrm{a}}+\Omega_{\mathrm{a}}=\Omega \text {. }
$$

This condition determines the value of the proper frequency $\omega_{\mathrm{a}}$ of each black hole appropriate to the corotation state. When expanded to 2PN order the condition (285) leads to Eq. (247)

\footnotetext{
59 The first law (280) has also been generalized for binary systems of point masses moving along generic stable bound (eccentric) orbits in Ref. [286].

${ }^{60}$ In the case of extended material bodies, $\mu_{\mathrm{a}}$ would represent the baryonic mass of the bodies.
} 
that we have already used in Section 8.1. With Eq. (285) imposed, the first law (282) simplifies considerably:

$$
\delta \mathrm{M}-\Omega \delta \mathrm{J}=\sum_{\mathrm{a}} c_{\mathrm{a}} z_{\mathrm{a}} \delta \mu_{\mathrm{a}}
$$

This is almost identical to the first law for non-spinning binaries given by Eq. (280); indeed it simply differs from it by the substitutions $c_{\mathrm{a}} \rightarrow 1$ and $\mu_{\mathrm{a}} \rightarrow m_{\mathrm{a}}$. Since the irreducible mass $\mu_{\mathrm{a}}$ of a rotating black hole is the spin-independent part of its total mass $m_{\mathrm{a}}$, this observation suggests that corotating binaries are very similar to non-spinning binaries, at least from the perspective of the first law. Finally we can easily reconcile the first law (286) for corotating systems with the known first law of binary black hole mechanics [208], namely

$$
\delta \mathrm{M}-\Omega \delta \mathrm{J}=\sum_{\mathrm{a}} \frac{\kappa_{\mathrm{a}}}{8 \pi} \delta A_{\mathrm{a}}
$$

Indeed it suffices to make the formal identification in Eq. (286) of $c_{\mathrm{a}} z_{\mathrm{a}}$ with $4 \mu_{\mathrm{a}} \kappa_{\mathrm{a}}$, where $\kappa_{\mathrm{a}}$ denotes the constant surface gravity, and using the surface areas $A_{\mathrm{a}}=16 \pi \mu_{\mathrm{a}}^{2}$ instead of the irreducible masses of the black holes. This shows that the heuristic model based on the constitutive relations (284) is able to capture the physics of corotating black hole binary systems.

\subsection{Post-Newtonian approximation versus gravitational self-force}

The high-accuracy predictions from general relativity we have drawn up to now are well suited to describe the inspiralling phase of compact binaries, when the post-Newtonian parameter (1) is small independently of the mass ratio $q \equiv m_{1} / m_{2}$ between the compact bodies. In this section we investigate how well does the post-Newtonian expansion compare with another very important approximation scheme in general relativity: The gravitational self-force approach, based on black-hole perturbation theory, which gives an accurate description of extreme mass ratio binaries having $q \ll 1$ or equivalently $\nu \ll 1$, even in the strong field regime. The gravitational self-force analysis [317, 360, 178, 231] (see [348, 177, 23] for reviews) is thus expected to provide templates for extreme mass ratio inspirals (EMRI) anticipated to be present in the bandwidth of space-based detectors.

Consider a system of two (non-spinning) compact objects with $q=m_{1} / m_{2} \ll 1$; we shall call the smaller mass $m_{1}$ the "particle", and the larger mass $m_{2}$ the "black hole". The orbit of the particle around the black hole is supposed to be exactly circular as we neglect the radiation-reaction effects. With circular orbits and no dissipation, we are considering the conservative part of the dynamics, and the geometry admits the HKV field (273). Note that in self-force theory there is a clean split between the dissipative and conservative parts of the dynamics (see e.g., [22]). This split is particularly transparent for an exact circular orbit, since the radial component (along $r$ ) is the only non-vanishing component of the conservative self-force, while the dissipative part of the self-force are the components along $t$ and $\varphi$.

The problem of the comparison between the post-Newtonian and perturbative self-force analyses in their common domain of validity, that of the slow-motion and weak-field regime of an extreme mass ratio binary, is illustrated in Figure 2. This problem has been tackled by Detweiler [176], who computed numerically within the self-force (SF) approach the redshift observable $u_{1}^{T}$ associated with the particle, and compared it with the $2 \mathrm{PN}$ prediction extracted from existing post-Newtonian results [76]. This comparison proved to be successful, and was later systematically implemented and extended to higher post-Newtonian orders in Refs. [68, 67]. In this section we review the works [68, 67] which have demonstrated an excellent agreement between the analytical post-Newtonian result derived through $3 \mathrm{PN}$ order, with inclusion of specific logarithmic terms at 4PN and 5PN orders, and the exact numerical SF result. 


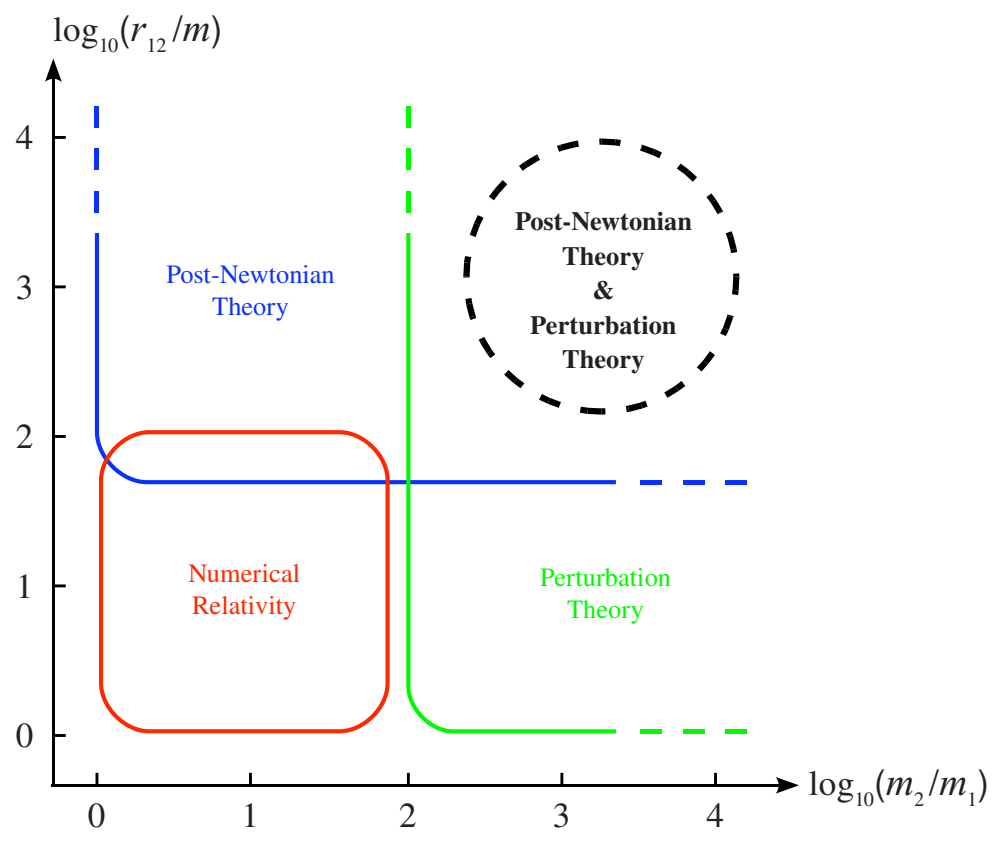

Figure 2: Different analytical approximation schemes and numerical techniques to study black hole binaries, depending on the mass ratio $q=m_{1} / m_{2}$ and the post-Newtonian parameter $\epsilon^{2} \sim v^{2} / c^{2} \sim$ $G m /\left(c^{2} r_{12}\right)$. Post-Newtonian theory and perturbative self-force analysis can be compared in the postNewtonian regime $\left(\epsilon \ll 1\right.$ thus $\left.r_{12} \gg G m / c^{2}\right)$ of an extreme mass ratio $\left(m_{1} \ll m_{2}\right)$ binary.

For the PN-SF comparison, we require two physical quantities which are precisely defined in the context of each of the approximation schemes. The orbital frequency $\Omega$ of the circular orbit as measured by a distant observer is one such quantity and has been introduced in Eq. (273); the second quantity is the redshift observable $u_{1}^{T}$ (or equivalently $z_{1}=1 / u_{1}^{T}$ ) associated with the smaller mass $m_{1} \ll m_{2}$ and defined by Eqs. (274) or (275). The truly coordinate and perturbative-gauge independent properties of $\Omega$ and the redshift observable $u_{1}^{T}$ play a crucial role in this comparison. In the perturbative self-force approach we use Schwarzschild coordinates for the background, and we refer to "gauge invariance" as a property which holds within the restricted class of gauges for which (273) is a helical Killing vector. In all other respects, the gauge choice is arbitrary. In the post-Newtonian approach we work with harmonic coordinates and compute the explicit expression (276) of the redshift observable.

The main difficulty in the post-Newtonian calculation is the control to high PN order of the near-zone metric $\left(g_{\mu \nu}\right)_{1}$ entering the definition of the redshift observable (276), and which has to be regularized at the location of the particle by means of dimensional regularization (see Sections 6.36.4). Up to $2.5 \mathrm{PN}$ order the Hadamard regularization is sufficient and the regularized metric has been provided in Eqs. (242). Here we report the end result of the post-Newtonian computation of the redshift observable including all terms up to the $3 \mathrm{PN}$ order, and augmented by the logarithmic contributions up to the 5PN order (and also the known Schwarzschild limit) [68, 67, 289]:

$$
\begin{aligned}
u_{1}^{T}=1 & +\left(\frac{3}{4}-\frac{3}{4} \Delta-\frac{\nu}{2}\right) x+\left(\frac{27}{16}-\frac{27}{16} \Delta-\frac{5}{2} \nu+\frac{5}{8} \Delta \nu+\frac{1}{24} \nu^{2}\right) x^{2} \\
& +\left(\frac{135}{32}-\frac{135}{32} \Delta-\frac{37}{4} \nu-\frac{67}{16} \Delta \nu+\frac{115}{32} \nu^{2}-\frac{5}{32} \Delta \nu^{2}+\frac{1}{48} \nu^{3}\right) x^{3}
\end{aligned}
$$




$$
\begin{aligned}
& +\left(\frac{2835}{256}-\frac{2835}{256} \Delta+\left[-\frac{2183}{48}+\frac{41}{64} \pi^{2}\right] \nu+\left[\frac{12199}{384}-\frac{41}{64} \pi^{2}\right] \Delta \nu\right. \\
& \left.+\left[\frac{17201}{576}-\frac{41}{192} \pi^{2}\right] \nu^{2}-\frac{795}{128} \Delta \nu^{2}-\frac{2827}{864} \nu^{3}-\frac{25}{1728} \Delta \nu^{3}+\frac{35}{10368} \nu^{4}\right) x^{4} \\
& +\left(\frac{15309}{512}-\frac{15309}{512} \Delta+\nu\left[u_{4}(\nu)+\Delta v_{4}(\nu)\right]+\left[-\frac{32}{5}+\frac{32}{5} \Delta-\frac{32}{15} \nu\right] \nu \ln x\right) x^{5} \\
& +\left(\frac{168399}{2048}-\frac{168399}{2048} \Delta+\nu\left[u_{5}(\nu)+\Delta v_{5}(\nu)\right]\right. \\
& \left.+\left[\frac{478}{105}-\frac{478}{105} \Delta+\frac{10306}{105} \nu-36 \Delta \nu-\frac{296}{15} \nu^{2}\right] \nu \ln x\right) x^{6}+\mathcal{O}\left(\frac{1}{c^{14}}\right) .
\end{aligned}
$$

We recall that $x$ denotes the post-Newtonian parameter (230), $\nu$ is the mass ratio (215), and $\Delta=\left(m_{1}-m_{2}\right) / m$. The redshift observable of the other particle is deduced by setting $\Delta \rightarrow-\Delta$.

In Eq. (288) we denote by $u_{4}(\nu), v_{4}(\nu)$ and $u_{5}(\nu), v_{5}(\nu)$ some unknown $4 \mathrm{PN}$ and $5 \mathrm{PN}$ coefficients, which are however polynomials of the symmetric mass ratio $\nu$. They can be entirely determined from the related coefficients $e_{4}(\nu), j_{4}(\nu)$ and $e_{5}(\nu), j_{5}(\nu)$ in the expressions of the energy and angular momentum in Eqs. (233) and (234). To this aim it suffices to apply the differential first law (280) up to 5PN order; see Ref. [289] for more details.

The post-Newtonian result (288) is valid for any mass ratio, and for comparison purpose with the SF calculation we now investigate the small mass ratio regime $q \ll 1$. We introduce a postNewtonian parameter appropriate to the small mass limit of the "particle",

$$
y \equiv\left(\frac{G m_{2} \Omega}{c^{3}}\right)^{2 / 3}=x(1+q)^{-2 / 3} .
$$

We express the symmetric mass ratio in terms of the asymmetric one: $\nu=q /(1+q)^{2}$, together with $\Delta=(q-1) /(q+1)$. Then Eq. (288), expanded through first order in $q$, which means including only the linear self-force level, reads

$$
u_{1}^{T}=u_{\mathrm{Schw}}^{T}+q u_{\mathrm{SF}}^{T}+\mathcal{O}\left(q^{2}\right) .
$$

The Schwarzschildean result is known in closed form as

$$
u_{\text {Schw }}^{T}=\frac{1}{\sqrt{1-3 y}},
$$

and for the self-force contribution one obtains ${ }^{61}$

$$
\begin{aligned}
u_{\mathrm{SF}}^{T}(y) & =-y-2 y^{2}-5 y^{3}+\left(-\frac{121}{3}+\frac{41}{32} \pi^{2}\right) y^{4} \\
& +\left(\alpha_{4}-\frac{64}{5} \ln y\right) y^{5}+\left(\alpha_{5}+\frac{956}{105} \ln y\right) y^{6}+o\left(y^{6}\right) .
\end{aligned}
$$

The analytic coefficients were determined up to $2 \mathrm{PN}$ order in Ref. [176]; the 3PN term was computed in Ref. [68] making full use of dimensional regularization; the logarithmic contributions at the $4 \mathrm{PN}$ and $5 \mathrm{PN}$ orders were added in Refs. [67, 146].

The coefficients $\alpha_{4}$ and $\alpha_{5}$ represent some pure numbers at the 4PN and 5PN orders. By an analytic self-force calculation [36] the coefficient $\alpha_{4}$ has been obtained as

$$
\alpha_{4}=-\frac{1157}{15}+\frac{677}{512} \pi^{2}-\frac{256}{5} \ln 2-\frac{128}{5} \gamma_{\mathrm{E}}
$$

\footnotetext{
61 Since there are logarithms in this expansion we use the Landau $o$-symbol for remainders; see the footnote 20.
} 
Using the first law (280), we know how to deduce from the PN coefficients in the redshift variable the corresponding PN coefficients in the energy function (233). Thus, the result reported in Eq. (236) for the 4PN term in the energy function for circular orbits has been deduced from Eq. (293) by application of the first law.

On the self-force front the main problem is to control the numerical resolution of the computation of the redshift observable in order to distinguish more accurately the contributions of very high order PN terms. The comparison of the post-Newtonian expansion (292) with the numerical SF data has confirmed with high precision the determination of the $3 \mathrm{PN}$ coefficient [68, 67]: Witness Table 1 where the agreement with the analytical value involves 7 significant digits. Notice that such agreement provides an independent check of the dimensional regularization procedure invoked in the PN expansion scheme (see Sections 6.3-6.4). It is remarkable that such procedure is equivalent to the procedure of subtraction of the singular field in the SF approach [178].

Table 1: Numerically determined value of the 3PN coefficient for the SF part of the redshift observable defined by Eq. (292). The analytic post-Newtonian computation [68] is confirmed with many significant digits.

\begin{tabular}{cc}
\hline 3PN coefficient & SF value \\
\hline$\alpha_{3} \equiv-\frac{121}{3}+\frac{41}{32} \pi^{2}=-27.6879026 \cdots$ & $-27.6879034 \pm 0.0000004$ \\
\hline
\end{tabular}

Table 2: Numerically determined values of higher-order PN coefficients for the SF part of the redshift observable defined by Eq. (292). The uncertainty in the last digit (or two last digits) is indicated in parentheses. The 4PN numerical value agrees with the analytical expression (293).

\begin{tabular}{cl}
\hline PN coefficient & \multicolumn{1}{c}{ SF value } \\
\hline$\alpha_{4}$ & $-114.34747(5)$ \\
$\alpha_{5}$ & $-245.53(1)$ \\
$\alpha_{6}$ & $-695(2)$ \\
$\beta_{6}$ & $+339.3(5)$ \\
$\alpha_{7}$ & $-5837(16)$ \\
\hline
\end{tabular}

Furthermore the PN-SF comparison has permitted to measure the coefficients $\alpha_{4}$ and $\alpha_{5}$ with at least 8 significant digits for the $4 \mathrm{PN}$ coefficient, and 5 significant digits for the $5 \mathrm{PN}$ one. In Table 2 we report the result of the analysis performed in Refs. [68,67] by making maximum use of the analytical coefficients available at the time, i.e., all the coefficients up to $3 \mathrm{PN}$ order plus the logarithmic contributions at 4PN and 5PN orders. One uses a set of five basis functions corresponding to the unknown non-logarithmic $4 \mathrm{PN}$ and $5 \mathrm{PN}$ coefficients $\alpha_{4}, \alpha_{5}$ in Eq. (292), and augmented by the $6 \mathrm{PN}$ and $7 \mathrm{PN}$ non-logarithmic coefficients $\alpha_{6}, \alpha_{7}$ plus a coefficient $\beta_{6}$ for the logarithm at $6 \mathrm{PN}$. A contribution $\beta_{7}$ from a logarithm at $7 \mathrm{PN}$ order is likely to confound with the $\alpha_{7}$ coefficient. There is also the possibility of the contribution of a logarithmic squared at $7 \mathrm{PN}$ order, but such a small effect is not permitted in this fit.

Gladly we discover that the more recent analytical value of the 4PN coefficient, Eq. (293), matches the numerical value which was earlier measured in Ref. [67] (see Table 2). This highlights the predictive power of perturbative self-force calculations in determining numerically new postNewtonian coefficients [176, 68, 67]. This ability is obviously due to the fact (illustrated in Figure 2) that perturbation theory is legitimate in the strong field regime of the coalescence of black hole binary systems, which is inaccessible to the post-Newtonian method. Of course, the limitation 
of the self-force approach is the small mass-ratio limit; in this respect it is taken over by the post-Newtonian approximation.

More recently, the accuracy of the numerical computation of the self-force, and the comparison with the post-Newtonian expansion, have been drastically improved by Shah, Friedman \& Whiting [383]. The PN coefficients of the redshift observable were obtained to very high $10.5 \mathrm{PN}$ order both numerically and also analytically, for a subset of coefficients that are either rational or made of the product of $\pi$ with a rational. The analytical values of the coefficients up to $6 \mathrm{PN}$ order have also been obtained from an alternative self-force calculation [38, 37]. An interesting feature of the post-Newtonian expansion at high order is the appearance of half-integral PN coefficients (i.e., of the type $\frac{n}{2} \mathrm{PN}$ where $n$ is an odd integer) in the conservative dynamics of binary point particles, moving on exactly circular orbits. This is interesting because any instantaneous (non-tail) term at any half-integral PN order will be zero for circular orbits, as can be shown by a simple dimensional argument [77]. Therefore half-integral coefficients can appear only due to truly hereditary (tail) integrals. Using standard post-Newtonian methods it has been proved in Refs. [77, 78] that the dominant half-integral PN term in the redshift observable (292) occurs at the 5.5PN order (confirming the earlier finding of Ref. [383]) and originates from the non-linear "tail-of-tail" integrals investigated in Section 3.2. The results for the 5.5PN coefficient in Eq. (292), and also for the next-to-leading $6.5 \mathrm{PN}$ and $7.5 \mathrm{PN}$ ones, are

$$
\alpha_{5.5}=-\frac{13696}{525} \pi, \quad \alpha_{6.5}=\frac{81077}{3675} \pi, \quad \alpha_{7.5}=\frac{82561159}{467775} \pi
$$

and fully agree between the PN and SF computations. We emphasize that the results (294) are achieved by the traditional PN approach, which is completely general (contrary to various analytical and numerical SF calculations [383, 38, 37, 268]), i.e., is not tuned to a particular type of source but is applicable to any extended PN source (see Part A). Note that Eqs. (294) represent the complete coefficients as there are no logarithms at these orders.

To conclude, the consistency of this "cross-cultural" comparison between the analytical postNewtonian and the perturbative self-force approaches confirms the soundness of both approximations in describing the dynamics of compact binaries. Furthermore this interplay between PN and SF efforts (which is now rapidly growing [383]) is important for the synthesis of template waveforms of EMRIs to be analysed by space-based gravitational wave detectors, and has also an impact on efforts of numerical relativity in the case of comparable masses.

\section{Gravitational Waves from Compact Binaries}

We pointed out that the 3.5PN equations of motion, Eqs. (203) or (219) - (220), are merely 1PN as regards the radiative aspects of the problem, because the radiation reaction force starts at the $2.5 \mathrm{PN}$ order. A solution would be to extend the precision of the equations of motion so as to include the full relative $3 \mathrm{PN}$ or $3.5 \mathrm{PN}$ precision into the radiation reaction force, but the equations of motion up to the $5.5 \mathrm{PN}$ or $6 \mathrm{PN}$ order are beyond the present state-of-the-art. The much better alternative solution is to apply the wave-generation formalism described in Part A, and to determine by its means the work done by the radiation reaction force directly as a total energy flux at future null infinity. ${ }^{62}$ In this approach, we replace the knowledge of the higher-order radiation reaction force by the computation of the total flux $\mathcal{F}$, and we apply the energy balance equation

$$
\frac{\mathrm{d} E}{\mathrm{~d} t}=-\mathcal{F} .
$$

Therefore, the result (232) that we found for the 3.5PN binary's center-of-mass energy $E$ constitutes only "half" of the solution of the problem. The second "half" consists of finding the rate of decrease

62 In addition, the wave generation formalism will provide the waveform itself, see Sections 9.4 and 9.5. 
$\mathrm{d} E / \mathrm{d} t$, which by the balance equation is nothing but the total gravitational-wave flux $\mathcal{F}$ at the relative 3.5PN order beyond the Einstein quadrupole formula (4).

Because the orbit of inspiralling binaries is circular, the energy balance equation is sufficient, and there is no need to invoke the angular momentum balance equation for computing the evolution of the orbital period $\dot{P}$ and eccentricity $\dot{e}$, see Eqs. (9)-(13) in the case of the binary pulsar. Furthermore the time average over one orbital period as in Eqs. (9) is here irrelevant, and the energy and angular momentum fluxes are related by $\mathcal{F}=\Omega \mathcal{G}$. This all sounds good, but it is important to remind that we shall use the balance equation (295) at the very high 3.5PN order, and that at such order one is missing a complete proof of it (following from first principles in general relativity). Nevertheless, in addition to its physically obvious character, Eq. (295) has been verified by radiation-reaction calculations, in the cases of point-particle binaries [258, 259] and extended post-Newtonian fluids [43,47], at the 1PN order and even at 1.5PN, the latter order being especially important because of the first appearance of wave tails; see Section 5.4. One should also quote here Refs. [260, 336, 278, 322, 254] for the 3.5PN terms in the binary's equations of motion, fully consistent with the balance equations.

Obtaining the energy flux $\mathcal{F}$ can be divided into two equally important steps: Computing the source multipole moments $\mathrm{I}_{L}$ and $\mathrm{J}_{L}$ of the compact binary system with due account of a self-field regularization; and controlling the tails and related non-linear effects occurring in the relation between the binary's source moments and the radiative ones $\mathrm{U}_{L}$ and $\mathrm{V}_{L}$ observed at future null infinity (cf. the general formalism of Part A).

\subsection{The binary's multipole moments}

The general expressions of the source multipole moments given by Theorem 6, Eqs. (123), are worked out explicitly for general fluid systems at the $3.5 \mathrm{PN}$ order. For this computation one uses the formula (126), and we insert the 3.5PN metric coefficients (in harmonic coordinates) expressed in Eqs. (144) by means of the retarded-type elementary potentials (146) - (148). Then we specialize each of the (quite numerous) terms to the case of point-particle binaries by inserting, for the matter stress-energy tensor $T^{\alpha \beta}$, the standard expression made out of Dirac delta-functions. In Section 11 we shall consider spinning point particle binaries, and in that case the stress-energy tensor is given by Eq. (378). The infinite self-field of point-particles is removed by means of the Hadamard regularization; and, as we discussed in Section 6.4, dimensional regularization is used to fix the values of a few ambiguity parameters. This computation has been performed in Ref. [86] at the $1 \mathrm{PN}$ order, and in [64] at the $2 \mathrm{PN}$ order; we report below the most accurate $3 \mathrm{PN}$ results obtained in Refs. [81, 80, 62, 63] for the flux and [11, 74, 197] for the waveform.

A difficult part of the analysis is to find the closed-form expressions, fully explicit in terms of the particle's positions and velocities, of many non-linear integrals. Let us give a few examples of the type of technical formulas that are employed in this calculation. Typically we have to compute some integrals like

$$
\stackrel{(p, q)}{Y_{L}}\left(\boldsymbol{y}_{1}, \boldsymbol{y}_{2}\right)=-\frac{1}{2 \pi} \mathcal{F} \mathcal{P} \int \mathrm{d}^{3} \mathbf{x} \hat{x}_{L} r_{1}^{p} r_{2}^{q}
$$

where $r_{1}=\left|\mathbf{x}-\boldsymbol{y}_{1}\right|$ and $r_{2}=\left|\mathbf{x}-\boldsymbol{y}_{2}\right|$. When $p>-3$ and $q>-3$, this integral is perfectly welldefined, since the finite part $\mathcal{F P}$ deals with the IR regularization of the bound at infinity. When $p \leqslant-3$ or $q \leqslant-3$, we cure the UV divergencies by means of the Hadamard partie finie defined by Eq. (162); so a partie finie prescription Pf is implicit in Eq. (296). An example of closed-form formula we get is

$$
\stackrel{(-1,-1)}{Y_{L}}=\frac{r_{12}}{\ell+1} \sum_{k=0}^{\ell} y_{1}^{\langle L-K} y_{2}^{K\rangle}
$$


where we pose $y_{1}^{L-K} \equiv y_{1}^{i_{1}} \cdots y_{1}^{i_{\ell-k}}$ and $y_{2}^{K} \equiv y_{2}^{i_{\ell-k+1}} \cdots y_{2}^{i_{\ell}}$, the brackets surrounding indices denoting the STF projection. Another example, in which the $\mathcal{F P}$ regularization is crucial, is (in the quadrupole case $\ell=2$ )

$$
\stackrel{(-2,-1)}{Y_{i j}}=y_{1}^{\langle i j\rangle}\left[\frac{16}{15} \ln \left(\frac{r_{12}}{r_{0}}\right)-\frac{188}{225}\right]+y_{1}^{\langle i} y_{2}^{j\rangle}\left[\frac{8}{15} \ln \left(\frac{r_{12}}{r_{0}}\right)-\frac{4}{225}\right]+y_{2}^{\langle i j\rangle}\left[\frac{2}{5} \ln \left(\frac{r_{12}}{r_{0}}\right)-\frac{2}{25}\right],
$$

where the IR scale $r_{0}$ is defined in Eq. (42). Still another example, which necessitates both the $\mathcal{F P}$ and a UV partie finie regularization at the point 1 , is

$$
\stackrel{(-3,0)}{Y_{i j}}=\left[2 \ln \left(\frac{s_{1}}{r_{0}}\right)+\frac{16}{15}\right] y_{1}^{\langle i j\rangle}
$$

where $s_{1}$ is the Hadamard-regularization constant introduced in Eq. (162).

The most important input for the computation of the waveform and flux is the mass quadrupole moment $\mathrm{I}_{i j}$, since this moment necessitates the full post-Newtonian precision. Here we give the mass quadrupole moment complete to order 3.5PN, for non-spinning compact binaries on circular orbits, as

$$
\mathrm{I}_{i j}=\mu\left(A x_{\langle i j\rangle}+B \frac{r^{2}}{c^{2}} v^{\langle i j\rangle}+\frac{48}{7} \frac{G^{2} m^{2} \nu}{c^{5} r} C x_{\langle i} v_{j\rangle}\right)+\mathcal{O}\left(\frac{1}{c^{8}}\right)
$$

where $\boldsymbol{x}=\boldsymbol{y}_{1}-\boldsymbol{y}_{2}=\left(x_{i}\right)$ and $\boldsymbol{v}=\boldsymbol{v}_{1}-\boldsymbol{v}_{2}=\left(v_{i}\right)$ are the orbital separation and relative velocity. The third term with coefficient $C$ is a radiation-reaction term, which will affect the waveform at orders 2.5PN and 3.5PN; however it does not contribute to the energy flux for circular orbits. The two conservative coefficients are $A$ and $B$. All those coefficients are [81, 74, 197]

$$
\begin{aligned}
A & =1+\gamma\left(-\frac{1}{42}-\frac{13}{14} \nu\right)+\gamma^{2}\left(-\frac{461}{1512}-\frac{18395}{1512} \nu-\frac{241}{1512} \nu^{2}\right) \\
& +\gamma^{3}\left(\frac{395899}{13200}-\frac{428}{105} \ln \left(\frac{r_{12}}{r_{0}}\right)+\left[\frac{3304319}{166320}-\frac{44}{3} \ln \left(\frac{r_{12}}{r_{0}^{\prime}}\right)\right] \nu+\frac{162539}{16632} \nu^{2}+\frac{2351}{33264} \nu^{3}\right) \\
B & =\frac{11}{21}-\frac{11}{7} \nu+\gamma\left(\frac{1607}{378}-\frac{1681}{378} \nu+\frac{229}{378} \nu^{2}\right) \\
& +\gamma^{2}\left(-\frac{357761}{19800}+\frac{428}{105} \ln \left(\frac{r_{12}}{r_{0}}\right)-\frac{92339}{5544} \nu+\frac{35759}{924} \nu^{2}+\frac{457}{5544} \nu^{3}\right) \\
C & =1+\gamma\left(-\frac{256}{135}-\frac{1532}{405} \nu\right) .
\end{aligned}
$$

These expressions are valid in harmonic coordinates via the post-Newtonian parameter $\gamma=\frac{G m}{r c^{2}}$ defined in Eq. (225). As we see, there are two types of logarithms at 3PN order in the quadrupole moment: One type involves the UV length scale $r_{0}^{\prime}$ related by Eq. (221) to the two gauge constants $r_{1}^{\prime}$ and $r_{2}^{\prime}$ present in the 3PN equations of motion; the other type contains the IR length scale $r_{0}$ coming from the general formalism of Part $\mathrm{A}$ - indeed, recall that there is a $\mathcal{F} \mathcal{P}$ operator in front of the source multipole moments in Theorem 6. As we know, the UV scale $r_{0}^{\prime}$ is specific to the standard harmonic ( $\mathrm{SH}$ ) coordinate system and is pure gauge (see Section 7.3): It will disappear from our physical results at the end. On the other hand, we have proved that the multipole expansion outside a general post-Newtonian source is actually free of $r_{0}$, since the $r_{0}$ 's present in the two terms of Eq. (105) cancel out. Indeed we have already found in Eqs. (93) - (94) that the constant $r_{0}$ present in $\mathrm{I}_{i j}$ is compensated by the same constant coming from the non-linear wave "tails of tails" in the radiative moment $\mathrm{U}_{i j}$. For a while, the expressions (301) contained the ambiguity parameters $\xi, \kappa$ and $\zeta$, which have now been replaced by their correct values (173). 
Besides the 3.5PN mass quadrupole (300) - (301), we need also (for the 3PN waveform) the mass octupole moment $\mathrm{I}_{i j k}$ and current quadrupole moment $\mathrm{J}_{i j}$, both of them at the 2.5PN order; these are given for circular orbits by [81,74]

$$
\begin{aligned}
\mathrm{I}_{i j k}= & -\nu m \Delta\left\{x_{\langle i j k\rangle}\left[1-\gamma \nu-\gamma^{2}\left(\frac{139}{330}+\frac{11923}{660} \nu+\frac{29}{110} \nu^{2}\right)\right]\right. \\
& +\frac{r^{2}}{c^{2}} x_{\langle i} v_{j k\rangle}\left[1-2 \nu-\gamma\left(-\frac{1066}{165}+\frac{1433}{330} \nu-\frac{21}{55} \nu^{2}\right)\right] \\
& \left.+\frac{196}{15} \frac{r}{c} \gamma^{2} \nu x_{\langle i j} v_{k\rangle}\right\}+\mathcal{O}\left(\frac{1}{c^{6}}\right), \\
\mathrm{J}_{i j}= & -\nu m \Delta\left\{\epsilon_{a b\langle i} x_{j\rangle a} v_{b}\left[1+\gamma\left(\frac{67}{28}-\frac{2}{7} \nu\right)+\gamma^{2}\left(\frac{13}{9}-\frac{4651}{252} \nu-\frac{1}{168} \nu^{2}\right)\right]\right. \\
& \left.-\frac{188}{35} \frac{r}{c} \gamma^{2} \nu \epsilon_{a b\langle i} v_{j\rangle a} x_{b}\right\}+\mathcal{O}\left(\frac{1}{c^{6}}\right) .
\end{aligned}
$$

The list of required source moments for the $3 \mathrm{PN}$ waveform continues with the $2 \mathrm{PN}$ mass $2^{4}$-pole and current $2^{3}$-pole (octupole) moments, and so on. Here we give the most updated moments: ${ }^{63}$

$$
\begin{aligned}
\mathrm{I}_{i j k l}= & \nu m\left\{x _ { \langle i j k l \rangle } \left[1-3 \nu+\gamma\left(\frac{3}{110}-\frac{25}{22} \nu+\frac{69}{22} \nu^{2}\right)\right.\right. \\
& \left.+\gamma^{2}\left(-\frac{126901}{200200}-\frac{58101}{2600} \nu+\frac{204153}{2860} \nu^{2}+\frac{1149}{1144} \nu^{3}\right)\right] \\
& +\frac{r^{2}}{c^{2}} x_{\langle i j} v_{k l\rangle}\left[\frac{78}{55}\left(1-5 \nu+5 \nu^{2}\right)\right. \\
& \left.+\gamma\left(\frac{30583}{3575}-\frac{107039}{3575} \nu+\frac{8792}{715} \nu^{2}-\frac{639}{715} \nu^{3}\right)\right] \\
& \left.+\frac{71}{715} \frac{r^{4}}{c^{4}} v_{\langle i j k l\rangle}\left(1-7 \nu+14 \nu^{2}-7 \nu^{3}\right)\right\}+\mathcal{O}\left(\frac{1}{c^{5}}\right) \\
\mathrm{J}_{i j k}= & \nu m\left\{\epsilon _ { a b \langle i } x _ { j k \rangle a } v _ { b } \left[1-3 \nu+\gamma\left(\frac{181}{90}-\frac{109}{18} \nu+\frac{13}{18} \nu^{2}\right)\right.\right. \\
& \left.+\gamma^{2}\left(\frac{1469}{3960}-\frac{5681}{264} \nu+\frac{48403}{660} \nu^{2}-\frac{559}{3960} \nu^{3}\right)\right] \\
& \left.+\frac{r^{2}}{c^{2}} \epsilon_{a b\langle i} x_{a} v_{j k\rangle b}\left[\frac{7}{45}\left(1-5 \nu+5 \nu^{2}\right)+\gamma\left(\frac{1621}{990}-\frac{4879}{990} \nu+\frac{1084}{495} \nu^{2}-\frac{259}{990} \nu^{3}\right)\right]\right\} \\
& +\mathcal{O}\left(\frac{1}{c^{5}}\right) \cdot \\
\mathrm{I}_{i j k l m}= & -\nu m \Delta\left\{x_{\langle i j k l m\rangle}\left[1-2 \nu+\gamma\left(\frac{2}{39}-\frac{47}{39} \nu+\frac{28}{13} \nu^{2}\right)\right]\right. \\
& \left.+\frac{70}{39} \frac{r^{2}}{c^{2}} x_{\langle i j k} v_{l m\rangle}\left(1-4 \nu+3 \nu^{2}\right)\right\}+\mathcal{O}\left(\frac{1}{c^{4}}\right), \\
\mathrm{J}_{i j k l}= & -\nu m \Delta\left\{\epsilon_{a b\langle i} x_{j k l\rangle a} v_{b}\left[1-2 \nu+\gamma\left(\frac{20}{11}-\frac{155}{44} \nu+\frac{5}{11} \nu^{2}\right)\right]\right. \\
& \left.+\frac{4}{11} \frac{r^{2}}{c^{2}} \epsilon_{a b\langle i} x_{j a} v_{k l\rangle b}\left(1-4 \nu+3 \nu^{2}\right)\right\}+\mathcal{O}\left(\frac{1}{c^{4}}\right) .
\end{aligned}
$$

\footnotetext{
63 The STF projection \langle\rangle applies only on "living" indices $i j k l \cdots$ but not on the summed up indices $a$ and $b$.
} 


$$
\begin{aligned}
\mathrm{I}_{i j k l m n}= & \nu m\left\{x_{\langle i j k l m n\rangle}\left[1-5 \nu+5 \nu^{2}+\gamma\left(\frac{1}{14}-\frac{3}{2} \nu+6 \nu^{2}-\frac{11}{2} \nu^{3}\right)\right]\right. \\
& \left.+\frac{15}{7} \frac{r^{2}}{c^{2}} x_{\langle i j k l} v_{m n\rangle}\left(1-7 \nu+14 \nu^{2}-7 \nu^{3}\right)\right\}+\mathcal{O}\left(\frac{1}{c^{4}}\right), \\
\mathrm{J}_{i j k l m}= & \nu m\left\{\epsilon_{a b\langle i} x_{j k l m\rangle a} v_{b}\left[1-5 \nu+5 \nu^{2}+\gamma\left(\frac{1549}{910}-\frac{1081}{130} \nu+\frac{107}{13} \nu^{2}-\frac{29}{26} \nu^{3}\right)\right]\right. \\
& \left.+\frac{54}{91} \frac{r^{2}}{c^{2}} \epsilon_{a b\langle i} x_{j k a} v_{l m\rangle b}\left(1-7 \nu+14 \nu^{2}-7 \nu^{3}\right)\right\}+\mathcal{O}\left(\frac{1}{c^{4}}\right) . \\
\mathrm{I}_{i j k l m n o}= & -\nu m \Delta\left(1-4 \nu+3 \nu^{2}\right) x_{\langle i j k l m n o\rangle}+\mathcal{O}\left(\frac{1}{c^{2}}\right), \\
\mathrm{J}_{i j k l m n}= & -\nu m \Delta\left(1-4 \nu+3 \nu^{2}\right) \epsilon_{a b\langle i} x_{j k l m n\rangle a} v_{b}+\mathcal{O}\left(\frac{1}{c^{2}}\right) .
\end{aligned}
$$

All the other higher-order moments are required at the Newtonian order, at which they are trivial to compute with result $(\forall \ell \in \mathbb{N})$

$$
\begin{aligned}
\mathrm{I}_{L} & =\nu m \sigma_{\ell}(\nu) x_{\langle L\rangle}+\mathcal{O}\left(\frac{1}{c}\right), \\
\mathrm{J}_{L-1} & =\nu m \sigma_{\ell}(\nu) \epsilon_{a b\left\langle i_{l-1}\right.} x_{L-2\rangle a} v_{b}+\mathcal{O}\left(\frac{1}{c}\right) .
\end{aligned}
$$

Here we introduce the useful notation $\sigma_{\ell}(\nu) \equiv X_{2}^{\ell-1}+(-)^{\ell} X_{1}^{\ell-1}$, where $X_{1}=\frac{m_{1}}{m}$ and $X_{2}=\frac{m_{2}}{m}$ are such that $X_{1}+X_{2}=1, X_{1}-X_{2}=\Delta$ and $X_{1} X_{2}=\nu$. More explicit expressions are $(k \in \mathbb{N})$ :

$$
\begin{aligned}
\sigma_{2 k}(\nu) & =\sum_{p=0}^{k-1}(-)^{p} \frac{2 k-1}{2 k-1-p}\left(\begin{array}{c}
2 k-1-p \\
p
\end{array}\right) \nu^{p}, \\
\sigma_{2 k+1}(\nu) & =-\Delta \sum_{p=0}^{k-1}(-)^{p}\left(\begin{array}{c}
2 k-1-p \\
p
\end{array}\right) \nu^{p},
\end{aligned}
$$

where $\left(\begin{array}{l}n \\ p\end{array}\right)$ is the usual binomial coefficient.

\subsection{Gravitational wave energy flux}

The results (300) - (304) permit the control of the instantaneous part of the total energy flux, by which we mean that part of the flux which is generated solely by the source multipole moments, i.e., not counting the hereditary tail and related integrals. The instantaneous flux $\mathcal{F}_{\text {inst }}$ is defined by the replacement into the general expression of $\mathcal{F}$ given by Eq. (68a) of all the radiative moments $\mathrm{U}_{L}$ and $\mathrm{V}_{L}$ by the corresponding $\ell$-th time derivatives of the source moments $\mathrm{I}_{L}$ and $\mathrm{J}_{L}$. $\mathrm{Up}$ to the 3.5PN order we have

$$
\begin{aligned}
\mathcal{F}_{\text {inst }}=\frac{G}{c^{5}} & \left\{\frac{1}{5} \mathrm{I}_{i j}^{(3)} \mathrm{I}_{i j}^{(3)}+\frac{1}{c^{2}}\left[\frac{1}{189} \mathrm{I}_{i j k}^{(4)} \mathrm{I}_{i j k}^{(4)}+\frac{16}{45} \mathrm{~J}_{i j}^{(3)} \mathrm{J}_{i j}^{(3)}\right]+\frac{1}{c^{4}}\left[\frac{1}{9072} \mathrm{I}_{i j k m}^{(5)} \mathrm{I}_{i j k m}^{(5)}+\frac{1}{84} \mathrm{~J}_{i j k}^{(4)} \mathrm{J}_{i j k}^{(4)}\right]\right. \\
& \left.+\frac{1}{c^{6}}\left[\frac{1}{594000} \mathrm{I}_{i j k m n}^{(6)} \mathrm{I}_{i j k m n}^{(6)}+\frac{4}{14175} \mathrm{~J}_{i j k m}^{(5)} \mathrm{J}_{i j k m}^{(5)}\right]+\mathcal{O}\left(\frac{1}{c^{8}}\right)\right\} .
\end{aligned}
$$

in which we insert the explicit expressions (300)-(304) for the moments. The time derivatives of these source moments are computed by means of the circular-orbit equations of motion given by 
Eq. (226) together with (228). The net result is

$$
\begin{aligned}
\mathcal{F}_{\text {inst }}=\frac{32 c^{5}}{5 G} & \nu^{2} \gamma^{5}\left\{1+\left(-\frac{2927}{336}-\frac{5}{4} \nu\right) \gamma+\left(\frac{293383}{9072}+\frac{380}{9} \nu\right) \gamma^{2}\right. \\
+ & {\left[\frac{53712289}{1108800}-\frac{1712}{105} \ln \left(\frac{r_{12}}{r_{0}}\right)\right.} \\
& \left.\left.+\left(-\frac{50625}{112}+\frac{123}{64} \pi^{2}+\frac{110}{3} \ln \left(\frac{r_{12}}{r_{0}^{\prime}}\right)\right) \nu-\frac{383}{9} \nu^{2}\right] \gamma^{3}+\mathcal{O}\left(\frac{1}{c^{8}}\right)\right\} .
\end{aligned}
$$

The Newtonian approximation agrees with the prediction of the Einstein quadrupole formula (4), as reduced for quasi-circular binary orbits by Landau \& Lifshitz [285]. At the 3PN order in Eq. (307), there was some Hadamard regularization ambiguity parameters which have been replaced by their values computed with dimensional regularization.

To the instantaneous part of the flux, we must add the contribution of non-linear multipole interactions contained in the relationship between the source and radiative moments. The needed material has already been provided in Sections 3.3. Similar to the decomposition of the radiative quadrupole moment in Eq. (88), we can split the energy flux into the different terms

$$
\mathcal{F}=\mathcal{F}_{\text {inst }}+\mathcal{F}_{\text {tail }}+\mathcal{F}_{\text {tail-tail }}
$$

where $\mathcal{F}_{\text {inst }}$ has just been obtained in Eq. (307); $\mathcal{F}_{\text {tail }}$ is made of the quadratic multipolar tail

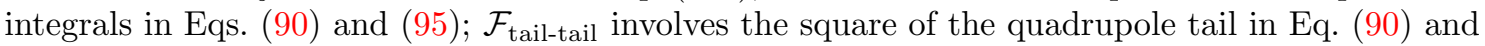
the quadrupole tail of tail given in Eq. (91).

We shall see that the tails play a crucial role in the predicted signal of compact binaries. It is quite remarkable that so small an effect as a "tail of tail" should be relevant to the data analysis of the current generation of gravitational wave detectors. By contrast, the non-linear memory effects, given by the integrals inside the 2.5PN and 3.5PN terms in Eq. (92), do not contribute to the gravitational-wave energy flux before the $4 \mathrm{PN}$ order in the case of circular-orbit binaries. Indeed the memory integrals are actually "instantaneous" in the flux, and a simple general argument based on dimensional analysis shows that instantaneous terms cannot contribute to the energy flux for circular orbits. ${ }^{64}$ Therefore the memory effect has rather poor observational consequences for future detections of inspiralling compact binaries.

Let us also recall that following the general formalism of Part A, the mass $M$ which appears in front of the tail integrals of Sections 3.2 and 3.3 represents the binary's mass monopole M or ADM mass. In a realistic model where the binary system has been formed as a close compact binary at a finite instant in the past, this mass is equal to the sum of the rest masses $m=m_{1}+m_{2}$, plus the total binary's mass-energy $E / c^{2}$ given for instance by Eq. (229). At 3.5PN order we need $2 \mathrm{PN}$ corrections in the tails and therefore $2 \mathrm{PN}$ also in the mass $M$, thus

$$
\mathrm{M}=m\left[1-\frac{\nu}{2} \gamma+\frac{\nu}{8}(7-\nu) \gamma^{2}+\mathcal{O}\left(\frac{1}{c^{6}}\right)\right] .
$$

Notice that $2 \mathrm{PN}$ order in $\mathrm{M}$ corresponds to $1 \mathrm{PN}$ order in $E$.

We give the two basic technical formulas needed when carrying out the reduction of the tail and tail-of-tail integrals to circular orbits (see e.g., [230]):

$$
\int_{0}^{+\infty} \mathrm{d} \tau \ln \left(\frac{\tau}{2 \tau_{0}}\right) e^{-\mathrm{i} \omega \tau}=\frac{\mathrm{i}}{\omega}\left(\ln \left(2 \omega \tau_{0}\right)+\gamma_{\mathrm{E}}+\mathrm{i} \frac{\pi}{2}\right),
$$

64 The same argument shows that the non-linear multipole interactions in Eq. (89) as well as those in Eqs. (97) and (98) do not contribute to the flux for circular orbits. 


$$
\int_{0}^{+\infty} \mathrm{d} \tau \ln ^{2}\left(\frac{\tau}{2 \tau_{0}}\right) e^{-\mathrm{i} \omega \tau}=-\frac{\mathrm{i}}{\omega}\left[\left(\ln \left(2 \omega \tau_{0}\right)+\gamma_{\mathrm{E}}+\mathrm{i} \frac{\pi}{2}\right)^{2}+\frac{\pi^{2}}{6}\right],
$$

where $\omega>0$ is a strictly positive frequency (a multiple of the orbital frequency $\Omega$ ), where $\tau_{0}=r_{0} / c$ and $\gamma_{\mathrm{E}}$ is the Euler constant.

Notice the important point that the tail (and tail-of-tail) integrals can be evaluated, thanks to these formulas, for a fixed (i.e., non-decaying) circular orbit. Indeed it can be shown [60, 87] that the "remote-past" contribution to the tail integrals is negligible; the errors due to the fact that the orbit has actually evolved in the past, and spiraled in by emission of gravitational radiation, are of the order of the radiation-reaction scale $\mathcal{O}\left(c^{-5}\right),{ }^{65}$ and do not affect the signal before the $4 \mathrm{PN}$ order. We then find, for the quadratic tails stricto sensu, the $1.5 \mathrm{PN}, 2.5 \mathrm{PN}$ and $3.5 \mathrm{PN}$ contributions

$$
\begin{aligned}
\mathcal{F}_{\text {tail }}=\frac{32 c^{5}}{5 G} \gamma^{5} \nu^{2} & \left\{4 \pi \gamma^{3 / 2}+\left(-\frac{25663}{672}-\frac{125}{8} \nu\right) \pi \gamma^{5 / 2}+\left(\frac{90205}{576}+\frac{505747}{1512} \nu+\frac{12809}{756} \nu^{2}\right) \pi \gamma^{7 / 2}\right. \\
& \left.+\mathcal{O}\left(\frac{1}{c^{8}}\right)\right\} .
\end{aligned}
$$

For the sum of squared tails and cubic tails of tails at $3 \mathrm{PN}$, we get

$$
\begin{aligned}
& \mathcal{F}_{\text {tail-tail }}=\frac{32 c^{5}}{5 G} \gamma^{5} \nu^{2}\left\{\left(-\frac{116761}{3675}+\frac{16}{3} \pi^{2}-\frac{1712}{105} \gamma_{\mathrm{E}}+\frac{1712}{105} \ln \left(\frac{r_{12}}{r_{0}}\right)-\frac{856}{105} \ln (16 \gamma)\right) \gamma^{3}\right. \\
&\left.+\mathcal{O}\left(\frac{1}{c^{8}}\right)\right\} .
\end{aligned}
$$

By comparing Eqs. (307) and (312) we observe that the constants $r_{0}$ cleanly cancel out. Adding together these contributions we obtain

$$
\begin{aligned}
\mathcal{F}=\frac{32 c^{5}}{5 G} \gamma^{5} \nu^{2} & \left\{1+\left(-\frac{2927}{336}-\frac{5}{4} \nu\right) \gamma+4 \pi \gamma^{3 / 2}\right. \\
+ & \left(\frac{293383}{9072}+\frac{380}{9} \nu\right) \gamma^{2}+\left(-\frac{25663}{672}-\frac{125}{8} \nu\right) \pi \gamma^{5 / 2} \\
+ & {\left[\frac{129386791}{7761600}+\frac{16 \pi^{2}}{3}-\frac{1712}{105} \gamma_{\mathrm{E}}-\frac{856}{105} \ln (16 \gamma)\right.} \\
& \left.+\left(-\frac{50625}{112}+\frac{110}{3} \ln \left(\frac{r_{12}}{r^{\prime}}\right)+\frac{123 \pi^{2}}{64}\right) \nu-\frac{383}{9} \nu^{2}\right] \gamma^{3} \\
& \left.+\left(\frac{90205}{576}+\frac{505747}{1512} \nu+\frac{12809}{756} \nu^{2}\right) \pi \gamma^{7 / 2}+\mathcal{O}\left(\frac{1}{c^{8}}\right)\right\} .
\end{aligned}
$$

The gauge constant $r_{0}^{\prime}$ has not yet disappeared because the post-Newtonian expansion is still parametrized by $\gamma$ instead of the frequency-related parameter $x$ defined by Eq. (230) - just as for $E$ when it was given by Eq. (229). After substituting the expression $\gamma(x)$ given by Eq. (231), we find that $r_{0}^{\prime}$ does cancel as well. Because the relation $\gamma(x)$ is issued from the equations of motion, the latter cancellation represents an interesting test of the consistency of the two computations, in harmonic coordinates, of the $3 \mathrm{PN}$ multipole moments and the $3 \mathrm{PN}$ equations of motion. At long last we obtain our end result: 66

$$
\mathcal{F}=\frac{32 c^{5}}{5 G} \nu^{2} x^{5}\left\{1+\left(-\frac{1247}{336}-\frac{35}{12} \nu\right) x+4 \pi x^{3 / 2}\right.
$$

\footnotetext{
65 Or, rather, $\mathcal{O}\left(c^{-5} \ln c\right)$ as shown in the Appendix of Ref. [87].

66 See Section 10 for the generalization of the flux of energy to eccentric binary orbits.
} 


$$
\begin{gathered}
+\left(-\frac{44711}{9072}+\frac{9271}{504} \nu+\frac{65}{18} \nu^{2}\right) x^{2}+\left(-\frac{8191}{672}-\frac{583}{24} \nu\right) \pi x^{5 / 2} \\
+\left[\frac{6643739519}{69854400}+\frac{16}{3} \pi^{2}-\frac{1712}{105} \gamma_{\mathrm{E}}-\frac{856}{105} \ln (16 x)\right. \\
\left.+\left(-\frac{134543}{7776}+\frac{41}{48} \pi^{2}\right) \nu-\frac{94403}{3024} \nu^{2}-\frac{775}{324} \nu^{3}\right] x^{3} \\
\left.+\left(-\frac{16285}{504}+\frac{214745}{1728} \nu+\frac{193385}{3024} \nu^{2}\right) \pi x^{7 / 2}+\mathcal{O}\left(\frac{1}{c^{8}}\right)\right\} .
\end{gathered}
$$

In the test-mass limit $\nu \rightarrow 0$ for one of the bodies, we recover exactly the result following from linear black-hole perturbations obtained by Tagoshi \& Sasaki [395] (see also [393, 397]). In particular, the rational fraction $\frac{6643739519}{69854400}$ comes out exactly the same as in black-hole perturbations. On the other hand, the ambiguity parameters discussed in Section 6.2 were part of the rational fraction $-\frac{134543}{7776}$, belonging to the coefficient of the term at $3 \mathrm{PN}$ order proportional to $\nu$ (hence this coefficient cannot be computed by linear black-hole perturbations).

The effects due to the spins of the two black holes arise at the $1.5 \mathrm{PN}$ order for the spin-orbit (SO) coupling, and at the $2 \mathrm{PN}$ order for the spin-spin (SS) coupling, for maximally rotating black holes. Spin effects will be discussed in Section 11. On the other hand, the terms due to the radiating energy flowing into the black-hole horizons and absorbed rather than escaping to infinity, have to be added to the standard post-Newtonian calculation based on point particles as presented here; such terms arise at the $4 \mathrm{PN}$ order for Schwarzschild black holes [349] and at 2.5PN order for Kerr black holes [392].

\subsection{Orbital phase evolution}

We shall now deduce the laws of variation with time of the orbital frequency and phase of an inspiralling compact binary from the energy balance equation (295). The center-of-mass energy $E$ is given by Eq. (232) and the total flux $\mathcal{F}$ by Eq. (314). For convenience we adopt the dimensionless time variable ${ }^{67}$

$$
\Theta \equiv \frac{\nu c^{3}}{5 G m}\left(t_{c}-t\right)
$$

where $t_{\mathrm{c}}$ denotes the instant of coalescence, at which the frequency formally tends to infinity, although evidently, the post-Newtonian method breaks down well before this point. We transform the balance equation into an ordinary differential equation for the parameter $x$, which is immediately integrated with the result

$$
\begin{aligned}
x=\frac{1}{4} \Theta^{-1 / 4} & \left\{1+\left(\frac{743}{4032}+\frac{11}{48} \nu\right) \Theta^{-1 / 4}-\frac{1}{5} \pi \Theta^{-3 / 8}\right. \\
+ & \left(\frac{19583}{254016}+\frac{24401}{193536} \nu+\frac{31}{288} \nu^{2}\right) \Theta^{-1 / 2}+\left(-\frac{11891}{53760}+\frac{109}{1920} \nu\right) \pi \Theta^{-5 / 8} \\
+ & {\left[-\frac{10052469856691}{6008596070400}+\frac{1}{6} \pi^{2}+\frac{107}{420} \gamma_{\mathrm{E}}-\frac{107}{3360} \ln \left(\frac{\Theta}{256}\right)\right.} \\
& \left.+\left(\frac{3147553127}{780337152}-\frac{451}{3072} \pi^{2}\right) \nu-\frac{15211}{442368} \nu^{2}+\frac{25565}{331776} \nu^{3}\right] \Theta^{-3 / 4} \\
+ & \left.\left(-\frac{113868647}{433520640}-\frac{31821}{143360} \nu+\frac{294941}{3870720} \nu^{2}\right) \pi \Theta^{-7 / 8}+\mathcal{O}\left(\frac{1}{c^{8}}\right)\right\} .
\end{aligned}
$$

\footnotetext{
67 Notice the "strange" post-Newtonian order of this time variable: $\Theta=\mathcal{O}\left(c^{+8}\right)$.
} 
The orbital phase is defined as the angle $\phi$, oriented in the sense of the motion, between the separation of the two bodies and the direction of the ascending node (called $\mathcal{N}$ in Section 9.4) within the plane of the sky. We have $\mathrm{d} \phi / \mathrm{d} t=\Omega$, which translates, with our notation, into $\mathrm{d} \phi / \mathrm{d} \Theta=-5 x^{3 / 2} / \nu$, from which we determine ${ }^{68}$

$$
\begin{aligned}
\phi=-\frac{1}{\nu} \Theta^{5 / 8} & \left\{1+\left(\frac{3715}{8064}+\frac{55}{96} \nu\right) \Theta^{-1 / 4}-\frac{3}{4} \pi \Theta^{-3 / 8}\right. \\
+ & \left(\frac{9275495}{14450688}+\frac{284875}{258048} \nu+\frac{1855}{2048} \nu^{2}\right) \Theta^{-1 / 2}+\left(-\frac{38645}{172032}+\frac{65}{2048} \nu\right) \pi \Theta^{-5 / 8} \ln \left(\frac{\Theta}{\Theta_{0}}\right) \\
+ & {\left[\frac{831032450749357}{57682522275840}-\frac{53}{40} \pi^{2}-\frac{107}{56} \gamma_{\mathrm{E}}+\frac{107}{448} \ln \left(\frac{\Theta}{256}\right)\right.} \\
& \left.+\left(-\frac{126510089885}{4161798144}+\frac{2255}{2048} \pi^{2}\right) \nu+\frac{154565}{1835008} \nu^{2}-\frac{1179625}{1769472} \nu^{3}\right] \Theta^{-3 / 4} \\
+ & \left.\left(\frac{188516689}{173408256 x}+\frac{488825}{516096} \nu-\frac{141769}{516096} \nu^{2}\right) \pi \Theta^{-7 / 8}+\mathcal{O}\left(\frac{1}{c^{8}}\right)\right\}
\end{aligned}
$$

where $\Theta_{0}$ is a constant of integration that can be fixed by the initial conditions when the wave frequency enters the detector. Finally we want also to dispose of the important expression of the phase in terms of the frequency $x$. For this we get

$$
\begin{aligned}
\phi=-\frac{x^{-5 / 2}}{32 \nu} & \left\{1+\left(\frac{3715}{1008}+\frac{55}{12} \nu\right) x-10 \pi x^{3 / 2}\right. \\
+ & \left(\frac{15293365}{1016064}+\frac{27145}{1008} \nu+\frac{3085}{144} \nu^{2}\right) x^{2}+\left(\frac{38645}{1344}-\frac{65}{16} \nu\right) \pi x^{5 / 2} \ln \left(\frac{x}{x_{0}}\right) \\
+ & {\left[\frac{12348611926451}{18776862720}-\frac{160}{3} \pi^{2}-\frac{1712}{21} \gamma_{\mathrm{E}}-\frac{856}{21} \ln (16 x)\right.} \\
& \left.\quad+\left(-\frac{15737765635}{12192768}+\frac{2255}{48} \pi^{2}\right) \nu+\frac{76055}{6912} \nu^{2}-\frac{127825}{5184} \nu^{3}\right] x^{3} \\
+ & \left.\left(\frac{77096675}{2032128}+\frac{378515}{12096} \nu-\frac{74045}{6048} \nu^{2}\right) \pi x^{7 / 2}+\mathcal{O}\left(\frac{1}{c^{8}}\right)\right\}
\end{aligned}
$$

where $x_{0}$ is another constant of integration. With the formula (318) the orbital phase is complete up to the 3.5PN order for non-spinning compact binaries. Note that the contributions of the quadrupole moments of compact objects which are induced by tidal effects, are expected from Eq. (16) to come into play only at the 5PN order.

As a rough estimate of the relative importance of the various post-Newtonian terms, we give in Table 3 their contributions to the accumulated number of gravitational-wave cycles $\mathcal{N}_{\text {cycle }}$ in the bandwidth of ground-based detectors. Note that such an estimate is only indicative, because a full treatment would require the knowledge of the detector's power spectral density of noise, and a complete simulation of the parameter estimation using matched filtering techniques [138, 350, 284]. We define $\mathcal{N}_{\text {cycle }}$ as

$$
\mathcal{N}_{\text {cycle }} \equiv \frac{\phi_{\text {ISCO }}-\phi_{\text {seismic }}}{\pi} .
$$

The frequency of the signal at the entrance of the bandwidth is the seismic cut-off frequency $f_{\text {seismic }}$ of ground-based detectors; the terminal frequency is assumed for simplicity to be given

68 This procedure for computing analytically the orbital phase corresponds to what is called in the jargon the "Taylor T2 approximant". We refer to Ref. [98] for discussions on the usefulness of defining several types of approximants for computing (in general numerically) the orbital phase. 
by the Schwarzschild innermost stable circular orbit: $f_{\mathrm{ISCO}}=\frac{c^{3}}{6^{3 / 2} \pi G m}$. Here we denote by $f=$ $\Omega / \pi=2 / P$ the signal frequency of the dominant harmonics at twice the orbital frequency. As we see in Table 3 , with the $3 \mathrm{PN}$ or $3.5 \mathrm{PN}$ approximations we reach an acceptable accuracy level of a few cycles say, that roughly corresponds to the demand made by data-analysists in the case of neutron-star binaries [139, 137, 138, 346, 105, 106]. Indeed, the above estimation suggests that the neglected 4PN terms will yield some systematic errors that are, at most, of the same order of magnitude, i.e., a few cycles, and perhaps much less.

Table 3: Post-Newtonian contributions to the accumulated number of gravitational-wave cycles $\mathcal{N}_{\text {cycle }}$ for compact binaries detectable in the bandwidth of LIGO-VIRGO detectors. The entry frequency is $f_{\text {seismic }}=10 \mathrm{~Hz}$ and the terminal frequency is $f_{\mathrm{ISCO}}=\frac{c^{3}}{6^{3 / 2} \pi G m}$. The main origin of the approximation (instantaneous vs. tail) is indicated. See also Table 4 in Section 11 below for the contributions of spin-orbit effects.

\begin{tabular}{ll|rrr}
\hline $\mathrm{PN}$ order & & $1.4 M_{\odot}+1.4 M_{\odot}$ & $10 M_{\odot}+1.4 M_{\odot}$ & $10 M_{\odot}+10 M_{\odot}$ \\
\hline $\mathrm{N}$ & (inst) & 15952.6 & 3558.9 & 598.8 \\
$1 \mathrm{PN}$ & (inst) & 439.5 & 212.4 & 59.1 \\
$1.5 \mathrm{PN}$ & (leading tail) & -210.3 & -180.9 & -51.2 \\
$2 \mathrm{PN}$ & (inst) & 9.9 & 9.8 & 4.0 \\
$2.5 \mathrm{PN}$ & $(1 \mathrm{PN}$ tail) & -11.7 & -20.0 & -7.1 \\
$3 \mathrm{PN}$ & (inst + tail-of-tail) & 2.6 & 2.3 & 2.2 \\
$3.5 \mathrm{PN}$ & $(2 \mathrm{PN}$ tail) & -0.9 & -1.8 & -0.8 \\
\hline
\end{tabular}

\subsection{Polarization waveforms for data analysis}

The theoretical templates of the compact binary inspiral follow from insertion of the previous solutions for the $3.5 \mathrm{PN}$-accurate orbital frequency and phase into the binary's two polarization waveforms $h_{+}$and $h_{\times}$defined with respect to a choice of two polarization vectors $\boldsymbol{P}=\left(P_{i}\right)$ and $\boldsymbol{Q}=\left(Q_{i}\right)$ orthogonal to the direction $\boldsymbol{N}$ of the observer; see Eqs. (69).

Our convention for the two polarization vectors is that they form with $\boldsymbol{N}$ a right-handed triad, and that $\boldsymbol{P}$ and $\boldsymbol{Q}$ lie along the major and minor axis, respectively, of the projection onto the plane of the sky of the circular orbit. This means that $\boldsymbol{P}$ is oriented toward the orbit's ascending node namely the point $\mathcal{N}$ at which the orbit intersects the plane of the sky and the bodies are moving toward the observer located in the direction $\boldsymbol{N}$. The ascending node is also chosen for the origin of the orbital phase $\phi$. We denote by $i$ the inclination angle between the direction of the detector $\boldsymbol{N}$ as seen from the binary's center-of-mass, and the normal to the orbital plane (we always suppose that the normal is right-handed with respect to the sense of motion, so that $0 \leqslant i \leqslant \pi$ ). We use the shorthands $c_{i} \equiv \cos i$ and $s_{i} \equiv \sin i$ for the cosine and sine of the inclination angle.

We shall include in $h_{+}$and $h_{\times}$all the harmonics, besides the dominant one at twice the orbital frequency, consistent with the $3 \mathrm{PN}$ approximation $[82,11,74]$. In Section 9.5 we shall give all the modes $(\ell, m)$ in a spherical-harmonic decomposition of the waveform, and shall extend the dominant quadrupole mode $(2,2)$ at $3.5 \mathrm{PN}$ order [197]. The post-Newtonian terms are ordered by means of the frequency-related variable $x=\left(\frac{G m \Omega}{c^{3}}\right)^{2 / 3}$; to ease the notation we pose

$$
h_{+, \times}=\frac{2 G \mu x}{c^{2} R} \sum_{p=0}^{+\infty} x^{p / 2} \underset{p / 2}{H_{+, \times}}\left(\psi, c_{i}, s_{i} ; \ln x\right)+\mathcal{O}\left(\frac{1}{R^{2}}\right) .
$$

Note that the post-Newtonian coefficients will involve the logarithm $\ln x$ starting at $3 \mathrm{PN}$ order; see Eq. (127). They depend on the binary's phase $\phi$, explicitly given at 3.5PN order by Eq. (318), 
through the very useful auxiliary phase variable $\psi$ that is "distorted by tails" [87, 11] and reads

$$
\psi \equiv \phi-\frac{2 G \mathrm{M} \Omega}{c^{3}} \ln \left(\frac{\Omega}{\Omega_{0}}\right)
$$

Here M denotes the binary's ADM mass and it is very important to include all its relevant postNewtonian contributions as given by Eq. (309). The constant frequency $\Omega_{0}$ can be chosen at will, for instance to be the entry frequency of some detector. For the plus polarization we have ${ }^{69}$

$$
\begin{aligned}
& \underset{0}{H_{+}}=-\left(1+c_{i}^{2}\right) \cos 2 \psi-\frac{1}{96} s_{i}^{2}\left(17+c_{i}^{2}\right), \\
& \underset{1 / 2}{H_{+}}=-s_{i} \Delta\left[\cos \psi\left(\frac{5}{8}+\frac{1}{8} c_{i}^{2}\right)-\cos 3 \psi\left(\frac{9}{8}+\frac{9}{8} c_{i}^{2}\right)\right], \\
& \underset{1}{H_{+}}=\cos 2 \psi\left[\frac{19}{6}+\frac{3}{2} c_{i}^{2}-\frac{1}{3} c_{i}^{4}+\nu\left(-\frac{19}{6}+\frac{11}{6} c_{i}^{2}+c_{i}^{4}\right)\right] \\
& -\cos 4 \psi\left[\frac{4}{3} s_{i}^{2}\left(1+c_{i}^{2}\right)(1-3 \nu)\right] \text {, } \\
& \underset{3 / 2}{H_{+}}=s_{i} \Delta \cos \psi\left[\frac{19}{64}+\frac{5}{16} c_{i}^{2}-\frac{1}{192} c_{i}^{4}+\nu\left(-\frac{49}{96}+\frac{1}{8} c_{i}^{2}+\frac{1}{96} c_{i}^{4}\right)\right] \\
& +\cos 2 \psi\left[-2 \pi\left(1+c_{i}^{2}\right)\right] \\
& +s_{i} \Delta \cos 3 \psi\left[-\frac{657}{128}-\frac{45}{16} c_{i}^{2}+\frac{81}{128} c_{i}^{4}+\nu\left(\frac{225}{64}-\frac{9}{8} c_{i}^{2}-\frac{81}{64} c_{i}^{4}\right)\right] \\
& +s_{i} \Delta \cos 5 \psi\left[\frac{625}{384} s_{i}^{2}\left(1+c_{i}^{2}\right)(1-2 \nu)\right] \text {, } \\
& \underset{2}{H}+=\pi s_{i} \Delta \cos \psi\left[-\frac{5}{8}-\frac{1}{8} c_{i}^{2}\right] \\
& +\cos 2 \psi\left[\frac{11}{60}+\frac{33}{10} c_{i}^{2}+\frac{29}{24} c_{i}^{4}-\frac{1}{24} c_{i}^{6}+\nu\left(\frac{353}{36}-3 c_{i}^{2}-\frac{251}{72} c_{i}^{4}+\frac{5}{24} c_{i}^{6}\right)\right. \\
& \left.+\nu^{2}\left(-\frac{49}{12}+\frac{9}{2} c_{i}^{2}-\frac{7}{24} c_{i}^{4}-\frac{5}{24} c_{i}^{6}\right)\right] \\
& +\pi s_{i} \Delta \cos 3 \psi\left[\frac{27}{8}\left(1+c_{i}^{2}\right)\right] \\
& +\frac{2}{15} s_{i}^{2} \cos 4 \psi\left[59+35 c_{i}^{2}-8 c_{i}^{4}-\frac{5}{3} \nu\left(131+59 c_{i}^{2}-24 c_{i}^{4}\right)+5 \nu^{2}\left(21-3 c_{i}^{2}-8 c_{i}^{4}\right)\right] \\
& +\cos 6 \psi\left[-\frac{81}{40} s_{i}^{4}\left(1+c_{i}^{2}\right)\left(1-5 \nu+5 \nu^{2}\right)\right] \\
& +s_{i} \Delta \sin \psi\left[\frac{11}{40}+\frac{5 \ln 2}{4}+c_{i}^{2}\left(\frac{7}{40}+\frac{\ln 2}{4}\right)\right] \\
& +s_{i} \Delta \sin 3 \psi\left[\left(-\frac{189}{40}+\frac{27}{4} \ln (3 / 2)\right)\left(1+c_{i}^{2}\right)\right] \text {, }
\end{aligned}
$$

69 Notice the obvious fact that the polarization waveforms remain invariant when we rotate by $\pi$ the separation direction between the particles and simultaneously exchange the labels of the two particles, i.e., when we apply the transformation $(\psi, \Delta) \rightarrow(\psi+\pi,-\Delta)$. Moreover, due to the parity invariance, the $H_{+}$'s are unchanged after the replacement $i \rightarrow \pi-i$, while the $H_{\times}$'s being the projection of $h_{i j}^{\mathrm{TT}}$ on a tensorial product of two vectors of inverse parity types, is changed into its opposite. 


$$
\begin{aligned}
& \underset{5 / 2}{H_{+}}=s_{i} \Delta \cos \psi\left[\frac{1771}{5120}-\frac{1667}{5120} c_{i}^{2}+\frac{217}{9216} c_{i}^{4}-\frac{1}{9216} c_{i}^{6}\right. \\
& +\nu\left(\frac{681}{256}+\frac{13}{768} c_{i}^{2}-\frac{35}{768} c_{i}^{4}+\frac{1}{2304} c_{i}^{6}\right) \\
& \left.+\nu^{2}\left(-\frac{3451}{9216}+\frac{673}{3072} c_{i}^{2}-\frac{5}{9216} c_{i}^{4}-\frac{1}{3072} c_{i}^{6}\right)\right] \\
& +\pi \cos 2 \psi\left[\frac{19}{3}+3 c_{i}^{2}-\frac{2}{3} c_{i}^{4}+\nu\left(-\frac{16}{3}+\frac{14}{3} c_{i}^{2}+2 c_{i}^{4}\right)\right] \\
& +s_{i} \Delta \cos 3 \psi\left[\frac{3537}{1024}-\frac{22977}{5120} c_{i}^{2}-\frac{15309}{5120} c_{i}^{4}+\frac{729}{5120} c_{i}^{6}\right. \\
& +\nu\left(-\frac{23829}{1280}+\frac{5529}{1280} c_{i}^{2}+\frac{7749}{1280} c_{i}^{4}-\frac{729}{1280} c_{i}^{6}\right) \\
& \left.+\nu^{2}\left(\frac{29127}{5120}-\frac{27267}{5120} c_{i}^{2}-\frac{1647}{5120} c_{i}^{4}+\frac{2187}{5120} c_{i}^{6}\right)\right] \\
& +\cos 4 \psi\left[-\frac{16 \pi}{3}\left(1+c_{i}^{2}\right) s_{i}^{2}(1-3 \nu)\right] \\
& +s_{i} \Delta \cos 5 \psi\left[-\frac{108125}{9216}+\frac{40625}{9216} c_{i}^{2}+\frac{83125}{9216} c_{i}^{4}-\frac{15625}{9216} c_{i}^{6}\right. \\
& +\nu\left(\frac{8125}{256}-\frac{40625}{2304} c_{i}^{2}-\frac{48125}{2304} c_{i}^{4}+\frac{15625}{2304} c_{i}^{6}\right) \\
& \left.+\nu^{2}\left(-\frac{119375}{9216}+\frac{40625}{3072} c_{i}^{2}+\frac{44375}{9216} c_{i}^{4}-\frac{15625}{3072} c_{i}^{6}\right)\right] \\
& +\Delta \cos 7 \psi\left[\frac{117649}{46080} s_{i}^{5}\left(1+c_{i}^{2}\right)\left(1-4 \nu+3 \nu^{2}\right)\right] \\
& +\sin 2 \psi\left[-\frac{9}{5}+\frac{14}{5} c_{i}^{2}+\frac{7}{5} c_{i}^{4}+\nu\left(32+\frac{56}{5} c_{i}^{2}-\frac{28}{5} c_{i}^{4}\right)\right] \\
& +s_{i}^{2}\left(1+c_{i}^{2}\right) \sin 4 \psi\left[\frac{56}{5}-\frac{32 \ln 2}{3}+\nu\left(-\frac{1193}{30}+32 \ln 2\right)\right], \\
& \underset{3}{H_{+}}=\pi \Delta s_{i} \cos \psi\left[\frac{19}{64}+\frac{5}{16} c_{i}^{2}-\frac{1}{192} c_{i}^{4}+\nu\left(-\frac{19}{96}+\frac{3}{16} c_{i}^{2}+\frac{1}{96} c_{i}^{4}\right)\right] \\
& +\cos 2 \psi\left[-\frac{465497}{11025}+\left(\frac{856}{105} \gamma_{\mathrm{E}}-\frac{2 \pi^{2}}{3}+\frac{428}{105} \ln (16 x)\right)\left(1+c_{i}^{2}\right)\right. \\
& -\frac{3561541}{88200} c_{i}^{2}-\frac{943}{720} c_{i}^{4}+\frac{169}{720} c_{i}^{6}-\frac{1}{360} c_{i}^{8} \\
& +\nu\left(\frac{2209}{360}-\frac{41 \pi^{2}}{96}\left(1+c_{i}^{2}\right)+\frac{2039}{180} c_{i}^{2}+\frac{3311}{720} c_{i}^{4}-\frac{853}{720} c_{i}^{6}+\frac{7}{360} c_{i}^{8}\right) \\
& +\nu^{2}\left(\frac{12871}{540}-\frac{1583}{60} c_{i}^{2}-\frac{145}{108} c_{i}^{4}+\frac{56}{45} c_{i}^{6}-\frac{7}{180} c_{i}^{8}\right) \\
& \left.+\nu^{3}\left(-\frac{3277}{810}+\frac{19661}{3240} c_{i}^{2}-\frac{281}{144} c_{i}^{4}-\frac{73}{720} c_{i}^{6}+\frac{7}{360} c_{i}^{8}\right)\right] \\
& +\pi \Delta s_{i} \cos 3 \psi\left[-\frac{1971}{128}-\frac{135}{16} c_{i}^{2}+\frac{243}{128} c_{i}^{4}+\nu\left(\frac{567}{64}-\frac{81}{16} c_{i}^{2}-\frac{243}{64} c_{i}^{4}\right)\right]
\end{aligned}
$$




$$
\begin{aligned}
& +s_{i}^{2} \cos 4 \psi\left[-\frac{2189}{210}+\frac{1123}{210} c_{i}^{2}+\frac{56}{9} c_{i}^{4}-\frac{16}{45} c_{i}^{6}\right. \\
& +\nu\left(\frac{6271}{90}-\frac{1969}{90} c_{i}^{2}-\frac{1432}{45} c_{i}^{4}+\frac{112}{45} c_{i}^{6}\right) \\
& \left.+\nu^{2}\left(-\frac{3007}{27}+\frac{3493}{135} c_{i}^{2}+\frac{1568}{45} c_{i}^{4}-\frac{224}{45} c_{i}^{6}\right)+\nu^{3}\left(\frac{161}{6}-\frac{1921}{90} c_{i}^{2}-\frac{184}{45} c_{i}^{4}+\frac{112}{45} c_{i}^{6}\right)\right] \\
& +\Delta \cos 5 \psi\left[\frac{3125 \pi}{384} s_{i}^{3}\left(1+c_{i}^{2}\right)(1-2 \nu)\right] \\
& +s_{i}^{4} \cos 6 \psi\left[\frac{1377}{80}+\frac{891}{80} c_{i}^{2}-\frac{729}{280} c_{i}^{4}+\nu\left(-\frac{7857}{80}-\frac{891}{16} c_{i}^{2}+\frac{729}{40} c_{i}^{4}\right)\right. \\
& \left.+\nu^{2}\left(\frac{567}{4}+\frac{567}{10} c_{i}^{2}-\frac{729}{20} c_{i}^{4}\right)+\nu^{3}\left(-\frac{729}{16}-\frac{243}{80} c_{i}^{2}+\frac{729}{40} c_{i}^{4}\right)\right] \\
& +\cos 8 \psi\left[-\frac{1024}{315} s_{i}^{6}\left(1+c_{i}^{2}\right)\left(1-7 \nu+14 \nu^{2}-7 \nu^{3}\right)\right] \\
& +\Delta s_{i} \sin \psi\left[-\frac{2159}{40320}-\frac{19 \ln 2}{32}+\left(-\frac{95}{224}-\frac{5 \ln 2}{8}\right) c_{i}^{2}+\left(\frac{181}{13440}+\frac{\ln 2}{96}\right) c_{i}^{4}\right. \\
& \left.+\nu\left(\frac{1369}{160}+\frac{19 \ln 2}{48}+\left(-\frac{41}{48}-\frac{3 \ln 2}{8}\right) c_{i}^{2}+\left(-\frac{313}{480}-\frac{\ln 2}{48}\right) c_{i}^{4}\right)\right] \\
& +\sin 2 \psi\left[-\frac{428 \pi}{105}\left(1+c_{i}^{2}\right)\right] \\
& +\Delta s_{i} \sin 3 \psi\left[\frac{205119}{8960}-\frac{1971}{64} \ln (3 / 2)+\left(\frac{1917}{224}-\frac{135}{8} \ln (3 / 2)\right) c_{i}^{2}\right. \\
& +\left(-\frac{43983}{8960}+\frac{243}{64} \ln (3 / 2)\right) c_{i}^{4} \\
& +\nu\left(-\frac{54869}{960}+\frac{567}{32} \ln (3 / 2)+\left(-\frac{923}{80}-\frac{81}{8} \ln (3 / 2)\right) c_{i}^{2}\right. \\
& \left.\left.+\left(\frac{41851}{2880}-\frac{243}{32} \ln (3 / 2)\right) c_{i}^{4}\right)\right] \\
& +\Delta s_{i}^{3}\left(1+c_{i}^{2}\right) \sin 5 \psi\left[-\frac{113125}{5376}+\frac{3125}{192} \ln (5 / 2)+\nu\left(\frac{17639}{320}-\frac{3125}{96} \ln (5 / 2)\right)\right] \text {. }
\end{aligned}
$$

For the cross polarizations we obtain

$$
\begin{aligned}
\underset{0}{H_{\times}} & =-2 c_{i} \sin 2 \psi \\
\underset{1 / 2}{H_{\times}} & =s_{i} c_{i} \Delta\left[-\frac{3}{4} \sin \psi+\frac{9}{4} \sin 3 \psi\right] \\
\underset{1}{H} & =c_{i} \sin 2 \psi\left[\frac{17}{3}-\frac{4}{3} c_{i}^{2}+\nu\left(-\frac{13}{3}+4 c_{i}^{2}\right)\right] \\
& +c_{i} s_{i}^{2} \sin 4 \psi\left[-\frac{8}{3}(1-3 \nu)\right], \\
\underset{3 / 2}{H_{\times}} & =s_{i} c_{i} \Delta \sin \psi\left[\frac{21}{32}-\frac{5}{96} c_{i}^{2}+\nu\left(-\frac{23}{48}+\frac{5}{48} c_{i}^{2}\right)\right] \\
& -4 \pi c_{i} \sin 2 \psi
\end{aligned}
$$




$$
\begin{aligned}
& +s_{i} c_{i} \Delta \sin 3 \psi\left[-\frac{603}{64}+\frac{135}{64} c_{i}^{2}+\nu\left(\frac{171}{32}-\frac{135}{32} c_{i}^{2}\right)\right] \\
& +s_{i} c_{i} \Delta \sin 5 \psi\left[\frac{625}{192}(1-2 \nu) s_{i}^{2}\right] \text {, } \\
& \underset{2}{H} \times s_{i} c_{i} \Delta \cos \psi\left[-\frac{9}{20}-\frac{3}{2} \ln 2\right] \\
& +s_{i} c_{i} \Delta \cos 3 \psi\left[\frac{189}{20}-\frac{27}{2} \ln (3 / 2)\right] \\
& -s_{i} c_{i} \Delta\left[\frac{3 \pi}{4}\right] \sin \psi \\
& +c_{i} \sin 2 \psi\left[\frac{17}{15}+\frac{113}{30} c_{i}^{2}-\frac{1}{4} c_{i}^{4}+\nu\left(\frac{143}{9}-\frac{245}{18} c_{i}^{2}+\frac{5}{4} c_{i}^{4}\right)+\nu^{2}\left(-\frac{14}{3}+\frac{35}{6} c_{i}^{2}-\frac{5}{4} c_{i}^{4}\right)\right] \\
& +s_{i} c_{i} \Delta \sin 3 \psi\left[\frac{27 \pi}{4}\right] \\
& +\frac{4}{15} c_{i} s_{i}^{2} \sin 4 \psi\left[55-12 c_{i}^{2}-\frac{5}{3} \nu\left(119-36 c_{i}^{2}\right)+5 \nu^{2}\left(17-12 c_{i}^{2}\right)\right] \\
& +c_{i} \sin 6 \psi\left[-\frac{81}{20} s_{i}^{4}\left(1-5 \nu+5 \nu^{2}\right)\right], \\
& \underset{5 / 2}{H} \times \frac{6}{5} s_{i}^{2} c_{i} \nu \\
& +c_{i} \cos 2 \psi\left[2-\frac{22}{5} c_{i}^{2}+\nu\left(-\frac{282}{5}+\frac{94}{5} c_{i}^{2}\right)\right] \\
& +c_{i} s_{i}^{2} \cos 4 \psi\left[-\frac{112}{5}+\frac{64}{3} \ln 2+\nu\left(\frac{1193}{15}-64 \ln 2\right)\right] \\
& +s_{i} c_{i} \Delta \sin \psi\left[-\frac{913}{7680}+\frac{1891}{11520} c_{i}^{2}-\frac{7}{4608} c_{i}^{4}\right. \\
& \left.+\nu\left(\frac{1165}{384}-\frac{235}{576} c_{i}^{2}+\frac{7}{1152} c_{i}^{4}\right)+\nu^{2}\left(-\frac{1301}{4608}+\frac{301}{2304} c_{i}^{2}-\frac{7}{1536} c_{i}^{4}\right)\right] \\
& +\pi c_{i} \sin 2 \psi\left[\frac{34}{3}-\frac{8}{3} c_{i}^{2}+\nu\left(-\frac{20}{3}+8 c_{i}^{2}\right)\right] \\
& +s_{i} c_{i} \Delta \sin 3 \psi\left[\frac{12501}{2560}-\frac{12069}{1280} c_{i}^{2}+\frac{1701}{2560} c_{i}^{4}\right. \\
& \left.+\nu\left(-\frac{19581}{640}+\frac{7821}{320} c_{i}^{2}-\frac{1701}{640} c_{i}^{4}\right)+\nu^{2}\left(\frac{18903}{2560}-\frac{11403}{1280} c_{i}^{2}+\frac{5103}{2560} c_{i}^{4}\right)\right] \\
& +s_{i}^{2} c_{i} \sin 4 \psi\left[-\frac{32 \pi}{3}(1-3 \nu)\right] \\
& +\Delta s_{i} c_{i} \sin 5 \psi\left[-\frac{101875}{4608}+\frac{6875}{256} c_{i}^{2}-\frac{21875}{4608} c_{i}^{4}\right. \\
& \left.+\nu\left(\frac{66875}{1152}-\frac{44375}{576} c_{i}^{2}+\frac{21875}{1152} c_{i}^{4}\right)+\nu^{2}\left(-\frac{100625}{4608}+\frac{83125}{2304} c_{i}^{2}-\frac{21875}{1536} c_{i}^{4}\right)\right] \\
& +\Delta s_{i}^{5} c_{i} \sin 7 \psi\left[\frac{117649}{23040}\left(1-4 \nu+3 \nu^{2}\right)\right] \text {, }
\end{aligned}
$$




$$
\begin{aligned}
& \underset{3}{H} \times s_{i} c_{i} \cos \psi\left[\frac{11617}{20160}+\frac{21}{16} \ln 2+\left(-\frac{251}{2240}-\frac{5}{48} \ln 2\right) c_{i}^{2}\right. \\
& \left.+\nu\left(-\frac{2419}{240}-\frac{5}{24} \ln 2+\left(\frac{727}{240}+\frac{5}{24} \ln 2\right) c_{i}^{2}\right)\right] \text {. } \\
& +c_{i} \cos 2 \psi\left[\frac{856 \pi}{105}\right] \\
& +\Delta s_{i} c_{i} \cos 3 \psi\left[-\frac{36801}{896}+\frac{1809}{32} \ln (3 / 2)+\left(\frac{65097}{4480}-\frac{405}{32} \ln (3 / 2)\right) c_{i}^{2}\right. \\
& \left.+\nu\left(\frac{28445}{288}-\frac{405}{16} \ln (3 / 2)+\left(-\frac{7137}{160}+\frac{405}{16} \ln (3 / 2)\right) c_{i}^{2}\right)\right] \\
& +\Delta s_{i}^{3} c_{i} \cos 5 \psi\left[\frac{113125}{2688}-\frac{3125}{96} \ln (5 / 2)+\nu\left(-\frac{17639}{160}+\frac{3125}{48} \ln (5 / 2)\right)\right] \\
& +\pi \Delta s_{i} c_{i} \sin \psi\left[\frac{21}{32}-\frac{5}{96} c_{i}^{2}+\nu\left(-\frac{5}{48}+\frac{5}{48} c_{i}^{2}\right)\right] \\
& +c_{i} \sin 2 \psi\left[-\frac{3620761}{44100}+\frac{1712}{105} \gamma_{\mathrm{E}}-\frac{4 \pi^{2}}{3}+\frac{856}{105} \ln (16 x)-\frac{3413}{1260} c_{i}^{2}+\frac{2909}{2520} c_{i}^{4}-\frac{1}{45} c_{i}^{6}\right. \\
& +\nu\left(\frac{743}{90}-\frac{41 \pi^{2}}{48}+\frac{3391}{180} c_{i}^{2}-\frac{2287}{360} c_{i}^{4}+\frac{7}{45} c_{i}^{6}\right) \\
& \left.+\nu^{2}\left(\frac{7919}{270}-\frac{5426}{135} c_{i}^{2}+\frac{382}{45} c_{i}^{4}-\frac{14}{45} c_{i}^{6}\right)+\nu^{3}\left(-\frac{6457}{1620}+\frac{1109}{180} c_{i}^{2}-\frac{281}{120} c_{i}^{4}+\frac{7}{45} c_{i}^{6}\right)\right] \\
& +\pi \Delta s_{i} c_{i} \sin 3 \psi\left[-\frac{1809}{64}+\frac{405}{64} c_{i}^{2}+\nu\left(\frac{405}{32}-\frac{405}{32} c_{i}^{2}\right)\right] \\
& +s_{i}^{2} c_{i} \sin 4 \psi\left[-\frac{1781}{105}+\frac{1208}{63} c_{i}^{2}-\frac{64}{45} c_{i}^{4}\right. \\
& +\nu\left(\frac{5207}{45}-\frac{536}{5} c_{i}^{2}+\frac{448}{45} c_{i}^{4}\right)+\nu^{2}\left(-\frac{24838}{135}+\frac{2224}{15} c_{i}^{2}-\frac{896}{45} c_{i}^{4}\right) \\
& \left.+\nu^{3}\left(\frac{1703}{45}-\frac{1976}{45} c_{i}^{2}+\frac{448}{45} c_{i}^{4}\right)\right] \\
& +\Delta \sin 5 \psi\left[\frac{3125 \pi}{192} s_{i}^{3} c_{i}(1-2 \nu)\right] \\
& +s_{i}^{4} c_{i} \sin 6 \psi\left[\frac{9153}{280}-\frac{243}{35} c_{i}^{2}+\nu\left(-\frac{7371}{40}+\frac{243}{5} c_{i}^{2}\right)\right. \\
& \left.+\nu^{2}\left(\frac{1296}{5}-\frac{486}{5} c_{i}^{2}\right)+\nu^{3}\left(-\frac{3159}{40}+\frac{243}{5} c_{i}^{2}\right)\right] \\
& +\sin 8 \psi\left[-\frac{2048}{315} s_{i}^{6} c_{i}\left(1-7 \nu+14 \nu^{2}-7 \nu^{3}\right)\right] \text {. }
\end{aligned}
$$

Notice the non-linear memory zero-frequency (DC) term present in the Newtonian plus polarization ${ }_{0} H_{+}$; see Refs. $[427,11,189]$ for the computation of this term. Notice also that there is another DC term in the $2.5 \mathrm{PN}$ cross polarization ${ }_{5 / 2} \mathrm{H}_{\times}$, first term in Eq. (323f).

The practical implementation of the theoretical templates in the data analysis of detectors follows from the standard matched filtering technique. The raw output of the detector $o(t)$ consists of the superposition of the real gravitational wave signal $h_{\text {real }}(t)$ and of noise $n(t)$. The noise is assumed to be a stationary Gaussian random variable, with zero expectation value, and with (supposedly known) frequency-dependent power spectral density $S_{n}(\omega)$. The experimenters construct 
the correlation between $o(t)$ and a filter $q(t)$, i.e.,

$$
c(t)=\int_{-\infty}^{+\infty} \mathrm{d} t^{\prime} o\left(t^{\prime}\right) q\left(t+t^{\prime}\right)
$$

and divide $c(t)$ by the square root of its variance, or correlation noise. The expectation value of this ratio defines the filtered signal-to-noise ratio (SNR). Looking for the useful signal $h_{\text {real }}(t)$ in the detector's output $o(t)$, the data analysists adopt for the filter

$$
\tilde{q}(\omega)=\frac{\tilde{h}(\omega)}{S_{n}(\omega)},
$$

where $\tilde{q}(\omega)$ and $\tilde{h}(\omega)$ are the Fourier transforms of $q(t)$ and of the theoretically computed template $h(t)$. By the matched filtering theorem, the filter (325) maximizes the SNR if $h(t)=h_{\text {real }}(t)$. The maximum SNR is then the best achievable with a linear filter. In practice, because of systematic errors in the theoretical modelling, the template $h(t)$ will not exactly match the real signal $h_{\text {real }}(t)$; however if the template is to constitute a realistic representation of nature the errors will be small. This is of course the motivation for computing high order post-Newtonian templates, in order to reduce as much as possible the systematic errors due to the unknown post-Newtonian remainder.

To conclude, the use of theoretical templates based on the preceding $3 \mathrm{PN} / 3.5 \mathrm{PN}$ waveforms, and having their frequency evolution built in via the $3.5 \mathrm{PN}$ phase evolution (318) [recall also the "tail-distorted" phase variable (321)], should yield some accurate detection and measurement of the binary signals, whose inspiral phase takes place in the detector's bandwidth $[105,106,159,156$, $3,18,111]$. Interestingly, it should also permit some new tests of general relativity, because we have the possibility of checking that the observed signals do obey each of the terms of the phasing formula (318) - particularly interesting are those terms associated with non-linear tails - exactly as they are predicted by Einstein's theory [84, 85, 15, 14]. Indeed, we don't know of any other physical systems for which it would be possible to perform such tests.

\subsection{Spherical harmonic modes for numerical relativity}

The spin-weighted spherical harmonic modes of the polarization waveforms have been defined in Eq. (71). They can be evaluated either from applying the angular integration formula (72), or alternatively from using the relations (73) -(74) giving the individual modes directly in terms of separate contributions of the radiative moments $\mathrm{U}_{L}$ and $\mathrm{V}_{L}$. The latter route is actually more interesting [272] if some of the radiative moments are known to higher PN order than others. In this case the comparison with the numerical calculation for these particular modes can be made with higher post-Newtonian accuracy.

A useful fact to remember is that for non-spinning binaries, the mode $h^{\ell m}$ is entirely given by the mass multipole moment $\mathrm{U}_{L}$ when $\ell+m$ is even, and by the current one $\mathrm{V}_{L}$ when $\ell+m$ is odd. This is valid in general for non-spinning binaries, regardless of the orbit being quasi-circular or elliptical. The important point is only that the motion of the two particles must be planar, i.e., takes place in a fixed plane. This is the case if the particles are non-spinning, but this will also be the case if, more generally, the spins are aligned or anti-aligned with the orbital angular momentum, since there is no orbital precession in this case. Thus, for any "planar" binaries, Eq. (73) splits to (see Ref. [197] for a proof)

$$
\begin{array}{ll}
h^{\ell m}=-\frac{G}{\sqrt{2} R c^{\ell+2}} \mathrm{U}^{\ell m} \quad(\text { when } \ell+m \text { is even }), \\
h^{\ell m}=\frac{G}{\sqrt{2} R c^{\ell+3}} \mathrm{iV}^{\ell m} \quad(\text { when } \ell+m \text { is odd }) .
\end{array}
$$


Let us factorize out in all the modes an overall coefficient including the appropriate phase factor $e^{-\mathrm{i} m \psi}$, where we recall that $\psi$ denotes the tail-distorted phase introduced in Eq. (321), and such that the dominant mode with $(\ell, m)=(2,2)$ conventionally starts with one at the Newtonian order. We thus pose

$$
h^{\ell m}=\frac{2 G m \nu x}{R c^{2}} \sqrt{\frac{16 \pi}{5}} \mathcal{H}^{\ell m} e^{-\mathrm{i} m \psi} .
$$

We now list all the known results for $\mathcal{H}^{\ell m}$. We assume $m \geqslant 0$; the modes having $m<0$ are easily deduced using $\mathcal{H}^{\ell,-m}=(-)^{\ell} \overline{\mathcal{H}}^{\ell m}$. The dominant mode $\mathcal{H}^{22}$, which is primarily important for numerical relativity comparisons, is known at $3.5 \mathrm{PN}$ order and reads $[74,197]$

$$
\begin{aligned}
\mathcal{H}^{22}= & +x\left(-\frac{107}{42}+\frac{55}{42} \nu\right)+2 \pi x^{3 / 2}+x^{2}\left(-\frac{2173}{1512}-\frac{1069}{216} \nu+\frac{2047}{1512} \nu^{2}\right) \\
+ & x^{5 / 2}\left(-\frac{107 \pi}{21}-24 \mathrm{i} \nu+\frac{34 \pi}{21} \nu\right)+x^{3}\left(\frac{27027409}{646800}-\frac{856}{105} \gamma_{\mathrm{E}}+\frac{428 \pi}{105} \mathrm{i}+\frac{2 \pi^{2}}{3}\right. \\
& \left.+\left(-\frac{278185}{33264}+\frac{41 \pi^{2}}{96}\right) \nu-\frac{20261}{2772} \nu^{2}+\frac{114635}{99792} \nu^{3}-\frac{428}{105} \ln (16 x)\right) \\
& +x^{7 / 2}\left(-\frac{2173 \pi}{756}+\left(-\frac{2495 \pi}{378}+\frac{14333}{162} \mathrm{i}\right) \nu+\left(\frac{40 \pi}{27}-\frac{4066}{945} \mathrm{i}\right) \nu^{2}\right)+\mathcal{O}\left(\frac{1}{c^{8}}\right) .
\end{aligned}
$$

Similarly, we report the subdominant modes $\mathcal{H}^{33}$ and $\mathcal{H}^{31}$ also known at 3.5PN order [195]

$$
\begin{aligned}
\mathcal{H}^{33}= & -\frac{3}{4} \mathrm{i} \sqrt{\frac{15}{14}} \Delta\left[x^{1 / 2}+x^{3 / 2}(-4+2 \nu)+x^{2}\left(3 \pi+\mathrm{i}\left[-\frac{21}{5}+6 \ln (3 / 2)\right]\right)\right. \\
+ & x^{5 / 2}\left(\frac{123}{110}-\frac{1838 \nu}{165}+\frac{887 \nu^{2}}{330}\right)+x^{3}\left(-12 \pi+\frac{9 \pi \nu}{2}\right. \\
& \left.+\mathrm{i}\left[\frac{84}{5}-24 \ln (3 / 2)+\nu\left(-\frac{48103}{1215}+9 \ln (3 / 2)\right)\right]\right) \\
+ & x^{7 / 2}\left(\frac{19388147}{280280}+\frac{492}{35} \ln (3 / 2)-18 \ln ^{2}(3 / 2)-\frac{78}{7} \gamma_{\mathrm{E}}+\frac{3}{2} \pi^{2}+6 \mathrm{i} \pi\left[-\frac{41}{35}+3 \ln (3 / 2)\right]\right. \\
& \left.\left.+\frac{\nu}{8}\left[-\frac{7055}{429}+\frac{41}{8} \pi^{2}\right]-\frac{318841}{17160} \nu^{2}+\frac{8237}{2860} \nu^{3}-\frac{39}{7} \ln (16 x)\right)\right]+\mathcal{O}\left(\frac{1}{c^{8}}\right), \\
\mathcal{H}^{31}= & \frac{\mathrm{i} \Delta}{12 \sqrt{14}}\left[x^{1 / 2}+x^{3 / 2}\left(-\frac{8}{3}-\frac{2 \nu}{3}\right)+x^{2}\left(\pi+\mathrm{i}\left[-\frac{7}{5}-2 \ln 2\right]\right)\right. \\
+ & x^{5 / 2}\left(\frac{607}{198}-\frac{136 \nu}{99}-\frac{247 \nu^{2}}{198}\right)+x^{3}\left(-\frac{8 \pi}{3}-\frac{7 \pi \nu}{6}\right. \\
& \left.+\mathrm{i}\left[\frac{56}{15}+\frac{16 \ln 2}{3}+\nu\left(-\frac{1}{15}+\frac{7 \ln 2}{3}\right)\right]\right) \\
+ & x^{7 / 2}\left(\frac{10753397}{1513512}-2 \ln 2\left[\frac{212}{105}+\ln 2\right]-\frac{26}{21} \gamma_{\mathrm{E}}+\frac{\pi^{2}}{6}-2 \mathrm{i} \pi\left[\frac{41}{105}+\ln 2\right]\right. \\
& \left.\left.+\frac{\nu}{8}\left(-\frac{1738843}{19305}+\frac{41}{8} \pi^{2}\right)+\frac{327059}{30888} \nu^{2}-\frac{17525}{15444} \nu^{3}-\frac{13}{21} \ln x\right)\right]+\mathcal{O}\left(\frac{1}{c^{8}}\right)
\end{aligned}
$$

The other modes are known with a precision consistent with 3PN order in the full waveform [74]:

$$
\mathcal{H}^{21}=\frac{\mathrm{i}}{3} \Delta\left[x^{1 / 2}+x^{3 / 2}\left(-\frac{17}{28}+\frac{5 \nu}{7}\right)+x^{2}\left(\pi+\mathrm{i}\left(-\frac{1}{2}-2 \ln 2\right)\right)\right.
$$




$$
\begin{aligned}
& +x^{5 / 2}\left(-\frac{43}{126}-\frac{509 \nu}{126}+\frac{79 \nu^{2}}{168}\right)+x^{3}\left(-\frac{17 \pi}{28}+\frac{3 \pi \nu}{14}\right. \\
& \left.\left.+\mathrm{i}\left(\frac{17}{56}+\nu\left(-\frac{353}{28}-\frac{3 \ln 2}{7}\right)+\frac{17 \ln 2}{14}\right)\right)\right]+\mathcal{O}\left(\frac{1}{c^{7}}\right) \\
& \mathcal{H}^{20}=-\frac{5}{14 \sqrt{6}}+\mathcal{O}\left(\frac{1}{c^{7}}\right) \\
& \mathcal{H}^{32}=\frac{1}{3} \sqrt{\frac{5}{7}}\left[x(1-3 \nu)+x^{2}\left(-\frac{193}{90}+\frac{145 \nu}{18}-\frac{73 \nu^{2}}{18}\right)+x^{5 / 2}\left(2 \pi-6 \pi \nu+\mathrm{i}\left(-3+\frac{66 \nu}{5}\right)\right)\right. \\
& \left.+x^{3}\left(-\frac{1451}{3960}-\frac{17387 \nu}{3960}+\frac{5557 \nu^{2}}{220}-\frac{5341 \nu^{3}}{1320}\right)\right]+\mathcal{O}\left(\frac{1}{c^{7}}\right) \text {, } \\
& \mathcal{H}^{30}=-\frac{2}{5} \mathrm{i} \sqrt{\frac{6}{7}} x^{5 / 2} \nu+\mathcal{O}\left(\frac{1}{c^{7}}\right), \\
& \mathcal{H}^{44}=-\frac{8}{9} \sqrt{\frac{5}{7}}\left[x(1-3 \nu)+x^{2}\left(-\frac{593}{110}+\frac{1273 \nu}{66}-\frac{175 \nu^{2}}{22}\right)\right. \\
& +x^{5 / 2}\left(4 \pi-12 \pi \nu+\mathrm{i}\left(-\frac{42}{5}+\nu\left(\frac{1193}{40}-24 \ln 2\right)+8 \ln 2\right)\right) \\
& \left.+x^{3}\left(\frac{1068671}{200200}-\frac{1088119 \nu}{28600}+\frac{146879 \nu^{2}}{2340}-\frac{226097 \nu^{3}}{17160}\right)\right]+\mathcal{O}\left(\frac{1}{c^{7}}\right) \text {, } \\
& \mathcal{H}^{43}=-\frac{9 \mathrm{i}}{4 \sqrt{70}} \Delta\left[x^{3 / 2}(1-2 \nu)+x^{5 / 2}\left(-\frac{39}{11}+\frac{1267 \nu}{132}-\frac{131 \nu^{2}}{33}\right)\right. \\
& \left.+x^{3}\left(3 \pi-6 \pi \nu+\mathrm{i}\left(-\frac{32}{5}+\nu\left(\frac{16301}{810}-12 \ln (3 / 2)\right)+6 \ln (3 / 2)\right)\right)\right]+\mathcal{O}\left(\frac{1}{c^{7}}\right) \text {, } \\
& \mathcal{H}^{42}=\frac{1}{63} \sqrt{5}\left[x(1-3 \nu)+x^{2}\left(-\frac{437}{110}+\frac{805 \nu}{66}-\frac{19 \nu^{2}}{22}\right)+x^{5 / 2}(2 \pi-6 \pi \nu\right. \\
& \left.\left.+\mathrm{i}\left(-\frac{21}{5}+\frac{84 \nu}{5}\right)\right)+x^{3}\left(\frac{1038039}{200200}-\frac{606751 \nu}{28600}+\frac{400453 \nu^{2}}{25740}+\frac{25783 \nu^{3}}{17160}\right)\right]+\mathcal{O}\left(\frac{1}{c^{7}}\right), \\
& \mathcal{H}^{41}=\frac{\mathrm{i}}{84 \sqrt{10}} \Delta\left[x^{3 / 2}(1-2 \nu)+x^{5 / 2}\left(-\frac{101}{33}+\frac{337 \nu}{44}-\frac{83 \nu^{2}}{33}\right)\right. \\
& \left.+x^{3}\left(\pi-2 \pi \nu+\mathrm{i}\left(-\frac{32}{15}-2 \ln 2+\nu\left(\frac{1661}{30}+4 \ln 2\right)\right)\right)\right]+\mathcal{O}\left(\frac{1}{c^{7}}\right), \\
& \mathcal{H}^{40}=-\frac{1}{504 \sqrt{2}}+\mathcal{O}\left(\frac{1}{c^{7}}\right) \\
& \mathcal{H}^{55}=\frac{625 \mathrm{i}}{96 \sqrt{66}} \Delta\left[x^{3 / 2}(1-2 \nu)+x^{5 / 2}\left(-\frac{263}{39}+\frac{688 \nu}{39}-\frac{256 \nu^{2}}{39}\right)\right. \\
& \left.+x^{3}\left(5 \pi-10 \pi \nu+\mathrm{i}\left(-\frac{181}{14}+\nu\left(\frac{105834}{3125}-20 \ln (5 / 2)\right)+10 \ln (5 / 2)\right)\right)\right]+\mathcal{O}\left(\frac{1}{c^{7}}\right) \text {, } \\
& \mathcal{H}^{54}=-\frac{32}{9 \sqrt{165}}\left[x^{2}\left(1-5 \nu+5 \nu^{2}\right)+x^{3}\left(-\frac{4451}{910}+\frac{3619 \nu}{130}-\frac{521 \nu^{2}}{13}+\frac{339 \nu^{3}}{26}\right)\right]+\mathcal{O}\left(\frac{1}{c^{7}}\right), \\
& \mathcal{H}^{53}=-\frac{9}{32} \mathrm{i} \sqrt{\frac{3}{110}} \Delta\left[x^{3 / 2}(1-2 \nu)+x^{5 / 2}\left(-\frac{69}{13}+\frac{464 \nu}{39}-\frac{88 \nu^{2}}{39}\right)\right.
\end{aligned}
$$




$$
\begin{aligned}
& \left.+x^{3}\left(3 \pi-6 \pi \nu+\mathrm{i}\left(-\frac{543}{70}+\nu\left(\frac{83702}{3645}-12 \ln (3 / 2)\right)+6 \ln (3 / 2)\right)\right)\right]+\mathcal{O}\left(\frac{1}{c^{7}}\right) \\
\mathcal{H}^{52} & =\frac{2}{27 \sqrt{55}}\left[x^{2}\left(1-5 \nu+5 \nu^{2}\right)+x^{3}\left(-\frac{3911}{910}+\frac{3079 \nu}{130}-\frac{413 \nu^{2}}{13}+\frac{231 \nu^{3}}{26}\right)\right]+\mathcal{O}\left(\frac{1}{c^{7}}\right) \\
\mathcal{H}^{51} & =\frac{\mathrm{i}}{288 \sqrt{385}} \Delta\left[x^{3 / 2}(1-2 \nu)+x^{5 / 2}\left(-\frac{179}{39}+\frac{352 \nu}{39}-\frac{4 \nu^{2}}{39}\right)\right. \\
& \left.+x^{3}\left(\pi-2 \pi \nu+\mathrm{i}\left(-\frac{181}{70}-2 \ln 2+\nu\left(\frac{626}{5}+4 \ln 2\right)\right)\right)\right]+\mathcal{O}\left(\frac{1}{c^{7}}\right) \\
\mathcal{H}^{50} & =\mathcal{O}\left(\frac{1}{c^{7}}\right) \\
\mathcal{H}^{66} & =\frac{54}{5 \sqrt{143}}\left[x^{2}\left(1-5 \nu+5 \nu^{2}\right)+x^{3}\left(-\frac{113}{14}+\frac{91 \nu}{2}-64 \nu^{2}+\frac{39 \nu^{3}}{2}\right)\right]+\mathcal{O}\left(\frac{1}{c^{7}}\right) \\
\mathcal{H}^{65} & =\frac{3125 i x^{5 / 2}}{504 \sqrt{429}} \Delta\left[1-4 \nu+3 \nu^{2}\right]+\mathcal{O}\left(\frac{1}{c^{7}}\right) \\
\mathcal{H}^{64} & =-\frac{128}{495} \sqrt{\frac{2}{39}}\left[x^{2}\left(1-5 \nu+5 \nu^{2}\right)+x^{3}\left(-\frac{93}{14}+\frac{71 \nu}{2}-44 \nu^{2}+\frac{19 \nu^{3}}{2}\right)\right]+\mathcal{O}\left(\frac{1}{c^{7}}\right) \\
\mathcal{H}^{60} & =\mathcal{O}\left(\frac{1}{c^{7}}\right) \\
\mathcal{H}^{63} & =-\frac{81 i x^{5 / 2}}{616 \sqrt{65}} \Delta\left[1-4 \nu+3 \nu^{2}\right]+\mathcal{O}\left(\frac{1}{c^{7}}\right) \\
\mathcal{H}^{62} & =\frac{2}{297 \sqrt{65}}\left[x^{2}\left(1-5 \nu+5 \nu^{2}\right)+x^{3}\left(-\frac{81}{14}+\frac{59 \nu}{2}-32 \nu^{2}+\frac{7 \nu^{3}}{2}\right)\right]+\mathcal{O}\left(\frac{1}{c^{7}}\right) \\
& \frac{i x^{5 / 2}}{8316 \sqrt{26}} \Delta\left[1-4 \nu+3 \nu^{2}\right]+\mathcal{O}\left(\frac{1}{c^{7}}\right) \\
& \\
\mathcal{H}^{6} &
\end{aligned}
$$

Notice that the modes with $m=0$ are zero except for the DC (zero-frequency) non-linear memory contributions. We already know that this effect arises at Newtonian order [see Eq. (322a)], hence the non zero values of the modes $\mathcal{H}^{20}$ and $\mathcal{H}^{40}$. See Ref. [189] for the DC memory contributions in the higher modes having $m=0$.

With the 3PN approximation all the modes with $\ell \geqslant 7$ can be considered as merely Newtonian. We give here the general Newtonian leading order expressions of any mode with arbitrary $\ell$ and non-zero $m$ (see the derivation in [272]):

$$
\begin{aligned}
\mathcal{H}^{\ell m} & =\frac{(-)^{(\ell-m+2) / 2}}{2^{\ell+1}\left(\frac{\ell+m}{2}\right) !\left(\frac{\ell-m}{2}\right) !(2 \ell-1) ! !}\left(\frac{5(\ell+1)(\ell+2)(\ell+m) !(\ell-m) !}{\ell(\ell-1)(2 \ell+1)}\right)^{1 / 2} \\
& \times \sigma_{\ell}(\nu)(\mathrm{i} m)^{\ell} x^{\ell / 2-1}+\mathcal{O}\left(\frac{1}{c^{\ell-2}}\right) \quad(\text { for } \ell+m \text { even }), \\
\mathcal{H}^{\ell m} & =\frac{(-)^{(\ell-m-1) / 2}}{2^{\ell-1}\left(\frac{\ell+m-1}{2}\right) !\left(\frac{\ell-m-1}{2}\right) !(2 \ell+1) ! !}\left(\frac{5(\ell+2)(2 \ell+1)(\ell+m) !(\ell-m) !}{\ell(\ell-1)(\ell+1)}\right)^{1 / 2} \\
& \times \sigma_{\ell+1}(\nu) \mathrm{i}(\mathrm{i} m)^{\ell} x^{(\ell-1) / 2}+\mathcal{O}\left(\frac{1}{c^{\ell-2}}\right) \quad(\text { for } \ell+m \text { odd }),
\end{aligned}
$$

in which we employ the function $\sigma_{\ell}(\nu)=X_{2}^{\ell-1}+(-)^{\ell} X_{1}^{\ell-1}$, also given by Eqs. (305). 


\section{Eccentric Compact Binaries}

Inspiralling compact binaries are usually modelled as moving in quasi-circular orbits since gravitational radiation reaction circularizes the orbit towards the late stages of inspiral [340,339], as we discussed in Section 1.2. Nevertheless, there is an increased interest in inspiralling binaries moving in quasi-eccentric orbits. Astrophysical scenarios currently exist which lead to binaries with nonzero eccentricity in the gravitational-wave detector bandwidth, both terrestrial and space-based. For instance, inner binaries of hierarchical triplets undergoing Kozai oscillations [283, 300] could not only merge due to gravitational radiation reaction but a fraction of them should have non negligible eccentricities when they enter the sensitivity band of advanced ground based interferometers [419]. On the other hand the population of stellar mass binaries in globular clusters is expected to have a thermal distribution of eccentricities [32]. In a study of the growth of intermediate black holes [235] in globular clusters it was found that the binaries have eccentricities between 0.1 and 0.2 in the $e$ LISA bandwidth. Though, supermassive black hole binaries are powerful gravitational wave sources for $e$ LISA, it is not known if they would be in quasi-circular or quasi-eccentric orbits. If a Kozai mechanism is at work, these supermassive black hole binaries could be in highly eccentric orbits and merge within the Hubble time [40]. Sources of the kind discussed above provide the prime motivation for investigating higher post-Newtonian order modelling for quasi-eccentric binaries.

\subsection{Doubly periodic structure of the motion of eccentric binaries}

In Section 7.3 we have given the equations of motion of non-spinning compact binary systems in the frame of the center-of-mass for general orbits at the $3 \mathrm{PN}$ and even $3.5 \mathrm{PN}$ order. We shall now investigate (in this section and the next one) the explicit solution to those equations. In particular, let us discuss the general "doubly-periodic" structure of the post-Newtonian solution, closely following Refs. [142, 143, 149].

The $3 \mathrm{PN}$ equations of motion admit, when neglecting the radiation reaction terms at $2.5 \mathrm{PN}$ order, ten first integrals of the motion corresponding to the conservation of energy, angular momentum, linear momentum, and center of mass position. When restricted to the frame of the center of mass, the equations admit four first integrals associated with the energy $E$ and the angular momentum vector $\mathbf{J}$, given in harmonic coordinates at 3PN order by Eqs. (4.8) - (4.9) of Ref. [79].

The motion takes place in the plane orthogonal to J. Denoting by $r=|\boldsymbol{x}|$ the binary's orbital separation in that plane, and by $\boldsymbol{v}=\boldsymbol{v}_{1}-\boldsymbol{v}_{2}$ the relative velocity, we find that $E$ and $\mathbf{J}$ are functions of $r, \dot{r}^{2}, v^{2}$ and $\boldsymbol{x} \times \boldsymbol{v}$. We adopt polar coordinates $(r, \phi)$ in the orbital plane, and express $E$ and the norm $\mathrm{J}=|\mathbf{J}|$, thanks to $v^{2}=\dot{r}^{2}+r^{2} \dot{\phi}^{2}$, as some explicit functions of $r, \dot{r}^{2}$ and $\dot{\phi}$. The latter functions can be inverted by means of a straightforward post-Newtonian iteration to give $\dot{r}^{2}$ and $\dot{\phi}$ in terms of $r$ and the constants of motion $E$ and J. Hence,

$$
\begin{aligned}
\dot{r}^{2} & =\mathcal{R}[r ; E, \mathrm{~J}], \\
\dot{\phi} & =\mathcal{S}[r ; E, \mathrm{~J}],
\end{aligned}
$$

where $\mathcal{R}$ and $\mathcal{S}$ denote certain polynomials in $1 / r$, the degree of which depends on the postNewtonian approximation in question; for instance it is seventh degree for both $\mathcal{R}$ and $\mathcal{S}$ at $3 \mathrm{PN}$ order [312]. The various coefficients of the powers of $1 / r$ are themselves polynomials in $E$ and $\mathrm{J}$, and also, of course, depend on the total mass $m$ and symmetric mass ratio $\nu$. In the case of bounded elliptic-like motion, one can prove [143] that the function $\mathcal{R}$ admits two real roots, say $r_{\mathrm{p}}$ and $r_{\mathrm{a}}$ such that $r_{\mathrm{p}} \leqslant r_{\mathrm{a}}$, which admit some non-zero finite Newtonian limits when $c \rightarrow \infty$, and represent respectively the radii of the orbit's periastron (p) and apastron (a). The other roots are complex and tend to zero when $c \rightarrow \infty$. 
Let us consider a given binary's orbital configuration, fully specified by some values of the integrals of motion $E$ and J corresponding to quasi-elliptic motion. ${ }^{70}$ The binary's orbital period, or time of return to the periastron, is obtained by integrating the radial motion as

$$
P=2 \int_{r_{\mathrm{p}}}^{r_{\mathrm{a}}} \frac{\mathrm{d} r}{\sqrt{\mathcal{R}[r]}} .
$$

We introduce the fractional angle (i.e., the angle divided by $2 \pi$ ) of the advance of the periastron per orbital revolution,

$$
K=\frac{1}{\pi} \int_{r_{\mathrm{p}}}^{r_{\mathrm{a}}} \mathrm{d} r \frac{\mathcal{S}[r]}{\sqrt{\mathcal{R}[r]}},
$$

which is such that the precession of the periastron per period is given by $\Delta \Phi=2 \pi(K-1)$. As $K$ tends to one in the limit $c \rightarrow \infty$ (as is easily checked from the usual Newtonian solution), it is often convenient to pose $k \equiv K-1$, which will then entirely describe the relativistic precession.

Let us then define the mean anomaly $\ell$ and the mean motion $n$ by

$$
\begin{aligned}
\ell & \equiv n\left(t-t_{\mathrm{p}}\right), \\
n & \equiv \frac{2 \pi}{P} .
\end{aligned}
$$

Here $t_{\mathrm{p}}$ denotes the instant of passage to the periastron. For a given value of the mean anomaly $\ell$, the orbital separation $r$ is obtained by inversion of the integral equation

$$
\ell=n \int_{r_{\mathrm{p}}}^{r} \frac{\mathrm{d} s}{\sqrt{\mathcal{R}[s]}} .
$$

This defines the function $r(\ell)$ which is a periodic function in $\ell$ with period $2 \pi$. The orbital phase $\phi$ is then obtained in terms of the mean anomaly $\ell$ by integrating the angular motion as

$$
\phi=\phi_{\mathrm{p}}+\frac{1}{n} \int_{0}^{\ell} \mathrm{d} l \mathcal{S}[r(l)],
$$

where $\phi_{\mathrm{p}}$ denotes the value of the phase at the instant $t_{\mathrm{p}}$. We may define the origin of the orbital phase at the ascending node $\mathcal{N}$ with respect to some observer. In the particular case of a circular orbit, $r=$ const, the phase evolves linearly with time, $\dot{\phi}=\mathcal{S}[r]=\Omega$, where $\Omega$ is the orbital frequency of the circular orbit given by

$$
\Omega=K n=(1+k) n .
$$

In the general case of a non-circular orbit it is convenient to keep that definition $\Omega=K n$ and to explicitly introduce the linearly growing part of the orbital phase (336) by writing it in the form

$$
\begin{aligned}
\phi & =\phi_{\mathrm{p}}+\Omega\left(t-t_{\mathrm{p}}\right)+W(\ell) \\
& =\phi_{\mathrm{p}}+K \ell+W(\ell) .
\end{aligned}
$$

Here $W(\ell)$ denotes a certain function of the mean anomaly which is periodic in $\ell$ with period $2 \pi$, hence periodic in time with period $P$. According to Eq. (336) this function is given in terms of the mean anomaly $\ell$ by

$$
W(\ell)=\frac{1}{n} \int_{0}^{\ell} \mathrm{d} l(\mathcal{S}[r(l)]-\Omega) .
$$

\footnotetext{
70 The dependence on $E$ and $\mathrm{J}$ will no longer be indicated but is always understood as implicit in what follows.
} 
Finally, the decomposition (338) exhibits clearly the nature of the compact binary motion, which may be called doubly periodic in that the mean anomaly $\ell$ is periodic with period $2 \pi$, and the periastron advance $K \ell$ is periodic with period $2 \pi K$. Notice however that, though standard, the term "doubly periodic" is misleading since the motion in physical space is not periodic in general. The radial motion $r(t)$ is periodic with period $P$ while the angular motion $\phi(t)$ is periodic [modulo $2 \pi$ ] with period $P / k$ where $k=K-1$. Only when the two periods are commensurable, i.e., when $k=1 / N$ where $N \in \mathbb{N}$, is the motion periodic in physical space (with period $N P$ ).

\subsection{Quasi-Keplerian representation of the motion}

The quasi-Keplerian (QK) representation of the motion of compact binaries is an elegant formulation of the solution of the $1 \mathrm{PN}$ equations of motion parametrized by the eccentric anomaly $u$ (entering a specific generalization of Kepler's equation) and depending on various orbital elements, such as three types of eccentricities. It was introduced by Damour \& Deruelle [149, 150] to study the problem of binary pulsar timing data including relativistic corrections at the $1 \mathrm{PN}$ order, where the relativistic periastron precession complicates the simpler Keplerian solution.

In the QK representation the radial motion is given in standard parametric form as

$$
r=a_{r}\left(1-e_{r} \cos u\right)
$$

where $u$ is the eccentric anomaly, with $a_{r}$ and $e_{r}$ denoting two constants representing the semimajor axis of the orbit and its eccentricity. However, these constants are labelled after the radial coordinate $r$ to remember that they enter (by definition) into the radial equation; in particular $e_{r}$ will differ from other kinds of eccentricities $e_{t}$ and $e_{\phi}$. The "time" eccentricity $e_{t}$ enters the Kepler equation which at the $1 \mathrm{PN}$ order takes the usual form

$$
\ell=u-e_{t} \sin u+\mathcal{O}\left(\frac{1}{c^{4}}\right),
$$

where the mean anomaly is proportional to the time elapsed since the instant $t_{\mathrm{p}}$ of passage at the periastron, $\ell=n\left(t-t_{\mathrm{p}}\right)$ where $n=2 \pi / P$ is the mean motion and $P$ is the orbital period; see Eqs. (334). The "angular" eccentricity $e_{\phi}$ enters the equation for the angular motion at $1 \mathrm{PN}$ order which is written in the form

$$
\frac{\phi-\phi_{\mathrm{p}}}{K}=v+\mathcal{O}\left(\frac{1}{c^{4}}\right)
$$

where the true anomaly $v$ is defined by ${ }^{71}$

$$
v \equiv 2 \arctan \left[\left(\frac{1+e_{\phi}}{1-e_{\phi}}\right)^{1 / 2} \tan \frac{u}{2}\right] .
$$

The constant $K$ is the advance of periastron per orbital revolution defined by Eq. (333); it may be written as $K=\frac{\Phi}{2 \pi}$ where $\Phi$ is the angle of return to the periastron.

Crucial to the formalism are the explicit expressions for the orbital elements $n, K, a_{r}, e_{r}, e_{t}$ and $e_{\phi}$ in terms of the conserved energy $E$ and angular momentum $\mathrm{J}$ of the orbit. For convenience we introduce two dimensionless parameters directly linked to $E$ and J by

$$
\varepsilon \equiv-\frac{2 E}{\mu c^{2}},
$$

${ }^{71}$ Comparing with Eqs. (338) we have also

$$
v=\ell+\frac{W(\ell)}{K}+\mathcal{O}\left(\frac{1}{c^{4}}\right) .
$$




$$
j \equiv-\frac{2 E h^{2}}{\mu^{3}}
$$

where $\mu=m \nu$ is the reduced mass with $m$ the total mass (recall that $E<0$ for bound orbits) and we have used the intermediate standard notation $h \equiv \frac{\mathrm{J}}{\mathrm{Gm}}$. The equations to follow will then appear as expansions in powers of the small post-Newtonian parameter $\varepsilon=\mathcal{O}\left(1 / c^{2}\right),{ }^{72}$ with coefficients depending on $j$ and the dimensionless reduced mass ratio $\nu$; notice that the parameter $j$ is at Newtonian order, $j=\mathcal{O}\left(1 / c^{0}\right)$. We have [149]

$$
\begin{aligned}
n & =\frac{\varepsilon^{3 / 2} c^{3}}{G m}\left\{1+\frac{\varepsilon}{8}(-15+\nu)+\mathcal{O}\left(\frac{1}{c^{4}}\right)\right\}, \\
K & =1+\frac{3 \varepsilon}{j}+\mathcal{O}\left(\frac{1}{c^{4}}\right), \\
a_{r} & =\frac{G m}{\varepsilon c^{2}}\left\{1+\frac{\varepsilon}{4}(-7+\nu)+\mathcal{O}\left(\frac{1}{c^{4}}\right)\right\}, \\
e_{r} & =\sqrt{1-j}+\frac{\varepsilon}{8 \sqrt{1-j}}[24-4 \nu+5 j(-3+\nu)]+\mathcal{O}\left(\frac{1}{c^{4}}\right), \\
e_{t} & =\sqrt{1-j}+\frac{\varepsilon}{8 \sqrt{1-j}}[-8+8 \nu+j(17-7 \nu)]+\mathcal{O}\left(\frac{1}{c^{4}}\right), \\
e_{\phi} & =\sqrt{1-j}+\frac{\varepsilon}{8 \sqrt{1-j}}[24+j(-15+\nu)]+\mathcal{O}\left(\frac{1}{c^{4}}\right) .
\end{aligned}
$$

The dependence of such relations on the coordinate system in use will be discussed later. Notice the interesting point that there is no dependence of the mean motion $n$ and the radial semi-major axis $a_{r}$ on the angular momentum $\mathrm{J}$ up to the 1PN order; such dependence will start only at $2 \mathrm{PN}$ order, see e.g., Eq. (347a).

The above QK representation of the compact binary motion at $1 \mathrm{PN}$ order has been generalized at the $2 \mathrm{PN}$ order in Refs. [170, 379, 420], and at the 3PN order by Memmesheimer, Gopakumar \& Schäfer [312]. The construction of a generalized QK representation at 3PN order exploits the fact that the radial equation given by Eq. (331a) is a polynomial in $1 / r$ (of seventh degree at 3PN order). However, this is true only in coordinate systems avoiding the appearance of terms with the logarithm $\ln r$; the presence of logarithms in the standard harmonic $(\mathrm{SH})$ coordinates at the 3PN order will obstruct the construction of the QK parametrization. Therefore Ref. [312] obtained it in the ADM coordinate system and also in the modified harmonic $(\mathrm{MH})$ coordinates, obtained by applying the gauge transformation given in Eq. (204) on the SH coordinates. The equations of motion in the center-of-mass frame in MH coordinates have been given in Eqs. (222); see also Ref. [9] for details about the transformation between $\mathrm{SH}$ and $\mathrm{MH}$ coordinates.

At the 3PN order the radial equation in ADM or MH coordinates is still given by Eq. (340). However, the Kepler equation (341) and angular equation (342) acquire extra contributions and now become

$$
\begin{aligned}
\ell & =u-e_{t} \sin u+f_{t} \sin v+g_{t}(v-u)+i_{t} \sin 2 v+h_{t} \sin 3 v+\mathcal{O}\left(\frac{1}{c^{8}}\right), \\
\frac{\phi-\phi_{\mathrm{p}}}{K} & =v+f_{\phi} \sin 2 v+g_{\phi} \sin 3 v+i_{\phi} \sin 4 v+h_{\phi} \sin 5 v+\mathcal{O}\left(\frac{1}{c^{8}}\right),
\end{aligned}
$$

in which the true anomaly $v$ is still given by Eq. (343). The new orbital elements $f_{t}, f_{\phi}, g_{t}, g_{\phi}$, $i_{t}, i_{\phi}, h_{t}$ and $h_{\phi}$ parametrize the $2 \mathrm{PN}$ and $3 \mathrm{PN}$ relativistic corrections. ${ }^{73}$ All the orbital elements

\footnotetext{
72 Note that this post-Newtonian parameter $\varepsilon$ is precisely specified by Eq. (344a), while we only intended to define $\epsilon$ in Eq. (1) as representing a post-Newtonian estimate.

73 More precisely, $f_{t}, f_{\phi}, g_{t}, g_{\phi}$ are composed of $2 \mathrm{PN}$ and $3 \mathrm{PN}$ terms, but $i_{t}, i_{\phi}, h_{t}, h_{\phi}$ start only at $3 \mathrm{PN}$ order.
} 
are now to be related, similarly to Eqs. (345), to the constants $\varepsilon$ and $j$ with $3 \mathrm{PN}$ accuracy in a given coordinate system. Let us make clear that in different coordinate systems such as $\mathrm{MH}$ and ADM coordinates, the QK representation takes exactly the same form as given by Eqs. (340) and (346). But, the relations linking the various orbital elements $a_{r}, e_{r}, e_{t}, e_{\phi}, f_{t}, f_{\phi}, \ldots$ to $E$ and $\mathrm{J}$ or $\varepsilon$ and $j$, are different, with the notable exceptions of $n$ and $K$.

Indeed, an important point related to the use of gauge invariant variables in the elliptical orbit case is that the functional forms of the mean motion $n$ and periastron advance $K$ in terms of the gauge invariant variables $\varepsilon$ and $j$ are identical in different coordinate systems like the MH and ADM coordinates [170]. Their explicit expressions at 3PN order read

$$
\begin{gathered}
n=\frac{\varepsilon^{3 / 2} c^{3}}{G m}\left\{1+\frac{\varepsilon}{8}(-15+\nu)+\frac{\varepsilon^{2}}{128}\left[555+30 \nu+11 \nu^{2}+\frac{192}{j^{1 / 2}}(-5+2 \nu)\right]\right. \\
+\frac{\varepsilon^{3}}{3072}\left[-29385-4995 \nu-315 \nu^{2}+135 \nu^{3}+\frac{5760}{j^{1 / 2}}\left(17-9 \nu+2 \nu^{2}\right)\right. \\
\left.\left.\quad+\frac{16}{j^{3 / 2}}\left(-10080+\left(13952-123 \pi^{2}\right) \nu-1440 \nu^{2}\right)\right]+\mathcal{O}\left(\frac{1}{c^{8}}\right)\right\} . \\
K=1+\frac{3 \varepsilon}{j}+\frac{\varepsilon^{2}}{4}\left[\frac{3}{j}(-5+2 \nu)+\frac{15}{j^{2}}(7-2 \nu)\right] \\
+\frac{\varepsilon^{3}}{128}\left[\frac{24}{j}\left(5-5 \nu+4 \nu^{2}\right)+\frac{1}{j^{2}}\left(-10080+\left(13952-123 \pi^{2}\right) \nu-1440 \nu^{2}\right)\right. \\
\left.\quad+\frac{5}{j^{3}}\left(7392+\left(-8000+123 \pi^{2}\right) \nu+336 \nu^{2}\right)\right]+\mathcal{O}\left(\frac{1}{c^{8}}\right) .
\end{gathered}
$$

Because of their gauge invariant meaning, it is natural to use $n$ and $K$ as two independent gaugeinvariant variables in the general orbit case. Actually, instead of working with the mean motion $n$ it is often preferable to use the orbital frequency $\Omega$ which has been defined for general quasi-elliptic orbits in Eq. (337). Moreover we can pose

$$
x=\left(\frac{G m \Omega}{c^{3}}\right)^{2 / 3} \quad(\text { with } \Omega=K n),
$$

which constitutes the obvious generalization of the gauge invariant variable $x$ used in the circular orbit case. The use of $x$ as an independent parameter will thus facilitate the straightforward reading out and check of the circular orbit limit. The parameter $x$ is related to the energy and angular momentum variables $\varepsilon$ and $j$ up to $3 \mathrm{PN}$ order by

$$
\begin{aligned}
& \frac{x}{\varepsilon}=1+\varepsilon {\left[-\frac{5}{4}+\frac{1}{12} \nu+\frac{2}{j}\right] } \\
&+\varepsilon^{2}\left[\frac{5}{2}+\frac{5}{24} \nu+\frac{1}{18} \nu^{2}+\frac{1}{j^{1 / 2}}(-5+2 \nu)+\frac{1}{j}\left(-5+\frac{7}{6} \nu\right)+\frac{1}{j^{2}}\left(\frac{33}{2}-5 \nu\right)\right] \\
&+\varepsilon^{3}\left[-\frac{235}{48}-\frac{25}{24} \nu-\frac{25}{576} \nu^{2}+\frac{35}{1296} \nu^{3}+\frac{1}{j}\left(\frac{35}{4}-\frac{5}{3} \nu+\frac{25}{36} \nu^{2}\right)\right. \\
&+\frac{1}{j^{1 / 2}}\left(\frac{145}{8}-\frac{235}{24} \nu+\frac{29}{12} \nu^{2}\right)+\frac{1}{j^{3 / 2}}\left(-45+\left(\frac{472}{9}-\frac{41}{96} \pi^{2}\right) \nu-5 \nu^{2}\right) \\
&+\frac{1}{j^{2}}\left(-\frac{565}{8}+\left(\frac{1903}{24}-\frac{41}{64} \pi^{2}\right) \nu-\frac{95}{12} \nu^{2}\right) \\
&\left.+\frac{1}{j^{3}}\left(\frac{529}{3}+\left(-\frac{610}{3}+\frac{205}{64} \pi^{2}\right) \nu+\frac{35}{4} \nu^{2}\right)\right]+\mathcal{O}\left(\frac{1}{c^{8}}\right) .
\end{aligned}
$$


Besides the very useful gauge-invariant quantities $n, K$ and $x$, the other orbital elements $a_{r}, e_{r}$, $e_{t}, e_{\phi}, f_{t}, g_{t}, i_{t}, h_{t}, f_{\phi}, g_{\phi}, i_{\phi}, h_{\phi}$ parametrizing Eqs. (340) and (346) are not gauge invariant; their expressions in terms of $\varepsilon$ and $j$ depend on the coordinate system in use. We refer to Refs. [312, 9] for the full expressions of all the orbital elements at 3PN order in both $\mathrm{MH}$ and ADM coordinate systems. Here, for future use, we only give the expression of the time eccentricity $e_{t}$ (squared) in $\mathrm{MH}$ coordinates:

$$
\begin{aligned}
& e_{t}^{2}=1-j+\frac{\varepsilon}{4}[-8+8 \nu+j(17-7 \nu)] \\
&+\frac{\varepsilon^{2}}{8}\left[12+72 \nu+20 \nu^{2}+j\left(-112+47 \nu-16 \nu^{2}\right)+24 j^{1 / 2}(5-2 \nu)\right. \\
&\left.+\frac{16}{j}(4-7 \nu)+\frac{24}{j^{1 / 2}}(-5+2 \nu)\right] \\
&+\frac{\varepsilon^{3}}{6720}\left[23520-464800 \nu+179760 \nu^{2}+16800 \nu^{3}+525 j\left(528-200 \nu+77 \nu^{2}-24 \nu^{3}\right)\right. \\
&+ 2520 j^{1 / 2}\left(-265+193 \nu-46 \nu^{2}\right)+\frac{6}{j}\left(-73920+\left(260272-4305 \pi^{2}\right) \nu-61040 \nu^{2}\right) \\
&+ \frac{70}{j^{1 / 2}}\left(16380+\left(-19964+123 \pi^{2}\right) \nu+3240 \nu^{2}\right) \\
&+ \frac{70}{j^{3 / 2}}\left(-10080+\left(13952-123 \pi^{2}\right) \nu-1440 \nu^{2}\right) \\
&\left.+\frac{8}{j^{2}}\left(53760+\left(-176024+4305 \pi^{2}\right) \nu+15120 \nu^{2}\right)\right]+\mathcal{O}\left(\frac{1}{c^{8}}\right)
\end{aligned}
$$

Again, with our notation (344), this appears as a post-Newtonian expansion in the small parameter $\varepsilon \rightarrow 0$ with fixed "Newtonian" parameter $j$.

In the case of a circular orbit, the angular momentum variable, say $j_{\text {circ }}$, is related to the constant of energy $\varepsilon$ by the $3 \mathrm{PN}$ gauge-invariant expansion

$j_{\text {circ }}=1+\left(\frac{9}{4}+\frac{\nu}{4}\right) \varepsilon+\left(\frac{81}{16}-2 \nu+\frac{\nu^{2}}{16}\right) \varepsilon^{2}+\left(\frac{945}{64}+\left[-\frac{7699}{192}+\frac{41}{32} \pi^{2}\right] \nu+\frac{\nu^{2}}{2}+\frac{\nu^{3}}{64}\right) \varepsilon^{3}+\mathcal{O}\left(\frac{1}{c^{8}}\right)$.

This permits to reduce various quantities to circular orbits, for instance, the periastron advance is found to be well defined in the limiting case of a circular orbit, and is given at $3 \mathrm{PN}$ order in terms of the PN parameter (230) [or (348)] by

$$
K_{\text {circ }}=1+3 x+\left(\frac{27}{2}-7 \nu\right) x^{2}+\left(\frac{135}{2}+\left[-\frac{649}{4}+\frac{123}{32} \pi^{2}\right] \nu+7 \nu^{2}\right) x^{3}+\mathcal{O}\left(\frac{1}{c^{8}}\right) .
$$

See Ref. [291] for a comparison between the PN prediction for the periastron advance of circular orbits and numerical calculations based on self-force theory in the small mass ratio limit.

\subsection{Averaged energy and angular momentum fluxes}

The gravitational wave energy and angular momentum fluxes from a system of two point masses in elliptic motion was first computed by Peters \& Mathews [340, 339] at Newtonian level. The $1 \mathrm{PN}$ and 1.5PN corrections to the fluxes were provided in Refs. [416, 86, 267, 87, 366] and used to study the associated secular evolution of orbital elements under gravitational radiation reaction using the QK representation of the binary's orbit at 1PN order [149]. These results were extended to $2 \mathrm{PN}$ order in Refs. [224, 225] for the instantaneous terms (leaving aside the tails) using the 
generalized QK representation [170, 379, 420]; the energy flux and waveform were in agreement with those of Ref. [424] obtained using a different method. Arun et al. [10, 9, 12] have fully generalized the results at 3PN order, including all tails and related hereditary contributions, by computing the averaged energy and angular momentum fluxes for quasi-elliptical orbits using the QK representation at 3PN order [312], and deriving the secular evolution of the orbital elements under $3 \mathrm{PN}$ gravitational radiation reaction. ${ }^{74}$

The secular evolution of orbital elements under gravitational radiation reaction is in principle only the starting point for constructing templates for eccentric binary orbits. To go beyond the secular evolution one needs to include in the evolution of the orbital elements, besides the averaged contributions in the fluxes, the terms rapidly oscillating at the orbital period. An analytic approach, based on an improved method of variation of constants, has been discussed in Ref. [153] for dealing with this issue at the leading 2.5PN radiation reaction order.

The generalized QK representation of the motion discussed in Section 10.2 plays a crucial role in the procedure of averaging the energy and angular momentum fluxes $\mathcal{F}$ and $\mathcal{G}_{i}$ over one orbit. $^{75}$ Actually the averaging procedure applies to the "instantaneous" parts of the fluxes, while the "hereditary" parts are treated separately for technical reasons [10, 9, 12]. Following the decomposition (308) we pose $\mathcal{F}=\mathcal{F}_{\text {inst }}+\mathcal{F}_{\text {hered }}$ where the hereditary part of the energy flux is composed of tails and tail-of-tails. For the angular momentum flux one needs also to include a contribution from the memory effect [12]. We thus have to compute for the instantaneous part

$$
\left\langle\mathcal{F}_{\text {inst }}\right\rangle=\frac{1}{P} \int_{0}^{P} \mathrm{~d} t \mathcal{F}_{\text {inst }}=\frac{1}{2 \pi} \int_{0}^{2 \pi} \mathrm{d} u \frac{\mathrm{d} \ell}{\mathrm{d} u} \mathcal{F}_{\text {inst }},
$$

and similarly for the instantaneous part of the angular momentum flux $\mathcal{G}_{i}$.

Thanks to the QK representation, we can express $\mathcal{F}_{\text {inst }}$, which is initially a function of the natural variables $r, \dot{r}$ and $v^{2}$, as a function of the varying eccentric anomaly $u$, and depending on two constants: The frequency-related parameter $x$ defined by (348), and the "time" eccentricity $e_{t}$ given by (350). To do so one must select a particular coordinate system - the MH coordinates for instance. The choice of $e_{t}$ rather than $e_{r}$ (say) is a matter of convenience; since $e_{t}$ appears in the Kepler-like equation (346a) at leading order, it will directly be dealt with when averaging over one orbit. We note that in the expression of the energy flux at the $3 \mathrm{PN}$ order there are some logarithmic terms of the type $\ln \left(r / r_{0}\right)$ even in $\mathrm{MH}$ coordinates. Indeed, as we have seen in Section 7.3, the $\mathrm{MH}$ coordinates permit the removal of the logarithms $\ln \left(r / r_{0}^{\prime}\right)$ in the equations of motion, where $r_{0}^{\prime}$ is the UV scale associated with Hadamard's self-field regularization [see Eq. (221)]; however there are still some logarithms $\ln \left(r / r_{0}\right)$ which involve the IR constant $r_{0}$ entering the definition of the multipole moments for general sources, see Theorem 6 where the finite part $\mathcal{F} \mathcal{P}$ contains the regularization factor (42). As a result we find that the general structure of $\mathcal{F}_{\text {inst }}$ (and similarly for $\mathcal{G}_{\text {inst }}$, the norm of the angular momentum flux) consists of a finite sum of terms of the type

$$
\mathcal{F}_{\text {inst }}=\frac{\mathrm{d} u}{\mathrm{~d} \ell} \sum_{l} \frac{\alpha_{l}\left(x, e_{t}\right)+\beta_{l}\left(x, e_{t}\right) \sin u+\gamma_{l}\left(x, e_{t}\right) \ln \left(1-e_{t} \cos u\right)}{\left(1-e_{t} \cos u\right)^{l+1}} .
$$

The factor $\mathrm{d} u / \mathrm{d} \ell$ has been inserted to prepare for the orbital average (351). The coefficients $\alpha_{l}$, $\beta_{l}$ and $\gamma_{l}$ are straightforwardly computed using the QK parametrization as functions of $x$ and the time eccentricity $e_{t}$. The $\beta_{l}$ 's correspond to $2.5 \mathrm{PN}$ radiation-reaction terms and will play no role, while the $\gamma_{l}$ 's correspond to the logarithmic terms $\ln \left(r / r_{0}\right)$ arising at the $3 \mathrm{PN}$ order. For

\footnotetext{
${ }^{74}$ On the other hand, for the computation of the gravitational waveform of eccentric binary orbits up to the $2 \mathrm{PN}$ order in the Fourier domain, see Refs. [401, 402].

${ }^{75}$ Recall that the fluxes are defined in a general way, for any matter system, in terms of the radiative multipole moments by the expressions (68).
} 
convenience the dependence on the constant $\ln r_{0}$ has been included into the coefficients $\alpha_{l}$ 's. To compute the average we dispose of the following integration formulas $(l \in \mathbb{N})^{76}$

$$
\begin{aligned}
& \int_{0}^{2 \pi} \frac{\mathrm{d} u}{2 \pi} \frac{\sin u}{\left(1-e_{t} \cos u\right)^{l+1}}=0 \\
& \int_{0}^{2 \pi} \frac{\mathrm{d} u}{2 \pi} \frac{1}{\left(1-e_{t} \cos u\right)^{l+1}}=\left.\frac{(-)^{l}}{l !}\left(\frac{\mathrm{d}^{l}}{\mathrm{~d} z^{l}}\left[\frac{1}{\sqrt{z^{2}-e_{t}^{2}}}\right]\right)\right|_{z=1}, \\
& \int_{0}^{2 \pi} \frac{\mathrm{d} u}{2 \pi} \frac{\ln \left(1-e_{t} \cos u\right)}{\left(1-e_{t} \cos u\right)^{l+1}}=\left.\frac{(-)^{l}}{l !}\left(\frac{\mathrm{d}^{l}}{\mathrm{~d} z^{l}}\left[\frac{Z\left(z, e_{t}\right)}{\sqrt{z^{2}-e_{t}^{2}}}\right]\right)\right|_{z=1} .
\end{aligned}
$$

In the right-hand sides of Eqs. (353b) and (353c) we have to differentiate $l$ times with respect to the intermediate variable $z$ before applying $z=1$. The equation (353c), necessary for dealing with the logarithmic terms, contains the not so trivial function

$$
Z\left(z, e_{t}\right)=\ln \left[\frac{\sqrt{1-e_{t}^{2}}+1}{2}\right]+2 \ln \left[1+\frac{\sqrt{1-e_{t}^{2}}-1}{z+\sqrt{z^{2}-e_{t}^{2}}}\right] .
$$

From Eq. (353a) we see that there will be no radiation-reaction terms at 2.5PN order in the final result; the $2.5 \mathrm{PN}$ contribution is proportional to $\dot{r}$ and vanishes after averaging since it involves only odd functions of $u$.

Finally, after implementing all the above integrations, the averaged instantaneous energy flux in $\mathrm{MH}$ coordinates at the $3 \mathrm{PN}$ order is obtained in the form [9]

$$
\left\langle\mathcal{F}_{\text {inst }}\right\rangle=\frac{32 c^{5}}{5 G} \nu^{2} x^{5}\left(\mathcal{I}_{0}+x \mathcal{I}_{1}+x^{2} \mathcal{I}_{2}+x^{3} \mathcal{I}_{3}\right)
$$

where we recall that the post-Newtonian parameter $x$ is defined by (348). The various instantaneous post-Newtonian pieces depend on the symmetric mass ratio $\nu$ and the time eccentricity $e_{t}$ in $\mathrm{MH}$ coordinates as

$$
\begin{aligned}
\mathcal{I}_{0} & =\frac{1}{\left(1-e_{t}^{2}\right)^{7 / 2}}\left\{1+\frac{73}{24} e_{t}^{2}+\frac{37}{96} e_{t}^{4}\right\} \\
\mathcal{I}_{1} & =\frac{1}{\left(1-e_{t}^{2}\right)^{9 / 2}}\left\{-\frac{1247}{336}-\frac{35}{12} \nu+e_{t}^{2}\left(\frac{10475}{672}-\frac{1081}{36} \nu\right)\right. \\
& \left.+e_{t}^{4}\left(\frac{10043}{384}-\frac{311}{12} \nu\right)+e_{t}^{6}\left(\frac{2179}{1792}-\frac{851}{576} \nu\right)\right\} \\
\mathcal{I}_{2} & =\frac{1}{\left(1-e_{t}^{2}\right)^{11 / 2}}\left\{-\frac{203471}{9072}+\frac{12799}{504} \nu+\frac{65}{18} \nu^{2}\right. \\
& +e_{t}^{2}\left(-\frac{3807197}{18144}+\frac{116789}{2016} \nu+\frac{5935}{54} \nu^{2}\right)+e_{t}^{4}\left(-\frac{268447}{24192}-\frac{2465027}{8064} \nu+\frac{247805}{864} \nu^{2}\right) \\
& +e_{t}^{6}\left(\frac{1307105}{16128}-\frac{416945}{2688} \nu+\frac{185305}{1728} \nu^{2}\right)+e_{t}^{8}\left(\frac{86567}{64512}-\frac{9769}{4608} \nu+\frac{21275}{6912} \nu^{2}\right) \\
& +\sqrt{1-e_{t}^{2}}\left[\frac{35}{2}-7 \nu+e_{t}^{2}\left(\frac{6425}{48}-\frac{1285}{24} \nu\right)\right.
\end{aligned}
$$

76 The second of these formulas can alternatively be written with the standard Legendre polynomial $P_{l}$ as

$$
\int_{0}^{2 \pi} \frac{\mathrm{d} u}{2 \pi} \frac{1}{\left(1-e_{t} \cos u\right)^{l+1}}=\frac{1}{\left(1-e_{t}^{2}\right)^{\frac{l+1}{2}}} P_{l}\left(\frac{1}{\sqrt{1-e_{t}^{2}}}\right) .
$$




$$
\begin{aligned}
+ & \left.\left.e_{t}^{4}\left(\frac{5065}{64}-\frac{1013}{32} \nu\right)+e_{t}^{6}\left(\frac{185}{96}-\frac{37}{48} \nu\right)\right]\right\} \\
\mathcal{I}_{3} & =\frac{1}{\left(1-e_{t}^{2}\right)^{13 / 2}}\left\{\frac{2193295679}{9979200}+\left[\frac{8009293}{54432}-\frac{41 \pi^{2}}{64}\right] \nu-\frac{209063}{3024} \nu^{2}-\frac{775}{324} \nu^{3}\right. \\
& +e_{t}^{2}\left(\frac{20506331429}{19958400}+\left[\frac{649801883}{272160}+\frac{4879 \pi^{2}}{1536}\right] \nu-\frac{3008759}{3024} \nu^{2}-\frac{53696}{243} \nu^{3}\right) \\
+ & e_{t}^{4}\left(-\frac{3611354071}{13305600}+\left[\frac{755536297}{136080}-\frac{29971 \pi^{2}}{1024}\right] \nu-\frac{179375}{576} \nu^{2}-\frac{10816087}{7776} \nu^{3}\right) \\
+ & e_{t}^{6}\left(\frac{4786812253}{26611200}+\left[\frac{1108811471}{1451520}-\frac{84501 \pi^{2}}{4096}\right] \nu+\frac{87787969}{48384} \nu^{2}-\frac{983251}{648} \nu^{3}\right) \\
+ & e_{t}^{8}\left(\frac{21505140101}{141926400}+\left[-\frac{32467919}{129024}-\frac{4059 \pi^{2}}{4096}\right] \nu+\frac{79938097}{193536} \nu^{2}-\frac{4586539}{15552} \nu^{3}\right) \\
+ & e_{t}^{10}\left(-\frac{8977637}{11354112}+\frac{9287}{48384} \nu+\frac{8977}{55296} \nu^{2}-\frac{567617}{124416} \nu^{3}\right) \\
+ & \sqrt{1-e_{t}^{2}}\left[-\frac{14047483}{151200}+\left[-\frac{165761}{1008}+\frac{287 \pi^{2}}{192}\right] \nu+\frac{455}{12} \nu^{2}\right. \\
+ & \left.+e_{t}^{8}\left(\frac{185}{48}-\frac{1073}{288} \nu+\frac{407}{288} \nu^{2}\right)\right] \\
+ & e_{t}^{2}\left(\frac{36863231}{100800}+\left[-\frac{14935421}{6048}+\frac{52685 \pi^{2}}{4608}\right] \nu+\frac{43559}{72} \nu^{2}\right) \\
+ & e_{t}^{4}\left(\frac{759524951}{403200}+\left[-\frac{31082483}{8064}+\frac{41533 \pi^{2}}{6144}\right] \nu+\frac{303985}{288} \nu^{2}\right) \\
+ & e_{t}^{6}\left(\frac{1399661203}{2419200}+\left[-\frac{40922933}{48384}+\frac{1517 \pi^{2}}{9216}\right] \nu+\frac{73357}{288} \nu^{2}\right)
\end{aligned}
$$

The Newtonian coefficient $\mathcal{I}_{0}$ is nothing but the Peters \& Mathews [340] enhancement function of eccentricity that enters in the orbital gravitational radiation decay of the binary pulsar; see Eq. (11). For ease of presentation we did not add a label on $e_{t}$ to indicate that it is the time eccentricity in $\mathrm{MH}$ coordinates; such MH-coordinates $e_{t}$ is given by Eq. (350). Recall that on the contrary $x$ is gauge invariant, so no such label is required on it.

The last term in the $3 \mathrm{PN}$ coefficient is proportional to some logarithm which directly arises from the integration formula (353c). Inside the logarithm we have posed

$$
x_{0} \equiv \frac{G m}{c^{2} r_{0}},
$$

exhibiting an explicit dependence upon the arbitrary length scale $r_{0}$; we recall that $r_{0}$ was introduced in the formalism through Eq. (42). Only after adding the hereditary contribution to the $3 \mathrm{PN}$ energy flux can we check the required cancellation of the constant $x_{0}$. The hereditary part is made of tails and tails-of-tails, and is of the form

$$
\left\langle\mathcal{F}_{\text {hered }}\right\rangle=\frac{32 c^{5}}{5 G} \nu^{2} x^{5}\left(x^{3 / 2} \mathcal{K}_{3 / 2}+x^{5 / 2} \mathcal{K}_{5 / 2}+x^{3} \mathcal{K}_{3}\right),
$$

where the post-Newtonian pieces, only at the $1.5 \mathrm{PN}, 2.5 \mathrm{PN}$ and $3 \mathrm{PN}$ orders, read [10]

$$
\mathcal{K}_{3 / 2}=4 \pi \varphi\left(e_{t}\right),
$$




$$
\begin{aligned}
\mathcal{K}_{5 / 2} & =-\frac{8191}{672} \pi \psi\left(e_{t}\right)-\frac{583}{24} \nu \pi \zeta\left(e_{t}\right), \\
\mathcal{K}_{3} & =-\frac{116761}{3675} \kappa\left(e_{t}\right)+\left[\frac{16}{3} \pi^{2}-\frac{1712}{105} \gamma_{\mathrm{E}}-\frac{1712}{105} \ln \left(\frac{4 x^{3 / 2}}{x_{0}}\right)\right] F\left(e_{t}\right),
\end{aligned}
$$

where $\varphi\left(e_{t}\right), \psi\left(e_{t}\right), \zeta\left(e_{t}\right), \kappa\left(e_{t}\right)$ and $F\left(e_{t}\right)$ are certain "enhancement" functions of the eccentricity.

Among them the four functions $\varphi\left(e_{t}\right), \psi\left(e_{t}\right), \zeta\left(e_{t}\right)$ and $\kappa\left(e_{t}\right)$ appearing in Eqs. (359) do not admit analytic closed-form expressions. They have been obtained in Refs. [10] (extending Ref. [87]) in the form of infinite series made out of quadratic products of Bessel functions. Numerical plots of these four enhancement factors as functions of eccentricity $e_{t}$ have been provided in Ref. [10]; we give in Figure 3 the graph of the function $\varphi\left(e_{t}\right)$ which enters the dominant 1.5PN tail term in Eq. (358).

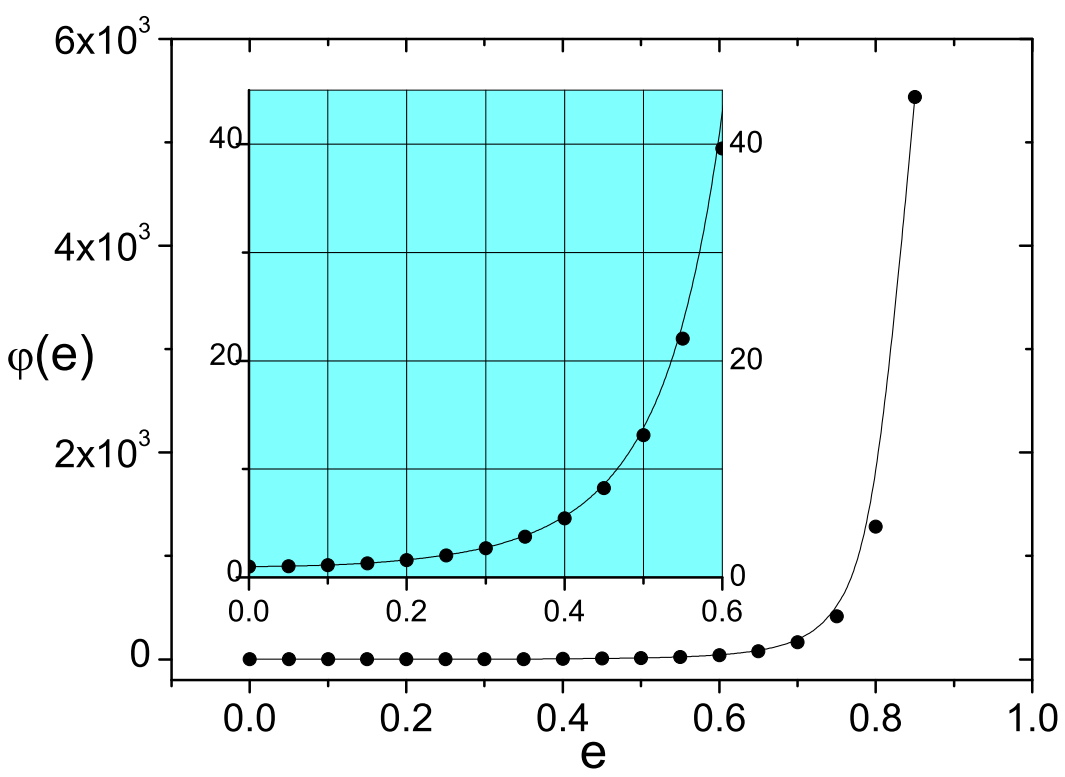

Figure 3: Variation of the enhancement factor $\varphi(e)$ with the eccentricity $e$. This function agrees with the numerical calculation of Ref. [87] modulo a trivial rescaling with the Peters-Mathews function (356a). The inset graph is a zoom of the function at a smaller scale. The dots represent the numerical computation and the solid line is a fit to the numerical points. In the circular orbit limit we have $\varphi(0)=1$.

Furthermore their leading correction term $e_{t}^{2}$ in the limit of small eccentricity $e_{t} \ll 1$ can be obtained analytically as [10]

$$
\begin{aligned}
& \varphi\left(e_{t}\right)=1+\frac{2335}{192} e_{t}^{2}+\mathcal{O}\left(e_{t}^{4}\right) \\
& \psi\left(e_{t}\right)=1-\frac{22988}{8191} e_{t}^{2}+\mathcal{O}\left(e_{t}^{4}\right) \\
& \zeta\left(e_{t}\right)=1+\frac{1011565}{48972} e_{t}^{2}+\mathcal{O}\left(e_{t}^{4}\right) \\
& \kappa\left(e_{t}\right)=1+\left(\frac{62}{3}-\frac{4613840}{350283} \ln 2+\frac{24570945}{1868176} \ln 3\right) e_{t}^{2}+\mathcal{O}\left(e_{t}^{4}\right) .
\end{aligned}
$$


On the other hand the function $F\left(e_{t}\right)$ in factor of the logarithm in the 3PN piece does admit some closed analytic form:

$$
F\left(e_{t}\right)=\frac{1}{\left(1-e_{t}^{2}\right)^{13 / 2}}\left[1+\frac{85}{6} e_{t}^{2}+\frac{5171}{192} e_{t}^{4}+\frac{1751}{192} e_{t}^{6}+\frac{297}{1024} e_{t}^{8}\right] .
$$

The latter analytical result is very important for checking that the arbitrary constant $x_{0}$ disappears from the final result. Indeed we immediately verify from comparing the last term in Eq. (356d) with Eq. (359c) that $x_{0}$ cancels out from the sum of the instantaneous and hereditary contributions in the 3PN energy flux. This fact was already observed for the circular orbit case in Ref. [81]; see also the discussions around Eqs. (93) - (94) and at the end of Section 4.2.

Finally we can check that the correct circular orbit limit, which is given by Eq. (314), is recovered from the sum $\left\langle\mathcal{F}_{\text {inst }}\right\rangle+\left\langle\mathcal{F}_{\text {hered }}\right\rangle$. The next correction of order $e_{t}^{2}$ when $e_{t} \rightarrow 0$ can be deduced from Eqs. (360)-(361) in analytic form; having the flux in analytic form may be useful for studying the gravitational waves from binary black hole systems with moderately high eccentricities, such as those formed in globular clusters [235].

Previously the averaged energy flux was represented using $x$ - the gauge invariant variable (348) - and the time eccentricity $e_{t}$ which however is gauge dependent. Of course it is possible to provide a fully gauge invariant formulation of the energy flux. The most natural choice is to express the result in terms of the conserved energy $E$ and angular momentum $\mathrm{J}$, or, rather, in terms of the pair of rescaled variables $(\varepsilon, j)$ defined by Eqs. (344). To this end it suffices to replace $e_{t}$ by its MH-coordinate expression (350) and to use Eq. (349) to re-express $x$ in terms of $\varepsilon$ and $j$. However, there are other possible choices for a couple of gauge invariant quantities. As we have seen the mean motion $n$ and the periastron precession $K$ are separately gauge invariant so we may define the pair of variables $(x, \iota)$, where $x$ is given by (348) and we pose

$$
\iota \equiv \frac{3 x}{K-1}
$$

Such choice would be motivated by the fact that $\iota$ reduces to the angular-momentum related variable $j$ in the limit $\varepsilon \rightarrow 0$. Note however that with the latter choices $(\varepsilon, j)$ or $(x, \iota)$ of gaugeinvariant variables, the circular-orbit limit is not directly readable from the result; this is why we have preferred to present it in terms of the gauge dependent couple of variables $\left(x, e_{t}\right)$.

As we are interested in the phasing of binaries moving in quasi-eccentric orbits in the adiabatic approximation, we require the orbital averages not only of the energy flux $\mathcal{F}$ but also of the angular momentum flux $\mathcal{G}_{i}$. Since the quasi-Keplerian orbit is planar, we only need to average the magnitude $\mathcal{G}$ of the angular momentum flux. The complete computation thus becomes a generalisation of the previous computation of the averaged energy flux requiring similar steps (see Ref. [12]): The angular momentum flux is split into instantaneous $\mathcal{G}_{\text {inst }}$ and hereditary $\mathcal{G}_{\text {hered }}$ contributions; the instantaneous part is averaged using the QK representation in either $\mathrm{MH}$ or ADM coordinates; the hereditary part is evaluated separately and defined by means of several types of enhancement functions of the time eccentricity $e_{t}$; finally these are obtained numerically as well as analytically to next-to-leading order $e_{t}^{2}$. At this stage we dispose of both the averaged energy and angular momentum fluxes $\langle\mathcal{F}\rangle$ and $\langle\mathcal{G}\rangle$.

The procedure to compute the secular evolution of the orbital elements under gravitational radiation-reaction is straightforward. Differentiating the orbital elements with respect to time, and using the heuristic balance equations, we equate the decreases of energy and angular momentum to the corresponding averaged fluxes $\langle\mathcal{F}\rangle$ and $\langle\mathcal{G}\rangle$ at 3PN order [12]. This extends earlier analyses at previous orders: Newtonian [339] as we have reviewed in Section 1.2; 1PN [86, 267]; 1.5PN [87, 366] and 2PN $[224,153]$. Let us take the example of the mean motion $n$. From Eq. (347a) together with the definitions (344) we know the function $n(E, \mathrm{~J})$ at $3 \mathrm{PN}$ order, where $E$ and $\mathrm{J}$ are the orbit's 
constant energy and angular momentum. Thus,

$$
\frac{\mathrm{d} n}{\mathrm{~d} t}=\frac{\partial n}{\partial E} \frac{\mathrm{d} E}{\mathrm{~d} t}+\frac{\partial n}{\partial \mathrm{J}} \frac{\mathrm{d} \mathrm{J}}{\mathrm{d} t} .
$$

The usual balance equations for energy and angular momentum

$$
\begin{aligned}
& \left\langle\frac{\mathrm{d} E}{\mathrm{~d} t}\right\rangle=-\langle\mathcal{F}\rangle, \\
& \left\langle\frac{\mathrm{d} J}{\mathrm{~d} t}\right\rangle=-\langle\mathcal{G}\rangle,
\end{aligned}
$$

have already been used at Newtonian order in Eqs. (9). Although heuristically assumed at 3PN order, they have been proved through 1.5PN order in Section 5.4. With the averaged fluxes known through $3 \mathrm{PN}$ order, we obtain the $3 \mathrm{PN}$ averaged evolution equation as

$$
\left\langle\frac{\mathrm{d} n}{\mathrm{~d} t}\right\rangle=-\frac{\partial n}{\partial E}\langle\mathcal{F}\rangle-\frac{\partial n}{\partial \mathrm{J}}\langle\mathcal{G}\rangle \text {. }
$$

We recall that this gives only the slow secular evolution under gravitational radiation reaction for eccentric orbits. The complete evolution includes also, superimposed on the averaged adiabatic evolution, some fast but smaller post-adiabatic oscillations at the orbital time scale [153, 279].

\section{Spinning Compact Binaries}

The post-Newtonian templates have been developed so far for compact binary systems which can be described with great precision by point masses without spins. Here by spin, we mean the intrinsic (classical) angular momentum $S$ of the individual compact body. However, including the effects of spins is essential, as the astrophysical evidence indicates that stellar-mass black holes [2, 390, 311, 227, 323] and supermassive black holes [188, 101, 102] (see Ref. [364] for a review) can be generically close to maximally spinning. The presence of spins crucially affects the dynamics of the binary, in particular leading to orbital plane precession if they are not aligned with the orbital angular momentum (see for instance $[138,8]$ ), and thereby to strong modulations in the observed signal frequency and phase.

In recent years an important effort has been undertaken to compute spin effects to high postNewtonian order in the dynamics and gravitational radiation of compact binaries:

1. Dynamics. The goal is to obtain the equations of motion and related conserved integrals of the motion, the equations of precession of the spins, and the post-Newtonian metric in the near zone. For this step we need a formulation of the dynamics of particles with spins (either Lagrangian or Hamiltonian);

2. Radiation. The mass and current radiative multipole moments, including tails and all hereditary effects, are to be computed. One then deduces the gravitational waveform and the fluxes, from which we compute the secular evolution of the orbital phase. This step requires plugging the previous dynamics into the general wave generation formalism of Part A.

We adopt a particular post-Newtonian counting for spin effects that actually refers to maximally spinning black holes. In this convention the two spin variables $S_{\mathrm{a}}(\mathrm{a}=1,2)$ have the dimension of an angular momentum multiplied by a factor $c$, and we pose

$$
S_{\mathrm{a}}=G m_{\mathrm{a}}^{2} \chi_{\mathrm{a}},
$$


where $m_{\mathrm{a}}$ is the mass of the compact body, and $\chi_{\mathrm{a}}$ is the dimensionless spin parameter, which equals one for maximally spinning Kerr black holes. Thus the spins $S_{\mathrm{a}}$ of the compact bodies can be considered as "Newtonian" quantities [there are no c's in Eq. (366)], and all spin effects will carry (at least) an explicit $1 / c$ factor with respect to non-spin effects. With this convention any post-Newtonian estimate is expected to be appropriate (i.e., numerically correct) in the case of maximal rotation. One should keep in mind that spin effects will be formally a factor $1 / c$ smaller for non-maximally spinning objects such as neutron stars; thus in this case a given post-Newtonian computation will actually be a factor $1 / c$ more accurate.

As usual we shall make a distinction between spin-orbit (SO) effects, which are linear in the spins, and spin-spin (SS) ones, which are quadratic. In this article we shall especially review the SO effects as they play the most important role in gravitational wave detection and parameter estimation. As we shall see a good deal is known on spin effects (both SO and SS), but still it will be important in the future to further improve our knowledge of the waveform and gravitationalwave phasing, by computing still higher post-Newtonian SO and SS terms, and to include at least the dominant spin-spin-spin (SSS) effect [305]. For the computations of SSS and even SSSS effects see Refs. [246, 245, 296, 305, 413].

The SO effects have been known at the leading level since the seminal works of Tulczyjew [411, 412], Barker \& O'Connell [27, 28] and Kidder et al. [275, 271]. With our post-Newtonian counting such leading level corresponds to the 1.5PN order. The SO terms have been computed to the next-to-leading level which corresponds to 2.5PN order in Refs. [394, 194, 165, 292, 352, 241] for the equations of motion or dynamics, and in Refs. [53, 54] for the gravitational radiation field. Note that Refs. [394, 194, 165, 241] employ traditional post-Newtonian methods (both harmonic-coordinates and Hamiltonian), but that Refs. [292, 352] are based on the effective field theory (EFT) approach. The next-to-next-to-leading SO level corresponding to 3.5PN order has been obtained in Refs. [242, 244] using the Hamiltonian method for the equations of motion, in Ref. [297] using the EFT, and in Refs. [307, 90] using the harmonic-coordinates method. Here we shall focus on the harmonic-coordinates approach [307, 90, 89, 306] which is in fact best formulated using a Lagrangian, see Section 11.1. With this approach the next-to-next-to-leading SO level was derived not only for the equations of motion including precession, but also for the radiation field (energy flux and orbital phasing) [89, 306]. An analytic solution for the SO precession effects will be presented in Section 11.2. Note that concerning the radiation field the highest known SO level actually contains specific tail-induced contributions at 3PN [54] and 4PN [306] orders, see Section 11.3.

The SS effects are known at the leading level corresponding to $2 \mathrm{PN}$ order from Barker \& O'Connell [27, 28] in the equations of motion (see [271, 351, 110] for subsequent derivations), and from Refs. [275, 271] in the radiation field. Next-to-leading SS contributions are at 3PN order and have been obtained with Hamiltonian [387, 389, 388, 247, 241], EFT [354, 356, 355, 293, 299] and harmonic-coordinates [88] techniques (with [88] obtaining also the next-to-leading SS terms in the gravitational-wave flux). With SS effects in a compact binary system one must make a distinction between the spin squared terms, involving the coupling between the two same spins $S_{1}$ or $S_{2}$, and the interaction terms, involving the coupling between the two different spins $S_{1}$ and $S_{2}$. The spin-squared terms $S_{1}^{2}$ and $S_{2}^{2}$ arise due to the effects on the dynamics of the quadrupole moments of the compact bodies that are induced by their spins [347]. They have been computed through 2PN order in the fluxes and orbital phase in Refs. [217, 218, 314]. The interaction terms $S_{1} \times S_{2}$ can be computed using a simple pole-dipole formalism like the one we shall review in Section 11.1. The interaction terms $S_{1} \times S_{2}$ between different spins have been derived to next-to-next-to-leading 4PN order for the equations of motion in Refs. [294, 298] (EFT) and [243] (Hamiltonian). In this article we shall generally neglect the SS effects and refer for these to the literature quoted above. 


\subsection{Lagrangian formalism for spinning point particles}

Some necessary material for constructing a Lagrangian for a spinning point particle in curved spacetime is presented here. The formalism is issued from early works [239, 19] and has also been developed in the context of the EFT approach [351]. Variants and alternatives (most importantly the associated Hamiltonian formalism) can be found in Refs. [389, 386, 25]. The formalism yields for the equations of motion of spinning particles and the equations of precession of the spins the classic results known in general relativity [411, 412, 310, 331, 135, 409, 179].

Let us consider a single spinning point particle moving in a given curved background metric $g_{\alpha \beta}(x)$. The particle follows the worldline $y^{\alpha}(\tau)$, with tangent four-velocity $u^{\alpha}=\mathrm{d} y^{\alpha} / \mathrm{d} \tau$, where $\tau$ is a parameter along the representative worldline. In a first stage we do not require that the four-velocity be normalized; thus $\tau$ needs not be the proper time elapsed along the worldline. To describe the internal degrees of freedom associated with the particle's spin, we introduce a moving orthonormal tetrad $e_{A}^{\alpha}(\tau)$ along the trajectory, which defines a "body-fixed" frame. ${ }^{77}$ The rotation tensor $\omega^{\alpha \beta}$ associated with the tetrad is defined by

$$
\frac{\mathrm{D} e_{A}^{\alpha}}{\mathrm{d} \tau}=-\omega^{\alpha \beta} e_{A \beta}
$$

where $\mathrm{D} / \mathrm{d} \tau \equiv u^{\beta} \nabla_{\beta}$ is the covariant derivative with respect to the parameter $\tau$ along the worldline; equivalently, we have

$$
\omega^{\alpha \beta}=e^{A \alpha} \frac{\mathrm{D} e_{A}{ }^{\beta}}{\mathrm{d} \tau} .
$$

Because of the normalization of the tetrad the rotation tensor is antisymmetric: $\omega^{\alpha \beta}=-\omega^{\beta \alpha}$.

We look for an action principle for the spinning particle. Following Refs. [239, 351] and the general spirit of effective field theories, we require the following symmetries to hold:

1. The action is a covariant scalar, i.e., behaves as a scalar with respect to general space-time diffeomorphisms;

2. It is a global Lorentz scalar, i.e., stays invariant under an arbitrary change of the tetrad vectors: $e_{A}^{\alpha}(\tau) \longrightarrow \Lambda_{A}^{B} e_{B}^{\alpha}(\tau)$ where $\Lambda_{A}^{B}$ is a constant Lorentz matrix;

3. It is reparametrization-invariant, i.e., its form is independent of the parameter $\tau$ used to follow the particle's worldline.

In addition to these symmetries we need to specify the dynamical degrees of freedom: These are chosen to be the particle's position $y^{\alpha}$ and the tetrad $e_{A}{ }^{\alpha}$. Furthermore we restrict ourselves to a Lagrangian depending only on the four-velocity $u^{\alpha}$, the rotation tensor $\omega^{\alpha \beta}$, and the metric $g_{\alpha \beta}$. Thus, the postulated action is of the type

$$
I\left[y^{\alpha}, e_{A}^{\alpha}\right]=\int_{-\infty}^{+\infty} \mathrm{d} \tau L\left(u^{\alpha}, \omega^{\alpha \beta}, g_{\alpha \beta}\right) .
$$

These assumptions confine the formalism to a "pole-dipole" model and to terms linear in the spins. An important point is that such a model is universal in the sense that it can be used for black holes as well as neutrons stars. Indeed, the internal structure of the spinning body appears only at the quadratic order in the spins, through the rotationally induced quadrupole moment.

77 The tetrad is orthonormal in the sense that $g_{\alpha \beta} e_{A}{ }^{\alpha} e_{B}{ }^{\beta}=\eta_{A B}$, where $\eta_{A B}=\operatorname{diag}(-1,1,1,1) \operatorname{denotes}$ a Minkowski metric. The indices $A B \cdots=0,1,2,3$ are the internal Lorentz indices, while as usual $\alpha \beta \ldots \mu \nu \ldots=$ $0,1,2,3$ are the space-time covariant indices. The inverse dual tetrad $e_{\alpha}^{A}$, defined by $e_{A}^{\beta} e^{A}=\delta_{\alpha}^{\beta}$, satisfies $\eta_{A B} e_{\alpha}^{A} e_{\beta}^{B}=g_{\alpha \beta}$. We have also the completeness relation $e_{A}{ }^{\beta} e^{B}{ }_{\beta}=\delta_{A}^{B}$. 
As it is written in (369), i.e., depending only on Lorentz scalars, $L$ is automatically a Lorentz scalar. By performing an infinitesimal coordinate transformation, one easily sees that the requirement that the Lagrangian be a covariant scalar specifies its dependence on the metric to be such that (see e.g., Ref. [19])

$$
2 \frac{\partial L}{\partial g_{\alpha \beta}}=p^{\alpha} u^{\beta}+S_{\gamma}^{\alpha} \omega^{\beta \gamma} .
$$

We have defined the conjugate linear momentum $p^{\alpha}$ and the antisymmetric spin tensor $S^{\alpha \beta}$ by

$$
\begin{gathered}
\left.p_{\alpha} \equiv \frac{\partial L}{\partial u^{\alpha}}\right|_{\omega, g}, \\
\left.S_{\alpha \beta} \equiv 2 \frac{\partial L}{\partial \omega^{\alpha \beta}}\right|_{u, g} .
\end{gathered}
$$

Note that the right-hand side of Eq. (370) is necessarily symmetric by exchange of the indices $\alpha$ and $\beta$. Finally, imposing the invariance of the action (369) by reparametrization of the worldline, we find that the Lagrangian must be a homogeneous function of degree one in the velocity $u^{\alpha}$ and rotation tensor $\omega^{\alpha \beta}$. Applying Euler's theorem to the function $L\left(u^{\alpha}, \omega^{\alpha \beta}\right)$ immediately gives

$$
L=p_{\alpha} u^{\alpha}+\frac{1}{2} S_{\alpha \beta} \omega^{\alpha \beta},
$$

where the functions $p_{\alpha}(u, \omega)$ and $S_{\alpha \beta}(u, \omega)$ must be reparametrization invariant. Note that, at this stage, their explicit expressions are not known. They will be specified only when a spin supplementary condition is imposed, see Eq. (379) below.

We now investigate the unconstrained variations of the action (369) with respect to the dynamical variables $e_{A}{ }^{\alpha}, y^{\alpha}$ and the metric. First, we vary it with respect to the tetrad $e_{A}{ }^{\alpha}$ while keeping the position $y^{\alpha}$ fixed. A worry is that we must have a way to distinguish intrinsic variations of the tetrad from variations which are induced by a change of the metric $g_{\alpha \beta}$. This is conveniently solved by decomposing the variation $\delta e_{A}{ }^{\beta}$ according to

$$
\delta e_{A}^{\beta}=e_{A \alpha}\left(\delta \theta^{\alpha \beta}+\frac{1}{2} \delta g^{\alpha \beta}\right)
$$

in which we have introduced the antisymmetric tensor $\delta \theta^{\alpha \beta} \equiv e^{A[\alpha} \delta e_{A}{ }^{\beta]}$, and where the corresponding symmetric part is simply given by the variation of the metric, i.e. $e^{A(\alpha} \delta e_{A}{ }^{\beta)} \equiv \frac{1}{2} \delta g^{\alpha \beta}$. Then we can consider the independent variations $\delta \theta^{\alpha \beta}$ and $\delta g^{\alpha \beta}$. Varying with respect to $\delta \theta^{\alpha \beta}$, but holding the metric fixed, gives the equation of spin precession which is found to be

$$
\frac{\mathrm{D} S_{\alpha \beta}}{\mathrm{d} \tau}=\omega_{\alpha}^{\gamma} S_{\beta \gamma}-\omega_{\beta}^{\gamma} S_{\alpha \gamma}
$$

or, alternatively, using the fact that the right-hand side of Eq. (370) is symmetric,

$$
\frac{\mathrm{D} S_{\alpha \beta}}{\mathrm{d} \tau}=p_{\alpha} u_{\beta}-p_{\beta} u_{\alpha}
$$

We next vary with respect to the particle's position $y^{\alpha}$ while holding the tetrad $e_{A}{ }^{\alpha}$ fixed. Operationally, this means that we have to parallel-transport the tetrad along the displacement vector, i.e., to impose

$$
\delta y^{\beta} \nabla_{\beta} e_{A}^{\alpha}=0 .
$$

A simple way to derive the result is to use locally inertial coordinates, such that the Christoffel symbols $\Gamma_{\beta \gamma}^{\alpha}=0$ along the particle's worldline $y^{\alpha}(\tau)$; then, Eq. (376) gives $\delta e_{A}^{\alpha}=\delta y^{\beta} \partial_{\beta} e_{A}^{\alpha}=$ 
$-\delta y^{\beta} \Gamma_{\beta \gamma}^{\alpha} e_{A}^{\gamma}=0$. The variation leads then to the well-known Mathisson-Papapetrou [310, 331, 135] equation of motion

$$
\frac{\mathrm{D} p_{\alpha}}{\mathrm{d} \tau}=-\frac{1}{2} u^{\beta} R_{\alpha \beta \mu \nu} S^{\mu \nu},
$$

which involves the famous coupling of the spin tensor to the Riemann curvature. ${ }^{78}$ With a little more work, the equation of motion (377) can also be derived using an arbitrary coordinate system, making use of the parallel transport equation (376). Finally, varying with respect to the metric while keeping $\delta \theta^{\alpha \beta}=0$, gives the stress-energy tensor of the spinning particle. We must again take into account the scalarity of the action, as imposed by Eq. (370). We obtain the standard pole-dipole result $[411,412,310,331,135,409,179]$ :

$$
T^{\alpha \beta}=\int_{-\infty}^{+\infty} \mathrm{d} \tau p^{(\alpha} u^{\beta)} \frac{\delta_{(4)}(x-y)}{\sqrt{-g}}-\nabla_{\gamma} \int_{-\infty}^{+\infty} \mathrm{d} \tau S^{\gamma(\alpha} u^{\beta)} \frac{\delta_{(4)}(x-y)}{\sqrt{-g}},
$$

where $\delta_{(4)}(x-y)$ denotes the four-dimensional Dirac function. It can easily be checked that the covariant conservation law $\nabla_{\beta} T^{\alpha \beta}=0$ holds as a consequence of the equation of motion (377) and the equation of spin precession (375).

Up to now we have considered unconstrained variations of the action (369), describing the particle's internal degrees of freedom by the six independent components of the tetrad $e_{A}^{\alpha}$ (namely a $4 \times 4$ matrix subject to the 10 constraints $\left.g_{\alpha \beta} e_{A}{ }^{\alpha} e_{B}{ }^{\beta}=\eta_{A B}\right)$. To correctly account for the number of degrees of freedom associated with the spin, we must impose three supplementary spin conditions (SSC). Several choices are possible for a sensible SSC. Notice that in the case of extended bodies the choice of a SSC corresponds to the choice of a central worldline inside the body with respect to which the spin angular momentum is defined (see Ref. [271] for a discussion). Here we adopt the Tulczyjew covariant SSC [411, 412]

$$
S^{\alpha \beta} p_{\beta}=0
$$

As shown by Hanson \& Regge [239] in the flat space-time case, it is possible to specify the Lagrangian in our original action (369) in such a way that the constraints (379) are directly the consequence of the equations derived from that Lagrangian. Here, for simplicity's sake, we shall simply impose the constraints (379) in the space of solutions of the Euler-Lagrange equations. From Eq. (379) we can introduce the covariant spin vector $S_{\mu}$ associated with the spin tensor by ${ }^{79}$

$$
S^{\alpha \beta} \equiv \frac{1}{m} \varepsilon^{\alpha \beta \mu \nu} p_{\mu} S_{\nu},
$$

where we have defined the mass of the particle by $m^{2} \equiv-g^{\mu \nu} p_{\mu} p_{\nu}$. By contracting Eq. (375) with $p^{\beta}$ and using the equation of motion (377), one obtains

$$
p_{\alpha}(p u)+m^{2} u_{\alpha}=\frac{1}{2} u^{\gamma} R_{\gamma \mu \nu}^{\beta} S^{\mu \nu} S_{\alpha \beta},
$$

where we denote $(p u) \equiv p_{\mu} u^{\mu}$. By further contracting Eq. (381) with $u^{\alpha}$ we obtain an explicit expression for $(p u)$, which can then be substituted back into (381) to provide the relation linking the four-momentum $p_{\alpha}$ to the four-velocity $u_{\alpha}$. It can be checked using (379) and (381) that the mass of the particle is constant along the particle's trajectory: $\mathrm{d} m / \mathrm{d} \tau=0$. Furthermore the four-dimensional magnitude $s$ of the spin defined by $s^{2} \equiv g^{\mu \nu} S_{\mu} S_{\nu}$ is also conserved: $\mathrm{d} s / \mathrm{d} \tau=0$.

\footnotetext{
78 Our conventions for the Riemann tensor $R_{\alpha \beta \mu \nu}$ follow those of MTW [319].

79 The four-dimensional Levi-Civita tensor is defined by $\varepsilon_{\alpha \beta \mu \nu} \equiv \sqrt{-g} \epsilon_{\alpha \beta \mu \nu}$ and $\varepsilon^{\alpha \beta \mu \nu} \equiv-\epsilon^{\alpha \beta \mu \nu} / \sqrt{-g}$; here $\epsilon_{\alpha \beta \mu \nu}=\epsilon^{\alpha \beta \mu \nu}$ denotes the completely anti-symmetric Levi-Civita symbol such that $\epsilon_{0123}=\epsilon^{0123}=1$. For convenience in this section we pose $c=1$.
} 
Henceforth we shall restrict our attention to spin-orbit (SO) interactions, which are linear in the spins. We shall also adopt for the parameter $\tau$ along the particle's worldline the proper time $\mathrm{d} \tau \equiv \sqrt{-g_{\mu \nu} \mathrm{d} y^{\mu} \mathrm{d} y^{\nu}}$, so that $g_{\mu \nu} u^{\mu} u^{\nu}=-1$. Neglecting quadratic spin-spin (SS) and higherorder interactions, the linear momentum is simply proportional to the normalized four-velocity: $p_{\alpha}=m u_{\alpha}+\mathcal{O}\left(S^{2}\right)$. Hence, from Eq. (375) we deduce that $\mathrm{D} S_{\alpha \beta} / \mathrm{d} \tau=\mathcal{O}\left(S^{2}\right)$. The equation for the spin covariant vector $S_{\alpha}$ then reduces at linear order to

$$
\frac{\mathrm{D} S_{\alpha}}{\mathrm{d} \tau}=\mathcal{O}\left(S^{2}\right)
$$

Thus the spin covector is parallel transported along the particle's trajectory at linear order in spin. We can also impose that the spin should be purely spatial for the comoving observer:

$$
S_{\alpha} u^{\alpha}=0 \text {. }
$$

From now on, we shall often omit writing the $\mathcal{O}\left(S^{2}\right)$ remainders.

In applications (e.g., the construction of gravitational wave templates for the compact binary inspiral) it is very useful to introduce new spin variables that are designed to have a conserved threedimensional Euclidean norm (numerically equal to $s$ ). Using conserved-norm spin vector variables is indeed the most natural choice when considering the dynamics of compact binaries reduced to the frame of the center of mass or to circular orbits [90]. Indeed the evolution equations of such spin variables reduces, by construction, to ordinary precession equations, and these variables are secularly constant (see Ref. [423]).

A standard, general procedure to define a (Euclidean) conserved-norm spin spatial vector consists of projecting the spin covector $S_{\alpha}$ onto an orthonormal tetrad $e_{A}{ }^{\alpha}$, which leads to the four scalar components $(A=0,1,2,3)$

$$
S_{A}=e_{A}{ }^{\alpha} S_{\alpha} .
$$

If we choose for the time-like tetrad vector the four-velocity itself, $e_{0}{ }^{\alpha}=u^{\alpha},{ }^{80}$ the time component tetrad projection $S_{0}$ vanishes because of the orthogonality condition (383). We have seen that $S_{\alpha} S^{\alpha}=s^{2}$ is conserved along the trajectory; because of (383) we can rewrite this as $\gamma^{\alpha \beta} S_{\alpha} S_{\beta}=$ $s^{2}$, in which we have introduced the projector $\gamma^{\alpha \beta}=g^{\alpha \beta}+u^{\alpha} u^{\beta}$ onto the spatial hypersurface orthogonal to $u^{\alpha}$. From the orthonormality of the tetrad and our choice $e_{0}^{\alpha}=u^{\alpha}$, we have $\gamma^{\alpha \beta}=\delta^{a b} e_{a}{ }^{\alpha} e_{b}{ }^{\beta}$ in which $a, b=1,2,3$ refer to the spatial values of the tetrad indices, i.e., $A=(0, a)$ and $B=(0, b)$. Therefore the conservation law $\gamma^{\alpha \beta} S_{\alpha} S_{\beta}=s^{2}$ becomes

$$
\delta^{a b} S_{a} S_{b}=s^{2}
$$

which is indeed the relation defining a Euclidean conserved-norm spin variable $S_{a} \cdot{ }^{81}$ However, note that the choice of the spin variable $S_{a}$ is still somewhat arbitrary, since a rotation of the tetrad vectors can freely be performed. We refer to $[165,90]$ for the definition of some "canonical" choice for the tetrad in order to fix this residual freedom. Such choice presents the advantage of providing a unique determination of the conserved-norm spin variable in a given gauge. This canonical choice will be the one adopted in all explicit results presented in Section 11.3.

The evolution equation (382) for the original spin variable $S_{\alpha}$ now translates into an ordinary precession equation for the tetrad components $S_{a}$, namely

$$
\frac{\mathrm{d} S_{a}}{\mathrm{~d} t}=\Omega_{a}{ }^{b} S_{b}
$$

\footnotetext{
80 Because of this choice, it is better to consider that the tetrad is not the same as the one we originally employed to construct the action (369).

81 Beware that here we employ the usual slight ambiguity in the notation when using the same carrier letter $S$ to denote the tetrad components (384) and the original spin covector. Thus, $S_{a}$ should not be confused with the spatial components $S_{i}$ (with $i=1,2,3$ ) of the covariant vector $S_{\alpha}$.
} 
where the precession tensor $\Omega_{a b}$ is related to the tetrad components $\omega_{A B}$ of the rotation tensor defined in (368) by $\Omega_{a b}=z \omega_{a b}$ where we pose $z \equiv \mathrm{d} \tau / \mathrm{d} t$, remembering the redshift variable (276). The antisymmetric character of the matrix $\Omega_{a b}$ guaranties that $S_{a}$ satisfies the Euclidean precession equation

$$
\frac{\mathrm{d} \boldsymbol{S}}{\mathrm{d} t}=\boldsymbol{\Omega} \times \boldsymbol{S}
$$

where we denote $\boldsymbol{S}=\left(S_{a}\right)$, and $\boldsymbol{\Omega}=\left(\Omega_{a}\right)$ with $\Omega_{a}=-\frac{1}{2} \epsilon_{a b c} \Omega^{b c}$. As a consequence of (387) the spin has a conserved Euclidean norm: $\boldsymbol{S}^{2}=s^{2}$. From now on we shall no longer make any distinction between the spatial tetrad indices $a b \ldots$ and the ordinary spatial indices $i j \ldots$ which are raised and lowered with the Kronecker metric. Explicit results for the equations of motion and gravitational wave templates will be given in Sections 11.2 and 11.3 using the canonical choice for the conserved-norm spin variable $\boldsymbol{S}$.

\subsection{Equations of motion and precession for spin-orbit effects}

The previous formalism can be generalized to self-gravitating systems consisting of two (or more generally $N$ ) spinning point particles. The metric generated by the system of particles, interacting only through gravitation, is solution of the Einstein field equations (18) with stress-energy tensor given by the sum of the individual stress-energy tensors (378) for each particles. The equations of motion of the particles are given by the Mathisson-Papapetrou equations (377) with "selfgravitating" metric evaluated at the location of the particles thanks to a regularization procedure (see Section 6). The precession equations of each of the spins are given by

$$
\frac{\mathrm{d} \boldsymbol{S}_{\mathrm{a}}}{\mathrm{d} t}=\boldsymbol{\Omega}_{\mathrm{a}} \times \boldsymbol{S}_{\mathrm{a}}
$$

where a $=1,2$ labels the particles. The spin variables $\boldsymbol{S}_{\mathrm{a}}$ are the conserved-norm spins defined in Section 11.1. In the following it is convenient to introduce two combinations of the individuals spins defined by (with $X_{\mathrm{a}} \equiv m_{\mathrm{a}} / m$ and $\left.\nu \equiv X_{1} X_{2}\right)^{82}$

$$
\begin{aligned}
\boldsymbol{S} & \equiv \boldsymbol{S}_{1}+\boldsymbol{S}_{2}, \\
\boldsymbol{\Sigma} & \equiv \frac{\boldsymbol{S}_{2}}{X_{2}}-\frac{\boldsymbol{S}_{1}}{X_{1}} .
\end{aligned}
$$

We shall investigate the case where the binary's orbit is quasi-circular, i.e., whose radius is constant apart from small perturbations induced by the spins (as usual we neglect the gravitational radiation damping effects). We denote by $\boldsymbol{x}=\boldsymbol{y}_{1}-\boldsymbol{y}_{2}$ and $\boldsymbol{v}=\mathrm{d} \boldsymbol{x} / \mathrm{d} t$ the relative position and velocity. ${ }^{83}$ We introduce an orthonormal moving triad $\{\boldsymbol{n}, \boldsymbol{\lambda}, \boldsymbol{\ell}\}$ defined by the unit separation vector $\boldsymbol{n}=\boldsymbol{x} / r$ (with $r=|\boldsymbol{x}|$ ) and the unit normal $\boldsymbol{\ell}$ to the instantaneous orbital plane given by $\boldsymbol{\ell}=\boldsymbol{n} \times \boldsymbol{v} /|\boldsymbol{n} \times \boldsymbol{v}|$; the orthonormal triad is then completed by $\boldsymbol{\lambda}=\boldsymbol{\ell} \times \boldsymbol{n}$. Those vectors are represented on Figure 4, which shows the geometry of the system. The orbital frequency $\Omega$ is defined for general orbits, not necessarily circular, by $\boldsymbol{v}=\dot{r} \boldsymbol{n}+r \Omega \boldsymbol{\lambda}$ where $\dot{\boldsymbol{r}}=\boldsymbol{n} \cdot \boldsymbol{v}$ represents

82 Notation adopted in Ref. [271]; the inverse formulas read

$$
\begin{aligned}
& \boldsymbol{S}_{1}=X_{1} \boldsymbol{S}-\nu \boldsymbol{\Sigma}, \\
& \boldsymbol{S}_{2}=X_{2} \boldsymbol{S}+\nu \boldsymbol{\Sigma} .
\end{aligned}
$$

83 Note that the individual particle's positions $\boldsymbol{y}_{\mathrm{a}}$ in the frame of the center-of-mass (defined by the cancellation of the center-of-mass integral of motion: $\boldsymbol{G}=0$ ) are related to the relative position and velocity $\boldsymbol{x}$ and $\boldsymbol{v}$ by some expressions similar to Eqs. (224) but containing spin effects starting at order 1.5PN. 
the derivative of $r$ with respect to the coordinate time $t$. The general expression for the relative acceleration $\boldsymbol{a} \equiv \mathrm{d} \boldsymbol{v} / \mathrm{d} t$ decomposed in the moving basis $\{\boldsymbol{n}, \boldsymbol{\lambda}, \boldsymbol{\ell}\}$ is

$$
\boldsymbol{a}=\left(\ddot{r}-r \Omega^{2}\right) \boldsymbol{n}+(r \dot{\Omega}+2 \dot{r} \Omega) \boldsymbol{\lambda}+r \varpi \Omega \boldsymbol{\ell} .
$$

Here we have introduced the orbital plane precession $\varpi$ of the orbit defined by $\varpi \equiv-\boldsymbol{\lambda} \cdot \mathrm{d} \boldsymbol{\ell} / \mathrm{d} t$. Next we impose the restriction to quasi-circular precessing orbits which is defined by the conditions $\dot{r}=\dot{\Omega}=\mathcal{O}\left(1 / c^{5}\right)$ and $\ddot{r}=\mathcal{O}\left(1 / c^{10}\right)$ so that $v^{2}=r^{2} \Omega^{2}+\mathcal{O}\left(1 / c^{10}\right)$; see Eqs. (227). Then $\boldsymbol{\lambda}$ represents the direction of the velocity, and the precession frequency $\varpi$ is proportional to the variation of $\ell$ in the direction of the velocity. In this way we find that the equations of the relative motion in the frame of the center-of-mass are

$$
\boldsymbol{a}=-r \Omega^{2} \boldsymbol{n}+r \varpi \Omega \boldsymbol{\ell}+\mathcal{O}\left(\frac{1}{c^{5}}\right) .
$$

Since we neglect the radiation reaction damping there is no component of the acceleration along $\boldsymbol{\lambda}$. This equation represents the generalization of Eq. (226) for spinning quasi-circular binaries with no radiation reaction. The orbital frequency $\Omega$ will contain spin effects in addition to the non-spin terms given by (228), while the precessional frequency $\varpi$ will entirely be due to spins.

Here we report the latest results for the spin-orbit $(\mathrm{SO})$ contributions into these quantities at the next-to-next-to-leading level corresponding to 3.5PN order [307, 90]. We project out the spins on the moving orthonormal basis, defining $\boldsymbol{S}=S_{n} \boldsymbol{n}+S_{\lambda} \boldsymbol{\lambda}+S_{\ell} \boldsymbol{\ell}$ and similarly for $\boldsymbol{\Sigma}$. We have

$$
\begin{aligned}
\Omega_{\mathrm{SO}}^{2} & =\frac{\gamma^{3 / 2}}{m r^{3}}\left\{-5 S_{\ell}-3 \Delta \Sigma_{\ell}+\gamma\left[\left(\frac{45}{2}-\frac{27}{2} \nu\right) S_{\ell}+\Delta\left(\frac{27}{2}-\frac{13}{2} \nu\right) \Sigma_{\ell}\right]\right. \\
& \left.+\gamma^{2}\left[\left(-\frac{495}{8}-\frac{561}{8} \nu-\frac{51}{8} \nu^{2}\right) S_{\ell}+\Delta\left(-\frac{297}{8}-\frac{341}{8} \nu-\frac{21}{8} \nu^{2}\right) \Sigma_{\ell}\right]\right\}+\mathcal{O}\left(\frac{1}{c^{8}}\right),
\end{aligned}
$$

which has to be added to the non-spin terms (228) up to 3.5PN order. We recall that the ordering post-Newtonian parameter is $\gamma=\frac{G m}{r c^{2}}$. On the other hand the next-to-next-to-leading SO effects into the precessional frequency read

$$
\begin{aligned}
\varpi & =\frac{c^{3} x^{3}}{G^{2} m^{3}}\left\{7 S_{n}+3 \Delta \Sigma_{n}+x\left[(-3-12 \nu) S_{n}+\Delta\left(-3-\frac{11}{2} \nu\right) \Sigma_{n}\right]\right. \\
& \left.+x^{2}\left[\left(-\frac{3}{2}-\frac{59}{2} \nu+9 \nu^{2}\right) S_{n}+\Delta\left(-\frac{3}{2}-\frac{77}{8} \nu+\frac{13}{3} \nu^{2}\right) \Sigma_{n}\right]\right\}+\mathcal{O}\left(\frac{1}{c^{8}}\right),
\end{aligned}
$$

where this time the ordering post-Newtonian parameter is $x \equiv\left(\frac{G m \Omega}{c^{3}}\right)^{2 / 3}$. The SO terms at the same level in the conserved energy associated with the equations of motion will be given in Eq. (415) below. In order to complete the evolution equations for quasi-circular orbits we need also the precession vectors $\boldsymbol{\Omega}_{\mathrm{a}}$ of the two spins as defined by Eq. (388). These are given by

$$
\begin{aligned}
\boldsymbol{\Omega}_{1} & =\frac{c^{3} x^{5 / 2}}{G m} \ell\left\{\frac{3}{4}+\frac{1}{2} \nu-\frac{3}{4} \Delta+x\left[\frac{9}{16}+\frac{5}{4} \nu-\frac{1}{24} \nu^{2}+\Delta\left(-\frac{9}{16}+\frac{5}{8} \nu\right)\right]\right. \\
& \left.+x^{2}\left[\frac{27}{32}+\frac{3}{16} \nu-\frac{105}{32} \nu^{2}-\frac{1}{48} \nu^{3}+\Delta\left(-\frac{27}{32}+\frac{39}{8} \nu-\frac{5}{32} \nu^{2}\right)\right]\right\}+\mathcal{O}\left(\frac{1}{c^{8}}\right) .
\end{aligned}
$$

We obtain $\boldsymbol{\Omega}_{2}$ from $\boldsymbol{\Omega}_{1}$ simply by exchanging the masses, $\Delta \rightarrow-\Delta$. At the linear SO level the precession vectors $\boldsymbol{\Omega}_{\mathrm{a}}$ are independent of the spins. ${ }^{84}$

\footnotetext{
${ }^{84}$ Beware of our inevitably slightly confusing notation: $\Omega$ is the binary's orbital frequency and $\Omega_{\mathrm{SO}}$ refers to the spin-orbit terms therein; $\Omega_{\mathrm{a}}$ is the precession frequency of the a-th spin while $\varpi$ is the precession frequency of the orbital plane; and $\omega_{\mathrm{a}}$ defined earlier in Eqs. (244) and (284) is the rotation frequency of the a-th black hole. Such different notions nicely mix up in the first law of spinning binary black holes in Section 8.3; see Eq. (282) and the corotation condition (285).
} 
We now investigate an analytical solution for the dynamics of compact spinning binaries on quasi-circular orbits, including the effects of spin precession $[54,306]$. This solution will be valid whenever the radiation reaction effects can be neglected, and is restricted to the linear SO level.

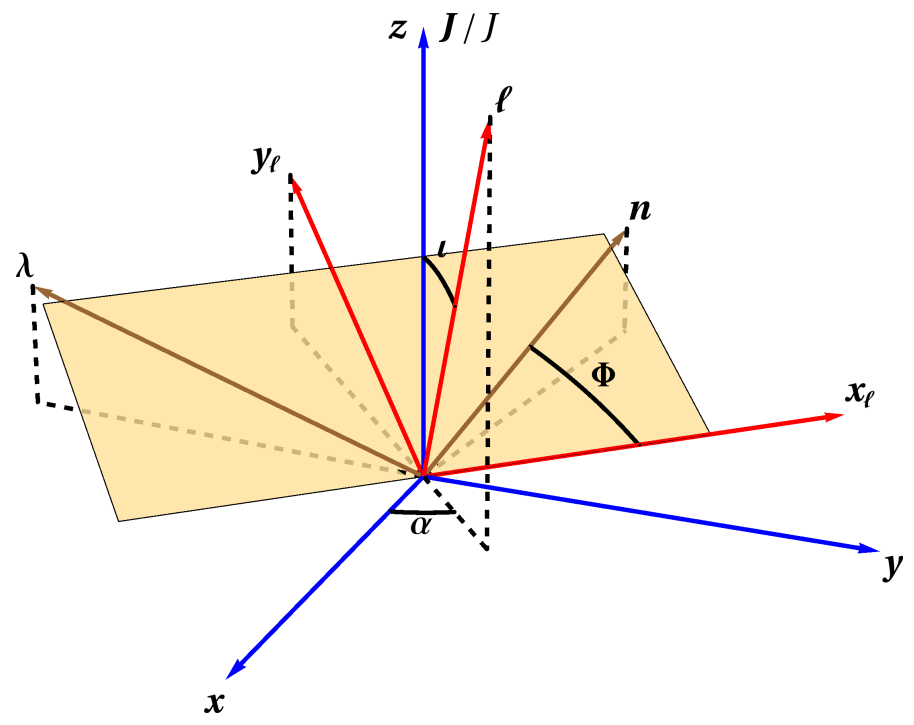

Figure 4: Geometric definitions for the precessional motion of spinning compact binaries [54, 306]. We show (i) the source frame defined by the fixed orthonormal basis $\{\boldsymbol{x}, \boldsymbol{y}, \boldsymbol{z}\}$; (ii) the instantaneous orbital plane which is described by the orthonormal basis $\left\{\boldsymbol{x}_{\ell}, \boldsymbol{y}_{\ell}, \boldsymbol{\ell}\right\}$; (iii) the moving triad $\{\boldsymbol{n}, \boldsymbol{\lambda}, \boldsymbol{\ell}\}$ and the associated three Euler angles $\alpha, \iota$ and $\Phi ;(\mathrm{v})$ the direction of the total angular momentum $\mathbf{J}$ which coincides with the $z$-direction. Dashed lines show projections into the $x-y$ plane.

In the following, we will extensively employ the total angular momentum of the system, that we denote by $\mathbf{J}$, and which is conserved when radiation-reaction effects are neglected,

$$
\frac{\mathrm{d} \mathbf{J}}{\mathrm{d} t}=0
$$

It is customary to decompose the conserved total angular momentum $\mathbf{J}$ as the sum of the orbital angular momentum $\boldsymbol{L}$ and of the two spins, ${ }^{85}$

$$
\mathbf{J}=\boldsymbol{L}+\frac{\boldsymbol{S}}{c} .
$$

This split between $\boldsymbol{L}$ and $\boldsymbol{S}=\boldsymbol{S}_{1}+\boldsymbol{S}_{2}$ is specified by our choice of spin variables, here the conserved-norm spins defined in Section 11.1. Note that although $\boldsymbol{L}$ is called the "orbital" angular momentum, it actually includes both non-spin and spin contributions. We refer to Eq. (4.7) in [90] for the expression of $\boldsymbol{L}$ at the next-to-next-to-leading SO level for quasi-circular orbits.

Our solution will consist of some explicit expressions for the moving triad $\{\boldsymbol{n}, \boldsymbol{\lambda}, \boldsymbol{\ell}\}$ at the SO level in the conservative dynamics for quasi-circular orbits. With the previous definitions of the orbital frequency $\Omega$ and the precessional frequency $\varpi$ we have the following system of equations for the time evolution of the triad vectors,

$$
\frac{\mathrm{d} \boldsymbol{n}}{\mathrm{d} t}=\Omega \boldsymbol{\lambda},
$$

\footnotetext{
85 Recall from Eq. (366) that in our convention the spins have the dimension of an angular momentum times $c$.
} 


$$
\begin{aligned}
& \frac{\mathrm{d} \boldsymbol{\lambda}}{\mathrm{d} t}=-\Omega \boldsymbol{n}+\varpi \boldsymbol{\ell}, \\
& \frac{\mathrm{d} \boldsymbol{\ell}}{\mathrm{d} t}=-\varpi \boldsymbol{\lambda} .
\end{aligned}
$$

Equivalently, introducing the orbital rotation vector $\boldsymbol{\Omega} \equiv \Omega \boldsymbol{\ell}$ and spin precession vector $\varpi \equiv \varpi \boldsymbol{n}$, these equations can be elegantly written as

$$
\begin{aligned}
& \frac{\mathrm{d} \boldsymbol{n}}{\mathrm{d} t}=\boldsymbol{\Omega} \times \boldsymbol{n}, \\
& \frac{\mathrm{d} \boldsymbol{\lambda}}{\mathrm{d} t}=(\boldsymbol{\Omega}+\varpi) \times \boldsymbol{\lambda}, \\
& \frac{\mathrm{d} \boldsymbol{\ell}}{\mathrm{d} t}=\varpi \times \boldsymbol{\ell} .
\end{aligned}
$$

Next we introduce a fixed (inertial) orthonormal basis $\{\mathbf{x}, \mathbf{y}, \mathbf{z}\}$ as follows: $\mathbf{z}$ is defined to be the normalized value $\mathbf{J} / \mathbf{J}$ of the total angular momentum $\mathbf{J} ; \mathbf{y}$ is orthogonal to the plane spanned by $\mathbf{z}$ and the direction $\boldsymbol{N}=\boldsymbol{X} / R$ of the detector as seen from the source (notation of Section 3.1) and is defined by $\mathbf{y}=\mathbf{z} \times \mathbf{N} /|\mathbf{z} \times \mathbf{N}|$; and $\mathbf{x}$ completes the triad - see Figure 4. Then, we introduce the standard spherical coordinates $(\alpha, \iota)$ of the vector $\ell$ measured in the inertial basis $\{\mathbf{x}, \mathbf{y}, \mathbf{z}\}$. Since $\iota$ is the angle between the total and orbital angular momenta, we have

$$
\sin \iota=\frac{|\mathbf{J} \times \ell|}{\mathrm{J}}
$$

where $\mathrm{J} \equiv|\mathbf{J}|$. The angles $(\alpha, \iota)$ are referred to as the precession angles.

We now derive from the time evolution of our triad vectors those of the precession angles $(\alpha, \iota)$, and of an appropriate phase $\Phi$ that specifies the position of $\boldsymbol{n}$ with respect to some reference direction in the orbital plane denoted $\mathbf{x}_{\ell}$. Following Ref. [13], we pose

$$
\begin{aligned}
& \mathbf{x}_{\ell}=\frac{\mathbf{J} \times \boldsymbol{\ell}}{|\mathbf{J} \times \boldsymbol{\ell}|}, \\
& \mathbf{y}_{\ell}=\boldsymbol{\ell} \times \mathbf{x}_{\ell},
\end{aligned}
$$

such that $\left\{\mathbf{x}_{\ell}, \mathbf{y}_{\ell}, \ell\right\}$ forms an orthonormal basis. The motion takes place in the instantaneous orbital plane spanned by $\boldsymbol{n}$ and $\boldsymbol{\lambda}$, and the phase angle $\Phi$ is such that (see Figure 4 ):

$$
\begin{aligned}
& \boldsymbol{n}=\cos \Phi \mathbf{x}_{\ell}+\sin \Phi \mathbf{y}_{\ell}, \\
& \boldsymbol{\lambda}=-\sin \Phi \mathbf{x}_{\ell}+\cos \Phi \mathbf{y}_{\ell},
\end{aligned}
$$

from which we deduce

$$
e^{-\mathrm{i} \Phi}=\mathbf{x}_{\ell} \cdot(\boldsymbol{n}+\mathrm{i} \boldsymbol{\lambda})=\frac{\mathrm{J}_{\lambda}-\mathrm{i} \mathrm{J}_{n}}{\sqrt{\mathrm{J}_{n}^{2}+\mathrm{J}_{\lambda}^{2}}} .
$$

Combining Eqs. (402) with (399) we also get

$$
\sin \iota e^{-\mathrm{i} \Phi}=\frac{\mathrm{J}_{\lambda}-\mathrm{i} \mathrm{J}_{n}}{\mathrm{~J}}
$$

By identifying the right-hand sides of (397) with the time-derivatives of the relations (401) we obtain the following system of equations for the variations of $\alpha, \iota$ and $\Phi$,

$$
\frac{\mathrm{d} \alpha}{\mathrm{d} t}=\varpi \frac{\sin \Phi}{\sin \iota},
$$




$$
\begin{aligned}
\frac{\mathrm{d} \iota}{\mathrm{d} t} & =\varpi \cos \Phi, \\
\frac{\mathrm{d} \Phi}{\mathrm{d} t} & =\Omega-\varpi \frac{\sin \Phi}{\tan \iota} .
\end{aligned}
$$

On the other hand, using the decompositon of the total angular momentum (396) together with the fact that the components of $\boldsymbol{L}$ projected along $\boldsymbol{n}$ and $\boldsymbol{\lambda}$ are of the order $\mathcal{O}(S)$, see e.g., Eq. (4.7) in Ref. [90], we deduce that $\sin \iota$ is itself a small quantity of order $\mathcal{O}(S)$. Since we also have $\varpi=\mathcal{O}(S)$, we conclude by direct integration of the sum of Eqs. (404a) and (404c) that

$$
\Phi+\alpha=\phi+\mathcal{O}\left(S^{2}\right)
$$

in which we have defined the "carrier" phase as

$$
\phi \equiv \int \Omega \mathrm{d} t=\Omega\left(t-t_{0}\right)+\phi_{0}
$$

with $\phi_{0}$ the value of the carrier phase at some arbitrary initial time $t_{0}$. An important point we have used when integrating (406) is that the orbital frequency $\Omega$ is constant at linear order in the spins. Indeed, from Eq. (392) we see that only the components of the conserved-norm spin vectors along $\boldsymbol{\ell}$ can contribute to $\Omega$ at linear order. As we show in Eq. (409c) below, these components are in fact constant at linear order in spins. Thus we can treat $\Omega$ as a constant for our purpose.

The combination $\Phi+\alpha$ being known by Eq. (405), we can further express the precession angles $\iota$ and $\alpha$ at linear order in spins in terms of the components $\mathrm{J}_{n}$ and $\mathrm{J}_{\lambda}$; from Eqs. (399) and (403):

$$
\begin{aligned}
\sin \iota & =\frac{\sqrt{\mathrm{J}_{n}^{2}+\mathrm{J}_{\lambda}^{2}}}{L_{\mathrm{NS}}}+\mathcal{O}\left(S^{2}\right), \\
e^{\mathrm{i} \alpha} & =\frac{\mathrm{J}_{\lambda}-\mathrm{i} \mathrm{J}_{n}}{\sqrt{\mathrm{J}_{n}^{2}+\mathrm{J}_{\lambda}^{2}}} e^{\mathrm{i} \phi}+\mathcal{O}\left(S^{2}\right),
\end{aligned}
$$

where we denote by $L_{\mathrm{NS}}$ the norm of the non-spin (NS) part of the orbital angular momentum $\boldsymbol{L}$.

It remains to obtain the explicit time variation of the components of the two individual spins $S_{n}^{\mathrm{a}}, S_{\lambda}^{\mathrm{a}}$ and $S_{\ell}^{\mathrm{a}}$ (with a $=1,2$ ). Using Eqs. (407) together with the decomposition (396) and the explicit expression of $\boldsymbol{L}$ in Ref. [90], we shall then be able to obtain the explicit time variation of the precession angles $(\alpha, \iota)$ and phase $\Phi$. Combining (388) and (397) we obtain

$$
\begin{aligned}
& \frac{\mathrm{d} S_{n}^{\mathrm{a}}}{\mathrm{d} t}=\left(\Omega-\Omega_{\mathrm{a}}\right) S_{\lambda}^{\mathrm{a}}, \\
& \frac{\mathrm{d} S_{\lambda}^{\mathrm{a}}}{\mathrm{d} t}=-\left(\Omega-\Omega_{\mathrm{a}}\right) S_{n}^{\mathrm{a}}+\varpi S_{\ell}^{\mathrm{a}}, \\
& \frac{\mathrm{d} S_{\ell}^{\mathrm{a}}}{\mathrm{d} t}=-\varpi S_{\lambda}^{\mathrm{a}},
\end{aligned}
$$

where $\Omega_{\mathrm{a}}$ is the norm of the precession vector of the a-th spin as given by (394), and the precession frequency $\varpi$ is explicitly given by (393). At linear order in spins these equations translate into

$$
\begin{aligned}
\frac{\mathrm{d} S_{n}^{\mathrm{a}}}{\mathrm{d} t} & =\left(\Omega-\Omega_{\mathrm{a}}\right) S_{\lambda}^{\mathrm{a}}, \\
\frac{\mathrm{d} S_{\lambda}^{\mathrm{a}}}{\mathrm{d} t} & =-\left(\Omega-\Omega_{\mathrm{a}}\right) S_{n}^{\mathrm{a}}+\mathcal{O}\left(S^{2}\right), \\
\frac{\mathrm{d} S_{\ell}^{\mathrm{a}}}{\mathrm{d} t} & =\mathcal{O}\left(S^{2}\right) .
\end{aligned}
$$


We see that, as stated before, the spin components along $\ell$ are constant, and so is the orbital frequency $\Omega$ given by (392). At the linear SO level, the equations (409) can be decoupled and integrated as

$$
\begin{aligned}
S_{n}^{\mathrm{a}} & =S_{\perp}^{\mathrm{a}} \cos \psi_{\mathrm{a}}, \\
S_{\lambda}^{\mathrm{a}} & =-S_{\perp}^{\mathrm{a}} \sin \psi_{\mathrm{a}}, \\
S_{\ell}^{\mathrm{a}} & =S_{\|}^{\mathrm{a}} .
\end{aligned}
$$

Here $S_{\perp}^{\mathrm{a}}$ and $S_{\|}^{\mathrm{a}}$ denote two quantities for each spins, that are constant up to terms $\mathcal{O}\left(S^{2}\right)$. The phase of the projection perpendicular to the direction $\ell$ of each of the spins is given by

$$
\psi_{\mathrm{a}}=\left(\Omega-\Omega_{\mathrm{a}}\right)\left(t-t_{0}\right)+\psi_{\mathrm{a}}^{0},
$$

where $\psi_{\mathrm{a}}^{0}$ is the constant initial phase at the reference time $t_{0}$

Finally we can give in an explicit way, to linear SO order, the triad $\{\boldsymbol{n}(t), \boldsymbol{\lambda}(t), \boldsymbol{\ell}(t)\}$ in terms of some reference triad $\left\{\boldsymbol{n}_{0}, \boldsymbol{\lambda}_{0}, \ell_{0}\right\}$ at the reference time $t_{0}$ in Eqs. (411) and (406). The best way to express the result is to introduce the complex null vector $\boldsymbol{m} \equiv \frac{1}{\sqrt{2}}(\boldsymbol{n}+\mathrm{i} \boldsymbol{\lambda})$ and its complex conjuguate $\overline{\boldsymbol{m}}$; the normalization is chosen so that $\boldsymbol{m} \cdot \overline{\boldsymbol{m}}=1$. We obtain

$$
\begin{aligned}
\boldsymbol{m} & =e^{-\mathrm{i}\left(\phi-\phi_{0}\right)} \boldsymbol{m}_{0}+\frac{\mathrm{i}}{\sqrt{2}}\left(\sin \iota e^{\mathrm{i} \alpha}-\sin \iota_{0} e^{\mathrm{i} \alpha_{0}}\right) e^{-\mathrm{i} \phi} \boldsymbol{\ell}_{0}+\mathcal{O}\left(S^{2}\right), \\
\boldsymbol{\ell} & =\boldsymbol{\ell}_{0}+\left[\frac{\mathrm{i}}{\sqrt{2}}\left(\sin \iota e^{-\mathrm{i} \alpha}-\sin \iota_{0} e^{-\mathrm{i} \alpha_{0}}\right) e^{\mathrm{i} \phi_{0}} \boldsymbol{m}_{0}+\text { c.c. }\right]+\mathcal{O}\left(S^{2}\right) .
\end{aligned}
$$

The precession effects in the dynamical solution for the evolution of the basis vectors $\{\boldsymbol{n}, \boldsymbol{\lambda}, \boldsymbol{\ell}\}$ are given by the second terms in these equations. They depend only in the combination $\sin \iota e^{\mathrm{i} \alpha}$ and its complex conjugate $\sin \iota e^{-\mathrm{i} \alpha}$, which follows from Eqs. (407) and the known spin and non-spin contributions to the total angular momentum $\mathbf{J}$. One can check that precession effects in the above dynamical solution (412) for the moving triad start at order $\mathcal{O}\left(1 / c^{3}\right)$.

\subsection{Spin-orbit effects in the gravitational wave flux and orbital phase}

Like in Section 9 our main task is to control up to high post-Newtonian order the mass and current radiative multipole moments $\mathrm{U}_{L}$ and $\mathrm{V}_{L}$ which parametrize the asymptotic waveform and gravitational fluxes far away from the source, cf. Eqs. (66) - (68). The radiative multipole moments are in turn related to the source multipole moments $\mathrm{I}_{L}$ and $\mathrm{J}_{L}$ through complicated relationships involving tails and related effects; see e.g., Eqs. (76). ${ }^{86}$

The source moments have been expressed in Eqs. (123) in terms of some source densities $\Sigma$, $\Sigma_{i}$ and $\Sigma_{i j}$ defined from the components of the post-Newtonian expansion of the pseudo-tensor, denoted $\bar{\tau}^{\alpha \beta}$. To lowest order the (PN expansion of the) pseudo-tensor reduces to the matter tensor $T^{\alpha \beta}$ which has compact support, and the source densities $\Sigma, \Sigma_{i}, \Sigma_{i j}$ reduce to the compact support quantities $\sigma, \sigma_{i} \sigma_{i j}$ given by Eqs. (145). Now, computing spin effects, the matter tensor $T^{\alpha \beta}$ has been found to be given by (378) in the framework of the pole-dipole approximation suitable for SO couplings (and sufficient also for SS interactions between different spins). Here, to give a flavor of the computation, we present the lowest order spin contributions (necessarily SO) to the general mass and current source multipole moments $(\forall \ell \in \mathbb{N})$ :

$$
\mathrm{I}_{L}^{\mathrm{SO}}=\frac{2 \ell \nu}{c^{3}(\ell+1)}\left\{\ell\left[\sigma_{\ell}(\nu)(\boldsymbol{v} \times \boldsymbol{S})^{\left\langle i_{\ell}\right.}-\sigma_{\ell+1}(\nu)(\boldsymbol{v} \times \boldsymbol{\Sigma})^{\left\langle i_{\ell}\right.}\right] x^{L-1\rangle}\right.
$$

${ }^{86}$ In this section we can neglect the gauge multipole moments $\mathrm{W}_{L}, \cdots, \mathrm{Z}_{L}$. 


$$
\begin{gathered}
\left.-(\ell-1)\left[\sigma_{\ell}(\nu)(\boldsymbol{x} \times \boldsymbol{S})^{\left\langle i_{\ell}\right.}-\sigma_{\ell+1}(\nu)(\boldsymbol{x} \times \boldsymbol{\Sigma})^{\left\langle i_{\ell}\right.}\right] v^{i_{\ell-1}} x^{L-2\rangle}\right\}+\mathcal{O}\left(\frac{1}{c^{5}}\right), \\
\mathrm{J}_{L}^{\mathrm{SO}}=\frac{(\ell+1) \nu}{2 c}\left[\sigma_{\ell-1}(\nu) S^{\left\langle i_{\ell}\right.}-\sigma_{\ell}(\nu) \Sigma^{\left\langle i_{\ell}\right.}\right] x^{L-1\rangle}+\mathcal{O}\left(\frac{1}{c^{3}}\right) .
\end{gathered}
$$

Paralleling the similar expressions (304) for the Newtonian approximation to the source moments in the non-spin case, we posed $\sigma_{\ell}(\nu) \equiv X_{2}^{\ell-1}+(-)^{\ell} X_{1}^{\ell-1}$ with $X_{\mathrm{a}}=m_{\mathrm{a}} / m$ [see also Eqs. (305)]. In Eqs. (413) we employ the notation (389) for the two spins and the ordinary cross product $\times$ of Euclidean vectors. Thus, the dominant level of spins is at the $1.5 \mathrm{PN}$ order in the mass-type moments $\mathrm{I}_{L}$, but only at the $0.5 \mathrm{PN}$ order in the current-type moments $\mathrm{J}_{L}$. It is then evident that the spin part of the current-type moments will always dominate over that of the mass-type moments. We refer to $[53,89]$ for higher order post-Newtonian expressions of the source moments. If we insert the expressions (413) into tail integrals like (76), we find that some spin contributions originate from tails starting at the $3 \mathrm{PN}$ order [54].

Finally, skipping details, we are left with the following highest-order known result for the SO contributions to the gravitational wave energy flux, which is currently 4PN order [53, 89, 306]:87

$$
\begin{aligned}
\mathcal{F}_{\mathrm{SO}}= & \frac{32 c^{5}}{5} \frac{\nu^{2} x^{13 / 2}}{G^{2} m^{2}}\left\{-4 S_{\ell}-\frac{5}{4} \Delta \Sigma_{\ell}\right. \\
+ & \left.+\left(-\frac{9}{2}+\frac{272}{9} \nu\right) S_{\ell}+\left(-\frac{13}{16}+\frac{43}{4} \nu\right) \Delta \Sigma_{\ell}\right] \\
& +x^{3 / 2}\left[-16 \pi S_{\ell}-\frac{31 \pi}{6} \Delta \Sigma_{\ell}\right] \\
& +x^{2}\left[\left(\frac{476645}{6804}+\frac{6172}{189} \nu-\frac{2810}{27} \nu^{2}\right) S_{\ell}+\left(\frac{9535}{336}+\frac{1849}{126} \nu-\frac{1501}{36} \nu^{2}\right) \Delta \Sigma_{\ell}\right] \\
& \left.+x^{5 / 2}\left[\left(-\frac{3485 \pi}{96}+\frac{13879 \pi}{72} \nu\right) S_{\ell}+\left(-\frac{7163 \pi}{672}+\frac{130583 \pi}{2016} \nu\right) \Delta \Sigma_{\ell}\right]+\mathcal{O}\left(\frac{1}{c^{6}}\right)\right\} .
\end{aligned}
$$

We recall that $S_{\ell} \equiv \boldsymbol{\ell} \cdot \mathbf{S}$ and $\Sigma_{\ell} \equiv \boldsymbol{\ell} \cdot \boldsymbol{\Sigma}$, with $\mathbf{S}$ and $\boldsymbol{\Sigma}$ denoting the combinations (389), and the individual spins are the specific conserved-norm spins that have been introduced in Section 11.1. The result (414) superposes to the non-spin contributions given by Eq. (314). Satisfyingly it is in complete agreement in the test-mass limit where $\nu \rightarrow 0$ with the result of black-hole perturbation theory on a Kerr background obtained in Ref. [396].

Finally we can compute the spin effects in the time evolution of the binary's orbital frequency $\Omega$. We rely as in Section 9 on the equation (295) balancing the total emitted energy flux $\mathcal{F}$ with the variation of the binary's center-of-mass energy $E$. The non-spin contributions in $E$ have been provided for quasi-circular binaries in Eq. (232); the SO contributions to next-to-next-to-leading order are given by [307, 90]

$$
\begin{aligned}
E_{\mathrm{SO}} & =-\frac{c^{2}}{2} \frac{\nu x^{5 / 2}}{G m}\left\{\frac{14}{3} S_{\ell}+2 \Delta \Sigma_{\ell}+x\left[\left(11-\frac{61}{9} \nu\right) S_{\ell}+\Delta\left(3-\frac{10}{3} \nu\right) \Sigma_{\ell}\right]\right. \\
& \left.+x^{2}\left[\left(\frac{135}{4}-\frac{367}{4} \nu+\frac{29}{12} \nu^{2}\right) S_{\ell}+\Delta\left(\frac{27}{4}-39 \nu+\frac{5}{4} \nu^{2}\right) \Sigma_{\ell}\right]\right\}+\mathcal{O}\left(\frac{1}{c^{8}}\right) .
\end{aligned}
$$

Using $E$ and $\mathcal{F}$ expressed as functions of the orbital frequency $\Omega$ (through $x$ ) and of the spin variables (through $S_{\ell}$ and $\Sigma_{\ell}$ ), we transform the balance equation into

$$
\dot{\Omega}_{\mathrm{SO}}=-\left(\frac{\mathcal{F}}{\mathrm{d} E / \mathrm{d} \Omega}\right)_{\mathrm{SO}} .
$$

87 Notice that the spin-orbit contributions due to the absorption by the black-hole horizons have to be added to this post-Newtonian result [349, 392, 5, 125]. 
However, in writing the latter equation it is important to justify that the spin quantities $S_{\ell}$ and $\Sigma_{\ell}$ are secularly constant, i.e., do not evolve on a gravitational radiation reaction time scale so we can neglect their variations when taking the time derivative of Eq. (415). Fortunately, this is the case of the conserved-norm spin variables, as proved in Ref. [423] up to relative 1PN order, i.e., considering radiation reaction effects up to 3.5PN order. Furthermore this can also be shown from the following structural general argument valid at linear order in spins $[54,89]$. In the centerof-mass frame, the only vectors at our disposal, except for the spins, are $\boldsymbol{n}$ and $\boldsymbol{v}$. Recalling that the spin vectors are pseudovectors regarding parity transformation, we see that the only way SO contributions can enter scalars such as the energy $E$ or the flux $\mathcal{F}$ is through the mixed products $\left(n, v, S_{\mathrm{a}}\right)$, i.e., through the components $S_{\ell}^{\mathrm{a}}$. Now, the same reasoning applies to the precession vectors $\boldsymbol{\Omega}_{\mathrm{a}}$ in Eqs. (388): They must be pseudovectors, and, at linear order in spin, they must only depend on $\boldsymbol{n}$ and $\boldsymbol{v}$; so that they must be proportional to $\boldsymbol{\ell}$, as can be explicitly seen for instance in Eq. (394). Now, the time derivative of the components along $\boldsymbol{\ell}$ of the spins are given by $\mathrm{d} S_{\ell}^{\mathrm{a}} / \mathrm{d} t=\mathbf{S}_{\mathrm{a}} \cdot\left(\mathrm{d} \boldsymbol{\ell} / \mathrm{d} t+\boldsymbol{\ell} \times \boldsymbol{\Omega}_{\mathrm{a}}\right)$. The second term vanishes because $\boldsymbol{\Omega}_{\mathrm{a}} \propto \boldsymbol{\ell}$, and since $\mathrm{d} \boldsymbol{\ell} / \mathrm{d} t=\mathcal{O}(S)$, we obtain that $S_{\ell}^{\mathrm{a}}$ is constant at linear order in the spins. We have already met an instance of this important fact in Eq. (409c). This argument is valid at any post-Newtonian order and for general orbits, but is limited to spin-orbit terms; furthermore it does not specify any time scale for the variation, so it applies to short time scales such as the orbital and precessional periods, as well as to the long gravitational radiation reaction time scale (see also Ref. [218] and references therein for related discussions).

Table 4: Spin-orbit contributions to the number of gravitational-wave cycles $\mathcal{N}_{\text {cycle }}$ [defined by Eq. (319)] for binaries detectable by ground-based detectors LIGO-VIRGO. The entry frequency is $f_{\text {seismic }}=10 \mathrm{~Hz}$ and the terminal frequency is $f_{\mathrm{ISCO}}=\frac{c^{3}}{6^{3 / 2} \pi G m}$. For each compact object the magnitude $\chi_{\mathrm{a}}$ and the orientation $\kappa_{\mathrm{a}}$ of the spin are defined by $\boldsymbol{S}_{\mathrm{a}}=G m_{\mathrm{a}}^{2} \chi_{\mathrm{a}} \hat{\boldsymbol{S}}_{\mathrm{a}}$ and $\kappa_{\mathrm{a}}=\hat{\boldsymbol{S}}_{\mathrm{a}} \cdot \boldsymbol{\ell}$; remind Eq. (366). The spin-spin (SS) terms are neglected.

\begin{tabular}{ll|ccc}
\hline PN order & & $1.4 M_{\odot}+1.4 M_{\odot}$ & $10 M_{\odot}+1.4 M_{\odot}$ & $10 M_{\odot}+10 M_{\odot}$ \\
\hline $1.5 \mathrm{PN}$ & (leading SO) & $65.6 \kappa_{1} \chi_{1}+65.6 \kappa_{2} \chi_{2}$ & $114.0 \kappa_{1} \chi_{1}+11.7 \kappa_{2} \chi_{2}$ & $16.0 \kappa_{1} \chi_{1}+16.0 \kappa_{2} \chi_{2}$ \\
$2.5 \mathrm{PN}$ & $(1 \mathrm{PN} \mathrm{SO})$ & $9.3 \kappa_{1} \chi_{1}+9.3 \kappa_{2} \chi_{2}$ & $33.8 \kappa_{1} \chi_{1}+2.9 \kappa_{2} \chi_{2}$ & $5.7 \kappa_{1} \chi_{1}+5.7 \kappa_{2} \chi_{2}$ \\
$3 \mathrm{PN}$ & (leading SO-tail) & $-3.2 \kappa_{1} \chi_{1}-3.2 \kappa_{2} \chi_{2}$ & $-13.2 \kappa_{1} \chi_{1}-1.3 \kappa_{2} \chi_{2}$ & $-2.6 \kappa_{1} \chi_{1}-2.6 \kappa_{2} \chi_{2}$ \\
$3.5 \mathrm{PN}$ & $(2 \mathrm{PN} \mathrm{SO})$ & $1.9 \kappa_{1} \chi_{1}+1.9 \kappa_{2} \chi_{2}$ & $11.1 \kappa_{1} \chi_{1}+0.8 \kappa_{2} \chi_{2}$ & $1.7 \kappa_{1} \chi_{1}+1.7 \kappa_{2} \chi_{2}$ \\
$4 \mathrm{PN}$ & $(1 \mathrm{PN} \mathrm{SO}-$ tail $)$ & $-1.5 \kappa_{1} \chi_{1}-1.5 \kappa_{2} \chi_{2}$ & $-8.0 \kappa_{1} \chi_{1}-0.7 \kappa_{2} \chi_{2}$ & $-1.5 \kappa_{1} \chi_{1}-1.5 \kappa_{2} \chi_{2}$ \\
\hline
\end{tabular}

We are then allowed to apply Eq. (416) with conserved-norm spin variables at the SO level. We thus obtain the secular evolution of $\Omega$ and from that we deduce by a further integration (following the Taylor approximant T2) the secular evolution of the carrier phase $\phi \equiv \int \Omega \mathrm{d} t$ :

$$
\begin{aligned}
\phi_{\mathrm{SO}}=- & \frac{x^{-1}}{32 G m^{2} \nu}\left\{\frac{235}{6} S_{\ell}+\frac{125}{8} \Delta \Sigma_{\ell}\right. \\
& +x \ln x\left[\left(-\frac{554345}{2016}-\frac{55}{8} \nu\right) S_{\ell}+\left(-\frac{41745}{448}+\frac{15}{8} \nu\right) \Delta \Sigma_{\ell}\right] \\
+ & x^{3 / 2}\left[\frac{940 \pi}{3} S_{\ell}+\frac{745 \pi}{6} \Delta \Sigma_{\ell}\right] \\
+ & x^{2}\left[\left(-\frac{8980424995}{6096384}+\frac{6586595}{6048} \nu-\frac{305}{288} \nu^{2}\right) S_{\ell}\right. \\
& \left.+\left(-\frac{170978035}{387072}+\frac{2876425}{5376} \nu+\frac{4735}{1152} \nu^{2}\right) \Delta \Sigma_{\ell}\right]
\end{aligned}
$$




$$
\left.+x^{5 / 2}\left[\left(\frac{2388425 \pi}{3024}-\frac{9925 \pi}{36} \nu\right) S_{\ell}+\left(\frac{3237995 \pi}{12096}-\frac{258245 \pi}{2016} \nu\right) \Delta \Sigma_{\ell}\right]+\mathcal{O}\left(\frac{1}{c^{6}}\right)\right\}
$$

This expression, when added to the expression for the non-spin terms given by Eq. (318), and considering also the SS terms, constitutes the main theoretical input needed for the construction of templates for spinning compact binaries. However, recall that in the case of precessional binaries, for which the spins are not aligned or anti-aligned with the orbital angular momentum, we must subtract to the carrier phase $\phi$ the precessional correction $\alpha$ arising from the precession of the orbital plane. Indeed the physical phase variable $\Phi$ which is defined in Figure 4 , has been proved to be given by $\Phi=\phi-\alpha$ at linear order in spins, cf. Eq. (405). The precessional correction $\alpha$ can be computed at linear order in spins from the results of Section 11.2.

As we have done in Table 3 for the non-spin terms, we evaluate in Table 4 the SO contributions to the number of gravitational-wave cycles accumulated in the bandwidth of LIGO-VIRGO detectors, Eq. (319). The results show that the SO terms up to 4PN order can be numerically important, for spins close to maximal and for suitable orientations. They can even be larger than the corresponding non-spin contributions at $3.5 \mathrm{PN}$ order and perhaps at $4 \mathrm{PN}$ order (but recall that the non-spin terms at 4PN order are not known); compare with Table 3. We thus conclude that the SO terms are relevant to be included in the gravitational wave templates for an accurate extraction of the binary parameters. Although numerically smaller, the SS terms are also relevant; for these we refer to the literature quoted at the beginning of Section 11.

\section{Acknowledgments}

The author's works reviewed in this article have been done in a series of collaborations with K.G. Arun, Alejandro Bohé, Alessandra Buonanno, Thibault Damour, Steven Detweiler, Gilles EspositoFarèse, Guillaume Faye, Bala R. Iyer, Alexandre Le Tiec, Sylvain Marsat, Samaya Nissanke, Olivier Poujade, B.S. Sathyaprakash, Gerhard Schäfer, Bernard F. Whiting, Clifford M. Will, and Alan G. Wiseman. 


\section{References}

[1] Abbott, B. P. et al. (LIGO Scientific Collaboration and Virgo Collaboration), "Observation of Gravitational Waves from a Binary Black Hole Merger", Phys. Rev. Lett., 116, 061102 (2016). [DOI], [ADS], [arXiv:1602.03837 [gr-qc]]. (Cited on page 7.)

[2] Abramowicz, M. A. and Kluźniak, W., "A precise determination of black hole spin in GRO J1655-40", Astron. Astrophys., 374, L19-L20 (2001). [DOI], [ADS], [astro-ph/0105077]. (Cited on page 146.)

[3] Ajith, P., Iyer, B. R., Robinson, C. A. K. and Sathyaprakash, B. S., "New class of post-Newtonian approximants to the waveform templates of inspiralling compact binaries: Test mass in the Schwarzschild spacetime", Phys. Rev. D, 71, 044029 (2005). [gr-qc/0412033]. (Cited on pages 65 and 131.)

[4] Ajith, P. et al., "Template bank for gravitational waveforms from coalescing binary black holes: Nonspinning binaries", Phys. Rev. D, 77, 104017 (2008). [DOI], [ADS], [arXiv:0710.2335 [gr-qc]]. Erratum: Phys. Rev. D, 79, 129901(E) (2009). (Cited on page 65.)

[5] Alvi, K., "Energy and angular momentum flow into a black hole in a binary", Phys. Rev. D, 64, 104020 (2001). [DOI], [arXiv:0107080 [gr-qc]]. (Cited on page 158.)

[6] Anderson, J. L. and DeCanio, T. C., "Equations of hydrodynamics in general relativity in the slow motion approximation", Gen. Relativ. Gravit., 6, 197-237 (1975). [DOI]. (Cited on pages 53, 57, and 58.)

[7] Anderson, J. L., Kates, R. E., Kegeles, L. S. and Madonna, R. G., "Divergent integrals of postNewtonian gravity: Nonanalytic terms in the near-zone expansion of a gravitationally radiating system found by matching", Phys. Rev. D, 25, 2038-2048 (1982). [DOI], [ADS]. (Cited on pages 11, 44, and 46.)

[8] Apostolatos, T. A., Cutler, C., Sussman, G. J. and Thorne, K. S., "Spin induced orbital precession and its modulation of the gravitational wave forms from merging binaries", Phys. Rev. D, 49, 62746297 (1994). [DOI]. (Cited on pages 21 and 146.)

[9] Arun, K. G., Blanchet, L., Iyer, B. R. and Qusailah, M. S., "Inspiralling compact binaries in quasi-elliptical orbits: The complete 3PN energy flux", Phys. Rev. D, 77, 064035 (2008). [DOI], [arXiv:0711.0302]. (Cited on pages 20, 92, 138, 140, 141, and 142.)

[10] Arun, K. G., Blanchet, L., Iyer, B. R. and Qusailah, M. S., "Tail effects in the 3PN gravitational wave energy flux of compact binaries in quasi-elliptical orbits", Phys. Rev. D, 77, 064034 (2008). [DOI], [arXiv:0711.0250]. (Cited on pages 20, 141, 143, and 144.)

[11] Arun, K. G., Blanchet, L., Iyer, B. R. and Qusailah, M. S. S., "The 2.5PN gravitational wave polarizations from inspiralling compact binaries in circular orbits", Class. Quantum Grav., 21, 37713801 (2004). [DOI], [gr-qc/0404185]. Erratum: Class. Quantum Grav., 22, 3115 (2005). (Cited on pages $20,21,117,125,126$, and 130.)

[12] Arun, K. G., Blanchet, L., Iyer, B. R. and Sinha, S., "Third post-Newtonian angular momentum flux and the secular evolution of orbital elements for inspiralling compact binaries in quasi-elliptical orbits", Phys. Rev. D, 80, 124018 (2009). [DOI], [arXiv:0908.3854]. (Cited on pages 20, 141, and 145.)

[13] Arun, K. G., Buonanno, A., Faye, G. and Ochsner, E., "Higher-order spin effects in the amplitude and phase of gravitational waveforms emitted by inspiraling compact binaries: Ready-to-use gravitational waveforms", Phys. Rev. D, 79, 104023 (2009). [DOI], [arXiv:0810.5336]. (Cited on page 155.)

[14] Arun, K. G., Iyer, B. R., Qusailah, M. S. S. and Sathyaprakash, B. S., "Probing the non-linear structure of general relativity with black hole binaries", Phys. Rev. D, 74, 024006 (2006). [DOI], [gr-qc/0604067]. (Cited on pages 16 and 131.) 
[15] Arun, K. G., Iyer, B. R., Qusailah, M. S. S. and Sathyaprakash, B. S., "Testing post-Newtonian theory with gravitational wave observations", Class. Quantum Grav., 23, L37-L43 (2006). [DOI], [ADS], [arXiv:gr-qc/0604018]. (Cited on pages 16 and 131.)

[16] Arun, K. G., Iyer, B. R., Sathyaprakash, B. S. and Sinha, S., "Higher harmonics increase LISA's mass reach for supermassive black holes", Phys. Rev. D, 75, 124002 (2007). [DOI], [arXiv:0704.1086]. (Cited on page 20.)

[17] Arun, K. G., Iyer, B. R., Sathyaprakash, B. S., Sinha, S. and Van Den Broeck, C., "Higher signal harmonics, LISA's angular resolution, and dark energy", Phys. Rev. D, 76, 104016 (2007). [DOI], [ADS], [arXiv:0707.3920]. (Cited on page 20.)

[18] Arun, K. G., Iyer, B. R., Sathyaprakash, B. S. and Sundararajan, P. A., "Parameter estimation of inspiralling compact binaries using 3.5 post-Newtonian gravitational wave phasing: The nonspinning case", Phys. Rev. D, 71, 084008 (2005). [DOI], [gr-qc/0411146]. (Cited on pages 65 and 131.)

[19] Bailey, I. and Israel, W., "Lagrangian dynamics of spinning particles and polarized media in general relativity", Commun. Math. Phys., 42, 65 (1975). [DOI]. (Cited on pages 148 and 149.)

[20] Baker, J. G., Centrella, J., Choi, D.-I., Koppitz, M., van Meter, J. and Miller, M. C., "Getting a kick out of numerical relativity", Astrophys. J., 653, L93-L96 (2006). [DOI], [astro-ph/0603204]. (Cited on page 22.)

[21] Baker, J. G., Centrella, J., Choi, D.-I., Koppitz, M. and van Meter, J. R., "Gravitational-Wave Extraction from an Inspiraling Configuration of Merging Black Holes", Phys. Rev. Lett., 96, 111102 (2006). [DOI], [ADS], [arXiv:gr-qc/0511103]. (Cited on page 8.)

[22] Barack, L., "Gravitational self-force in extreme mass-ratio inspirals", Class. Quantum Grav., 26, 213001 (2009). [DOI], [ADS], [arXiv:0908.1664 [gr-qc]]. (Cited on page 112.)

[23] Barack, L., "Computational Methods for the Self-Force in Black Hole Spacetimes", in Blanchet, L., Spallicci, A. and Whiting, B., eds., Mass and Motion in General Relativity, Lectures from the CNRS School on Mass held in Orléans, France, 23-25 June 2008, Fundamental Theories of Physics, 162, pp. 327-366, (Springer, Dordrecht; New York, 2011). [DOI], [ADS]. (Cited on pages 9 and 112.)

[24] Barack, L. and Sago, N., "Gravitational self-force correction to the innermost stable circular orbit of a Schwarzschild black hole", Phys. Rev. Lett., 102, 191101 (2009). [DOI], [arXiv:0902.0573]. (Cited on page 108.)

[25] Barausse, E., Racine, E. and Buonanno, A., "Hamiltonian of a spinning test particle in curved spacetime", Phys. Rev. D, 80, 104025 (2009). [DOI], [arXiv:0907.4745 [gr-qc]]. (Cited on page 148.)

[26] Bardeen, J. M., Carter, B. and Hawking, S. W., "The Four Laws of Black Hole Mechanics", Commun. Math. Phys., 31, 161-170 (1973). [DOI], [ADS]. (Cited on page 110.)

[27] Barker, B. M. and O'Connell, R. F., "Gravitational two-body problem with arbitrary masses, spins, and quadrupole moments", Phys. Rev. D, 12, 329-335 (1975). [DOI]. (Cited on pages 19, 102, and 147.)

[28] Barker, B. M. and O'Connell, R. F., "The Gravitational Interaction: Spin, Rotation, and Quantum Effects - A Review", Gen. Relativ. Gravit., 11, 149-175 (1979). [DOI]. (Cited on pages 19, 102, and 147.)

[29] Baumgarte, T. W., "Innermost stable circular orbit of binary black holes", Phys. Rev. D, 62, 024018 (2000). [DOI], [ADS]. (Cited on page 103.)

[30] Bekenstein, J. D., "Gravitational Radiation Recoil and Runaway Black Holes", Astrophys. J., 183, 657-664 (1973). [DOI], [ADS]. (Cited on pages 10 and 22.) 
[31] Bel, L., Damour, T., Deruelle, N., Ibáñez, J. and Martin, J., "Poincaré-Invariant Gravitational Field and Equations of Motion of two Pointlike Objects: The Postlinear Approximation of General Relativity", Gen. Relativ. Gravit., 13, 963-1004 (1981). [DOI]. (Cited on page 17.)

[32] Benacquista, M. J. and Downing, J. M. B., "Relativistic Binaries in Globular Clusters", Living Rev. Relativity, 16, lrr-2013-4 (2013). [DOI], [ADS], [arXiv:1110.4423]. URL (accessed 6 October 2013): http://www.livingreviews.org/lrr-2013-4. (Cited on page 135.)

[33] Bernard, L., Blanchet, L., Bohé, A., Faye, G. and Marsat, S., "Fokker action of non-spinning compact binaries at the fourth post-Newtonian approximation", arXiv, e-print, (2015). [arXiv:1512.02876 [grqc]]. (Cited on page 19.)

[34] Berti, E., Cardoso, V., Gonzalez, J. A., Sperhake, U., Hannam, M., Husa, S. and Brügmann, B., "Inspiral, merger, and ringdown of unequal mass black hole binaries: A multipolar analysis", Phys. Rev. D, 76, 064034 (2007). [DOI], [ADS], [arXiv:gr-qc/0703053]. (Cited on page 36.)

[35] Bertotti, B. and Plebański, J. F., "Theory of gravitational perturbations in the fast motion approximation", Ann. Phys. (N.Y.), 11, 169-200 (1960). [DOI]. (Cited on page 18.)

[36] Bini, D. and Damour, T., "Analytical determination of the two-body gravitational interaction potential at the fourth post-Newtonian approximation", Phys. Rev. D, 87, 121501 (2013). [DOI], [ADS], [arXiv:1305.4884 [gr-qc]]. (Cited on pages 19, 96, and 114.)

[37] Bini, D. and Damour, T., "Analytic determination of the eight-and-a-half post-Newtonian self-force contributions to the two-body gravitational interaction potential", Phys. Rev. D, 89, 104047 (2014). [DOI], [arXiv:1403.2366 [gr-qc]]. (Cited on page 116.)

[38] Bini, D. and Damour, T., "High-order post-Newtonian contributions to the two-body gravitational interaction potential from analytical gravitational self-force calculations", Phys. Rev. D, 89, 064063 (2014). [DOI], [arXiv:1312.2503 [gr-qc]]. (Cited on page 116.)

[39] Bini, D., Damour, T. and Geralico, A., "Confirming and improving post-Newtonian and effectiveone-body results from self-force computations along eccentric orbits around a Schwarzschild black hole", arXiv, e-print, (2015). [arXiv:1511.04533 [gr-qc]]. (Cited on page 19.)

[40] Blaes, O., Lee, M. H. and Socrates, A., "The Kozai Mechanism and the Evolution of Binary Supermassive Black Holes", Astrophys. J., 578, 775-786 (2002). [DOI], [ADS], [astro-ph/0203370]. (Cited on page 135.)

[41] Blanchet, L., "Radiative gravitational fields in general-relativity. II. Asymptotic-behaviour at future null infinity", Proc. R. Soc. London, Ser. A, 409, 383-399 (1987). [DOI]. (Cited on pages 10, 11, 33 , and 35.)

[42] Blanchet, L., Contribution à l'étude du rayonnement gravitationnel émis par un système isolé, Habil. thesis, (Université Paris VI, Paris, 1990). (Cited on pages 21 and 43.)

[43] Blanchet, L., "Time asymmetric structure of gravitational radiation", Phys. Rev. D, 47, 4392-4420 (1993). [DOI]. (Cited on pages 11, 18, 19, 46, 57, 58, 63, and 117.)

[44] Blanchet, L., "Second-post-Newtonian generation of gravitational radiation", Phys. Rev. D, 51, 2559-2583 (1995). [DOI], [gr-qc/9501030]. (Cited on pages 11, 12, 20, 37, 46, 48, 51, and 64.)

[45] Blanchet, L., "Energy losses by gravitational radiation in inspiralling compact binaries to five halves post-Newtonian order", Phys. Rev. D, 54, 1417-1438 (1996). [DOI], [gr-qc/9603048]. Erratum: Phys. Rev. D, 71, 129904(E) (2005). (Cited on pages 20 and 91.)

[46] Blanchet, L., "Gravitational radiation from relativistic sources", in Marck, J.-A. and Lasota, J.-P., eds., Relativistic Gravitation and Gravitational Radiation, Proceedings of the Les Houches School of Physics, held in Les Houches, Haute Savoie, 26 September-6 October, 1995, Cambridge Contemporary Astrophysics, pp. 33-66, (Cambridge University Press, Cambridge, 1997). [gr-qc/9609049], [Google Books]. (Cited on page 9.) 
[47] Blanchet, L., "Gravitational radiation reaction and balance equations to post-Newtonian order", Phys. Rev. D, 55, 714-732 (1997). [DOI], [gr-qc/9609049]. (Cited on pages 11, 18, 63, 64, and 117.)

[48] Blanchet, L., "Gravitational-wave tails of tails", Class. Quantum Grav., 15, 113-141 (1998). [DOI], [gr-qc/9710038]. Erratum: Class. Quantum Grav., 22, 3381 (2005). (Cited on pages 11, 20, 39, 40, 43, and 44.)

[49] Blanchet, L., "On the multipole expansion of the gravitational field", Class. Quantum Grav., 15, 1971-1999 (1998). [DOI], [gr-qc/9801101]. (Cited on pages 11, 12, 46, 48, and 51.)

[50] Blanchet, L., "Quadrupole-quadrupole gravitational waves", Class. Quantum Grav., 15, 89-111 (1998). [DOI], [gr-qc/9710037]. (Cited on pages 11, 21, 39, and 43.)

[51] Blanchet, L., "Innermost circular orbit of binary black holes at the third post-Newtonian approximation", Phys. Rev. D, 65, 124009 (2002). [DOI], [gr-qc/0112056]. (Cited on pages 101, 103, and 110.)

[52] Blanchet, L., "Post-Newtonian theory and the two-body problem", in Blanchet, L., Spallicci, A. and Whiting, B., eds., Mass and Motion in General Relativity, Lectures from the CNRS School on Mass held in Orléans, France, 23-25 June 2008, Fundamental Theories of Physics, pp. 125-166, (Springer, Dordrecht; New York, 2011). [DOI], [ADS], [arXiv:0907.3596 [gr-qc]]. (Cited on page 9.)

[53] Blanchet, L., Buonanno, A. and Faye, G., "Higher-order spin effects in the dynamics of compact binaries II. Radiation field", Phys. Rev. D, 74, 104034 (2006). [DOI], [gr-qc/0605140]. Erratum: Phys. Rev. D, 75, 049903 (2007). (Cited on pages 21, 147, and 158.)

[54] Blanchet, L., Buonanno, A. and Faye, G., "Tail-induced spin-orbit effect in the gravitational radiation of compact binaries", Phys. Rev. D, 84, 064041 (2011). [DOI], [arXiv:1104.5659 [gr-qc]]. (Cited on pages $21,147,154,158$, and 159.)

[55] Blanchet, L., Buonanno, A. and Le Tiec, A., "First law of mechanics for black hole binaries with spins", Phys. Rev. D, 87, 024030 (2013). [DOI], [arXiv:1211.1060 [gr-qc]]. (Cited on pages 102, 103, and 111.)

[56] Blanchet, L. and Damour, T., "Multipolar radiation reaction in general relativity", Phys. Lett. A, 104, 82-86 (1984). [DOI]. (Cited on page 63.)

[57] Blanchet, L. and Damour, T., "Radiative gravitational fields in general relativity I. General structure of the field outside the source", Philos. Trans. R. Soc. London, Ser. A, 320, 379-430 (1986). [DOI]. (Cited on pages 10, 11, 20, 26, 27, 29, 31, and 33.)

[58] Blanchet, L. and Damour, T., "Tail-transported temporal correlations in the dynamics of a gravitating system", Phys. Rev. D, 37, 1410-1435 (1988). [DOI]. (Cited on pages 11, 19, 43, 44, 46, 57, 58, 59, and 63.)

[59] Blanchet, L. and Damour, T., "Post-Newtonian generation of gravitational waves", Ann. Inst. Henri Poincare A, 50, 377-408 (1989). (Cited on pages 11, 37, 46, and 49.)

[60] Blanchet, L. and Damour, T., "Hereditary effects in gravitational radiation", Phys. Rev. D, 46, 4304-4319 (1992). [DOI]. (Cited on pages 11, 20, 21, 39, 43, 57, and 122.)

[61] Blanchet, L., Damour, T. and Esposito-Farèse, G., "Dimensional regularization of the third postNewtonian dynamics of point particles in harmonic coordinates", Phys. Rev. D, 69, 124007 (2004). [DOI], [gr-qc/0311052]. (Cited on pages 18, 70, 72, 74, and 75.)

[62] Blanchet, L., Damour, T., Esposito-Farèse, G. and Iyer, B. R., "Gravitational radiation from inspiralling compact binaries completed at the third post-Newtonian order", Phys. Rev. Lett., 93, 091101 (2004). [DOI], [gr-qc/0406012]. (Cited on pages 20, 71, 77, 78, and 117.) 
[63] Blanchet, L., Damour, T., Esposito-Farèse, G. and Iyer, B. R., "Dimensional regularization of the third post-Newtonian gravitational wave generation of two point masses", Phys. Rev. D, 71, 124004 (2005). [DOI], [ADS], [gr-qc/0503044]. (Cited on pages 20, 71, 77, 78, and 117.)

[64] Blanchet, L., Damour, T. and Iyer, B. R., "Gravitational waves from inspiralling compact binaries: Energy loss and wave form to second post-Newtonian order", Phys. Rev. D, 51, 5360-5386 (1995). [DOI], [gr-qc/9501029]. (Cited on pages 20, 97, and 117.)

[65] Blanchet, L., Damour, T. and Iyer, B. R., "Surface-integral expressions for the multipole moments of post-Newtonian sources and the boosted Schwarzschild solution", Class. Quantum Grav., 22, 155 (2005). [DOI], [gr-qc/0410021]. (Cited on pages 53 and 71.)

[66] Blanchet, L., Damour, T., Iyer, B. R., Will, C. M. and Wiseman, A. G., "Gravitational-Radiation Damping of Compact Binary Systems to Second Post-Newtonian Order", Phys. Rev. Lett., 74, 35153518 (1995). [DOI], [gr-qc/9501027]. (Cited on page 20.)

[67] Blanchet, L., Detweiler, S., Le Tiec, A. and Whiting, B. F., "Higher-order Post-Newtonian fit of the gravitational self-force for circular orbits in the Schwarzschild geometry", Phys. Rev. D, 81, 084033 (2010). [DOI], [ADS], [arXiv:1002.0726 [gr-qc]]. (Cited on pages 95, 112, 113, 114, and 115.)

[68] Blanchet, L., Detweiler, S., Le Tiec, A. and Whiting, B. F., "Post-Newtonian and numerical calculations of the gravitational self-force for circular orbits in the Schwarzschild geometry", Phys. Rev. D, 81, 064004 (2010). [DOI], [ADS], [arXiv:0910.0207 [gr-qc]]. (Cited on pages 60, 112, 113, 114, and 115.)

[69] Blanchet, L. and Faye, G., "Equations of motion of point-particle binaries at the third postNewtonian order", Phys. Lett. A, 271, 58-64 (2000). [DOI], [gr-qc/0004009]. (Cited on pages 17, $68,69,70,71,74,77,82$, and 95.)

[70] Blanchet, L. and Faye, G., "Hadamard regularization", J. Math. Phys., 41, 7675-7714 (2000). [DOI], [gr-qc/0004008]. (Cited on pages 17, 66, 67, 68, 69, 74, and 75.)

[71] Blanchet, L. and Faye, G., "General relativistic dynamics of compact binaries at the third postNewtonian order", Phys. Rev. D, 63, 062005 (2001). [DOI], [gr-qc/0007051]. (Cited on pages 17, $24,59,68,69,70,71,74,76,77$, and 82.)

[72] Blanchet, L. and Faye, G., "Lorentzian regularization and the problem of point-like particles in general relativity", J. Math. Phys., 42, 4391-4418 (2001). [DOI], [gr-qc/0006100]. (Cited on pages 17, $66,68,69$, and 75.$)$

[73] Blanchet, L., Faye, G., Iyer, B. R. and Joguet, B., "Gravitational-wave inspiral of compact binary systems to 7/2 post-Newtonian order", Phys. Rev. D, 65, 061501(R) (2002). [DOI], [gr-qc/0105099]. Erratum: Phys. Rev. D, 71, 129902(E) (2005). (Cited on pages 20 and 71.)

[74] Blanchet, L., Faye, G., Iyer, B. R. and Sinha, S., "The third post-Newtonian gravitational wave polarisations and associated spherical harmonic modes for inspiralling compact binaries in quasicircular orbits", Class. Quantum Grav., 25, 165003 (2008). [DOI], [arXiv:0802.1249]. (Cited on pages 11, 20, 31, 40,44,117, 118, 119, 125, and 132.)

[75] Blanchet, L., Faye, G. and Nissanke, S., "Structure of the post-Newtonian expansion in general relativity", Phys. Rev. D, 72, 044024 (2005). [DOI]. (Cited on pages 11, 54, 58, and 59.)

[76] Blanchet, L., Faye, G. and Ponsot, B., "Gravitational field and equations of motion of compact binaries to 5/2 post-Newtonian order", Phys. Rev. D, 58, 124002 (1998). [DOI], [gr-qc/9804079]. (Cited on pages 17, 68, 78, 97, and 112.)

[77] Blanchet, L., Faye, G. and Whiting, B. F., "Half-integral conservative post-Newtonian approximations in the redshift factor of black hole binaries", Phys. Rev. D, 89, 064026 (2014). [DOI], [arXiv:1312.2975 [gr-qc]]. (Cited on page 116.) 
[78] Blanchet, L., Faye, G. and Whiting, B. F., "High-order half-integral conservative post-Newtonian coefficients in the redshift factor of black hole binaries", Phys. Rev. D, 90, 044017 (2014). [DOI], [arXiv:1405.5151 [gr-qc]]. (Cited on page 116.)

[79] Blanchet, L. and Iyer, B. R., "Third post-Newtonian dynamics of compact binaries: Equations of motion in the center-of-mass frame", Class. Quantum Grav., 20, 755 (2003). [DOI], [gr-qc/0209089]. (Cited on pages 17, 90, 91, 94, 103, and 135.)

[80] Blanchet, L. and Iyer, B. R., "Hadamard regularization of the third post-Newtonian gravitational wave generation of two point masses", Phys. Rev. D, 71, 024004 (2005). [DOI], [gr-qc/0409094]. (Cited on pages 20, 71, 76, 77, 78, and 117.)

[81] Blanchet, L., Iyer, B. R. and Joguet, B., "Gravitational waves from inspiralling compact binaries: Energy flux to third post-Newtonian order", Phys. Rev. D, 65, 064005 (2002). [gr-qc/0105098]. Erratum: Phys. Rev. D, 71, 129903(E) (2005). (Cited on pages 20, 71, 76, 77, 78, 117, 118, 119, and 145.)

[82] Blanchet, L., Iyer, B. R., Will, C. M. and Wiseman, A. G., "Gravitational wave forms from inspiralling compact binaries to second-post-Newtonian order", Class. Quantum Grav., 13, 575-584 (1996). [DOI], [gr-qc/9602024]. (Cited on pages 20 and 125.)

[83] Blanchet, L., Qusailah, M. S. and Will, C. M., "Gravitational recoil of inspiraling black-hole binaries to second post-Newtonian order", Astrophys. J., 635, 508 (2005). [DOI], [astro-ph/0507692]. (Cited on page 22.)

[84] Blanchet, L. and Sathyaprakash, B. S., "Signal analysis of gravitational wave tails", Class. Quantum Grav., 11, 2807-2831 (1994). [DOI]. (Cited on pages 16 and 131.)

[85] Blanchet, L. and Sathyaprakash, B. S., "Detecting a Tail Effect in Gravitational-Wave Experiments", Phys. Rev. Lett., 74, 1067-1070 (1995). [DOI], [ADS]. (Cited on pages 16 and 131.)

[86] Blanchet, L. and Schäfer, G., "Higher order gravitational radiation losses in binary systems", Mon. Not. R. Astron. Soc., 239, 845-867 (1989). [DOI]. (Cited on pages 20, 117, 140, and 145.)

[87] Blanchet, L. and Schäfer, G., "Gravitational wave tails and binary star systems", Class. Quantum Grav., 10, 2699-2721 (1993). [DOI]. (Cited on pages 14, 20, 122, 126, 140, 144, and 145.)

[88] Bohé, A., Faye, G., Marsat, S. and Porter, E. K., "Quadratic-in-spin effects in the orbital dynamics and gravitational-wave energy flux of compact binaries at the 3PN order", Class. Quantum Grav., 32, 195010 (2015). [DOI], [arXiv:1501.01529 [gr-qc]]. (Cited on pages 21 and 147.)

[89] Bohé, A., Marsat, S. and Blanchet, L., "Next-to-next-to-leading order spin-orbit effects in the gravitational wave flux and orbital phasing of compact binaries", Class. Quantum Grav., 30, 135009 (2013). [arXiv:1303.7412]. (Cited on pages 21, 147, 158, and 159.)

[90] Bohé, A., Marsat, S., Faye, G. and Blanchet, L., "Next-to-next-to-leading order spin-orbit effects in the near-zone metric and precession equations of compact binary systems", Class. Quantum Grav., 30, 075017 (2013). [arXiv:1212.5520]. (Cited on pages 19, 147, 151, 153, 154, 156, and 158.)

[91] Bollini, C. G. and Giambiagi, J. J., "Lowest order 'divergent' graphs in v-dimensional space", Phys. Lett. B, 40, 566-568 (1972). [DOI]. (Cited on page 72.)

[92] Bonazzola, S., Gourgoulhon, E. and Marck, J.-A., "Numerical models of irrotational binary neutron stars in general relativity", Phys. Rev. Lett., 82, 892-895 (1999). [DOI], [ADS], [arXiv:gr-qc/9810072 [gr-qc]]. (Cited on page 101.)

[93] Bondi, H., van der Burg, M. G. J. and Metzner, A. W. K., "Gravitational Waves in General Relativity. VII. Waves from Axi-Symmetric Isolated Systems", Proc. R. Soc. London, Ser. A, 269, 21-52 (1962). [DOI], [ADS]. (Cited on pages 10,11,33, and 41.) 
[94] Bonnor, W. B., "Spherical gravitational waves", Philos. Trans. R. Soc. London, Ser. A, 251, 233-271 (1959). [DOI]. (Cited on pages 10 and 26.)

[95] Bonnor, W. B. and Rotenberg, M. A., "Transport of momentum by gravitational waves: Linear approximation", Proc. R. Soc. London, Ser. A, 265, 109-116 (1961). [DOI]. (Cited on pages 10 and 26.)

[96] Bonnor, W. B. and Rotenberg, M. A., "Gravitational waves from isolated sources", Proc. R. Soc. London, Ser. A, 289, 247-274 (1966). [DOI]. (Cited on pages 10 and 26.)

[97] Boyle, M., Brown, D. A., Kidder, L. E., Mroué, A. H., Pfeiffer, H. P., Scheel, M. A., Cook, G. B. and Teukolsky, S. A., "High-accuracy comparison of numerical relativity simulations with post-Newtonian expansions", Phys. Rev. D, 76, 124038 (2007). [DOI], [ADS], [arXiv:0710.0158 [gr-qc]]. (Cited on page 36.)

[98] Boyle, M., Buonanno, A., Kidder, L. E., Mroué, A. H., Pan, Y., Pfeiffer, H. P. and Scheel, M. A., "High-accuracy numerical simulation of black-hole binaries: Computation of the gravitational-wave energy flux and comparisons with post-Newtonian approximants", Phys. Rev. D, 78, 104020 (2008). [DOI], [arXiv:0804.4184 [gr-qc]]. (Cited on pages 36 and 124.)

[99] Braginsky, V. B. and Thorne, K. S., "Gravitational-wave bursts with memory and experimental prospects", Nature, 327, 123-125 (1987). [DOI]. (Cited on page 21.)

[100] Breitenlohner, P. and Maison, D., "Dimensional renormalization and the action principle", Commun. Math. Phys., 52, 11-38 (1977). [DOI]. (Cited on page 72.)

[101] Brenneman, L. W. and Reynolds, C. S., "Constraining Black Hole Spin via X-Ray Spectroscopy", Astrophys. J., 652, 1028-1043 (2006). [DOI], [ADS], [arXiv:astro-ph/0608502]. (Cited on page 146.)

[102] Brenneman, L. W. et al., "The Spin of the Supermassive Black Hole in NGC 3783", Astrophys. J., 736, 103 (2011). [DOI], [arXiv:1104.1172 [astro-ph.HE]]. (Cited on page 146.)

[103] Breuer, R. and Rudolph, E., "Radiation reaction and energy loss in the post-Newtonian approximation of general relativity", Gen. Relativ. Gravit., 13, 777 (1981). [DOI]. (Cited on page 13.)

[104] Bruhat, Y., "The Cauchy Problem", in Witten, L., ed., Gravitation: An Introduction to Current Research, pp. 130-168, (Wiley, New York; London, 1962). (Cited on page 7.)

[105] Buonanno, A., Chen, Y. and Vallisneri, M., "Detection template families for gravitational waves from the final stages of binary black-holes binaries: Nonspinning case", Phys. Rev. D, 67, 024016 (2003). [DOI], [gr-qc/0205122]. (Cited on pages 65, 71, 125, and 131.)

[106] Buonanno, A., Chen, Y. and Vallisneri, M., "Detection template families for precessing binaries of spinning compact binaries: Adiabatic limit", Phys. Rev. D, 67, 104025 (2003). [DOI], [grqc/0211087]. (Cited on pages 65, 71, 125, and 131.)

[107] Buonanno, A., Cook, G. B. and Pretorius, F., "Inspiral, merger, and ring-down of equal-mass blackhole binaries", Phys. Rev. D, 75, 124018 (2007). [DOI], [ADS], [gr-qc/0610122]. (Cited on page 36.)

[108] Buonanno, A. and Damour, T., "Effective one-body approach to general relativistic two-body dynamics", Phys. Rev. D, 59, 084006 (1999). [DOI], [ADS], [arXiv:gr-qc/9811091]. (Cited on page 65.)

[109] Buonanno, A. and Damour, T., "Transition from inspiral to plunge in binary black hole coalescences", Phys. Rev. D, 62, 064015 (2000). [DOI], [ADS], [arXiv:gr-qc/0001013]. (Cited on page 65.)

[110] Buonanno, A., Faye, G. and Hinderer, T., "Spin effects on gravitational waves from inspiralling compact binaries at second post-Newtonian order", Phys. Rev. D, 87, 044009 (2013). [DOI], [arXiv:1209.6349]. (Cited on page 147.) 
[111] Buonanno, A., Iyer, B. R., Pan, Y., Ochsner, E. and Sathyaprakash, B. S., "Comparison of postNewtonian templates for compact binary inspiral signals in gravitational-wave detectors", Phys. Rev. $D, \mathbf{8 0}, 084043$ (2009). [DOI], [arXiv:0907.0700 [gr-qc]]. (Cited on pages 65 and 131.)

[112] Buonanno, A., Pan, Y., Pfeiffer, H. P., Scheel, M. A., Buchman, L. T. and Kidder, L. E., "Effectiveone-body waveforms calibrated to numerical relativity simulations: Coalescence of nonspinning, equal-mass black holes", Phys. Rev. D, 79, 124028 (2009). [DOI], [ADS], [arXiv:0902.0790 [gr-qc]]. (Cited on page 65.)

[113] Burke, W. L., "Gravitational radiation damping of slowly moving systems calculated using matched asymptotic expansions", J. Math. Phys., 12, 401-418 (1971). [DOI], [ADS]. (Cited on pages 11, 13, 46, and 62.)

[114] Burke, W. L. and Thorne, K. S., "Gravitational Radiation Damping”, in Carmeli, M., Fickler, S. I. and Witten, L., eds., Relativity, Proceedings of the Relativity Conference in the Midwest, held at Cincinnati, Ohio, June 2-6, 1969, pp. 209-228, (Plenum Press, New York; London, 1970). (Cited on pages $11,13,46$, and 62.$)$

[115] Campanelli, M., "Understanding the fate of merging supermassive black holes", Class. Quantum Grav., 22, S387 (2005). [DOI], [astro-ph/0411744]. (Cited on page 22.)

[116] Campanelli, M., Lousto, C. O., Marronetti, P. and Zlochower, Y., "Accurate Evolutions of Orbiting Black-Hole Binaries without Excision”, Phys. Rev. Lett., 96, 111101 (2006). [DOI], [ADS], [arXiv:grqc/0511048]. (Cited on page 8.)

[117] Campanelli, M., Lousto, C. O., Zlochower, Y. and Merritt, D., "Large merger recoils and spin flips from generic black-hole binaries", Astrophys. J. Lett., 659, L5-L8 (2007). [DOI], [arXiv:grqc/0701164 [gr-qc]]. (Cited on page 22.)

[118] Campbell, W. B., Macek, J. and Morgan, T. A., "Relativistic time-dependent multipole analysis for scalar, electromagnetic, and gravitational fields", Phys. Rev. D, 15, 2156-2164 (1977). [DOI]. (Cited on page 10.)

[119] Campbell, W. B. and Morgan, T. A., "Debye Potentials For Gravitational Field", Physica, 53(2), 264 (1971). [DOI]. (Cited on page 10.)

[120] Carmeli, M., "The equations of motion of slowly moving particles in the general theory of relativity", Nuovo Cimento, 37, 842 (1965). [DOI]. (Cited on page 97.)

[121] Caudill, M., Cook, G. B., Grigsby, J. D. and Pfeiffer, H. P., "Circular orbits and spin in black-hole initial data", Phys. Rev. D, 74, 064011 (2006). [DOI], [ADS], [gr-qc/0605053]. (Cited on pages 101, 103, 104, and 110.)

[122] Chandrasekhar, S., "The Post-Newtonian Equations of Hydrodynamics in General Relativity", Astrophys. J., 142, 1488-1540 (1965). [DOI], [ADS]. (Cited on pages 9 and 53.)

[123] Chandrasekhar, S. and Esposito, F. P., "The $2 \frac{1}{2}$-Post-Newtonian Equations of Hydrodynamics and Radiation Reaction in General Relativity", Astrophys. J., 160, 153-179 (1970). [DOI]. (Cited on pages 9 and 53.)

[124] Chandrasekhar, S. and Nutku, Y., "The Second Post-Newtonian Equations of Hydrodynamics in General Relativity", Astrophys. J., 158, 55-79 (1969). [DOI]. (Cited on pages 9 and 53.)

[125] Chatziioannou, K., Poisson, E. and Yunes, N., "Tidal heating and torquing of a Kerr black hole to next-to-leading order in the tidal coupling", Phys. Rev. D, 87, 044022 (2013). [DOI], [ADS], [arXiv:1211.1686 [gr-qc]]. (Cited on page 158.)

[126] Chicone, C., Kopeikin, S. M., Mashhoon, B. and Retzloff, D. G., "Delay equations and radiation damping", Phys. Lett. A, 285, 17-26 (2001). [DOI], [gr-qc/0101122]. (Cited on page 55.) 
[127] Christodoulou, D., "Reversible and irreversible transformations in black-hole physics", Phys. Rev. Lett., 25, 1596 (1970). [DOI]. (Cited on page 101.)

[128] Christodoulou, D., "Nonlinear Nature of Gravitation and Gravitational-Wave Experiments", Phys. Rev. Lett., 67, 1486-1489 (1991). [DOI]. (Cited on pages 21 and 43.)

[129] Christodoulou, D. and Ruffini, R., "Reversible transformations of a charged black hole", Phys. Rev. $D, 4,3552-3555$ (1971). [DOI]. (Cited on page 101.)

[130] Christodoulou, D. and Schmidt, B. G., "Convergent and Asymptotic Iteration Methods in General Relativity", Commun. Math. Phys., 68, 275-289 (1979). [DOI]. (Cited on page 28.)

[131] Collins, J. C., Renormalization: An introduction to renormalization, the renormalization group, and the operator-product expansion, (Cambridge University Press, Cambridge; New York, 1984). [Google Books]. (Cited on page 72.)

[132] Cook, G. B., "Three-dimensional initial data for the collision of two black holes. II. Quasicircular orbits for equal-mass black holes", Phys. Rev. D, 50, 5025-5032 (1994). [DOI], [ADS]. (Cited on pages 103 and 104.)

[133] Cook, G. B. and Pfeiffer, H. P., "Excision boundary conditions for black hole initial data", Phys. Rev. D, 70, 104016 (2004). [DOI], [ADS]. (Cited on pages 101, 102, 103, 104, and 110.)

[134] Cooperstock, F. I. and Booth, D. J., "Angular-Momentum Flux For Gravitational Radiation to Octupole Order", Nuovo Cimento B, 62(1), 163-170 (1969). [DOI]. (Cited on page 10.)

[135] Corinaldesi, E. and Papapetrou, A., "Spinning test-particles in general relativity. II", Proc. R. Soc. London, Ser. A, 209, 259-268 (1951). [DOI]. (Cited on pages 148 and 150.)

[136] Crowley, R. J. and Thorne, K. S., "Generation of gravitational waves. II. Post-linear formalism revisited", Astrophys. J., 215, 624-635 (1977). [DOI]. (Cited on page 10.)

[137] Cutler, C., Finn, L. S., Poisson, E. and Sussman, G. J., "Gravitational radiation from a particle in circular orbit around a black hole. II. Numerical results for the nonrotating case", Phys. Rev. D, 47, 1511-1518 (1993). [DOI]. (Cited on pages 16, 65, and 125.)

[138] Cutler, C. and Flanagan, É. É., "Gravitational waves from merging compact binaries: How accurately can one extract the binary's parameters from the inspiral wave form?", Phys. Rev. D, 49, 2658-2697 (1994). [DOI], [arXiv:gr-qc/9402014]. (Cited on pages 14, 16, 65, 124, 125, and 146.)

[139] Cutler, C. et al., "The Last Three Minutes: Issues in Gravitational-Wave Measurements of Coalescing Compact Binaries", Phys. Rev. Lett., 70, 2984-2987 (1993). [DOI], [astro-ph/9208005]. (Cited on pages $14,16,65$, and 125.)

[140] D'Alembert, J., Traité de Dynamique, (David L'Aine, Paris, 1743). [Google Books]. (Cited on page 7.)

[141] Damour, T., "Problème des deux corps et freinage de rayonnement en relativité générale", $C . R$. Acad. Sci. Ser. II, 294, 1355-1357 (1982). (Cited on page 17.)

[142] Damour, T., "Gravitational radiation and the motion of compact bodies", in Deruelle, N. and Piran, T., eds., Rayonnement Gravitationnel / Gravitational Radiation, Proceedings of the Les Houches Summer School, $2-21$ June 1982, pp. 59-144, (North-Holland, Amsterdam, 1983). (Cited on pages $7,9,16,17,65$, and 135 .)

[143] Damour, T., "Gravitational radiation reaction in the binary pulsar and the quadrupole formula controvercy", Phys. Rev. Lett., 51, 1019-1021 (1983). [DOI]. (Cited on pages 7, 17, and 135.) 
[144] Damour, T., "An Introduction to the Theory of Gravitational Radiation", in Carter, B. and Hartle, J. B., eds., Gravitation in Astrophysics: Cargèse 1986, Proceedings of a NATO Advanced Study Institute on Gravitation in Astrophysics, Cargése, France, 15-31 July, 1986, NATO ASI Series B, 156, pp. 3-62, (Plenum Press, New York, 1987). (Cited on page 9.)

[145] Damour, T., "The problem of motion in Newtonian and Einsteinian gravity", in Hawking, S. W. and Israel, W., eds., Three Hundred Years of Gravitation, pp. 128-198, (Cambridge University Press, Cambridge; New York, 1987). [ADS]. (Cited on pages 9 and 70.)

[146] Damour, T., "Gravitational self-force in a Schwarzschild background and the effective one-body formalism", Phys. Rev. D, 81, 024017 (2010). [DOI], [arXiv:0910.5533 [gr-qc]]. (Cited on page 114.)

[147] Damour, T. and Deruelle, N., "Lagrangien généralisé du système de deux masses ponctuelles, à l'approximation post-post-newtonienne de la relativité générale", C. R. Acad. Sci. Ser. II, 293, 537-540 (1981). (Cited on pages 7, 17, and 84.)

[148] Damour, T. and Deruelle, N., "Radiation reaction and angular momentum loss in small angle gravitational scattering", Phys. Lett. A, 87, 81-84 (1981). [DOI]. (Cited on pages 7 and 17.)

[149] Damour, T. and Deruelle, N., "General relativistic celestial mechanics of binary systems I. The postNewtonian motion", Ann. Inst. Henri Poincare A, 43, 107-132 (1985). Online version (accessed 17 October 2013):

http://www.numdam.org/item?id=AIHPA_1985_-43_1_107_0. (Cited on pages 135, 137, 138, and 140.)

[150] Damour, T. and Deruelle, N., "General relativistic celestial mechanics of binary systems II. The post-Newtonian timing formula", Ann. Inst. Henri Poincare A, 44, 263-292 (1986). Online version (accessed 17 October 2013):

http://www.numdam.org/item?id=AIHPA_1986_-44_3_263_0. (Cited on page 137.)

[151] Damour, T. and Esposito-Farèse, G., "Testing gravity to second post-Newtonian order: A fieldtheory approach", Phys. Rev. D, 53, 5541-5578 (1996). [DOI], [ADS], [gr-qc/9506063]. (Cited on pages 18 and 71.$)$

[152] Damour, T. and Gopakumar, A., "Gravitational recoil during binary black hole coalescence using the effective one body approach", Phys. Rev. D, 73, 124006 (2006). [DOI], [gr-qc/0602117]. (Cited on page 22.)

[153] Damour, T., Gopakumar, A. and Iyer, B. R., "Phasing of gravitational waves from inspiralling eccentric binaries", Phys. Rev. D, 70, 064028 (2004). [DOI], [gr-qc/0404128]. (Cited on pages 141, 145, and 146.)

[154] Damour, T. and Iyer, B. R., "Multipole analysis for electromagnetism and linearized gravity with irreducible Cartesian tensors", Phys. Rev. D, 43, 3259-3272 (1991). [DOI]. (Cited on pages 10 and 51.)

[155] Damour, T. and Iyer, B. R., "Post-Newtonian generation of gravitational waves. II. The spin moments", Ann. Inst. Henri Poincare A, 54, 115-164 (1991). Online version (accessed 17 October 2013): http://www.numdam.org/item?id=AIHPA_1991__54_2_115_0. (Cited on pages 11 and 46.)

[156] Damour, T., Iyer, B. R., Jaranowski, P. and Sathyaprakash, B. S., "Gravitational waves from black hole binary inspiral and merger: The span of third post-Newtonian effective-one-body templates", Phys. Rev. D, 67, 064028 (2003). [DOI], [gr-qc/0211041]. (Cited on pages 65, 71, and 131.)

[157] Damour, T., Iyer, B. R. and Sathyaprakash, B. S., "Improved filters for gravitational waves from inspiraling compact binaries", Phys. Rev. D, 57, 885-907 (1998). [DOI], [gr-qc/9708034]. (Cited on pages 16 and 65 .) 
[158] Damour, T., Iyer, B. R. and Sathyaprakash, B. S., "Frequency-domain P-approximant filters for time-truncated inspiral gravitational wave signals from compact binaries", Phys. Rev. D, 62, 084036 (2000). [DOI], [gr-qc/0001023]. (Cited on page 65.)

[159] Damour, T., Iyer, B. R. and Sathyaprakash, B. S., "Comparison of search templates for gravitational waves from binary inspiral: 3.5PN update", Phys. Rev. D, 66, 027502 (2002). [DOI], [gr-qc/0207021]. (Cited on pages 65, 71, and 131.)

[160] Damour, T., Jaranowski, P. and Schäfer, G., "Dynamical invariants for general relativistic twobody systems at the third post-Newtonian approximation", Phys. Rev. D, 62, 044024 (2000). [grqc/9912092]. (Cited on pages 95 and 110.)

[161] Damour, T., Jaranowski, P. and Schäfer, G., "On the determination of the last stable orbit for circular general relativistic binaries at the third post-Newtonian approximation", Phys. Rev. D, 62, 084011 (2000). [ADS], [gr-qc/0005034]. (Cited on page 65.)

[162] Damour, T., Jaranowski, P. and Schäfer, G., "Poincaré invariance in the ADM Hamiltonian approach to the general relativistic two-body problem", Phys. Rev. D, 62, 021501(R) (2000). [gr-qc/0003051]. Erratum: Phys. Rev. D, 63, 029903(E) (2000). (Cited on pages 17, 18, 69, 88, and 93.)

[163] Damour, T., Jaranowski, P. and Schäfer, G., "Dimensional regularization of the gravitational interaction of point masses", Phys. Lett. B, 513, 147-155 (2001). [DOI], [gr-qc/0105038]. (Cited on pages $18,70,74$, and 76.$)$

[164] Damour, T., Jaranowski, P. and Schäfer, G., "Equivalence between the ADM-Hamiltonian and the harmonic-coordinates approaches to the third post-Newtonian dynamics of compact binaries", Phys. Rev. D, 63, 044021 (2001). [DOI], [gr-qc/0010040]. Erratum: Phys. Rev. D, 66, 029901(E) (2002). (Cited on pages 17,18 , and 70. )

[165] Damour, T., Jaranowski, P. and Schäfer, G., "Hamiltonian of two spinning compact bodies with next-to-leading order gravitational spin-orbit coupling", Phys. Rev. D, 77, 064032 (2008). [DOI], [arXiv:0711.1048]. (Cited on pages 19, 147, and 151.)

[166] Damour, T., Jaranowski, P. and Schäfer, G., "Non-local-in-time action for the fourth postNewtonian conservative dynamics of two-body systems", Phys. Rev. D, 89, 064058 (2014). [DOI], [arXiv:1401.4548 [gr-qc]]. (Cited on page 19.)

[167] Damour, T., Jaranowski, P. and Schäfer, G., "Fourth post-Newtonian effective one-body dynamics", Phys. Rev. D, 91, 084024 (2015). [DOI], [arXiv:1502.07245 [gr-qc]]. (Cited on page 19.)

[168] Damour, T. and Nagar, A., "The Effective One-Body description of the Two-Body Problem", in Blanchet, L., Spallicci, A. and Whiting, B., eds., Mass and Motion in General Relativity, Lectures from the CNRS School on Mass held in Orléans, France, 23-25 June 2008, Fundamental Theories of Physics, 162, pp. 211-252, (Springer, Dordrecht; New York, 2011). [DOI], [ADS]. (Cited on page 65.)

[169] Damour, T. and Schäfer, G., "Lagrangians for $n$ Point Masses at the Second Post-Newtonian Approximation of General Relativity", Gen. Relativ. Gravit., 17, 879-905 (1985). [DOI]. (Cited on pages 17 and 85.$)$

[170] Damour, T. and Schäfer, G., "Higher-Order Relativistic Periastron Advances in Binary Pulsars", Nuovo Cimento B, 101, 127-176 (1988). [DOI]. (Cited on pages 138, 139, and 141.)

[171] Damour, T. and Schmidt, B. G., "Reliability of perturbation theory in general relativity", J. Math. Phys., 31, 2441-2458 (1990). [DOI]. (Cited on page 28.)

[172] Damour, T., Soffel, M. and Xu, C., "General-relativistic celestial mechanics. I. Method and definition of reference systems", Phys. Rev. D, 43, 3273-3307 (1991). [DOI], [ADS]. (Cited on page 70.) 
[173] Damour, T. and Taylor, J. H., "On the Orbital Period Change of the Binary Pulsar PSR 1913+16", Astrophys. J., 366, 501-511 (1991). [DOI], [ADS]. (Cited on page 17.)

[174] de Andrade, V. C., Blanchet, L. and Faye, G., "Third post-Newtonian dynamics of compact binaries: Noetherian conserved quantities and equivalence between the harmonic-coordinate and ADMHamiltonian formalisms", Class. Quantum Grav., 18, 753-778 (2001). [DOI], [gr-qc/0011063]. (Cited on pages $17,18,71,85,86,87,88$, and 90.$)$

[175] Deruelle, N., Sur les équations du mouvement et le rayonnement gravitationnel d'un système binaire en Relativité Générale, Ph.D. thesis, (Université Pierre et Marie Curie, Paris, 1982). (Cited on page 17.)

[176] Detweiler, S., "Consequence of the gravitational self-force for circular orbits of the Schwarzschild geometry", Phys. Rev. D, 77, 124026 (2008). [DOI], [arXiv:0804.3529]. (Cited on pages 109, 112, 114, and 115.)

[177] Detweiler, S., "Elementary Development of the Gravitational Self-Force", in Blanchet, L., Spallicci, A. and Whiting, B., eds., Mass and Motion in General Relativity, Lectures from the CNRS School on Mass held in Orléans, France, 23 - 25 June 2008, Fundamental Theories of Physics, 162, pp. 271-307, (Springer, Dordrecht; New York, 2011). [DOI], [ADS]. (Cited on pages 9 and 112.)

[178] Detweiler, S. and Whiting, B. F., "Self-force via a Green's function decomposition", Phys. Rev. D, 67, 024025 (2003). [DOI], [arXiv:gr-qc/0202086]. (Cited on pages 112 and 115.)

[179] Dixon, W. G., "Extended bodies in general relativity: Their description and motion", in Ehlers, J., ed., Isolated Gravitating Systems in General Relativity (Sistemi gravitazionali isolati in relatività generale), Proceedings of the International School of Physics 'Enrico Fermi', Course 67, Varenna on Lake Como, Villa Monastero, Italy, 28 June-10 July, 1976, pp. 156-219, (North-Holland, Amsterdam; New York, 1979). (Cited on pages 148 and 150.)

[180] Eder, E., "Existence, uniqueness and iterative construction of motions of charged particles with retarded interactions", Ann. Inst. Henri Poincare A, 39, 1-27 (1983). Online version (accessed 17 October 2013): http://www.numdam.org/item?id=AIHPA_1983_-39_1_1_0. (Cited on page 38.)

[181] Ehlers, J., "Isolated systems in general relativity", Ann. N.Y. Acad. Sci., 336, 279-294 (1980). [DOI]. (Cited on pages 53 and 57.)

[182] Ehlers, J., Rosenblum, A., Goldberg, J. N. and Havas, P., "Comments on gravitational radiation damping and energy loss in binary systems", Astrophys. J. Lett., 208, L77-L81 (1976). [DOI], [ADS]. (Cited on page 54.)

[183] Einstein, A., "Über Gravitationswellen", Sitzungsber. K. Preuss. Akad. Wiss., 1918, 154-167 (1918). [ADS]. Online version (accessed 31 January 2014): http://echo.mpiwg-berlin.mpg.de/MPIWG:8HSP60BU. (Cited on pages 9 and 12.)

[184] Einstein, A., Infeld, L. and Hoffmann, B., "The Gravitational Equations and the Problem of Motion", Ann. Math. (2), 39, 65-100 (1938). [DOI]. (Cited on pages 7, 17, and 70.)

[185] Epstein, R. and Wagoner, R. V., "Post-Newtonian Generation of Gravitational Waves", Astrophys. J., 197, 717-723 (1975). [DOI], [ADS]. (Cited on pages 11, 20, and 50.)

[186] Esposito, L. W. and Harrison, E. R., "Properties of the Hulse-Taylor binary pulsar system", Astrophys. J. Lett., 196, L1-L2 (1975). [DOI]. (Cited on page 13.)

[187] Faber, J. A. and Rasio, F. A., "Binary Neutron Star Mergers", Living Rev. Relativity, 15, lrr-2012-8 (2012). [DOI], [ADS], [arXiv:1204.3858 [gr-qc]]. URL (accessed 6 October 2013): http://www.livingreviews.org//rr-2012-8. (Cited on pages 8 and 16.) 
[188] Fabian, A. C. and Miniutti, G., "The X-ray spectra of accreting Kerr black holes", in Wiltshire, D. L., Visser, M. and Scott, S. M., eds., The Kerr Spacetime: Rotating Black Holes in General Relativity, 9, (Cambridge University Press, Cambridge; New York, 2009). [ADS], [arXiv:astro-ph/0507409]. (Cited on page 146.)

[189] Favata, M., "Post-Newtonian corrections to the gravitational-wave memory for quasicircular, inspiralling compact binaries", Phys. Rev. D, 80, 024002 (2009). [DOI], [ADS], [arXiv:0812.0069]. (Cited on pages $21,43,130$, and 134.)

[190] Favata, M., "Conservative corrections to the innermost stable circular orbit (ISCO) of a Kerr black hole: a new gauge-invariant post-Newtonian ISCO condition, and the ISCO shift due to test-particle spin and the gravitational self-force", Phys. Rev. D, 83, 024028 (2011). [DOI], [arXiv:1010.2553]. (Cited on page 108.)

[191] Favata, M., "Conservative self-force correction to the innermost stable circular orbit: comparison with multiple post-Newtonian-based methods", Phys. Rev. D, 83, 024027 (2011). [DOI], [arXiv:1008.4622]. (Cited on page 108.)

[192] Favata, M., "The gravitational-wave memory from eccentric binaries", Phys. Rev. D, 84, 124013 (2011). [DOI], [arXiv:1108.3121]. (Cited on pages 21 and 43.)

[193] Faye, G., Equations du mouvement d'un système binaire d'objets compact à l'approximation postnewtonienne, Ph.D. thesis, (Université Paris VI, Paris, 1999). (Cited on page 75.)

[194] Faye, G., Blanchet, L. and Buonanno, A., "Higher-order spin effects in the dynamics of compact binaries I. Equations of motion", Phys. Rev. D, 74, 104033 (2006). [DOI], [gr-qc/0605139]. (Cited on pages 19 and 147.)

[195] Faye, G., Blanchet, L. and Iyer, B. R., "Non-linear multipole interactions and gravitational-wave octupole modes for inspiralling compact binaries to third-and-a-half post-Newtonian order", Class. Quantum Grav., 32, 045016 (2015). [arXiv:1409.3546 [gr-qc]]. (Cited on page 132.)

[196] Faye, G., Jaranowski, P. and Schäfer, G., "Skeleton approximate solution of the Einstein field equations for multiple black-hole systems", Phys. Rev. D, 69, 124029 (2004). [DOI], [gr-qc/0311018]. (Cited on page 103.)

[197] Faye, G., Marsat, S., Blanchet, L. and Iyer, B. R., "The third and a half post-Newtonian gravitational wave quadrupole mode for quasi-circular inspiralling compact binaries", Class. Quantum Grav., 29, 175004 (2012). [DOI], [arXiv:1204.1043]. (Cited on pages 11, 20, 31, 40, 44, 45, 117, 118, 125, 131, and 132.)

[198] Finn, L. S. and Chernoff, D. F., "Observing binary inspiral in gravitational radiation: One interferometer", Phys. Rev. D, 47, 2198-2219 (1993). [DOI], [arXiv:gr-qc/9301003]. (Cited on pages 16 and 65.)

[199] Fitchett, M. J., "The influence of gravitational wave momentum losses on the centre of mass motion of a Newtonian binary system", Mon. Not. R. Astron. Soc., 203, 1049-1062 (1983). [ADS]. (Cited on page 22.)

[200] Flanagan, É.É. and Hinderer, T., "Constraining neutron star tidal Love numbers with gravitational wave detectors", Phys. Rev. D, 77, 021502 (2008). [DOI], [ADS], [arXiv:0709.1915 [astro-ph]]. (Cited on page 16.)

[201] Fock, V. A., "On motion of finite masses in general relativity", J. Phys. (Moscow), 1(2), 81-116 (1939). (Cited on page 17.)

[202] Fock, V. A., Theory of space, time and gravitation, (Pergamon, London, 1959). (Cited on pages 33 and 97.) 
[203] Foffa, S. and Sturani, R., "Effective field theory calculation of conservative binary dynamics at third post-Newtonian order", Phys. Rev. D, 84, 044031 (2011). [DOI], [arXiv:1104.1122 [gr-qc]]. (Cited on pages 18 and 71.)

[204] Foffa, S. and Sturani, R., "The dynamics of the gravitational two-body problem in the postNewtonian approximation at quadratic order in the Newton's constant", Phys. Rev. D, 87, 064011 (2012). [arXiv:1206.7087 [gr-qc]]. (Cited on pages 19 and 96.)

[205] Foffa, S. and Sturani, R., "Tail terms in gravitational radiation reaction via effective field theory", Phys. Rev. D, 87, 044056 (2013). [DOI], [arXiv:1111.5488 [gr-qc]]. (Cited on page 19.)

[206] Foffa, S. and Sturani, R., "Effective field theory methods to model compact binaries", Class. Quantum Grav., 31, 043001 (2014). [DOI], [ADS], [arXiv:1309.3474 [gr-qc]]. (Cited on page 18.)

[207] Fokker, A. D., "Ein invarianter Variationssatz für die Bewegung mehrerer elektrischer Massenteilchen", Z. Phys., 58, 386-393 (1929). [DOI], [ADS]. (Cited on page 18.)

[208] Friedman, J. L., Uryū, K. and Shibata, M., "Thermodynamics of binary black holes and neutron stars", Phys. Rev. D, 65, 064035 (2002). [DOI]. Erratum: Phys. Rev. D, 70, 129904(E) (2004). (Cited on pages 108, 110, and 112.)

[209] Fujita, R., "Gravitational Radiation for Extreme Mass Ratio Inspirals to the 14th Post-Newtonian Order", Prog. Theor. Phys., 127, 583-590 (2012). [DOI], [arXiv:1104.5615 [gr-qc]]. (Cited on page 21.)

[210] Fujita, R., "Gravitational Waves from a Particle in Circular Orbits around a Schwarzschild Black Hole to the 22nd Post-Newtonian Order", Prog. Theor. Phys., 128, 971-992 (2012). [DOI], [ADS], [arXiv:1211.5535 [gr-qc]]. (Cited on page 21.)

[211] Futamase, T., "Gravitational radiation reaction in the Newtonian limit", Phys. Rev. D, 28, 23732381 (1983). [DOI]. (Cited on page 54.)

[212] Futamase, T., "Strong-field point-particle limit and the equations of motion in the binary pulsar", Phys. Rev. D, 36, 321-329 (1987). [DOI]. (Cited on page 70.)

[213] Futamase, T. and Itoh, Y., "The Post-Newtonian Approximation for Relativistic Compact Binaries", Living Rev. Relativity, 10, lrr-2007-2 (2007). [DOI], [ADS]. URL (accessed 6 October 2013): http://www.livingreviews.org/lrr-2007-2. (Cited on page 17.)

[214] Futamase, T. and Schutz, B. F., "Newtonian and post-Newtonian approximations are asymptotic to general relativity", Phys. Rev. D, 28, 2363-2372 (1983). [DOI]. (Cited on page 54.)

[215] Galley, C. R., Leibovich, A. K., Porto, R. A. and Ross, A., "The tail effect in gravitational radiation-reaction: time non-locality and renormalization group evolution", arXiv, e-print, (2015). [arXiv:1511.07379 [gr-qc]]. (Cited on page 19.)

[216] Gal'tsov, D. V., Matiukhin, A. A. and Petukhov, V. I., "Relativistic corrections to the gravitational radiation of a binary system and the fine structure of the spectrum", Phys. Lett. A, 77, 387-390 (1980). [DOI]. (Cited on page 21.)

[217] Gergely, L.Á., "Spin-spin effects in radiating compact binaries", Phys. Rev. D, 61, 024035 (1999). [DOI], [gr-qc/9911082]. (Cited on page 147.)

[218] Gergely, L.Á., "Second post-Newtonian radiative evolution of the relative orientations of angular momenta in spinning compact binaries", Phys. Rev. D, 62, 024007 (2000). [DOI], [gr-qc/0003037]. (Cited on pages 147 and 159.)

[219] Geroch, R., "Multipole Moments. II. Curved Space", J. Math. Phys., 11, 2580-2588 (1970). [DOI], [ADS]. (Cited on page 10.) 
[220] Geroch, R. and Horowitz, G. T., "Asymptotically simple does not imply asymptotically Minkowskian", Phys. Rev. Lett., 40, 203-206 (1978). (Cited on pages 10, 11, 34, and 35.)

[221] Goldberger, W. D. and Ross, A., "Gravitational radiative corrections from effective field theory", Phys. Rev. D, 81, 124015 (2010). [DOI], [arXiv:0912.4254]. (Cited on page 20.)

[222] Goldberger, W. D., Ross, A. and Rothstein, I. Z., "Black hole mass dynamics and renormalization group evolution", Phys. Rev. D, 89, 124033 (2014). [DOI], [ADS], [arXiv:1211.6095 [hep-th]]. (Cited on pages 18 and 44.)

[223] Goldberger, W. D. and Rothstein, I. Z., "Effective field theory of gravity for extended objects", Phys. Rev. D, 73, 104029 (2006). [DOI], [arXiv:hep-th/0409156 [hep-th]]. (Cited on pages 18, 71, and 72.)

[224] Gopakumar, A. and Iyer, B. R., "Gravitational waves from inspiraling compact binaries: Angular momentum flux, evolution of the orbital elements and the waveform to the second post-Newtonian order", Phys. Rev. D, 56, 7708-7731 (1997). [DOI], [arXiv:gr-qc/9710075]. (Cited on pages 20, 140, and 145.)

[225] Gopakumar, A. and Iyer, B. R., "Second post-Newtonian gravitational wave polarizations for compact binaries in elliptical orbits", Phys. Rev. D, 65, 084011 (2002). [DOI], [arXiv:gr-qc/0110100]. (Cited on page 140.)

[226] Gopakumar, A., Iyer, B. R. and Iyer, S., "Second post-Newtonian gravitational radiation reaction for two-body systems: Nonspinning bodies", Phys. Rev. D, 55, 6030-6053 (1997). [DOI], [arXiv:grqc/9703075]. (Cited on page 18.)

[227] Gou, L. et al., "The extreme spin of the black hole in Cygnus X-1", Astrophys. J., 742, 85 (2011). [DOI], [arXiv:1106.3690 [astro-ph.HE]]. (Cited on page 146.)

[228] Gourgoulhon, E., Grandclément, P. and Bonazzola, S., "Binary black holes in circular orbits. I. A global spacetime approach", Phys. Rev. D, 65, 044020 (2002). [DOI], [ADS], [gr-qc/0106015]. (Cited on pages 101, 103, 104, and 110.)

[229] Gourgoulhon, E., Grandclément, P., Taniguchi, K., Marck, J.-A. and Bonazzola, S., "Quasiequilibrium sequences of synchronized and irrotational binary neutron stars in general relativity", Phys. Rev. D, 63, 064029 (2001). [DOI], [gr-qc/0007028]. (Cited on page 101.)

[230] Gradshteyn, I. S. and Ryzhik, I. M., Table of Integrals, Series and Products, (Academic Press, San Diego; London, 1980). (Cited on page 121.)

[231] Gralla, S. E. and Wald, R. M., "A rigorous derivation of gravitational self-force", Class. Quantum Grav., 25, 205009 (2008). [DOI], [arXiv:0806.3293]. (Cited on page 112.)

[232] Grandclément, P., Gourgoulhon, E. and Bonazzola, S., "Binary black holes in circular orbits. II. Numerical methods and first results", Phys. Rev. D, 65, 044021 (2002). [DOI], [ADS]. (Cited on pages 101, 102, 103, 104, and 110.)

[233] Grandclément, P. and Novak, J., "Spectral Methods for Numerical Relativity", Living Rev. Relativity, 12, lrr-2009-1 (2009). [DOI], [ADS], [arXiv:0706.2286 [gr-qc]]. URL (accessed 6 October 2013): http://www.livingreviews.org/lrr-2009-1. (Cited on page 8.)

[234] Grishchuk, L. P. and Kopeikin, S. M., "Equations of motion for isolated bodies with relativistic corrections including the radiation-reaction force", in Kovalevsky, J. and Brumberg, V. A., eds., Relativity in Celestial Mechanics and Astrometry: High Precision Dynamical Theories and Observational Verifications, Proceedings of the 114th Symposium of the International Astronomical Union, held in Leningrad, USSR, May 28-31, 1985, pp. 19-34, (Reidel, Dordrecht; Boston, 1986). (Cited on pages 17 and 70.$)$ 
[235] Gultekin, K., Miller, M. C. and Hamilton, D. P., "Growth of Intermediate-Mass Black Holes in Globular Clusters", Astrophys. J., 616, 221 (2004). [DOI], [astro-ph/0402532]. (Cited on pages 135 and 145.)

[236] Hadamard, J., Le problème de Cauchy et les équations aux dérivées partielles linéaires hyperboliques, (Hermann, Paris, 1932). (Cited on pages 7 and 66.)

[237] Hannam, M., Husa, S., González, J. A., Sperhake, U. and Brügmann, B., "Where post-Newtonian and numerical-relativity waveforms meet", Phys. Rev. D, 77, 044020 (2008). [DOI], [ADS], [arXiv:0706.1305]. (Cited on page 36.)

[238] Hansen, R. O., "Multipole moments of stationary space-times", J. Math. Phys., 15, 46-52 (1974). [DOI], [ADS]. (Cited on page 10.)

[239] Hanson, A. J. and Regge, T., "The Relativistic Spherical Top", Ann. Phys. (N.Y.), 87, 498-566 (1974). [DOI]. (Cited on pages 148 and 150.)

[240] Hari Dass, N. D. and Soni, V., "Feynman graph derivation of the Einstein quadrupole formula", J. Phys. A: Math. Gen., 15, 473-492 (1982). [DOI]. (Cited on page 18.)

[241] Hartung, J. and Steinhoff, J., "Next-to-leading order spin-orbit and spin(a)-spin(b) Hamiltonians for $n$ gravitating spinning compact objects", Phys. Rev. D, 83, 044008 (2011). [DOI], [arXiv:1011.1179 [gr-qc]]. (Cited on pages 19 and 147.)

[242] Hartung, J. and Steinhoff, J., "Next-to-next-to-leading order post-Newtonian spin-orbit Hamiltonian for self-gravitating binaries", Ann. Phys. (Berlin), 523, 783-790 (2011). [DOI], [ADS], [arXiv:1104.3079 [gr-qc]]. (Cited on pages 19 and 147.)

[243] Hartung, J. and Steinhoff, J., "Next-to-next-to-leading order post-Newtonian spin(1)-spin(2) Hamiltonian for self-gravitating binaries", Ann. Phys. (Berlin), 523, 919-924 (2011). [DOI], [ADS], [arXiv:1107.4294 [gr-qc]]. (Cited on pages 19 and 147.)

[244] Hartung, J., Steinhoff, J. and Schäfer, G., "Next-to-next-to-leading order post-Newtonian linear-in-spin binary Hamiltonians", Ann. Phys. (Berlin), 525, 359-394 (2013). [DOI], [ADS], [arXiv:1302.6723 [gr-qc]]. (Cited on pages 19 and 147.)

[245] Hergt, S. and Schäfer, G., "Higher-order-in-spin interaction Hamiltonians for binary black holes from Poincaré invariance", Phys. Rev. D, 78, 124004 (2008). [DOI], [arXiv:0809.2208 [gr-qc]]. (Cited on page 147.)

[246] Hergt, S. and Schäfer, G., "Higher-order-in-spin interaction Hamiltonians for binary black holes from source terms of Kerr geometry in approximate ADM coordinates", Phys. Rev. D, 77, 104001 (2008). [DOI], [arXiv:0712.1515 [gr-qc]]. (Cited on page 147.)

[247] Hergt, S., Steinhoff, J. and Schäfer, G., "The reduced Hamiltonian for next-to-leading-order spinsquared dynamics of general compact binaries", Class. Quantum Grav., 27, 135007 (2010). [DOI], [arXiv:1002.2093 [gr-qc]]. (Cited on pages 19 and 147.)

[248] Hopper, S., Kavanagh, C. and Ottewill, A. C., "Analytic self-force calculations in the post-Newtonian regime: eccentric orbits on a Schwarzschild background", Phys. Rev. D, 93, 044010 (2016). [DOI], [arXiv:1512.01556 [gr-qc]]. (Cited on page 19.)

[249] Hotokezaka, K., Kyutoku, K. and Shibata, M., "Exploring tidal effects of coalescing binary neutron stars in numerical relativity", Phys. Rev. D, 87, 044001 (2013). [DOI], [arXiv:1301.3555 [gr-qc]]. (Cited on page 16.)

[250] Hulse, R. A. and Taylor, J. H., "Discovery of a pulsar in a binary system", Astrophys. J., 195, L51-L53 (1975). [DOI], [ADS]. (Cited on page 7.) 
[251] Hunter, A. J. and Rotenberg, M. A., "The double-series approximation method in general relativity. I. Exact solution of the (24) approximation. II. Discussion of "wave tails' in the (2s) approximation", J. Phys. A: Math. Gen., 2, 34-49 (1969). [DOI]. (Cited on pages 10 and 26.)

[252] Isaacson, R. A. and Winicour, J., "Harmonic and Null Descriptions of Gravitational Radiation", Phys. Rev., 168, 1451-1456 (1968). [DOI]. (Cited on page 33.)

[253] Itoh, Y., "Equation of motion for relativistic compact binaries with the strong field point particle limit: Third post-Newtonian order", Phys. Rev. D, 69, 064018 (2004). [DOI]. (Cited on pages 17, 18, 70, and 71.)

[254] Itoh, Y., "Third-and-a-half order post-Newtonian equations of motion for relativistic compact binaries using the strong field point particle limit", Phys. Rev. D, 80, 124003 (2009). [DOI], [arXiv:0911.4232 [gr-qc]]. (Cited on pages 18, 70, 78, 92, and 117.)

[255] Itoh, Y. and Futamase, T., "New derivation of a third post-Newtonian equation of motion for relativistic compact binaries without ambiguity", Phys. Rev. D, 68, 121501(R) (2003). [DOI], [grqc/0310028]. (Cited on pages 17, 18, 70, and 71.)

[256] Itoh, Y...., Futamase, T. and Asada, H., "Equation of motion for relativistic compact binaries with the strong field point particle limit: Formulation, the first post-Newtonian order, and multipole terms", Phys. Rev. D, 62, 064002 (2000). [DOI], [gr-qc/9910052]. (Cited on pages 17, 18, and 70.)

[257] Itoh, Y., Futamase, T. and Asada, H., "Equation of motion for relativistic compact binaries with the strong field point particle limit: The second and half post-Newtonian order", Phys. Rev. D, 63, 064038 (2001). [DOI], [gr-qc/0101114]. (Cited on pages 17, 18, and 70.)

[258] Iyer, B. R. and Will, C. M., "Post-Newtonian gravitational radiation reaction for two-body systems", Phys. Rev. Lett., 70, 113-116 (1993). [DOI]. (Cited on pages 18, 64, 78, 84, 92, and 117.)

[259] Iyer, B. R. and Will, C. M., "Post-Newtonian gravitational radiation reaction for two-body systems: Nonspinning bodies", Phys. Rev. D, 52, 6882-6893 (1995). [DOI]. (Cited on pages 18, 64, 78, 84, 92, and 117.)

[260] Jaranowski, P. and Schäfer, G., "Radiative 3.5 post-Newtonian ADM Hamiltonian for many-body point-mass systems", Phys. Rev. D, 55, 4712-4722 (1997). [DOI]. (Cited on pages 18, 78, 92, and 117.)

[261] Jaranowski, P. and Schäfer, G., "Third post-Newtonian higher order ADM Hamilton dynamics for two-body point-mass systems", Phys. Rev. D, 57, 7274-7291 (1998). [DOI], [gr-qc/9712075]. (Cited on pages $17,68,69,86,87,88$, and 93.)

[262] Jaranowski, P. and Schäfer, G., "Binary black-hole problem at the third post-Newtonian approximation in the orbital motion: Static part", Phys. Rev. D, 60, 124003 (1999). [DOI], [gr-qc/9906092]. (Cited on pages $17,68,69,86,87,88$, and 93.)

[263] Jaranowski, P. and Schäfer, G., "The binary black-hole dynamics at the third post-Newtonian order in the orbital motion", Ann. Phys. (Berlin), 9, 378-383 (2000). [DOI], [gr-qc/0003054]. (Cited on pages 17,68 , and 88 .)

[264] Jaranowski, P. and Schäfer, G., "Towards the fourth post-Newtonian Hamiltonian for two-point-mass systems", Phys. Rev. D, 86, 061503(R) (2012). [DOI], [arXiv:1207.5448 [gr-qc]]. (Cited on pages 19 and 96.)

[265] Jaranowski, P. and Schäfer, G., "Dimensional regularization of local singularities in the 4th post-Newtonian two-point-mass Hamiltonian", Phys. Rev. D, 87, 081503(R) (2013). [DOI], [arXiv:1303.3225 [gr-qc]]. (Cited on pages 19 and 96.) 
[266] Jaranowski, P. and Schäfer, G., "Derivation of the local-in-time fourth post-Newtonian ADM Hamiltonian for spinless compact binaries", Phys. Rev. D, 92, 124043 (2015). [DOI], [arXiv:1508.01016 [gr-qc]]. (Cited on page 19.)

[267] Junker, W. and Schäfer, G., "Binary systems: higher order gravitational radiation damping and wave emission", Mon. Not. R. Astron. Soc., 254, 146-164 (1992). [ADS]. (Cited on pages 140 and 145.)

[268] Kavanagh, C., Ottewill, A. C. and Wardell, B., "Analytical high-order post-Newtonian expansions for extreme mass ratio binaries", Phys. Rev. D, 92, 084025 (2015). [DOI], [ADS], [arXiv:1503.02334 [gr-qc]]. (Cited on page 116.)

[269] Kerlick, G. D., "Finite reduced hydrodynamic equations in the slow-motion approximation to general relativity. Part I. First post-Newtonian equations", Gen. Relativ. Gravit., 12, 467-482 (1980). [DOI]. (Cited on pages 53 and 57.)

[270] Kerlick, G. D., "Finite reduced hydrodynamic equations in the slow-motion approximation to general relativity. Part II. Radiation reaction and higher-order divergent terms", Gen. Relativ. Gravit., 12, 521-543 (1980). [DOI]. (Cited on pages 53 and 57.)

[271] Kidder, L. E., "Coalescing binary systems of compact objects to (post) ${ }^{5 / 2}$-Newtonian order. V. Spin effects", Phys. Rev. D, 52, 821-847 (1995). [DOI], [ADS]. (Cited on pages 19, 21, 22, 102, 147, 150, and 152.)

[272] Kidder, L. E., "Using full information when computing modes of post-Newtonian waveforms from inspiralling compact binaries in circular orbits", Phys. Rev. D, 77, 044016 (2008). [DOI], [arXiv:0710.0614]. (Cited on pages 20, 36, 37, 131, and 134.)

[273] Kidder, L. E., Blanchet, L. and Iyer, B. R., "A note on the radiation reaction in the 2.5PN waveform from inspiralling binaries in quasi-circular orbits", Class. Quantum Grav., 24, 5307 (2007). [DOI], [arXiv:0706.0726]. (Cited on page 20.)

[274] Kidder, L. E., Will, C. M. and Wiseman, A. G., "Coalescing binary systems of compact objects to (post) $)^{5 / 2}$-Newtonian order. III. Transition from inspiral to plunge", Phys. Rev. D, 47, 3281-3291 (1993). [DOI], [ADS]. (Cited on page 103.)

[275] Kidder, L. E., Will, C. M. and Wiseman, A. G., "Spin effects in the inspiral of coalescing compact binaries", Phys. Rev. D, 47, R4183-R4187 (1993). [DOI]. (Cited on pages 19, 21, 102, 104, 105, and 147.)

[276] Kochanek, C. S., "Coalescing binary neutron stars", Astrophys. J., 398, 234-247 (1992). [DOI], [ADS]. (Cited on page 15.)

[277] Kol, B. and Smolkin, M., "Non-relativistic gravitation: From Newton to Einstein and back", Class. Quantum Grav., 25, 145011 (2008). [DOI], [arXiv:0712.4116 [hep-th]]. (Cited on page 18.)

[278] Königsdörffer, C., Faye, G. and Schäfer, G., "The binary black-hole dynamics at the third-and-a-half post-Newtonian order in the ADM-formalism", Phys. Rev. D, 68, 044004 (2003). [DOI], [astroph/0305048]. (Cited on pages 18, 78, 92, and 117.)

[279] Königsdörffer, C. and Gopakumar, A., "Phasing of gravitational waves from inspiralling eccentric binaries at the third-and-a-half post-Newtonian order", Phys. Rev. D, 73, 124012 (2006). [DOI], [ADS], [gr-qc/0603056]. (Cited on page 146.)

[280] Kopeikin, S. M., "The equations of motion of extended bodies in general-relativity with conservative corrections and radiation damping taken into account", Astron. Zh., 62, 889-904 (1985). (Cited on pages 17 and 70.)

[281] Kopeikin, S. M., "Celestial Coordinate Reference Systems in Curved Spacetime", Celest. Mech., 44, 87 (1988). [DOI]. (Cited on page 70.) 
[282] Kopeikin, S. M., Schäfer, G., Gwinn, C. R. and Eubanks, T. M., "Astrometric and timing effects of gravitational waves from localized sources", Phys. Rev. D, 59, 084023 (1999). [DOI], [ADS], [grqc/9811003]. (Cited on page 12.)

[283] Kozai, Y., "Secular perturbations of asteroids with high inclination and eccentricity", Astron. J., 67, 591-598 (1962). [DOI], [ADS]. (Cited on pages 15 and 135.)

[284] Królak, A., Kokkotas, K. D. and Schäfer, G., "Estimation of the post-Newtonian parameters in the gravitational-wave emission of a coalescing binary", Phys. Rev. D, 52, 2089-2111 (1995). [DOI], [gr-qc/9503013]. (Cited on pages 16, 65, and 124.)

[285] Landau, L. D. and Lifshitz, E. M., The classical theory of fields, (Pergamon Press, Oxford; New York, 1971), 3rd edition. (Cited on pages 9, 12, and 121.)

[286] Le Tiec, A., "First law of mechanics for compact binaries on eccentric orbits", Phys. Rev. D, 92, 084021 (2015). [DOI], [arXiv:1506.05648 [gr-qc]]. (Cited on page 111.)

[287] Le Tiec, A., Barausse, E. and Buonanno, A., "Gravitational Self-Force Correction to the Binding Energy of Compact Binary Systems", Phys. Rev. Lett., 108, 131103 (2012). [DOI], [arXiv:1111.5609 [gr-qc]]. (Cited on pages 96 and 108.)

[288] Le Tiec, A. and Blanchet, L., "The Close-Limit Approximation for Black Hole Binaries with PostNewtonian Initial Conditions", Class. Quantum Grav., 27, 045008 (2010). [DOI], [arXiv:0901.4593 [gr-qc]]. (Cited on page 22.)

[289] Le Tiec, A., Blanchet, L. and Whiting, B. F., "First law of binary black hole mechanics in general relativity and post-Newtonian theory", Phys. Rev. D, 85, 064039 (2012). [DOI], [arXiv:1111.5378 [gr-qc]]. (Cited on pages 95, 96, 108, 110, 113, and 114.)

[290] Le Tiec, A., Blanchet, L. and Will, C. M., "Gravitational-Wave Recoil from the Ringdown Phase of Coalescing Black Hole Binaries", Class. Quantum Grav., 27, 012001 (2010). [DOI], [arXiv:0901.4594 [gr-qc]]. (Cited on page 22.)

[291] Le Tiec, A., Mroué, A. H., Barack, L., Buonanno, A., Pfeiffer, H. P., Sago, N. and Taracchini, A., "Periastron Advance in Black-Hole Binaries", Phys. Rev. Lett., 107, 141101 (2011). [arXiv:1106.3278 [gr-qc]]. (Cited on page 140.)

[292] Levi, M., "Next-to-leading order gravitational spin-orbit coupling in an effective field theory approach", Phys. Rev. D, 82, 104004 (2010). [DOI], [arXiv:1006.4139 [gr-qc]]. (Cited on pages 19 and 147.)

[293] Levi, M., "Next-to-leading order gravitational spin1-spin2 coupling with Kaluza-Klein reduction", Phys. Rev. D, 82, 064029 (2010). [DOI], [ADS], [arXiv:0802.1508 [gr-qc]]. (Cited on pages 19 and 147.)

[294] Levi, M., "Binary dynamics from spin1-spin2 coupling at fourth post-Newtonian order", Phys. Rev. D, 85, 064043 (2012). [DOI], [ADS], [arXiv:1107.4322]. (Cited on pages 19 and 147.)

[295] Levi, M. and Steinhoff, J., "Equivalence of ADM Hamiltonian and Effective Field Theory approaches at next-to-next-to-leading order spin1-spin2 coupling of binary inspirals", J. Cosmol. Astropart. Phys., 2014(12), 003 (2014). [DOI], [arXiv:1408.5762 [gr-qc]]. (Cited on page 19.)

[296] Levi, M. and Steinhoff, J., "Leading order finite size effects with spins for inspiralling compact binaries", J. High Energy Phys., 2015(06), 059 (2015). [DOI], [arXiv:1410.2601 [gr-qc]]. (Cited on page 147.)

[297] Levi, M. and Steinhoff, J., "Next-to-next-to-leading order gravitational spin-orbit coupling via the effective field theory for spinning objects in the post-Newtonian scheme", arXiv, e-print, (2015). [arXiv:1506.05056 [gr-qc]]. (Cited on pages 19 and 147.) 
[298] Levi, M. and Steinhoff, J., "Next-to-next-to-leading order gravitational spin-squared potential via the effective field theory for spinning objects in the post-Newtonian scheme", arXiv, e-print, (2015). [arXiv:1506.05794 [gr-qc]]. (Cited on pages 19 and 147.)

[299] Levi, M. and Steinhoff, J., "Spinning gravitating objects in the effective field theory in the postNewtonian scheme", J. High Energy Phys., 2015(09), 219 (2015). [DOI], [arXiv:1501.04956 [gr-qc]]. (Cited on pages 19 and 147.)

[300] Lidov, M. L., "The evolution of orbits of artificial satellites of planets under the action of gravitational perturbations of external bodies", Planet. Space Sci., 9, 719 (1962). [DOI]. (Cited on pages 15 and 135.)

[301] Limousin, F., Gondek-Rosinska, D. and Gourgoulhon, E., "Last orbits of binary strange quark stars", Phys. Rev. D, 71, 064012 (2005). [DOI], [ADS], [arXiv:gr-qc/0411127 [gr-qc]]. (Cited on page 101.)

[302] Lincoln, C. W. and Will, C. M., "Coalescing binary systems of compact objects to (post) (5/2 $^{5 /}$ Newtonian order: Late-time evolution and gravitational-radiation emission", Phys. Rev. D, 42, 1123-1143 (1990). [DOI], [ADS]. (Cited on page 92.)

[303] Lorentz, H. A. and Droste, J., "The motion of a system of bodies under the influence of their mutual attraction, according to Einstein's theory", in The Collected Papers of H.A. Lorentz, Vol. 5, pp. 330-355, (Nijhoff, The Hague, 1937). [DOI], Translated from Versl. K. Akad. Wet. Amsterdam, 26, 392 and 649 (1917). (Cited on pages 7 and 17.)

[304] Madore, J., "Gravitational radiation from a bounded source. I", Ann. Inst. Henri Poincare, 12, 285-305 (1970). Online version (accessed 6 October 2013):

http://www .numdam.org/item?id=AIHPA_1970_-12_3_285_0. (Cited on pages 33 and 34.)

[305] Marsat, S., "Cubic order spin effects in the dynamics and gravitational wave energy flux of compact object binaries", Class. Quantum Grav., 32, 085008 (2015). [DOI], [arXiv:1411.4118 [gr-qc]]. (Cited on page 147.)

[306] Marsat, S., Bohé, A., Blanchet, L. and Buonanno, A., "Next-to-leading tail-induced spin-orbit effects in the gravitational radiation of compact binaries", Class. Quantum Grav., 31, 025023 (2013). [DOI], [arXiv:1307.6793 [gr-qc]]. (Cited on pages 21, 147, 154, and 158.)

[307] Marsat, S., Bohé, A., Faye, G. and Blanchet, L., "Next-to-next-to-leading order spin-orbit effects in the equations of motion of compact binary systems", Class. Quantum Grav., 30, 055007 (2013). [DOI], [arXiv:1210.4143]. (Cited on pages 19, 147, 153, and 158.)

[308] Martin, J. and Sanz, J. L., "Slow motion approximation in predictive relativistic mechanics. II. Non-interaction theorem for interactions derived from the classical field-theory", J. Math. Phys., 20, 25-34 (1979). [DOI]. (Cited on page 85.)

[309] Mathews, J., "Gravitational multipole radiation", J. Soc. Ind. Appl. Math., 10, 768-780 (1962). [DOI]. (Cited on page 10.)

[310] Mathisson, M., "Republication of: New mechanics of material systems", Gen. Relativ. Gravit., 42, 1011-1048 (2010). [DOI]. (Cited on pages 148 and 150.)

[311] McClintock, J. E., Shafee, R., Narayan, R., Remillard, R. A., Davis, S. W. and Li, L.-X., "The Spin of the Near-Extreme Kerr Black Hole GRS 1915+105", Astrophys. J., 652, 518-539 (2006). [DOI], [ADS], [arXiv:astro-ph/0606076]. (Cited on page 146.)

[312] Memmesheimer, R., Gopakumar, A. and Schäfer, G., "Third post-Newtonian accurate generalized quasi-Keplerian parametrization for compact binaries in eccentric orbits", Phys. Rev. D, 70, 104011 (2004). [DOI], [gr-qc/0407049]. (Cited on pages 135, 138, 140, and 141.) 
[313] Merritt, D., Milosavljević, M., Favata, M., Hughes, S. A. and Holz, D. E., "Consequences of Gravitational Radiation Recoil", Astrophys. J. Lett., 607, L9-L12 (2004). [DOI], [ADS], [astro-ph/0402057]. (Cited on page 21.)

[314] Mikóczi, B., Vasúth, M. and Gergely, L.Á., "Self-interaction spin effects in inspiralling compact binaries", Phys. Rev. D, 71, 124043 (2005). [DOI], [astro-ph/0504538]. (Cited on pages 21 and 147.)

[315] Miller, M. C. and Hamilton, D. P., "Four-Body Effects in Globular Cluster Black Hole Coalescence", Astrophys. J., 576, 894 (2002). [DOI], [astro-ph/0202298]. (Cited on page 15.)

[316] Mino, Y., Sasaki, M., Shibata, M., Tagoshi, H. and Tanaka, T., "Black Hole Perturbation", Prog. Theor. Phys. Suppl., 128, 1-121 (1997). [DOI], [gr-qc/9712057]. (Cited on page 21.)

[317] Mino, Y., Sasaki, M. and Tanaka, T., "Gravitational radiation reaction to a particle motion", Phys. Rev. D, 55, 3457-3476 (1997). [DOI], [arXiv:gr-qc/9606018]. (Cited on page 112.)

[318] Mirshekari, S. and Will, C. M., "Compact binary systems in scalar-tensor gravity: Equations of motion to 2.5 post-Newtonian order", Phys. Rev. D, 87, 084070 (2013). [DOI], [ADS], [arXiv:1301.4680 [gr-qc]]. (Cited on page 17.)

[319] Misner, C. W., Thorne, K. S. and Wheeler, J. A., Gravitation, (W. H. Freeman, San Francisco, 1973). [ADS]. (Cited on pages 13, 14, and 150.)

[320] Mora, T. and Will, C. M., "Post-Newtonian diagnostic of quasi-equilibrium binary configurations of compact objects", Phys. Rev. D, 69, 104021 (2004). [DOI], [arXiv:gr-qc/0312082]. (Cited on pages 16,92, and 93.)

[321] Moritz, H., Advanced Physical Geodesy, (H. Wichmann, Karlsruhe, 1980). (Cited on page 15.)

[322] Nissanke, S. and Blanchet, L., "Gravitational radiation reaction in the equations of motion of compact binaries to 3.5 post-Newtonian order", Class. Quantum Grav., 22, 1007-1031 (2005). [DOI], [grqc/0412018]. (Cited on pages 18, 78, 92, and 117.)

[323] Nowak, M. A., Wilms, J., Pottschmidt, K., Schulz, N., Miller, J. and Maitra, D., "Suzaku observations of $4 \mathrm{U} 1957+11$ : The most rapidly spinning black hole in the galaxy?", in Petre, R., Mitsuda, K. and Angelini, L., eds., SUZAKU 2011. Exploring the X-ray Universe: Suzaku and Beyond (SUZAKU 2011), Palo Alto, California, USA, 20 - 22 July 2011, AIP Conference Proceedings, 1427, pp. 48-51, (AIP Publishing, Melville, NY, 2012). [DOI], [ADS]. (Cited on page 146.)

[324] Ohta, T., Okamura, H., Kimura, T. and Hiida, K., "Physically acceptable solution of Einstein's equation for many-body system", Prog. Theor. Phys., 50, 492-514 (1973). [DOI]. (Cited on pages 17, 18, and 97.)

[325] Ohta, T., Okamura, H., Kimura, T. and Hiida, K., "Coordinate Condition and Higher Order Gravitational Potential in Canocical Formalism", Prog. Theor. Phys., 51, 1598-1612 (1974). [DOI]. (Cited on pages 17 and 18.)

[326] Ohta, T., Okamura, H., Kimura, T. and Hiida, K., "Higher-order gravitational potential for manybody system", Prog. Theor. Phys., 51, 1220-1238 (1974). [DOI]. (Cited on pages 17 and 18.)

[327] Okamura, H., Ohta, T., Kimura, T. and Hiida, K., "Perturbation calculation of gravitational potentials", Prog. Theor. Phys., 50, 2066-2079 (1973). [DOI]. (Cited on pages 17 and 18.)

[328] Owen, B. J., Tagoshi, H. and Ohashi, A., "Nonprecessional spin-orbit effects on gravitational waves from inspiraling compact binaries to second post-Newtonian order", Phys. Rev. D, 57, 6168-6175 (1998). [DOI], [gr-qc/9710134]. (Cited on page 21.) 
[329] Pan, Y., Buonanno, A., Buchman, L. T., Chu, T., Kidder, L. E., Pfeiffer, H. P. and Scheel, M. A., "Effective-one-body waveforms calibrated to numerical relativity simulations: Coalescence of non-precessing, spinning, equal-mass black holes", Phys. Rev. D, 81, 084041 (2010). [DOI], [ADS], [arXiv:0912.3466 [gr-qc]]. (Cited on page 65.)

[330] Papapetrou, A., "Equations of motion in general relativity", Proc. Phys. Soc. London, Sect. B, 64, 57-75 (1951). (Cited on page 17.)

[331] Papapetrou, A., "Spinning Test-Particles in General Relativity. I", Proc. R. Soc. London, Ser. A, 209, 248-258 (1951). [DOI]. (Cited on pages 148 and 150.)

[332] Papapetrou, A., "Relativité - une formule pour le rayonnement gravitationnel en première approximation", C. R. Acad. Sci. Ser. II, 255, 1578 (1962). (Cited on page 10.)

[333] Papapetrou, A., "Étude systématique du rayonnement gravitationnel 4-polaire. Énergie-impulsion et moment cinétique du rayonnement", Ann. Inst. Henri Poincare, XIV, 79 (1971). (Cited on page 10.)

[334] Papapetrou, A. and Linet, B., "Equation of motion including the reaction of gravitational radiation", Gen. Relativ. Gravit., 13, 335 (1981). [DOI]. (Cited on pages 53 and 57.)

[335] Pati, M. E. and Will, C. M., "Post-Newtonian gravitational radiation and equations of motion via direct integration of the relaxed Einstein equations: Foundations", Phys. Rev. D, 62, 124015 (2000). [DOI], [gr-qc/0007087]. (Cited on pages 11 and 50.)

[336] Pati, M. E. and Will, C. M., "Post-Newtonian gravitational radiation and equations of motion via direct integration of the relaxed Einstein equations. II. Two-body equations of motion to second postNewtonian order, and radiation reaction to 3.5 post-Newtonian order", Phys. Rev. D, 65, 104008 (2002). [DOI], [ADS], [gr-qc/0201001]. (Cited on pages 11, 18, 78, 92, and 117.)

[337] Penrose, R., "Asymptotic Properties of Fields and Space-Times", Phys. Rev. Lett., 10, 66-68 (1963). [DOI], [ADS]. (Cited on pages 10, 11, 33, and 34.)

[338] Penrose, R., "Zero Rest-Mass Fields Including Gravitation: Asymptotic Behaviour", Proc. R. Soc. London, Ser. A, 284, 159-203 (1965). [DOI], [ADS]. (Cited on pages 10, 11, 33, and 34.)

[339] Peters, P. C., "Gravitational Radiation and the Motion of Two Point Masses", Phys. Rev., 136, B1224-B1232 (1964). [DOI], [ADS]. (Cited on pages 10, 13, 14, 135, 140, and 145.)

[340] Peters, P. C. and Mathews, J., "Gravitational Radiation from Point Masses in a Keplerian Orbit", Phys. Rev., 131, 435-440 (1963). [DOI], [ADS]. (Cited on pages 13, 14, 135, 140, and 143.)

[341] Petrova, N. M., "Ob Uravnenii Dvizheniya i Tenzore Materii dlya Sistemy Konechnykh Mass v Obshchei Teorii Otnositielnosti", J. Exp. Theor. Phys., 19(11), 989-999 (1949). (Cited on page 17.)

[342] Pfeiffer, H. P., Teukolsky, S. A. and Cook, G. B., "Quasicircular orbits for spinning binary black holes", Phys. Rev. D, 62, 104018 (2000). [DOI], [ADS], [gr-qc/0006084]. (Cited on pages 103 and 104.)

[343] Pirani, F. A. E., "Introduction to Gravitational Radiation Theory", in Trautman, A., Pirani, F. A. E. and Bondi, H., eds., Lectures on General Relativity, Vol. 1, Brandeis Summer Institute in Theoretical Physics 1964, pp. 249-373, (Prentice-Hall, Englewood Cliffs, NJ, 1965). [ADS]. (Cited on page 10.)

[344] Plebański, J. F. and Bażański, S. L., "The general Fokker action principle and its application in general relativity theory", Acta Phys. Pol., 18, 307-345 (1959). (Cited on page 53.)

[345] Poisson, E., "Gravitational radiation from a particle in circular orbit around a black hole. I. Analytic results for the nonrotating case", Phys. Rev. D, 47, 1497-1510 (1993). [DOI], [ADS]. (Cited on pages 21 and 38.) 
[346] Poisson, E., "Gravitational radiation from a particle in circular orbit around a black-hole. VI. Accuracy of the post-Newtonian expansion", Phys. Rev. D, 52, 5719-5723 (1995). [DOI], [gr-qc/9505030]. Erratum: Phys. Rev. D, 55, 7980 (1997). (Cited on pages 16, 65, and 125.)

[347] Poisson, E., "Gravitational waves from inspiraling compact binaries: The quadrupole-moment term", Phys. Rev. D, 57, 5287-5290 (1997). [DOI], [gr-qc/9709032]. (Cited on page 147.)

[348] Poisson, E., Pound, A. and Vega, I., "The Motion of Point Particles in Curved Spacetime", Living Rev. Relativity, 14, lrr-2011-7 (2011). [DOI], [ADS], [arXiv:1102.0529 [gr-qc]]. URL (accessed 6 October 2013):

http://www.livingreviews.org//rr-2011-7. (Cited on pages 9 and 112.)

[349] Poisson, E. and Sasaki, M., "Gravitational radiation from a particle in circular orbit around a black hole. V. Black-hole absorption and tail corrections", Phys. Rev. D, 51, 5753-5767 (1995). [DOI], [gr-qc/9412027]. (Cited on pages 123 and 158.)

[350] Poisson, E. and Will, C. M., "Gravitational waves from inspiraling compact binaries: Parameter estimation using second-post-Newtonian wave forms", Phys. Rev. D, 52, 848-855 (1995). [DOI], [arXiv:gr-qc/9502040]. (Cited on pages 16, 65, and 124.)

[351] Porto, R. A., "Post-Newtonian corrections to the motion of spinning bodies in NRGR", Phys. Rev. $D, \mathbf{7 3}, 104031$ (2006). [DOI], [gr-qc/0511061]. (Cited on pages 147 and 148.)

[352] Porto, R. A., "Next-to-leading-order spin-orbit effects in the motion of inspiralling compact binaries", Class. Quantum Grav., 27, 205001 (2010). [DOI], [arXiv:1005.5730 [gr-qc]]. (Cited on pages 19 and 147.)

[353] Porto, R. A., Ross, A. and Rothstein, I. Z., "Spin induced multipole moments for the gravitational wave flux from binary inspirals to third Post-Newtonian order", J. Cosmol. Astropart. Phys., 2011(3), 009 (2011). [DOI], [arXiv:1007.1312 [gr-qc]]. (Cited on page 21.)

[354] Porto, R. A. and Rothstein, I. Z., "Calculation of the first nonlinear contribution to the generalrelativistic spin-spin interaction for binary systems", Phys. Rev. Lett., 97, 021101 (2006). [DOI], [arXiv:gr-qc/0604099]. (Cited on pages 19 and 147.)

[355] Porto, R. A. and Rothstein, I. Z., "Next to leading order spin(1)spin(1) effects in the motion of inspiralling compact binaries", Phys. Rev. D, 78, 044013 (2008). [DOI], [ADS], [arXiv:0804.0260 [gr-qc]]. (Cited on pages 19 and 147.)

[356] Porto, R. A. and Rothstein, I. Z., "Spin(1)spin(2) effects in the motion of inspiralling compact binaries at third order in the post-Newtonian expansion", Phys. Rev. D, 78, 044012 (2008). [DOI], [arXiv:0802.0720 [gr-qc]]. (Cited on pages 19 and 147.)

[357] Poujade, O. and Blanchet, L., "Post-Newtonian approximation for isolated systems calculated by matched asymptotic expansions", Phys. Rev. D, 65, 124020 (2002). [DOI], [gr-qc/0112057]. (Cited on pages $11,54,55,56,57$, and 59.)

[358] Press, W. H., "Gravitational Radiation from Sources Which Extend Into Their Own Wave Zone", Phys. Rev. D, 15, 965-968 (1977). [DOI]. (Cited on page 10.)

[359] Pretorius, F., "Evolution of Binary Black-Hole Spacetimes", Phys. Rev. Lett., 95, 121101 (2005). [DOI], [ADS], [arXiv:gr-qc/0507014]. (Cited on page 8.)

[360] Quinn, T. C. and Wald, R. M., "Axiomatic approach to electromagnetic and gravitational radiation reaction of particles in curved spacetime", Phys. Rev. D, 56, 3381-3394 (1997). [DOI], [arXiv:grqc/9610053]. (Cited on page 112.)

[361] Rendall, A. D., "Convergent and divergent perturbation series and the post-Minkowskian scheme", Class. Quantum Grav., 7, 803 (1990). [DOI]. (Cited on pages 9 and 28.) 
[362] Rendall, A. D., "On the definition of post-Newtonian approximations", Proc. R. Soc. London, Ser. A, 438, 341-360 (1992). [DOI]. (Cited on pages 9, 28, and 54.)

[363] Rendall, A. D., "The Newtonian limit for asymptotically flat solutions of the Einstein-Vlasov system", Commun. Math. Phys., 163, 89-112 (1994). [DOI], [gr-qc/9303027]. (Cited on pages 9 and 28.)

[364] Reynolds, C. S., "Measuring Black Hole Spin Using X-Ray Reflection Spectroscopy", Space Sci. Rev., 183, 277-294 (2014). [DOI], [ADS], [arXiv:1302.3260 [astro-ph.HE]]. (Cited on page 146.)

[365] Riesz, M., "L’intégrale de Riemann-Liouville et le problème de Cauchy", Acta Math., 81, 1-218 (1949). [DOI]. (Cited on page 67.)

[366] Rieth, R. and Schäfer, G., "Spin and tail effects in the gravitational-wave emission of compact binaries", Class. Quantum Grav., 14, 2357 (1997). [DOI]. (Cited on pages 140 and 145.)

[367] Sachs, R. K., "Gravitational waves in general relativity. VI. The outgoing radiation condition", Proc. R. Soc. London, Ser. A, 264, 309-338 (1961). [DOI]. (Cited on page 10.)

[368] Sachs, R. K., "Gravitational Waves in General Relativity. VIII. Waves in Asymptotically Flat SpaceTime", Proc. R. Soc. London, Ser. A, 270, 103-126 (1962). [DOI], [ADS]. (Cited on pages 10, 11, and 33.)

[369] Sachs, R. K. and Bergmann, P. G., "Structure of Particles in Linearized Gravitational Theory", Phys. Rev., 112, 674-680 (1958). [DOI]. (Cited on page 10.)

[370] Sago, N., Barack, L. and Detweiler, S., "Two approaches for the gravitational self force in black hole spacetime: Comparison of numerical results", Phys. Rev. D, 78, 124024 (2008). [DOI], [arXiv:0810.2530]. (Cited on page 109.)

[371] Santamaría, L. et al., "Matching post-Newtonian and numerical relativity waveforms: Systematic errors and a new phenomenological model for non-precessing black hole binaries", Phys. Rev. D, 82, 064016 (2010). [DOI], [ADS], [arXiv:1005.3306 [gr-qc]]. (Cited on page 65.)

[372] Sasaki, M., "Post-Newtonian Expansion of the Ingoing-Wave Regge-Wheeler Function", Prog. Theor. Phys., 92, 17-36 (1994). [DOI]. (Cited on page 21.)

[373] Sasaki, M. and Tagoshi, H., "Analytic Black Hole Perturbation Approach to Gravitational Radiation", Living Rev. Relativity, 6, lrr-2003-6 (2003). [DOI], [ADS], [arXiv:gr-qc/0306120]. URL (accessed 6 October 2013): http://www.livingreviews.org/lrr-2003-6. (Cited on page 9.)

[374] Schäfer, G., "Acceleration-dependent Lagrangians in general relativity", Phys. Lett. A, 100, 128 (1984). [DOI]. (Cited on page 62.)

[375] Schäfer, G., "The Gravitational Quadrupole Radiation-Reaction Force and the Canonical Formalism of ADM", Ann. Phys. (N.Y.), 161, 81-100 (1985). [DOI]. (Cited on page 17.)

[376] Schäfer, G., "The ADM Hamiltonian at the Postlinear Approximation", Gen. Relativ. Gravit., 18, 255-270 (1986). [DOI]. (Cited on page 17.)

[377] Schäfer, G., "Three-body Hamiltonian in general relativity", Phys. Lett., 123, 336-339 (1987). [DOI]. (Cited on page 97.)

[378] Schäfer, G., "Post-Newtonian Methods: Analytic Results on the Binary Problem", in Blanchet, L., Spallicci, A. and Whiting, B., eds., Mass and Motion in General Relativity, Lectures from the CNRS School on Mass held in Orléans, France, 23-25 June 2008, Fundamental Theories of Physics, 162, pp. 167-210, (Springer, Dordrecht; New York, 2011). [DOI], [ADS]. (Cited on pages 9 and 108.)

[379] Schäfer, G. and Wex, N., "Second post-Newtonian motion of compact binaries", Phys. Lett. A, 174, 196-205 (1993). [DOI]. Erratum: Phys. Lett. A, 177, 461 (1993). (Cited on pages 138 and 141.) 
[380] Schwartz, L., "Sur l'impossibilité de la multiplication des distributions", C. R. Acad. Sci. Ser. II, 239, 847-848 (1954). (Cited on pages 68 and 69.)

[381] Schwartz, L., Théorie des distributions, (Hermann, Paris, 1978). (Cited on pages 66, 67, and 75.)

[382] Sellier, A., "Hadamard's finite part concept in dimension $n \geq 2$, distributional definition, regularization forms and distributional derivatives", Proc. R. Soc. London, Ser. A, 445, 69-98 (1994). [DOI]. (Cited on page 66.)

[383] Shah, A., Friedmann, J. and Whiting, B. F., "Finding high-order analytic post-Newtonian parameters from a high-precision numerical self-force calculation", Phys. Rev. D, 89, 064042 (2014). [DOI], [arXiv:1312.1952 [gr-qc]]. (Cited on page 116.)

[384] Simon, W. and Beig, R., "The multipole structure of stationary space-times", J. Math. Phys., 24, 1163-1171 (1983). [DOI]. (Cited on page 10.)

[385] Sopuerta, C. F., Yunes, N. and Laguna, P., "Gravitational Recoil from Binary Black Hole Mergers: the Close-Limit Approximation", Phys. Rev. D, 74, 124010 (2006). [DOI], [astro-ph/0608600]. (Cited on page 22.)

[386] Steinhoff, J., "Canonical formulation of spin in general relativity", Ann. Phys. (Berlin), 523, 296 (2011). [DOI], [arXiv:1106.4203 [gr-qc]]. (Cited on page 148.)

[387] Steinhoff, J., Hergt, S. and Schäfer, G., "Next-to-leading order gravitational spin(1)-spin(2) dynamics in Hamiltonian form", Phys. Rev. D, 77, 081501(R) (2008). [arXiv:0712.1716 [gr-qc]]. (Cited on pages 19 and 147.)

[388] Steinhoff, J., Hergt, S. and Schäfer, G., "Spin-squared Hamiltonian of next-to-leading order gravitational interaction", Phys. Rev. D, 78, 101503(R) (2008). [arXiv:0809.2200 [gr-qc]]. (Cited on pages 19 and 147.)

[389] Steinhoff, J., Schäfer, G. and Hergt, S., "ADM canonical formalism for gravitating spinning objects", Phys. Rev. D, 77, 104018 (2008). [DOI], [arXiv:0805.3136 [gr-qc]]. (Cited on pages 19, 147, and 148.)

[390] Strohmayer, T. E., "Discovery of a $450 \mathrm{~Hz}$ quasi-periodic oscillation from the microquasar GRO J1655-40 with the Rossi X-ray Timing Explorer", Astrophys. J. Lett., 552, L49-L53 (2001). [DOI], [ADS]. (Cited on page 146.)

[391] 't Hooft, G. and Veltman, M., "Regularization and renormalization of gauge fields", Nucl. Phys. B, 44, 139 (1972). [DOI]. (Cited on page 72.)

[392] Tagoshi, H., Mano, S. and Takasugi, E., "Post-Newtonian Expansion of Gravitational Waves from a Particle in Circular Orbits around a Rotating Black Hole", Prog. Theor. Phys., 98, 829 (1997). [DOI], [gr-qc/9711072]. (Cited on pages 123 and 158.)

[393] Tagoshi, H. and Nakamura, T., "Gravitational waves from a point particle in circular orbit around a black hole: Logarithmic terms in the post-Newtonian expansion", Phys. Rev. D, 49, 4016-4022 (1994). [DOI]. (Cited on pages 16, 21, 65, and 123.)

[394] Tagoshi, H., Ohashi, A. and Owen, B. J., "Gravitational field and equations of motion of spinning compact binaries to 2.5-post-Newtonian order", Phys. Rev. D, 63, 044006 (2001). [DOI], [grqc/0010014]. (Cited on pages 19 and 147.)

[395] Tagoshi, H. and Sasaki, M., "Post-Newtonian Expansion of Gravitational Waves from a Particle in Circular Orbit around a Schwarzschild Black Hole", Prog. Theor. Phys., 92, 745-771 (1994). [DOI], [gr-qc/9405062]. (Cited on pages 21 and 123.)

[396] Tagoshi, H., Shibata, M., Tanaka, T. and Sasaki, M., "Post-Newtonian expansion of gravitational waves from a particle in circular orbit around a rotating black hole: Up to $O\left(v^{8}\right)$ beyond the quadrupole formula", Phys. Rev. D, 54, 1439-1459 (1996). [DOI]. (Cited on page 158.) 
[397] Tanaka, T., Tagoshi, H. and Sasaki, M., "Gravitational Waves by a Particle in Circular Orbit around a Schwarzschild Black Hole: 5.5 Post-Newtonian Formula", Prog. Theor. Phys., 96, 1087-1101 (1996). [DOI], [gr-qc/9701050]. (Cited on pages 21 and 123.)

[398] Taylor, J. H., "Pulsar timing and relativistic gravity", Class. Quantum Grav., 10, 167-174 (1993). [DOI]. (Cited on pages 7, 13, and 14.)

[399] Taylor, J. H., Fowler, L. A. and McCulloch, P. M., "Measurements of general relativistic effects in the binary pulsar PSR 1913+16", Nature, 277, 437-440 (1979). [DOI]. (Cited on pages 7, 13, and 14.)

[400] Taylor, J. H. and Weisberg, J. M., "A New Test of General Relativity: Gravitational Radiation and the Binary Pulsar PSR 1913+16", Astrophys. J., 253, 908-920 (1982). [DOI]. (Cited on pages 7, 13, and 14.)

[401] Tessmer, M. and Schäfer, G., "Full-analytic frequency-domain 1PN-accurate gravitational wave forms from eccentric compact binaries", Phys. Rev. D, 82, 124064 (2010). [DOI], [arXiv:1006.3714 [gr-qc]]. (Cited on page 141.)

[402] Tessmer, M. and Schäfer, G., "Full-analytic frequency-domain gravitational wave forms from eccentric compact binaries to 2PN accuracy", Ann. Phys. (Berlin), 523, 813 (2011). [DOI], [arXiv:1012.3894 [gr-qc]]. (Cited on page 141.)

[403] Thorne, K. S., "Multipole expansions of gravitational radiation", Rev. Mod. Phys., 52, 299-339 (1980). [DOI], [ADS]. (Cited on pages 10, 11, 20, 26, 27, 35, 36, 37, and 50.)

[404] Thorne, K. S., "The theory of gravitational radiation: An introductory review", in Deruelle, N. and Piran, T., eds., Gravitational Radiation, NATO Advanced Study Institute, Centre de physique des Houches, $2-21$ June 1982, pp. 1-57, (North-Holland; Elsevier, Amsterdam; New York, 1983). (Cited on page 9.)

[405] Thorne, K. S., "Gravitational radiation", in Hawking, S. W. and Israel, W., eds., Three Hundred Years of Gravitation, pp. 330-458, (Cambridge University Press, Cambridge; New York, 1987). [Google Books]. (Cited on page 9.)

[406] Thorne, K. S., "Gravitational-wave bursts with memory: The Christodoulou effect", Phys. Rev. D, 45, 520 (1992). [DOI]. (Cited on pages 21 and 43.)

[407] Thorne, K. S. and Hartle, J. B., "Laws of motion and precession for black holes and other bodies", Phys. Rev. D, 31, 1815-1837 (1985). [DOI]. (Cited on page 70.)

[408] Thorne, K. S. and Kovàcs, S. J., "Generation of gravitational waves. I. Weak-field sources", Astrophys. J., 200, 245-262 (1975). [DOI]. (Cited on page 10.)

[409] Trautman, A., "Lectures on General Relativity", Gen. Relativ. Gravit., 34, 721-762 (2002). [DOI]. Lectures delivered at King's College in London in May-June 1958. (Cited on pages 148 and 150.)

[410] Trias, M. and Sintes, A. M., "LISA observations of supermassive black holes: Parameter estimation using full post-Newtonian inspiral waveforms", Phys. Rev. D, 77, 024030 (2008). [DOI], [ADS], [arXiv:0707.4434 [gr-qc]]. (Cited on page 20.)

[411] Tulczyjew, W., "On the energy-momentum tensor density for simple pole particles", Bull. Acad. Polon. Sci. Cl. III, 5, 279 (1957). (Cited on pages 147, 148, and 150.)

[412] Tulczyjew, W., "Motion of multipole particles in general relativity theory", Acta Phys. Pol., 18, 37 (1959). (Cited on pages 147, 148, and 150.)

[413] Vaidya, V., "Gravitational spin Hamiltonians from the $S$ matrix", Phys. Rev. D, 91, 024017 (2015). [DOI], [arXiv:1410.5348 [hep-th]]. (Cited on page 147.) 
[414] Vines, J., Hinderer, T. and Flanagan, É.É., "Post-1-Newtonian tidal effects in the gravitational waveform from binary inspirals", Phys. Rev. D, 83, 084051 (2011). [DOI], [arXiv:1101.1673 [gr-qc]]. (Cited on page 16.)

[415] Wagoner, R. V., "Test for Existence of Gravitational Radiation", Astrophys. J. Lett., 196, L63-L65 (1975). [DOI]. (Cited on page 13.)

[416] Wagoner, R. V. and Will, C. M., "Post-Newtonian gravitational radiation from orbiting point masses", Astrophys. J., 210, 764-775 (1976). [DOI]. (Cited on pages 20 and 140.)

[417] Wald, R. M., "On perturbations of a Kerr black hole", J. Math. Phys., 14, 1453-1461 (1973). [DOI]. (Cited on page 110.)

[418] Walker, M. and Will, C. M., "The approximation of radiative effects in relativistic gravity: Gravitational radiation reaction and energy loss in nearly Newtonian systems", Astrophys. J. Lett., 242, L129-L133 (1980). [DOI], [ADS]. (Cited on page 54.)

[419] Wen, L., "On the Eccentricity Distribution of Coalescing Black Hole Binaries Driven by the Kozai Mechanism in Globular Clusters", Astrophys. J., 598, 419 (2003). [DOI], [astro-ph/0211492]. (Cited on page 135.)

[420] Wex, N., "The second post-Newtonian motion of compact binary-star systems with spin", Class. Quantum Grav., 12, 983-1005 (1995). [DOI]. (Cited on pages 138 and 141.)

[421] Will, C. M., "Gravitational Waves from Inspiralling Compact Binaries: A Post-Newtonian Approach", in Sasaki, M., ed., Relativistic Cosmology, Proceedings of the 8th Nishinomiya-Yukawa Memorial Symposium, Shukugawa City Hall, Nishinomiya, Hyogo, Japan, 28-29 October, 1993, NYMSS, 8, pp. 83-98, (Universal Academy Press, Tokyo, 1993). [gr-qc/9403033]. (Cited on page 9.)

[422] Will, C. M., "Generation of post-Newtonian gravitational radiation via direct integration of the relaxed Einstein equations", Prog. Theor. Phys. Suppl., 136, 158-167 (1999). [DOI], [gr-qc/9910057]. (Cited on pages 11, 20, and 50.)

[423] Will, C. M., "Post-Newtonian gravitational radiation and equations of motion via direct integration of the relaxed Einstein equations. III. Radiation reaction for binary systems with spinning bodies", Phys. Rev. D, 71, 084027 (2005). [DOI], [gr-qc/0502039]. (Cited on pages 151 and 159.)

[424] Will, C. M. and Wiseman, A. G., "Gravitational radiation from compact binary systems: Gravitational waveforms and energy loss to second post-Newtonian order", Phys. Rev. D, 54, 4813-4848 (1996). [DOI], [gr-qc/9608012]. (Cited on pages 11, 12, 20, 50, 51, and 141.)

[425] Wiseman, A. G., "Coalescing binary systems of compact objects to (post) ${ }^{5 / 2}$-Newtonian order. II. Higher-order wave forms and radiation recoil", Phys. Rev. D, 46, 1517-1539 (1992). [DOI], [ADS]. (Cited on page 22.)

[426] Wiseman, A. G., "Coalescing binary systems of compact objects to (post) ${ }^{5 / 2}$-Newtonian order. IV. The gravitational wave tail", Phys. Rev. D, 48, 4757-4770 (1993). [DOI], [ADS]. (Cited on page 20.)

[427] Wiseman, A. G. and Will, C. M., "Christodoulou's nonlinear gravitational-wave memory: Evaluation in the quadrupole approximation", Phys. Rev. D, 44, R2945-R2949 (1991). [DOI]. (Cited on pages 21, 43, and 130.)

[428] Zeng, J. and Will, C. M., "Application of energy and angular momentum balance to gravitational radiation reaction for binary systems with spin-orbit coupling", Gen. Relativ. Gravit., 39, 1661 (2007). [DOI], [arXiv:0704.2720]. (Cited on page 18.) 